\title{
Selective Functionalization of Pyridines via Heterocyclic Phosphonium Salts
}

\author{
Michael C. Hilton, ${ }^{\ddagger}$ Ryan D. Dolewski, ${ }^{\ddagger}$ and Andrew McNally* \\ Department of Chemistry, Colorado State University, \\ Fort Collins, Colorado 80523, United States. \\ *andy.mcnally@colostate.edu
}

\section{Table of Contents}

1. General Information $S 2$

2. Base and Temperature Effects During Heterocyclic Phosphonium S4 Salt Formation

3. Preparation of Heterocyclic Phosphonium Salt Precursors S6

4. Preparation of Heterocyclic Phosphonium Salts S9

5. Preparation of Heteroaryl Ethers $S 31$

6. Comparative Literature Syntheses of Heteroaryl Ethers S58

7. Counterion Study S59

8. References $S 60$

9. ${ }^{1} \mathrm{H},{ }^{13} \mathrm{C},{ }^{19} \mathrm{~F}$ and ${ }^{31} \mathrm{P}$ Spectra $\quad S 62$ 


\section{General Information}

Proton nuclear magnetic resonance $\left({ }^{1} \mathrm{H}\right.$ NMR $)$ spectra were recorded at ambient temperature on a Varian $400 \mathrm{MR}$ spectrometer (400 MHz) or an Agilent Inova 400 (400 MHz) spectrometer. Chemical shifts $(\delta)$ are reported in ppm and quoted to the nearest $0.01 \mathrm{ppm}$ relative to the residual protons in $\mathrm{CDCl}_{3}$ (7.26 ppm), $\mathrm{C}_{6} \mathrm{D}_{6}(7.16 \mathrm{ppm}),\left(\mathrm{CD}_{3}\right)_{2} \mathrm{SO}$ (2.50 ppm), $\mathrm{CD}_{3} \mathrm{OD}$ (3.31 ppm) or $\mathrm{CD}_{3} \mathrm{CN}(1.94 \mathrm{ppm})$ and coupling constants $(J)$ are quoted in Hertz $(\mathrm{Hz})$. Data are reported as follows: Chemical shift (number of protons, multiplicity, coupling constants, proton assignment). Coupling constants were quoted to the nearest $0.1 \mathrm{~Hz}$ and multiplicity reported according to the following convention: $\mathrm{s}=$ singlet, $\mathrm{d}=$ doublet, $\mathrm{t}=$ triplet, $\mathrm{q}=$ quartet, $\mathrm{qn}=$ quintet, $\mathrm{sext}=$ sextet, $\mathrm{sp}=$ septet, $\mathrm{m}=$ multiplet, $\mathrm{br}=$ broad. Where coincident coupling constants have been observed, the apparent (app) multiplicity of the proton resonance has been reported. Carbon nuclear magnetic resonance $\left({ }^{13} \mathrm{C} \mathrm{NMR}\right)$ spectra were recorded at ambient temperature on a Varian $400 \mathrm{MR}$ spectrometer (100 MHz) or an Agilent Inova $400(100 \mathrm{MHz})$ spectrometer. ${ }^{*}$ Chemical shift $(\delta)$ was measured in ppm and quoted to the nearest $0.1 \mathrm{ppm}$ relative to the residual solvent peaks in $\mathrm{CDCl}_{3}(77.00 \mathrm{ppm}), \mathrm{C}_{6} \mathrm{D}_{6}(128.06 \mathrm{ppm}),\left(\mathrm{CD}_{3}\right)_{2} \mathrm{SO}$ (39.51 ppm), $\mathrm{CD}_{3} \mathrm{OD}(49.00 \mathrm{ppm})$ or $\mathrm{CD}_{3} \mathrm{CN}$ (1.32 ppm). DEPT135, NOE experiments and 2-dimensional experiments (COSY, HMBC and HSQC) were used to support assignments where appropriate.

Low-resolution mass spectra (LRMS) were measured on an Agilent 6310 Quadrupole Mass Spectrometer. Infared (IR) spectra were recorded on a Bruker Tensor 27 FT-IR spectrometer as either solids or neat films, either through direct application or deposited in $\mathrm{CHCl}_{3}$, with absorptions reported in wavenumbers $\left(\mathrm{cm}^{-1}\right)$.

Analytical thin layer chromatography (TLC) was performed using pre-coated Merck glass backed silica gel plates (Silicagel 60 F254). Flash column chromatography was undertaken on Fluka or Material Harvest silica gel (230-400 mesh) under a positive pressure of air. Visualization was achieved using ultraviolet light $(254 \mathrm{~nm})$ and chemical staining with ceric ammonium molybdate or basic potassium permanganate solutions as appropriate.

\footnotetext{
${ }^{*}$ Artifact from instrument with chemical shift of $194 \mathrm{ppm}$ is observed in several ${ }^{13} \mathrm{C}$ NMR spectra (see $S 56$ for an example).
} 
Tetrahydrofuran (THF), toluene, hexane, diethyl ether and dichloromethane were dried and distilled using standard methods. ${ }^{1}$ 1,2-Dichloroethane (DCE), 1,4-dioxane, chloroform, chlorobenzene and acetone were purchased anhydrous from Sigma Aldrich chemical company. All reagents were purchased at the highest commercial quality and used without further purification. Reactions were carried out under an atmosphere of nitrogen unless otherwise stated. All reactions were monitored by TLC, ${ }^{1} \mathrm{H}$ NMR spectra taken from reaction samples, gas chromatography (GC) and gas chromatography-mass spectrometry (GCMS) using a Agilent 5977A fitted with a an Agilent J\&W HP-5ms Ultra Inert Column (30 m, $0.25 \mathrm{~mm}, 0.25 \mu \mathrm{m}$ film) for MS analysis and an Agilent J\&W VF-5ms column ( $10 \mathrm{~m}, 0.15 \mathrm{~mm}, 0.15 \mu \mathrm{m}$ film) for FID analysis or liquid chromatography mass spectrometry (LCMS) using an Agilent 6310 Quadrupole Mass Spectrometer. Melting points (mp) were recorded using a Büchi B-450 melting point apparatus and are reported uncorrected.

$\mathrm{PPh}_{3}(99 \%)$ was purchased from Oakwood Chemical and is most effective when crushed to a powder before use. $\mathrm{Tf}_{2} \mathrm{O}(99 \%)$ was purchased from Oakwood Chemical and used without further purification but was routinely stored in a $-20{ }^{\circ} \mathrm{C}$ fridge. $\mathrm{NEt}_{3}$ and $\mathrm{DBU}$ were distilled before use. $\mathrm{NaH}$ (60\% in mineral oil) was purchased from Sigma Aldrich and was typically distributed into vials and stored in a desiccator. 


\section{Base and Temperature Effects During Heterocyclic Phosphonium Salt Formation}

The choice of base can have a large effect on the yield of heterocyclic phosphonium salt for certain types of substrates. Shown below are representative examples of this effect. In general, either $\mathrm{NEt}_{3}$ or DBU give optimal results.

Table S1. Effect of Base on Heterocyclic Phosphonium Salt Formation

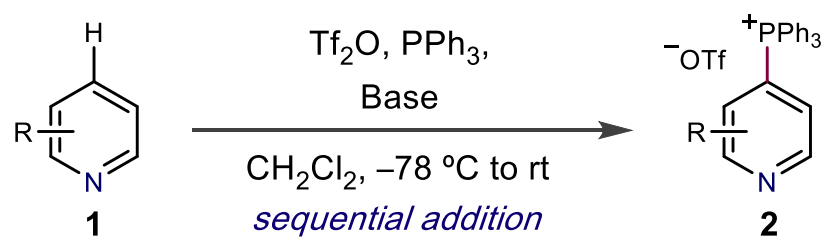

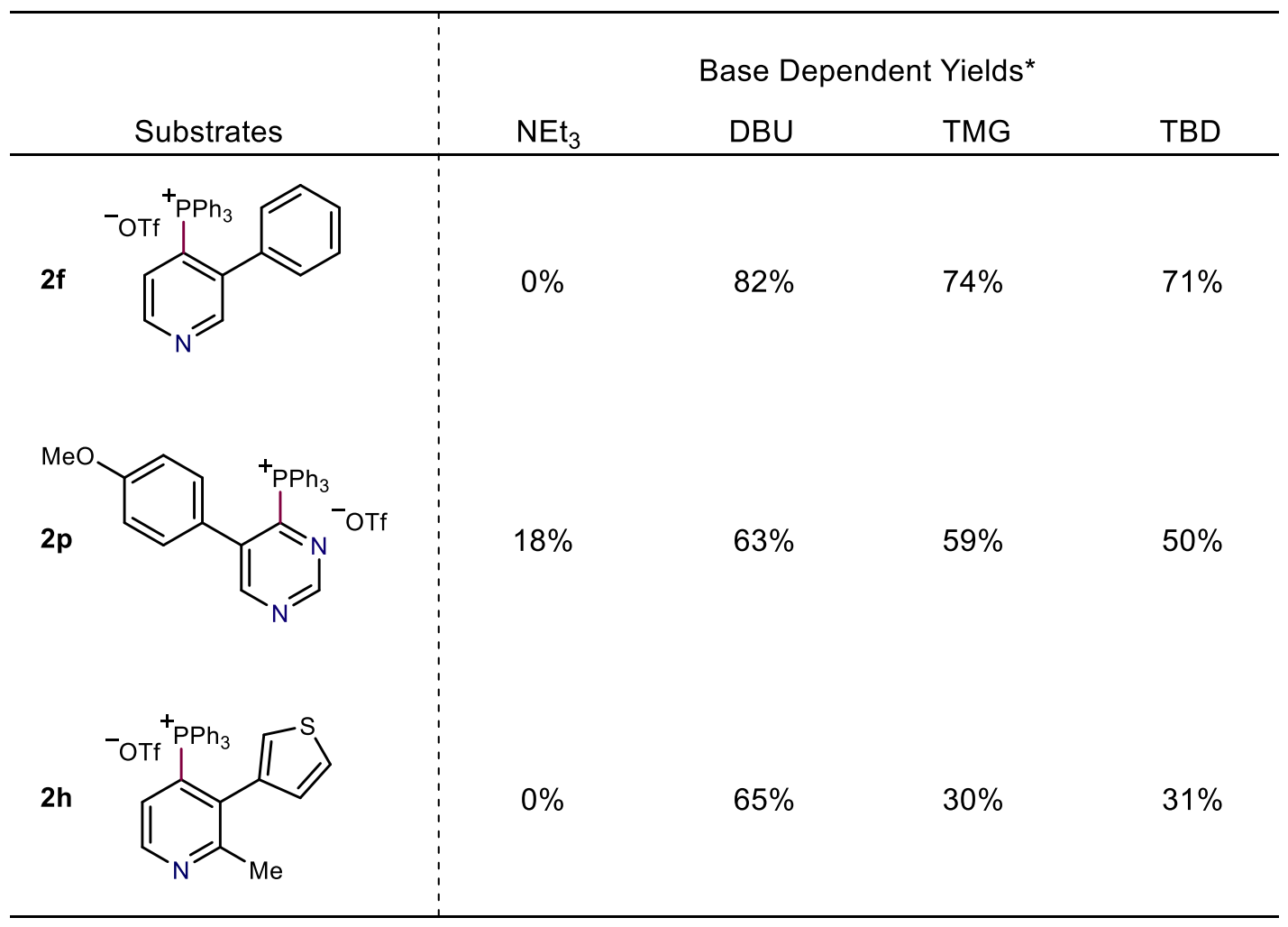

${ }^{* 1} \mathrm{H}$ NMR yields shown using 1,3,5-trimethoxybenzene as an internal standard.

For the majority of examples $-78{ }^{\circ} \mathrm{C}$ during the $\mathrm{Tf}_{2} \mathrm{O}, \mathrm{PPh}_{3}$ and base stages of the reaction is optimal. Table S2 shows that yields can decrease if the temperature deviates from $-78{ }^{\circ} \mathrm{C}$. However, for a small number of substrates we found that optimal yields were obtained at different 
temperatures during the $\mathrm{PPh}_{3}$ or base addition stages. These examples (2b, 10 and $\left.\mathbf{1 2}\right)$ are also shown in Table S2 and are explicitly described in the Section 4.

Table S2. Effect of Temperature on Heterocyclic Phosphonium Salt Formation

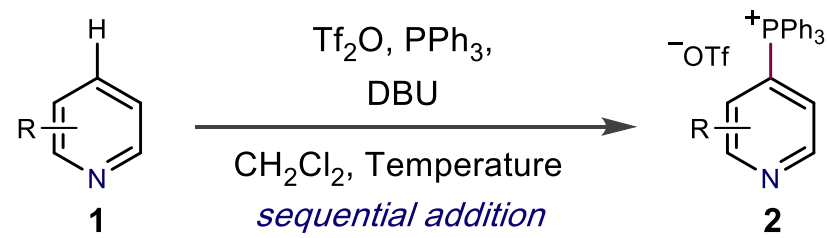

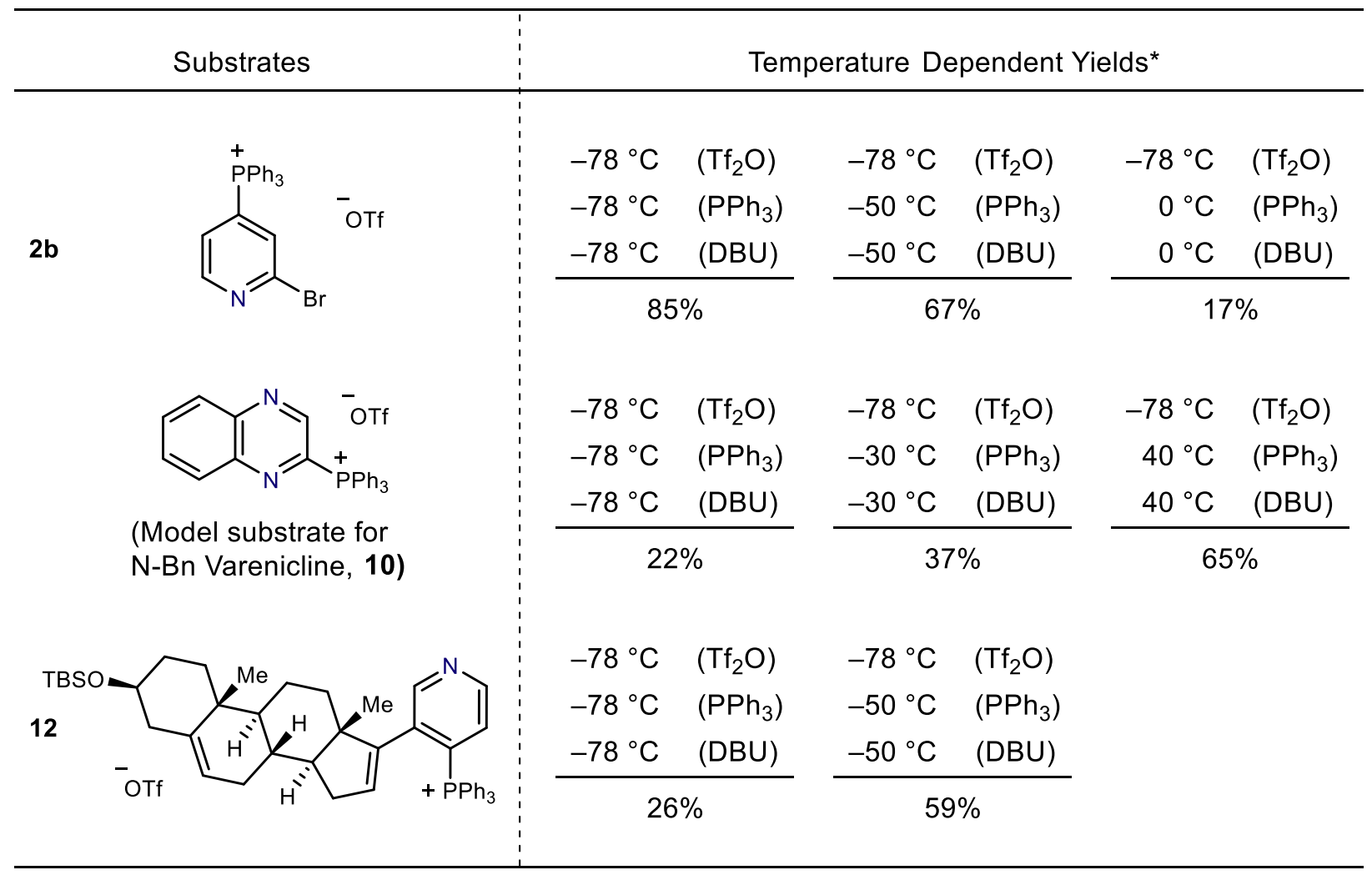

${ }^{* 1} \mathrm{H}$ NMR yields shown using 1,3,5-trimethoxybenzene as an internal standard. 


\section{Preparation of Heterocyclic Phosphonium Salt Precursors}

\section{2-Methyl-3-(thiophen-3-yl)pyridine}

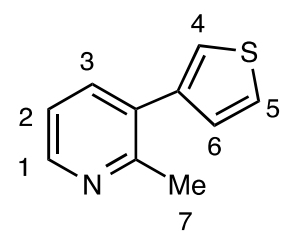

An oven dried $250 \mathrm{~mL}$ round bottom flask was charged with 2-methyl-3pyridyltrifluoromethanesulfonate (1.45 g, $6.00 \mathrm{mmol}), 3$-thienylboronic acid ( $1.54 \mathrm{~g}, 12.00 \mathrm{mmol})$, $\mathrm{Pd}\left(\mathrm{PPh}_{3}\right)_{4}(693 \mathrm{mg}, 0.60 \mathrm{mmol})$, and $\mathrm{K}_{3} \mathrm{PO}_{4}(2.55 \mathrm{~g}, 12.00 \mathrm{mmol})$. The flask was subjected to three cycles of vacuum/nitrogen backfill before addition of 1,4-dioxane $(144 \mathrm{~mL})$. The mixture was heated at $85{ }^{\circ} \mathrm{C}$ for 12 hours, cooled to room temperature and diluted with $\mathrm{Et}_{2} \mathrm{O}$. The suspension was washed with a saturated aqueous solution of $\mathrm{K}_{2} \mathrm{CO}_{3}$. The organic layer was separated and the aqueous layer was extracted with $\mathrm{Et}_{2} \mathrm{O}(3 \times 25 \mathrm{~mL})$. The combined organic extracts were dried $\left(\mathrm{MgSO}_{4}\right)$, filtered and concentrated in vacuo. The crude material was purified by flash chromatography (silica gel: $30 \%$ EtOAc in hexanes) to provide the title compound as a purple/brown oil (893 mg, $5.10 \mathrm{mmol}, 85 \%$ yield). IR $v_{\max } / \mathrm{cm}^{-1}$ (film): 3100, 3044, 2988, 2961, 1567, 1431, 863, 776, 734, 653; ${ }^{1} \mathrm{H}$ NMR (400 MHz, $\left.\mathrm{CDCl}_{3}\right) \delta: 8.46\left(1 \mathrm{H}, \mathrm{dd}, J=4.9,1.8 \mathrm{~Hz}, \mathrm{H}_{1}\right)$, $7.56\left(1 \mathrm{H}, \mathrm{dd}, J=7.7,1.8 \mathrm{~Hz}, \mathrm{H}_{3}\right), 7.18-7.10\left(2 \mathrm{H}, \mathrm{m}, \mathrm{H}_{2}\right.$ and $\left.\mathrm{H}_{5}\right), 7.39(1 \mathrm{H}, \mathrm{dd}, J=4.9,3.1 \mathrm{~Hz}$, $\left.\mathrm{H}_{6}\right), \quad 7.28-7.21\left(1 \mathrm{H}, \quad \mathrm{m}, \mathrm{H}_{4}\right), \quad 2.56\left(3 \mathrm{H}, \quad \mathrm{s}, \quad \mathrm{H}_{7}\right) ;{ }^{13} \mathrm{C} \quad \mathrm{NMR}\left(100 \mathrm{MHz}, \mathrm{CDCl}_{3}\right)$ $\delta: 155.93,147.73,139.99,136.97,131.77,128.35,125.60,132.28,120.93,23.57 \quad ; m / z$ LRMS $(\mathrm{ESI}+\mathrm{APCI})$ found $[\mathrm{M}+\mathrm{H}]^{+} 176.0, \mathrm{C}_{10} \mathrm{H}_{10} \mathrm{NS}^{+}$requires 176.0.

\section{2-Butyl-5-(trifluoromethyl)pyridine}

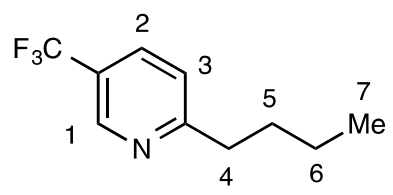


An oven-dried $100 \mathrm{~mL}$ round bottomed flask was charged with $\mathrm{ZnCl}_{2}$ (1.36 g, $\left.10.00 \mathrm{mmol}\right)$ and anhydrous THF $(20 \mathrm{~mL})$. The colorless solution was cooled to $0{ }^{\circ} \mathrm{C}, n$-BuLi $(2.5 \mathrm{M}$ in hexanes, $4.00 \mathrm{~mL}, 10.00 \mathrm{mmol}$ ) was added dropwise, and the reaction mixture was stirred for 1 hour. The resulting $n$-butylzinc chloride solution $(10.00 \mathrm{mmol})$ was added dropwise to a $50 \mathrm{~mL}$ round

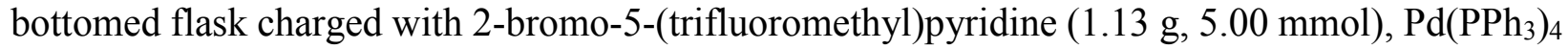
(58 mg, $0.025 \mathrm{mmol}$ ), and $5 \mathrm{~mL}$ of anhydrous THF. After 3 hours of stirring the reaction mixture was quenched with $\mathrm{H}_{2} \mathrm{O}(20 \mathrm{~mL})$, organic layer separated, and aqueous layer extracted with $\mathrm{CH}_{2} \mathrm{Cl}_{2}(3 \times 50 \mathrm{~mL})$. The combined organic extracts were dried $\left(\mathrm{MgSO}_{4}\right)$, filtered and concentrated in vacuo. The crude material was purified by flash chromatography (silica gel: $30 \% \mathrm{CH}_{2} \mathrm{Cl}_{2}$ in hexanes) to provide the title compound as a colorless oil (864 mg, $4.26 \mathrm{mmol}, 85 \%$ yield). IR $\mathrm{n}_{\max } / \mathrm{cm}^{-1}$ (film): 2960, 1609, 1325, 1161, 1124, 1080, 1016; ${ }^{1} \mathrm{H}$ NMR (400 MHz, $\mathrm{CDCl}_{3}$ ) $\delta: 8.77$ $\left(1 \mathrm{H}, \mathrm{s}, \mathrm{H}_{1}\right), 7.79\left(1 \mathrm{H}, \mathrm{dd}, J=8.2,1.8 \mathrm{~Hz}, \mathrm{H}_{2}\right), 7.29-7.21\left(1 \mathrm{H}, \mathrm{m}, \mathrm{H}_{3}\right), 2.84\left(2 \mathrm{H}, \mathrm{t}, J=7.5 \mathrm{~Hz}, \mathrm{H}_{4}\right)$, $1.71\left(2 \mathrm{H}\right.$, qn, $\left.J=7.5 \mathrm{~Hz}, \mathrm{H}_{5}\right), 1.37\left(2 \mathrm{H}\right.$, sext, $\left.J=7.5 \mathrm{~Hz}, \mathrm{H}_{6}\right), 0.92\left(3 \mathrm{H}, \mathrm{t}, J=7.6 \mathrm{~Hz}, \mathrm{H}_{7}\right) ;{ }^{13} \mathrm{C}$ $\operatorname{NMR}\left(100 \mathrm{MHz}, \mathrm{CDCl}_{3}\right) \delta: 166.60,146.12$ (q, $\left.J=4.1 \mathrm{~Hz}\right), 133.23$ (q, $\left.J=3.5 \mathrm{~Hz}\right), 123.99$ (q, $J=$ $32.8 \mathrm{~Hz}), 123.77$ (q, $J=271.2 \mathrm{~Hz}), 122.36,38.08,31.70,22.37,12.82 ;{ }^{19} \mathrm{~F}$ NMR (365 MHz, $\mathrm{CDCl}_{3}$ ) $\delta$ : $-62.32 ; m / z$ LRMS (ESI + APCI) found $[\mathrm{M}+\mathrm{H}]^{+} 204.1, \mathrm{C}_{10} \mathrm{H}_{13} \mathrm{~F}_{3} \mathrm{~N}^{+}$requires 204.1.

\section{8-benzyl-7,8,9,10-tetrahydro-6H-6,10-methanoazepino[4,5-g]quinoxaline}

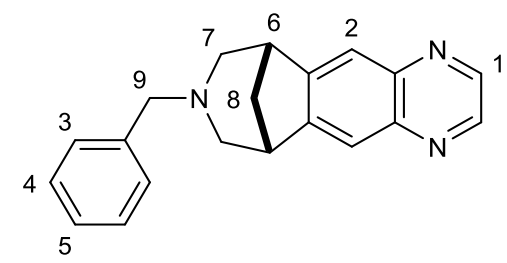

An oven dried $8 \mathrm{~mL}$ vial was charged with Varenicline (7,8,9,10-tetrahydro-6H-6,10methanoazepino[4,5-g]quinoxaline) $(211 \mathrm{mg}, 1.00 \mathrm{mmol})$, benzaldehyde (112 $\mu \mathrm{L}, 1.10 \mathrm{mmol})$, and 1,2-dichloroethane $(3.5 \mathrm{~mL})$. Sodium triacetoxyborohydride $(318 \mathrm{mg}, 1.50 \mathrm{mmol})$ was then added in one portion. The flask was subjected to three cycles of vacuum/nitrogen backfill. The 
mixture was stirred at room temperature for 12 hours. ${ }^{\dagger}$ The reaction mixture was diluted with $\mathrm{CH}_{2} \mathrm{Cl}_{2}$ and washed with a saturated solution of brine. The organic layer was separated and the aqueous layer was extracted with $\mathrm{CH}_{2} \mathrm{Cl}_{2}(3 \times 25 \mathrm{~mL})$. The combined organic extracts were dried $\left(\mathrm{MgSO}_{4}\right)$, filtered and concentrated in vacuo. The crude material was purified by flash chromatography (silica gel: 75\% EtOAc in hexanes) to provide the title compound as a yellow oil (278 mg, $0.92 \mathrm{mmol}, 92 \%$ yield). IR $v_{\max } / \mathrm{cm}^{-1}$ (film): 3057, 3027, 2944, 2787, 2750, 1472, 1453, 1358, 1186, 1025, 921, 885, 735, 697; ${ }^{1} \mathrm{H} \mathrm{NMR}\left(400 \mathrm{MHz}, \mathrm{CDCl}_{3}\right) \delta: 8.77\left(2 \mathrm{H}, \mathrm{s}, \mathrm{H}_{1}\right), 7.79(2 \mathrm{H}$, $\left.\mathrm{s}, \mathrm{H}_{2}\right), 7.16-7.06\left(3 \mathrm{H}, \mathrm{m}, \mathrm{H}_{4}\right.$ and $\left.\mathrm{H}_{5}\right), 6.89-6.79\left(2 \mathrm{H}, \mathrm{m}, \mathrm{H}_{3}\right), 3.48\left(2 \mathrm{H}, \mathrm{s}, \mathrm{H}_{9}\right), 3.41-3.30(2 \mathrm{H}, \mathrm{m}$, $\left.\mathrm{H}_{6}\right), 3.06-2.94\left(2 \mathrm{H}, \mathrm{m}, \mathrm{H}_{7}\right), 2.57\left(2 \mathrm{H}, \mathrm{d}, J=10.2 \mathrm{~Hz}, \mathrm{H}_{7}\right), 2.39-2.28\left(1 \mathrm{H}, \mathrm{m}, \mathrm{H}_{8}\right), 1.86(1 \mathrm{H}, \mathrm{d}, J=$ $\left.10.8 \quad \mathrm{~Hz}, \quad \mathrm{H}_{8}\right) ; \quad{ }^{13} \mathrm{C} \quad \mathrm{NMR} \quad\left(100 \quad \mathrm{MHz}, \quad \mathrm{CDCl}_{3}\right)$ $\delta: 150.86,143.31,143.25,138.08,128.23,127.93,126.59,120.38,61.45,57.32,43.08,41.2$ 0; $m / z$ LRMS (ESI + APCI) found $[\mathrm{M}+\mathrm{H}]^{+} 302.02, \mathrm{C}_{20} \mathrm{H}_{20} \mathrm{~N}_{3}{ }^{+}$requires 302.2.

3-((8R,9S,10R,13S,14S)-3-((tert-butyldimethylsilyl)oxy)-10,13-dimethyl2,3,4,7,8,9,10,11,12,13,14,15-dodecahydro-1H-cyclopenta[a]phenanthren-17-yl)pyridine

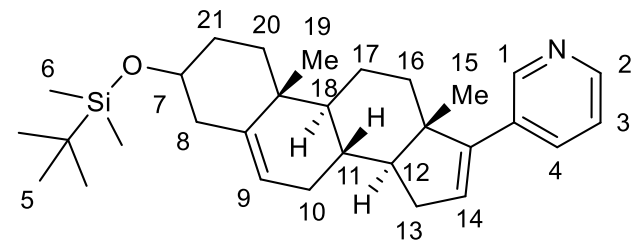

An oven dried $8 \mathrm{~mL}$ vial was charged with Abiraterone acetate (methyl $(8 R, 9 S, 10 R, 13 S, 14 S)$ 10,13-dimethyl-17-(pyridin-3-yl)-2,3,4,7,8,9,10,11,12,13,14,15-dodecahydro- $1 H$ cyclopenta[a]phenanthrene-3-carboxylate) (391 mg, $1.00 \mathrm{mmol})$, methanol $(5.00 \mathrm{~mL})$, and THF $(1.25 \mathrm{~mL})$. Potassium hydroxide $(56 \mathrm{mg}, 1.00 \mathrm{mmol})$ was added in one portion and the vial was subjected to three cycles of vacuum/nitrogen backfill and was heated at $30^{\circ} \mathrm{C}$ for 1 hour. The reaction mixture was concentrated in vacuo, diluted with ethyl acetate $(10 \mathrm{~mL})$, washed with water $(10 \mathrm{~mL})$ and a saturated solution of brine $(10 \mathrm{~mL})$. The organic layer was dried $\left(\mathrm{Na}_{2} \mathrm{SO}_{4}\right)$, filtered

\footnotetext{
${ }^{\dagger}$ An additional 0.75 equivalence of sodium triacetoxyborohydride (159 mg, $0.75 \mathrm{mmol}$ ) was added to the reaction mixture after 6 hours.
} 
and concentrated in vacuo. The resulting oil was transferred to an oven dried $8 \mathrm{~mL}$ vial that was charged with imidazole (102 mg, $1.50 \mathrm{mmol})$, tert-butyldimethylsilyl chloride (188 mg, 1.25 $\mathrm{mmol})$, and dimethylformamide $(9.00 \mathrm{~mL})$. The reaction mixture was stirred at room temperature for 10 hours before additional amounts of imidazole $(51 \mathrm{mg}, 0.75 \mathrm{mmol})$, tert-butyldimethylsilyl chloride (94 mg, $0.62 \mathrm{mmol})$, and dimethylformamide $(1.00 \mathrm{~mL})$ were added. The reaction was stirred for a further 5 hours at room temperature before being quenched with water $(10 \mathrm{~mL})$ and diluted with ethyl acetate $(10 \mathrm{~mL})$. The organic layer was separated and the aqueous layer was extracted with ethyl acetate $(3 \times 10 \mathrm{~mL})$, the combined organic extracts were dried $\left(\mathrm{Na}_{2} \mathrm{SO}_{4}\right)$, filtered and concentrated in vacuo. The crude material was purified by flash chromatography (silica gel: 50\% EtOAc in hexanes) to provide the title compound as a white solid (419 mg, 0.90 mmol, 90\% yield over 2 steps). ${ }^{1} \mathrm{H}$ NMR $\left(400 \mathrm{MHz}, \mathrm{CDCl}_{3}\right) \delta: 8.80-8.23(2 \mathrm{H}, \mathrm{m}), 7.63(1 \mathrm{H}, \mathrm{d}, J$ $=7.8 \mathrm{~Hz}), 7.29-7.13(1 \mathrm{H}, \mathrm{m}), 5.97(1 \mathrm{H}, \mathrm{br} \mathrm{s}), 5.33(1 \mathrm{H}, \mathrm{d}, J=4.0 \mathrm{~Hz}), 3.46(1 \mathrm{H}, \mathrm{m}), 2.33-2.11$ $(3 \mathrm{H}, \mathrm{m}), 2.10-1.93(3 \mathrm{H}, \mathrm{m}), 1.85-1.37(9 \mathrm{H}, \mathrm{m}), 1.11-0.94(8 \mathrm{H}, \mathrm{m}), 0.86(9 \mathrm{H}, \mathrm{s}), 0.03(6 \mathrm{H}, \mathrm{s}) ;{ }^{13} \mathrm{C}$ $\operatorname{NMR}\left(100 \mathrm{MHz}, \mathrm{CDCl}_{3}\right) \delta:$ 151.61, 147.63, 141.88, 133.80, 129.37, 122.90, 120.77, 72.52, 57.57, $50.40,47.32$, 42.81, 37.27, 36.76, 35.23, 32.03, 31.80, 31.53, 30.42, 25.92, 20.83, 19.35, 16.55, 4.60. The spectroscopic data is in agreement with a reported synthesis. ${ }^{2} m / z$ LRMS (ESI + APCI) found $[\mathrm{M}+\mathrm{H}]^{+} 464.4, \mathrm{C}_{30} \mathrm{H}_{46} \mathrm{NOSi}^{+}$requires 464.3 .

\section{Preparation of Heterocyclic Phosphonium Salts}

\section{General Procedure A}

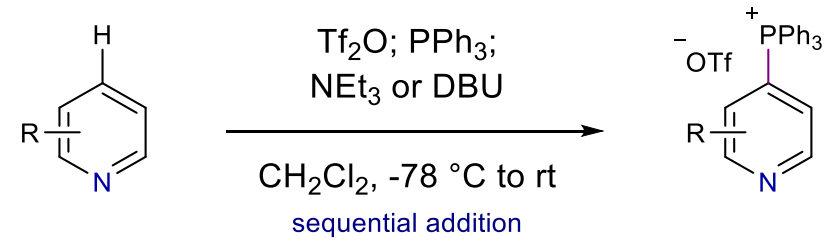

An oven dried $8 \mathrm{~mL}$ vial $(\leq 0.5 \mathrm{mmol}$ scale $)$ or a round bottom flask ( $>0.5 \mathrm{mmol}$ scale $)$ equipped with a stir bar was charged with the heterocycle (1.0 equiv) and placed under a nitrogen atmosphere. $\mathrm{CH}_{2} \mathrm{Cl}_{2}(0.1 \mathrm{M})$ was added, the reaction vessel cooled to $-78{ }^{\circ} \mathrm{C}$ and $\mathrm{Tf}_{2} \mathrm{O}$ (1.0 equiv) was added dropwise over 5 minutes. The reaction was stirred for 30 minutes before $\mathrm{PPh}_{3}(1.1$ 
equiv) was added in one portion. The reaction was subjected to three rapid cycles of vacuum/nitrogen backfill and was stirred for a further 30 minutes at $-78{ }^{\circ} \mathrm{C}$. The stated organic base ( $\mathrm{NEt}_{3}$ or DBU, 1.0 equiv) was added dropwise via syringe, the cooling bath was removed and the reaction was allowed to warm to room temperature while stirring (approximately 15-30 minutes). The reaction mixture was quenched with $\mathrm{H}_{2} \mathrm{O}$ (approximately the same volume as $\mathrm{CH}_{2} \mathrm{Cl}_{2}$ ) and the mixture was transferred to a separatory funnel. The mixture was diluted $\mathrm{CH}_{2} \mathrm{Cl}_{2}$ and the resulting organic layer was washed three times with $\mathrm{H}_{2} \mathrm{O}$. The organic layer was dried $\left(\mathrm{MgSO}_{4}\right)$, filtered and concentrated in vacuo to approximately 2-10 $\mathrm{mL}$ (depending on the scale of the reaction). An excess of chilled $\mathrm{Et}_{2} \mathrm{O}\left(0^{\circ} \mathrm{C}\right)$ was added to the concentrated solution that was then placed in a $-20^{\circ} \mathrm{C}$ refrigerator for approximately 1 hour. The resulting suspension was filtered on a frit, the solid washed with chilled $\mathrm{Et}_{2} \mathrm{O}\left(0^{\circ} \mathrm{C}\right)$ and dried in vacuo to provide the pure phosphonium salt.

\section{Notes.}

1) $\mathrm{PPh}_{3}$ was crushed into a powder prior to use.

2) Certain substrates require longer periods for the precipitation step and specific cases are indicated below.

3) In a small number of cases, residual $\mathrm{CH}_{2} \mathrm{Cl}_{2}$ can become trapped in the phosphonium salt products. In these cases, heating the salts under vacuum $\left(50-100^{\circ} \mathrm{C}\right)$ removed the solvent.

4) In order to evaluate regioselectivity from the crude reaction mixtures, a duplicate reaction was performed and aliquots taken after addition of the organic base and warming to room temperature. These aliquots were concentrated in vacuo and analyzed by ${ }^{1} \mathrm{H}$ and ${ }^{31} \mathrm{P} \mathrm{NMR}$.

\section{(2-Chloropyridin-4-yl)triphenylphosphonium trifluoromethanesulfonate (2a)}

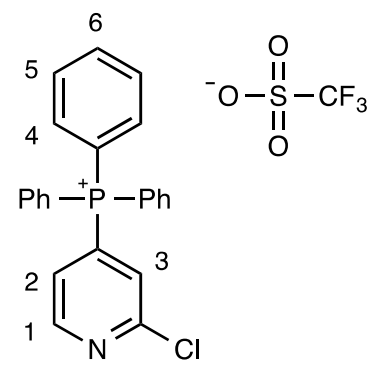


Prepared according to general procedure A using 2-chloropyridine ( $473 \mu \mathrm{L}, 5.00 \mathrm{mmol}), \mathrm{Tf}_{2} \mathrm{O}$ (845 $\mu \mathrm{L}, 5.00 \mathrm{mmol}), \mathrm{PPh}_{3}(1.44 \mathrm{~g}, 5.50 \mathrm{mmol}), \mathrm{NEt}_{3}(697 \mu \mathrm{L}, 5.00 \mathrm{mmol})$ and $\mathrm{CH}_{2} \mathrm{Cl}_{2}(50 \mathrm{~mL})$. After the purification procedure, the title compound was isolated as a white solid (2.14 g, 4.08 mmol, $82 \%$ yield). $\mathrm{mp} 158-160^{\circ} \mathrm{C}$; IR $v_{\max } / \mathrm{cm}^{-1}$ (film): 3087, 3061, 3028, 1459, 1264, 1137, 1110 , 1031, 749, 726, 634; ${ }^{1} \mathrm{H}$ NMR (400 MHz, $\left.\mathrm{CDCl}_{3}\right) \delta: 8.82\left(1 \mathrm{H}\right.$, app t, $\left.J=5.1 \mathrm{~Hz}, \mathrm{H}_{1}\right), 7.98-7.89$ $\left(3 \mathrm{H}, \mathrm{m}, \mathrm{H}_{6}\right), 7.86-7.77\left(6 \mathrm{H}, \mathrm{m}, \mathrm{H}_{5}\right), 7.75-7.62\left(7 \mathrm{H}, \mathrm{m}, \mathrm{H}_{2}\right.$ and $\left.\mathrm{H}_{4}\right), 7.40\left(1 \mathrm{H}, \mathrm{d}, J=13.2 \mathrm{~Hz}, \mathrm{H}_{3}\right)$; ${ }^{13} \mathrm{C}$ NMR $\left(100 \mathrm{MHz}, \mathrm{CDCl}_{3}\right) \delta: 153.38(\mathrm{~d}, J=15.1 \mathrm{~Hz}), 152.31(\mathrm{~d}, J=11.3 \mathrm{~Hz}), 136.41(\mathrm{~d}, J=$ $3.0 \mathrm{~Hz}), 134.49$ (d, $J=10.4 \mathrm{~Hz}), 132.01$ (d, $J=83.2 \mathrm{~Hz}), 131.11$ (d, $J=13.0 \mathrm{~Hz}), 127.41$ (d, $J=$ $9.5 \mathrm{~Hz}), 126.28$ (d, $J=8.4 \mathrm{~Hz}), 120.72(\mathrm{q}, J=320.4 \mathrm{~Hz}), 115.02(\mathrm{~d}, J=90.0 \mathrm{~Hz}) ;{ }^{19} \mathrm{~F}$ NMR $(365$ $\left.\mathrm{MHz}, \mathrm{CDCl}_{3}\right) \delta:-78.19 ;{ }^{31} \mathrm{P} \mathrm{NMR}\left(162 \mathrm{MHz}, \mathrm{CDCl}_{3}\right) \delta: 22.27 ; \mathrm{m} / z$ LRMS (ESI + APCI) found $[\mathrm{M}-\mathrm{OTf}]^{+} 374.1, \mathrm{C}_{23} \mathrm{H}_{18} \mathrm{ClNP}^{+}$requires 374.1 .

\section{(2-Bromopyridin-4-yl)triphenylphosphonium trifluoromethanesulfonate (2b)}

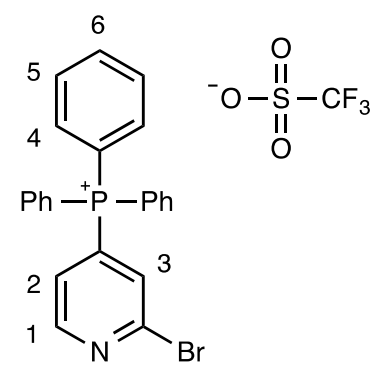

Prepared according to general procedure A using 2-bromopyridine ( $954 \mu \mathrm{L}, 10.00 \mathrm{mmol}$ ), $\mathrm{Tf}_{2} \mathrm{O}$ (1.69 mL, $10.00 \mathrm{mmol}), \mathrm{PPh}_{3}(2.89 \mathrm{~g}, 11.00 \mathrm{mmol}), \mathrm{DBU}(1.52 \mathrm{~mL}, 10.00 \mathrm{mmol})$ and $\mathrm{CH}_{2} \mathrm{Cl}_{2}$ $(100 \mathrm{~mL})$. After the purification procedure, the title compound was isolated as a white solid (4.84 g, $8.52 \mathrm{mmol}, 85 \%$ yield). $\mathrm{mp} 129-136{ }^{\circ} \mathrm{C}$; IR $v_{\max } / \mathrm{cm}^{-1}$ (film): $3099,3059,3027,2996,1264$, 1136, 1109, 1030, 634; ${ }^{1} \mathrm{H}$ NMR (400 MHz, $\left.\mathrm{CDCl}_{3}\right) \delta: 8.81\left(1 \mathrm{H}\right.$, app t, $\left.J=5.1 \mathrm{~Hz}, \mathrm{H}_{1}\right), 7.99-7.89$ $\left(3 \mathrm{H}, \mathrm{m}, \mathrm{H}_{6}\right), 7.87-7.73\left(7 \mathrm{H}, \mathrm{m}, \mathrm{H}_{2}\right.$ and $\left.\mathrm{H}_{5}\right), 7.72-7.60\left(6 \mathrm{H}, \mathrm{m}, \mathrm{H}_{4}\right), 7.54\left(1 \mathrm{H}, \mathrm{d}, J=12.9 \mathrm{~Hz}, \mathrm{H}_{3}\right)$; ${ }^{13} \mathrm{C}$ NMR $\left(100 \mathrm{MHz}, \mathrm{CDCl}_{3}\right) \delta: 152.63(\mathrm{~d}, J=10.8 \mathrm{~Hz}), 143.77(\mathrm{~d}, J=14.0 \mathrm{~Hz}), 136.15(\mathrm{~d}, J=$ $3.1 \mathrm{~Hz}), 134.47$ (d, $J=10.6 \mathrm{~Hz}), 131.67$ (d, $J=82.9 \mathrm{~Hz}), 131.11$ (d, $J=13.2 \mathrm{~Hz}), 130.81$ (d, $J=$ $9.2 \mathrm{~Hz}), 126.58(\mathrm{~d}, J=8.3 \mathrm{~Hz}), 120.71(\mathrm{~d}, J=320.9 \mathrm{~Hz}), 115.01(\mathrm{~d}, J=89.7 \mathrm{~Hz}) ;{ }^{19} \mathrm{~F}$ NMR $(365$ 
$\left.\mathrm{MHz}, \mathrm{CDCl}_{3}\right) \delta:-78.19 ;{ }^{31} \mathrm{P} \mathrm{NMR}\left(162 \mathrm{MHz}, \mathrm{CDCl}_{3}\right) \delta: 22.00 ; \mathrm{m} / z$ LRMS (ESI + APCI) found $[\mathrm{M}-\mathrm{OTf}]^{+} 418.1, \mathrm{C}_{23} \mathrm{H}_{18} \mathrm{BrNP}^{+}$requires 418.0.

\section{(2-Fluoropyridin-4-yl)triphenylphosphonium trifluoromethanesulfonate (2c)}

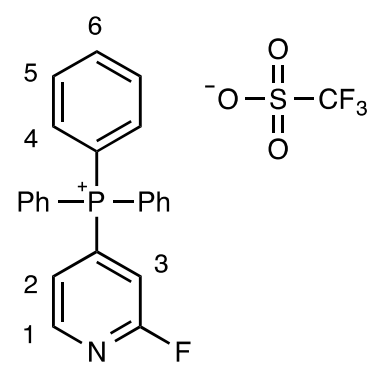

Prepared according to general procedure A using 2-fluoropyridine ( $859 \mu \mathrm{L}, 10.00 \mathrm{mmol}), \mathrm{Tf}_{2} \mathrm{O}$ (1.69 mL, $10.00 \mathrm{mmol}), \mathrm{PPh}_{3}(2.89 \mathrm{~g}, 11.00 \mathrm{mmol}), \mathrm{DBU}(1.52 \mathrm{~mL}, 10.00 \mathrm{mmol})$ and $\mathrm{CH}_{2} \mathrm{Cl}_{2}$ $(100 \mathrm{~mL})$. After the purification procedure, the title compound was isolated as a white solid (3.96 g, $7.80 \mathrm{mmol}, 79 \%$ yield). $\mathrm{mp}$ 192-196 ${ }^{\circ} \mathrm{C}$; IR $v_{\max } / \mathrm{cm}^{-1}$ (film): 3087, 3064, 1586, 1437, 1388, 1262, 1227, 1142, 1108, 1031, 726, 718, 687, 634; ${ }^{1} \mathrm{H}$ NMR (400 MHz, $\left.\mathrm{CDCl}_{3}\right)$ 8: 8.67 (1H, app $\left.\mathrm{t}, J=5.3 \mathrm{~Hz}, \mathrm{H}_{1}\right), 7.99-7.88\left(3 \mathrm{H}, \mathrm{m}, \mathrm{H}_{6}\right), 7.87-7.75\left(6 \mathrm{H}, \mathrm{m}, \mathrm{H}_{5}\right), 7.74-7.60\left(7 \mathrm{H}, \mathrm{m}, \mathrm{H}_{2}\right.$ and $\left.\mathrm{H}_{4}\right)$, $7.10\left(1 \mathrm{H}, \mathrm{d}, J=13.4 \mathrm{~Hz}, \mathrm{H}_{3}\right) ;{ }^{13} \mathrm{C} \mathrm{NMR}\left(100 \mathrm{MHz}, \mathrm{CDCl}_{3}\right) \delta: 163.28$ (dd, $\left.J=245.4,16.9 \mathrm{~Hz}\right)$, $150.63(\mathrm{dd}, J=13.7,12.7 \mathrm{~Hz}), 136.09$ (d, $J=3.2 \mathrm{~Hz}), 134.21(\mathrm{~d}, J=10.7 \mathrm{~Hz}), 133.95(\mathrm{dd}, J=$ 84.4, $6.6 \mathrm{~Hz}), 130.81(\mathrm{~d}, J=13.8 \mathrm{~Hz}), 125.18$ (dd, $J=8.2,5.1 \mathrm{~Hz}), 122.44$ (q, $J=321.6 \mathrm{~Hz})$, $114.81(\mathrm{~d}, J=90.1 \mathrm{~Hz}), 114.19(\mathrm{dd}, J=40.3,10.2 \mathrm{~Hz}) ;{ }^{19} \mathrm{~F}$ NMR $\left(365 \mathrm{MHz}, \mathrm{CDCl}_{3}\right) \delta$ : $-61.56(\mathrm{~d}$, $J=10.3 \mathrm{~Hz}),-78.20 ;{ }^{31} \mathrm{P}$ NMR $\left(162 \mathrm{MHz}, \mathrm{CDCl}_{3}\right) \delta: 22.15,(\mathrm{~d}, J=9.9 \mathrm{~Hz}) ; m / z \mathrm{LRMS}(\mathrm{ESI}+$ $\mathrm{APCI}$ ) found $[\mathrm{M}-\mathrm{OTf}]^{+} 358.1, \mathrm{C}_{23} \mathrm{H}_{18} \mathrm{FNP}^{+}$requires 358.1 . 


\section{Triphenyl(2-phenylpyridin-4-yl)phosphonium trifluoromethanesulfonate (2d)}

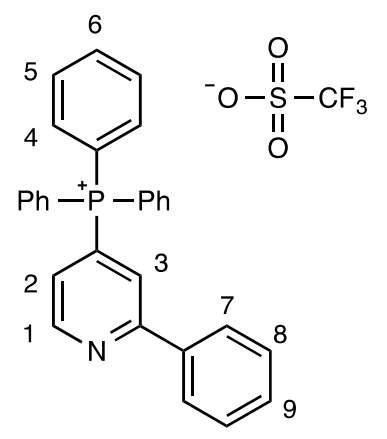

Prepared according to general procedure A using 2-phenylpyridine (2.57 mL, $18.00 \mathrm{mmol}), \mathrm{Tf}_{2} \mathrm{O}$ (3.04 mL, $18.00 \mathrm{mmol}), \mathrm{PPh}_{3}(5.19 \mathrm{~g}, 19.8 \mathrm{mmol}), \mathrm{NEt}_{3}(2.51 \mathrm{~mL}, 18.00 \mathrm{mmol})$ and $\mathrm{CH}_{2} \mathrm{Cl}_{2}(180$ $\mathrm{mL})$. After the purification procedure, the title compound was isolated as a white solid (8.53 $\mathrm{g}$, $15.08 \mathrm{mmol}, 84 \%$ yield). $\mathrm{mp} 169-171^{\circ} \mathrm{C}$; IR $v_{\max } / \mathrm{cm}^{-1}$ (film): 3087, 3066, 3011, 1584, 1570, 1471, 1439, 1374, 1079, 997, 751; ${ }^{1} \mathrm{H} \mathrm{NMR}\left(400 \mathrm{MHz}, \mathrm{CDCl}_{3}\right) \delta: 9.01\left(1 \mathrm{H}\right.$, app t, $\left.J=5.1 \mathrm{~Hz}, \mathrm{H}_{1}\right), 7.93-$ $7.54\left(18 \mathrm{H}, \mathrm{m}, \mathrm{H}_{3}, \mathrm{H}_{4}, \mathrm{H}_{5}, \mathrm{H}_{6}\right.$, and $\left.\mathrm{H}_{7}\right), 7.50\left(1 \mathrm{H}, \mathrm{ddd}, J=17.8,5.1,1.1 \mathrm{~Hz}, \mathrm{H}_{2}\right), 7.42-7.36(3 \mathrm{H}, \mathrm{m}$, $\mathrm{H}_{8}$ and $\left.\mathrm{H}_{9}\right) ;{ }^{13} \mathrm{C} \mathrm{NMR}\left(100 \mathrm{MHz}, \mathrm{CDCl}_{3}\right) \delta: 159.09(\mathrm{~d}, J=9.9 \mathrm{~Hz}), 151.63(\mathrm{~d}, J=10.7 \mathrm{~Hz}), 136.74$ $(\mathrm{d}, J=1.5 \mathrm{~Hz}), 136.14$ (d, $J=3.2 \mathrm{~Hz}), 134.30$ (d, $J=9.8 \mathrm{~Hz}), 130.91$ (d, $J=13.0 \mathrm{~Hz}), 130.35$, $129.23(\mathrm{~d}, J=84.1 \mathrm{~Hz}), 128.98,127.00,125.25$ (d, $J=7.8 \mathrm{~Hz}), 123.08$, (d, $J=8.4 \mathrm{~Hz}), 120.68$ (q, $J=321.1 \mathrm{~Hz}), 115.49(\mathrm{~d}, J=89.1 \mathrm{~Hz}) ;{ }^{19} \mathrm{~F} \mathrm{NMR}\left(365 \mathrm{MHz}, \mathrm{CDCl}_{3}\right) \delta:-78.1 ;{ }^{31} \mathrm{P} \mathrm{NMR}(162 \mathrm{MHz}$, $\left.\mathrm{CDCl}_{3}\right) \delta: 22.7 ; m / z \mathrm{LRMS}(\mathrm{ESI}+\mathrm{APCI})$ found $[\mathrm{M}-\mathrm{OTf}]^{+} 416.2, \mathrm{C}_{29} \mathrm{H}_{23} \mathrm{NP}^{+}$requires 416.2.

\section{[2,2'-Bipyridin]-4-yltriphenylphosphonium trifluoromethanesulfonate (2e)}

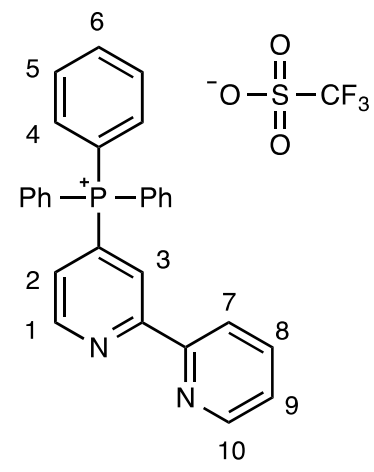


Prepared according to general procedure A using 2,2'-bipyridine (929 mg, $5.95 \mathrm{mmol}), \mathrm{Tf}_{2} \mathrm{O}$ (1.00 $\mathrm{mL}, 5.95 \mathrm{mmol}), \mathrm{PPh}_{3}(1.73 \mathrm{~g}, 6.55 \mathrm{mmol}), \mathrm{NEt}_{3}(830 \mu \mathrm{L}, 5.99 \mathrm{mmol})$ and $\mathrm{CH}_{2} \mathrm{Cl}_{2}(60 \mathrm{~mL})$. After the purification procedure, the title compound was isolated as a white solid $(2.92 \mathrm{~g}, 5.15 \mathrm{mmol}$, 87\% yield). mp 179-182 ${ }^{\circ} \mathrm{C}$; IR $v_{\max } / \mathrm{cm}^{-1}$ (film): 3060, 3014, 1576, 1438, 1261, 1142, 1106, 1030; ${ }^{1} \mathrm{H}$ NMR $\left(400 \mathrm{MHz}, \mathrm{CDCl}_{3}\right) \delta: 9.06\left(1 \mathrm{H}\right.$, app t, $\left.J=5.1 \mathrm{~Hz}, \mathrm{H}_{1}\right), 8.65\left(1 \mathrm{H}, \mathrm{d}, J=13.8 \mathrm{~Hz}, \mathrm{H}_{3}\right)$, $8.55\left(1 \mathrm{H}, \mathrm{d}, J=4.4 \mathrm{~Hz}, \mathrm{H}_{10}\right), 8.46\left(1 \mathrm{H}, \mathrm{d}, J=7.9 \mathrm{~Hz}, \mathrm{H}_{7}\right), 7.96-7.88\left(3 \mathrm{H}, \mathrm{m}, \mathrm{H}_{6}\right), 7.87-7.74(7 \mathrm{H}$, $\mathrm{m}, \mathrm{H}_{5}$ and $\left.\mathrm{H}_{8}\right), 7.72-7.55\left(7 \mathrm{H}, \mathrm{m}, \mathrm{H}_{2}\right.$ and $\left.\mathrm{H}_{4}\right), 7.35\left(1 \mathrm{H}, \mathrm{dd}, J=7.7,4.5 \mathrm{~Hz}, \mathrm{H}_{9}\right) ;{ }^{13} \mathrm{C}$ NMR $(100$ MHz, $\left.\mathrm{CDCl}_{3}\right) \delta: 157.80$ (d, $\left.J=9.9 \mathrm{~Hz}\right), 153.36$ (d, $\left.J=2.3 \mathrm{~Hz}\right), 151.37$ (d, $\left.J=10.7 \mathrm{~Hz}\right), 149.34$, 137.34, 136.17 (d, $J=3.1 \mathrm{~Hz}), 134.42$ (d, $J=9.9 \mathrm{~Hz}), 130.97$ (d, $J=13.0 \mathrm{~Hz}), 129.29$ (d, $J=83.9$ Hz), 126.91 (d, $J=8.4 \mathrm{~Hz}), 125.08,123.89$ (d, $J=9.2 \mathrm{~Hz}), 121.65,120.80$ (q, $J=321.2 \mathrm{~Hz}$ ), $115.75(\mathrm{~d}, J=89.3 \mathrm{~Hz}) ;{ }^{19} \mathrm{~F}$ NMR $\left(365 \mathrm{MHz}, \mathrm{CDCl}_{3}\right) \delta:-78.10 ;{ }^{31} \mathrm{P}$ NMR (162 MHz, $\left.\mathrm{CDCl}_{3}\right) \delta:$ 22.64; $m / z$ LRMS (ESI + APCI) found [M - OTf] $]^{+}$417.2, $\mathrm{C}_{28} \mathrm{H}_{22} \mathrm{~N}_{2} \mathrm{P}^{+}$requires 417.2.

Triphenyl(3-phenylpyridin-4-yl)phosphonium trifluoromethanesulfonate (2f)

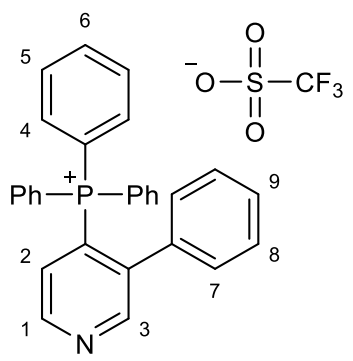

Prepared according to general procedure A (except that the stirring time after addition of $\mathrm{PPh}_{3}$ was 1 hour instead of 30 minutes) using 3-phenylpyridine ( $400 \mathrm{mg}, 2.58 \mathrm{mmol}$ ), $\mathrm{Tf}_{2} \mathrm{O}$ (435 $\mu \mathrm{L}, 2.58$ $\mathrm{mmol}), \mathrm{PPh}_{3}(744 \mathrm{mg}, 2.84 \mathrm{mmol}), \mathrm{DBU}(285 \mu \mathrm{L}, 2.58 \mathrm{mmol})$ and $\mathrm{CH}_{2} \mathrm{Cl}_{2}(26 \mathrm{~mL})$. After the purification procedure, the title compound was isolated as a white solid $(1.05 \mathrm{~g}, 1.86 \mathrm{mmol}, 72 \%$ yield). mp 185-188 ${ }^{\circ} \mathrm{C}$; IR $v_{\max } / \mathrm{cm}^{-1}$ (film): 3032, 2980, 2948, 1518, 1407, 1292, 1249, 1186, 825; ${ }^{1} \mathrm{H}$ NMR $\left(400 \mathrm{MHz}, \mathrm{CDCl}_{3}\right) \delta: 8.95\left(1 \mathrm{H}\right.$, app t, $\left.J=4.7 \mathrm{~Hz}, \mathrm{H}_{1}\right), 8.74\left(1 \mathrm{H}, \mathrm{d}, J=6.8 \mathrm{~Hz}, \mathrm{H}_{3}\right), 7.85-$ $7.73\left(3 \mathrm{H}, \mathrm{m}, \mathrm{H}_{6}\right), 7.73-7.40\left(13 \mathrm{H}, \mathrm{m}, \mathrm{H}_{2}, \mathrm{H}_{4}\right.$, and $\left.\mathrm{H}_{5}\right), 7.11\left(1 \mathrm{H}, \mathrm{t}, J=7.6 \mathrm{~Hz}, \mathrm{H}_{9}\right), 6.91(2 \mathrm{H}$, app $\left.\mathrm{t}, J=7.6 \mathrm{~Hz}, \mathrm{H}_{8}\right), 6.71\left(2 \mathrm{H}, \mathrm{d}, J=7.5 \mathrm{~Hz}, \mathrm{H}_{7}\right) ;{ }^{13} \mathrm{C} \mathrm{NMR}\left(100 \mathrm{MHz}, \mathrm{CDCl}_{3}\right) \delta: 153.63(\mathrm{~d}, J=8.0$ Hz), 149.97 (d, $J=10.4 \mathrm{~Hz}), 141.68$ (d, $J=7.3 \mathrm{~Hz}), 135.43$ (d, $J=3.0 \mathrm{~Hz}), 134.41$ (d, $J=4.5 \mathrm{~Hz})$, 
$134.18(\mathrm{~d}, J=10.3 \mathrm{~Hz}), 130.59$ ( d, $J=13.0 \mathrm{~Hz}), 129.21,128.89,128.30,128.20,126.35$ (d, $J=$ $83.4 \mathrm{~Hz}), 120.82(\mathrm{q}, J=321.2 \mathrm{~Hz}), 116.89(\mathrm{~d}, J=89.2 \mathrm{~Hz}) ;{ }^{19} \mathrm{~F}$ NMR $(365 \mathrm{MHz}$, $\left.\mathrm{CDCl}_{3}\right) \delta:-77.68 ;{ }^{31} \mathrm{P}$ NMR $\left(162 \mathrm{MHz}, \mathrm{CDCl}_{3}\right) \delta: 21.73 ; \mathrm{m} / z$ LRMS (ESI + APCI ) found $[\mathrm{M}-$ $\mathrm{OTf}^{+}$416.2, $\mathrm{C}_{29} \mathrm{H}_{23} \mathrm{NP}^{+}$requires 416.2.

Triphenyl(5,6,7,8-tetrahydroquinolin-4-yl)phosphonium trifluoromethanesulfonate (2g)

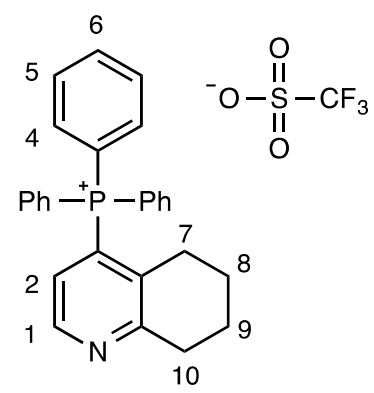

Prepared according to general procedure A (except the product suspension was placed in a $-20^{\circ} \mathrm{C}$ refrigerator for approximately 12 hours instead of 1 hour) using 5,6,7,8-tetrahydroquinoline (773 $\mu \mathrm{L}, 5.95 \mathrm{mmol}), \mathrm{Tf}_{2} \mathrm{O}(1.00 \mathrm{~mL}, 5.95 \mathrm{mmol}), \mathrm{PPh}_{3}(1.73 \mathrm{~g}, 6.55 \mathrm{mmol}), \mathrm{DBU}(890 \mu \mathrm{L}, 5.95 \mathrm{mmol})$ and $\mathrm{CH}_{2} \mathrm{Cl}_{2}(60 \mathrm{~mL})$. After the purification procedure, the title compound was isolated as a pale tan solid (2.24 g, $4.13 \mathrm{mmol}, 69 \%$ yield). $\mathrm{mp} 248-251^{\circ} \mathrm{C}$; IR $v_{\max } / \mathrm{cm}^{-1}$ (film): 3019, 2954, 1442, 1259, 1144, 1029; ${ }^{1} \mathrm{H}$ NMR (400 MHz, DMSO-d $)$ 8: $8.74\left(1 \mathrm{H}\right.$, app t, $\left.J=5.1 \mathrm{~Hz}, \mathrm{H}_{1}\right), 8.07-7.93$ $\left(3 \mathrm{H}, \mathrm{m}, \mathrm{H}_{6}\right), 7.92-7.71\left(12 \mathrm{H}, \mathrm{m}, \mathrm{H}_{4}\right.$ and $\left.\mathrm{H}_{5}\right), 6.94\left(1 \mathrm{H}, \mathrm{dd}, J=15.3,5.1 \mathrm{~Hz}, \mathrm{H}_{2}\right), 3.12-2.97(2 \mathrm{H}$, $\left.\mathrm{m}, \mathrm{H}_{10}\right), 2.21-2.04\left(2 \mathrm{H}, \mathrm{m}, \mathrm{H}_{7}\right), 1.84-1.71\left(2 \mathrm{H}, \mathrm{m}, \mathrm{H}_{9}\right), 1.60-1.44\left(2 \mathrm{H}, \mathrm{m}, \mathrm{H}_{8}\right) ;{ }^{13} \mathrm{C} \mathrm{NMR}(100 \mathrm{MHz}$, $\left.\mathrm{CDCl}_{3}\right) \delta: 160.25(\mathrm{~d}, J=8.4 \mathrm{~Hz}), 148.20(\mathrm{~d}, J=11.4 \mathrm{~Hz}), 135.48$ (d, $\left.J=7.6 \mathrm{~Hz}\right), 135.27$ (d, $J=$ $3.1 \mathrm{~Hz}), 134.06$ (d, $J=10.7 \mathrm{~Hz}), 130.50(\mathrm{~d}, J=13.0 \mathrm{~Hz}), 126.18$ (d, $J=9.9 \mathrm{~Hz}), 125.51$ (d, $J=$ $82.4 \mathrm{~Hz}), 120.40$ (q, $J=322.0 \mathrm{~Hz}), 116.34$ (d, $J=87.7 \mathrm{~Hz}), 32.01$ (d, $J=2.3 \mathrm{~Hz}), 29.66$ (d, $J=$ $5.3 \mathrm{~Hz}), 21.03,20.54 ;{ }^{19} \mathrm{~F}$ NMR (365 $\left.\mathrm{MHz}, \mathrm{CDCl}_{3}\right) \delta:-77.75 ;{ }^{31} \mathrm{P}$ NMR $(162 \mathrm{MHz}$, $\left.\mathrm{CDCl}_{3}\right) \delta: 20.85 ; \mathrm{m} / z$ LRMS (ESI + APCI) found [M - OTf] $]^{+} 394.2, \mathrm{C}_{27} \mathrm{H}_{25} \mathrm{NP}^{+}$requires 394.2. 


\section{(2-Methyl-3-(thiophen-3-yl)pyridin-4-yl)triphenylphosphonium trifluoromethanesulfonate}

(2h)

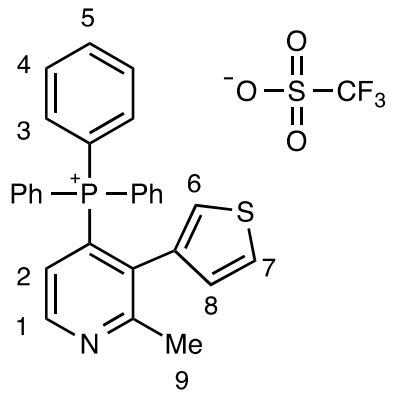

Prepared according to general procedure A (except that the stirring time after addition of $\mathrm{PPh}_{3}$ was 1 hour instead of 30 minutes and an additional hour of stirring at room temperature after addition of DBU) using 2-methyl-3-(thiophen-3-yl)pyridine (518 mg, $3.00 \mathrm{mmol}), \mathrm{Tf}_{2} \mathrm{O}(500 \mu \mathrm{L}, 3.00$ mmol), $\mathrm{PPh}_{3}(775 \mathrm{mg}, 3.30 \mathrm{mmol}), \mathrm{DBU}(448 \mu \mathrm{L}, 3.00 \mathrm{mmol})$ and $\mathrm{CH}_{2} \mathrm{Cl}_{2}(30 \mathrm{~mL})$. After the purification procedure, ${ }^{\ddagger}$ the title compound was isolated as a tan solid $(1.04 \mathrm{~g}, 1.78 \mathrm{mmol}, 59 \%$ yield). mp $182-185^{\circ} \mathrm{C}$; IR $v_{\max } / \mathrm{cm}^{-1}$ (film): $3112,3072,2976,1272,1262,1168,1153,1027,632$; ${ }^{1} \mathrm{H}$ NMR $\left(400 \mathrm{MHz}, \mathrm{CDCl}_{3}\right) \delta: 8.82\left(1 \mathrm{H}\right.$, app t, $\left.J=5.0 \mathrm{~Hz}, \mathrm{H}_{1}\right), 7.86-7.77\left(3 \mathrm{H}, \mathrm{m}, \mathrm{H}_{5}\right), 7.75-7.65$ $\left(6 \mathrm{H}, \mathrm{m}, \mathrm{H}_{4}\right), 7.63-7.52\left(6 \mathrm{H}, \mathrm{m}, \mathrm{H}_{3}\right), 7.28-7.18\left(1 \mathrm{H}, \mathrm{m}, \mathrm{H}_{2}\right), 6.85\left(1 \mathrm{H}, \mathrm{dd}, J=5.1,2.9 \mathrm{~Hz}, \mathrm{H}_{8}\right), 6.58$ $\left(1 \mathrm{H}, \mathrm{dd}, J=2.9,1.1 \mathrm{~Hz}, \mathrm{H}_{6}\right), 6.20\left(1 \mathrm{H}, \mathrm{dd}, J=5.1,1.2 \mathrm{~Hz}, \mathrm{H}_{7}\right), 2.31\left(3 \mathrm{H}, \mathrm{s}, \mathrm{H}_{9}\right) ;{ }^{13} \mathrm{C}$ NMR $(100$ $\left.\mathrm{MHz}, \mathrm{CDCl}_{3}\right) \delta: 161.95(\mathrm{~d}, J=8.1 \mathrm{~Hz}), 149.44(\mathrm{~d}, J=10.8 \mathrm{~Hz}), 135.84$ (d, $\left.J=7.6 \mathrm{~Hz}\right), 135.21$ (d, $J=3.0 \mathrm{~Hz}), 134.56(\mathrm{~d}, J=5.3 \mathrm{~Hz}), 133.80(\mathrm{~d}, J=9.9 \mathrm{~Hz}), 130.49(\mathrm{~d}, J=12.9 \mathrm{~Hz}), 128.05,127.68$ (d, $J=84.8 \mathrm{~Hz}), 126.48,125.97,125.86$ (d, $J=9.6 \mathrm{~Hz}), 120.69$ (q, $J=320.9 \mathrm{~Hz}), 117.16$ (d, $J=$ $89.6 \mathrm{~Hz}), 23.54(\mathrm{~d}, J=2.3 \mathrm{~Hz}) ;{ }^{19} \mathrm{~F}$ NMR $\left(365 \mathrm{MHz}, \mathrm{CDCl}_{3}\right) \delta:-78.03 ;{ }^{31} \mathrm{P} \mathrm{NMR}(162 \mathrm{MHz}$, $\left.\mathrm{CDCl}_{3}\right) \delta: 21.38 ; m / z$ LRMS (ESI + APCI) found [M - OTf $]^{+} 436.1, \mathrm{C}_{28} \mathrm{H}_{23} \mathrm{NPS}^{+}$requires 436.1 .

\footnotetext{
₹ The concentrated $\mathrm{CH}_{2} \mathrm{Cl}_{2}$ solution of crude product was added dropwise to an excess of chilled $\mathrm{Et}_{2} \mathrm{O}\left(0^{\circ} \mathrm{C}\right)$ instead of the order of addition in General Procedure A. The mixture was then placed in a $-20^{\circ} \mathrm{C}$ refrigerator for approximately 1 hour. The resulting suspension was filtered on a frit and the solid was washed with chilled $\mathrm{Et}_{2} \mathrm{O}\left(0^{\circ} \mathrm{C}\right)$. The solid was redissolved in approximately 10 $\mathrm{mL}$ of $\mathrm{CH}_{2} \mathrm{Cl}_{2}$ and was precipitated a second time via dropwise addition to an excess of chilled $\mathrm{Et}_{2} \mathrm{O}\left(0{ }^{\circ} \mathrm{C}\right)$. The resulting suspension was filtered on a frit, the solid washed with chilled $\mathrm{Et}_{2} \mathrm{O}(0$ ${ }^{\circ} \mathrm{C}$ ) and dried in vacuo to provide the pure phosphonium salt.
} 


\section{(2-Methyl-5-(phenylethynyl)pyridin-4-yl)triphenylphosphonium trifluoromethanesulfonate}

(2i)

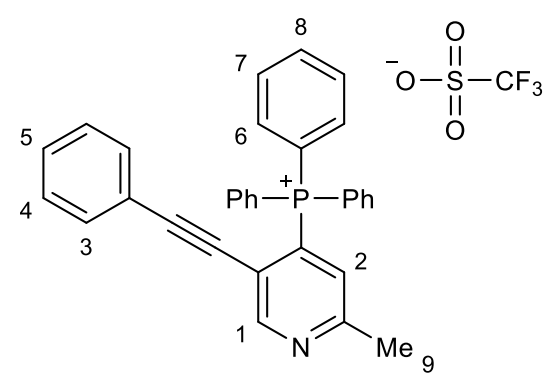

Prepared according to general procedure A using 2-methyl-5-(phenylethynyl)pyridine (860 mg, $4.45 \mathrm{mmol}), \mathrm{Tf}_{2} \mathrm{O}(749 \mu \mathrm{L}, 4.45 \mathrm{mmol}), \mathrm{PPh}_{3}(1.29 \mathrm{~g}, 4.90 \mathrm{mmol}), \mathrm{DBU}(619 \mathrm{~mL}, 4.45 \mathrm{mmol})$ and $\mathrm{CH}_{2} \mathrm{Cl}_{2}(40 \mathrm{~mL})$. After the purification procedure, the title compound was isolated as an offwhite solid (1.60 g, $2.65 \mathrm{mmol}, 60 \%$ yield). $\mathrm{mp} 188-190^{\circ} \mathrm{C}$; IR $v_{\max } / \mathrm{cm}^{-1}$ (film): 3098, 2214, 1584, 1462, 1260, 1028, 761; ${ }^{1} \mathrm{H}$ NMR (400 MHz, $\left.\mathrm{CDCl}_{3}\right) \delta: 8.96\left(1 \mathrm{H}, \mathrm{d}, J=2.8 \mathrm{~Hz}, \mathrm{H}_{1}\right), 7.86(3 \mathrm{H}, \mathrm{m}$, $\left.\mathrm{H}_{8}\right), 7.80-7.68\left(13 \mathrm{H}, \mathrm{m}, \mathrm{H}_{7}, \mathrm{H}_{6}\right.$, and $\left.\mathrm{H}_{5}\right), 7.17\left(2 \mathrm{H}\right.$, app t, $\left.J=7.7 \mathrm{~Hz}, \mathrm{H}_{4}\right), 7.09(1 \mathrm{H}, \mathrm{d}, J=15.1$ $\left.\mathrm{Hz}, \mathrm{H}_{2}\right), 6.66\left(2 \mathrm{H}, \mathrm{d}, J=7.4 \mathrm{~Hz}, \mathrm{H}_{3}\right), 2.66\left(3 \mathrm{H}, \mathrm{s}, \mathrm{H}_{9}\right) ;{ }^{13} \mathrm{C} \mathrm{NMR}\left(100 \mathrm{MHz}, \mathrm{CDCl}_{3}\right) \delta: 160.40$ (d, $J=10.4 \mathrm{~Hz}), 154.04(\mathrm{~d}, J=7.1 \mathrm{~Hz}), 135.72(\mathrm{~d}, J=3.0 \mathrm{~Hz}), 134.22(\mathrm{~d}, J=10.5 \mathrm{~Hz}), 130.83,130.67$ $(\mathrm{d}, J=13.2 \mathrm{~Hz}), 129.87,128.65$ (d, $J=85.9 \mathrm{~Hz}), 128.32,128.11(\mathrm{~d}, J=8.8 \mathrm{~Hz}), 120.75(\mathrm{q}, J=$ $321.2 \mathrm{~Hz}), 120.37$ (d, $J=4.5 \mathrm{~Hz}), 119.90,115.82$ (d, $J=90.3 \mathrm{~Hz}), 103.30,83.97$ (d, $J=6.1 \mathrm{~Hz})$, 24.83; ${ }^{19} \mathrm{~F}$ NMR $\left(365 \mathrm{MHz}, \mathrm{CDCl}_{3}\right) \delta:-78.1 ;{ }^{31} \mathrm{P} \mathrm{NMR}\left(162 \mathrm{MHz}, \mathrm{CDCl}_{3}\right) \delta: 22.8 ; \mathrm{m} / z \mathrm{LRMS}$ $(\mathrm{ESI}+\mathrm{APCI})$ found $[\mathrm{M}-\mathrm{OTf}]^{+} 454.2, \mathrm{C}_{32} \mathrm{H}_{25} \mathrm{NP}^{+}$requires 454.2.

\section{(2-Fluoro-5-methylpyridin-4-yl)triphenylphosphonium trifluoromethanesulfonate (2j)}

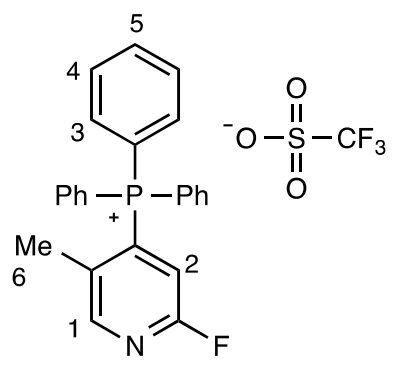


Prepared according to general procedure A using 2-fluoro-5-methylpyridine ( $620 \mu \mathrm{L}, 5.98 \mathrm{mmol})$, $\mathrm{Tf}_{2} \mathrm{O}(1.00 \mathrm{~mL}, 5.95 \mathrm{mmol}), \mathrm{PPh}_{3}(1.73 \mathrm{~g}, 6.55 \mathrm{mmol})$, DBU $(890 \mu \mathrm{L}, 5.95 \mathrm{mmol})$ and $\mathrm{CH}_{2} \mathrm{Cl}_{2}$ $(60 \mathrm{~mL})$. After the purification procedure, the title compound was isolated as a white solid (2.54 g, $4.87 \mathrm{mmol}, 82 \%$ yield). $\mathrm{mp} 160-161{ }^{\circ} \mathrm{C}$; IR $v_{\max } / \mathrm{cm}^{-1}$ (film): 3066, 1438, 1261, 1142, 1032, $720 ;{ }^{1} \mathrm{H}$ NMR $\left(400 \mathrm{MHz}, \mathrm{CDCl}_{3}\right) \delta: 8.43\left(1 \mathrm{H}, \mathrm{d}, J=6.8 \mathrm{~Hz}, \mathrm{H}_{1}\right), 7.96-7.88\left(3 \mathrm{H}, \mathrm{m}, \mathrm{H}_{5}\right), 7.87-7.79$ (6H, m, $\left.\mathrm{H}_{4}\right), 7.75-7.67\left(6 \mathrm{H}, \mathrm{m}, \mathrm{H}_{3}\right), 6.72\left(1 \mathrm{H}, \mathrm{dd}, 15.6,2.8 \mathrm{~Hz}, \mathrm{H}_{2}\right), 2.00\left(3 \mathrm{H}, \mathrm{s}, \mathrm{H}_{6}\right) ;{ }^{13} \mathrm{C}$ NMR $\left(100 \mathrm{MHz}, \mathrm{CDCl}_{3}\right) \delta: 162.44(\mathrm{dd}, J=244.1,17.6 \mathrm{~Hz}), 152.46$ (dd, $\left.J=13.7,10.7 \mathrm{~Hz}\right), 136.14(\mathrm{~d}$, $J=3.1 \mathrm{~Hz}), 134.41(\operatorname{app~t}, J=6.4 \mathrm{~Hz}), 134.11(\mathrm{~d}, J=10.7 \mathrm{~Hz}), 131.74(\mathrm{dd}, J=83.1,6.9 \mathrm{~Hz})$, 131.20 (d, $J=13.0 \mathrm{~Hz}), 120.71$ (q, $J=320.4 \mathrm{~Hz}), 115.43$ (dd, $J=40.4,11.4 \mathrm{~Hz}), 115.26$ (d, $J=$ $89.27 \mathrm{~Hz}), 18.97(\mathrm{~d}, J=3.8 \mathrm{~Hz}) ;{ }^{19} \mathrm{~F}$ NMR $\left(365 \mathrm{MHz}, \mathrm{CDCl}_{3}\right) \delta$ : $-66.58(\mathrm{~d}, J=9.4 \mathrm{~Hz}),-78.17$; ${ }^{31} \mathrm{P}$ NMR $\left(162 \mathrm{MHz}, \mathrm{CDCl}_{3}\right) \delta: 21.40(\mathrm{~d}, J=9.4 \mathrm{~Hz}) ; \mathrm{m} / z$ LRMS (ESI + APCI) found [M - OTf] 372.2, $\mathrm{C}_{24} \mathrm{H}_{20} \mathrm{FNP}^{+}$requires 372.1 .

(5-Methoxy-2-methylpyridin-4-yl)triphenylphosphonium trifluoromethanesulfonate (2k) and (3-methoxy-6-methylpyridin-2-yl)triphenylphosphonium trifluoromethanesulfonate (2k')

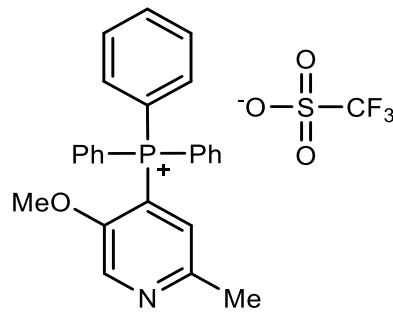

Major

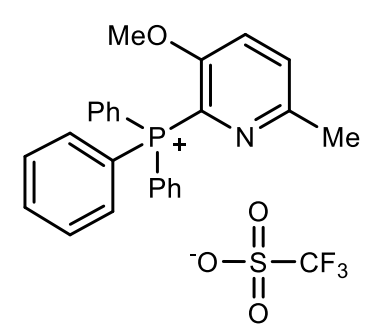

Minor

10:1 Mixture of Regioisomers

Prepared according to general procedure A using 5-methoxy-2-methylpyridine (397 mg, 3.23 $\mathrm{mmol}), \mathrm{Tf}_{2} \mathrm{O}(542 \mu \mathrm{L}, 3.23 \mathrm{mmol}), \mathrm{PPh}_{3}(931 \mathrm{mg}, 3.35 \mathrm{mmol}), \mathrm{DBU}(482 \mu \mathrm{L}, 3.23 \mathrm{mmol})$ and $\mathrm{CH}_{2} \mathrm{Cl}_{2}(32 \mathrm{~mL})$. After the purification procedure, the title compounds (10:1 mixture of regioisomers) were isolated as a white solid (1.29 g, $2.43 \mathrm{mmol}, 75 \%$ combined yield). An 
analytically pure sample of the major regioisomer was obtained by recrystallization from $\mathrm{CH}_{2} \mathrm{Cl}_{2}$ and $\mathrm{Et}_{2} \mathrm{O}$.

(5-Methoxy-2-methylpyridin-4-yl)triphenylphosphonium trifluoromethanesulfonate (2k)

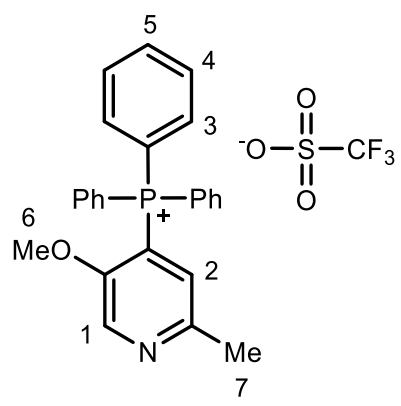

mp 211-212 ${ }^{\circ} \mathrm{C}$; IR $v_{\max } / \mathrm{cm}^{-1}$ (film): 3069, 2952, 1486, 1438, 1261, 1224, 1108, 1031; ${ }^{1} \mathrm{H}$ NMR $\left(400 \mathrm{MHz}, \mathrm{CDCl}_{3}\right) \delta: 8.52\left(1 \mathrm{H}, \mathrm{d}, J=6.9 \mathrm{~Hz}, \mathrm{H}_{1}\right), 7.95-7.40\left(15 \mathrm{H}, \mathrm{m}, \mathrm{H}_{3}, \mathrm{H}_{4}\right.$, and $\left.\mathrm{H}_{5}\right), 6.79(1 \mathrm{H}$, $\left.\mathrm{d}, J=15.0 \mathrm{~Hz}, \mathrm{H}_{2}\right), 3.62\left(3 \mathrm{H}, \mathrm{s}, \mathrm{H}_{6}\right), 2.46\left(3 \mathrm{H}, \mathrm{s}, \mathrm{H}_{7}\right) ;{ }^{13} \mathrm{C} \mathrm{NMR}\left(100 \mathrm{MHz}, \mathrm{CDCl}_{3}\right) \delta: 153.72$, $153.05(\mathrm{~d}, J=10.7 \mathrm{~Hz}), 135.62(\mathrm{~d}, J=4.6 \mathrm{~Hz}), 135.46(\mathrm{~d}, J=3.1 \mathrm{~Hz}), 133.71(\mathrm{~d}, J=10.7 \mathrm{~Hz})$, $130.51(\mathrm{~d}, J=13.0 \mathrm{~Hz}), 127.12(\mathrm{~d}, J=6.9 \mathrm{~Hz}), 120.72(\mathrm{q}, J=321.21 \mathrm{~Hz}), 116.46(\mathrm{~d}, J=91.6 \mathrm{~Hz})$, $115.62(\mathrm{~d}, J=87.0 \mathrm{~Hz}), 57.01,23.55 ;{ }^{19} \mathrm{~F} \mathrm{NMR}\left(365 \mathrm{MHz}, \mathrm{CDCl}_{3}\right) \delta:-78.13 ;{ }^{31} \mathrm{P} \mathrm{NMR}(162 \mathrm{MHz}$, $\left.\mathrm{CDCl}_{3}\right) \delta: 21.36 ; \mathrm{m} / z$ LRMS (ESI + APCI) found $[\mathrm{M}-\mathrm{OTf}]^{+} 384.2, \mathrm{C}_{25} \mathrm{H}_{23} \mathrm{NOP}^{+}$requires 384.2.

(2-Butyl-5-(trifluoromethyl)pyridin-4-yl)triphenylphosphonium trifluoromethanesulfonate (2l)

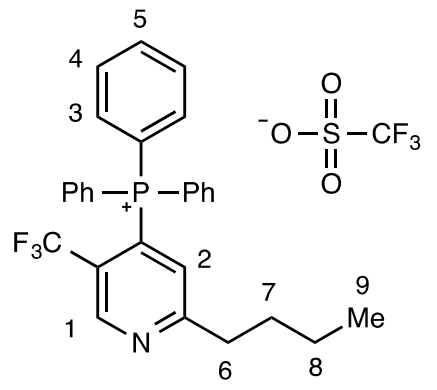


Prepared according to general procedure A using 2-butyl-5-(trifluoromethyl)pyridine (1.02 g, 5.00 $\mathrm{mmol}), \mathrm{Tf}_{2} \mathrm{O}(845 \mu \mathrm{L}, 5.00 \mathrm{mmol}), \mathrm{PPh}_{3}(1.44 \mathrm{~g}, 5.50 \mathrm{mmol}), \mathrm{DBU}(758 \mu \mathrm{L}, 5.00 \mathrm{mmol})$ and $\mathrm{CH}_{2} \mathrm{Cl}_{2}(50 \mathrm{~mL})$. After the purification procedure, the title compound was isolated as a white solid (2.55 g, $4.16 \mathrm{mmol}, 83 \%$ yield). $\mathrm{mp} 152-154{ }^{\circ} \mathrm{C}$; IR $v_{\max } / \mathrm{cm}^{-1}$ (film): 3057, 2931, 1717, 1437 , 1414, 1260, 1222, 1147, 1105, 1029; ${ }^{1} \mathrm{H} \mathrm{NMR}\left(400 \mathrm{MHz}, \mathrm{CDCl}_{3}\right) \delta: 9.16\left(1 \mathrm{H}, \mathrm{d}, J=6.8 \mathrm{~Hz}, \mathrm{H}_{1}\right)$, 7.92-7.87 (3H, m, H5), 7.80-7.76 (6H, m, H4), 7.73-7.67 (6H, m, H3), $7.18(1 \mathrm{H}, \mathrm{d}, J=17.2 \mathrm{~Hz}$, $\left.\mathrm{H}_{2}\right), 2.93\left(2 \mathrm{H}, \mathrm{t}, J=7.6 \mathrm{~Hz}, \mathrm{H}_{6}\right), 1.69-1.62\left(2 \mathrm{H}, \mathrm{m}, \mathrm{H}_{7}\right), 1.37-1.27\left(2 \mathrm{H}, \mathrm{m}, \mathrm{H}_{8}\right), 0.88(3 \mathrm{H}, \mathrm{t}, J=$ $\left.7.2 \mathrm{~Hz}, \mathrm{H}_{9}\right) ;{ }^{13} \mathrm{C} \mathrm{NMR}\left(100 \mathrm{MHz}, \mathrm{CDCl}_{3}\right) \delta: 169.99$ (d, $\left.J=9.7 \mathrm{~Hz}\right), 150.06(\mathrm{~m}), 135.96(\mathrm{~d}, J=3.1$ Hz), 134.41 (d, $J=10.4 \mathrm{~Hz}), 130.74$ (d, $J=13.0 \mathrm{~Hz}), 129.77$ (d, $J=8.5 \mathrm{~Hz}), 125.90$ (d, $J=80.1$, $1.0 \mathrm{~Hz}), 124.42(\mathrm{qd}, J=33.1,4.0 \mathrm{~Hz}), 122.49(\mathrm{qd}, J=275.1,2.9 \mathrm{~Hz}), 120.76(\mathrm{q}, J=321.2 \mathrm{~Hz})$, $116.40(\mathrm{~d}, J=90.4 \mathrm{~Hz}), 37.93,30.35,22.11,13.63 ;{ }^{19} \mathrm{~F} \mathrm{NMR}\left(365 \mathrm{MHz}, \mathrm{CDCl}_{3}\right) \delta$ : -78.27, -

53.55; ${ }^{31} \mathrm{P}$ NMR (162 MHz, $\left.\mathrm{CDCl}_{3}\right) \delta: 27.4(\mathrm{~d}, J=2.3 \mathrm{~Hz}) ; m / z$ LRMS found $[\mathrm{M}]^{+}$305.2, $\mathrm{C}_{28} \mathrm{H}_{26} \mathrm{~F}_{3} \mathrm{NP}^{+}$requires 305.2.

\section{Triphenyl(4-(trifluoromethyl)pyridin-2-yl)phosphonium trifluoromethanesulfonate (2m)}

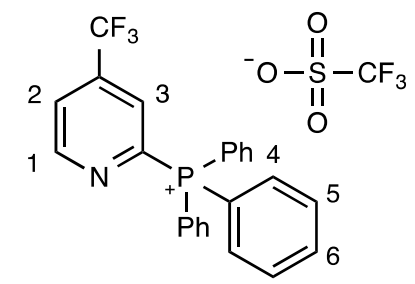

Prepared according to general procedure A using 4-(trifluoromethyl)pyridine (926 $\mu \mathrm{L}, 8.00$ $\mathrm{mmol}), \mathrm{Tf}_{2} \mathrm{O}$ (1.35 mL, $\left.8.00 \mathrm{mmol}\right), \mathrm{PPh}_{3}(2.31 \mathrm{~g}, 8.80 \mathrm{mmol}), \mathrm{DBU}(1.20 \mathrm{~mL}, 8.00 \mathrm{mmol})$ and $\mathrm{CH}_{2} \mathrm{Cl}_{2}(80 \mathrm{~mL})$. After the purification procedure, the title compound was isolated as an off-white solid (3.69 g, $6.62 \mathrm{mmol}, 83 \%$ yield). $\mathrm{mp} 110-112{ }^{\circ} \mathrm{C}$; IR $v_{\max } / \mathrm{cm}^{-1}$ (film): 3071, 1588, 1485 , 1390, 1223, 1180, 998, 572, 535; ${ }^{1} \mathrm{H}$ NMR (400 MHz, $\left.\mathrm{CDCl}_{3}\right) \delta: 9.33\left(1 \mathrm{H}, \mathrm{d}, J=2.8 \mathrm{~Hz}, \mathrm{H}_{1}\right), 8.02$ $\left(1 \mathrm{H}, \mathrm{m}, \mathrm{H}_{2}\right), 7.92\left(3 \mathrm{H}, \mathrm{m}, \mathrm{H}_{6}\right), 7.82-7.69\left(13 \mathrm{H}, \mathrm{m}, \mathrm{H}_{3}, \mathrm{H}_{4}\right.$, and $\left.\mathrm{H}_{5}\right) ;{ }^{13} \mathrm{C} \mathrm{NMR}\left(100 \mathrm{MHz}, \mathrm{CDCl}_{3}\right)$ $\delta: 154.20(\mathrm{~d}, J=19.9 \mathrm{~Hz}), 147.00(\mathrm{~d}, J=121.0 \mathrm{~Hz}), 139.92(\mathrm{qd}, J=35.8 \mathrm{~Hz}, 11.3 \mathrm{~Hz}), 136.02$ (d, $J=2.9 \mathrm{~Hz}), 134.55(\mathrm{~d}, J=10.2 \mathrm{~Hz}), 130.70(\mathrm{~d}, J=13.1 \mathrm{~Hz}), 126.05$ (dq, $J=25.9,3.6 \mathrm{~Hz}), 124.36$ (m), $121.47(\mathrm{qd}, J=274.1,3.0 \mathrm{~Hz}), 120.70(\mathrm{q}, J=320.5 \mathrm{~Hz}), 115.93(\mathrm{~d}, J=90.0 \mathrm{~Hz}){ }^{19} \mathrm{~F}$ NMR 
$\left(365 \mathrm{MHz}, \mathrm{CDCl}_{3}\right) \delta:-64.7,-78.2 ;{ }^{31} \mathrm{P}$ NMR $\left(162 \mathrm{MHz}, \mathrm{CDCl}_{3}\right) \delta: 16.2 ; \mathrm{m} / z$ LRMS (ESI + $\mathrm{APCI}$ ) found [M - OTf $]^{+} 408.1, \mathrm{C}_{24} \mathrm{H}_{18} \mathrm{~F}_{3} \mathrm{NP}^{+}$requires 408.1 .

\section{(4-bromopyridin-2-yl)triphenylphosphonium trifluoromethanesulfonate (2n)}

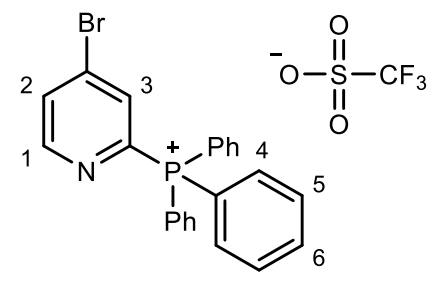

Prepared according to general procedure $\mathrm{A}^{\S}$ using 4-bromopyridine (198 $\left.\mu \mathrm{L}, 2.00 \mathrm{mmol}\right), \mathrm{Tf}_{2} \mathrm{O}$ (338 $\mu \mathrm{L}, 2.00 \mathrm{mmol}), \mathrm{PPh}_{3}(577 \mathrm{mg}, 2.20 \mathrm{mmol}), \mathrm{DBU}(303 \mu \mathrm{L}, 2.00 \mathrm{mmol})$ and $\mathrm{CH}_{2} \mathrm{Cl}_{2}(40 \mathrm{~mL})$. After the purification procedure, ${ }^{* *}$ the title compound was isolated as a tan solid (677 mg, 1.20 mmol, $60 \%$ yield). $\mathrm{mp} 147-149^{\circ} \mathrm{C}$; IR $v_{\max } / \mathrm{cm}^{-1}$ (film): 3087, 3061, 1553, 1436, 1258, 1137, 1110 , 1028, 635; ${ }^{1} \mathrm{H}$ NMR $\left(400 \mathrm{MHz}, \mathrm{CDCl}_{3}\right) \delta: 8.88\left(1 \mathrm{H}, \mathrm{d}, J=5.1 \mathrm{~Hz}, \mathrm{H}_{1}\right), 8.03-7.84\left(4 \mathrm{H}, \mathrm{m}, \mathrm{H}_{3}\right.$ and $\left.\mathrm{H}_{6}\right), 7.84-7.59\left(13 \mathrm{H}, \mathrm{m}, \mathrm{H}_{2}, \mathrm{H}_{4}\right.$, and $\left.\mathrm{H}_{5}\right) ;{ }^{13} \mathrm{C} \mathrm{NMR}\left(100 \mathrm{MHz}, \mathrm{CDCl}_{3}\right) \delta: 153.28(\mathrm{~d}, J=20.6 \mathrm{~Hz})$, 146.37 (d, $J=119.1 \mathrm{~Hz}), 135.97$ (d, $J=3.1 \mathrm{~Hz}), 135.16$ (d, $J=14.4 \mathrm{~Hz}), 134.55$ (d, $J=10.8 \mathrm{~Hz})$, $134.00(\mathrm{~d}, J=25.2 \mathrm{~Hz}), 131.88(\mathrm{~d}, J=3.1 \mathrm{~Hz}), 130.69$ (d, $J=13.6 \mathrm{~Hz}), 120.80$ (q, $J=321.0 \mathrm{~Hz})$, $116.21(\mathrm{~d}, J=89.2 \mathrm{~Hz}) ;{ }^{19} \mathrm{~F}$ NMR $\left(365 \mathrm{MHz}, \mathrm{CDCl}_{3}\right) \delta:-78.1 ;{ }^{31} \mathrm{P}$ NMR $(162 \mathrm{MHz}$, $\left.\mathrm{CDCl}_{3}\right) \delta: 15.6 ; \mathrm{m} / z \mathrm{LRMS}(\mathrm{ESI}+\mathrm{APCI})$ found $[\mathrm{M}-\mathrm{OTf}]^{+} 418.1, \mathrm{C}_{23} \mathrm{H}_{18} \mathrm{BrNP}^{+}$requires 418.0.

\footnotetext{
${ }^{\S}$ A solution of 4-bromopyridine in $\mathrm{CH}_{2} \mathrm{Cl}_{2}(20 \mathrm{~mL})$ was added to a solution of $\mathrm{Tf}_{2} \mathrm{O}$ in $\mathrm{CH}_{2} \mathrm{Cl}_{2}$ $(20 \mathrm{~mL})$ at $-78^{\circ} \mathrm{C}$ over 10 minutes instead of the order of addition in General Procedure A. ** Once the organic layer was separated, dried $\left(\mathrm{MgSO}_{4}\right)$, filtered, and concentrated in vacuo, the title compound was isolated by recrystallization from $\mathrm{CH}_{2} \mathrm{Cl}_{2}$ and $\mathrm{Et}_{2} \mathrm{O}$, instead of precipitated according to General Procedure A.
} 


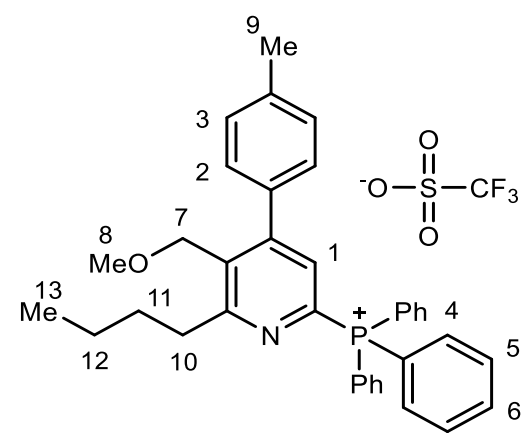

Prepared according to general procedure A using 2-butyl-3-(methoxymethyl)-4-( $p$-tolyl)pyridine (483 mg, $1.79 \mathrm{mmol}), \mathrm{Tf}_{2} \mathrm{O}(300 \mu \mathrm{L}, 1.79 \mathrm{mmol}), \mathrm{PPh}_{3}(517 \mathrm{mg}, 1.97 \mathrm{mmol}), \mathrm{DBU}(268 \mu \mathrm{L}, 1.79$ $\mathrm{mmol})$ and $\mathrm{CH}_{2} \mathrm{Cl}_{2}(60 \mathrm{~mL})$. After the purification procedure, the title compound was isolated as a white solid (909 mg, $1.34 \mathrm{mmol}, 75 \%$ yield). $\mathrm{mp} 149-150{ }^{\circ} \mathrm{C}$; IR $v_{\max } / \mathrm{cm}^{-1}$ (film): 3059, 2956, 2869, 1568, 1438, 1261, 1151, $1031 ;{ }^{1} \mathrm{H}$ NMR (400 MHz, $\left.\mathrm{CDCl}_{3}\right)$ 8: 7.91-7.82 (3H, m, H6), 7.78$7.62\left(12 \mathrm{H}, \mathrm{m}, \mathrm{H}_{5}\right.$ and $\left.\mathrm{H}_{4}\right), 7.35\left(1 \mathrm{H}, \mathrm{d}, J=6.4 \mathrm{~Hz}, \mathrm{H}_{1}\right), 7.26-7.23\left(4 \mathrm{H}, \mathrm{m}, \mathrm{H}_{3}\right.$ and $\left.\mathrm{H}_{2}\right), 4.35(2 \mathrm{H}$, s, $\left.\mathrm{H}_{7}\right), 3.38\left(3 \mathrm{H}, \mathrm{s}, \mathrm{H}_{8}\right), 3.06\left(2 \mathrm{H}, \mathrm{t}, J=7.7 \mathrm{~Hz}, \mathrm{H}_{10}\right), 2.35\left(3 \mathrm{H}, \mathrm{s}, \mathrm{H}_{9}\right), 1.71(2 \mathrm{H}, \mathrm{qn}, J=7.7 \mathrm{~Hz}$, $\left.\mathrm{H}_{11}\right), 1.33\left(2 \mathrm{H}\right.$, sext, $\left.J=7.6 \mathrm{~Hz}, \mathrm{H}_{12}\right), 0.87\left(3 \mathrm{H}, \mathrm{t}, J=7.5 \mathrm{~Hz}, \mathrm{H}_{13}\right) ;{ }^{13} \mathrm{C} \mathrm{NMR}\left(100 \mathrm{MHz}, \mathrm{CDCl}_{3}\right)$ $\delta: 167.00(\mathrm{~d}, J=19.1 \mathrm{~Hz}), 152.58(\mathrm{~d}, J=11.4 \mathrm{~Hz}), 143.18,141.99,139.39,135.63(\mathrm{~d}, J=3.1 \mathrm{~Hz})$, $134.47(\mathrm{~d}, J=10.7 \mathrm{~Hz}), 133.60(\mathrm{~d}, J=1.5 \mathrm{~Hz}), 133.19$ (d, $J=3.8 \mathrm{~Hz}), 130.42$ (d, $J=13.0 \mathrm{~Hz})$, $129.91(\mathrm{~d}, J=25.2 \mathrm{~Hz}), 129.04$ (d, $J=84.7 \mathrm{~Hz}), 120.83$ (q, $J=321.2 \mathrm{~Hz}), 117.07$ (d, $J=89.27$ $\mathrm{Hz}), 67.93,58.68,34.27,30.58,22.31,21.08,13.83 ;{ }^{19} \mathrm{~F} \mathrm{NMR}\left(365 \mathrm{MHz}, \mathrm{CDCl}_{3}\right) \delta:-78.07 ;{ }^{31} \mathrm{P}$ NMR (162 MHz, $\left.\mathrm{CDCl}_{3}\right) \delta: 14.49 ; m / z$ LRMS (ESI + APCI) found [M - OTf] $]^{+} 530.3, \mathrm{C}_{36} \mathrm{H}_{37} \mathrm{NOP}^{+}$ requires 530.3 . 


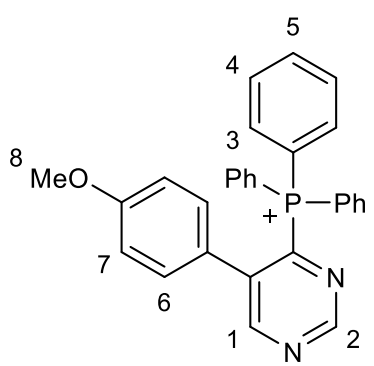

Major
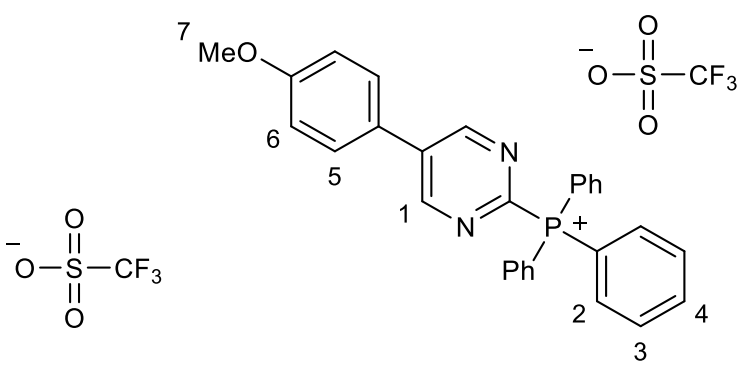

Minor

24:1 Mixture of Regioisomers

Prepared according to general procedure A (except that the stirring time after addition of $\mathrm{PPh}_{3}$ was 1 hour instead of 30 minutes) using 5-(4-methoxyphenyl)pyrimidine (450 mg, $2.42 \mathrm{mmol}$ ), $\mathrm{Tf}_{2} \mathrm{O}$ (409 $\mu \mathrm{L}, 2.42 \mathrm{mmol}), \mathrm{PPh}_{3}(698 \mathrm{mg}, 2.66 \mathrm{mmol})$, DBU (367 $\left.\mu \mathrm{L}, 2.42 \mathrm{mmol}\right)$ and $\mathrm{CH}_{2} \mathrm{Cl}_{2}(24 \mathrm{~mL})$. After the purification procedure ${ }^{\dagger \dagger}$ the title compound was isolated as a tan solid $(724 \mathrm{mg}, 1.21$ mmol, 50\% combined yield). Both isomers, IR $v_{\max } / \mathrm{cm}^{-1}$ (film): 3063, 2936, 1396, 1258, 1222, 1146, 1105, 1028, 634; Major isomer, ${ }^{1} \mathrm{H} \mathrm{NMR}\left(400 \mathrm{MHz}, \mathrm{CDCl}_{3}\right) \delta: 9.44\left(1 \mathrm{H}, \mathrm{s}, \mathrm{H}_{2}\right), 8.98(1 \mathrm{H}$, $\left.\mathrm{d}, J=9.0 \mathrm{~Hz}, \mathrm{H}_{1}\right), 7.80-7.70\left(3 \mathrm{H}, \mathrm{m}, \mathrm{H}_{5}\right), 7.67-7.56\left(12 \mathrm{H}, \mathrm{m}, \mathrm{H}_{3}\right.$ and $\left.\mathrm{H}_{4}\right), 6.91(2 \mathrm{H}, \mathrm{d}, J=8.7 \mathrm{~Hz}$, $\left.\mathrm{H}_{6}\right), 6.55\left(2 \mathrm{H}, \mathrm{d}, J=8.7 \mathrm{~Hz}, \mathrm{H}_{7}\right), 3.72\left(3 \mathrm{H}, \mathrm{s}, \mathrm{H}_{8}\right)$; Minor isomer, ${ }^{1} \mathrm{H}$ NMR $\left(400 \mathrm{MHz}, \mathrm{CDCl}_{3}\right) \delta$ : $9.23\left(2 \mathrm{H}, \mathrm{s}, \mathrm{H}_{1}\right), 7.80-7.70\left(3 \mathrm{H}, \mathrm{m}, \mathrm{H}_{4}\right), 7.70\left(2 \mathrm{H}, \mathrm{d}, J=8.7 \mathrm{~Hz}, \mathrm{H}_{5}\right), 7.67-7.56\left(12 \mathrm{H}, \mathrm{m}, \mathrm{H}_{2}\right.$ and $\left.\mathrm{H}_{3}\right), 7.09\left(2 \mathrm{H}, \mathrm{d}, J=8.6 \mathrm{~Hz}, \mathrm{H}_{6}\right), 3.88\left(3 \mathrm{H}, \mathrm{s}, \mathrm{H}_{7}\right)$; Major isomer, ${ }^{13} \mathrm{C} \mathrm{NMR}\left(100 \mathrm{MHz}, \mathrm{CDCl}_{3}\right)$ $\delta: 161.84(\mathrm{~d}, J=5.3 \mathrm{~Hz}), 160.53,156.97(\mathrm{~d}, J=16.8 \mathrm{~Hz}), 149.74(\mathrm{~d}, J=114.5 \mathrm{~Hz}), 142.72(\mathrm{~d}, J$ $=19.2 \mathrm{~Hz}), 135.22(\mathrm{~d}, J=3.1 \mathrm{~Hz}), 134.67(\mathrm{~d}, J=10.2 \mathrm{~Hz}), 130.60,130.25(\mathrm{~d}, J=13.1 \mathrm{~Hz}), 123.61$,

\footnotetext{
${ }^{++}$The concentrated $\mathrm{CH}_{2} \mathrm{Cl}_{2}$ solution of crude product was added dropwise to an excess of chilled $\mathrm{Et}_{2} \mathrm{O}\left(0^{\circ} \mathrm{C}\right)$ instead of the order of addition in General Procedure A. The mixture was then placed in a $-20^{\circ} \mathrm{C}$ refrigerator for approximately 1 hour. The resulting suspension was filtered on a frit and the solid was washed with chilled $\mathrm{Et}_{2} \mathrm{O}\left(0^{\circ} \mathrm{C}\right)$. The solid was redissolved in approximately 10 $\mathrm{mL}$ of $\mathrm{CH}_{2} \mathrm{Cl}_{2}$ and was precipitated a second time via dropwise addition to an excess of chilled $\mathrm{Et}_{2} \mathrm{O}\left(0^{\circ} \mathrm{C}\right)$. The resulting suspension was filtered on a frit, the solid washed with chilled $\mathrm{Et}_{2} \mathrm{O}(0$ ${ }^{\circ} \mathrm{C}$ ) and dried in vacuo to provide the pure phosphonium salt.
} 
120.82 (q, $J=321.3 \mathrm{~Hz}), 117.10(\mathrm{~d}, J=88.6 \mathrm{~Hz}), 114.37,55.42$; Both isomers, ${ }^{19} \mathrm{~F}$ NMR $(365$ $\left.\mathrm{MHz}, \mathrm{CDCl}_{3}\right) \delta:-78.01$; Major isomer, ${ }^{31} \mathrm{P}$ NMR $\left(162 \mathrm{MHz}, \mathrm{CDCl}_{3}\right) \delta: 17.84 ; m / z$ LRMS (ESI + APCI) found [M - OTf] $]^{+} 447.2, \mathrm{C}_{29} \mathrm{H}_{24} \mathrm{~N}_{2} \mathrm{OP}^{+}$requires 447.2.

Triphenyl(pyrazin-2-yl)phosphonium trifluoromethanesulfonate (2q)

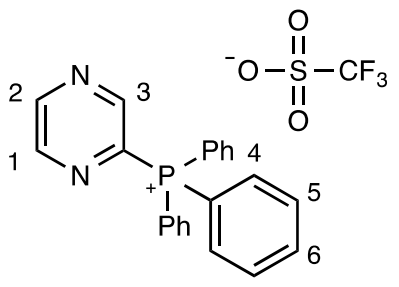

Prepared according to general procedure A using pyrazine (120 mg, $1.50 \mathrm{mmol}), \mathrm{Tf}_{2} \mathrm{O}(252 \mu \mathrm{L}$, $1.50 \mathrm{mmol}), \mathrm{PPh}_{3}(432 \mathrm{mg}, 1.65 \mathrm{mmol}), \mathrm{DBU}(224 \mu \mathrm{L}, 1.50 \mathrm{mmol})$ and $\mathrm{CH}_{2} \mathrm{Cl}_{2}(13.0 \mathrm{~mL})$. After the purification procedure, the title compound was isolated as an off-white solid (525 mg, 1.07 mmol, $80 \%$ yield). $\mathrm{mp} 185-188^{\circ} \mathrm{C}$; IR $v_{\max } / \mathrm{cm}^{-1}$ (film): 3066, 1587, 1484, 1395, 1186, 617, 571; ${ }^{1} \mathrm{H} \mathrm{NMR}\left(400 \mathrm{MHz}, \mathrm{CDCl}_{3}\right)$ 8: $9.09(1 \mathrm{H}, \mathrm{br} \mathrm{s}), 9.06(1 \mathrm{H}, \mathrm{br} \mathrm{s}), 8.86(1 \mathrm{H}, \mathrm{br} \mathrm{s}), 7.91(3 \mathrm{H}, \mathrm{m}), 7.82-$ $7.71(12 \mathrm{H}, \mathrm{m}) ;{ }^{13} \mathrm{C} \mathrm{NMR}\left(100 \mathrm{MHz}, \mathrm{CDCl}_{3}\right) \delta: 149.72(\mathrm{~d}, J=24.0 \mathrm{~Hz}), 149.51(\mathrm{~d}, J=3.4 \mathrm{~Hz})$, 147.25 (d, $J=14.7 \mathrm{~Hz}), 141.37$ (d, $J=115.4 \mathrm{~Hz}), 136.02$ (d, $J=3.1 \mathrm{~Hz}), 134.54$ (d, $J=10.4 \mathrm{~Hz})$, $130.71(\mathrm{~d}, J=13.1 \mathrm{~Hz}), 120.69$ (q, $J=321.1 \mathrm{~Hz}), 115.71$ (d, $J=89.3 \mathrm{~Hz}) ;{ }^{19} \mathrm{~F}$ NMR $(365 \mathrm{MHz}$, $\left.\mathrm{CDCl}_{3}\right) \delta:-78.2 ;{ }^{31} \mathrm{P} \mathrm{NMR}\left(162 \mathrm{MHz}, \mathrm{CDCl}_{3}\right) \delta: 13.9 ; \mathrm{m} / z \mathrm{LRMS}(\mathrm{ESI}+\mathrm{APCI})$ found [M - OTf ${ }^{+}$ 341.1, $\mathrm{C}_{22} \mathrm{H}_{18} \mathrm{~N}_{2} \mathrm{P}^{+}$requires 341.1.

\section{(S)-(3-(1-methylpyrrolidin-2-yl)pyridin-4-yl)triphenylphosphonium}

\section{trifluoromethanesulfonate (4)}

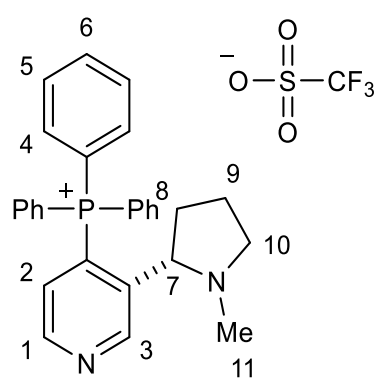


Prepared according to general procedure A using (-)-nicotine ( $803 \mu \mathrm{L}, 5.00 \mathrm{mmol}), \mathrm{Tf}_{2} \mathrm{O}(845 \mu \mathrm{L}$, $5.00 \mathrm{mmol}), \mathrm{PPh}_{3}(1.44 \mathrm{~g}, 5.50 \mathrm{mmol}), \mathrm{DBU}(748 \mu \mathrm{L}, 5.00 \mathrm{mmol})$ and $\mathrm{CH}_{2} \mathrm{Cl}_{2}(50 \mathrm{~mL})$. After the purification procedure, the title compound was isolated as a tan solid $(2.19 \mathrm{~g}, 3.82 \mathrm{mmol}, 76 \%$ yield). mp $178-181^{\circ} \mathrm{C}$; IR $v_{\max } / \mathrm{cm}^{-1}$ (film): 3066, 2971, 2758, 1338, 1264, 1223, 1142, 1108, 1102 , 1031, 719, 636; ${ }^{1} \mathrm{H}$ NMR $\left(400 \mathrm{MHz}, \mathrm{CDCl}_{3}\right) \delta: 9.37\left(1 \mathrm{H}, \mathrm{d}, J=6.9 \mathrm{~Hz}, \mathrm{H}_{3}\right), 8.79(1 \mathrm{H}$, app t, $J=$ 4.6 Hz, $\left.\mathrm{H}_{1}\right), 7.96-7.62\left(15 \mathrm{H}, \mathrm{m}, \mathrm{H}_{4}, \mathrm{H}_{5}\right.$, and $\left.\mathrm{H}_{6}\right), 7.12\left(1 \mathrm{H}, \mathrm{dd}, J=15.5,5.1 \mathrm{~Hz}, \mathrm{H}_{2}\right), 3.10-2.93$ $\left(2 \mathrm{H}, \mathrm{m}, \mathrm{H}_{7}\right.$ and $\left.\mathrm{H}_{10}\right), 1.99\left(1 \mathrm{H}\right.$, app q, $\left.J=8.5 \mathrm{~Hz}, \mathrm{H}_{10}\right), 1.87-1.67$ (4H, $\mathrm{m}, \mathrm{H}_{9}$ and $\left.\mathrm{H}_{11}\right), 1.49-1.23$ $\left(2 \mathrm{H}, \mathrm{m}, \mathrm{H}_{8}\right.$ and $\left.\mathrm{H}_{9}\right), 1.01-0.83\left(1 \mathrm{H}, \mathrm{m}, \mathrm{H}_{8}\right) ;{ }^{13} \mathrm{C} \mathrm{NMR}\left(100 \mathrm{MHz}, \mathrm{CDCl}_{3}\right) \delta: 153.01$ (d, $\left.J=8.1 \mathrm{~Hz}\right)$, $149.96(\mathrm{~d}, J=10.6 \mathrm{~Hz}), 144.24$ (d, $J=6.7 \mathrm{~Hz}), 135.99$ (d, $J=3.0 \mathrm{~Hz}), 134.39$ (d, $J=10.2 \mathrm{~Hz})$, $131.05(\mathrm{~d}, J=13.0 \mathrm{~Hz}), 127.53(\mathrm{~d}, J=9.9 \mathrm{~Hz}), 126.24$ (d, $J=81.6 \mathrm{~Hz}), 120.83$ (q, $J=321.3 \mathrm{~Hz})$, $116.74(\mathrm{~d}, J=88.5 \mathrm{~Hz}), 66.03$ (d, $J=4.9 \mathrm{~Hz}), 55.97,39.39,35.43,22.93 ;{ }^{19} \mathrm{~F}$ NMR $(365 \mathrm{MHz}$, $\left.\mathrm{CDCl}_{3}\right) \delta:-77.01 ;{ }^{31} \mathrm{P} \mathrm{NMR}\left(162 \mathrm{MHz}, \mathrm{CDCl}_{3}\right) \delta: 20.69 ; \mathrm{m} / z$ LRMS (ESI + APCI) found [M $\mathrm{OTf}]^{+}$423.2, $\mathrm{C}_{28} \mathrm{H}_{28} \mathrm{~N}_{2} \mathrm{P}^{+}$requires 423.2 .

\section{(8-Chloro-11-(1-(ethoxycarbonyl)piperidin-4-ylidene)-6,11-dihydro-5 $\mathrm{H}$ -}

benzo[5,6]cyclohepta[1,2-b]pyridin-4-yl)triphenylphosphonium trifluoromethanesulfonate (6)

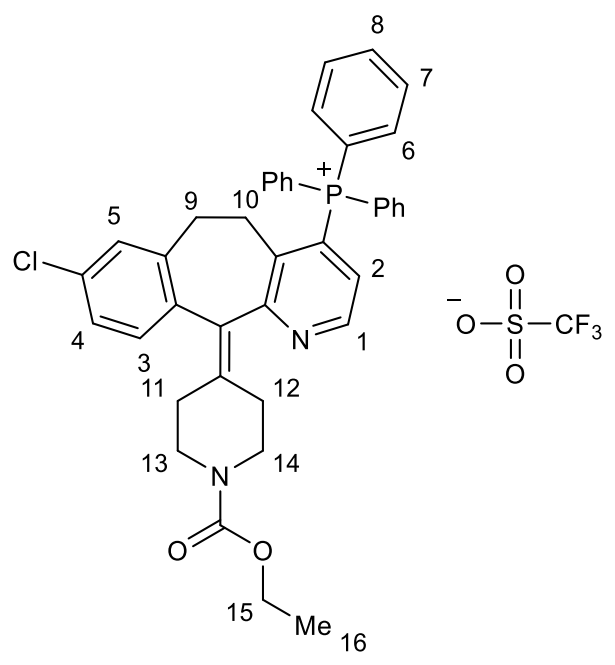

Prepared according to general procedure A using Loratadine (ethyl-4-(8-chloro-5,6-dihydro-11Hbenzo[5,6]cyclohepta[1,2-b]pyridine-11-ylidene)-1-piperidinecarboxylate) (750 mg, $1.96 \mathrm{mmol}$ ), 
$\mathrm{Tf}_{2} \mathrm{O}(329 \mu \mathrm{L}, 1.96 \mathrm{mmol}), \mathrm{PPh}_{3}(565 \mathrm{mg}, 2.15 \mathrm{mmol})$, DBU $(300 \mu \mathrm{L}, 1.96 \mathrm{mmol})$ and $\mathrm{CH}_{2} \mathrm{Cl}_{2}$ $(20 \mathrm{~mL})$. After the purification procedure, the title compound was isolated as a white solid (1.37 g, $1.72 \mathrm{mmol}, 88 \%$ yield). mp 129-136 ${ }^{\circ} \mathrm{C}$; IR $v_{\max } / \mathrm{cm}^{-1}$ (film): 3061, 2978, 2908, 2868, 1437 , 1261, 1221, 1147, 1106, 1029, 635; ${ }^{1} \mathrm{H} \mathrm{NMR}\left(400 \mathrm{MHz}, \mathrm{CDCl}_{3}\right) \delta: 8.73(1 \mathrm{H}$, app t, $J=5.0 \mathrm{~Hz}$, $\left.\mathrm{H}_{1}\right)$, 7.97-7.87 (3H, m, $\left.\mathrm{H}_{8}\right), 7.86-7.74\left(6 \mathrm{H}, \mathrm{m}, \mathrm{H}_{7}\right), 7.73-7.60\left(6 \mathrm{H}, \mathrm{m}, \mathrm{H}_{6}\right)$, 7.16-7.01 (3H, m, $\mathrm{H}_{2}$, $\mathrm{H}_{3}$, and $\left.\mathrm{H}_{4}\right), 6.71\left(1 \mathrm{H}, \mathrm{s}, \mathrm{H}_{5}\right), 4.14\left(2 \mathrm{H}, \mathrm{q}, J=7.0 \mathrm{~Hz}, \mathrm{H}_{15}\right), 3.84-3.61\left(2 \mathrm{H}, \mathrm{m}, \mathrm{H}_{13}\right.$ or $\left.\mathrm{H}_{14}\right), 3.45-$ $3.20\left(3 \mathrm{H}, \mathrm{m}, \mathrm{H}_{10}\right.$ and $\mathrm{H}_{13}$ or $\left.\mathrm{H}_{14}\right), 2.75\left(1 \mathrm{H}, \mathrm{dt}, J=17.4,4.7 \mathrm{~Hz}, \mathrm{H}_{9}\right), 2.58(1 \mathrm{H}, \mathrm{dt}, J=14.9,4.7$ $\left.\mathrm{Hz}, \mathrm{H}_{10}\right), 2.53-2.30\left(3 \mathrm{H}, \mathrm{m}, \mathrm{H}_{11}\right.$ or $\left.\mathrm{H}_{12}\right), 2.26-2.09$ (1H, m, $\mathrm{H}_{11}$ or $\left.\mathrm{H}_{12}\right), 1.60-1.43\left(1 \mathrm{H}, \mathrm{m}, \mathrm{H}_{9}\right), 1.25$ $\left(3 \mathrm{H}, \mathrm{t}, J=7.2 \mathrm{~Hz}, \mathrm{H}_{16}\right) ;{ }^{13} \mathrm{C} \mathrm{NMR}\left(100 \mathrm{MHz} \mathrm{CDCl}_{3}\right) \delta: 163.64(\mathrm{~d}, J=8.3 \mathrm{~Hz}), 155.37,149.08$ (d, $J=11.4 \mathrm{~Hz}), 139.23,136.84,136.66(\mathrm{~d}, J=6.8 \mathrm{~Hz}), 136.06(\mathrm{~d}, J=3.1 \mathrm{~Hz}), 134.21(\mathrm{~d}, J=10.7$ $\mathrm{Hz}), 133.95,133.57,132.37,131.58,131.13$ (d, $J=13.0 \mathrm{~Hz}), 129.85,127.22$ ( d, $J=10.0 \mathrm{~Hz}$ ), 127.01 (d, $J=82.2 \mathrm{~Hz}), 126.43,120.78$ (q, $J=321.3 \mathrm{~Hz}), 116.42$ (d, $J=88.5 \mathrm{~Hz}), 61.39$, 44.65, 44.41, 30.74, 30.46, 30.39, 29.39, 14.59; ${ }^{19} \mathrm{~F}$ NMR (365 MHz, $\left.\mathrm{CDCl}_{3}\right) \delta:-78.16 ;{ }^{31} \mathrm{P}$ NMR (162 $\left.\mathrm{MHz}, \mathrm{CDCl}_{3}\right) \delta: 21.17 ; \mathrm{m} / \mathrm{z} \operatorname{LRMS}(\mathrm{ESI}+\mathrm{APCI})$ found $[\mathrm{M}-\mathrm{OTf}]^{+} 643.2, \mathrm{C}_{40} \mathrm{H}_{37} \mathrm{ClN}_{2} \mathrm{O}_{2} \mathrm{P}^{+}$ requires 643.2 .

\section{(2-(1-(4-Chlorophenyl)-3-(dimethylamino)propyl)pyridin-4-yl)triphenylphosphonium}

\section{trifluoromethanesulfonate (8)}

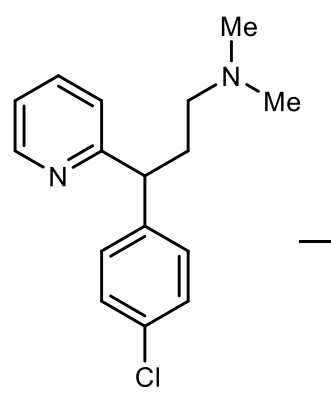

Chlorphenamine
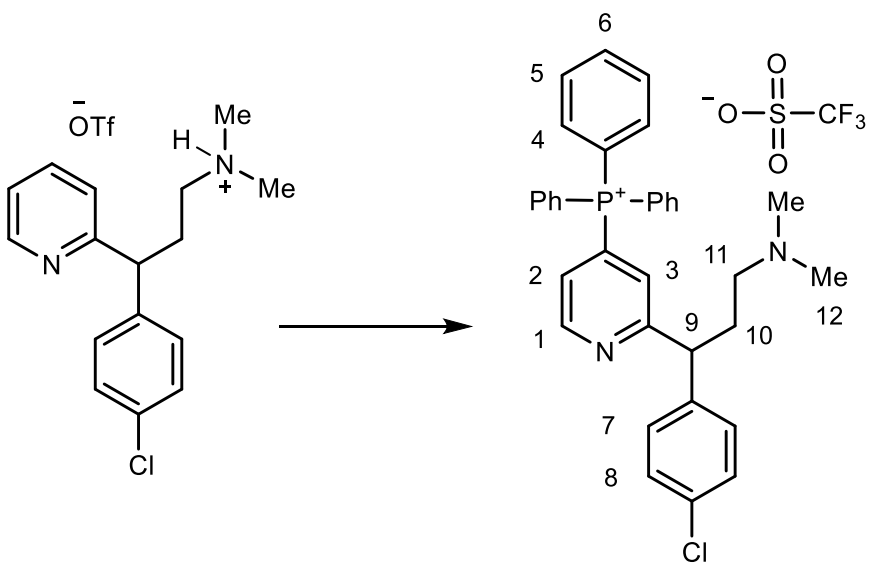

Chlorphenamine $(1.64 \mathrm{~g}, 5.95 \mathrm{mmol})$ was dissolved in $\mathrm{Et}_{2} \mathrm{O}(10 \mathrm{~mL})$ and cooled to $0{ }^{\circ} \mathrm{C}$. Trifluoromethanesulfonic acid $(527 \mu \mathrm{L}, 5.95 \mathrm{mmol})$ was added dropwise, the ice bath was 
removed and the solution was stirred for 10 minutes at room temperature. The solution was concentrated in vacuo and the resulting acid salt was subjected to general procedure A (expect that the product was precipitated a second time using the same protocol and the product suspension was placed in a $-20{ }^{\circ} \mathrm{C}$ refrigerator for 12 hours instead of 1) using $\mathrm{Tf}_{2} \mathrm{O}(1.0 \mathrm{~mL}, 5.95 \mathrm{mmol})$, $\mathrm{PPh}_{3}(1.73 \mathrm{~g}, 6.55 \mathrm{mmol}), \mathrm{DBU}(1.78 \mathrm{~mL}, 11.90 \mathrm{mmol})$ and $\mathrm{CH}_{2} \mathrm{Cl}_{2}(60 \mathrm{~mL})$. After the purification procedure, the title compound was isolated as a yellow solid (1.63 g, $2.38 \mathrm{mmol}, 40 \%$ yield). $\mathrm{mp}$ 52-58 ${ }^{\circ} \mathrm{C}$; IR $v_{\max } / \mathrm{cm}^{-1}$ (film): 3062, 2941, 2818, 2770, 1438, 1260, 1108, 1029; ${ }^{1} \mathrm{H}$ NMR (400 $\left.\mathrm{MHz}, \mathrm{CDCl}_{3}\right) \delta: 8.97\left(1 \mathrm{H}\right.$, app t, $\left.J=5.1 \mathrm{~Hz}, \mathrm{H}_{1}\right), 7.93-7.86\left(3 \mathrm{H}, \mathrm{m}, \mathrm{H}_{6}\right), 7.80-7.70\left(6 \mathrm{H}, \mathrm{m}, \mathrm{H}_{5}\right)$, 7.61-7.50 (6H, m, $\left.\mathrm{H}_{4}\right), 7.39\left(1 \mathrm{H}\right.$, ddd, $\left.J=12.8,5.1,1.5 \mathrm{~Hz}, \mathrm{H}_{2}\right), 7.25-7.16\left(5 \mathrm{H}, \mathrm{m}, \mathrm{H}_{3}, \mathrm{H}_{7}\right.$, and $\left.\mathrm{H}_{8}\right), 4.28\left(1 \mathrm{H}\right.$, app t, $\left.J=6.8 \mathrm{~Hz}, \mathrm{H}_{9}\right), 2.56-2.43\left(1 \mathrm{H}, \mathrm{m}, \mathrm{H}_{10}\right), 2.32-2.11\left(9 \mathrm{H}, \mathrm{m}, \mathrm{H}_{10}, \mathrm{H}_{11}\right.$, and $\left.\mathrm{H}_{12}\right)$; ${ }^{13} \mathrm{C}$ NMR $\left(100 \mathrm{MHz}, \mathrm{CDCl}_{3}\right) \delta: 165.55(\mathrm{~d}, J=9.9 \mathrm{~Hz}), 150.97(\mathrm{~d}, J=9.9 \mathrm{~Hz}), 140.26,135.82(\mathrm{~d}$, $J=3.1 \mathrm{~Hz}), 134.02(\mathrm{~d}, J=10.7 \mathrm{~Hz}), 132.25,130.61(\mathrm{~d}, J=13.0 \mathrm{~Hz}), 128.92(\mathrm{~d}, J=85.5 \mathrm{~Hz})$, 128.75, 127.92, 126.26 (d, $J=8.4 \mathrm{~Hz}), 124.42$ (d, $J=7.6 \mathrm{~Hz}), 120.46$ (q, $J=321.2 \mathrm{~Hz}), 115.31$ $(\mathrm{d}, J=89.3 \mathrm{~Hz}), 56.73,49.77,44.88,31.99 ;{ }^{19} \mathrm{~F}$ NMR $\left(365 \mathrm{MHz}, \mathrm{CDCl}_{3}\right) \delta:-78.14 ;{ }^{31} \mathrm{P}$ NMR $(162$ $\mathrm{MHz}, \mathrm{CDCl}_{3}$ ) $\delta: 22.38 ; m / z$ LRMS (ESI + APCI) found [M - OTf] ${ }^{+} 535.2, \mathrm{C}_{34} \mathrm{H}_{33} \mathrm{ClN}_{2} \mathrm{P}+$ requires 535.2 .

((rac)-8-benzyl-7,8,9,10-tetrahydro-6H-6,10-methanoazepino[4,5-g]quinoxalin-2yl)triphenylphosphonium trifluoromethanesulfonate (10)

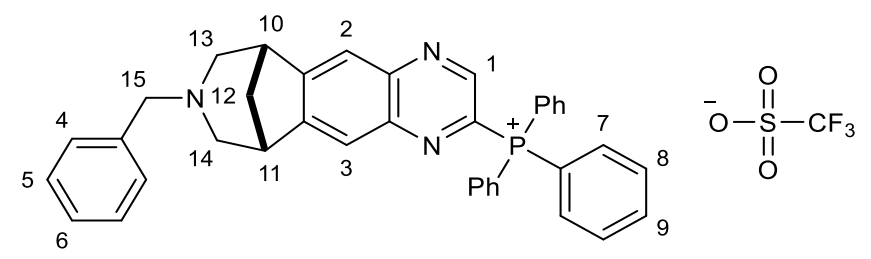

Prepared according to general procedure A (except that after the addition of $\mathrm{PPh}_{3}$ the reaction mixture was heated to $40^{\circ} \mathrm{C}$ for 1 hour, followed by addition of DBU at $40{ }^{\circ} \mathrm{C}$ instead of $-78^{\circ} \mathrm{C}$ ) using 8-benzyl-7,8,9,10-tetrahydro-6 $H$-6,10-methanoazepino[4,5-g]quinoxaline (115 mg, 0.38 $\mathrm{mmol}), \mathrm{Tf}_{2} \mathrm{O}(64 \mu \mathrm{L}, 0.38 \mathrm{mmol}), \mathrm{PPh}_{3}(110 \mathrm{mg}, 0.42 \mathrm{mmol}), \mathrm{DBU}(57 \mu \mathrm{L}, 0.38 \mathrm{mmol})$ and 
$\mathrm{CH}_{2} \mathrm{Cl}_{2}(3.8 \mathrm{~mL})$. After the purification procedure, ${ }^{+1}$ the title compound was isolated as a tan solid (158 mg, $0.22 \mathrm{mmol}, 58 \%$ yield). $\mathrm{mp} 101-106^{\circ} \mathrm{C}$; IR $v_{\max } / \mathrm{cm}^{-1}$ (film): 3061, 2949, 2900,2797, 1439, 1262, 1148, 1109, 1029, 636; ${ }^{1} \mathrm{H}$ NMR (400 MHz, DMSO-d 6 ) $\delta: 9.09$ (1H, s, H1), 8.10-7.72 $\left(17 \mathrm{H}, \mathrm{m}, \mathrm{H}_{2}, \mathrm{H}_{3}, \mathrm{H}_{7}, \mathrm{H}_{8}\right.$, and $\left.\mathrm{H}_{9}\right), 7.20-7.05$ (3H, $\mathrm{m}, \mathrm{H}_{5}$ and $\left.\mathrm{H}_{6}\right), 6.92-6.80\left(2 \mathrm{H}, \mathrm{m}, \mathrm{H}_{4}\right), 3.55-3.27$ (4H, m, $\mathrm{H}_{15}, \mathrm{H}_{10}$, and $\left.\mathrm{H}_{11}\right), 3.03-2.87$ (2H, m, $\mathrm{H}_{13}$ or $\left.\mathrm{H}_{14}\right)$, 2.66-2.42 (2H, m, $\mathrm{H}_{13}$ or $\left.\mathrm{H}_{14}\right), 2.29-2.15$ $\left(1 \mathrm{H}, \mathrm{m}, \mathrm{H}_{12}\right), 1.87\left(1 \mathrm{H}, \mathrm{d}, J=10.8 \mathrm{~Hz}, \mathrm{H}_{12}\right) ;{ }^{13} \mathrm{C} \mathrm{NMR}\left(100 \mathrm{MHz}, \mathrm{CDCl}_{3}\right)^{\S \S} \delta: 144.90(\mathrm{~d}, J=23.5$ Hz), 144.21, 143.45 (d, $J=16.9 \mathrm{~Hz}), 137.51$ (br s), 136.00 (d, $J=2.9 \mathrm{~Hz}), 134.55$ (d, $J=10.9 \mathrm{~Hz})$, $130.72(\mathrm{~d}, J=13.0 \mathrm{~Hz}), 129.20-126.21$ (3C, m), 120.69 (br s), 120.67 (q, $J=321.5 \mathrm{~Hz}), 116.42$ $(\mathrm{d}, J=88.3 \mathrm{~Hz}), 61.29,57.90-56.06$ (2C, m), 43.14-40.43 (3C, m); ${ }^{19} \mathrm{~F}$ NMR (365 MHz, DMSO$\left.\mathrm{d}_{6}\right) \delta:-77.80 ;{ }^{31} \mathrm{P}$ NMR (162 MHz, DMSO-d 6 ) $\delta: 13.81 ; \mathrm{m} / z$ LRMS found $[\mathrm{M}+\mathrm{H}]^{+} 562.2$, $\mathrm{C}_{38} \mathrm{H}_{33} \mathrm{~N}_{3} \mathrm{P}^{+}$requires 562.2 .

\section{(3-((8R,9S,10R,13S,14S)-3-((tert-butyldimethylsilyl)oxy)-10,13-dimethyl-}

\section{2,3,4,7,8,9,10,11,12,13,14,15-dodecahydro-1 $H$-cyclopenta[a]phenanthren-17-yl)pyridin-4-}

\section{yl)triphenylphosphonium trifluoromethanesulfonate (12)}

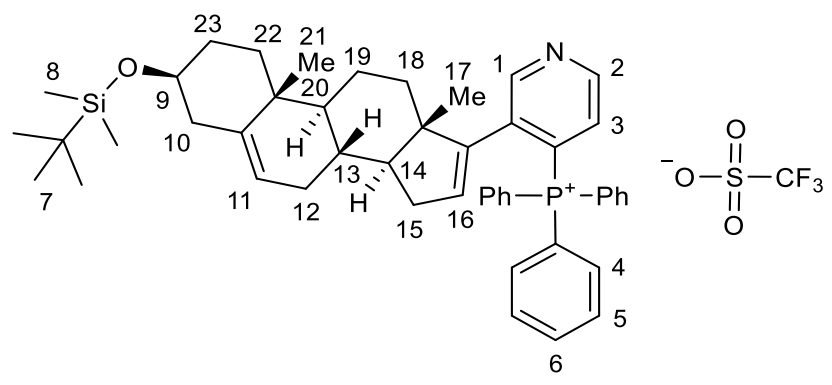

\footnotetext{
¥¥ The concentrated $\mathrm{CH}_{2} \mathrm{Cl}_{2}$ solution of crude product was added dropwise to an excess of chilled $\mathrm{Et}_{2} \mathrm{O}\left(0^{\circ} \mathrm{C}\right)$ instead of the order of addition in General Procedure A. The mixture was then placed in a $-20{ }^{\circ} \mathrm{C}$ refrigerator for approximately 1 hour. The resulting suspension was filtered on a frit and the solid was washed with chilled $\mathrm{Et}_{2} \mathrm{O}\left(0^{\circ} \mathrm{C}\right)$. The solid was redissolved in approximately 10 $\mathrm{mL}$ of $\mathrm{CH}_{2} \mathrm{Cl}_{2}$ and was precipitated a second time via dropwise addition to an excess of chilled $\mathrm{Et}_{2} \mathrm{O}\left(0{ }^{\circ} \mathrm{C}\right)$. The resulting suspension was filtered on a frit, the solid washed with chilled $\mathrm{Et}_{2} \mathrm{O}(0$ ${ }^{\circ} \mathrm{C}$ ) and dried in vacuo to provide the pure phosphonium salt.

$\S \S$ Please note that the ${ }^{13} \mathrm{C}$ NMR contains a number of broad peaks that account for the lower than expected number of carbon resonances. ${ }^{13} \mathrm{C}$ NMR does not improve with an increase in number of scans or by varying concentration.
} 
Prepared according to general procedure A (except that the reaction was warmed to $-50^{\circ} \mathrm{C}$ before the addition of $\mathrm{PPh}_{3}$, was stirred at $-50{ }^{\circ} \mathrm{C}$ for 1 hour, and DBU was added at $-50{ }^{\circ} \mathrm{C}$ instead of -78 ${ }^{\circ} \mathrm{C}$. During the purification stage the product suspension was placed in a $-20{ }^{\circ} \mathrm{C}$ refrigerator for approximately 12 hours instead of 1) using 3-((8R,9S,10R,13S,14S)-3-((tertbutyldimethylsilyl)oxy)-10,13-dimethyl-2,3,4,7,8,9,10,11,12,13,14,15-dodecahydro- $1 H$ cyclopenta[a]phenanthren-17-yl)pyridine (295 mg, $0.64 \mathrm{mmol}), \mathrm{Tf}_{2} \mathrm{O}$ (107 $\left.\mu \mathrm{L}, 0.64 \mathrm{mmol}\right), \mathrm{PPh}_{3}$ (184 mg, $0.70 \mathrm{mmol}), \mathrm{DBU}(95 \mu \mathrm{L}, 0.64 \mathrm{mmol})$ and $\mathrm{CH}_{2} \mathrm{Cl}_{2}(6.5 \mathrm{~mL})$. After the purification procedure, the title compound was isolated as a white solid (412 mg, $0.47 \mathrm{mmol}, 74 \%$ yield). $\mathrm{mp}$ 158-163 ${ }^{\circ} \mathrm{C}$; IR $v_{\max } / \mathrm{cm}^{-1}$ (film): 3062, 2929, 1442, 1259, 1096, 1030, 909, 725; ${ }^{1} \mathrm{H}$ NMR (400 $\left.\mathrm{MHz} \mathrm{CDCl}_{3}\right) \delta: 9.02(1 \mathrm{H}, \mathrm{d}, J=7.2 \mathrm{~Hz}), 8.75(1 \mathrm{H}, \mathrm{t}, J=4.6 \mathrm{~Hz}), 7.92-7.83(3 \mathrm{H}, \mathrm{m}), 7.83-7.73$ $(6 \mathrm{H}, \mathrm{m}), 7.72-7.61(6 \mathrm{H}, \mathrm{m}), 7.33-7.24(1 \mathrm{H}, \mathrm{m}), 5.57(1 \mathrm{H}, \mathrm{br} \mathrm{s}), 5.24(1 \mathrm{H}, \mathrm{d}, J=4.4 \mathrm{~Hz}), 3.49(1 \mathrm{H}$, m), 2.30-2.10 (2H, m), 1.89-1.66 (6H, m), 1.62-0.71 $(22 \mathrm{H}, \mathrm{m}), 0.65-0.51(1 \mathrm{H}, \mathrm{dt}, J=11.7 \mathrm{~Hz}$, $4.92 \mathrm{~Hz}), 0.06(6 \mathrm{H}, \mathrm{s}),-0.12-(-0.23)(1 \mathrm{H}, \mathrm{m}) ;{ }^{13} \mathrm{C} \mathrm{NMR}\left(100 \mathrm{MHz}, \mathrm{CDCl}_{3}\right) \delta: 150.77(\mathrm{~d}, J=7.6$ $\mathrm{Hz}), 149.14(\mathrm{~d}, J=3.8 \mathrm{~Hz}), 148.73$ (d, $J=10.7 \mathrm{~Hz}), 141.78,139.29,137.51$ (d, $J=6.1 \mathrm{~Hz}), 135.66$ (d, $J=3.1 \mathrm{~Hz}) 134.31$ (d, $J=9.9 \mathrm{~Hz}), 130.93$ (d, $J=13.0 \mathrm{~Hz}), 129.99$ (d, $J=10.7 \mathrm{~Hz}), 125.29$ (d, $J=83.9 \mathrm{~Hz}), 120.84$ (q, $J=328.8 \mathrm{~Hz}), 120.18,118.03$ (d, $J=90.0 \mathrm{~Hz}), 72.31,55.45,49.91,48.91$, 42.68, 37.24, 36.58, 33.70, 32.64, 31.92, 31.17, 29.97, 25.92, 20.42, 19.20, 18.79, 18.24, -4.58; ${ }^{19} \mathrm{~F}$ NMR $\left(365 \mathrm{MHz}, \mathrm{CDCl}_{3}\right) \delta:-78.11 ;{ }^{31} \mathrm{P} \mathrm{NMR}\left(162 \mathrm{MHz}, \mathrm{CDCl}_{3}\right) \delta: 22.78 ; \mathrm{m} / z \mathrm{LRMS}$ found $[\mathrm{M}+\mathrm{H}]^{+}$724.4, $\mathrm{C}_{48} \mathrm{H}_{59} \mathrm{NOPSi}^{+}$requires 724.4. 


\section{(4-((R)-(Benzyloxy)((1S,2S,4S,5R)-5-vinylquinuclidin-2-yl)methyl)quinolin-2-}

\section{yl)triphenylphosphonium trifluoromethanesulfonate (14)}

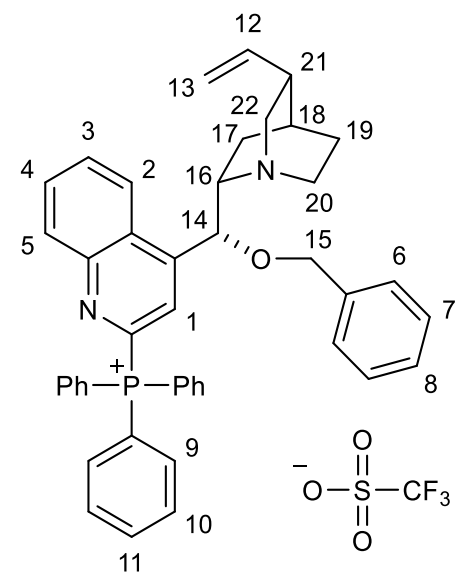

Prepared according to general procedure A (except that the stirring time after addition of $\mathrm{PPh}_{3} \mathrm{was}$ 1 hour instead of 30 minutes) using (1S,2S,4S,5R)-2-((R)-(benzyloxy)(quinolin-4-yl)methyl)-5vinylquinuclidine (472 mg, $1.23 \mathrm{mmol}), \mathrm{Tf}_{2} \mathrm{O}$ (207 $\left.\mu \mathrm{L}, 1.23 \mathrm{mmol}\right), \mathrm{PPh}_{3}(354 \mathrm{mg}, 1.35 \mathrm{mmol})$, $\mathrm{NEt}_{3}(171 \mu \mathrm{L}, 1.23 \mathrm{mmol})$ and $\mathrm{CH}_{2} \mathrm{Cl}_{2}(12 \mathrm{~mL})$. After the purification procedure, ${ }^{* * *}$ the title compound was isolated as a yellow solid (507 mg, $0.638 \mathrm{mmol}, 52 \%$ yield). $\mathrm{mp} \mathrm{92-98}{ }^{\circ} \mathrm{C}$; IR $v_{\max } / \mathrm{cm}^{-1}$ (film): 3062, 3031, 2940, 2866, 1144, 1108, 1029, 635; ${ }^{1} \mathrm{H}$ NMR (400 MHz, $\left.\mathrm{CDCl}_{3}\right) \delta:$ $8.54\left(1 \mathrm{H}, \mathrm{d}, J=8.1 \mathrm{~Hz}, \mathrm{H}_{5}\right), 8.26\left(1 \mathrm{H}, \mathrm{d}, J=8.6 \mathrm{~Hz}, \mathrm{H}_{2}\right), 7.94\left(1 \mathrm{H}, \mathrm{t}, J=7.2 \mathrm{~Hz}, \mathrm{H}_{3}\right), 7.91-7.60$ $\left(17 \mathrm{H}, \mathrm{m}, \mathrm{H}_{1}, \mathrm{H}_{4}, \mathrm{H}_{9}, \mathrm{H}_{10}\right.$, and $\left.\mathrm{H}_{11}\right), 7.29-7.02\left(3 \mathrm{H}, \mathrm{m}, \mathrm{H}_{7}\right.$ and $\left.\mathrm{H}_{8}\right), 7.14-7.02\left(2 \mathrm{H}, \mathrm{m}, \mathrm{H}_{6}\right), 5.83-$ $5.45\left(2 \mathrm{H}, \mathrm{m}, \mathrm{H}_{12}\right.$ and $\left.\mathrm{H}_{14}\right), 5.07-4.89\left(2 \mathrm{H}, \mathrm{m}, \mathrm{H}_{13}\right), 4.60\left(1 \mathrm{H}, \mathrm{d}, J=11.4 \mathrm{~Hz}, \mathrm{H}_{15}\right), 4.33(1 \mathrm{H}, \mathrm{d}, J$ $\left.=11.4 \mathrm{~Hz}, \mathrm{H}_{15}\right), 3.52-3.06\left(3 \mathrm{H}, \mathrm{m}, \mathrm{H}_{16}, \mathrm{H}_{20}\right.$, and $\left.\mathrm{H}_{22}\right), 2.84-2.62\left(2 \mathrm{H}, \mathrm{m}, \mathrm{H}_{20}\right.$ and $\left.\mathrm{H}_{22}\right), 2.49-2.33$ (1H, br s, $\left.\mathrm{H}_{21}\right), 2.06-1.47\left(5 \mathrm{H}, \mathrm{m}, \mathrm{H}_{17}, \mathrm{H}_{18}, \mathrm{H}_{19}\right) ;{ }^{13} \mathrm{C} \mathrm{NMR}\left(100 \mathrm{MHz}, \mathrm{CDCl}_{3}\right) \delta: 149.20(\mathrm{~d}, J=$ $22.1 \mathrm{~Hz}), 148.45,145.10,143.93,139.54,136.58,135.68$ (d, $J=3.0 \mathrm{~Hz}), 134.53$ (d, $J=10.0 \mathrm{~Hz})$, 131.99-131.79 (3C, m), 131.23, 130.44 (d, $J=13.2 \mathrm{~Hz}), 128.40,127.89,127.22,126.71$ (d, $J=$ $3.1 \mathrm{~Hz}), 126.08,123.78,120.64(\mathrm{q}, J=320.3 \mathrm{~Hz}), 116.99$ (d, $J=87.7 \mathrm{~Hz}), 115.55,71.81,60.71$,

\footnotetext{
*** The concentrated $\mathrm{CH}_{2} \mathrm{Cl}_{2}$ solution of crude product was added dropwise to an excess of chilled hexanes $\left(0^{\circ} \mathrm{C}\right)$ instead of the order of addition in General Procedure A. The mixture was then placed in a $-20{ }^{\circ} \mathrm{C}$ refrigerator for approximately 1 hour. The resulting suspension was filtered on a frit and the solid was washed with chilled hexanes $\left(0{ }^{\circ} \mathrm{C}\right)$. The solid was redissolved in approximately $10 \mathrm{~mL}$ of $\mathrm{CH}_{2} \mathrm{Cl}_{2}$ and was precipitated a second time via dropwise addition to an excess of chilled hexanes $\left(0^{\circ} \mathrm{C}\right)$. The resulting suspension was filtered on a frit, the solid washed with chilled hexanes $\left(0^{\circ} \mathrm{C}\right)$ and dried in vacuo to provide the pure phosphonium salt.
} 
55.61, 43.04, 38.32, 26.89, 25.83; ${ }^{19} \mathrm{~F}$ NMR (365 MHz, $\left.\mathrm{CDCl}_{3}\right) \delta:-78.15 ;{ }^{31} \mathrm{P}$ NMR $(162 \mathrm{MHz}$, $\mathrm{CDCl}_{3}$ ) $\delta: 14.92 ; m / z$ LRMS (ESI + APCI) found [M - OTf] ${ }^{+} 645.3, \mathrm{C}_{44} \mathrm{H}_{42} \mathrm{~N}_{2} \mathrm{OP}^{+}$requires 645.3.

\section{Preparation of Heteroaryl Ethers}

\section{General procedure B}
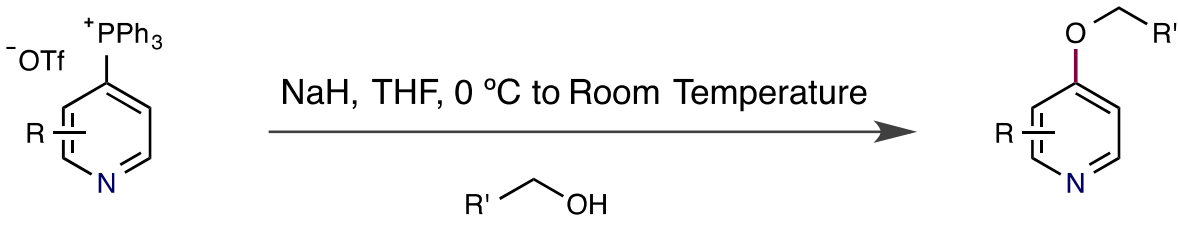

An oven dried $8 \mathrm{~mL}$ vial with a septa cap was charged with sodium hydride (60\% dispersion in mineral oil, 1.5 equiv) and placed under a nitrogen atmosphere. THF $(0.5 \mathrm{M})$ was added, the suspension was cooled to $0{ }^{\circ} \mathrm{C}$ and the alcohol (1.5 equiv) was added dropwise over 5 minutes (if the alcohol was a solid or viscous liquid, it was added as a $1.0 \mathrm{M}$ solution in THF to an equivalent volume 1.0 M solution of $\mathrm{NaH}$ in THF). The reaction was stirred for 30 minutes before the septa cap was briefly removed and the phosphonium salt (1.0 equiv) was added in one portion. The reaction was subjected to three rapid cycles of vacuum/nitrogen backfillitit the ice bath removed and the reaction stirred for 12 hours while warming to room temperature. The reaction was quenched with $\mathrm{H}_{2} \mathrm{O}$, the aqueous layer was separated and extracted with $\mathrm{Et}_{2} \mathrm{O}(3 \mathrm{x})$. The combined organic extracts were washed with a saturated aqueous solution of brine, dried $\left(\mathrm{MgSO}_{4}\right)$, filtered and concentrated in vacuo. The residue was purified by flash column chromatography under the stated conditions to provide the heteroaryl ether product.

\footnotetext{
${ }^{+++}$Vacuum was applied very briefly (less than a second) using a Schlenk manifold so that negligible solvent loss occurs.
} 


\section{4-(Hexyloxy)-2-phenylpyridine (3da)}

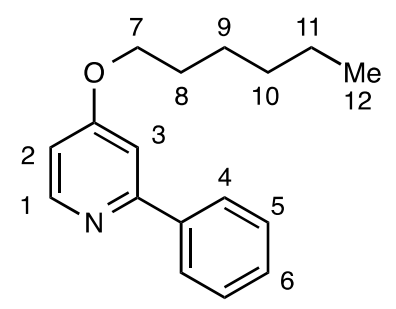

Prepared according to general procedure B using sodium hydride (60\% dispersion in mineral oil, $30 \mathrm{mg}, 0.75 \mathrm{mmol}), n$-hexanol (94 $\mu \mathrm{L}, 0.75 \mathrm{mmol})$, triphenyl(2-phenylpyridin-4-yl)phosphonium trifluoromethanesulfonate $(283 \mathrm{mg}, 0.50 \mathrm{mmol})$ and THF $(1.0 \mathrm{~mL})$. Flash column chromatography (silica gel, gradient elution: 5\% EtOAc in hexanes to 10\% EtOAc in hexanes) afforded the title compound as a white amorphous solid (92 mg, $0.36 \mathrm{mmol}, 72 \%$ yield). mp 36-37 ${ }^{\circ} \mathrm{C}$; IR $v_{\max } / \mathrm{cm}^{-}$ ${ }^{1}$ (film):3068, 2942, 1563, 1467, 1323, 1219, 1021; ${ }^{1} \mathrm{H}$ NMR (400 MHz, $\left.\mathrm{CDCl}_{3}\right) \delta: 8.50(1 \mathrm{H}, \mathrm{d}, J$ $\left.=5.5 \mathrm{~Hz}, \mathrm{H}_{1}\right), 7.97\left(2 \mathrm{H}, \mathrm{d}, J=7.4 \mathrm{~Hz}, \mathrm{H}_{4}\right), 7.46\left(2 \mathrm{H}, \mathrm{t}, J=7.4 \mathrm{~Hz}, \mathrm{H}_{5}\right), 7.40(1 \mathrm{H}, \mathrm{t}, J=7.4 \mathrm{~Hz}$, $\left.\mathrm{H}_{6}\right), 7.22\left(1 \mathrm{H}, \mathrm{d}, J=2.4 \mathrm{~Hz}, \mathrm{H}_{3}\right), 6.74\left(1 \mathrm{H}, \mathrm{dd}, J=5.7,2.2 \mathrm{~Hz}, \mathrm{H}_{2}\right), 4.05\left(2 \mathrm{H}, \mathrm{t}, J=6.5 \mathrm{~Hz}, \mathrm{H}_{7}\right)$ $1.82\left(2 \mathrm{H}, \mathrm{qn}, J=6.5 \mathrm{~Hz}, \mathrm{H}_{8}\right), 1.48\left(2 \mathrm{H}, \mathrm{qn}, J=6.7 \mathrm{~Hz}, \mathrm{H}_{9}\right), 1.40-1.31\left(4 \mathrm{H}, \mathrm{m}, \mathrm{H}_{10}\right.$ and $\left.\mathrm{H}_{11}\right), 0.92$ $\left(3 \mathrm{H}, \mathrm{t}, J=7.0 \mathrm{~Hz}, \mathrm{H}_{12}\right) ;{ }^{13} \mathrm{C} \mathrm{NMR}\left(100 \mathrm{MHz}, \mathrm{CDCl}_{3}\right) \delta: 165.83,159.08,150.79,139.49128 .84$, 128.57, 126.87, 108.45, 107.25, 67.92, 31.45, 28.86, 25.57, 22.52, 13.95; m/z LRMS (ESI + APCI) found $[\mathrm{M}+\mathrm{H}]^{+} 256.2, \mathrm{C}_{17} \mathrm{H}_{22} \mathrm{NO}^{+}$requires 256.2 .

\section{4-(benzyloxy)-2-phenylpyridine 2-phenylpyridin-4-ol (3db)}

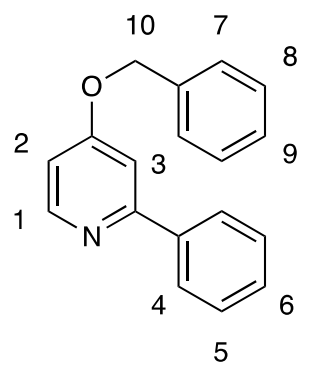

Prepared according to general procedure $\mathrm{B}$ (except that $\mathrm{CH}_{2} \mathrm{Cl}_{2}$ was used instead of $\mathrm{Et}_{2} \mathrm{O}$ for the extraction stage of the workup) using sodium hydride (60\% dispersion in mineral oil, $30 \mathrm{mg}, 0.75$ 
mmol), benzyl alcohol (78 $\mu \mathrm{L}, 0.75 \mathrm{mmol})$, triphenyl(2-phenylpyridin-4-yl)phosphonium trifluoromethanesulfonate $(282 \mathrm{mg}, 0.50 \mathrm{mmol})$ and THF $(1.0 \mathrm{~mL})$. Flash column chromatography (silica gel, gradient elution: $5 \%$ to $15 \%$ EtOAc in hexanes) to afford the title compound as a faintly purple amorphous solid (94 mg, $0.36 \mathrm{mmol}, 72 \%$ yield). $\mathrm{mp} 71-73{ }^{\circ} \mathrm{C}$; IR $v_{\max } / \mathrm{cm}^{-1}$ (film): 3030 , 2937, 2871, 1961, 1495, 1421, 1250, 1121, 1079, 1029, 646, 548; ${ }^{1} \mathrm{H}$ NMR (400 MHz, $\left.\mathrm{CDCl}_{3}\right) \delta$ : $8.53\left(1 \mathrm{H}, \mathrm{d}, J=5.7 \mathrm{~Hz}, \mathrm{H}_{1}\right), 7.95\left(2 \mathrm{H}, \mathrm{d}, J=7.6 \mathrm{~Hz}, \mathrm{H}_{4}\right), 7.50-7.35\left(8 \mathrm{H}, \mathrm{m}, \mathrm{H}_{5}, \mathrm{H}_{6}, \mathrm{H}_{7}, \mathrm{H}_{8}, \mathrm{H}_{9}\right)$, $7.32\left(1 \mathrm{H}, \mathrm{d}, J=1.7 \mathrm{~Hz}, \mathrm{H}_{3}\right), 6.85\left(1 \mathrm{H}, \mathrm{dd}, J=5.4,2.2 \mathrm{~Hz}, \mathrm{H}_{2}\right), 5.18\left(2 \mathrm{H}, \mathrm{s}, \mathrm{H}_{10}\right) ;{ }^{13} \mathrm{C}$ NMR $(100$ $\mathrm{MHz}$,

$\delta: 165.44,159.16,150.87,139 . .29,135.66,128.93,128.67,128.59,128.30,127.46,126.86$, $108.70,107.57,69.78$. The spectroscopic data is in agreement with a reported synthesis. ${ }^{3} \mathrm{~m} / \mathrm{z}$ LRMS (ESI + APCI) found $[\mathrm{M}+\mathrm{H}]^{+} 262.1, \mathrm{C}_{18} \mathrm{H}_{16} \mathrm{NO}^{+}$requires 262.1 .

\section{2-Phenyl-4-(pyridin-3-ylmethoxy)pyridine (3dc)}

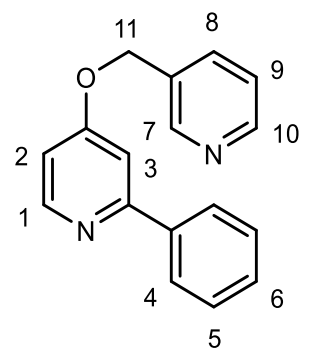

Prepared according to general procedure $\mathrm{B}$ (except that $\mathrm{CH}_{2} \mathrm{Cl}_{2}$ was used instead of $\mathrm{Et}_{2} \mathrm{O}$ for the extraction stage of the workup) using sodium hydride (60\% dispersion in mineral oil, $30 \mathrm{mg}, 0.75$ mmol), pyridin-3-ylmethanol (73 $\mu \mathrm{L}, 0.75 \mathrm{mmol})$, triphenyl(2-phenylpyridin-4-yl)phosphonium trifluoromethanesulfonate $(283 \mathrm{mg}, 0.50 \mathrm{mmol})$ and THF $(1.0 \mathrm{~mL})$. Prior to flash chromatography the crude reaction product was dissolved in methanol $(5.0 \mathrm{~mL})$ and a methanolic solution of hydrochloric acid $(5.0 \mathrm{~mL}$ of $4.2 \mathrm{M}$ solution) was added dropwise. The resulting mixture was concentrated in vacuo and dry loaded onto a silica gel column (elution: $2.5 \% \mathrm{MeOH}$ in $\mathrm{CH}_{2} \mathrm{Cl}_{2}$, then $30 \mathrm{~mL}$ of $\mathrm{NEt}_{3}$, then EtOAc) afforded the title compound as a pink amorphous solid ( $84 \mathrm{mg}$, $0.32 \mathrm{mmol}, 64 \%$ yield). $\mathrm{mp} 90-91{ }^{\circ} \mathrm{C}$; IR $v_{\max } / \mathrm{cm}^{-1}$ (film): 3027, 2984, 1561, 1207, 1013, 695; ${ }^{1} \mathrm{H}$ NMR $\left(400 \mathrm{MHz}, \mathrm{CDCl}_{3}\right) \delta: 8.70\left(1 \mathrm{H}, \mathrm{s}, \mathrm{H}_{7}\right), 8.61\left(1 \mathrm{H}, \mathrm{d}, J=4.6 \mathrm{~Hz}, \mathrm{H}_{10}\right), 8.52(1 \mathrm{H}, \mathrm{d}, J=5.7$ 
$\left.\mathrm{Hz}, \mathrm{H}_{1}\right), 7.94\left(2 \mathrm{H}, \mathrm{d}, J=7.5 \mathrm{~Hz}, \mathrm{H}_{4}\right), 7.76\left(1 \mathrm{H}, \mathrm{d}, J=7.7 \mathrm{~Hz}, \mathrm{H}_{8}\right), 7.48-7.36\left(3 \mathrm{H}, \mathrm{m}, \mathrm{H}_{5}\right.$ and $\left.\mathrm{H}_{6}\right)$, $7.33\left(1 \mathrm{H}, \mathrm{dd}, J=7.7,4.7 \mathrm{~Hz}, \mathrm{H}_{9}\right), 7.29\left(1 \mathrm{H}, \mathrm{d}, J=2.3 \mathrm{~Hz}, \mathrm{H}_{3}\right), 6.81\left(1 \mathrm{H}, \mathrm{dd}, J=5.7,2.3 \mathrm{~Hz}, \mathrm{H}_{2}\right)$, $5.15\left(2 \mathrm{H}, \mathrm{s}, \mathrm{H}_{11}\right) ;{ }^{13} \mathrm{C} \mathrm{NMR}\left(100 \mathrm{MHz}, \mathrm{CDCl}_{3}\right) \delta: 165.07,159.28,150.95,149.78,148.93,139.07$, 135.23, 131.26, 129.05, 128.62, 126.84, 123.53, 108.52, 107.36, 67.27; m/z LRMS (ESI + APCI) found $[\mathrm{M}+\mathrm{H}]^{+}$263.2, $\mathrm{C}_{17} \mathrm{H}_{15} \mathrm{~N}_{2} \mathrm{O}^{+}$requires 263.1 .

\section{2-Phenyl-4-(2,2,2-trifluoroethoxy)pyridine (3dd)}

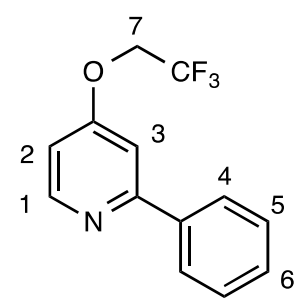

Prepared according to general procedure B using sodium hydride (60\% dispersion in mineral oil, $30 \mathrm{mg}, 0.75 \mathrm{mmol}), 2,2,2$-trifluoroethan-1-ol $(57 \mu \mathrm{L}, 0.75 \mathrm{mmol})$, triphenyl(2-phenylpyridin-4yl)phosphonium trifluoromethanesulfonate $(283 \mathrm{mg}, 0.50 \mathrm{mmol})$ and THF (1.0 mL). Flash column chromatography (silica gel, gradient elution: 10\% EtOAc in hexanes to 15\% EtOAc in hexanes) afforded the title compound as an off white amorphous solid (100 mg, $0.40 \mathrm{mmol}, 79 \%$ yield). $\mathrm{mp}$ 50-51 ${ }^{\circ} \mathrm{C}$; IR $v_{\max } / \mathrm{cm}^{-1}$ (film): 3095, 2975, 1568, 1271, 1164, 978, 776; ${ }^{1} \mathrm{H}$ NMR (400 MHz, $\left.\mathrm{CDCl}_{3}\right) \delta: 8.56\left(1 \mathrm{H}, \mathrm{d}, J=5.6 \mathrm{~Hz}, \mathrm{H}_{1}\right), 7.96\left(2 \mathrm{H}, \mathrm{d}, J=7.4 \mathrm{~Hz}, \mathrm{H}_{4}\right), 7.49-7.40\left(3 \mathrm{H}, \mathrm{m}, \mathrm{H}_{5}\right.$ and $\left.\mathrm{H}_{6}\right)$, $7.26\left(1 \mathrm{H}, \mathrm{d}, J=2.4 \mathrm{~Hz}, \mathrm{H}_{3}\right), 6.77\left(1 \mathrm{H}, \mathrm{dd}, J=5.6,2.4 \mathrm{~Hz}, \mathrm{H}_{2}\right), 4.43\left(2 \mathrm{H}, \mathrm{q}, J=7.9 \mathrm{~Hz}, \mathrm{H}_{7}\right) ;{ }^{13} \mathrm{C}$ $\operatorname{NMR}\left(100 \mathrm{MHz}, \mathrm{CDCl}_{3}\right) \delta: 163.99,159.68,151.21,138.79,129.31,128.71,126.90,122.9(\mathrm{q}, J=$ $227.7 \mathrm{~Hz}), 108.07,107.15,64.9$ (q, $J=35.9 \mathrm{~Hz}$ ); ${ }^{19} \mathrm{~F}$ NMR (365 MHz, $\left.\mathrm{CDCl}_{3}\right)$ 8: -73.70. The spectroscopic data is in agreement with a reported synthesis. ${ }^{4} \mathrm{~m} / z$ LRMS (ESI + APCI) found $[\mathrm{M}+\mathrm{H}]^{+}$254.1, $\mathrm{C}_{13} \mathrm{H}_{11} \mathrm{~F}_{3} \mathrm{NO}^{+}$requires 254.1. 


\section{2-Phenyl-4-(prop-2-yn-1-yloxy)pyridine (3de)}

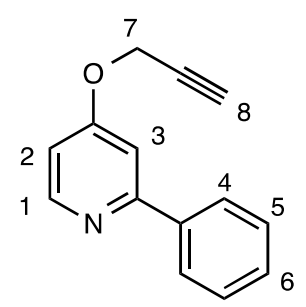

Prepared according to general procedure B using sodium hydride (60\% dispersion in mineral oil, $30 \mathrm{mg}, 0.75 \mathrm{mmol}$ ), prop-2-yn-1-ol (43 $\mu \mathrm{L}, 0.75 \mathrm{mmol})$, triphenyl-(2-phenylpyridin-4yl)phosphonium trifluoromethanesulfonate $(283 \mathrm{mg}, 0.50 \mathrm{mmol})$ and THF $(1.0 \mathrm{~mL})$. Flash column chromatography (silica gel: 5\% EtOAc in hexanes) afforded the title compound as a yellow oil (68 mg, 0.33 mmol, 65\% yield). IR $v_{\max } / \mathrm{cm}^{-1}$ (film): 3296, 3055, 2923, 1589, 1474, 1196, 1019; ${ }^{1} \mathrm{H}$ NMR (400 MHz, $\left.\mathrm{CDCl}_{3}\right) \delta: 8.54\left(1 \mathrm{H}, \mathrm{d}, J=5.6 \mathrm{~Hz}, \mathrm{H}_{1}\right), 7.96\left(2 \mathrm{H}, \mathrm{d}, J=7.4 \mathrm{~Hz}, \mathrm{H}_{4}\right), 7.50-7.38$ $\left(3 \mathrm{H}, \mathrm{m}, \mathrm{H}_{5}\right.$ and $\left.\mathrm{H}_{6}\right), 7.31\left(1 \mathrm{H}, \mathrm{d}, J=2.4 \mathrm{~Hz}, \mathrm{H}_{3}\right), 6.84\left(1 \mathrm{H}, \mathrm{dd}, J=5.6,2.4 \mathrm{~Hz}, \mathrm{H}_{2}\right), 4.78(2 \mathrm{H}, \mathrm{d}, J$ $\left.=2.3 \mathrm{~Hz}, \mathrm{H}_{7}\right), 2.60\left(1 \mathrm{H}, \mathrm{t}, J=2.3 \mathrm{~Hz}, \mathrm{H}_{8}\right) ;{ }^{13} \mathrm{C} \mathrm{NMR}\left(100 \mathrm{MHz}, \mathrm{CDCl}_{3}\right) \delta: 164.29,159.27,150.88$, $139.21,129.03,128.65,126.91,108.69,107.45,77.27,76.62,55.49$. The spectroscopic data is in agreement with a reported synthesis. ${ }^{5} \mathrm{~m} / z$ LRMS (ESI + APCI) found $[\mathrm{M}+\mathrm{H}]^{+} 210.1, \mathrm{C}_{14} \mathrm{H}_{12} \mathrm{NO}^{+}$ requires 210.1 .

\section{4-(Cyclohexyloxy)-2-phenylpyridine (3df)}

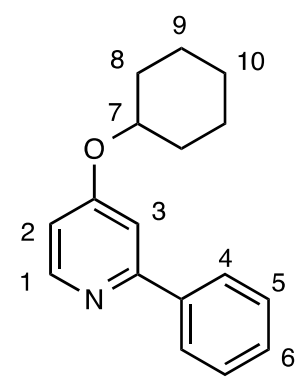

Prepared according to general procedure B using sodium hydride (60\% dispersion in mineral oil, $30 \mathrm{mg}, \quad 0.75 \mathrm{mmol})$, cyclohexanol (78 $\mu \mathrm{L}, 0.75 \mathrm{mmol})$, triphenyl(2-phenylpyridin-4yl)phosphonium trifluoromethanesulfonate $(282 \mathrm{mg}, 0.50 \mathrm{mmol})$ and THF $(1.0 \mathrm{~mL})$. The reaction 
was stirred for 13 hours after the addition of the phosphonium salt. Flash column chromatography (basic alumina: 6\% EtOAc in hexanes) afforded the title compound as an amorphous white solid (107 mg, $0.42 \mathrm{mmol}, 85 \%$ yield). $\mathrm{mp} 49-50{ }^{\circ} \mathrm{C}$; IR $v_{\max } / \mathrm{cm}^{-1}$ (film): 3032, 2939, 1559, 1476, 1211, 1018; ${ }^{1} \mathrm{H}$ NMR (400 MHz, $\left.\mathrm{CDCl}_{3}\right) \delta: 8.49\left(1 \mathrm{H}, \mathrm{d}, J=5.7 \mathrm{~Hz}, \mathrm{H}_{1}\right), 7.95\left(2 \mathrm{H}, \mathrm{d}, J=7.5 \mathrm{~Hz}, \mathrm{H}_{4}\right)$, 7.49-7.37 (3H, m, $\mathrm{H}_{5}$ and $\left.\mathrm{H}_{6}\right), 7.21\left(1 \mathrm{H}, \mathrm{d}, J=2.2 \mathrm{~Hz}, \mathrm{H}_{3}\right), 6.74\left(1 \mathrm{H}, \mathrm{dd}, J=5.7,2.2 \mathrm{~Hz}, \mathrm{H}_{2}\right), 4.42$ $\left(1 \mathrm{H}, \mathrm{m}, \mathrm{H}_{7}\right), 2.07-1.96\left(2 \mathrm{H}, \mathrm{m}, \mathrm{H}_{8}\right), 1.89-1.77\left(2 \mathrm{H}, \mathrm{m}, \mathrm{H}_{9}\right), 1.67-1.53\left(3 \mathrm{H}, \mathrm{m}, \mathrm{H}_{8}\right.$ and $\left.\mathrm{H}_{10}\right), 1.48-$ $1.30\left(3 \mathrm{H}, \mathrm{m}, \mathrm{H}_{9}\right.$ and $\left.\mathrm{H}_{10}\right) ;{ }^{13} \mathrm{C} \mathrm{NMR}\left(100 \mathrm{MHz}, \mathrm{CDCl}_{3}\right) \delta: 164.70,159.17,150.80,139.56,128.80$, 128.56, 126.88, 109.18, 108.30, 75.16, 31.40, 25.38, 23.51; m/z LRMS (ESI + APCI) found $[\mathrm{M}+\mathrm{H}]^{+}$254.2, $\mathrm{C}_{17} \mathrm{H}_{20} \mathrm{NO}^{+}$requires 254.2.

\section{4-((1-Benzylpyrrolidin-3-yl)oxy)-2-phenylpyridine (3dg)}

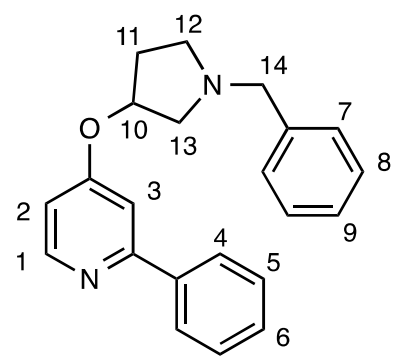

Prepared according to general procedure B using sodium hydride (60\% dispersion in mineral oil, $30 \mathrm{mg}, 0.75 \mathrm{mmol})$, 1-benzylpyrrolidin-3-ol (124 $\mu \mathrm{L}, 0.75 \mathrm{mmol})$, triphenyl(2-phenylpyridin-4yl)phosphonium trifluoromethanesulfonate $(283 \mathrm{mg}, 0.50 \mathrm{mmol})$ and THF $(1.0 \mathrm{~mL})$. Flash column chromatography (basic alumina, gradient elution: 10\% EtOAc in hexanes to 30\% EtOAc in hexanes) afforded the title compound as a yellow oil (125 mg, $0.38 \mathrm{mmol}, 75 \%$ yield). IR $v_{\max } / \mathrm{cm}^{-}$ 1 (film): 3029, 2920, 2792, 1559,1207, 694; ${ }^{1} \mathrm{H}$ NMR (400 MHz, $\left.\mathrm{CDCl}_{3}\right) \delta: 8.49$ (1H, d, $J=5.7$ $\left.\mathrm{Hz}, \mathrm{H}_{1}\right), 7.96\left(2 \mathrm{H}, \mathrm{d}, J=7.5 \mathrm{~Hz}, \mathrm{H}_{4}\right), 7.52-7.23\left(8 \mathrm{H}, \mathrm{m}, \mathrm{H}_{5}, \mathrm{H}_{6}, \mathrm{H}_{7}, \mathrm{H}_{8}\right.$, and $\left.\mathrm{H}_{9}\right), 7.19$ (1H, d, $J=$ $\left.2.1 \mathrm{~Hz}, \mathrm{H}_{3}\right), 6.68\left(1 \mathrm{H}, \mathrm{dd}, J=5.7,2.1 \mathrm{~Hz}, \mathrm{H}_{2}\right), 4.98-4.86\left(1 \mathrm{H}, \mathrm{m}, \mathrm{H}_{10}\right), 3.72(1 \mathrm{H}, \mathrm{d}, J=12.7 \mathrm{~Hz}$, $\left.\mathrm{H}_{14}\right), 3.66\left(1 \mathrm{H}, \mathrm{d}, J=12.7 \mathrm{~Hz}, \mathrm{H}_{14}\right), 2.99\left(1 \mathrm{H}, \mathrm{dd}, J=10.6,6.1 \mathrm{~Hz}, \mathrm{H}_{13}\right), 2.88-2.73\left(2 \mathrm{H}, \mathrm{m}, \mathrm{H}_{12}\right.$ and $\left.\mathrm{H}_{13}\right), 2.59\left(1 \mathrm{H}\right.$, app q, $\left.J=7.6 \mathrm{~Hz}, \mathrm{H}_{12}\right), 2.42-2.29\left(1 \mathrm{H}, \mathrm{m}, \mathrm{H}_{11}\right), 2.09-1.96\left(1 \mathrm{H}, \mathrm{m}, \mathrm{H}_{11}\right) ;{ }^{13} \mathrm{C}$ NMR (100 MHz, $\left.\mathrm{CDCl}_{3}\right)$ 8: 164.53, 159.07, 150.78, 139.34, 138.31, 128.87, 128.71, 128.56, 
128.23, 127.04, 126.82, 108.99, 108.02, 76.78, 60.06, 59.77, 52.56, 32.05; $\mathrm{m} / z$ LRMS (ESI + APCI) found $[\mathrm{M}+\mathrm{H}]^{+} 331.2, \mathrm{C}_{22} \mathrm{H}_{23} \mathrm{~N}_{2} \mathrm{O}^{+}$requires 331.2 .

\section{4-((1-Benzhydrylazetidin-3-yl)oxy)-2-phenylpyridine (3dh)}

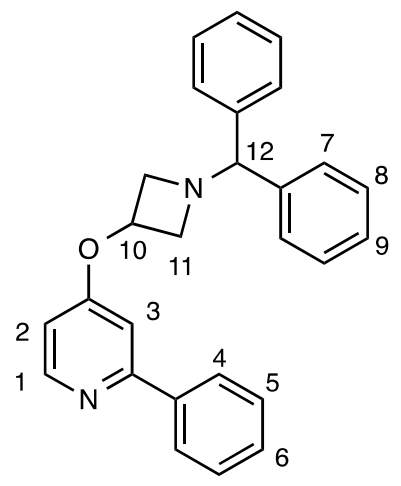

Prepared according to general procedure B using sodium hydride (60\% dispersion in mineral oil, $30 \mathrm{mg}, 0.75 \mathrm{mmol}$ ), 1-benzhydrylazetidin-3-ol (180 mg, $0.75 \mathrm{mmol})$, triphenyl(2-phenylpyridin4-yl)phosphonium trifluoromethanesulfonate $(283 \mathrm{mg}, 0.50 \mathrm{mmol})$ and THF $(1.0 \mathrm{~mL})$. Flash column chromatography (silica gel, gradient elution: 10\% EtOAc in hexanes to 30\% EtOAc in hexanes) afforded the title compound as a yellow oil (158 $\mathrm{mg}, 0.40 \mathrm{mmol}, 80 \%$ yield). IR $v_{\max } / \mathrm{cm}^{-}$ ${ }^{1}$ (film): 3027, 2954, 2838, 1592, 1211, 906, 729; ${ }^{1} \mathrm{H}$ NMR (400 MHz, $\left.\mathrm{CDCl}_{3}\right) \delta: 8.47$ (1H, d, $J=$ $\left.5.5 \mathrm{~Hz}, \mathrm{H}_{1}\right), 7.92\left(2 \mathrm{H}, \mathrm{d}, J=7.4 \mathrm{~Hz}, \mathrm{H}_{4}\right), 7.48-7.38\left(7 \mathrm{H}, \mathrm{m}, \mathrm{H}_{5}, \mathrm{H}_{6}\right.$, and $\left.\mathrm{H}_{7}\right), 7.30(4 \mathrm{H}$, app t, $J=$ $\left.7.4 \mathrm{~Hz}, \mathrm{H}_{8}\right), 7.21\left(2 \mathrm{H}, \mathrm{t}, J=7.4 \mathrm{~Hz}, \mathrm{H}_{9}\right), 7.10\left(1 \mathrm{H}, \mathrm{d}, J=2.3 \mathrm{~Hz}, \mathrm{H}_{3}\right), 6.61(1 \mathrm{H}, \mathrm{dd}, J=5.5,2.3$ $\left.\mathrm{Hz}, \mathrm{H}_{2}\right), 4.93\left(1 \mathrm{H}\right.$, app qn, $\left.J=5.6 \mathrm{~Hz}, \mathrm{H}_{10}\right), 4.45\left(1 \mathrm{H}, \mathrm{s}, \mathrm{H}_{12}\right), 3.76\left(2 \mathrm{H}\right.$, app t, $\left.J=5.6 \mathrm{~Hz}, \mathrm{H}_{11}\right)$, $3.18\left(2 \mathrm{H}\right.$, app t, $\left.J=5.6 \mathrm{~Hz}, \mathrm{H}_{11}\right) ;{ }^{13} \mathrm{C} \mathrm{NMR}\left(100 \mathrm{MHz}, \mathrm{CDCl}_{3}\right) \delta: 163.96,159.39,150.98,141.67$, $139.26,129.05,128.68,128.55,127.39,127.34,126.90,108.68,107.31,78.28,66.32,60.16 ; \mathrm{m} / \mathrm{z}$ LRMS (ESI + APCI) found $[\mathrm{M}+\mathrm{H}]^{+} 393.2, \mathrm{C}_{27} \mathrm{H}_{25} \mathrm{~N}_{2} \mathrm{O}^{+}$requires 393.2. 


\section{4-((1-Ethynylcyclopentyl)oxy)-2-phenylpyridine (3di)}

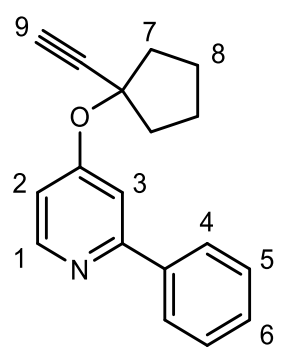

Prepared according to general procedure B using sodium hydride (60\% dispersion in mineral oil, $30 \mathrm{mg}, 0.75 \mathrm{mmol}), 1$-ethynylcyclopentan-1-ol (85 $\mu \mathrm{L}, 0.75 \mathrm{mmol})$, triphenyl(2-phenylpyridin-4yl)phosphonium trifluoromethanesulfonate $(283 \mathrm{mg}, 0.50 \mathrm{mmol})$ and THF $(1.0 \mathrm{~mL})$. Flash column chromatography (silica gel: 10\% EtOAc in hexanes) afforded the title compound as a yellow oil (93 mg, $0.35 \mathrm{mmol}, 71 \%$ yield). IR $v_{\max } / \mathrm{cm}^{-1}$ (film): 3292, 3032, 2960, 1590, 1472, 1189, 992; ${ }^{1} \mathrm{H}$ NMR (400 MHz, $\left.\mathrm{CDCl}_{3}\right) \delta: 8.51\left(1 \mathrm{H}, \mathrm{d}, J=5.6 \mathrm{~Hz}, \mathrm{H}_{1}\right), 7.96\left(2 \mathrm{H}, \mathrm{d}, J=7.8 \mathrm{~Hz}, \mathrm{H}_{4}\right), 7.55-7.35$ $\left(4 \mathrm{H}, \mathrm{m}, \mathrm{H}_{3}, \mathrm{H}_{5}\right.$ and $\left.\mathrm{H}_{6}\right), 7.05\left(1 \mathrm{H}, \mathrm{dd}, J=5.6,2.4 \mathrm{~Hz}, \mathrm{H}_{2}\right), 2.73\left(1 \mathrm{H}, \mathrm{s}, \mathrm{H}_{9}\right), 2.43-2.33\left(2 \mathrm{H}, \mathrm{m}, \mathrm{H}_{7}\right)$, 2.25-2.15 (2H, m, H7), 1.86-1.78 (4H, m, H8); $\left.{ }^{13} \mathrm{C} \mathrm{NMR} \mathrm{(100} \mathrm{MHz,} \mathrm{CDCl}_{3}\right) \delta: 162.79,158.76$, $150.34,139.51,128.80,128.58,126.85,111.58,110.10,84.05,80.99,75.58,40.73,23.79 ; \mathrm{m} / z$ LRMS (ESI + APCI) found $[\mathrm{M}+\mathrm{H}]^{+} 264.2, \mathrm{C}_{18} \mathrm{H}_{18} \mathrm{NO}^{+}$requires 264.1 .

\section{2-Chloro-4-(hexyloxy)pyridine (3aa)}

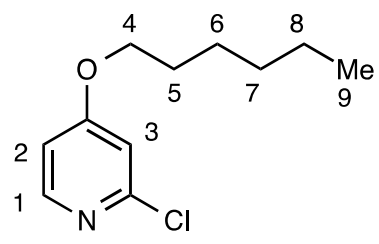

Prepared according to general procedure B using sodium hydride (60\% dispersion in mineral oil, $30 \mathrm{mg}, 0.75 \mathrm{mmol}), n$-hexanol $(94 \mu \mathrm{L}, 0.75 \mathrm{mmol}),(2$-chloropyridin-4-yl)triphenylphosphonium trifluoromethanesulfonate $(262 \mathrm{mg}, 0.50 \mathrm{mmol})$ and THF $(1.0 \mathrm{~mL})$. Flash column chromatography (silica gel, gradient elution: 4\% EtOAc in hexanes to 8\% EtOAc in hexanes) afforded the title compound as a yellow oil (82 mg, $0.34 \mathrm{mmol}, 77 \%$ yield). IR $v_{\max } / \mathrm{cm}^{-1}$ (film): 2930, 1584, 1460, 
1304, 1070, 835; ${ }^{1} \mathrm{H}$ NMR (400 MHz, $\left.\mathrm{CDCl}_{3}\right) \delta: 8.15\left(1 \mathrm{H}, \mathrm{d}, J=5.7 \mathrm{~Hz}, \mathrm{H}_{1}\right), 6.80(1 \mathrm{H}, \mathrm{d}, J=2.2$ $\left.\mathrm{Hz}, \mathrm{H}_{3}\right), 6.71\left(1 \mathrm{H}, \mathrm{dd}, J=5.7,2.2 \mathrm{~Hz}, \mathrm{H}_{2}\right), 3.98\left(2 \mathrm{H}, \mathrm{t}, J=6.7 \mathrm{~Hz}, \mathrm{H}_{4}\right), 1.77(2 \mathrm{H}, \mathrm{qn}, J=6.9 \mathrm{~Hz}$, $\left.\mathrm{H}_{5}\right), 1.43\left(2 \mathrm{H}, \mathrm{qn}, J=6.9 \mathrm{~Hz}, \mathrm{H}_{6}\right), 1.37-1.27\left(4 \mathrm{H}, \mathrm{m}, \mathrm{H}_{7}\right.$ and $\left.\mathrm{H}_{8}\right), 0.89\left(3 \mathrm{H}, \mathrm{t}, J=6.8 \mathrm{~Hz}, \mathrm{H}_{9}\right) ;{ }^{13} \mathrm{C}$ NMR (100 MHz, $\left.\mathrm{CDCl}_{3}\right) \delta: 166.76,152.52,150.13,110.03,109.78,68.53,31.37,28.66,25.46$, 22.48, 13.92. The spectroscopic data is in agreement with a reported synthesis. ${ }^{6} \mathrm{~m} / \mathrm{z}$ LRMS (ESI $+\mathrm{APCI}$ ) found $[\mathrm{M}+\mathrm{H}]^{+}$214.1, $\mathrm{C}_{11} \mathrm{H}_{17} \mathrm{ClNO}^{+}$requires 214.1 .

\section{2-Bromo-4-(hexyloxy)pyridine (3ba)}

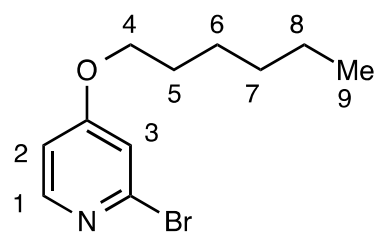

Prepared according to general procedure B using sodium hydride (60\% dispersion in mineral oil, $30 \mathrm{mg}, 0.75 \mathrm{mmol}), n$-hexanol $(94 \mu \mathrm{L}, 0.75 \mathrm{mmol}),(2$-bromopyridin-4-yl)triphenylphosphonium trifluoromethanesulfonate $(284 \mathrm{mg}, 0.50 \mathrm{mmol})$ and THF $(1.0 \mathrm{~mL})$. Flash column chromatography (silica gel, gradient elution: 2.5\% EtOAc in hexanes to 10\% EtOAc in hexanes) afforded the title compound as a pale orange oil (103 mg, $0.40 \mathrm{mmol}, 80 \%$ yield). IR $v_{\max } / \mathrm{cm}^{-1}$ (film): 2929, 1580, 1302, 1062, 834; ${ }^{1} \mathrm{H}$ NMR (400 MHz, $\left.\mathrm{CDCl}_{3}\right) \delta: 8.13\left(1 \mathrm{H}, \mathrm{d}, J=5.7 \mathrm{~Hz}, \mathrm{H}_{1}\right) 6.96(1 \mathrm{H}, \mathrm{d}, J=2.2$ $\left.\mathrm{Hz}, \mathrm{H}_{3}\right) 6.74\left(1 \mathrm{H}, \mathrm{dd}, J=5.7,2.2 \mathrm{~Hz}, \mathrm{H}_{2}\right), 3.97\left(2 \mathrm{H}, \mathrm{t}, J=6.6 \mathrm{~Hz}, \mathrm{H}_{4}\right), 1.77(2 \mathrm{H}, \mathrm{qn}, J=6.8 \mathrm{~Hz}$, $\left.\mathrm{H}_{5}\right), 1.43\left(2 \mathrm{H}, \mathrm{qn}, J=6.8 \mathrm{~Hz}, \mathrm{H}_{6}\right), 1.37-1.28\left(4 \mathrm{H}, \mathrm{m}, \mathrm{H}_{7}\right.$ and $\left.\mathrm{H}_{8}\right), 0.89\left(3 \mathrm{H}, \mathrm{t}, J=6.8 \mathrm{~Hz}, \mathrm{H}_{9}\right) ;{ }^{13} \mathrm{C}$ NMR (100 MHz, $\left.\mathrm{CDCl}_{3}\right) \delta: 166.32,150.51,142.92,113.59,110.46,68.52,31.37,28.66,25.46$, 22.49, 13.94. The spectroscopic data is in agreement with a reported synthesis. ${ }^{7} \mathrm{~m} / z$ LRMS (ESI $+\mathrm{APCI})$ found $[\mathrm{M}+\mathrm{H}]^{+} 258.0, \mathrm{C}_{11} \mathrm{H}_{17} \mathrm{BrNO}^{+}$requires 258.0. 


\section{2-Fluoro-4-(hexyloxy)pyridine (3ca)}

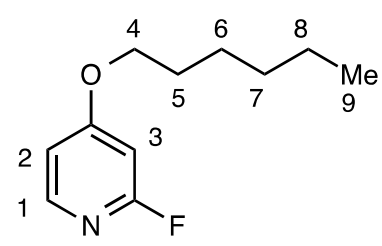

Prepared according to general procedure B using sodium hydride (60\% dispersion in mineral oil, $30 \mathrm{mg}, 0.75 \mathrm{mmol}$ ), $n$-hexanol (94 $\mu \mathrm{L}, 0.75 \mathrm{mmol}$ ), (2-fluoropyridin-4-yl)triphenylphosphonium trifluoromethanesulfonate $(254 \mathrm{mg}, 0.50 \mathrm{mmol})$ and THF $(1.0 \mathrm{~mL})$. Flash column chromatography (silica gel, gradient elution: 2.5\% EtOAc in hexanes to 10\% EtOAc in hexanes) afforded the title compound as a yellow oil (67 mg, $0.34 \mathrm{mmol}, 68 \%$ yield). IR $v_{\max } / \mathrm{cm}^{-1}$ (film): 2955, 1609, 1467 , 1417, 1159; ${ }^{1} \mathrm{H}$ NMR $\left(400 \mathrm{MHz}, \mathrm{CDCl}_{3}\right) \delta: 7.99\left(1 \mathrm{H}, \mathrm{d}, J=5.6 \mathrm{~Hz}, \mathrm{H}_{1}\right) 6.68(1 \mathrm{H}, \mathrm{dd}, J=5.7,2.1$ Hz, H2) $6.35\left(1 \mathrm{H}, \mathrm{d}, J=2.1 \mathrm{~Hz}, \mathrm{H}_{3}\right) 3.99\left(2 \mathrm{H}, \mathrm{t}, J=6.5 \mathrm{~Hz}, \mathrm{H}_{4}\right) 1.79\left(2 \mathrm{H}, \mathrm{qn}, J=6.7 \mathrm{~Hz}, \mathrm{H}_{5}\right)$, $1.44\left(2 \mathrm{H}, \mathrm{qn}, J=6.7 \mathrm{~Hz}, \mathrm{H}_{6}\right) 1.38-1.28\left(4 \mathrm{H}, \mathrm{m}, \mathrm{H}_{7}\right.$ and $\left.\mathrm{H}_{8}\right), 0.90\left(3 \mathrm{H}, \mathrm{t}, J=6.7 \mathrm{~Hz}, \mathrm{H}_{9}\right)$; ${ }^{13} \mathrm{C} \mathrm{NMR}$ $\left(100 \mathrm{MHz}, \mathrm{CDCl}_{3}\right) \delta: 169.00(\mathrm{~d}, J=12.0 \mathrm{~Hz}), 165.31(\mathrm{~d}, J=235.0 \mathrm{~Hz}), 147.90(\mathrm{~d}, J=19.1 \mathrm{~Hz})$, $109.38(\mathrm{~d}, J=3.8 \mathrm{~Hz}), 94.43$ (d, $J=41.6 \mathrm{~Hz}), 68.69$, 31.41, 28.69, 25.50, 22.51, 13.95; ${ }^{19} \mathrm{~F} \mathrm{NMR}$ $\left(365 \mathrm{MHz}, \mathrm{CDCl}_{3}\right) \delta:-67.03 ; \mathrm{m} / z$ LRMS (ESI + APCI) found $[\mathrm{M}+\mathrm{H}]^{+}$198.2, $\mathrm{C}_{11} \mathrm{H}_{17} \mathrm{FNO}^{+}$ requires 198.1 .

\section{4-(Hexyloxy)-2,2'-bipyridine (3ea)}

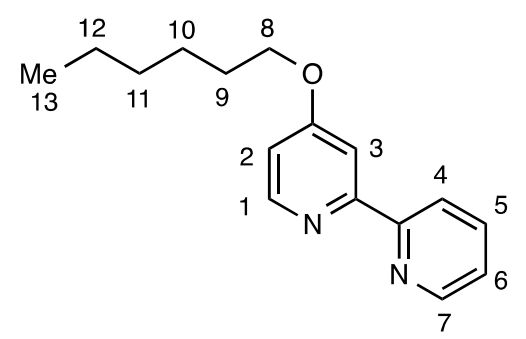

Prepared according to general procedure B using sodium hydride (60\% dispersion in mineral oil, $30 \mathrm{mg}, 0.75 \mathrm{mmol}), n$-hexanol (94 $\mu \mathrm{L}, 0.75 \mathrm{mmol})$, [2,2'-bipyridin]-4-yltriphenylphosphonium trifluoromethanesulfonate $(283 \mathrm{mg}, 0.50 \mathrm{mmol})$ and THF $(1.0 \mathrm{~mL})$. Flash column chromatography 
(basic alumina, gradient elution: 5\% EtOAc in hexanes to 20\% EtOAc in hexanes) afforded the title compound as a pink oil (72 mg, $0.28 \mathrm{mmol}, 56 \%$ yield). IR $v_{\max } / \mathrm{cm}^{-1}$ (film): 3058, 2929, 1582 , 1458, 1302, 1209, 793; ${ }^{1} \mathrm{H}$ NMR $\left(400 \mathrm{MHz}, \mathrm{CDCl}_{3}\right) \delta: 8.63\left(1 \mathrm{H}, \mathrm{d}, J=4.7 \mathrm{~Hz}, \mathrm{H}_{7}\right), 8.43(1 \mathrm{H}, \mathrm{d}$, $\left.J=5.7 \mathrm{~Hz}, \mathrm{H}_{1}\right), 8.36\left(1 \mathrm{H}, \mathrm{d}, J=7.7 \mathrm{~Hz}, \mathrm{H}_{4}\right), 7.92\left(1 \mathrm{H}, \mathrm{d}, J=2.4 \mathrm{~Hz}, \mathrm{H}_{3}\right), 7.76(1 \mathrm{H}, \mathrm{t}, J=7.7 \mathrm{~Hz}$, $\left.\mathrm{H}_{5}\right), 7.25\left(1 \mathrm{H}, \mathrm{dd}, J=7.7,4.7 \mathrm{~Hz}, \mathrm{H}_{6}\right), 6.78\left(1 \mathrm{H}, \mathrm{dd}, J=5.7,2.4 \mathrm{~Hz}, \mathrm{H}_{2}\right), 4.08(2 \mathrm{H}, \mathrm{t}, J=6.6 \mathrm{~Hz}$, $\left.\mathrm{H}_{8}\right), 1.78\left(2 \mathrm{H}, \mathrm{qn}, J=6.8 \mathrm{~Hz}, \mathrm{H}_{9}\right), 1.48-1.25\left(6 \mathrm{H}, \mathrm{m}, \mathrm{H}_{10}, \mathrm{H}_{11}\right.$, and $\left.\mathrm{H}_{12}\right), 0.87(3 \mathrm{H}, \mathrm{t}, J=6.5 \mathrm{~Hz}$, $\left.\mathrm{H}_{13}\right) ;{ }^{13} \mathrm{C} \mathrm{NMR}\left(100 \mathrm{MHz}, \mathrm{CDCl}_{3}\right) \delta: 166.17,157.86,156.03,150.18,148.97,136.79,123.69$, 121.18, 111.09, 106.56, 68.01, 31.44, 28.88, 25.56, 22.52, 13.95; m/z LRMS (ESI + APCI) found $[\mathrm{M}+\mathrm{H}]^{+} 257.2, \mathrm{C}_{16} \mathrm{H}_{20} \mathrm{~N}_{2} \mathrm{O}^{+}$requires 257.2.

\section{4-(Hexyloxy)-3-phenylpyridine (3fa)}

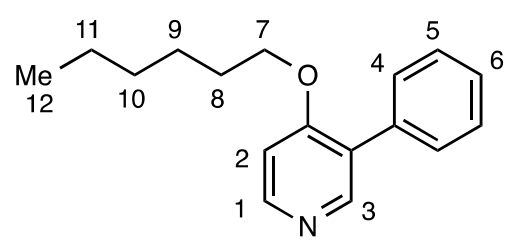

Prepared according to general procedure B using sodium hydride (60\% dispersion in mineral oil, $30 \mathrm{mg}, 0.75 \mathrm{mmol}$ ), $n$-hexanol ( $94 \mu \mathrm{L}, 0.75 \mathrm{mmol}$ ), triphenyl(3-phenylpyridin-4-yl)phosphonium trifluoromethanesulfonate $(283 \mathrm{mg}, 0.50 \mathrm{mmol})$ and THF $(1.0 \mathrm{~mL})$. Flash column chromatography (silica gel, gradient elution: 10\% EtOAc in hexanes to 40\% EtOAc in hexanes) afforded the title compound as a yellow oil (92 mg, $0.36 \mathrm{mmol}, 72 \%$ yield). IR $v_{\max } / \mathrm{cm}^{-1}$ (film): 3055, 2930, 1584 , 1280, 1007, 697; ${ }^{1} \mathrm{H}$ NMR (400 MHz, $\left.\mathrm{CDCl}_{3}\right)$ 8: 8.50-8.38 (2H, m, $\mathrm{H}_{1}$ and $\left.\mathrm{H}_{3}\right), 7.53(2 \mathrm{H}, \mathrm{d}, J=$ $\left.7.5 \mathrm{~Hz}, \mathrm{H}_{4}\right), 7.42\left(2 \mathrm{H}, \mathrm{t}, J=7.5 \mathrm{~Hz}, \mathrm{H}_{5}\right), 7.35\left(1 \mathrm{H}, \mathrm{t}, J=7.5 \mathrm{~Hz}, \mathrm{H}_{6}\right), 6.86\left(1 \mathrm{H}, \mathrm{d}, J=5.5 \mathrm{~Hz}, \mathrm{H}_{2}\right)$, $4.03\left(2 \mathrm{H}, \mathrm{t}, J=6.5 \mathrm{~Hz}, \mathrm{H}_{7}\right), 1.75\left(2 \mathrm{H}, \mathrm{qn}, J=6.5 \mathrm{~Hz}, \mathrm{H}_{8}\right), 1.41\left(2 \mathrm{H}, \mathrm{qn}, J=6.7 \mathrm{~Hz}, \mathrm{H}_{9}\right), 1.35-1.24$ $\left(4 \mathrm{H}, \mathrm{m}, \mathrm{H}_{10}\right.$ and $\left.\mathrm{H}_{11}\right), 0.88\left(3 \mathrm{H}, \mathrm{t}, J=6.7, \mathrm{H}_{12}\right) ;{ }^{13} \mathrm{C} \mathrm{NMR}\left(100 \mathrm{MHz}, \mathrm{CDCl}_{3}\right) \delta: 161.89,150.82$, 150.28, 134.91, 129.42, 128.04, 127.42, 126.38, 107.07, 68.09, 31.27, 28.61, 25.52, 22.44, 13.86; $m / z$ LRMS (ESI + APCI) found $[\mathrm{M}+\mathrm{H}]^{+} 256.2, \mathrm{C}_{17} \mathrm{H}_{22} \mathrm{NO}^{+}$requires 256.2 . 


\section{4-(Hexyloxy)-5,6,7,8-tetrahydroquinoline (3ga)}

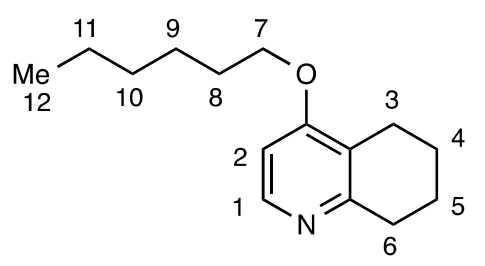

Prepared according to general procedure B using sodium hydride (60\% dispersion in mineral oil, $30 \mathrm{mg}, 0.75 \mathrm{mmol}), n$-hexanol $(94 \mu \mathrm{L}, 0.75 \mathrm{mmol})$, triphenyl(5,6,7,8-tetrahydroquinolin-4yl)phosphonium trifluoromethanesulfonate $(272 \mathrm{mg}, 0.50 \mathrm{mmol})$ and THF (1.0 mL). Flash column chromatography (neutralized silica gel: 20\% EtOAc in hexanes) afforded the title compound as a yellow oil (93 mg, $0.40 \mathrm{mmol}, 79 \%$ yield). IR $v_{\max } / \mathrm{cm}^{-1}$ (film): 2929, 2870, 1574, 1463, 1289, 1105; ${ }^{1} \mathrm{H} \mathrm{NMR}\left(400 \mathrm{MHz}, \mathrm{CDCl}_{3}\right) \delta: 8.20\left(1 \mathrm{H}, \mathrm{d}, J=5.5 \mathrm{~Hz}, \mathrm{H}_{1}\right), 6.51\left(1 \mathrm{H}, \mathrm{d}, J=5.5 \mathrm{~Hz}, \mathrm{H}_{2}\right)$, $3.94\left(2 \mathrm{H}, \mathrm{t}, J=6.4 \mathrm{~Hz}, \mathrm{H}_{7}\right), 2.83\left(2 \mathrm{H}, \mathrm{t}, J=6.1 \mathrm{~Hz}, \mathrm{H}_{6}\right), 2.60\left(2 \mathrm{H}, \mathrm{t}, J=6.1 \mathrm{~Hz}, \mathrm{H}_{3}\right), 1.88-1.67$ $\left(6 \mathrm{H}, \mathrm{m}, \mathrm{H}_{4}, \mathrm{H}_{5}\right.$, and $\left.\mathrm{H}_{8}\right), 1.43\left(2 \mathrm{H}, \mathrm{qn}, J=6.7 \mathrm{~Hz}, \mathrm{H}_{9}\right), 1.37-1.24\left(4 \mathrm{H}, \mathrm{m}, \mathrm{H}_{10}\right.$ and $\left.\mathrm{H}_{11}\right), 0.88(3 \mathrm{H}$, $\left.\mathrm{t}, J=6.7 \mathrm{~Hz}, \mathrm{H}_{12}\right) ;{ }^{13} \mathrm{C} \mathrm{NMR}\left(100 \mathrm{MHz}, \mathrm{CDCl}_{3}\right) \delta: 162.82,157.70,147.66,121.04,103.70,67.68$, $32.45,31.40,28.86,25.61,22.73,22.49,22.17,22.14,13.90 ; m / z$ LRMS (ESI + APCI) found $[\mathrm{M}+\mathrm{H}]^{+}$234.2, $\mathrm{C}_{15} \mathrm{H}_{24} \mathrm{NO}^{+}$requires 234.2.

\section{4-(Hexyloxy)-2-methyl-3-(thiophen-3-yl)pyridine (3ha)}

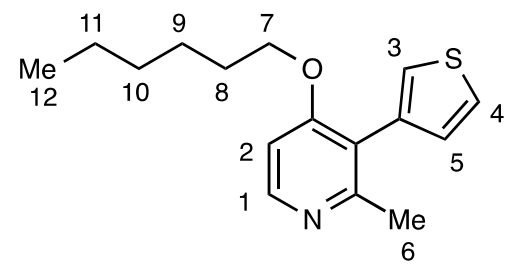

Prepared according to general procedure B using sodium hydride (60\% dispersion in mineral oil, $30 \mathrm{mg}, 0.75 \mathrm{mmol}), n$-hexanol (94 $\mu \mathrm{L}, 0.75 \mathrm{mmol})$, (2-methyl-3-(thiophen-3-yl)pyridin-4yl)triphenylphosphonium trifluoromethanesulfonate $(293 \mathrm{mg}, 0.50 \mathrm{mmol})$ and THF $(1.0 \mathrm{~mL})$. Flash column chromatography (basic alumina, gradient elution: 15\% EtOAc in hexanes to 25\% EtOAc in hexanes) afforded the title compound as a yellow oil (100 mg, $0.36 \mathrm{mmol}, 73 \%$ yield). 
IR $v_{\max } / \mathrm{cm}^{-1}$ (film): 2928, 1571, 1462, 1292, 1075, 752; ${ }^{1} \mathrm{H}$ NMR (400 MHz, $\left.\mathrm{CDCl}_{3}\right) \delta: 8.32(1 \mathrm{H}$, d, $\left.J=5.7 \mathrm{~Hz}, \mathrm{H}_{1}\right), 7.33\left(1 \mathrm{H}, \mathrm{dd}, J=5.0,2.7 \mathrm{~Hz}, \mathrm{H}_{4}\right), 7.15\left(1 \mathrm{H}, \mathrm{d}, J=2.7 \mathrm{~Hz}, \mathrm{H}_{3}\right), 7.02(1 \mathrm{H}, \mathrm{d}, J$ $\left.=5.0 \mathrm{~Hz}, \mathrm{H}_{5}\right), 6.68\left(1 \mathrm{H}, \mathrm{d}, J=5.7 \mathrm{~Hz}, \mathrm{H}_{2}\right), 3.92\left(2 \mathrm{H}, \mathrm{t}, J=6.4 \mathrm{~Hz}, \mathrm{H}_{7}\right), 2.38\left(3 \mathrm{H}, \mathrm{s}, \mathrm{H}_{6}\right), 1.64(2 \mathrm{H}$, qn, $\left.J=6.5 \mathrm{~Hz}, \mathrm{H}_{8}\right), 1.34-1.17\left(6 \mathrm{H}, \mathrm{m}, \mathrm{H}_{9}, \mathrm{H}_{10}\right.$, and $\left.\mathrm{H}_{11}\right), 0.84\left(3 \mathrm{H}, \mathrm{t}, J=6.7 \mathrm{~Hz}, \mathrm{H}_{12}\right) ;{ }^{13} \mathrm{C} \mathrm{NMR}$ $\left(100 \mathrm{MHz}, \mathrm{CDCl}_{3}\right)$ 8: 162.79, 157.75, 149.13, 134.76, 129.34, 124.26, 123.95, 120.93, 104.79, 68.03, 31.17, 28.53, 25.35, 23.36, 22.40, 13.85; $\mathrm{m} / \mathrm{z}$ LRMS (ESI + APCI) found $[\mathrm{M}+\mathrm{H}]^{+} 276.2$, $\mathrm{C}_{16} \mathrm{H}_{22} \mathrm{NOS}^{+}$requires 276.1 .

\section{4-(Hexyloxy)-2-methyl-5-(phenylethynyl)pyridine (3ia)}

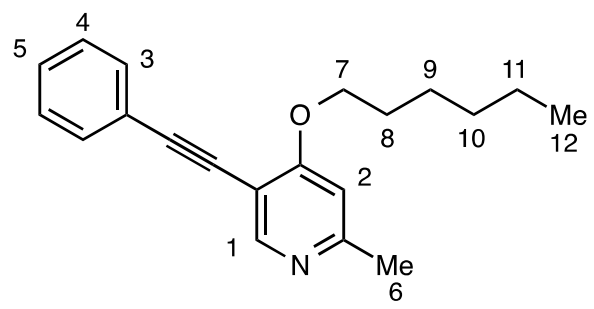

Prepared according to general procedure B using sodium hydride (60\% dispersion in mineral oil, $30 \mathrm{mg}, 0.75 \mathrm{mmol}$ ), $n$-hexanol $(94 \mu \mathrm{L}, 0.75 \mathrm{mmol}$ ), (2-methyl-5-(phenylethynyl)pyridin-4yl)triphenylphosphonium trifluoromethanesulfonate $(302 \mathrm{mg}, 0.50 \mathrm{mmol})$ and THF $(1.0 \mathrm{~mL})$. Flash column chromatography (silica gel: 15\% EtOAc in hexanes) afforded the title compound as a white solid (91 mg, $0.31 \mathrm{mmol}, 62 \%$ yield). $\mathrm{mp} 66-68^{\circ} \mathrm{C}$; IR $v_{\max } / \mathrm{cm}^{-1}$ (film): 3055, 3035, 3019, 2954, 2930, 2871, 2855, 2731, 2594, 2224, 2161, 2050, 1979, 1966, 1947, 1893, 1822, 1802, 1667, 1544, 1426, 1325, 1237, 1147, 1126, 1068, 911, 768, 723, 678; ${ }^{1} \mathrm{H}$ NMR (400 MHz, $\left.\mathrm{CDCl}_{3}\right) \delta$ : $8.48\left(1 \mathrm{H}, \mathrm{s}, \mathrm{H}_{1}\right), 7.53\left(2 \mathrm{H}, \mathrm{dd}, J=4.1,7.0 \mathrm{~Hz}, \mathrm{H}_{4}\right), 7.38-7.31\left(3 \mathrm{H}, \mathrm{m}, \mathrm{H}_{3}\right.$ and $\left.\mathrm{H}_{5}\right), 6.66(1 \mathrm{H}, \mathrm{s}$, $\left.\mathrm{H}_{2}\right), 4.08\left(2 \mathrm{H}, \mathrm{t}, J=6.4 \mathrm{~Hz}, \mathrm{H}_{7}\right), 2.55\left(3 \mathrm{H}, \mathrm{s}, \mathrm{H}_{6}\right), 1.88\left(2 \mathrm{H}, \mathrm{qn}, 7.0 \mathrm{~Hz}, \mathrm{H}_{8}\right), 1.54(2 \mathrm{H}, \mathrm{qn}, 7.0 \mathrm{~Hz}$, $\left.\mathrm{H}_{9}\right), 1.44-1.31\left(4 \mathrm{H}, \mathrm{m}, \mathrm{H}_{10}\right.$ and $\left.\mathrm{H}_{11}\right), 0.90(3 \mathrm{H}, \mathrm{t}, J=6.9 \mathrm{~Hz}) ;{ }^{13} \mathrm{C} \mathrm{NMR}\left(100 \mathrm{MHz}, \mathrm{CDCl}_{3}\right)$

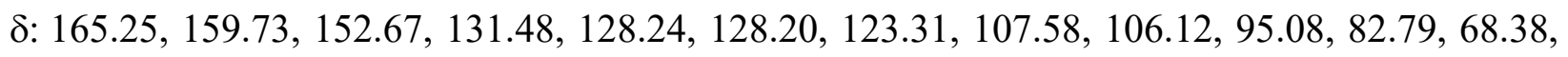
31.43, 28.76, 25.54, 24.91, 22.54, 13.94; $\mathrm{m} / \mathrm{z}$ LRMS (ESI + APCI) found $[\mathrm{M}+\mathrm{H}]^{+}$294.2, $\mathrm{C}_{20} \mathrm{H}_{24} \mathrm{NO}^{+}$requires 294.2. 


\section{2-Fluoro-4-(hexyloxy)-5-methylpyridine (3ja)}

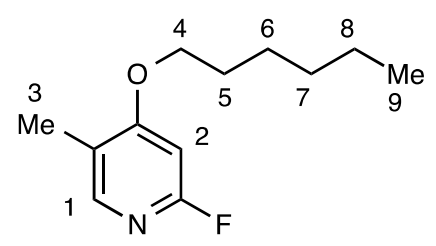

Prepared according to general procedure B using sodium hydride (60\% dispersion in mineral oil, $20 \mathrm{mg}, \quad 0.50 \mathrm{mmol}$ ), $n$-hexanol (63 $\mu \mathrm{L}, \quad 0.50 \mathrm{mmol}$ ), (2-fluoro-5-methylpyridin-4yl)triphenylphosphonium trifluoromethanesulfonate $(261 \mathrm{mg}, 0.50 \mathrm{mmol})$ and THF (1.0 mL). Flash column chromatography (silica gel: $2.5 \%$ EtOAc in hexanes) afforded the title compound as a white amorphous solid (64 mg, $0.30 \mathrm{mmol}, 60 \%$ yield). $\mathrm{mp} 30-32{ }^{\circ} \mathrm{C}$; IR $v_{\max } / \mathrm{cm}^{-1}$ (film): 3073 ,

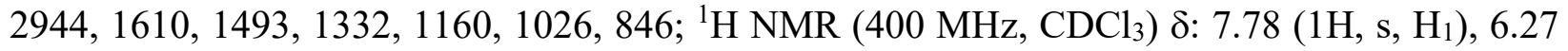
$\left(1 \mathrm{H}, \mathrm{s}, \mathrm{H}_{2}\right), 3.98\left(2 \mathrm{H}, \mathrm{t}, J=6.5 \mathrm{~Hz}, \mathrm{H}_{4}\right), 2.10\left(3 \mathrm{H}, \mathrm{s}, \mathrm{H}_{3}\right), 1.81\left(2 \mathrm{H}, \mathrm{qn}, J=6.5 \mathrm{~Hz}, \mathrm{H}_{5}\right), 1.54-1.27$ $\left(6 \mathrm{H}, \mathrm{m}, \mathrm{H}_{6}, \mathrm{H}_{7}\right.$, and $\left.\mathrm{H}_{8}\right), 0.90\left(3 \mathrm{H}, \mathrm{t}, J=6.8 \mathrm{~Hz}, \mathrm{H}_{9}\right) ;{ }^{13} \mathrm{C} \mathrm{NMR}\left(100 \mathrm{MHz}, \mathrm{CDCl}_{3}\right) \delta: 166.82(\mathrm{~d}$, $J=10.7 \mathrm{~Hz}), 163.77(\mathrm{~d}, J=233.5 \mathrm{~Hz}), 146.46(\mathrm{~d}, J=17.6 \mathrm{~Hz}), 120.38$ (d, $J=4.6 \mathrm{~Hz}), 91.45$ (d, $J=42.7 \mathrm{~Hz}), 68.47,31.40,28.66,25.57,22.51,13.93,12.59 ;{ }^{19} \mathrm{~F} \mathrm{NMR}\left(365 \mathrm{MHz}, \mathrm{CDCl}_{3}\right) \delta$ : 69.11; $m / z$ LRMS (ESI + APCI) found $[\mathrm{M}+\mathrm{H}]^{+} 212.2, \mathrm{C}_{12} \mathrm{H}_{19} \mathrm{FNO}^{+}$requires 212.1.

\section{4-(Hexyloxy)-5-methoxy-2-methylpyridine (3ka)}

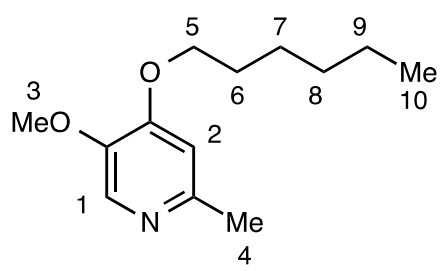

Prepared according to general procedure B using sodium hydride (60\% dispersion in mineral oil, $30 \mathrm{mg}, 0.75 \mathrm{mmol}), n$-hexanol (94 $\mu \mathrm{L}, 0.75 \mathrm{mmol})$, a 7:1 mixture of (5-methoxy-2-methylpyridin4-yl)triphenylphosphonium trifluoromethanesulfonate and (3-methoxy-6-methylpyridin-2yl)triphenylphosphonium trifluoromethanesulfonate $(267 \mathrm{mg}, 0.50 \mathrm{mmol})$ and THF $(1.0 \mathrm{~mL})$. Flash column chromatography (silica gel was packed in hexanes and neutralized with $\mathrm{NEt}_{3}$ then 
gradient elution: $40 \%$ EtOAc in hexanes to 50\% EtOAc in hexanes) afforded the title compound as a tan amorphous solid (51 mg, $0.23 \mathrm{mmol}, 46 \%$ yield). $\mathrm{mp} 41-42{ }^{\circ} \mathrm{C}$; IR $v_{\max } / \mathrm{cm}^{-1}$ (film): 3068 , 3004, 2938, 1590, 1511, 1231, 1026; ${ }^{1} \mathrm{H}$ NMR $\left(400 \mathrm{MHz}, \mathrm{CDCl}_{3}\right) \delta: 7.96\left(1 \mathrm{H}, \mathrm{s}, \mathrm{H}_{1}\right), 6.61(1 \mathrm{H}, \mathrm{s}$, $\left.\mathrm{H}_{2}\right), 4.00\left(2 \mathrm{H}, \mathrm{t}, J=7.2 \mathrm{~Hz}, \mathrm{H}_{5}\right), 3.86\left(3 \mathrm{H}, \mathrm{s}, \mathrm{H}_{3}\right), 2.43\left(3 \mathrm{H}, \mathrm{s}, \mathrm{H}_{4}\right), 1.82\left(2 \mathrm{H}, \mathrm{qn}, J=7.2 \mathrm{~Hz}, \mathrm{H}_{6}\right)$, $1.43\left(2 \mathrm{H}, \mathrm{qn}, J=7.1 \mathrm{~Hz}, \mathrm{H}_{7}\right), 1.35-1.26\left(4 \mathrm{H}, \mathrm{m}, \mathrm{H}_{8}\right.$ and $\left.\mathrm{H}_{9}\right), 0.87\left(3 \mathrm{H}, \mathrm{t}, J=7.0 \mathrm{~Hz}, \mathrm{H}_{10}\right) ;{ }^{13} \mathrm{C}$ NMR (100 MHz, $\left.\mathrm{CDCl}_{3}\right) \delta: 154.99,152.62$ 143.98, 133.25, 106.80, 68.47, 56.85, 31.42, 28.70, 25.48, 24.10, 22.47, 13.92; $\mathrm{m} / z$ LRMS (ESI + APCI) found $[\mathrm{M}+\mathrm{H}]^{+} 224.2, \mathrm{C}_{13} \mathrm{H}_{22} \mathrm{NO}_{2}{ }^{+}$requires 224.2 .

\section{2-Butyl-4-(hexyloxy)-5-(trifluoromethyl)pyridine (3la)}

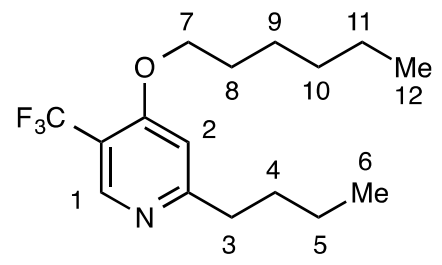

Prepared according to general procedure B using sodium hydride (60\% dispersion in mineral oil, $30 \mathrm{mg}, 0.75 \mathrm{mmol}$ ), $n$-hexanol $(94 \mu \mathrm{L}, 0.75 \mathrm{mmol}$ ), (2-butyl-5-(trifluoromethyl)pyridin-4yl)triphenylphosphonium trifluoromethanesulfonate $(307 \mathrm{mg}, 0.50 \mathrm{mmol})$ and THF $(1.0 \mathrm{~mL})$. Flash column chromatography (silica gel: $\mathrm{CH}_{2} \mathrm{Cl}_{2}$ ) afforded the title compound as a yellow oil (112 mg, $0.37 \mathrm{mmol}, 74 \%$ yield). IR $v_{\max } / \mathrm{cm}^{-1}$ (film): 2957, 2861, 1603, 1325, 1129, 1043; ${ }^{1} \mathrm{H}$ $\operatorname{NMR}\left(400 \mathrm{MHz}, \mathrm{CDCl}_{3}\right) \delta: 8.52\left(1 \mathrm{H}, \mathrm{s}, \mathrm{H}_{1}\right), 6.71\left(1 \mathrm{H}, \mathrm{s}, \mathrm{H}_{2}\right), 4.07\left(2 \mathrm{H}, \mathrm{t}, J=6.5 \mathrm{~Hz}, \mathrm{H}_{7}\right), 2.76$ $\left(2 \mathrm{H}, \mathrm{t}, J=7.6 \mathrm{~Hz}, \mathrm{H}_{3}\right), 1.81\left(2 \mathrm{H}, \mathrm{qn}, J=6.5 \mathrm{~Hz}, \mathrm{H}_{8}\right), 1.69\left(2 \mathrm{H}, \mathrm{qn}, J=7.7 \mathrm{~Hz}, \mathrm{H}_{4}\right), 1.50-1.25(8 \mathrm{H}$, $\mathrm{m}, \mathrm{H}_{5}, \mathrm{H}_{9}, \mathrm{H}_{10}$, and $\left.\mathrm{H}_{11}\right), 0.95-0.85\left(6 \mathrm{H}, \mathrm{m}, \mathrm{H}_{6}\right.$ and $\left.\mathrm{H}_{12}\right) ;{ }^{13} \mathrm{C} \mathrm{NMR}\left(100 \mathrm{MHz}, \mathrm{CDCl}_{3}\right) \delta$ : 168.62, $163.29,147.39$ (q, $J=5.8 \mathrm{~Hz}), 123.42(\mathrm{q}, J=272.4 \mathrm{~Hz}), 113.06(\mathrm{q}, J=31.3 \mathrm{~Hz}), 106.25,68.64$, $38.59,31.74,31.28,28.57,25.33,22.46,22.44,13.86,13.83 ;{ }^{19} \mathrm{~F} \mathrm{NMR}\left(365 \mathrm{MHz}, \mathrm{CDCl}_{3}\right) \delta:-$ $62.29 ; m / z$ LRMS (ESI + APCI) found $[\mathrm{M}+\mathrm{H}]^{+} 304.2, \mathrm{C}_{16} \mathrm{H}_{25} \mathrm{~F}_{3} \mathrm{NO}^{+}$requires 304.2. 


\section{2-(Hexyloxy)-4-(trifluoromethyl)pyridine (3ma)}

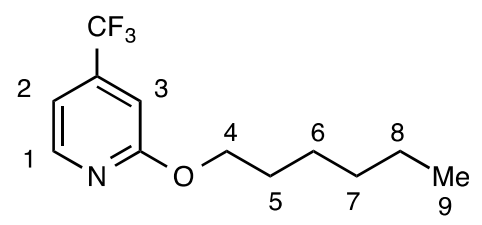

Prepared according to general procedure B using sodium hydride (60\% dispersion in mineral oil, $30 \mathrm{mg}, 0.75 \mathrm{mmol}), n$-hexanol $(94 \mu \mathrm{L}, 0.75 \mathrm{mmol})$, triphenyl(4-(trifluoromethyl)pyridin-2yl)phosphonium trifluoromethanesulfonate $(279 \mathrm{mg}, 0.50 \mathrm{mmol})$ and THF $(1.0 \mathrm{~mL})$. Flash column chromatography (silica gel: 2.5\% EtOAc in hexanes) afforded the title compound as a yellow oil (79 mg, $0.32 \mathrm{mmol}, 64 \%$ yield). IR $v_{\max } / \mathrm{cm}^{-1}$ (film): 2932, 1571, 1423, 1375, 1335, 1171, 1138; ${ }^{1} \mathrm{H}$ NMR (400 MHz, $\left.\mathrm{CDCl}_{3}\right) \delta: 8.28\left(1 \mathrm{H}, \mathrm{d}, J=5.3 \mathrm{~Hz}, \mathrm{H}_{1}\right), 7.02\left(1 \mathrm{H}, \mathrm{d}, J=5.3 \mathrm{~Hz}, \mathrm{H}_{2}\right), 6.94$ $\left(1 \mathrm{H}, \mathrm{s}, \mathrm{H}_{3}\right), 4.32\left(2 \mathrm{H}, \mathrm{t}, J=6.6 \mathrm{~Hz}, \mathrm{H}_{4}\right), 1.77\left(2 \mathrm{H}, \mathrm{qn}, J=6.7 \mathrm{~Hz}, \mathrm{H}_{5}\right), 1.44(2 \mathrm{H}, \mathrm{qn}, J=6.7 \mathrm{~Hz}$, $\left.\mathrm{H}_{6}\right), 1.38-1.28\left(4 \mathrm{H}, \mathrm{m}, \mathrm{H}_{7}\right.$ and $\left.\mathrm{H}_{8}\right), 0.90\left(3 \mathrm{H}, \mathrm{t}, J=6.8 \mathrm{~Hz}, \mathrm{H}_{9}\right) ;{ }^{13} \mathrm{C} \mathrm{NMR}\left(100 \mathrm{MHz}, \mathrm{CDCl}_{3}\right)$ $\delta: 164.48,148.26,140.76(\mathrm{q}, J=33.6 \mathrm{~Hz}), 122.72$ (q, $J=273.1 \mathrm{~Hz}), 111.88(\mathrm{q}, J=3.1 \mathrm{~Hz}), 107.76$ (q, $J=4.0 \mathrm{~Hz}), 66.77,31.57,28.88,25.70,22.60,13.98 ;{ }^{19} \mathrm{~F} \mathrm{NMR}\left(365 \mathrm{MHz}, \mathrm{CDCl}_{3}\right) \delta:-65.16$; $m / z$ LRMS (ESI + APCI) found $[\mathrm{M}+\mathrm{H}]^{+} 248.1, \mathrm{C}_{12} \mathrm{H}_{17} \mathrm{~F}_{3} \mathrm{NO}^{+}$requires 248.1.

\section{4-bromo-2-(hexyloxy)pyridine (3na)}

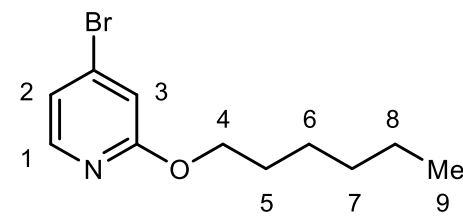

Prepared according to general procedure B (except that the reaction was allowed to stir for 14 hours after the addition of the phosphonium salt and $\mathrm{CH}_{2} \mathrm{Cl}_{2}$ was used instead of $\mathrm{Et}_{2} \mathrm{O}$ for the extraction stage of the workup) using sodium hydride (60\% dispersion in mineral oil, $11 \mathrm{mg}, 0.28$ mmol), hexanol (35 $\mu \mathrm{L}, 0.28 \mu \mathrm{mmol}$ ), (4-bromopyridin-2-yl)triphenylphosphonium trifluoromethanesulfonate $(142 \mathrm{mg}, 0.25 \mathrm{mmol})$ and THF $(0.5 \mathrm{~mL})$. Flash column chromatography (silica gel, gradient elution: $10 \%$ toluene in hexanes to $20 \%$ toluene in hexanes) to afford the title 
compound as a clear oil (33 mg, $0.13 \mathrm{mmol}, 52 \%$ yield). IR $v_{\max } / \mathrm{cm}^{-1}$ (film): 2927, 1577, 1553, 1466, 1410, 1351, 1308, 1221, 1016, 982; ${ }^{1} \mathrm{H}$ NMR $\left(400 \mathrm{MHz}, \mathrm{CDCl}_{3}\right) \delta: 7.96(1 \mathrm{H}, \mathrm{d}, J=5.7 \mathrm{~Hz}$, $\left.\mathrm{H}_{1}\right), 6.99\left(1 \mathrm{H}, \mathrm{dd}, J=5.7,1.5 \mathrm{~Hz}, \mathrm{H}_{2}\right), 6.93\left(1 \mathrm{H}, \mathrm{d}, J=1.5 \mathrm{~Hz}, \mathrm{H}_{3}\right), 4.26\left(2 \mathrm{H}, \mathrm{t}, J=6.7 \mathrm{~Hz}, \mathrm{H}_{4}\right)$, $1.75\left(2 \mathrm{H}, \mathrm{qn}, J=7.3 \mathrm{~Hz}, \mathrm{H}_{5}\right), 1.49-1.28\left(6 \mathrm{H}, \mathrm{m}, \mathrm{H}_{6}, \mathrm{H}_{7}\right.$, and $\left.\mathrm{H}_{8}\right), 0.90\left(3 \mathrm{H}, \mathrm{m}, \mathrm{H}_{9}\right) ;{ }^{13} \mathrm{C}$ NMR (100 $\left.\mathrm{MHz}, \mathrm{CDCl}_{3}\right)$ 164.69, 147.44, 133.69, 119.97, 114.26, 66.61, 31.54, 28.91, 25.67, 22.58, 14.01; $\mathrm{m} / \mathrm{z}$ LRMS (ESI + APCI) found $[\mathrm{M}+\mathrm{H}]^{+} 258.1, \mathrm{C}_{11} \mathrm{H}_{17} \mathrm{BrNO}^{+}$requires 258.0.

\section{2-Butyl-6-(hexyloxy)-3-(methoxymethyl)-4-(p-tolyl)pyridine (3oa)}

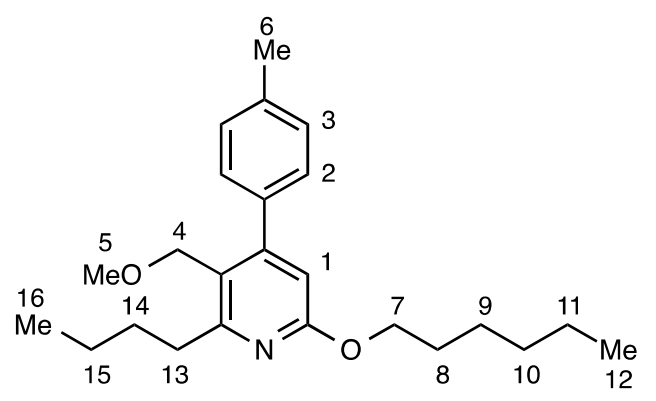

Prepared according to general procedure B using sodium hydride (60\% dispersion in mineral oil, $15 \mathrm{mg}, 0.38 \mathrm{mmol}$ ), $n$-hexanol (47 $\mu \mathrm{L}, 0.38 \mathrm{mmol})$, (6-butyl-5-(methoxymethyl)-4-( $p$ tolyl)pyridin-2-yl)triphenylphosphonium trifluoromethanesulfonate $(170 \mathrm{mg}, 0.25 \mathrm{mmol})$ and THF $\left(0.5 \mathrm{~mL}\right.$ ). Flash column chromatography (silica gel, gradient elution: $20 \%$ hexanes in $\mathrm{CH}_{2} \mathrm{Cl}_{2}$ to $\mathrm{CH}_{2} \mathrm{Cl}_{2}$ ) afforded the title compound as a yellow oil ( $69 \mathrm{mg}, 0.19 \mathrm{mmol}, 76 \%$ yield). IR $v_{\max } / \mathrm{cm}^{-}$ ${ }^{1}$ (film): 2927, 1594, 1342 1185, 1089, 821; ${ }^{1} \mathrm{H}$ NMR (400 MHz, $\left.\mathrm{CDCl}_{3}\right) \delta: 7.32$ (2H, d, $J=7.7$ $\left.\mathrm{Hz}, \mathrm{H}_{2}\right), 7.23\left(2 \mathrm{H}, \mathrm{d}, J=7.7 \mathrm{~Hz}, \mathrm{H}_{3}\right), 6.47\left(1 \mathrm{H}, \mathrm{s}, \mathrm{H}_{1}\right), 4.31\left(2 \mathrm{H}, \mathrm{t}, J=6.7 \mathrm{~Hz}, \mathrm{H}_{7}\right), 4.20(2 \mathrm{H}, \mathrm{s}$, $\left.\mathrm{H}_{4}\right), 3.32\left(3 \mathrm{H}, \mathrm{s}, \mathrm{H}_{5}\right), 2.85\left(2 \mathrm{H}, \mathrm{t}, J=7.8 \mathrm{~Hz}, \mathrm{H}_{13}\right), 2.41\left(3 \mathrm{H}, \mathrm{s}, \mathrm{H}_{6}\right), 1.84-1.72\left(4 \mathrm{H}, \mathrm{m}, \mathrm{H}_{14}\right.$ and $\left.\mathrm{H}_{8}\right), 1.52-1.41\left(4 \mathrm{H}, \mathrm{m}, \mathrm{H}_{15}\right.$ and $\left.\mathrm{H}_{9}\right), 1.40-1.29\left(4 \mathrm{H}, \mathrm{m}, \mathrm{H}_{10}\right.$ and $\left.\mathrm{H}_{11}\right), 0.99\left(3 \mathrm{H}, \mathrm{t}, J=7.4 \mathrm{~Hz}, \mathrm{H}_{16}\right)$, $0.91\left(3 \mathrm{H}, \mathrm{t}, J=7.0 \mathrm{~Hz}, \mathrm{H}_{12}\right) ;{ }^{13} \mathrm{C}$ NMR $\left(100 \mathrm{MHz}, \mathrm{CDCl}_{3}\right) \delta: 162.64,161.30,154.16,137.59$, 136.74, 128.80, 128.71, 121.05, 107.82, 68.36, 65.95, 57.86, 34.45, 31.67, 31.64, 29.12, 25.76, 22.87, 22.61, 21.17, 14.09, 14.02; m/z LRMS (ESI + APCI) found $[\mathrm{M}+\mathrm{H}]^{+} 370.3, \mathrm{C}_{24} \mathrm{H}_{36} \mathrm{NO}_{2}{ }^{+}$ requires 370.3 . 


\section{4-(Hexyloxy)-5-(4-methoxyphenyl)pyrimidine (3pa)}

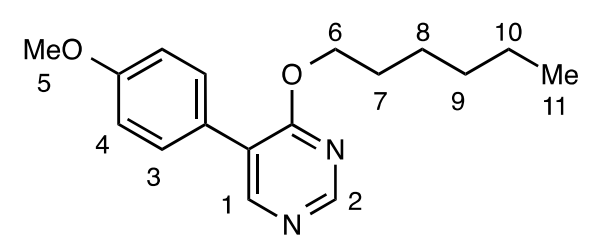

Prepared according to general procedure B (except that the reaction was allowed to stir for 30 hours after the addition of the phosphonium salt) using sodium hydride ( $60 \%$ dispersion in mineral oil, $30 \mathrm{mg}, 0.75 \mathrm{mmol}), n$-hexanol (94 $\mu \mathrm{L}, 0.75 \mathrm{mmol})$, (5-(4-methoxyphenyl)pyrimidin-4yl)triphenylphosphonium trifluoromethanesulfonate $(298 \mathrm{mg}, 0.50 \mathrm{mmol})$ and THF (1.0 mL). Flash column chromatography (silica gel, gradient elution: 10\% EtOAc in hexanes to 20\% EtOAc in hexanes) afforded the title compound as a yellow oil $\left(85 \mathrm{mg}, 0.30 \mathrm{mmol}, 60 \%\right.$ yield). IR $v_{\max } / \mathrm{cm}^{-}$ ${ }^{1}$ (film): 3038, 2930, 1554, 1448, 1306, 1249, 995, 753; ${ }^{1} \mathrm{H}$ NMR (400 MHz, $\left.\mathrm{CDCl}_{3}\right) \delta: 8.68(1 \mathrm{H}$, $\left.\mathrm{s}, \mathrm{H}_{2}\right), 8.45\left(1 \mathrm{H}\right.$, br s, $\left.\mathrm{H}_{1}\right), 7.52-7.47\left(2 \mathrm{H}, \mathrm{m}, \mathrm{H}_{3}\right), 6.99-6.93\left(2 \mathrm{H}, \mathrm{m}, \mathrm{H}_{4}\right), 4.41(2 \mathrm{H}, \mathrm{t}, J=6.7 \mathrm{~Hz}$, $\left.\mathrm{H}_{6}\right), 3.84\left(3 \mathrm{H}, \mathrm{s}, \mathrm{H}_{5}\right), 1.77\left(2 \mathrm{H}, \mathrm{qn}, J=6.8 \mathrm{~Hz}, \mathrm{H}_{7}\right), 1.41\left(2 \mathrm{H}, \mathrm{qn}, J=6.8 \mathrm{~Hz}, \mathrm{H}_{8}\right), 1.36-1.27(4 \mathrm{H}$, $\mathrm{m}, \mathrm{H}_{9}$ and $\left.\mathrm{H}_{10}\right), 0.87\left(3 \mathrm{H}, \mathrm{t}, J=6.9 \mathrm{~Hz}, \mathrm{H}_{11}\right) ;{ }^{13} \mathrm{C} \mathrm{NMR}\left(100 \mathrm{MHz}, \mathrm{CDCl}_{3}\right) \delta: 165.87,159.57$, 156.36, 155.36, 130.06, 125.29, 121.84, 113.87, 66.87, 55.22, 31.35, 28.53, 25.59, 22.46, 13.90; $m / z$ LRMS (ESI + APCI) found $[\mathrm{M}+\mathrm{H}]^{+} 287.2, \mathrm{C}_{17} \mathrm{H}_{23} \mathrm{~N}_{2} \mathrm{O}_{2}{ }^{+}$requires 287.2.

\section{2-(Hexyloxy)pyrazine (3qa)}

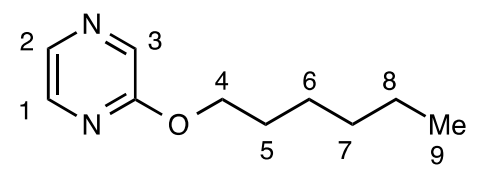

Prepared according to general procedure B using sodium hydride (60\% dispersion in mineral oil, $30 \mathrm{mg}, 0.75 \mathrm{mmol}$ ), $n$-hexanol (94 $\mu \mathrm{L}, 0.75 \mathrm{mmol}$ ), triphenyl(pyrazin-2-yl)phosphonium trifluoromethanesulfonate $(245 \mathrm{mg}, 0.5 \mathrm{mmol})$ and THF $(1.0 \mathrm{~mL})$. Flash column chromatography (silica gel: 10\% EtOAc in hexanes) afforded the title compound as a yellow oil (70 $\mathrm{mg}, 0.39 \mathrm{mmol}$, $78 \%$ yield). IR $v_{\max } / \mathrm{cm}^{-1}$ (film): 3060, 2923, 1532, 1414, 1284, 1005; ${ }^{1} \mathrm{H} \mathrm{NMR}\left(400 \mathrm{MHz}, \mathrm{CDCl}_{3}\right)$ $\delta: 8.19\left(1 \mathrm{H}\right.$, br s, $\left.\mathrm{H}_{1}\right), 8.10-7.97\left(2 \mathrm{H}\right.$, br s, $\mathrm{H}_{2}$ and $\left.\mathrm{H}_{3}\right), 4.28\left(2 \mathrm{H}, \mathrm{t}, J=6.7 \mathrm{~Hz}, \mathrm{H}_{4}\right), 1.76(2 \mathrm{H}, \mathrm{qn}$, 
$\left.J=6.8 \mathrm{~Hz}, \mathrm{H}_{5}\right), 1.42\left(2 \mathrm{H}, \mathrm{qn}, J=6.8 \mathrm{~Hz}, \mathrm{H}_{6}\right), 1.37-1.25\left(4 \mathrm{H}, \mathrm{m}, \mathrm{H}_{7}\right.$ and $\left.\mathrm{H}_{8}\right), 0.88(3 \mathrm{H}, \mathrm{t}, J=6.8$ $\left.\mathrm{Hz}, \mathrm{H}_{9}\right) ;{ }^{13} \mathrm{C}$ NMR (100 MHz, $\left.\mathrm{CDCl}_{3}\right) \delta: 160.46,140.49,136.18,136.02,66.40,31.49,28.74$, $25.60,22.54,13.97$. The spectroscopic data is in agreement with a reported synthesis. ${ }^{8} \mathrm{~m} / z$ LRMS $(\mathrm{ESI}+\mathrm{APCI})$ found $[\mathrm{M}+\mathrm{H}]^{+} 181.2, \mathrm{C}_{10} \mathrm{H}_{17} \mathrm{~N}_{2} \mathrm{O}^{+}$requires 181.1 .

\section{(S)-4-methoxy-3-(1-methylpyrrolidin-2-yl)pyridine (5)}

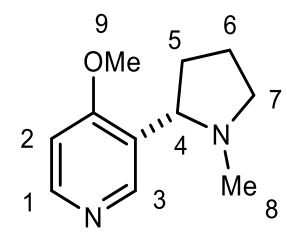

Prepared according to general procedure $\mathrm{B}$ (except that $\mathrm{CH}_{2} \mathrm{Cl}_{2}$ was used instead of $\mathrm{Et}_{2} \mathrm{O}$ for the extraction stage of the workup) using sodium hydride (60\% dispersion in mineral oil, $30 \mathrm{mg}, 0.75$ mmol), methanol (30 $\mu \mathrm{L}, \quad 0.75 \quad \mathrm{mmol}), \quad(S)$-(3-(1-methylpyrrolidin-2-yl)pyridin-4yl)triphenylphosphonium trifluoromethanesulfonate $(286 \mathrm{mg}, 0.50 \mathrm{mmol})$ and THF $(1.0 \mathrm{~mL})$. Flash column chromatography (silica gel was packed in hexanes and neutralized with $\mathrm{NEt}_{3}$ then gradient elution: $1 \% \mathrm{MeOH}$ in $\mathrm{CH}_{2} \mathrm{Cl}_{2}$ to $10 \% \mathrm{MeOH}$ in $\mathrm{CH}_{2} \mathrm{Cl}_{2}$ ) followed by a second flash column (basic alumina: $2 \% \mathrm{MeOH}$ in $\mathrm{CH}_{2} \mathrm{Cl}_{2}$ ) afforded the title compound as a tan amorphous solid (76 mg, $0.40 \mathrm{mmol}, 79 \%$ yield). $\mathrm{mp} 73-75^{\circ} \mathrm{C}$; IR $v_{\max } / \mathrm{cm}^{-1}$ (film): 3031, 2969, 2780, 1589 , $1455,1274,1023,805 ;{ }^{1} \mathrm{H}$ NMR $\left(400 \mathrm{MHz}, \mathrm{CDCl}_{3}\right) \delta: 8.51\left(1 \mathrm{H}, \mathrm{br} \mathrm{s}, \mathrm{H}_{3}\right), 8.34\left(1 \mathrm{H}, \mathrm{br} \mathrm{s}, \mathrm{H}_{1}\right)$, $6.70\left(1 \mathrm{H}, \mathrm{d}, J=5.7 \mathrm{~Hz}, \mathrm{H}_{2}\right), 3.81\left(3 \mathrm{H}, \mathrm{s}, \mathrm{H}_{9}\right), 3.44\left(1 \mathrm{H}\right.$, app t, $\left.J=8.3 \mathrm{~Hz}, \mathrm{H}_{4}\right), 3.19(1 \mathrm{H}$, app t, $J$ $\left.=8.5 \mathrm{~Hz}, \mathrm{H}_{7}\right), 2.30-2.14\left(5 \mathrm{H}, \mathrm{m}, \mathrm{H}_{5}, \mathrm{H}_{7}\right.$, and $\left.\mathrm{H}_{8}\right), 1.93-1.80\left(1 \mathrm{H}, \mathrm{m}, \mathrm{H}_{6}\right), 1.80-1.69\left(1 \mathrm{H}, \mathrm{m}, \mathrm{H}_{6}\right)$, 1.64-1.52 (1H, m, $\left.\mathrm{H}_{5}\right) ;{ }^{13} \mathrm{C}$ NMR (100 MHz, $\left.\mathrm{CDCl}_{3}\right) \delta: 163.51,149.64,148.92,126.87,105.58$, $62.64,56.96,55.14,40.73,33.02,22.66$. The spectroscopic data is in agreement with a reported synthesis. ${ }^{9} \mathrm{~m} / z$ LRMS (ESI + APCI) found $[\mathrm{M}+\mathrm{H}]^{+}$193.1, $\mathrm{C}_{11} \mathrm{H}_{17} \mathrm{~N}_{2} \mathrm{O}^{+}$requires 193.1. 


\section{Ethyl 4-(8-chloro-4-(hexyloxy)-5,6-dihydro-11 $H$-benzo[5,6]cyclohepta[1,2-b]pyridin-11-}

ylidene)piperidine-1-carboxylate (7)

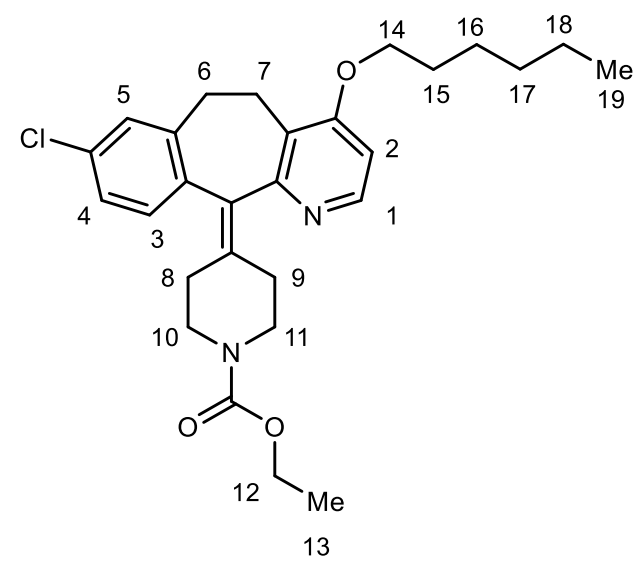

Prepared according to general procedure B (except that the reaction was allowed to stir for 15 hours after the addition of the phosphonium salt and $\mathrm{CH}_{2} \mathrm{Cl}_{2}$ was used instead of $\mathrm{Et}_{2} \mathrm{O}$ for the extraction stage of the workup) using sodium hydride (60\% dispersion in mineral oil, $15 \mathrm{mg}, 0.38$ mmol), $n$-hexanol (47 $\mu \mathrm{L}, 0.38 \mathrm{mmol})$, (8-chloro-11-(1-(ethoxycarbonyl)piperidin-4-ylidene)6,11-dihydro-5H-benzo[5,6]cyclohepta[1,2-b]pyridin-4-yl)triphenylphosphonium trifluoromethanesulfonate $(198 \mathrm{mg}, 0.25 \mathrm{mmol})$ and THF $(0.5 \mathrm{~mL})$. Flash column chromatography (silica gel was packed in hexanes and neutralized with $\mathrm{NEt}_{3}$ then: $1 \% \mathrm{NEt}_{3}, 50 \% \mathrm{CH}_{2} \mathrm{Cl}_{2}$ in hexanes) followed by a second flash column (basic alumina, gradient elution: 30\% EtOAc in hexanes to 50\% EtOAc in hexanes) $)^{\text {t* }}$ afforded the title compound as a yellow oil (64 mg, 0.13 mmol, 53\% yield). IR $v_{\max } / \mathrm{cm}^{-1}$ (film): 3010, 2929, 1688, 1567, 1435, 1230, $748{ }^{1} \mathrm{H}$ NMR (400 $\left.\mathrm{MHz}, \mathrm{CDCl}_{3}\right) \delta: 8.27\left(1 \mathrm{H}, \mathrm{d}, J=5.6 \mathrm{~Hz}, \mathrm{H}_{1}\right), 7.18\left(1 \mathrm{H}, \mathrm{s}, \mathrm{H}_{5}\right), 7.14-7.07\left(2 \mathrm{H}, \mathrm{m}, \mathrm{H}_{3}\right.$ and $\left.\mathrm{H}_{4}\right), 6.61$ $\left(1 \mathrm{H}, \mathrm{d}, J=5.6 \mathrm{~Hz}, \mathrm{H}_{2}\right), 4.12\left(2 \mathrm{H}, \mathrm{q}, J=7.1 \mathrm{~Hz}, \mathrm{H}_{12}\right), 4.01-3.69\left(4 \mathrm{H}, \mathrm{m}, \mathrm{H}_{10}\right.$ or $\mathrm{H}_{11}$ and $\left.\mathrm{H}_{12}\right), 3.40-$ $3.28\left(1 \mathrm{H}, \mathrm{m}, \mathrm{H}_{6}\right), 3.17-2.98\left(3 \mathrm{H}, \mathrm{m}, \mathrm{H}_{7}\right.$ and $\mathrm{H}_{10}$ or $\left.\mathrm{H}_{11}\right), 2.92-2.71\left(2 \mathrm{H}, \mathrm{m}, \mathrm{H}_{6}\right.$ and $\left.\mathrm{H}_{7}\right), 2.58-2.45$ $\left(1 \mathrm{H}, \mathrm{m}, \mathrm{H}_{8}\right.$ or $\left.\mathrm{H}_{9}\right), 2.43-2.18\left(3 \mathrm{H}, \mathrm{m}, \mathrm{H}_{8}\right.$ or $\left.\mathrm{H}_{9}\right), 1.77\left(2 \mathrm{H}, \mathrm{qn}, J=6.9 \mathrm{~Hz}, \mathrm{H}_{15}\right), 1.50-1.28(6 \mathrm{H}, \mathrm{m}$, $\mathrm{H}_{16}, \mathrm{H}_{17}$, and $\left.\mathrm{H}_{18}\right), 1.27-1.17\left(3 \mathrm{H}, \mathrm{m}, \mathrm{H}_{12}\right), 0.89\left(3 \mathrm{H}, \mathrm{t}, J=6.9 \mathrm{~Hz}, \mathrm{H}_{19}\right) ;{ }^{13} \mathrm{C} \mathrm{NMR}(100 \mathrm{MHz}$,

¥¥¥ To remove an impurity the product was dissolved in $\mathrm{Et}_{2} \mathrm{O}(10 \mathrm{~mL})$ and trifluoroacetic acid $(0.5$ $\mathrm{mL}$ ) was added dropwise at $0{ }^{\circ} \mathrm{C}$ and then concentrated in vacuo. The residue was dissolved in $\mathrm{CH}_{2} \mathrm{Cl}_{2}$ and washed with a saturated aqueous solution of sodium bicarbonate $(10 \mathrm{~mL})$. The organic layer was washed with a saturated aqueous solution of brine, dried $\left(\mathrm{MgSO}_{4}\right)$, filtered and concentrated in vacuo. 
$\left.\mathrm{CDCl}_{3}\right) \delta: 163.41,156.51,155.43,147.69,140.30,138.79,137.37,133.98,132.73,129.73$, $128.28,126.05,122.03,104.98,68.08,61.22,44.78,44.70,31.40,30.70,30.56,29.64,28.86$, 25.67, 25.33, 22.49, 14.63, 13.94; $\mathrm{m} / z$ LRMS (ESI + APCI) found $[\mathrm{M}+\mathrm{H}]^{+} 483.3, \mathrm{C}_{28} \mathrm{H}_{36} \mathrm{ClN}_{2} \mathrm{O}_{3}{ }^{+}$ requires 483.2 .

3-(4-Chlorophenyl)-3-(4-methoxypyridin-2-yl)- $N, N$-dimethylpropan-1-amine (9)

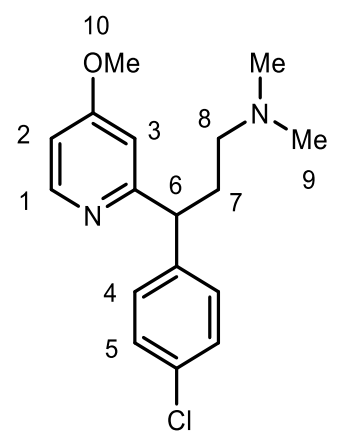

Prepared according to general procedure B (except that the reaction was allowed to stir for 18 hours after the addition of the phosphonium salt and $\mathrm{CH}_{2} \mathrm{Cl}_{2}$ was used instead of $\mathrm{Et}_{2} \mathrm{O}$ for the extraction stage of the workup) using sodium hydride (60\% dispersion in mineral oil, $15 \mathrm{mg}, 0.38$

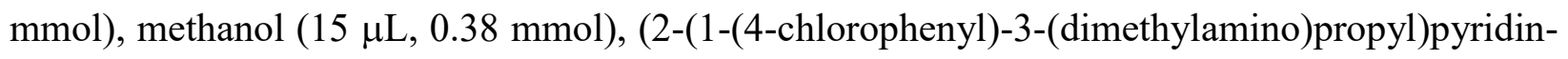
4-yl)triphenylphosphonium trifluoromethanesulfonate $(171 \mathrm{mg}, 0.25 \mathrm{mmol})$ and THF $(0.5 \mathrm{~mL})$. Flash column chromatography (silica gel was packed in hexanes and neutralized with $\mathrm{NEt}_{3}$ then: $1 \% \mathrm{MeOH}, 1 \% \mathrm{NEt}_{3}$ in $\mathrm{CH}_{2} \mathrm{Cl}_{2}$ ) followed by a second flash column (basic alumina: $2 \% \mathrm{MeOH}$ in $\mathrm{CH}_{2} \mathrm{Cl}_{2}$ ) afforded the title compound as a tan oil (35 mg, $0.12 \mathrm{mmol}, 46 \%$ yield). IR $v_{\max } / \mathrm{cm}^{-1}$ (film): 3011, 2940, 2768, 1593, 1567, 1488, 1304, 1037, 749; ${ }^{1} \mathrm{H}$ NMR (400 MHz, $\left.\mathrm{CDCl}_{3}\right) \delta: 8.35$ $\left(1 \mathrm{H}, \mathrm{d}, J=5.6 \mathrm{~Hz}, \mathrm{H}_{1}\right), 7.28-7.18\left(4 \mathrm{H}, \mathrm{m}, \mathrm{H}_{4}\right.$ and $\left.\mathrm{H}_{5}\right), 6.64\left(1 \mathrm{H}, \mathrm{d}, J=2.4 \mathrm{~Hz}, \mathrm{H}_{3}\right), 6.60(1 \mathrm{H}, \mathrm{dd}$, $\left.J=5.6,2.4 \mathrm{~Hz}, \mathrm{H}_{2}\right), 4.05-4.00\left(1 \mathrm{H}, \mathrm{m}, \mathrm{H}_{6}\right), 3.75\left(3 \mathrm{H}, \mathrm{s}, \mathrm{H}_{10}\right), 2.44-2.30\left(1 \mathrm{H}, \mathrm{m}, \mathrm{H}_{7}\right), 2.21-2.06$ (9H, $\mathrm{m}, \mathrm{H}_{7}, \mathrm{H}_{8}$, and $\left.\mathrm{H}_{9}\right) ;{ }^{13} \mathrm{C} \mathrm{NMR}\left(100 \mathrm{MHz}, \mathrm{CDCl}_{3}\right) \delta: 166.01,164.65,150.60,142.03,132.15$, 129.33, 128.54, 108.86, 107.51, 57.59, 54.98, 50.60, 45.36, 32.69; $\mathrm{m} / z$ LRMS (ESI + APCI) found $[\mathrm{M}+\mathrm{H}]^{+}$305.2, $\mathrm{C}_{17} \mathrm{H}_{22} \mathrm{ClN}_{2} \mathrm{O}^{+}$requires 305.1. 
(rac)-2-(Hexyloxy)-7,8,9,10-tetrahydro-6H-6,10-methanoazepino[4,5-g]quinoxaline (11)

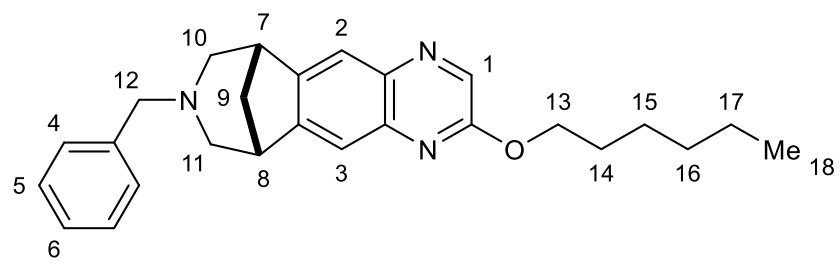

Prepared according to general procedure B using sodium hydride (60\% dispersion in mineral oil, $11 \mathrm{mg}, 0.28 \mathrm{mmol}), n$-hexanol (35 $\mu \mathrm{L}, 0.28 \mathrm{mmol})$, triphenyl(( $\mathrm{rac})-7,8,9,10$-tetrahydro- $6 \mathrm{H}-6,10$ methanoazepino[4,5-g]quinoxalin-2-yl)phosphonium trifluoromethanesulfonate (130 mg, 0.18 $\mathrm{mmol})$ and THF (0.4 mL). Flash column chromatography (silica gel: 20\% EtOAc in hexanes) afforded the title compound as a tan oil (18 $\mathrm{mg}, 0.045 \mathrm{mmol}, 25 \%$ yield).; IR $v_{\max } / \mathrm{cm}^{-1}$ (film): 3026, 2950, 1570, 1473, 1345, 1300, 1204; ${ }^{1} \mathrm{H}$ NMR (400 MHz, $\left.\mathrm{CDCl}_{3}\right)$ 8: $8.40\left(1 \mathrm{H}, \mathrm{s}, \mathrm{H}_{1}\right), 7.69$ $\left(1 \mathrm{H}, \mathrm{s}, \mathrm{H}_{2}\right) 7.56\left(1 \mathrm{H}, \mathrm{s}, \mathrm{H}_{3}\right), 7.15-7.09\left(3 \mathrm{H}, \mathrm{m}, \mathrm{H}_{5}\right.$ and $\left.\mathrm{H}_{6}\right), 6.90-6.82\left(2 \mathrm{H}, \mathrm{m}, \mathrm{H}_{4}\right), 4.47(2 \mathrm{H}, \mathrm{t}, J=$ $\left.6.8 \mathrm{~Hz}, \mathrm{H}_{13}\right), 3.48\left(2 \mathrm{H}, \mathrm{s}, \mathrm{H}_{12}\right)$ 3.32-3.25 (2H, br s, $\mathrm{H}_{7}$ and $\left.\mathrm{H}_{8}\right), 2.95\left(2 \mathrm{H}, \mathrm{m}, \mathrm{H}_{10}\right.$ or $\left.\mathrm{H}_{11}\right), 2.53$ (2H, m, $\mathrm{H}_{10}$ or $\left.\mathrm{H}_{11}\right), 2.37-2.27$ (1H, m, $\left.\mathrm{H}_{9}\right), 1.91-1.78$ (3H, m, $\mathrm{H}_{9}$ and $\left.\mathrm{H}_{14}\right), 1.57-1.45$ (2H, m, $\left.\mathrm{H}_{15}\right), 1.44-1.32\left(4 \mathrm{H}, \mathrm{m}, \mathrm{H}_{16}\right.$ and $\left.\mathrm{H}_{17}\right), 0.93\left(3 \mathrm{H}, \mathrm{t}, J=7.0 \mathrm{~Hz}, \mathrm{H}_{18}\right) ;{ }^{13} \mathrm{C}$ NMR $\left(100 \mathrm{MHz}, \mathrm{CDCl}_{3}\right)$ $\delta: 157.19,150.56,146.56,140.35,138.59,138.38,137.42,128.34,127.99,126.58,120.32$, $118.83,66.29,61.59,57.31,57.12,43.51,41.38,40.98,31.58,28.83,25.75,22.61,14.06 ; \mathrm{m} / \mathrm{z}$ LRMS (ESI + APCI) found $[\mathrm{M}+\mathrm{H}]^{+} 402.2, \mathrm{C}_{26} \mathrm{H}_{32} \mathrm{~N}_{3} \mathrm{O}^{+}$requires 402.3 .

\section{3-((8R,9S,10R,13S,14S)-3-((Tert-butyldimethylsilyl)oxy)-10,13-dimethyl-}

\section{2,3,4,7,8,9,10,11,12,13,14,15-dodecahydro- $1 H$-cyclopenta[a]phenanthren-17-yl)-4-} (hexyloxy)pyridine (13)

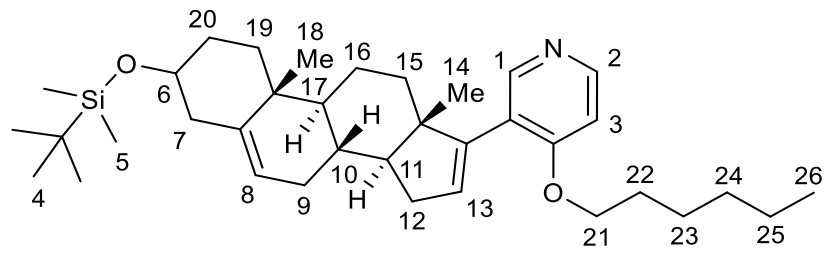


Prepared according to general procedure B using sodium hydride (60\% dispersion in mineral oil, $15 \mathrm{mg}, 0.38 \mathrm{mmol}), \quad n$-hexanol (47 $\mu \mathrm{L}, 0.38 \mathrm{mmol}), \quad(3-((8 R, 9 S, 10 R, 13 S, 14 S)-3-(($ tertbutyldimethylsilyl)oxy)-10,13-dimethyl-2,3,4,7,8,9,10,11,12,13,14,15-dodecahydro-1Hcyclopenta[a]phenanthren-17-yl)pyridin-4-yl)triphenylphosphonium trifluoromethanesulfonate (219 mg, $0.25 \mathrm{mmol})$ and THF $(0.5 \mathrm{~mL})$. Flash column chromatography (silica gel, gradient elution: $30 \%$ EtOAc in hexanes to $40 \%$ EtOAc in hexanes) afforded the title compound as a white solid (82 mg, $0.15 \mathrm{mmol}, 60 \%$ yield). mp 59-64 ${ }^{\circ} \mathrm{C}$; IR $v_{\max } / \mathrm{cm}^{-1}$ (film): 2928, 1577, 1496, 1382, 1279, 1250, 1077, 1022, 888; ${ }^{1} \mathrm{H}$ NMR (400 MHz, $\left.\mathrm{CDCl}_{3}\right)$ 8: 8.40-8.15 (2H, m), 6.76 ( $1 \mathrm{H}, \mathrm{d}, J=$ $5.6 \mathrm{~Hz}), 5.89-5.84(1 \mathrm{H}, \mathrm{dd}, J=3.0,1.5 \mathrm{~Hz}), 5.38-5.32(1 \mathrm{H}, \mathrm{m}), 3.98(2 \mathrm{H}, \mathrm{t}, J=6.6 \mathrm{~Hz}), 3.49(1 \mathrm{H}$, m), 2.34-2.14 (3H, m), 2.12-1.99 (2H, m), 1.85-1.27 (18H, m), 1.12-0.81 (20H, m), 0.05 (6H, s); ${ }^{13} \mathrm{C}$ NMR (100 MHz, $\left.\mathrm{CDCl}_{3}\right) \delta: 163.00,150.04,149.49,148.60,141.88,130.91,123.01,120.90$, 106.78, 72.57, 68.01, 57.21, 50.59, 48.61, 42.83, 37.29, 36.79, 34.99, 32.14, 32.05, 31.63, 31.38, $30.71,28.74,25.92,25.66,22.56,20.84,19.35,18.24,16.12,13.98,-4.60 ; \mathrm{m} / z$ LRMS (ESI + APCI) found $[\mathrm{M}+\mathrm{H}]^{+}$564.4, $\mathrm{C}_{36} \mathrm{H}_{58} \mathrm{NO}_{2} \mathrm{Si}^{+}$requires 564.4.

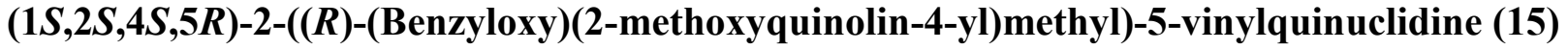

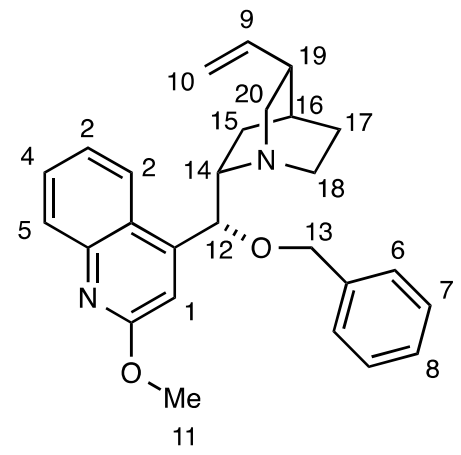

Prepared according to general procedure B (except that the reaction was allowed to stir for 11 hours after the addition of the phosphonium salt and $\mathrm{CH}_{2} \mathrm{Cl}_{2}$ was used instead of $\mathrm{Et}_{2} \mathrm{O}$ for the extraction stage of the workup) using sodium hydride (60\% dispersion in mineral oil, $15 \mathrm{mg}, 0.38$ mmol), methanol (15 $\mu \mathrm{L}, 0.38 \mathrm{mmol})$, (4-((R)-(benzyloxy)((1S,2S,4S,5R)-5-vinylquinuclidin-2yl)methyl)quinolin-2-yl)triphenylphosphonium trifluoromethanesulfonate (199 mg, $0.25 \mathrm{mmol}$ ) and THF $(0.5 \mathrm{~mL})$. Flash column chromatography (silica gel was packed in hexanes and 
neutralized with $\mathrm{NEt}_{3}$ then: $1 \% \mathrm{NEt}_{3}, 50 \% \mathrm{CH}_{2} \mathrm{Cl}_{2}$ in hexanes) afforded the title compound as a pale yellow oil (60 mg, $0.14 \mathrm{mmol}, 58 \%$ yield). IR $v_{\max } / \mathrm{cm}^{-1}$ (film): 3065, 2938, 1609, 1382, 1338, 1236, 1045, 754; ${ }^{1} \mathrm{H}$ NMR (400 MHz, $\left.\mathrm{CDCl}_{3}\right) \delta: 8.00\left(1 \mathrm{H}, \mathrm{d}, J=8.0 \mathrm{~Hz}, \mathrm{H}_{2}\right), 7.91(1 \mathrm{H}, \mathrm{d}, J=8.2$ $\left.\mathrm{Hz}, \mathrm{H}_{5}\right), 7.64\left(1 \mathrm{H}, \mathrm{t}, J=7.5 \mathrm{~Hz}, \mathrm{H}_{4}\right), 7.45-7.27\left(6 \mathrm{H}, \mathrm{m}, \mathrm{H}_{6}, \mathrm{H}_{7}, \mathrm{H}_{8}\right.$ and $\left.\mathrm{H}_{3}\right), 7.06\left(1 \mathrm{H} \mathrm{s}, \mathrm{H}_{1}\right), 5.83-$ $5.64\left(1 \mathrm{H}, \mathrm{m}, \mathrm{H}_{9}\right), 5.31-5.15\left(1 \mathrm{H}\right.$, br s, $\left.\mathrm{H}_{12}\right), 5.00-4.85\left(2 \mathrm{H}, \mathrm{m}, \mathrm{H}_{10}\right), 4.50\left(1 \mathrm{H}, \mathrm{d}, J=11.3 \mathrm{~Hz}, \mathrm{H}_{13}\right)$, $4.40\left(1 \mathrm{H}, \mathrm{d}, J=11.3 \mathrm{~Hz}, \mathrm{H}_{13}\right), 4.07\left(3 \mathrm{H}, \mathrm{s}, \mathrm{H}_{11}\right), 3.45-3.31\left(1 \mathrm{H}, \mathrm{m}, \mathrm{H}_{18}\right), 3.18-3.03\left(2 \mathrm{H}, \mathrm{m}, \mathrm{H}_{20}\right.$ and $\left.\mathrm{H}_{14}\right), 2.72-2.55\left(2 \mathrm{H}, \mathrm{m}, \mathrm{H}_{18}\right.$ and $\left.\mathrm{H}_{20}\right), 2.30-2.18\left(1 \mathrm{H}, \mathrm{m}, \mathrm{H}_{19}\right), 1.89-1.40\left(5 \mathrm{H}, \mathrm{m}, \mathrm{H}_{15}, \mathrm{H}_{16}\right.$, and $\left.\mathrm{H}_{17}\right) ;{ }^{13} \mathrm{C} \mathrm{NMR}\left(100 \mathrm{MHz}, \mathrm{CDCl}_{3}\right) \delta: 162.30,148.70,147.35,141.69,137.83,129.26,128.38$, $128.17,127.65,127.61,124.10,123.38,123.23,114.29,110.34,80.78,71.23,60.30,56.94,53.24$, 43.17, 39.89, 27.89, 27.55, 22.07; $m / z$ LRMS (ESI + APCI) found $[\mathrm{M}+\mathrm{H}]^{+} 415.3, \mathrm{C}_{27} \mathrm{H}_{31} \mathrm{~N}_{2} \mathrm{O}_{2}{ }^{+}$ requires 415.2 .

\section{(S)-4-(benzylthio)-3-(1-methylpyrrolidin-2-yl)pyridine (16)}

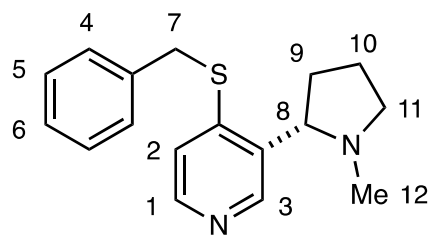

Prepared according to general procedure B (except that the reaction was allowed to stir for 18 hours after the addition of the phosphonium salt and $\mathrm{CH}_{2} \mathrm{Cl}_{2}$ was used instead of $\mathrm{Et}_{2} \mathrm{O}$ for the extraction stage of the workup) using sodium hydride (60\% dispersion in mineral oil, $15 \mathrm{mg}, 0.38$ mmol), benzyl mercaptan (47 $\mu \mathrm{L}, 0.38 \mathrm{mmol})$, (S)-(3-(1-methylpyrrolidin-2-yl)pyridin-4yl)triphenylphosphonium trifluoromethanesulfonate $(143 \mathrm{mg}, 0.25 \mathrm{mmol})$ and $\mathrm{THF}(1.0 \mathrm{~mL})$. Flash column chromatography (silica gel, gradient elution: $2.5 \% \mathrm{MeOH}$ in $\mathrm{CH}_{2} \mathrm{Cl}_{2}$ to $5 \% \mathrm{MeOH}$ in $\mathrm{CH}_{2} \mathrm{Cl}_{2}$ ) to afford the title compound as tan solid (33 mg, $0.12 \mathrm{mmol}, 48 \%$ yield). m.p. 87-89 ${ }^{\circ} \mathrm{C}$; IR $v_{\max } / \mathrm{cm}^{-1}$ (film): 3035, 2938, 2774, 1572, 1455, 1408, 1181, 1087, 1049; ${ }^{1} \mathrm{H}$ NMR (400 $\left.\mathrm{MHz} \mathrm{CDCl}_{3}\right) \delta: 8.63\left(1 \mathrm{H}\right.$, br s, $\left.\mathrm{H}_{3}\right), 8.31\left(1 \mathrm{H}\right.$, br s, $\left.\mathrm{H}_{1}\right), 7.40-7.22\left(5 \mathrm{H}, \mathrm{m}, \mathrm{H}_{4}, \mathrm{H}_{5}\right.$, and $\left.\mathrm{H}_{6}\right), 7.10$ $\left(1 \mathrm{H}, \mathrm{d}, J=4.2 \mathrm{~Hz}, \mathrm{H}_{2}\right), 4.18\left(1 \mathrm{H}, \mathrm{d}, J=12.7 \mathrm{~Hz}, \mathrm{H}_{7}\right), 4.14\left(1 \mathrm{H}, \mathrm{d}, J=12.7 \mathrm{~Hz}, \mathrm{H}_{7}\right), 3.46(1 \mathrm{H}$, app t, $\left.J=8.3 \mathrm{~Hz}, \mathrm{H}_{8}\right), 3.23\left(1 \mathrm{H}\right.$, app t, $\left.J=8.4 \mathrm{~Hz}, \mathrm{H}_{11}\right), 2.36-2.24\left(2 \mathrm{H}, \mathrm{m}, \mathrm{H}_{9}\right.$ and $\left.\mathrm{H}_{11}\right), 2.19(3 \mathrm{H}, \mathrm{s}$, $\left.\mathrm{H}_{12}\right), 1.98-1.85\left(1 \mathrm{H}, \mathrm{m}, \mathrm{H}_{10}\right), 1.83-1.71\left(1 \mathrm{H}, \mathrm{m}, \mathrm{H}_{10}\right), 1.68-1.54\left(1 \mathrm{H}, \mathrm{m}, \mathrm{H}_{9}\right) ;{ }^{13} \mathrm{C} \mathrm{NMR}(100 \mathrm{MHz}$, 
$\left.\mathrm{CDCl}_{3}\right)_{147.74}, 147.64,147.59,135.39,128.81,128.71,127.64,119.21,65.74,56.70,40.37$, $36.28,32.79,22.71 ; \mathrm{m} / \mathrm{z}$ LRMS (ESI + APCI) found $[\mathrm{M}+\mathrm{H}]^{+} 285.2, \mathrm{C}_{17} \mathrm{H}_{21} \mathrm{~N}_{2} \mathrm{~S}^{+}$requires 285.1.

\section{[2,2'-bipyridin]-4-amine (17)}

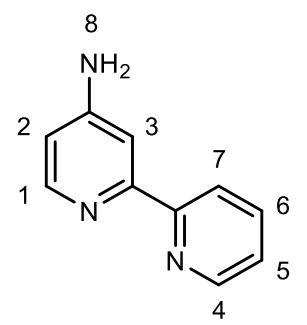

An oven dried $8 \mathrm{~mL}$ vial was charged with [2,2'-Bipyridin]-4-yltriphenylphosphonium trifluoromethanesulfonate (142 $\mathrm{mg}, 0.25 \mathrm{mmol})$ and sodium azide ( $16.3 \mathrm{mg}, 0.25 \mathrm{mmol})$. The vial was subjected to three cycles of vacuum/nitrogen backfill before addition of DMSO $(0.5 \mathrm{~mL})$ and heated at $120{ }^{\circ} \mathrm{C}$ for 19 hours. The reaction mixture was cooled to rt, quenched with $\mathrm{H}_{2} \mathrm{O}$ and diluted with EtOAc. The organic was layer separated, and the aqueous layer was extracted with EtOAc $(3 \times 10 \mathrm{~mL})$. The combined organic extracts was dried $\left(\mathrm{MgSO}_{4}\right)$, filtered and concentrated in vacuo. The oil was transferred to an oven dried $8 \mathrm{~mL}$ vial and subjected to three cycles of vacuum/nitrogen backfill before addition of DMF: $\mathrm{H}_{2} \mathrm{O}(9: 1)(5.0 \mathrm{~mL})$. After heating at $100{ }^{\circ} \mathrm{C}$ for 11 hours, the reaction mixture was cooled to $\mathrm{rt}$ and quenched with $\mathrm{H}_{2} \mathrm{O}$ and diluted with EtOAc. The organic was layer separated and the aqueous layer was extracted with EtOAc $(3 \times 10 \mathrm{~mL})$. The combined organic extracts were dried $\left(\mathrm{MgSO}_{4}\right)$, filtered and concentrated in vacuo. The crude material was purified by flash chromatography (silica gel: $10 \% \mathrm{MeOH}$ in $\mathrm{CH}_{2} \mathrm{Cl}_{2}$ ) to provide the title compound as a white solid ( $23 \mathrm{mg}, 0.13 \mathrm{mmol}, 52 \%$ yield). The spectroscopic data is in agreement with a reported synthesis. ${ }^{10} \mathrm{~m} / z$ LRMS (ESI + APCI) found $[\mathrm{M}+\mathrm{H}]^{+} 172.1, \mathrm{C}_{10} \mathrm{H}_{10} \mathrm{~N}_{3}$ ${ }^{+}$requires 172.1. 


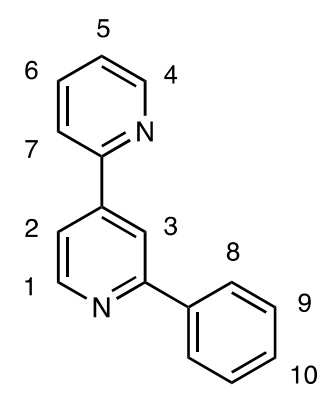

An oven dried $8 \mathrm{~mL}$ vial was charged with 2-bromopyridine ( $52 \mu \mathrm{L}, 0.55 \mathrm{mmol})$, and subjected to three cycles of vacuum/nitrogen backfill before addition of THF $(2.5 \mathrm{~mL})$. After the solution was cooled to $-78{ }^{\circ} \mathrm{C}, n$ - $\mathrm{BuLi}(2.5 \mathrm{M}$ in hexanes, $200 \mu \mathrm{L}, 0.50 \mathrm{mmol})$ was added dropwise over 5 minutes, and the solution stirred for 1 hour. A solution of Triphenyl(2-phenylpyridin-4yl)phosphonium trifluoromethanesulfonate in THF was added dropwise over 5 minutes to the solution of pyridin-2-yllithium at $-78^{\circ} \mathrm{C}$. The mixture was warmed to $0{ }^{\circ} \mathrm{C}$ and stirred for 4 hours, quenched with $\mathrm{H}_{2} \mathrm{O}$, and diluted with $\mathrm{CH}_{2} \mathrm{Cl}_{2}$. The organic was layer separated and the aqueous layer was extracted with $\mathrm{CH}_{2} \mathrm{Cl}_{2}(3 \times 10 \mathrm{~mL})$. The combined organic extracts were dried $\left(\mathrm{MgSO}_{4}\right)$, filtered and concentrated in vacuo. The crude material was purified by flash column chromatography ( silica gel was packed in hexanes and neutralized with $\mathrm{NEt}_{3}$ then gradient elution: $15 \%$ EtOAc in hexanes to $25 \%$ EtOAc in hexanes) to afford the title compound as tan oil (39 $\mathrm{mg}$, $0.17 \mathrm{mmol}, 68 \%$ yield). IR $v_{\max } / \mathrm{cm}^{-1}$ (film): 3052, 1597, 1584, 1545, 1463, 1444, 1434, 1388; ${ }^{1} \mathrm{H}$

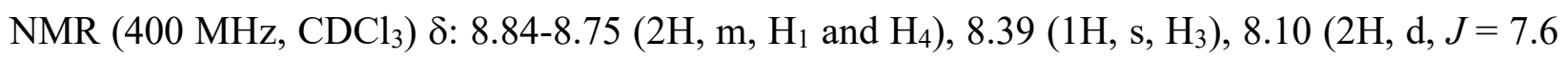
$\left.\mathrm{Hz}, \mathrm{H}_{8}\right), 7.90-7.78\left(3 \mathrm{H}, \mathrm{m}, \mathrm{H}_{2}, \mathrm{H}_{6}\right.$, and $\left.\mathrm{H}_{7}\right), 7.54-7.40\left(3 \mathrm{H}, \mathrm{m}, \mathrm{H}_{9}\right.$ and $\left.\mathrm{H}_{10}\right), 7.39-7.32\left(1 \mathrm{H}, \mathrm{m}, \mathrm{H}_{5}\right)$; ${ }^{13} \mathrm{C}$ NMR (100 MHz, $\left.\mathrm{CDCl}_{3}\right)$ 158.18, 154.82, 150.13, 150.07, 147.30, 139.24, 137.03, 129.06, 128.71, 127.05, 123.77, 120.93, 119.49, 118.13; m/z LRMS (ESI + APCI) found [M+H] $]^{+} 233.1$, $\mathrm{C}_{16} \mathrm{H}_{13} \mathrm{~N}_{2}^{+}$ requires 233.1 . 


\section{5-(2-phenylpyridin-4-yl)pyrimidine (19)}

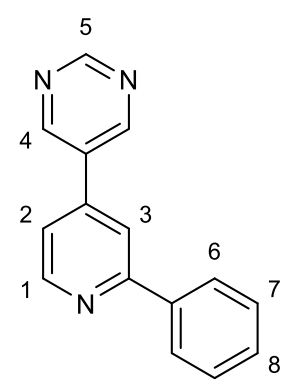

An oven dried $8 \mathrm{~mL}$ vial was charged with 5-bromopyrimidine (90 mg, $0.55 \mathrm{mmol}$ ), and subjected to three cycles of vacuum/nitrogen backfill before addition of THF (2.5 mL). After the solution was cooled to $-78^{\circ} \mathrm{C}, n$-BuLi $(2.5 \mathrm{M}$ in hexanes, $200 \mu \mathrm{L}, 0.50 \mathrm{mmol})$ was added dropwise over 5 minutes, and the solution stirred for 1 hour. A solution of Triphenyl(2-phenylpyridin-4yl)phosphonium trifluoromethanesulfonate in THF was added dropwise over 5 minutes to the solution of pyrimidin-5-yllithium at $-78{ }^{\circ} \mathrm{C}$. The mixture was warmed to $0{ }^{\circ} \mathrm{C}$ and stirred for 4 hours, quenched with $\mathrm{H}_{2} \mathrm{O}$, and diluted with $\mathrm{CH}_{2} \mathrm{Cl}_{2}$. The organic layer was separated and the aqueous layer was extracted with $\mathrm{CH}_{2} \mathrm{Cl}_{2}(3 \times 10 \mathrm{~mL})$. The combined organic extracts were dried $\left(\mathrm{MgSO}_{4}\right)$, filtered and concentrated in vacuo. The crude material was purified by flash chromatography ( silica gel was packed in hexanes and neutralized with $\mathrm{NEt}_{3}$ then gradient elution: 20\% EtOAc in hexanes to 50\% EtOAc in hexanes) to provide the title compound as a white solid (42.3 mg, $0.18 \mathrm{mmol}, 73 \%$ yield). IR $v_{\max } / \mathrm{cm}^{-1}$ (film): 3054, 3025, 2920, 1602, 1562, 1415, 1381, 1191, 688, 626; ${ }^{1} \mathrm{H}$ NMR (400 MHz, $\left.\mathrm{CDCl}_{3}\right) \delta: 9.32\left(1 \mathrm{H}, \mathrm{s}, \mathrm{H}_{5}\right), 9.06\left(2 \mathrm{H}, \mathrm{s}, \mathrm{H}_{4}\right), 8.84(1 \mathrm{H}, \mathrm{d}, J=$ $\left.5.1 \mathrm{~Hz}, \mathrm{H}_{1}\right), 8.06\left(2 \mathrm{H}, \mathrm{d}, J=7.4 \mathrm{~Hz}, \mathrm{H}_{6}\right), 7.91\left(1 \mathrm{H}, \mathrm{s}, \mathrm{H}_{3}\right), 7.58-7.40\left(4 \mathrm{H}, \mathrm{m}, \mathrm{H}_{2}, \mathrm{H}_{7}\right.$, and $\left.\mathrm{H}_{8}\right) ;{ }^{13} \mathrm{C}$ NMR

$\delta: 158.86,158.75,154.98,150.67,142.66,138.65,132.14,129.50,128.89,126.99,119.68,118$. 19; $m / z$ LRMS (ESI + APCI) found $[\mathrm{M}+\mathrm{H}]^{+} 234.1, \mathrm{C}_{15} \mathrm{H}_{12} \mathrm{~N}_{3}{ }^{+}$requires 234.1 . 


\section{Comparative Literature Syntheses of Heteroaryl Ethers}

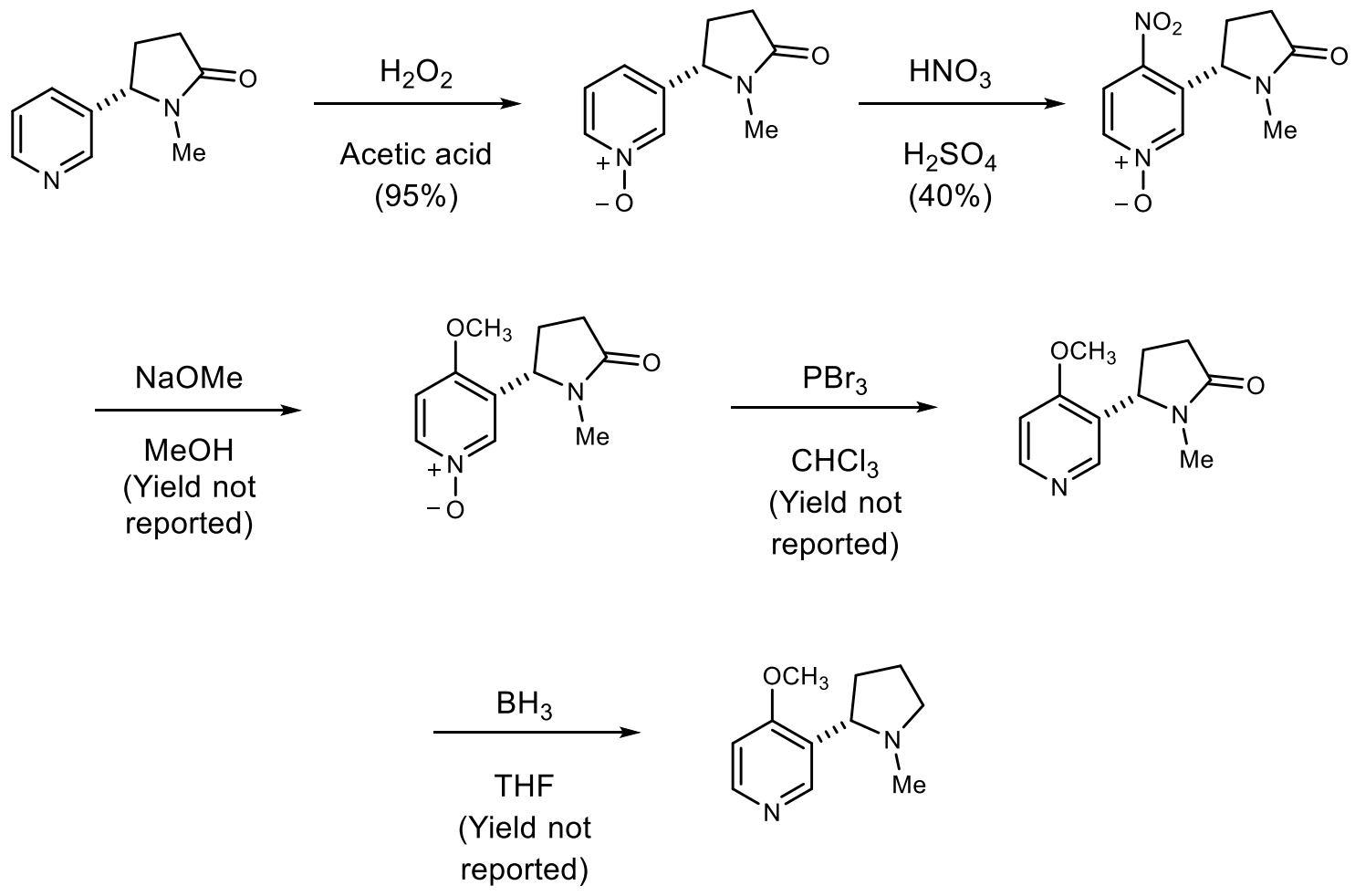

Scheme S1. Literature Synthesis of Nicotine derivative 5., 911,12<smiles>CO[n+]1ccc(-c2ccccn2)cc1-c1ccccn1</smiles><smiles>COc1ccnc(-c2ccccn2)c1</smiles>

Scheme S1. Literature Synthesis of Structure 3ea analog. ${ }^{13}$ 

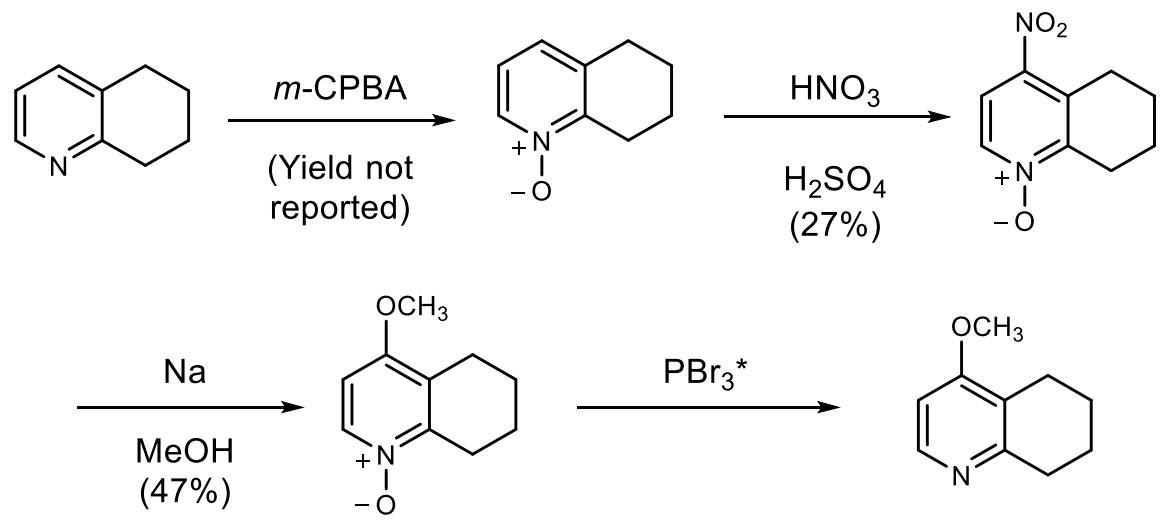

${ }^{*}$ Reduction step not reported in this manuscript but related analogs were reduced using $\mathrm{PBr}_{3}$

Scheme S2. Literature Synthesis of Substrate 3ga analog. ${ }^{14}$

\section{Counterion Study}
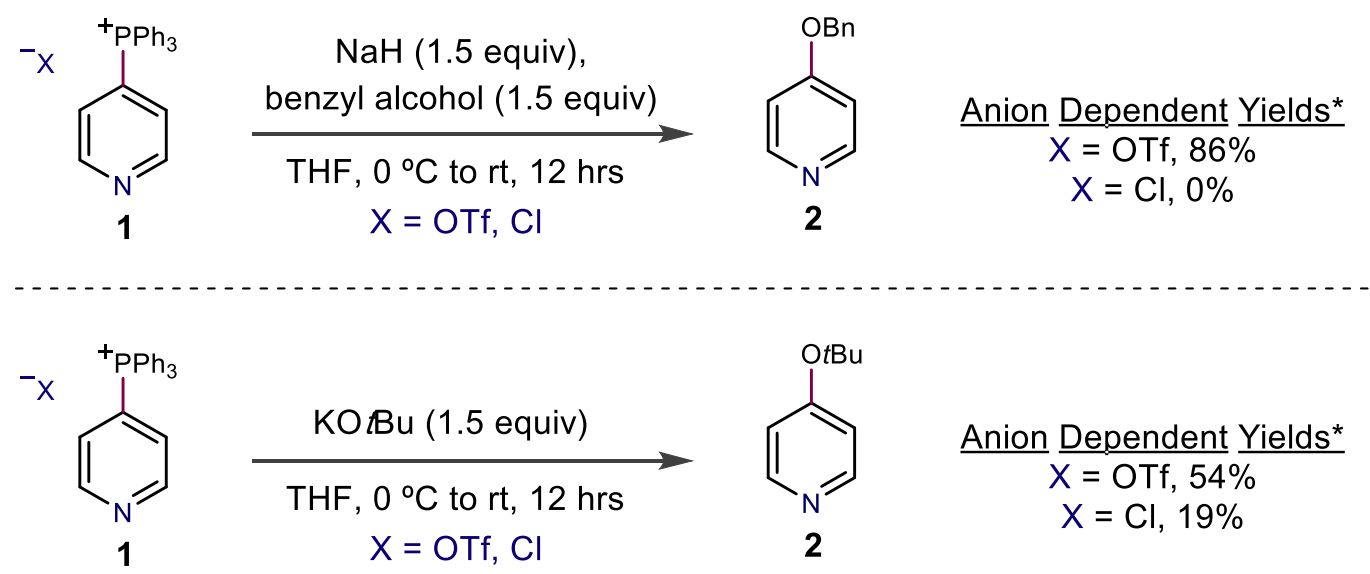

${ }^{* 1} \mathrm{H}$ NMR yields shown using 1,3,5-trimethoxybenzene as an internal standard.

\section{4-(tert-butoxy)pyridine}<smiles>CC(C)(C)Oc1ccncc1</smiles> 
An oven dried $8 \mathrm{~mL}$ vial was charged with potassium tert-butoxide ( $25 \mathrm{mg}, 0.22 \mathrm{mmol}$ ) and the respective phosphonium salt $(0.15 \mathrm{mmol})$ under $\mathrm{N}_{2}$. The vial was placed in a $0{ }^{\circ} \mathrm{C}$ ice bath before THF $(0.3 \mathrm{~mL})$ was added. The ice bath was removed and the reaction stirred for 12 hours while warming to room temperature. The reaction was quenched with $\mathrm{H}_{2} \mathrm{O}$, the aqueous layer was separated and extracted with $\mathrm{CH}_{2} \mathrm{Cl}_{2}(3 \times 10 \mathrm{~mL})$. The combined organic extracts were washed with a saturated aqueous solution of brine, dried $\left(\mathrm{MgSO}_{4}\right)$, filtered and concentrated in vacuo. The residue was purified by flash column chromatography (silica gel was packed in hexanes and neutralized with $\mathrm{NEt}_{3}$ then gradient elution: 20\% EtOAc in hexanes to 30\% EtOAc in hexanes) afforded the title compound as a clear oil. ${ }^{1} \mathrm{H} \mathrm{NMR}\left(400 \mathrm{MHz}, \mathrm{CDCl}_{3}\right) \delta: 8.35(2 \mathrm{H}, \mathrm{d}, J=5.5 \mathrm{~Hz}$, $\left.\mathrm{H}_{1}\right), 6.81\left(2 \mathrm{H}, \mathrm{d}, J=5.5 \mathrm{~Hz}, \mathrm{H}_{2}\right), 1.41\left(9 \mathrm{H}, \mathrm{s}, \mathrm{H}_{3}\right) ;{ }^{13} \mathrm{C} \mathrm{NMR}\left(100 \mathrm{MHz}, \mathrm{CDCl}_{3}\right)$ 162.74, 150.69, $115.91,79.57,28.59$.

\section{References}

S1. D. D. Perrin, W. L. F. Amarego, Purification of Laboratory Chemicals (Pergamon, Press, Oxford. ed. 3, 1988).

S2. Kuo, L.-H.; Fan, H.-P.; Wu, M.-F.; Chang, Y.-S. Process for the Preparation of Abiraterone and Intermediates Thereof. WO 2014/207567 A2, December, 31, 2014.

S3. Andersson, H.; Das, S.; Gustafsson, M.; Olsson, R.; Almqvist, F. Synthesis of Substituted 4Pyridones and 4-Aminopyridinium Salts via a One-Pot Pyridine Synthesis. Tet. Lett., 2010, 51, pp 4218-4220.

S4. Huang, Y.; Huang, R.; Weng, Z. Transition-Metal-Mediated Synthesis of Trifluoroethyl Aryl Ethers. Synlett., 2015, 26 (17), pp 2327-2331.

S5. Mizuno, H.; Sakamoto, N. Preparation of Pyridines and Pesticides Containing Them. JP 2003146971A, May, 21, 2003.

S6. Laskar, I. R.; Hsu, S-F.; Chen, T-M. Investigatin Photoluminescence and Electroluminescence of Iridium (III)-based Blue-Emitting Phosphors. Polyhedron., 2006, 25, 1167-1176. 
S7. Zhou, M.; Wang, P.; Zhang, J.; Feng, X. Blue Light Organic Electrophosphorescent Material Iridium Metal Complex, Preparation Method Thereof and Organic Electroluminescent Device. CN 104945439A, September, 30, 2015.

S8. Ward, J. S.; Merrit, L.; Calligaro, D. O.; Bymaster, F. P.; Shannon, H. E.; Sawyer, B. D.; Mitch, C. H.; Detter, J. B.; Peters, S. C. Functionally Selective M1 Muscarinic Agonists. 3. Side Chain and Azacycles Contributing to Functional Muscarinic Selectivity Among Pyrazinylazacycles. J. Med. Chem., 1995, 38 (18) pp 3469-3481.

S9. Dukat, M.; Ramunno, A.; Banzi, R.; Damaj, M. I.; Martin, B.; Glennon, R. A. 3-(2Aminoethyl)pyridine Analogs as $a 4 \mathrm{~b} 2$ Nicotinic Cholinergic Receptor Ligands. Bioorg. Med. Chem. Lett., 2005, 15, 4308-4312.

S10. Duric, S.; Tzschucke, C. C. Synthesis of Unsymmetrically Substituted Bipyridines by Palladium-Catalyzed Direct C-H Arylation of Pyridine N-Oxides. Org. Lett., 2011, 13 (9), pp 2310-2313.

S11.Shibagaki, M.; Matsushita, H.; Kaneko, H. The Synthesis of 4-Aminonicotine and 4Aminocotinine. Heterocycles., 1985, 23, 1681-1684.

S12. Ochiai, E. Recent Japanese Work on the Chemistry of Pyridine 1-oxide and Related Compounds. J. Org. Chem., 1953, 18, 534-551.

S13. Woodward, R. B.; Wenkert, D. Studies of 2,2'-Bipyridyl N,N'-Dioxides. J. Org. Chem., 1983, $48,283-289$.

S14. Uchida, M.; Mortia, S.; Chihiro, M.; Kanbe, T.; Yamasaki, K.; Yabuuchi, Y.; Nakagawa, K. Studies on Proton Pump Inihibitors. I. Synthesis of 8-[(2-Benzimidazolyl)sulfinyl]-5,6,7,8tetrahydroquinolines and Related Compounds. Chem. Pharm. Bull., 1989, 37, 1517-1523. 

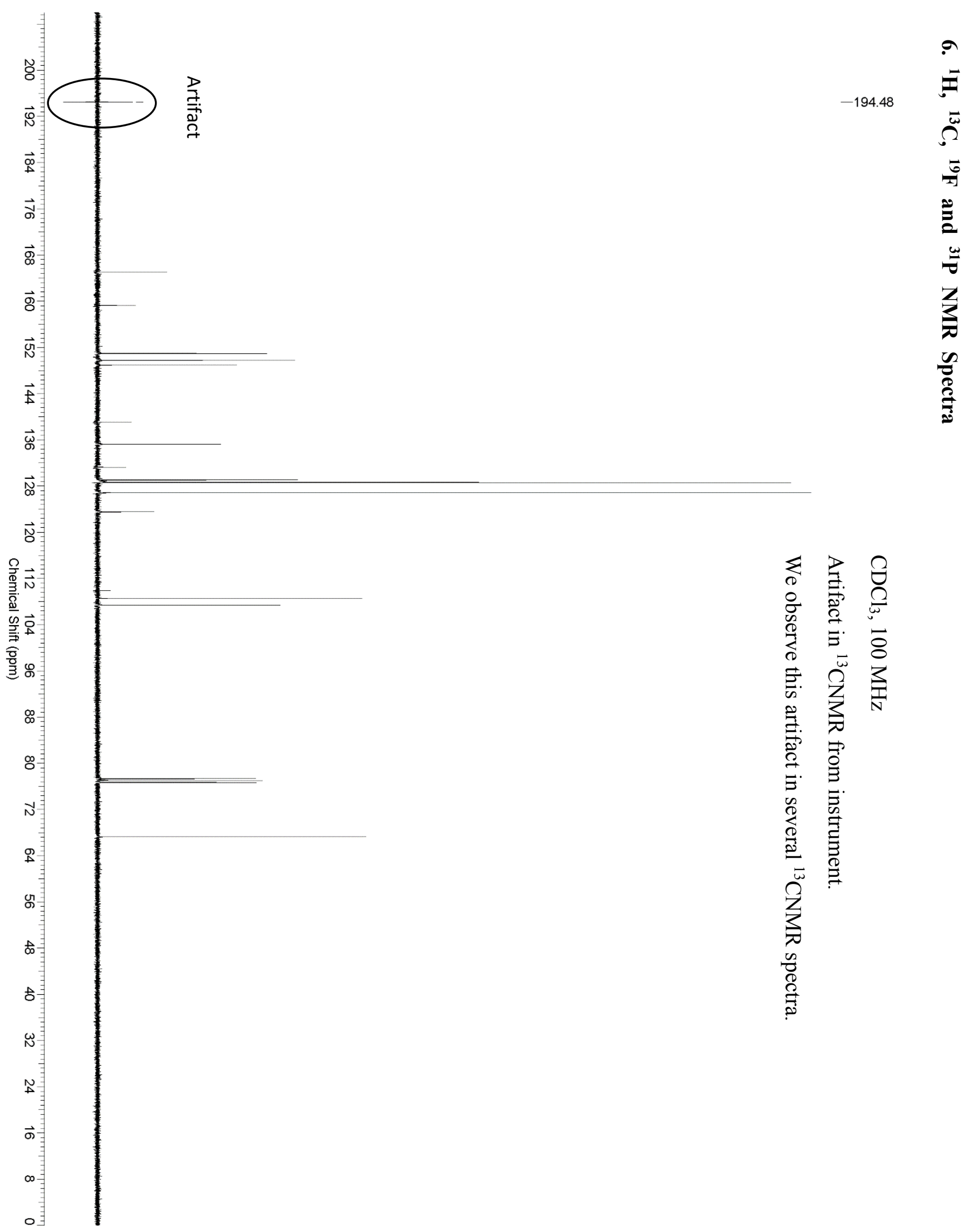


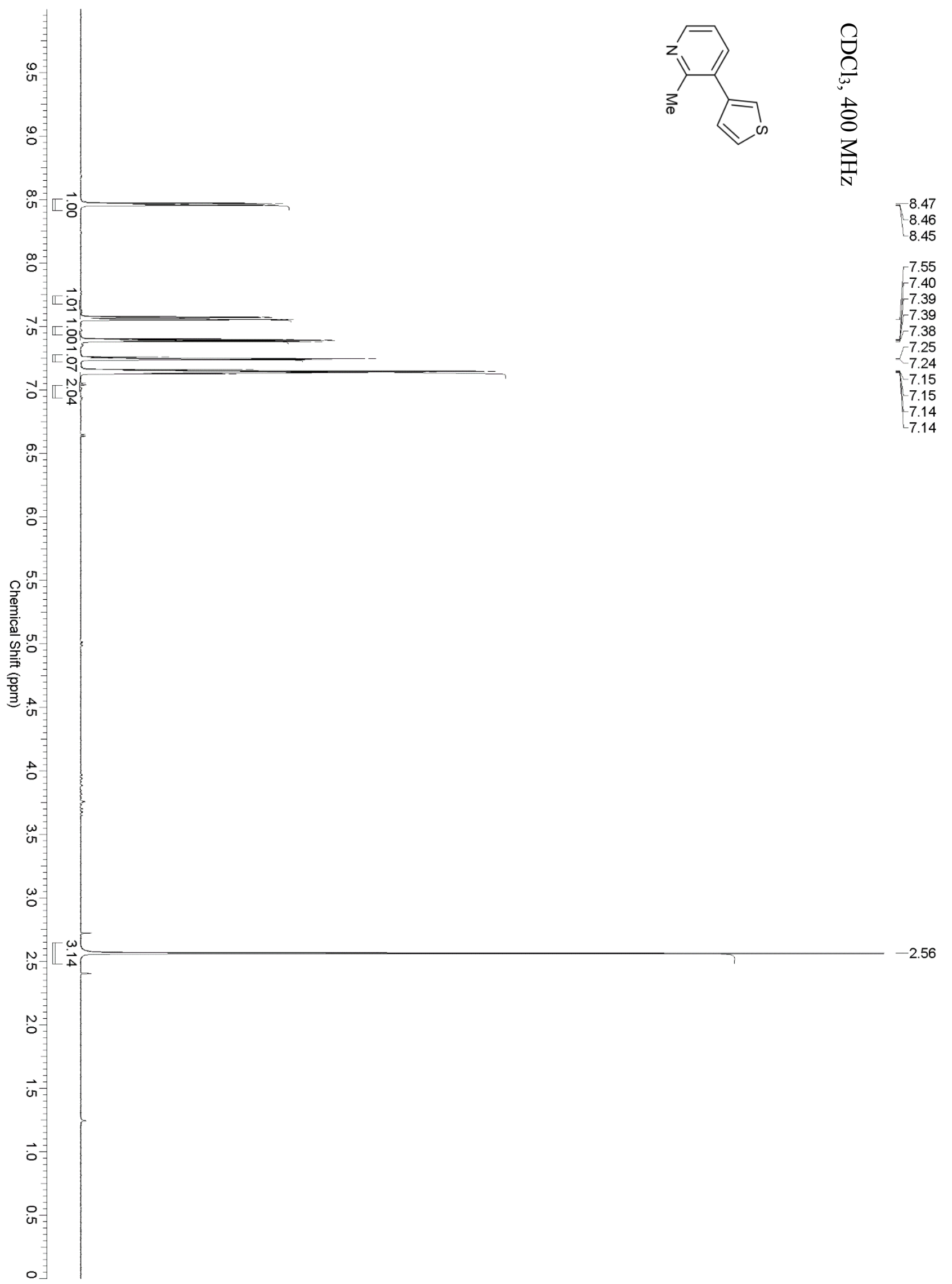




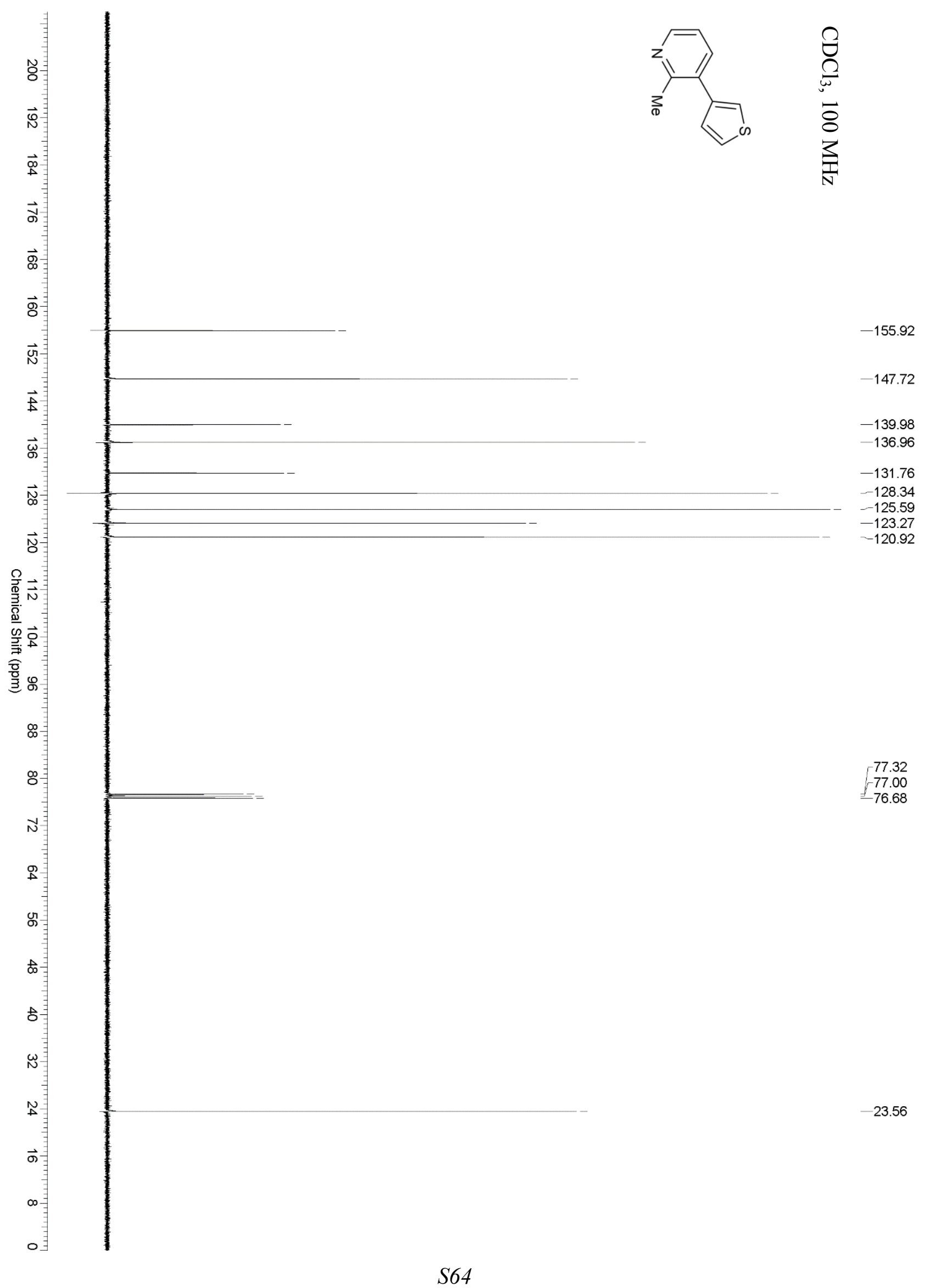




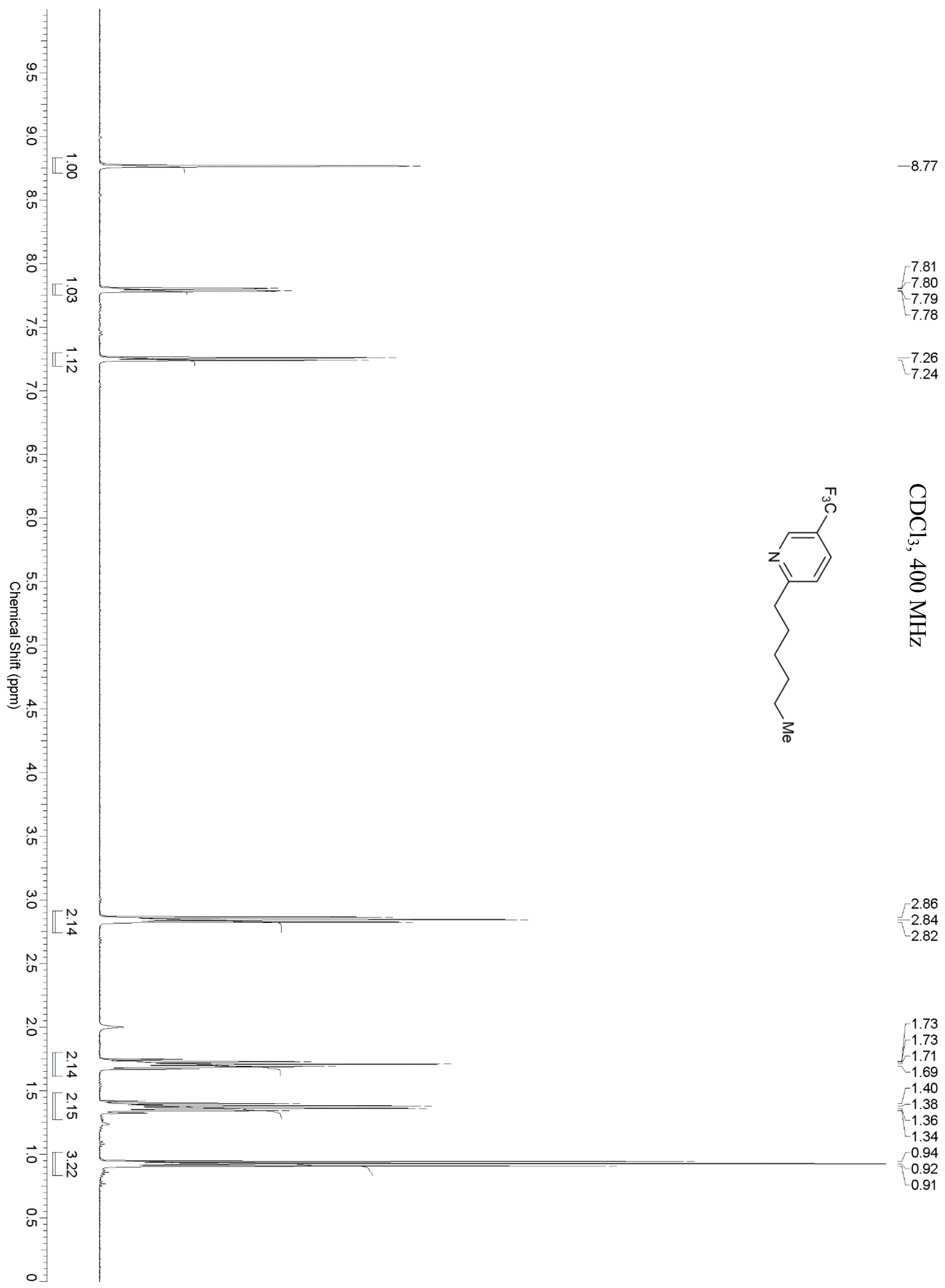




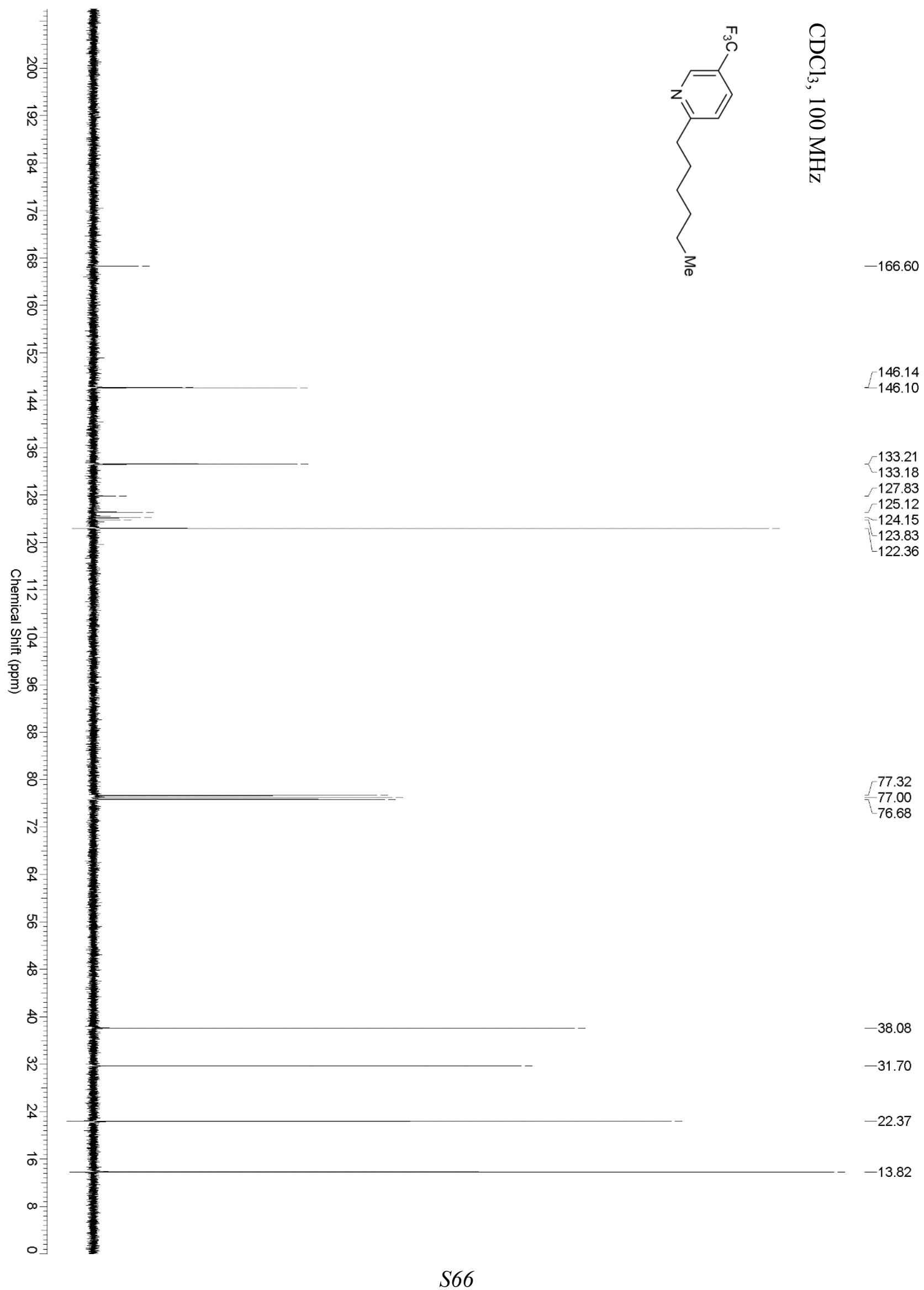




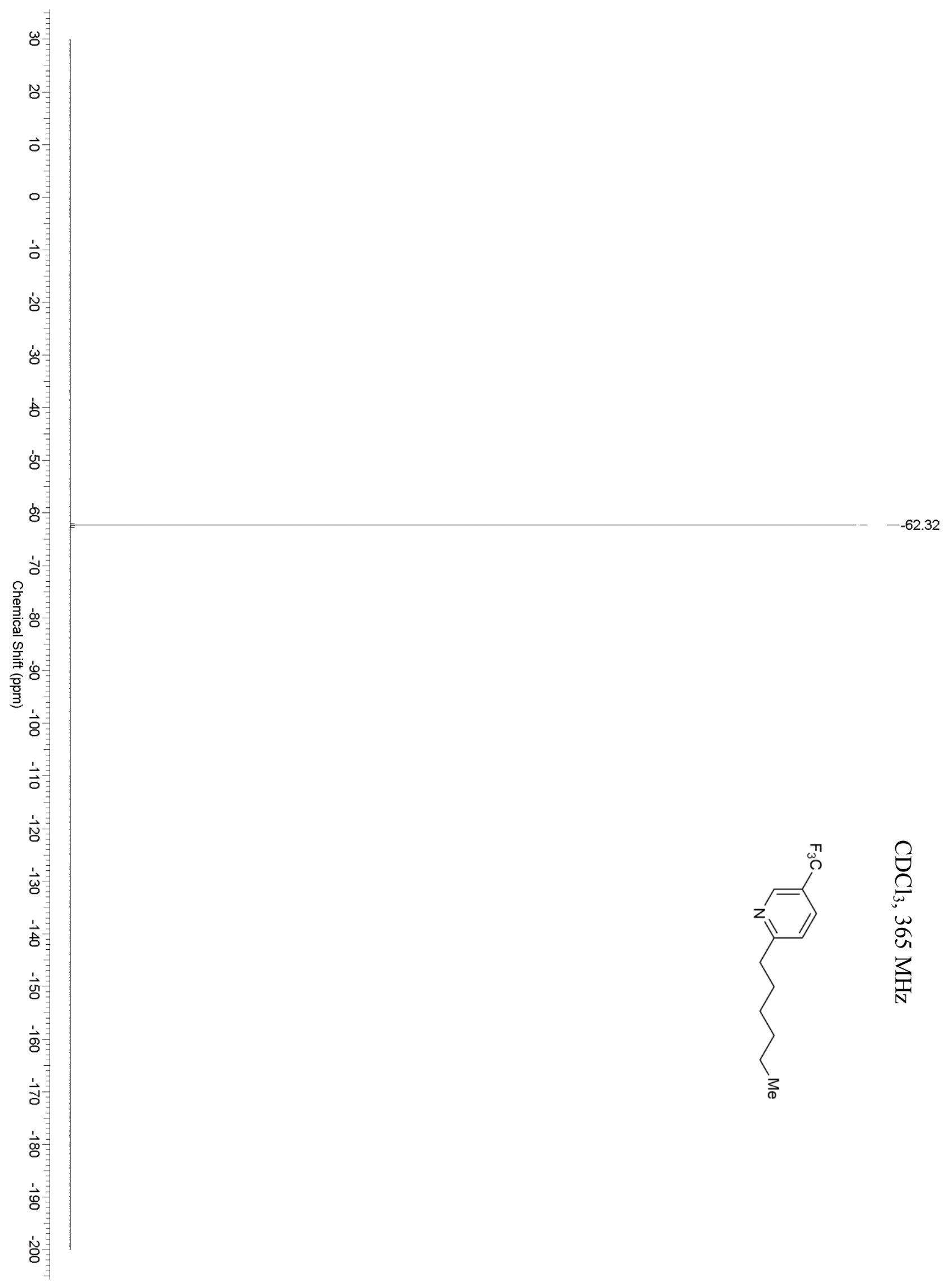




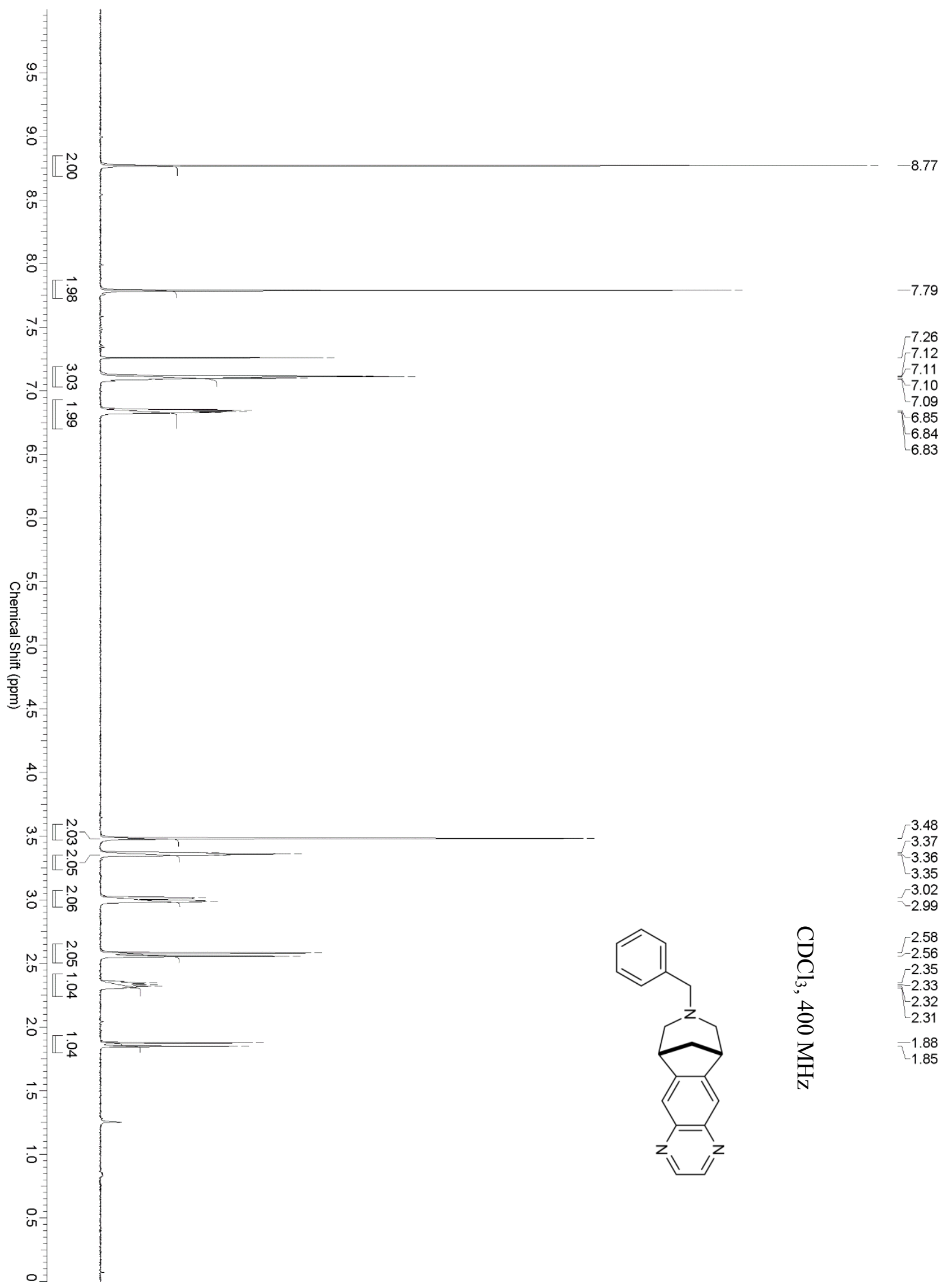




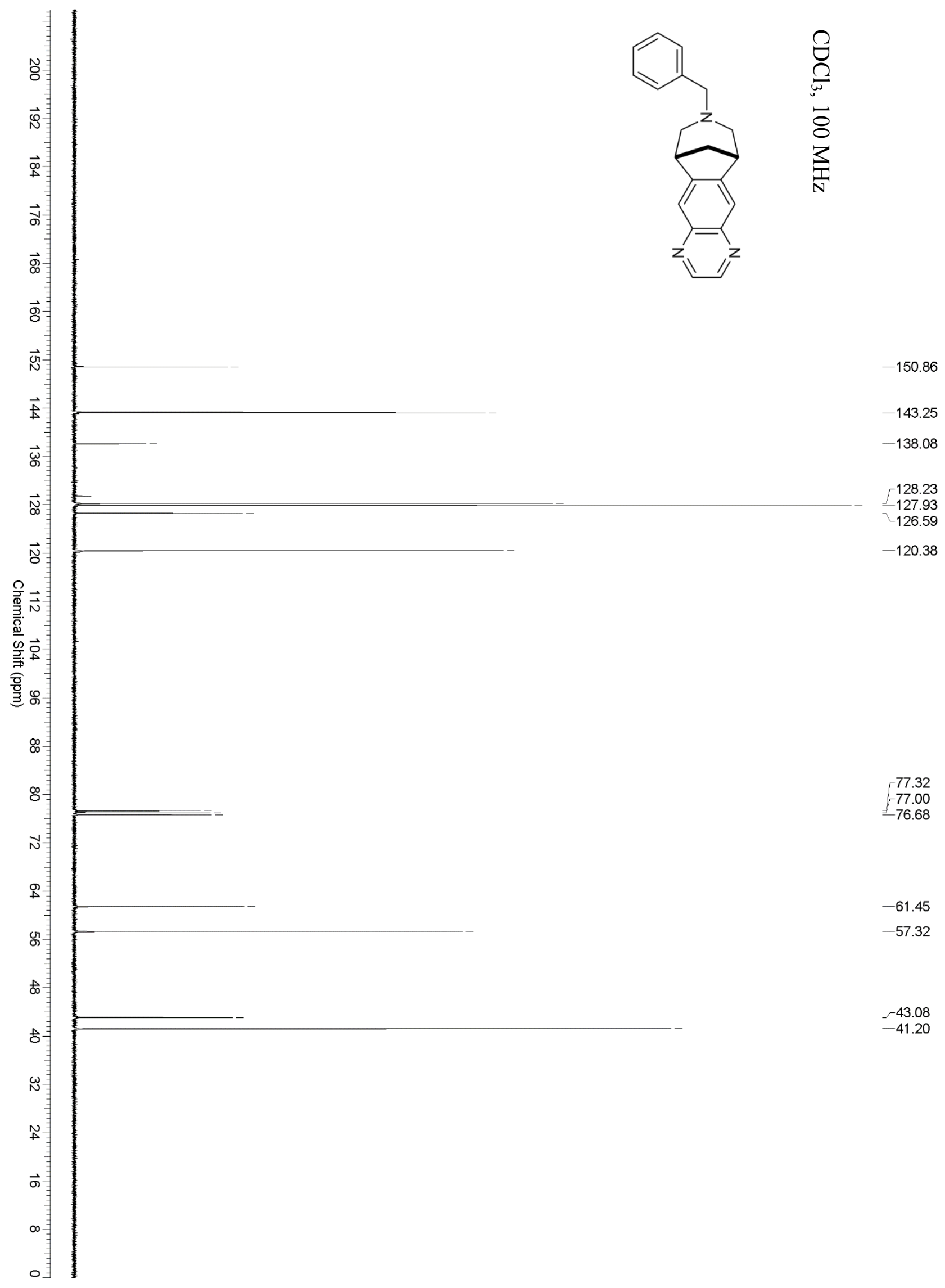




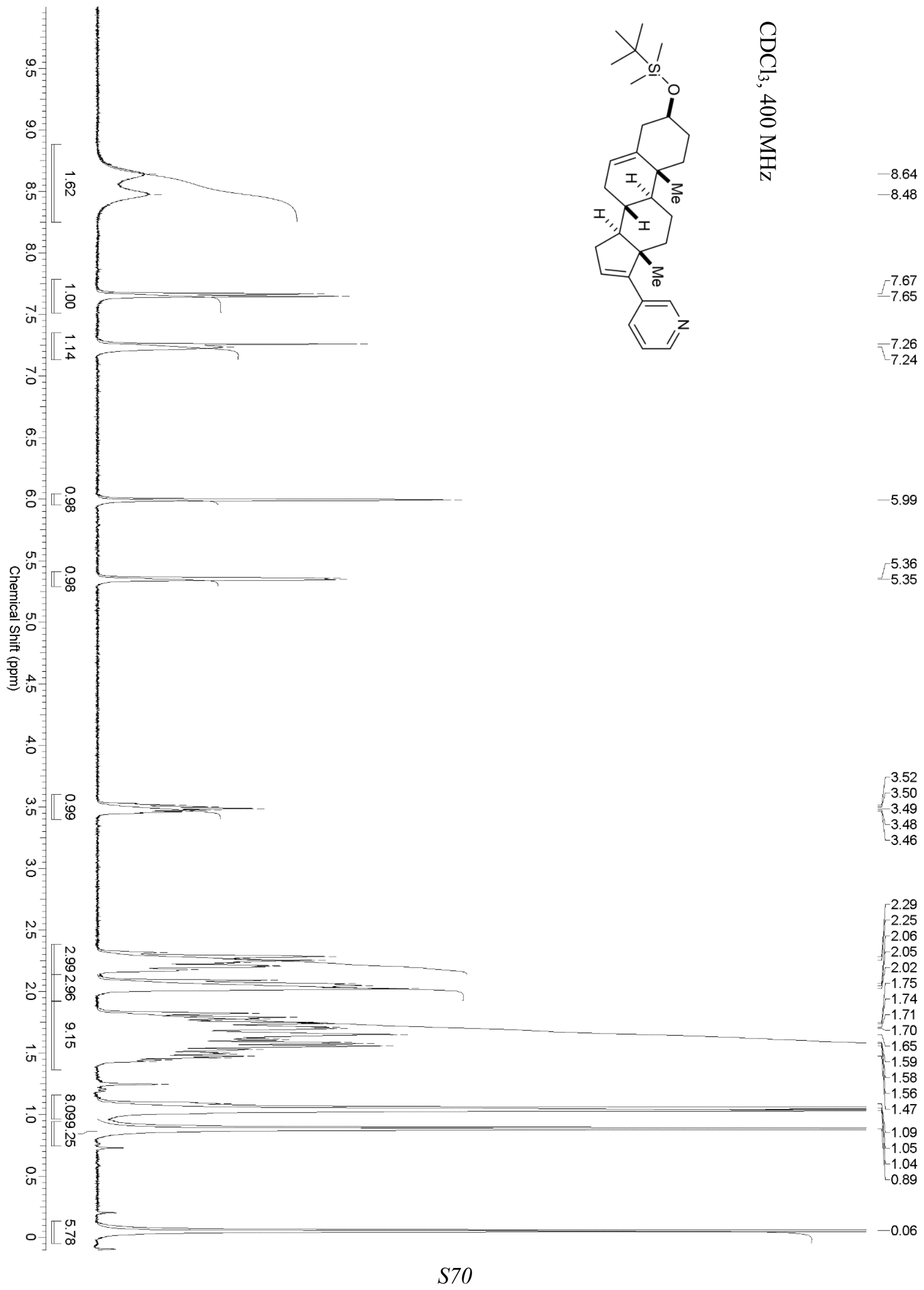




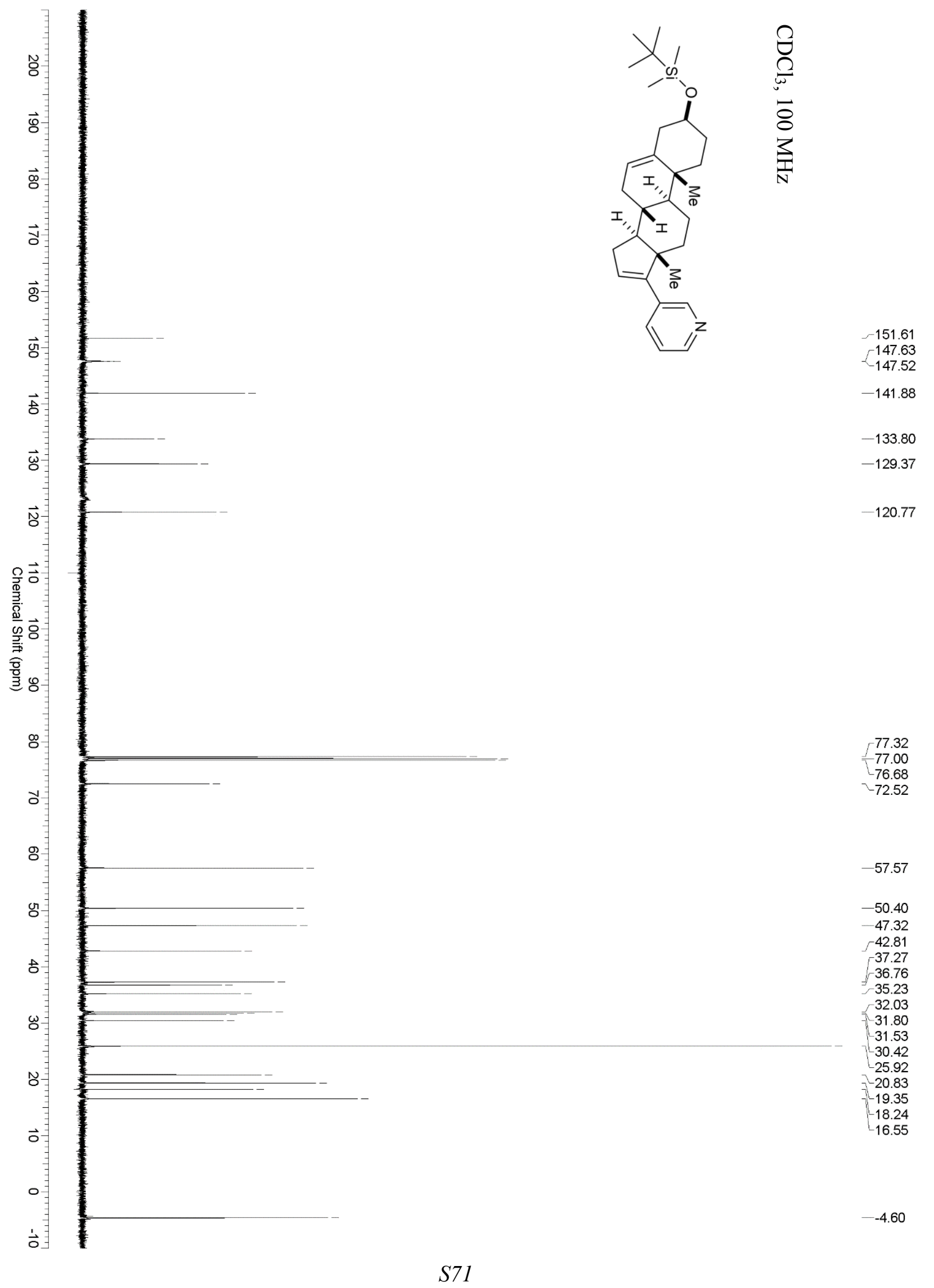




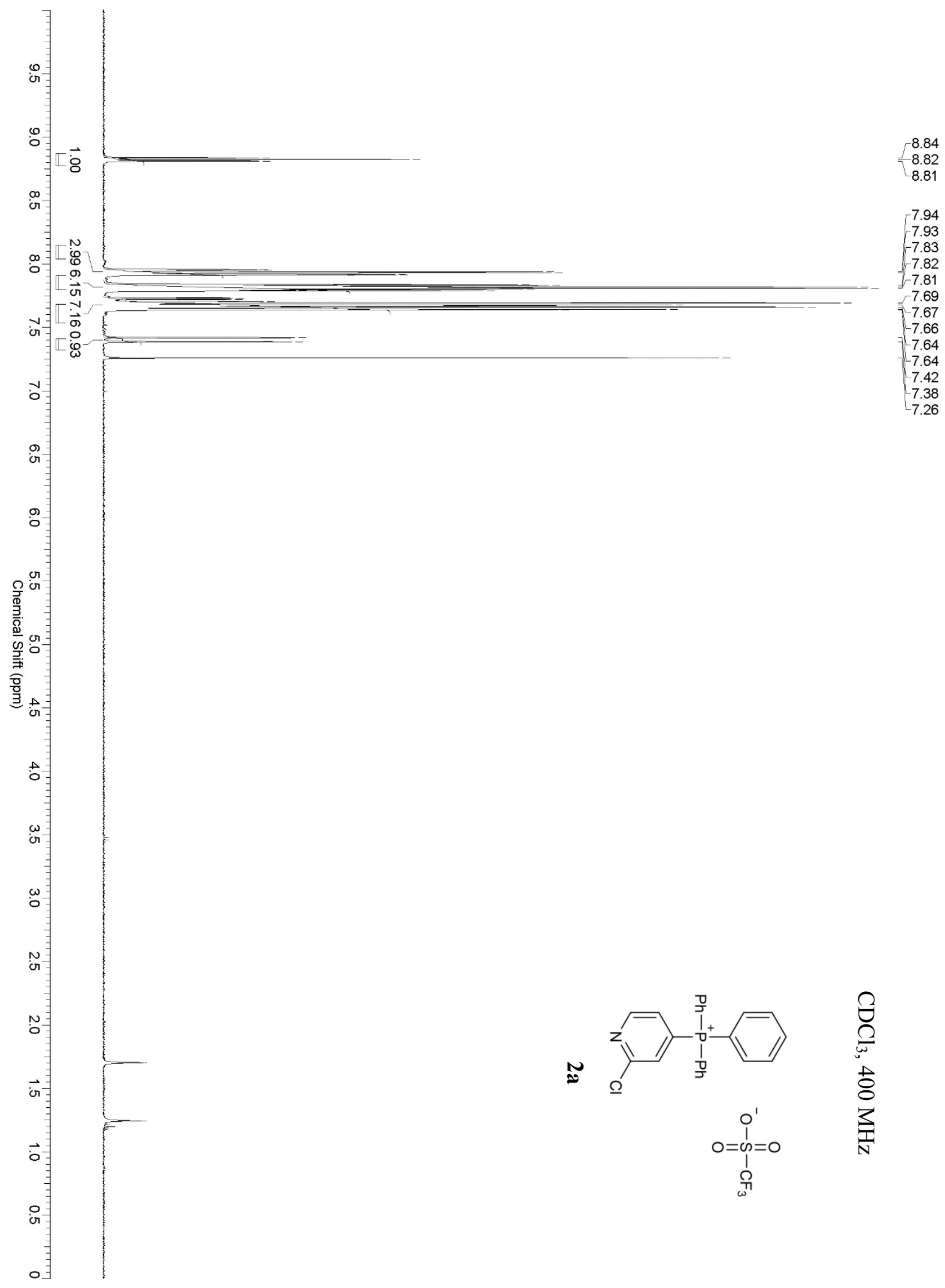




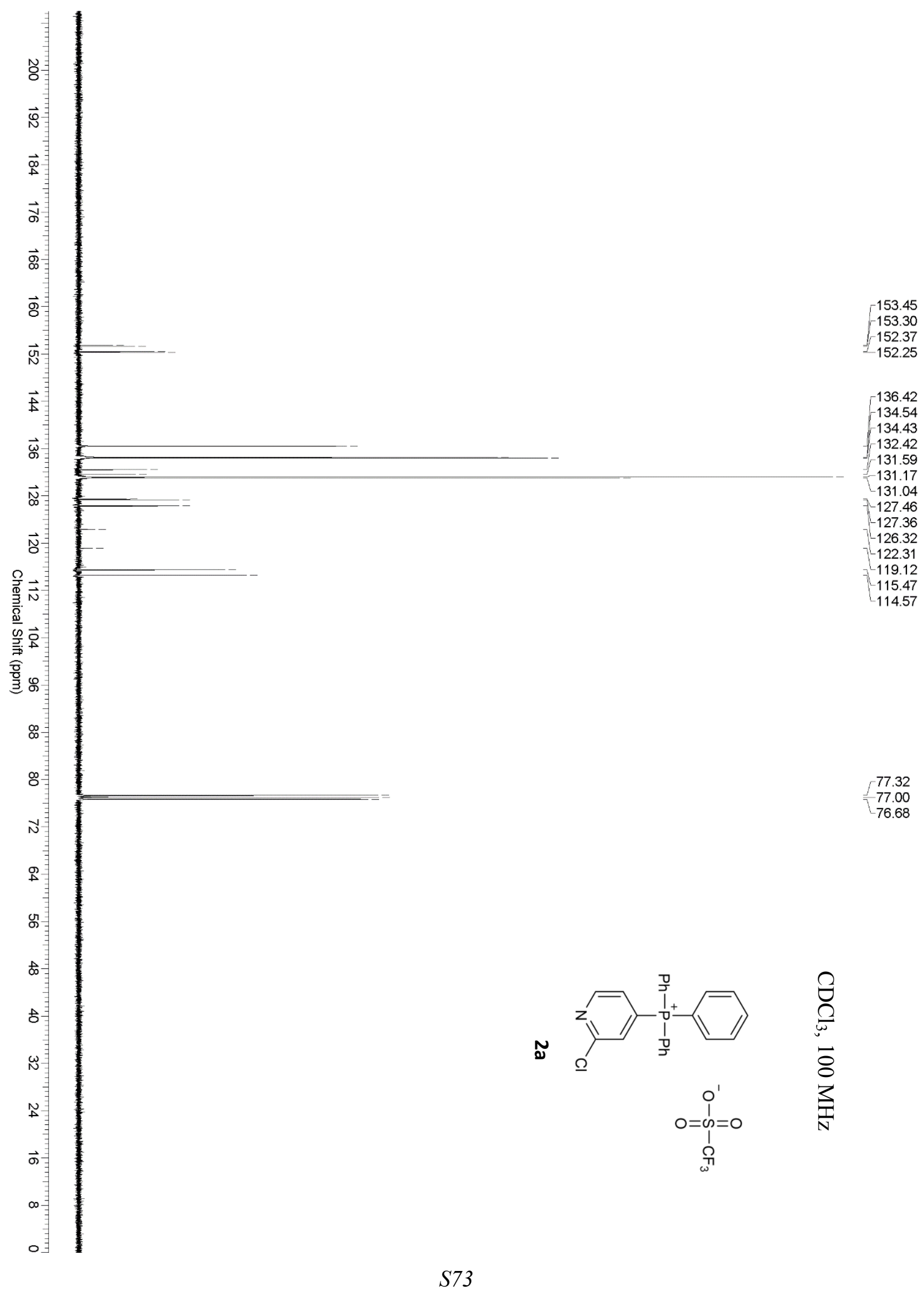




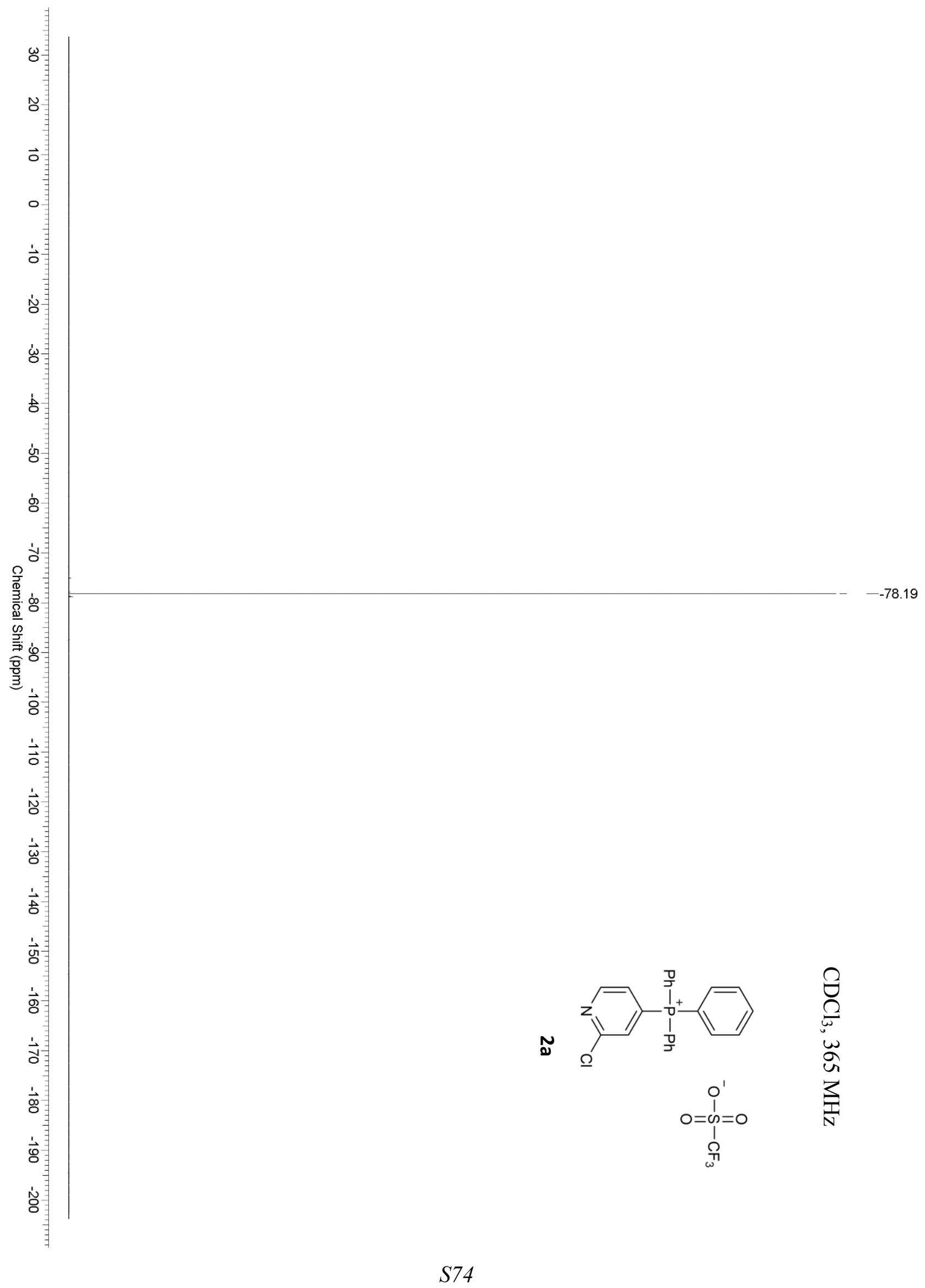




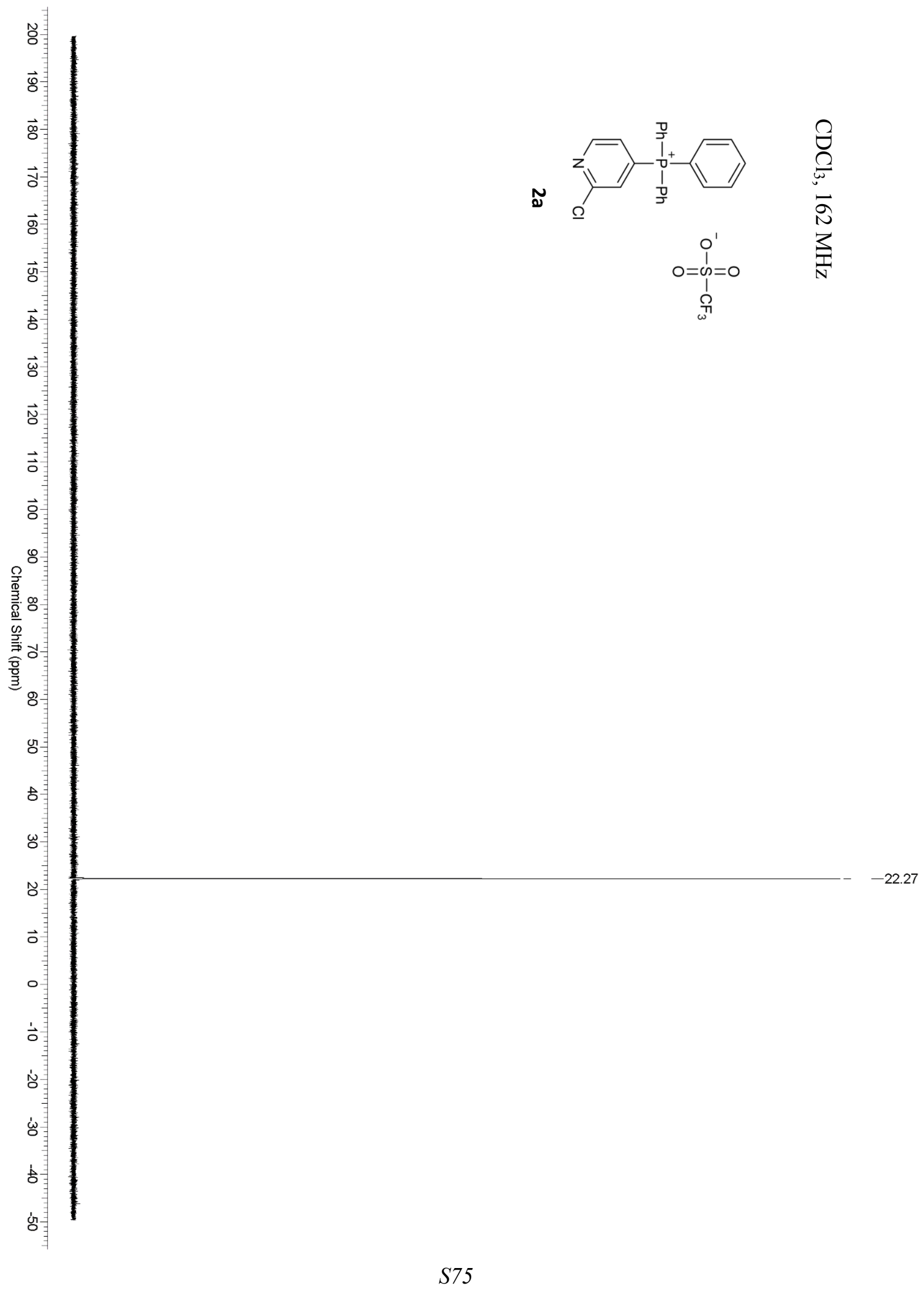




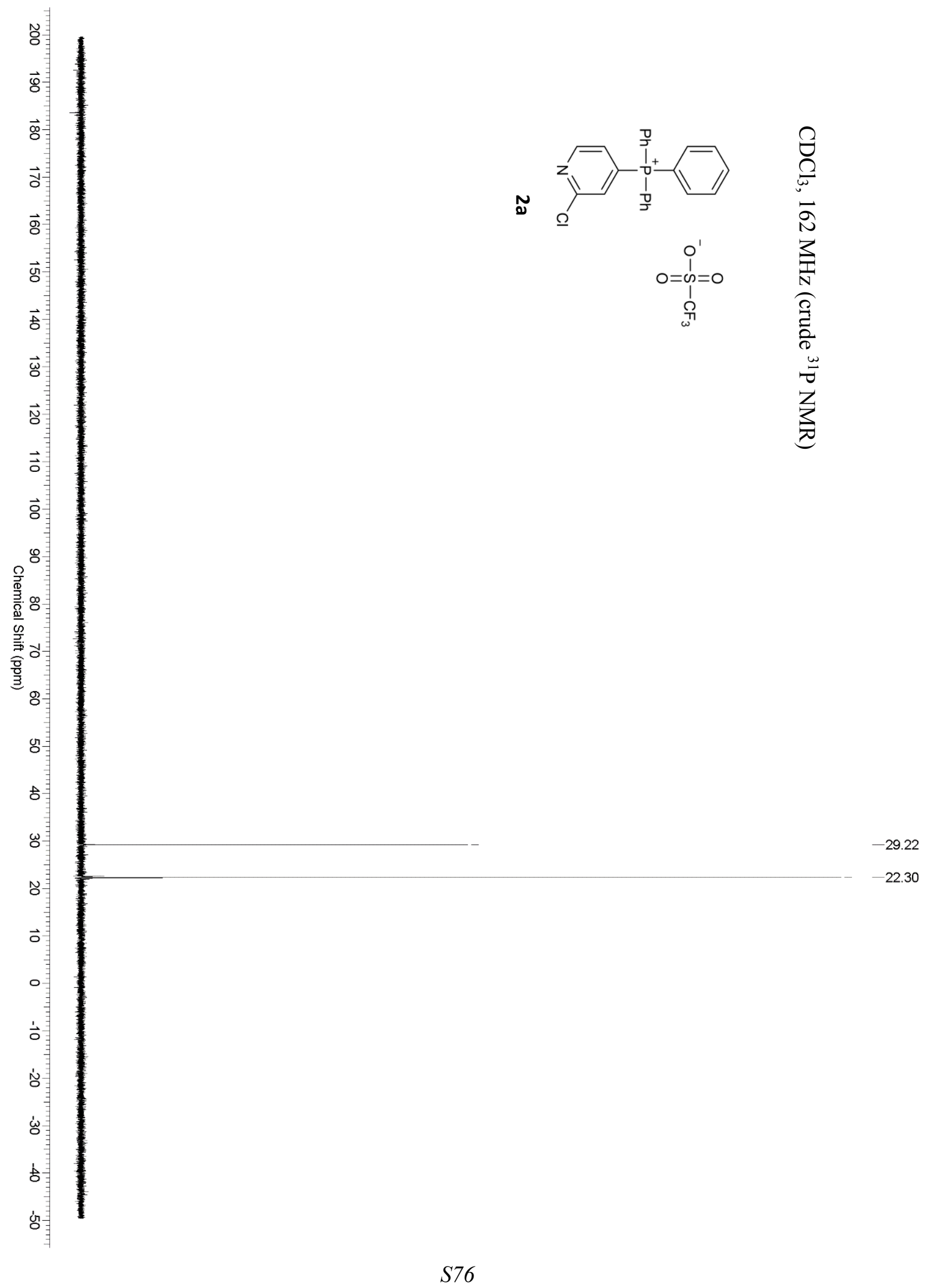




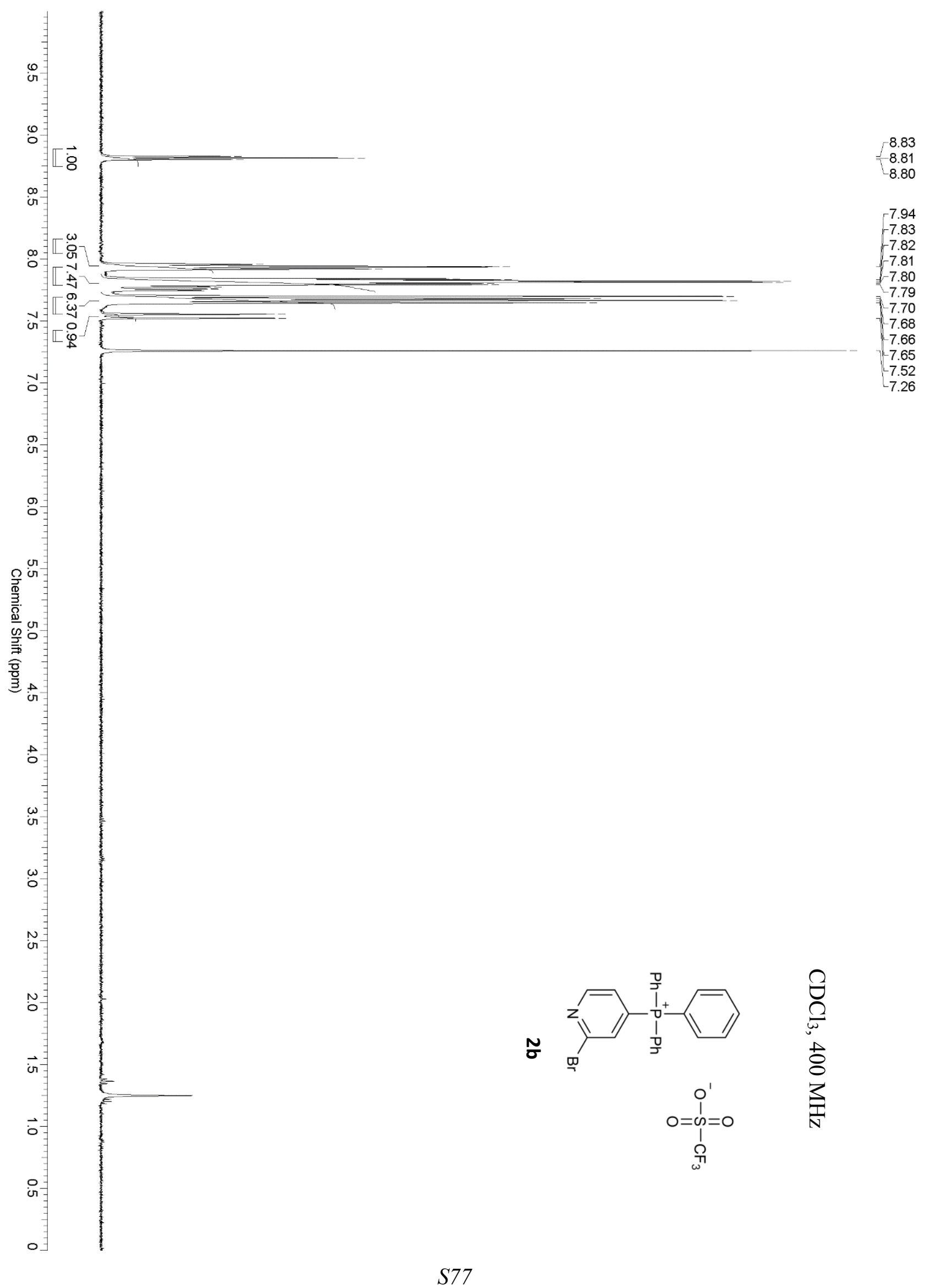




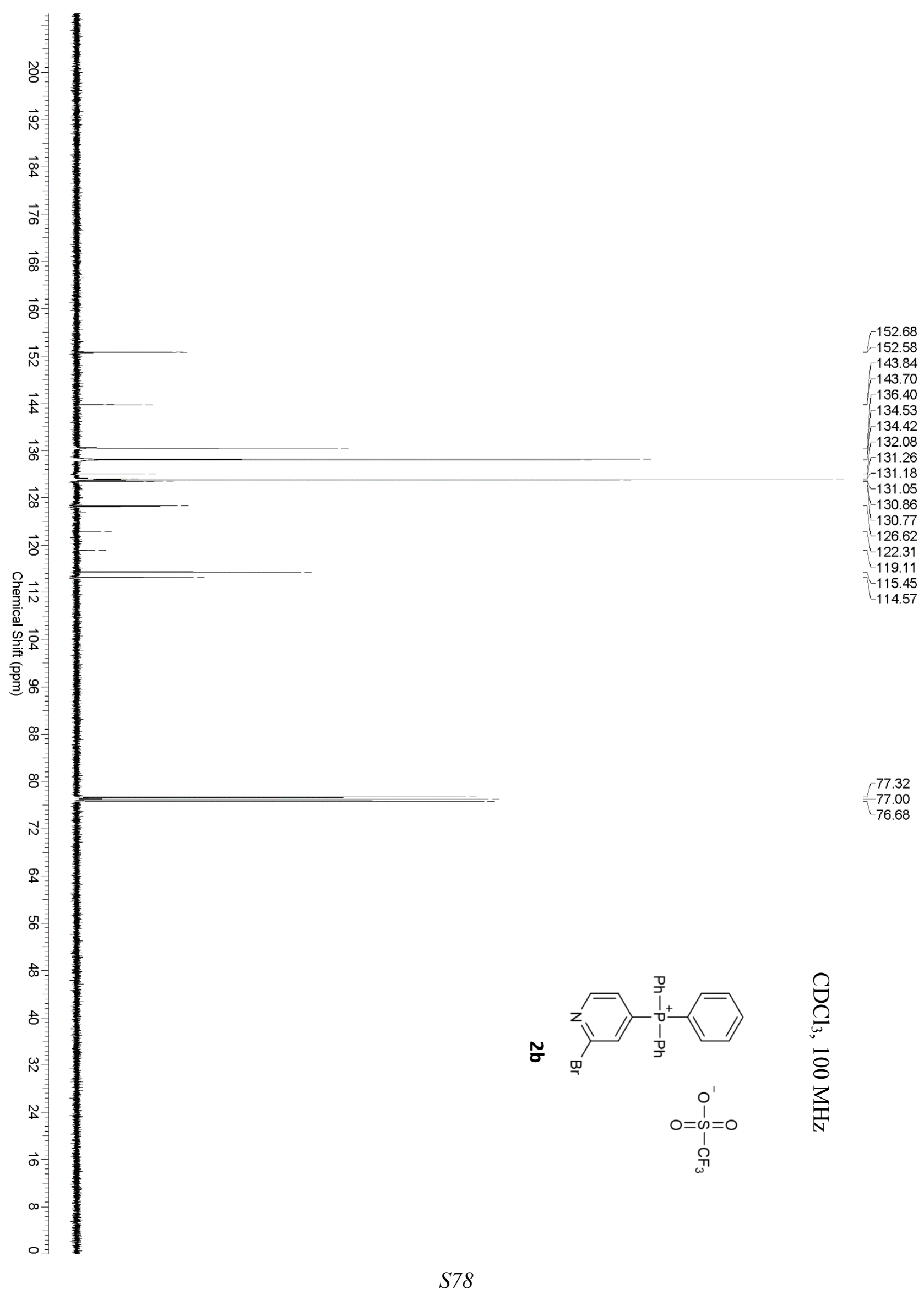




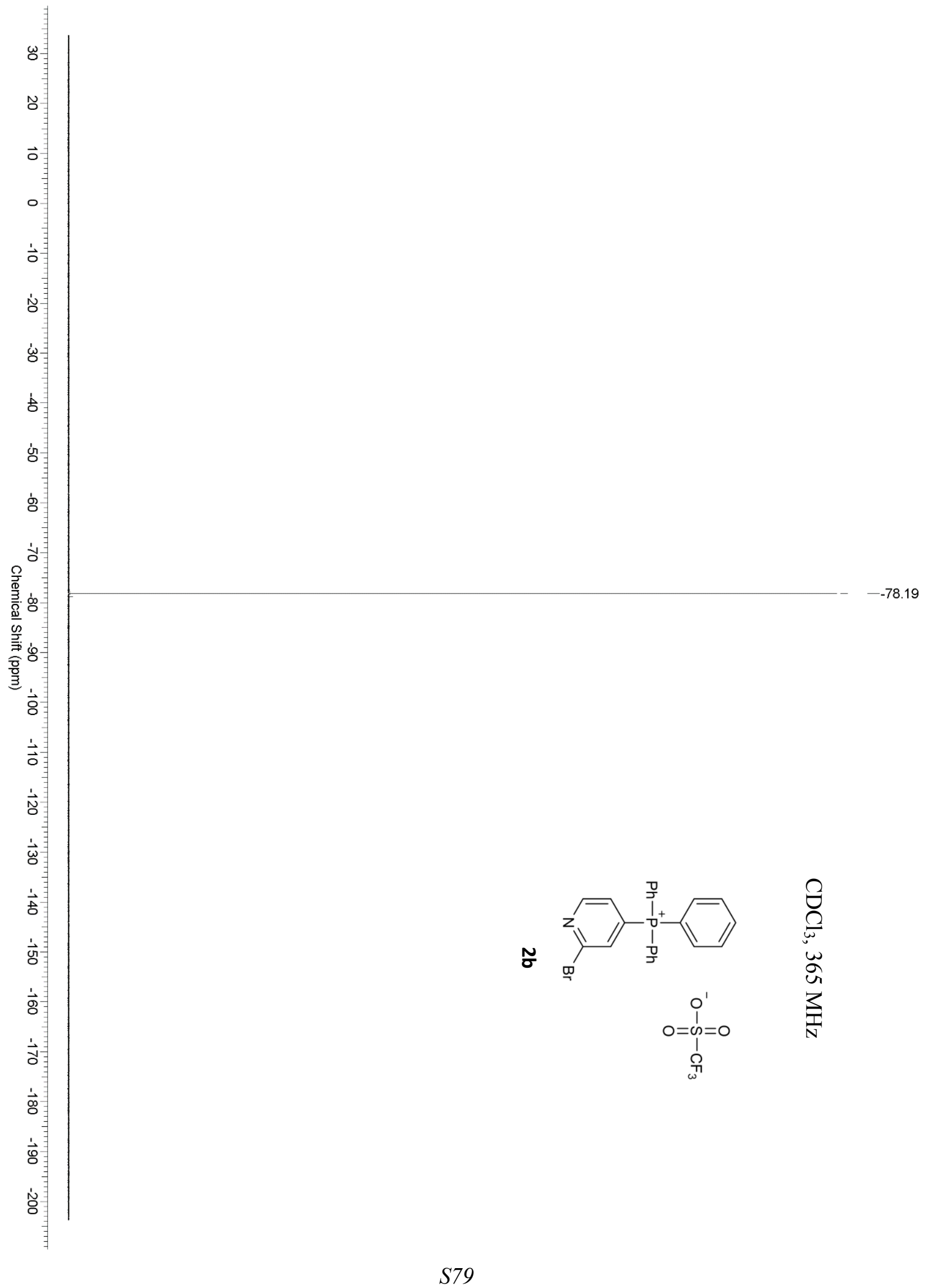




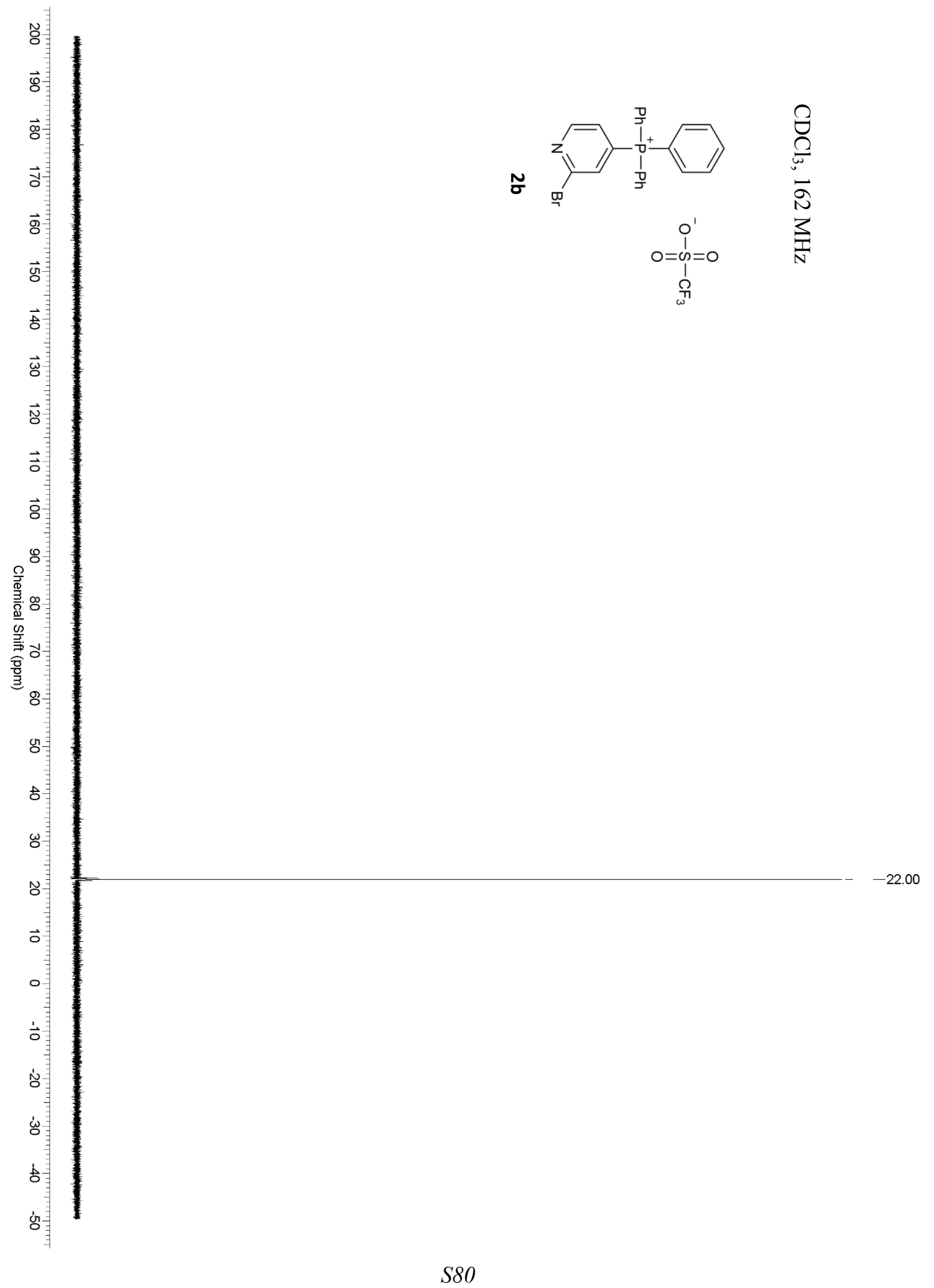




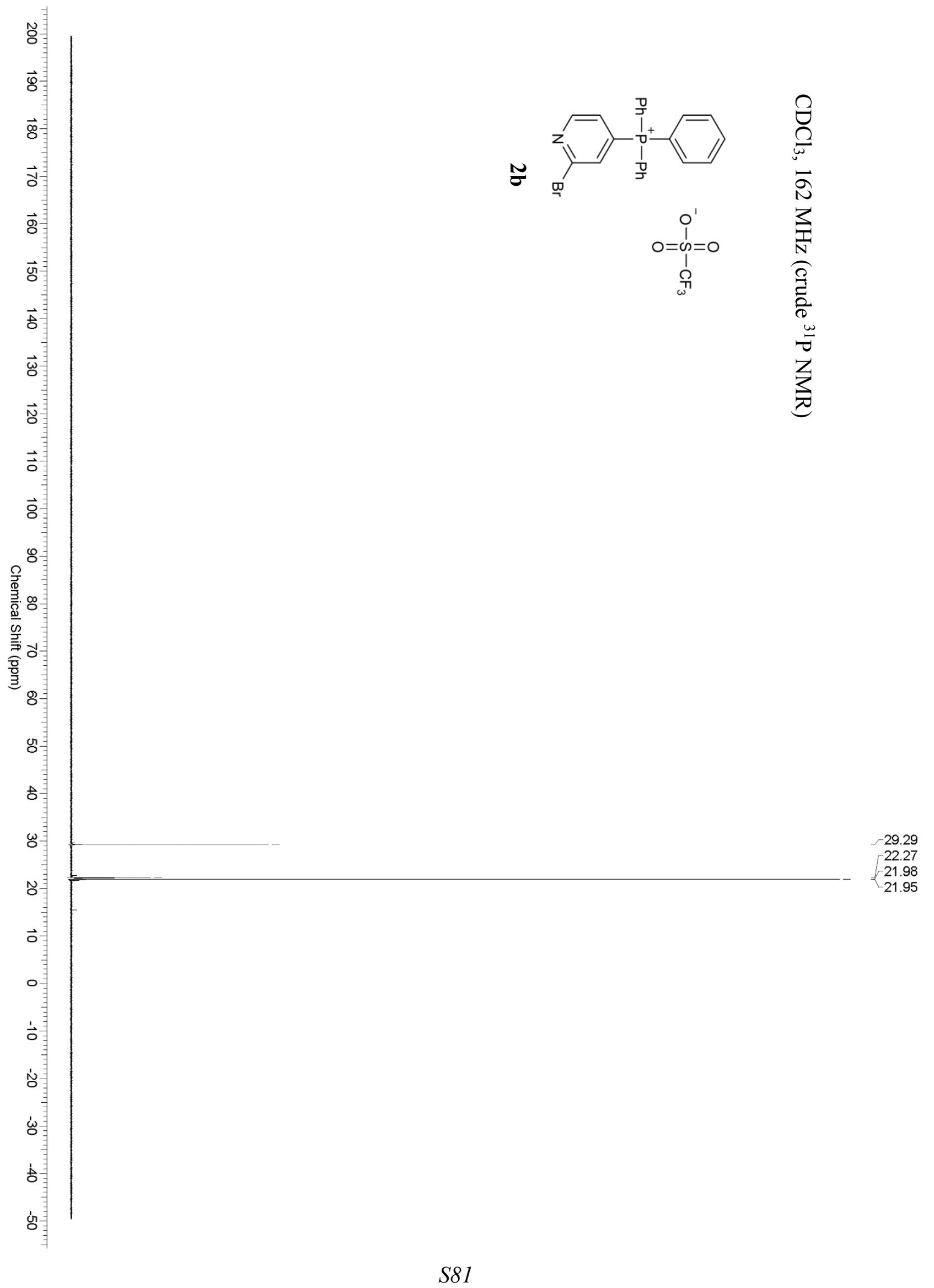




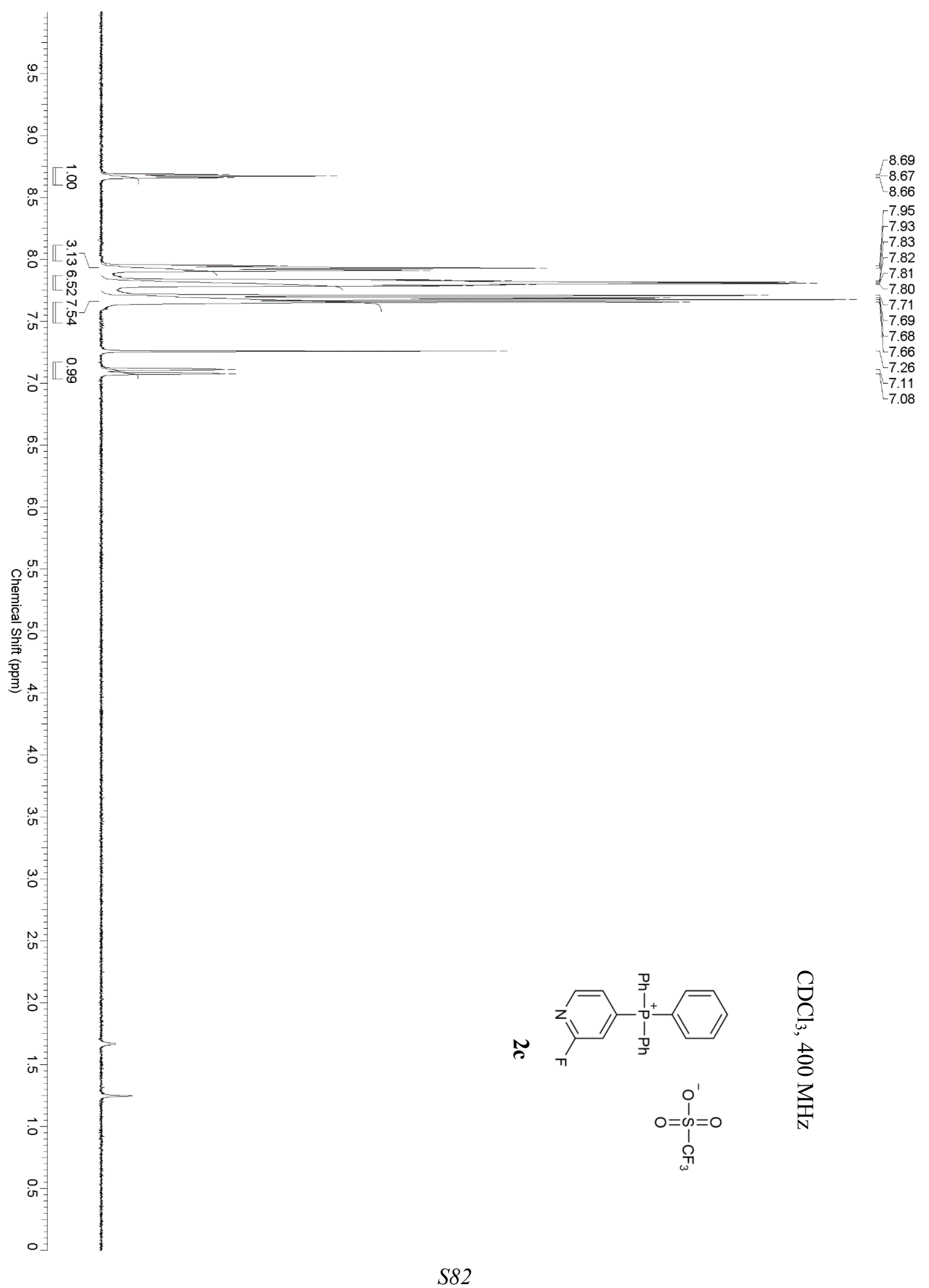




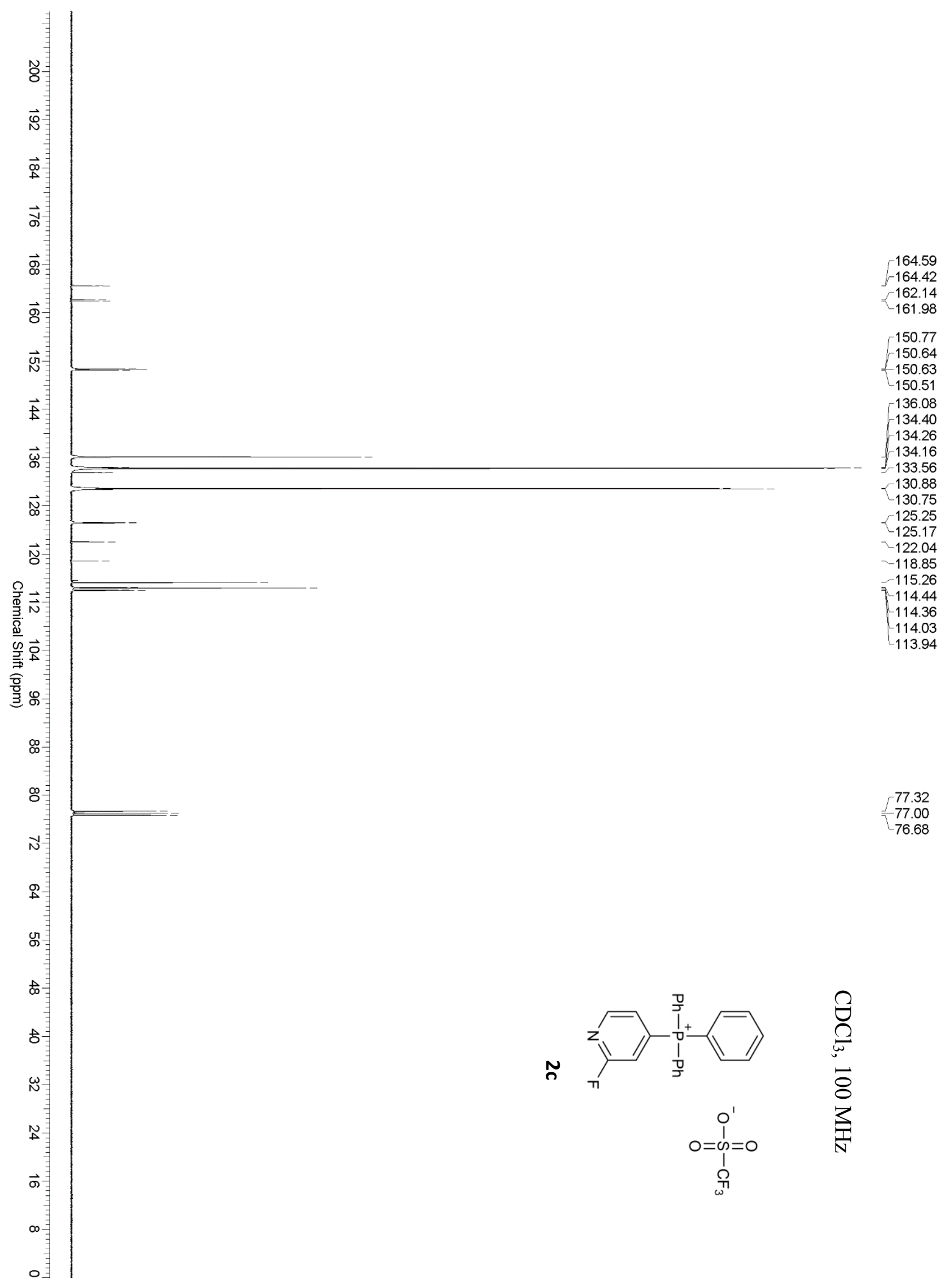




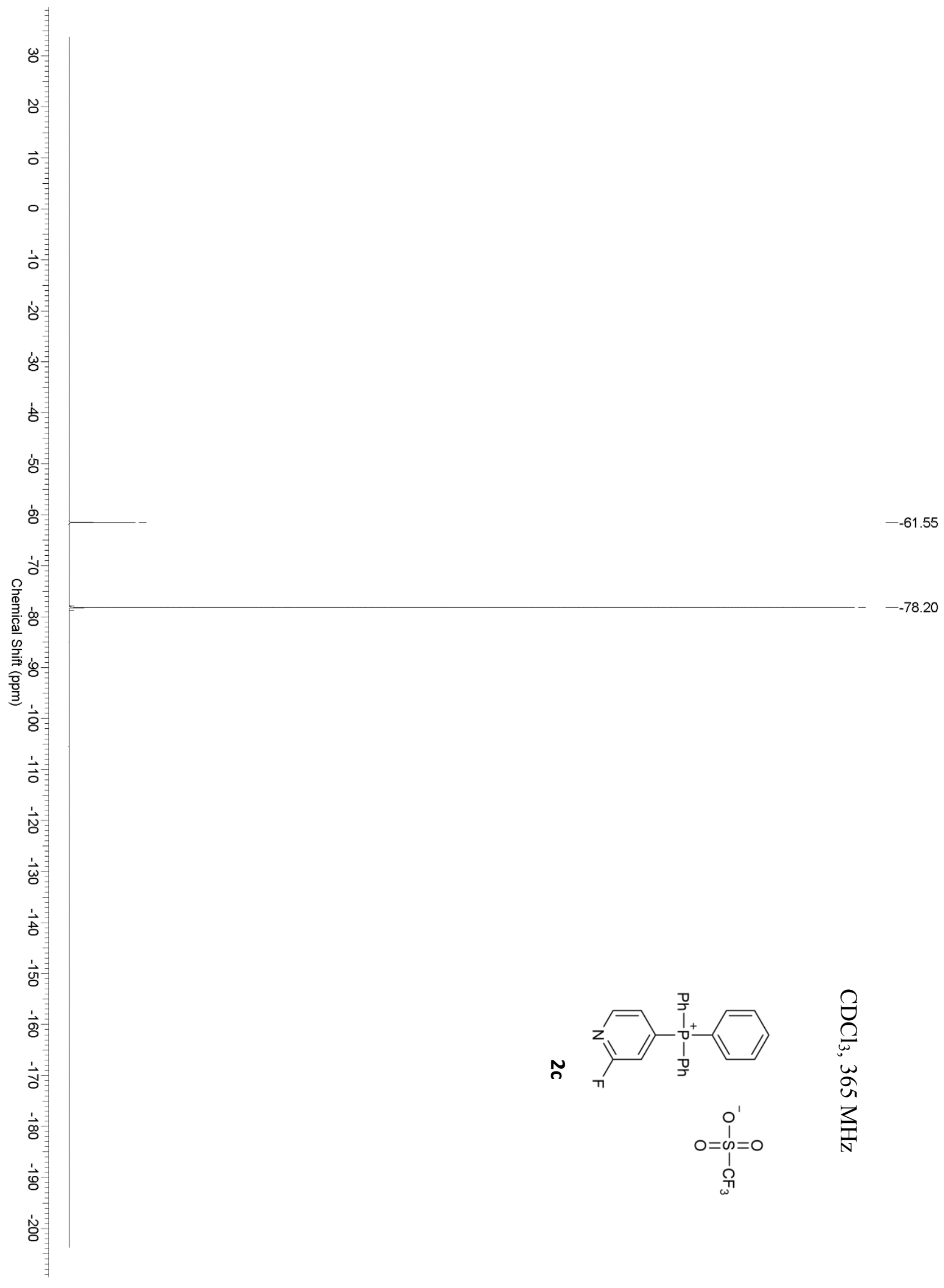




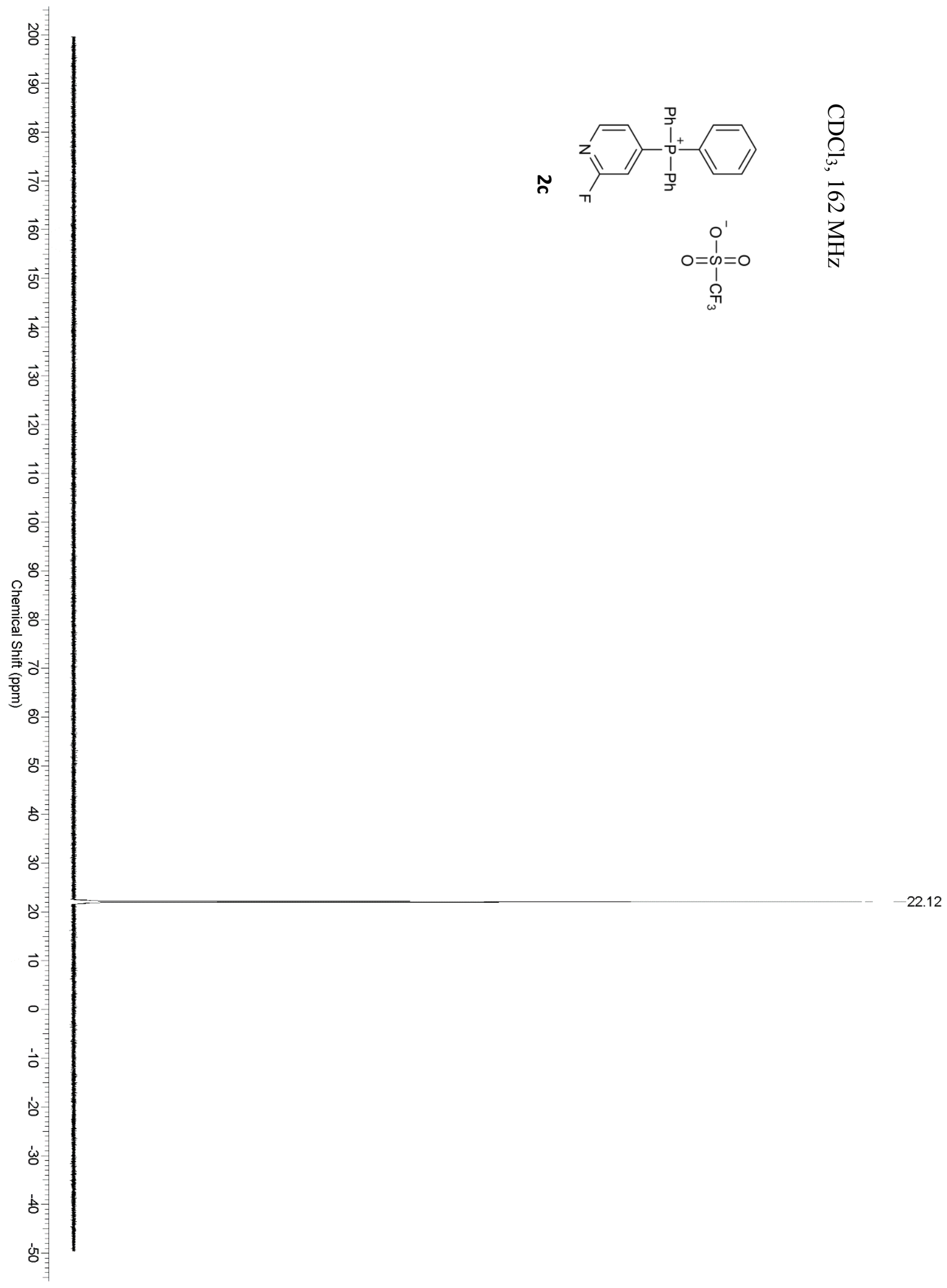




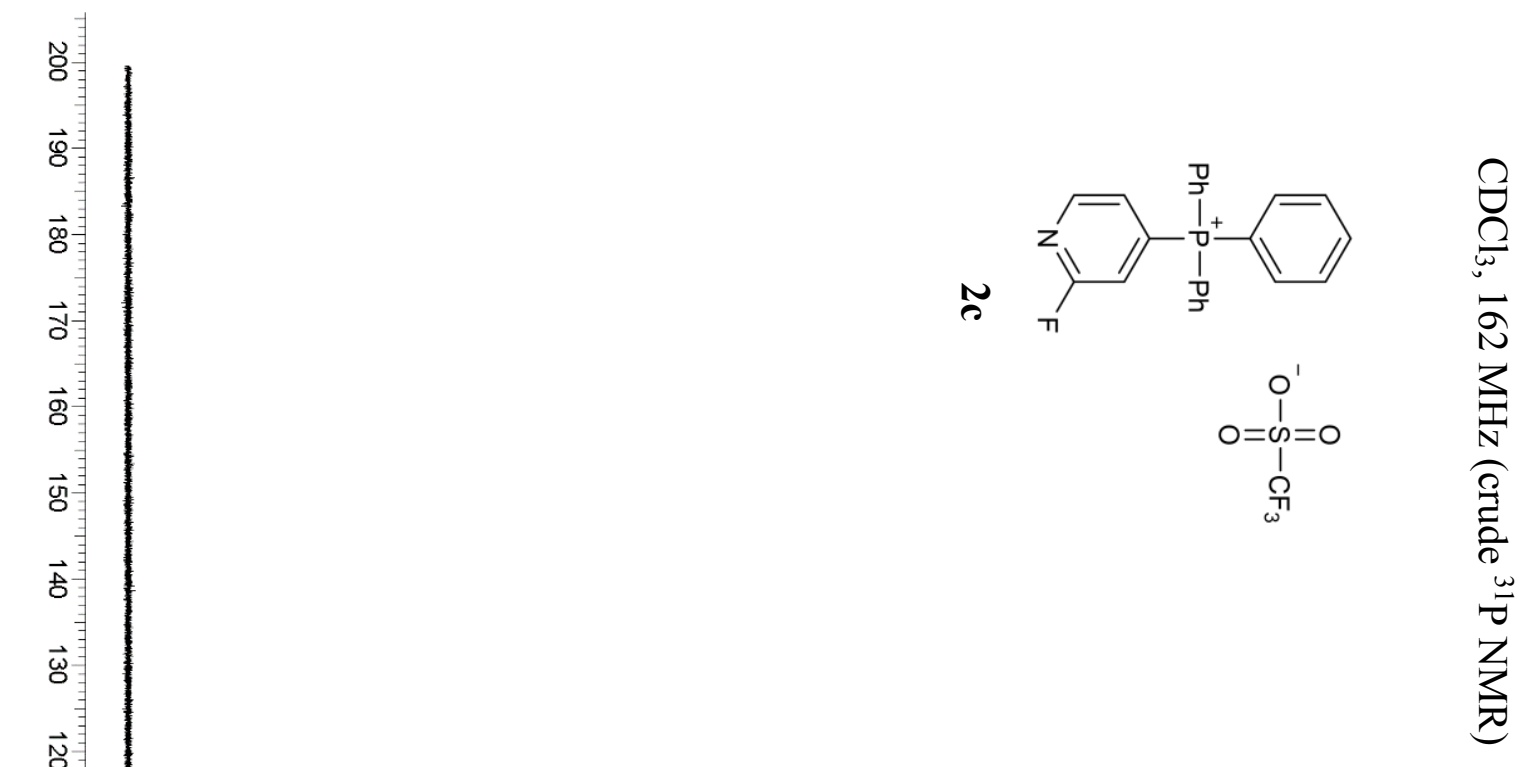

${ }^{\frac{9}{0}}{ }^{\frac{0}{0}}=$

$\overrightarrow{\mathrm{o}}$

$\overrightarrow{8}$

In

古

$\omega$

青泰

$-29.25$

o

○

它夆

o

กิ่

ผ

古

败丰 


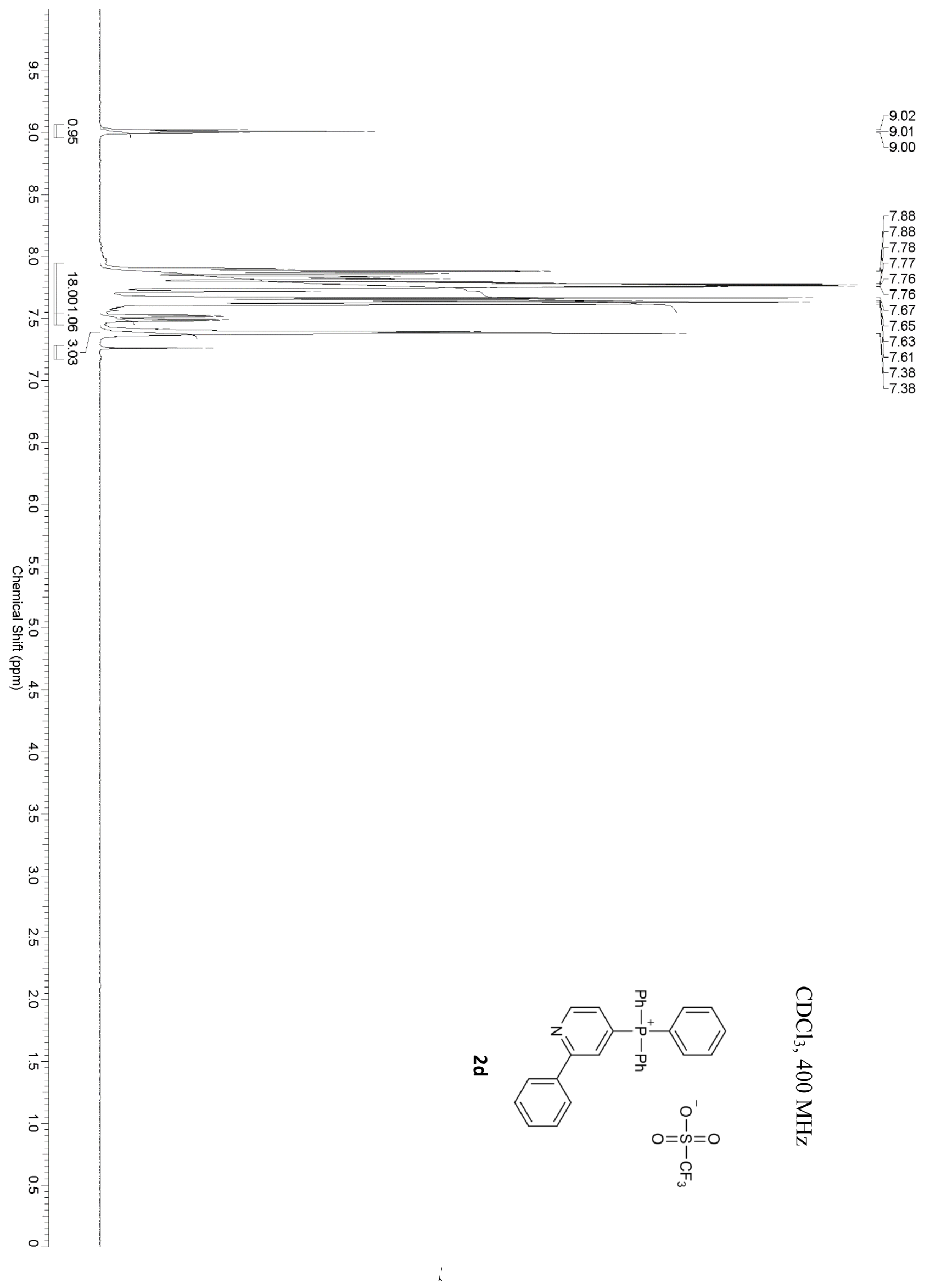




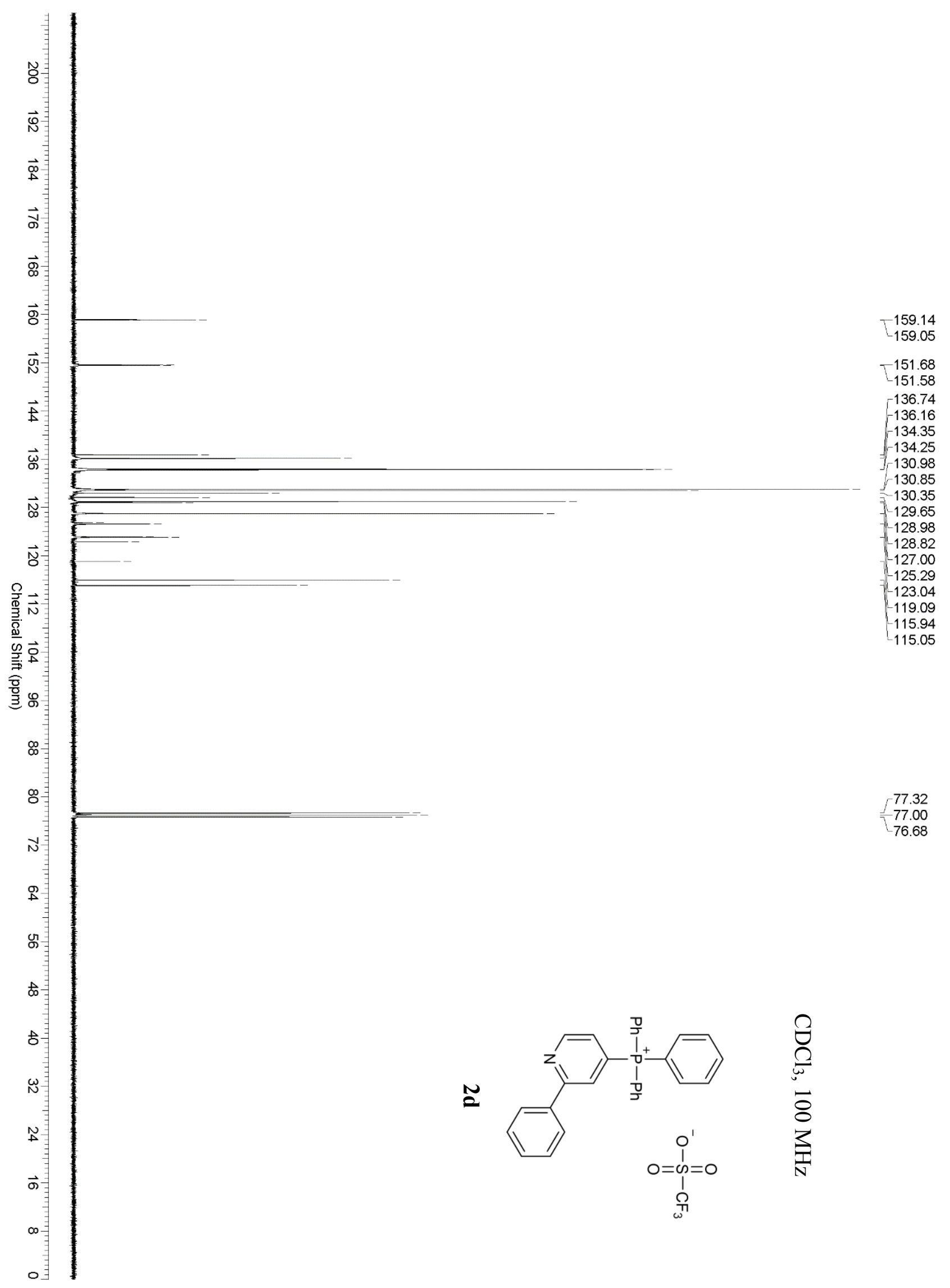




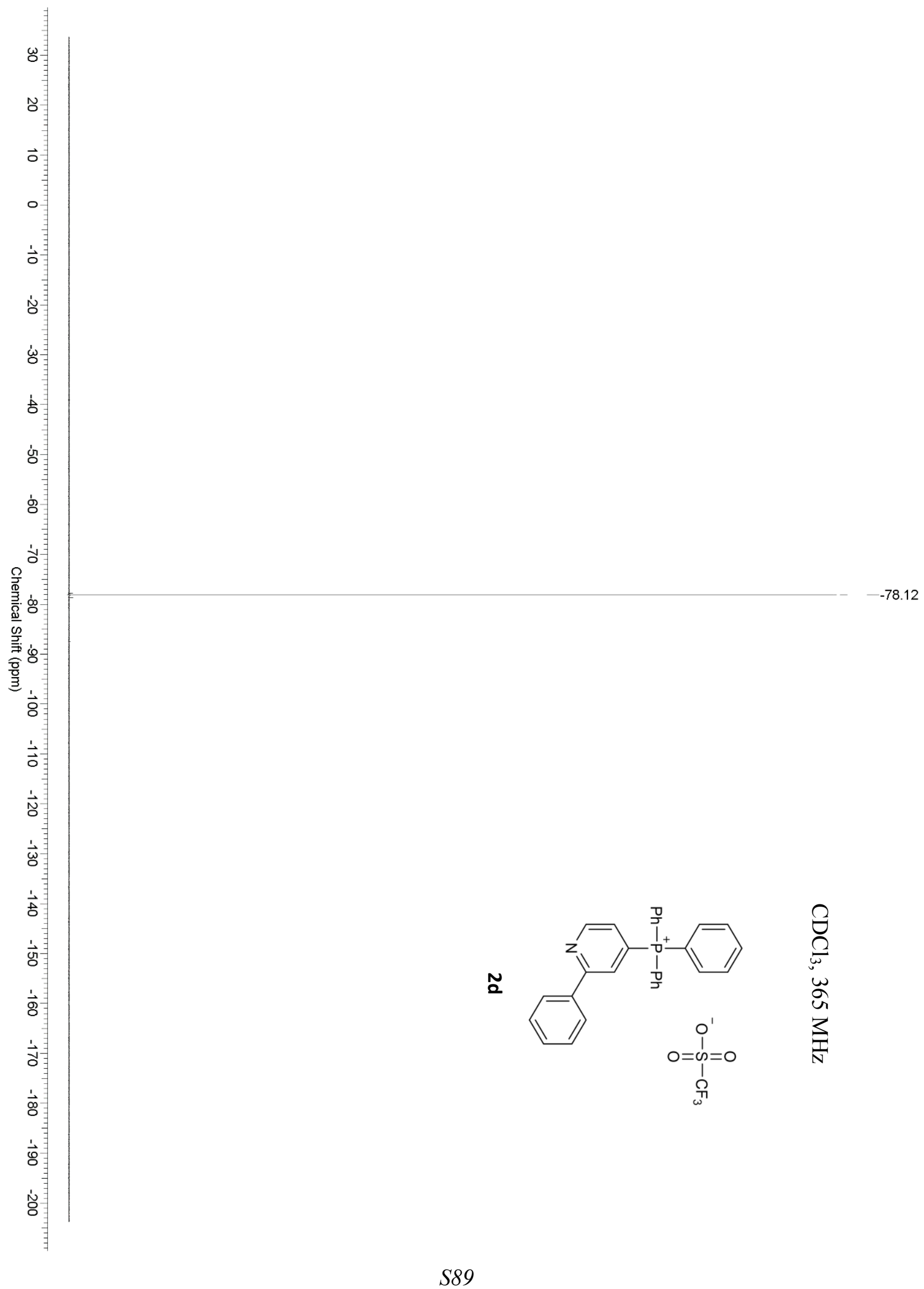




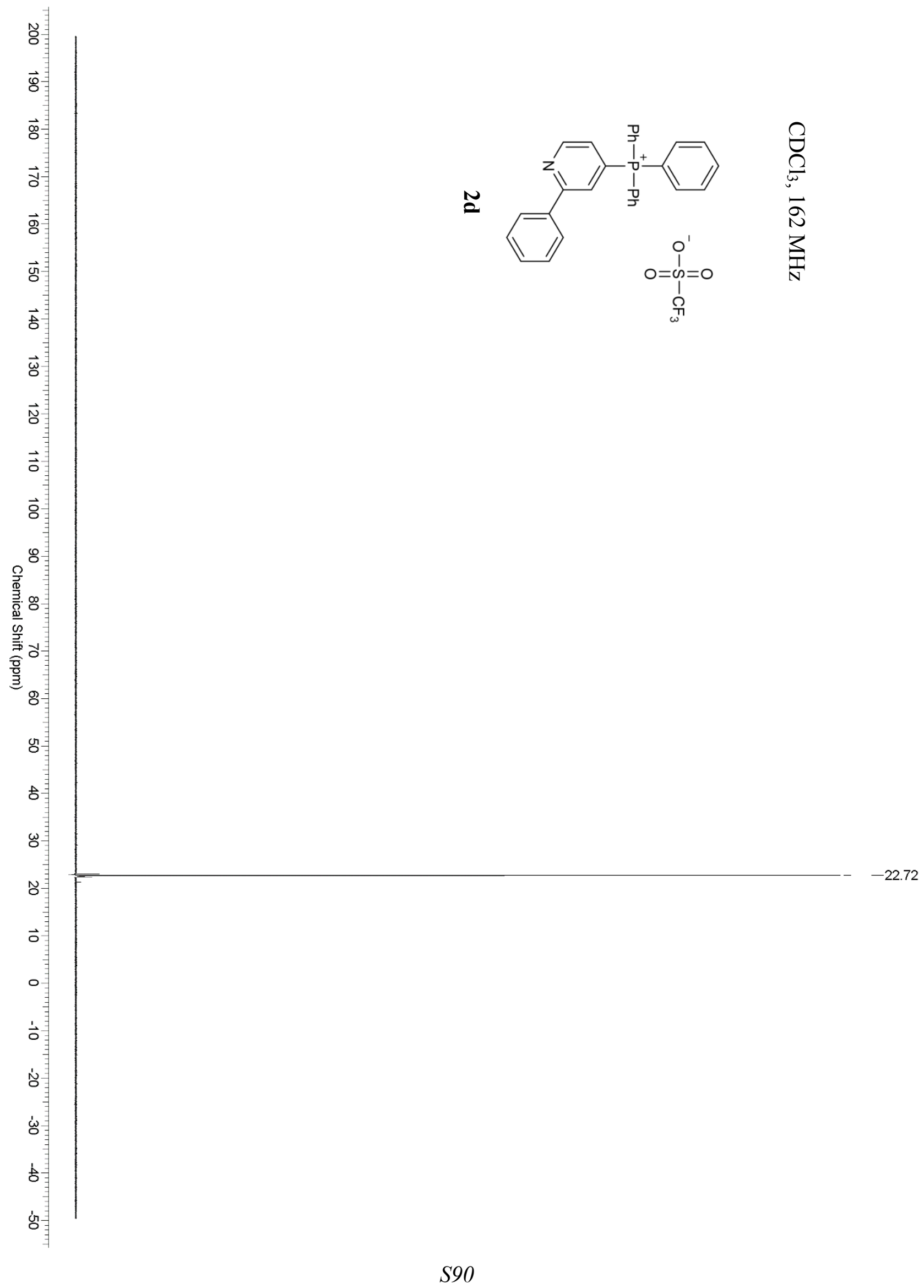




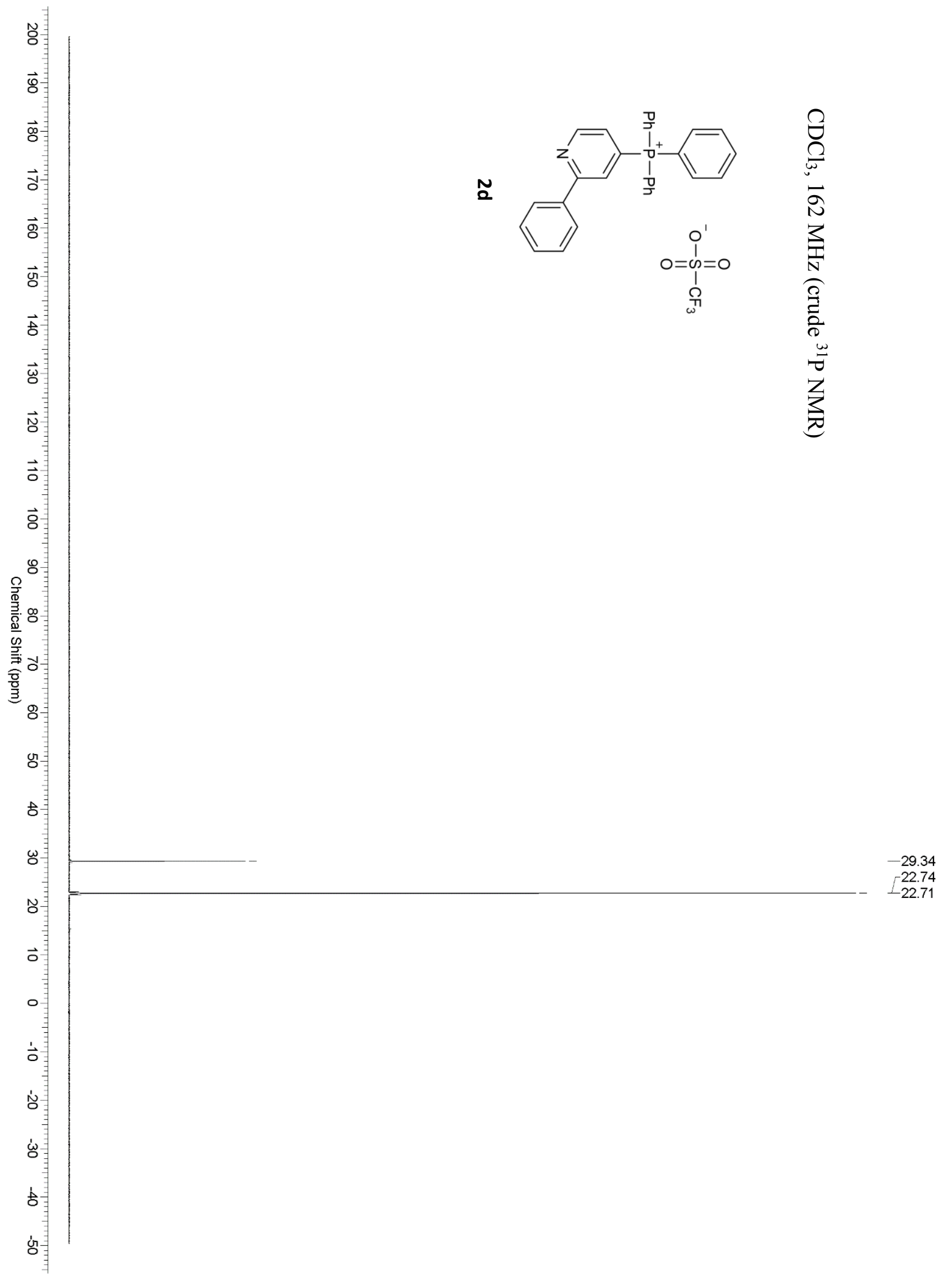




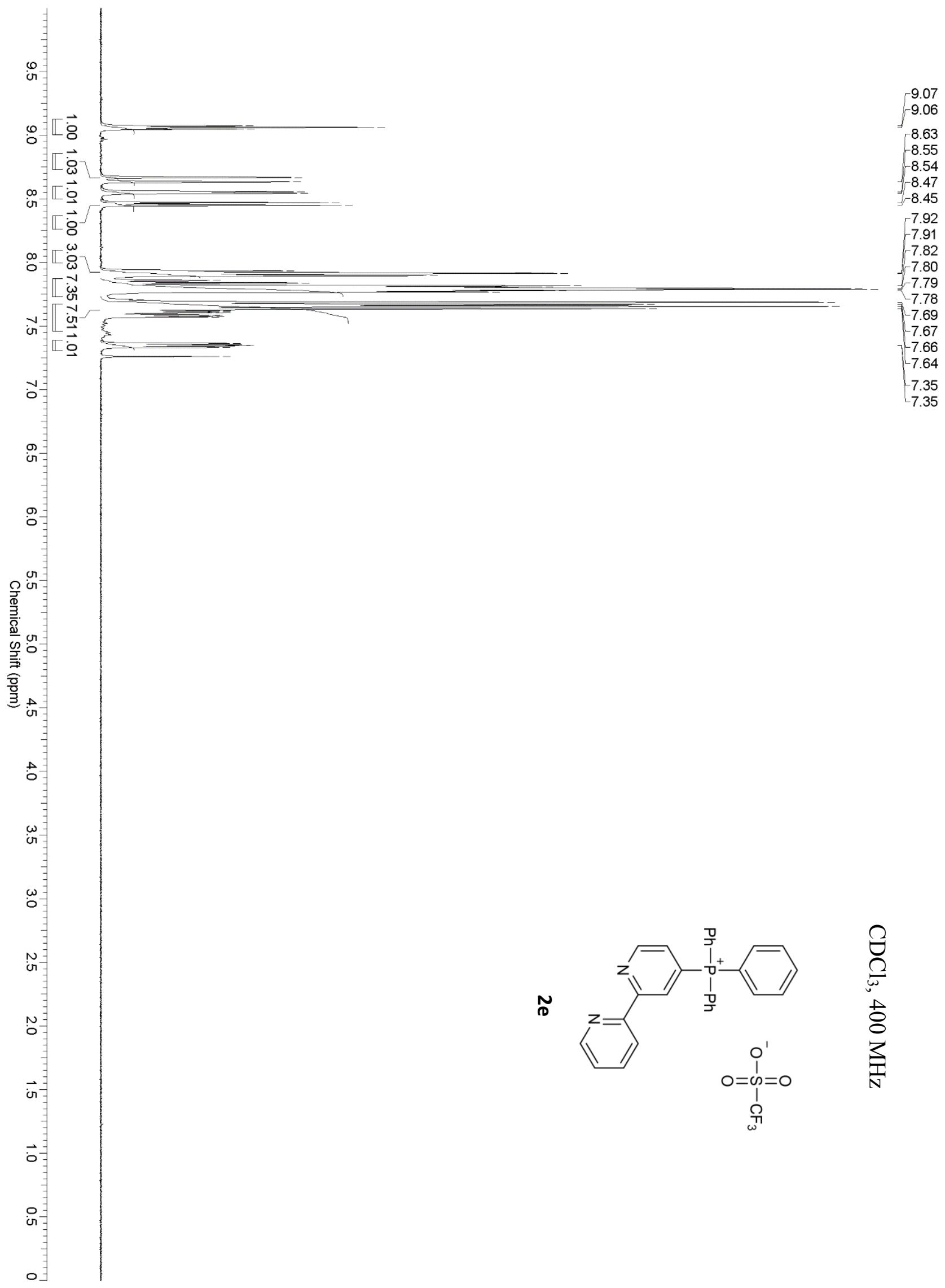




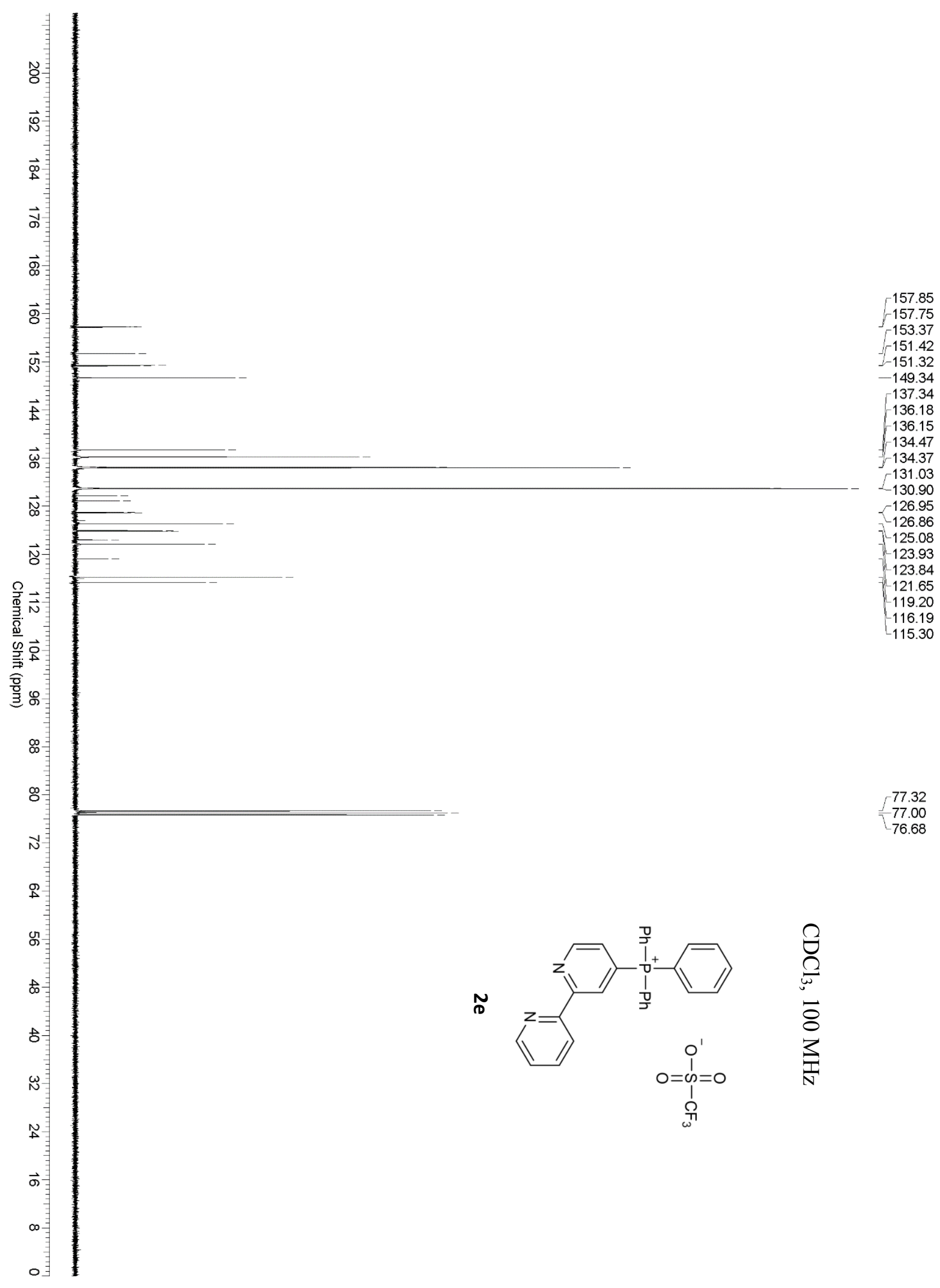




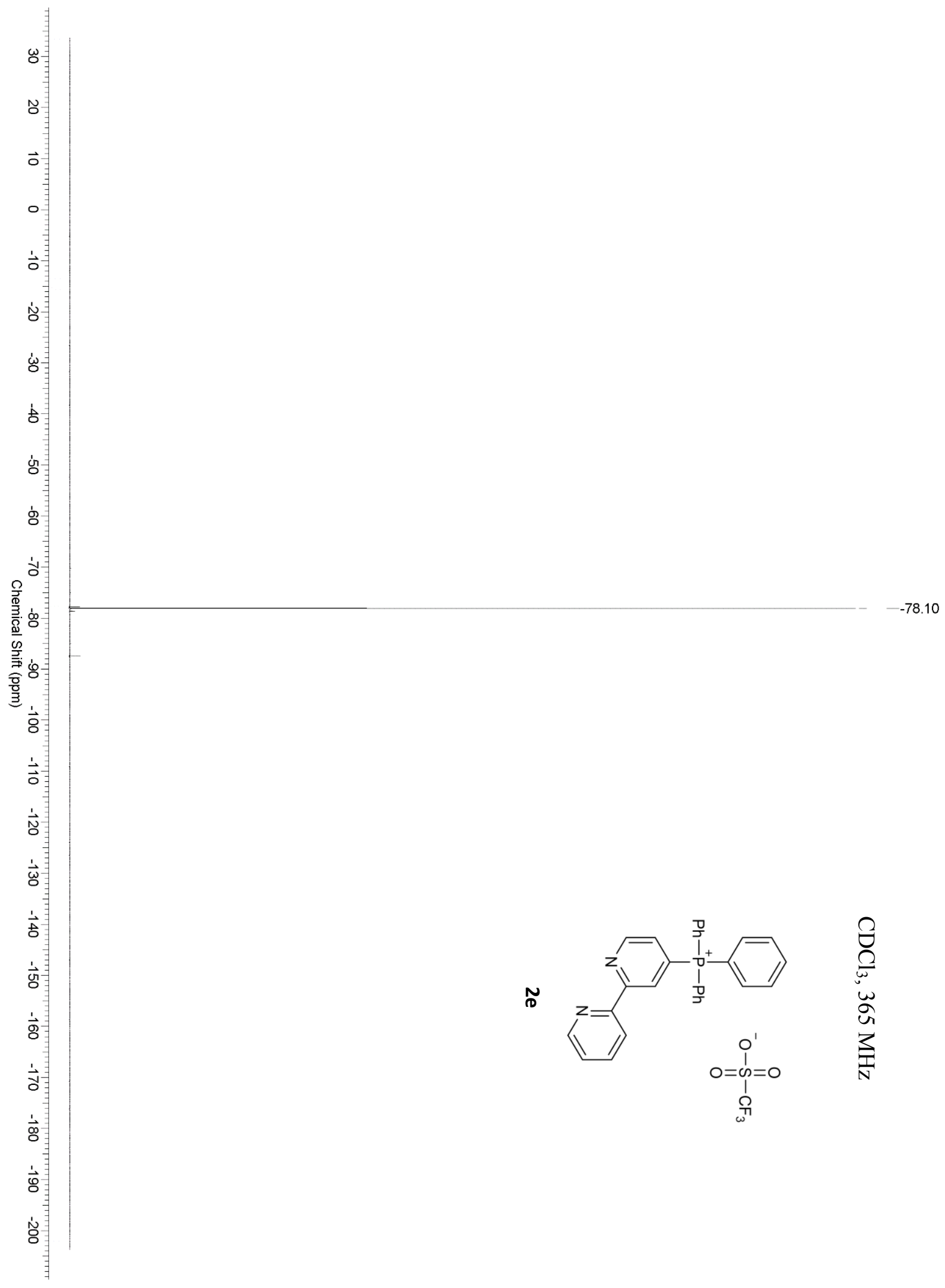




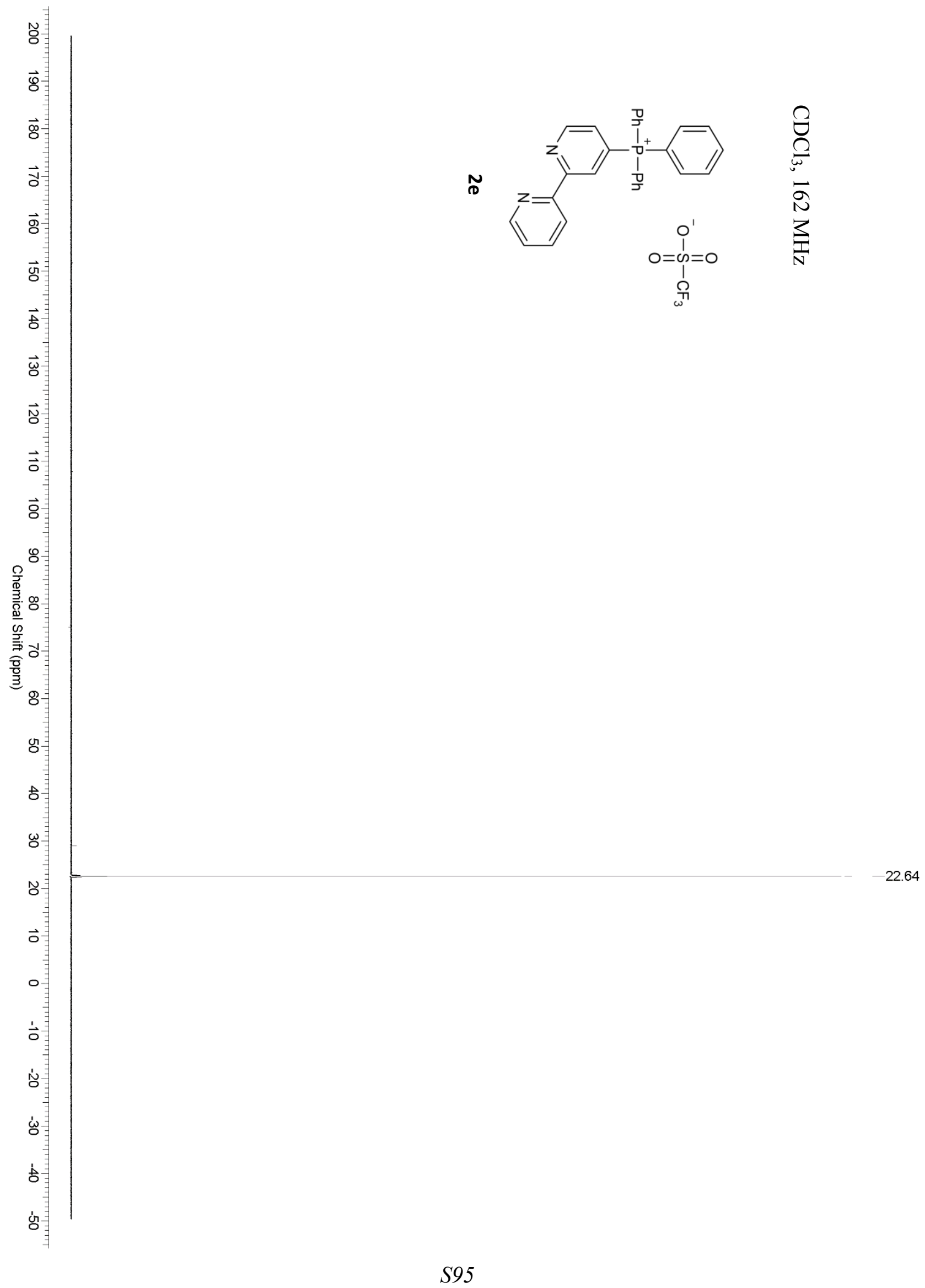




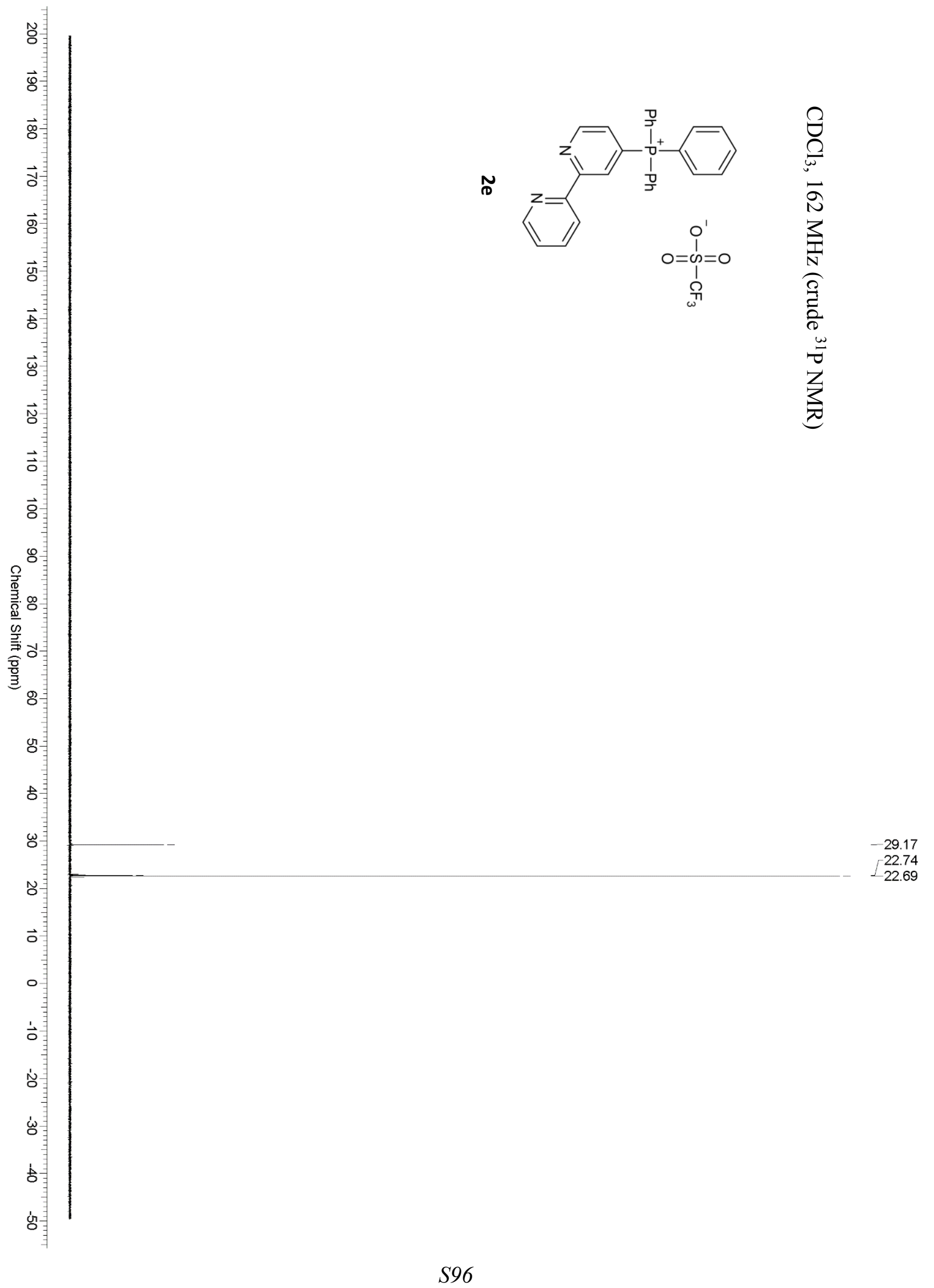




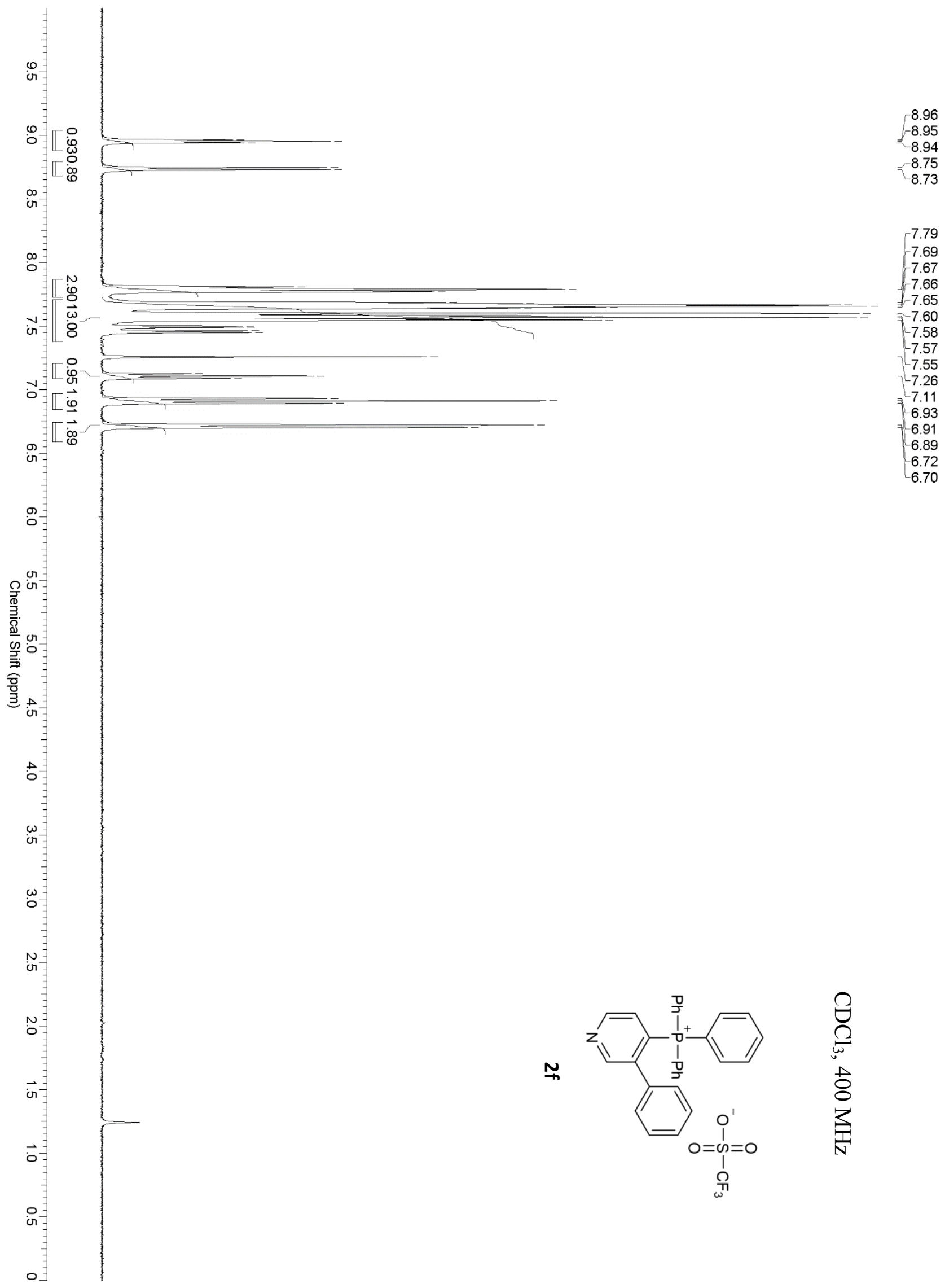




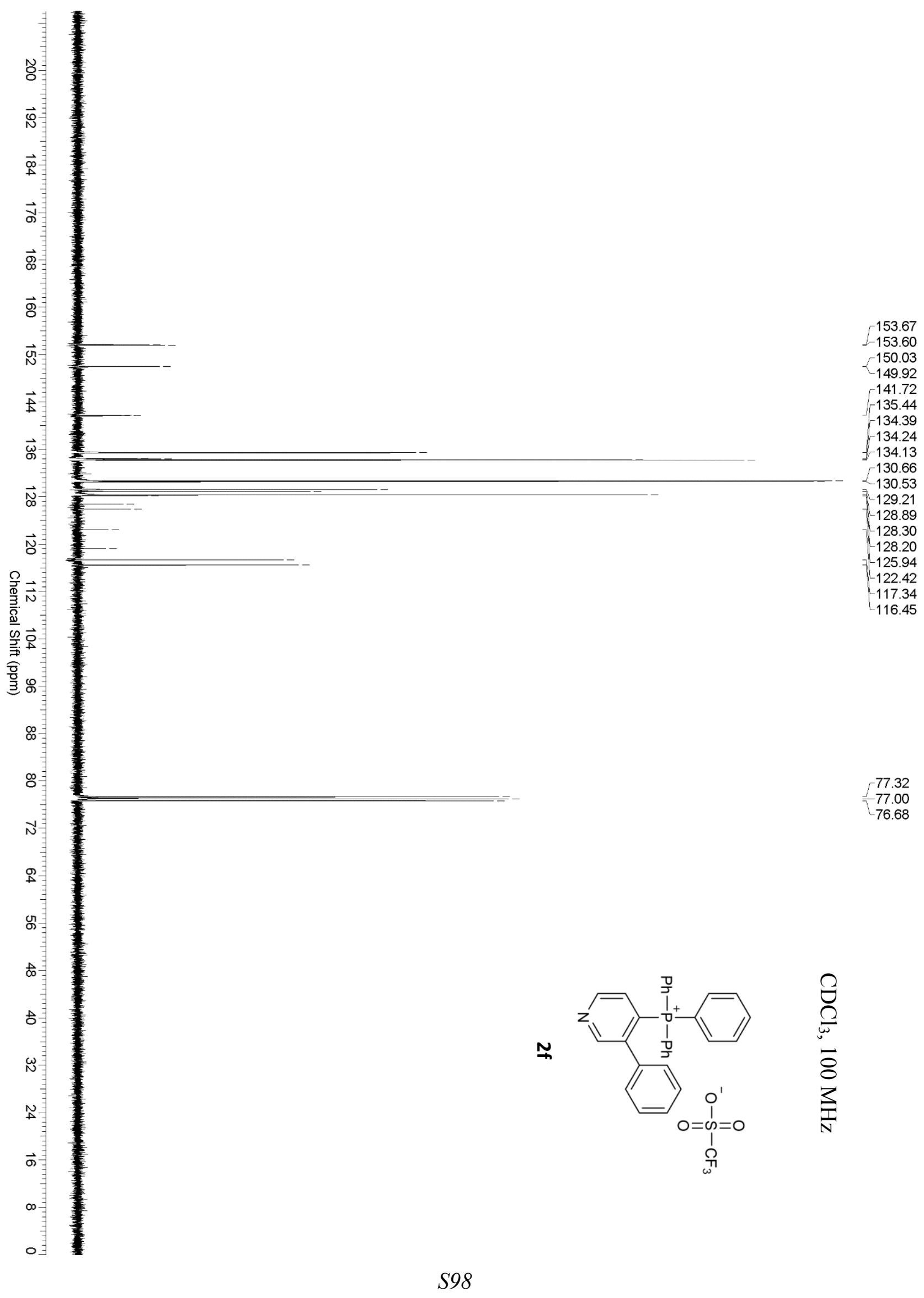




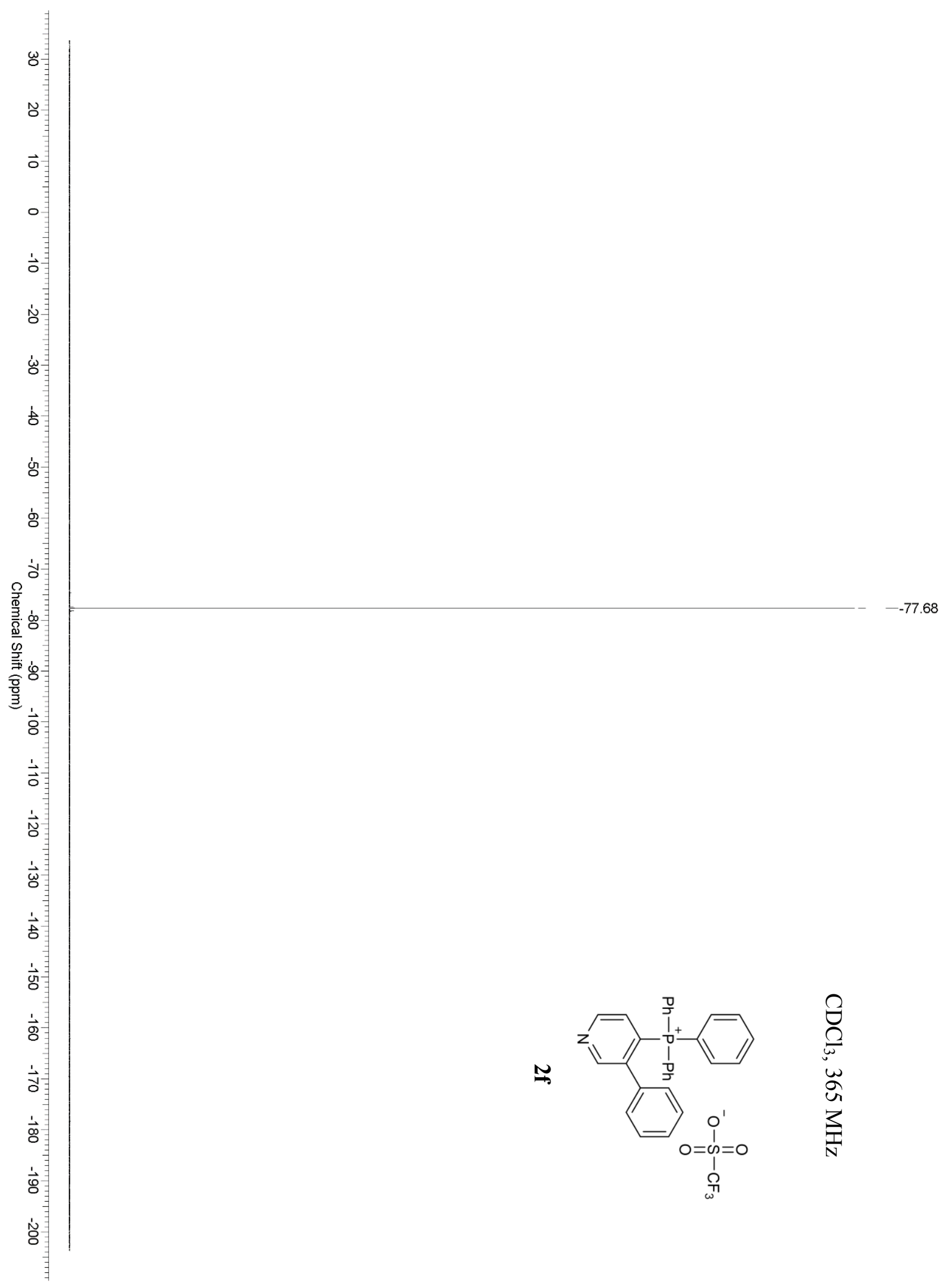




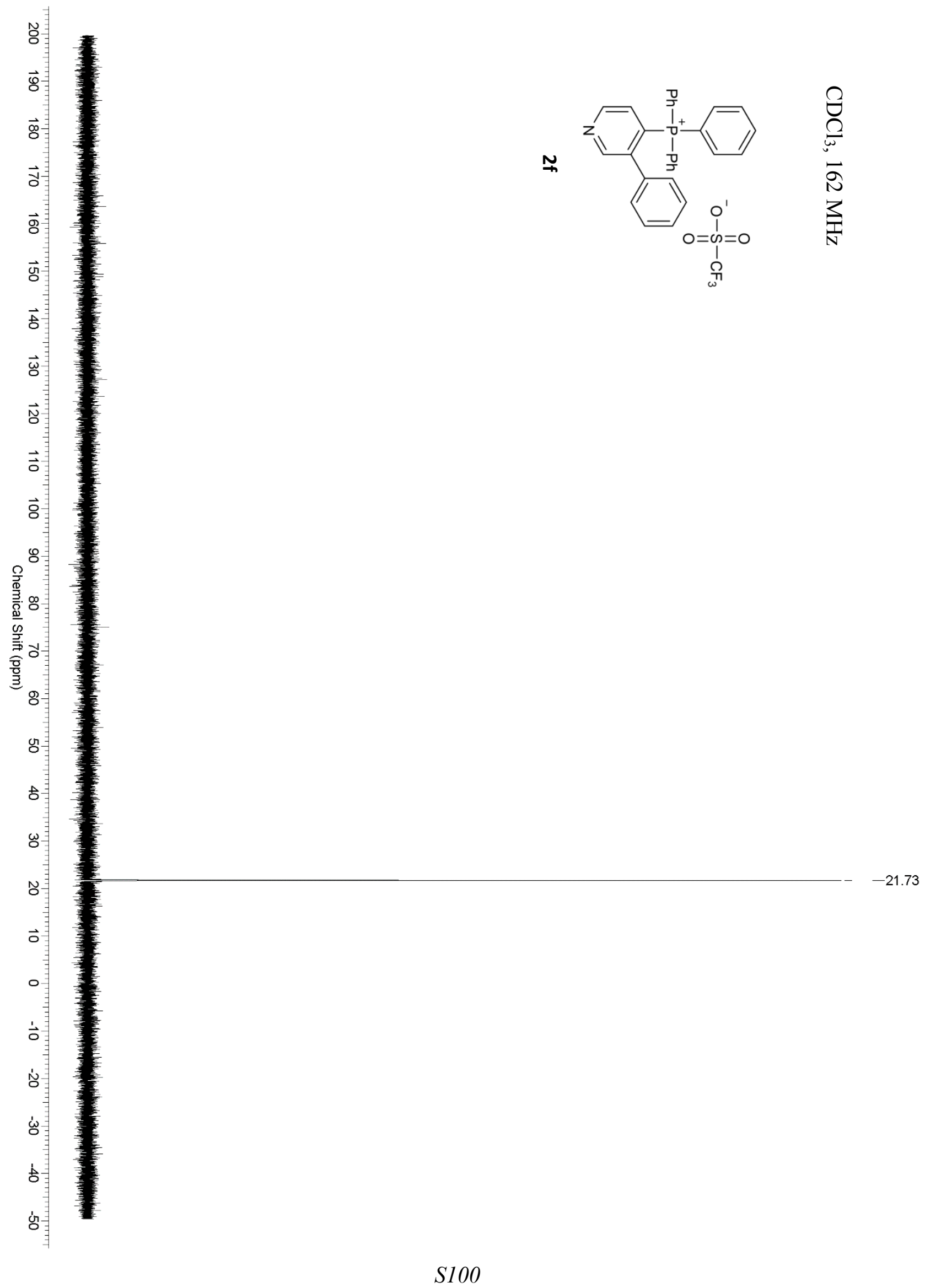




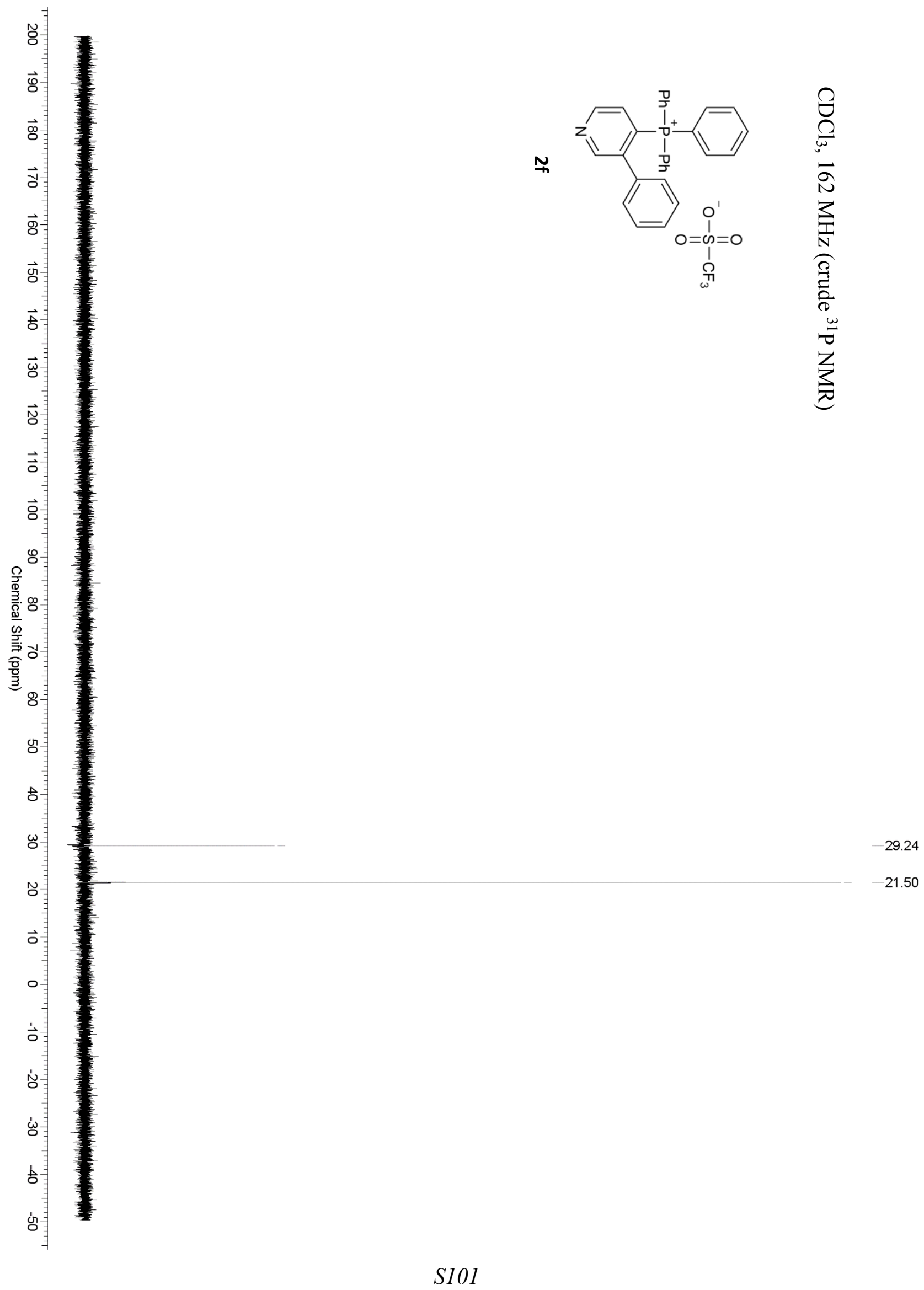




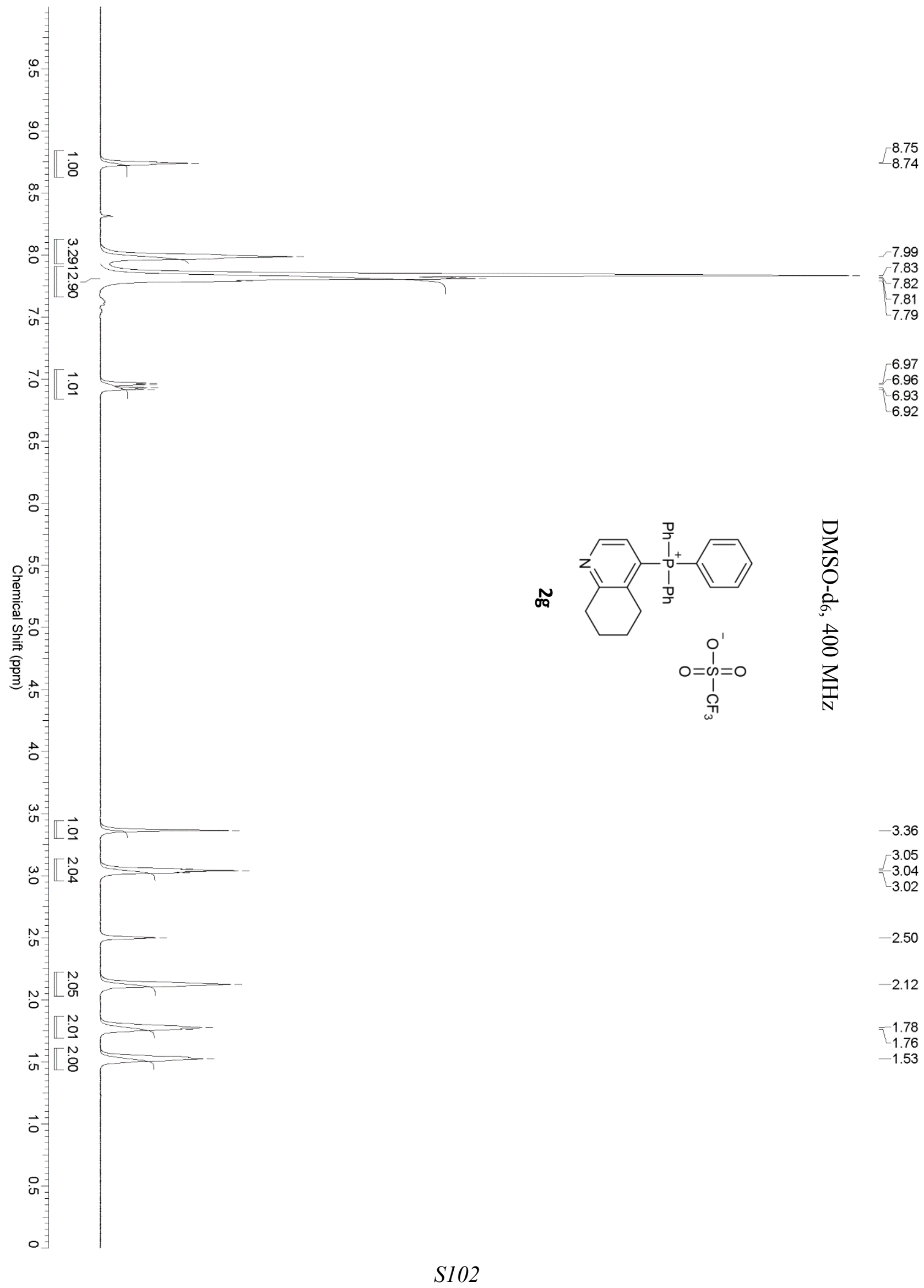




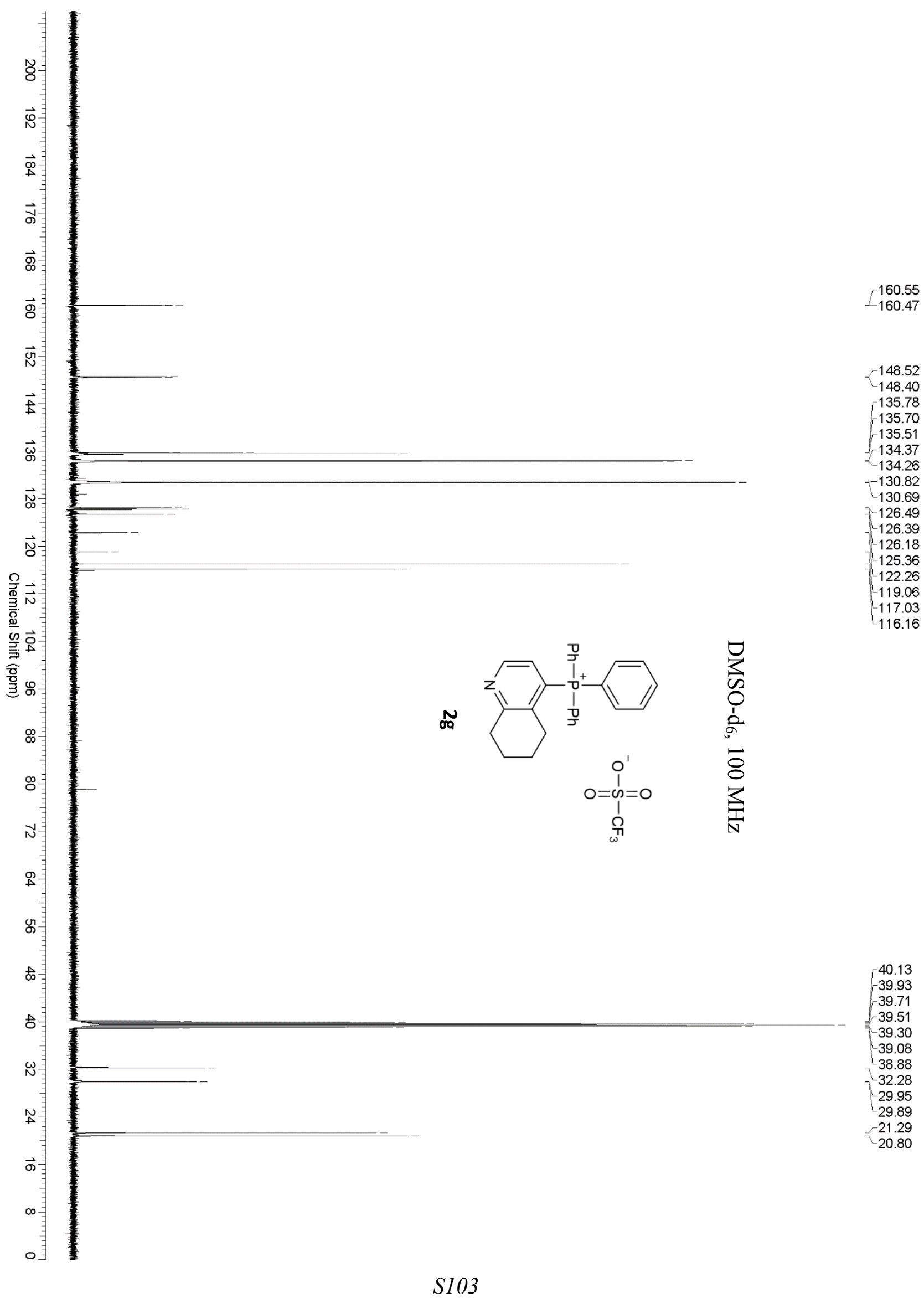




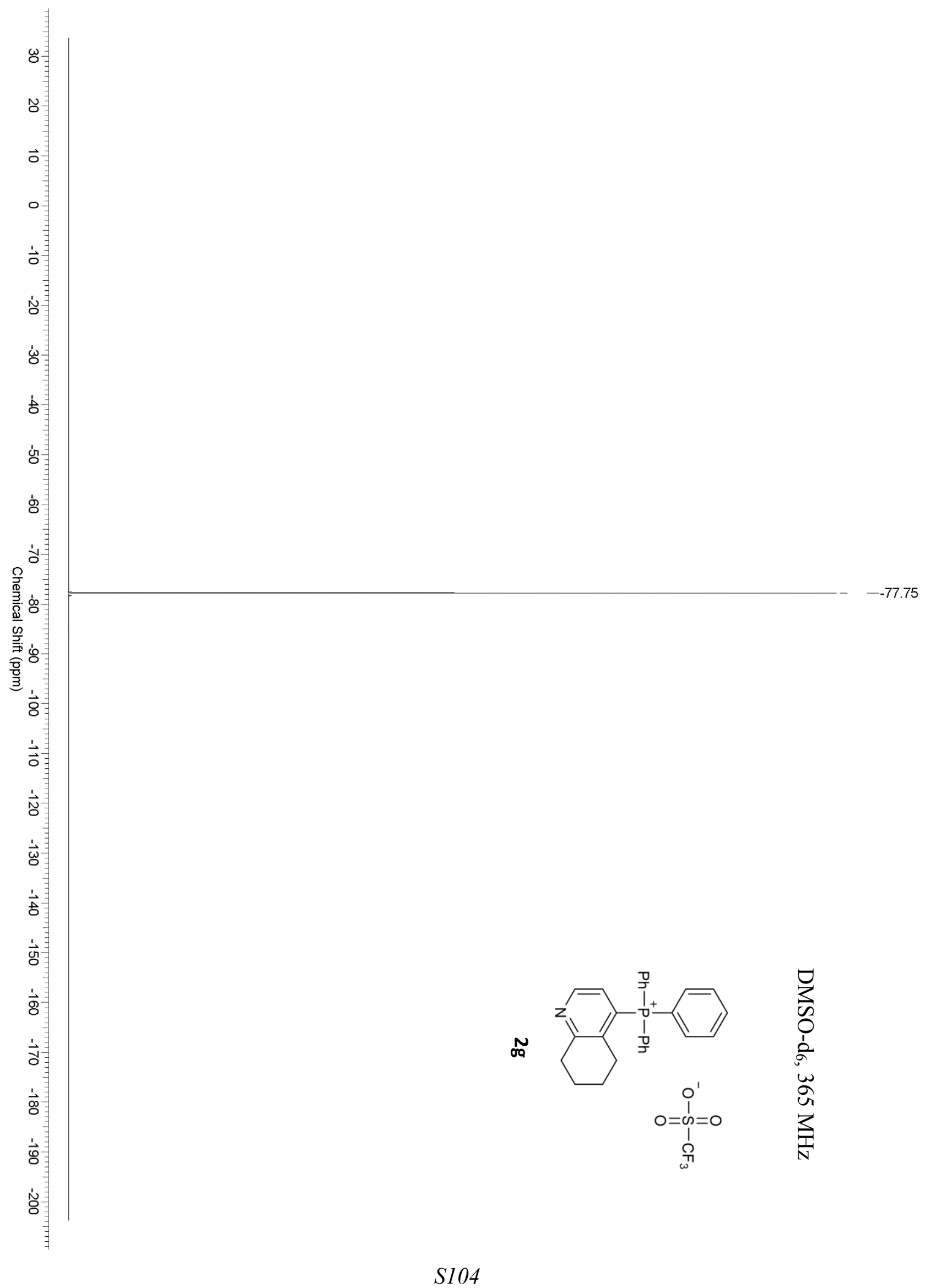




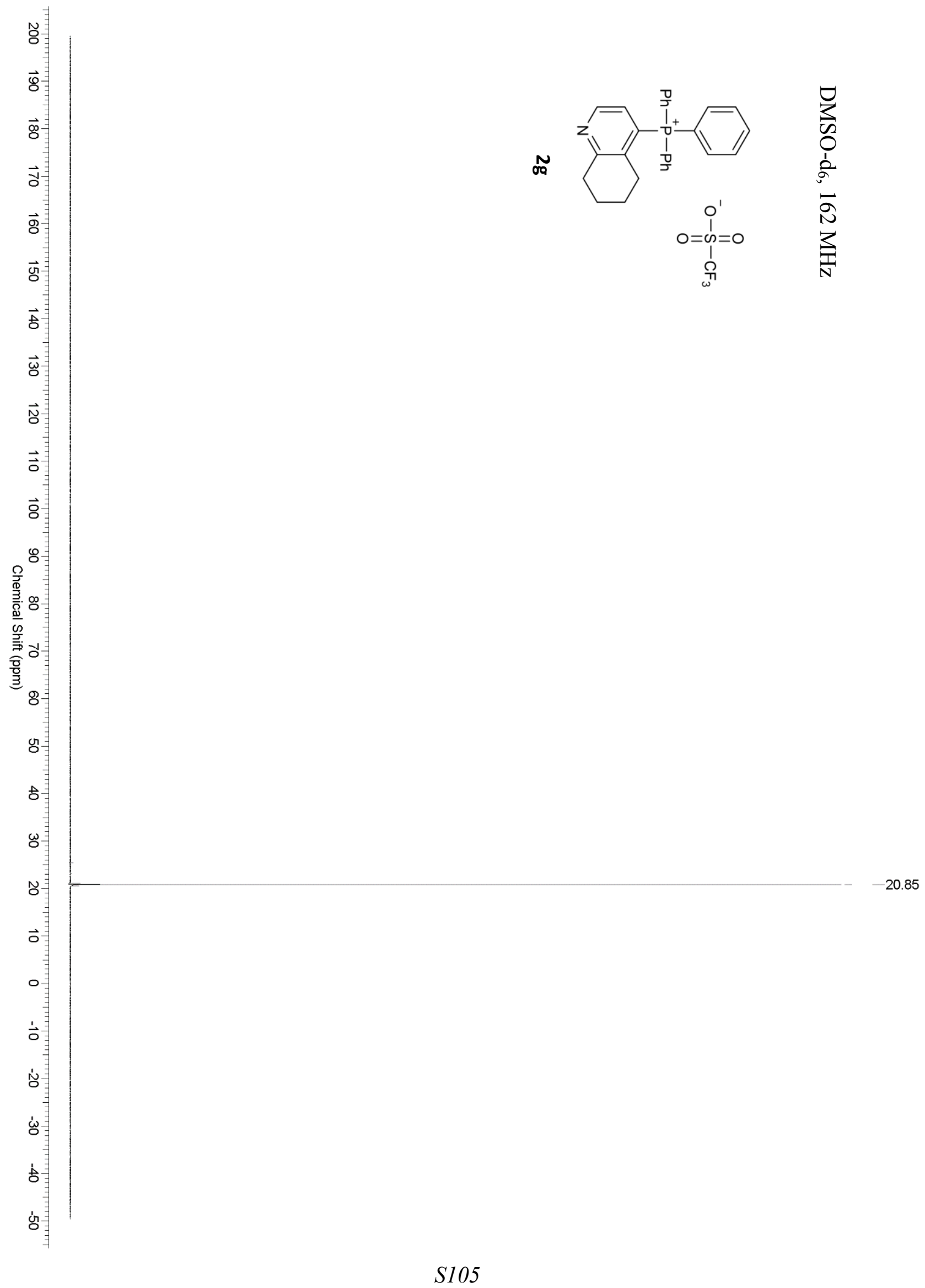




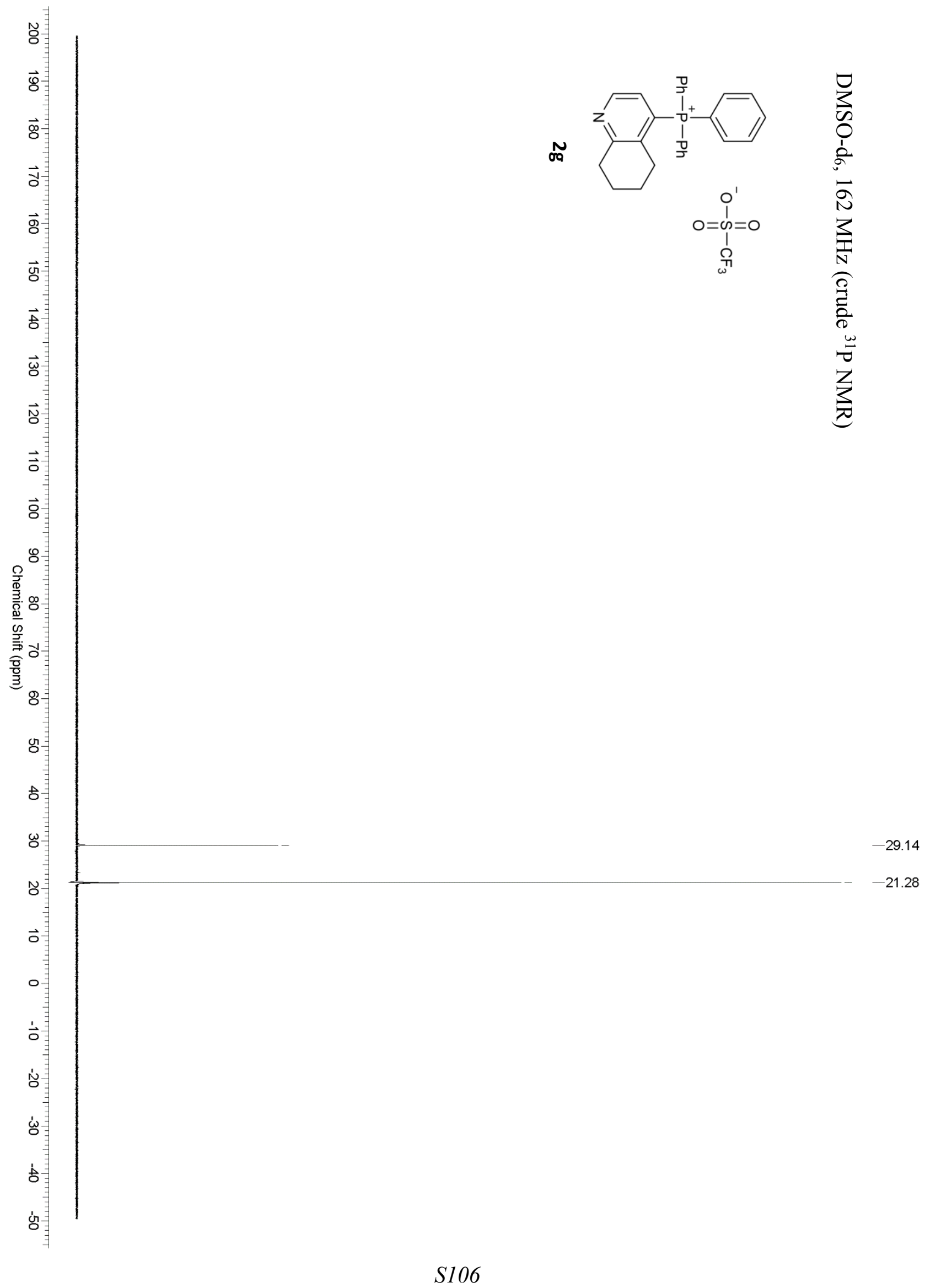




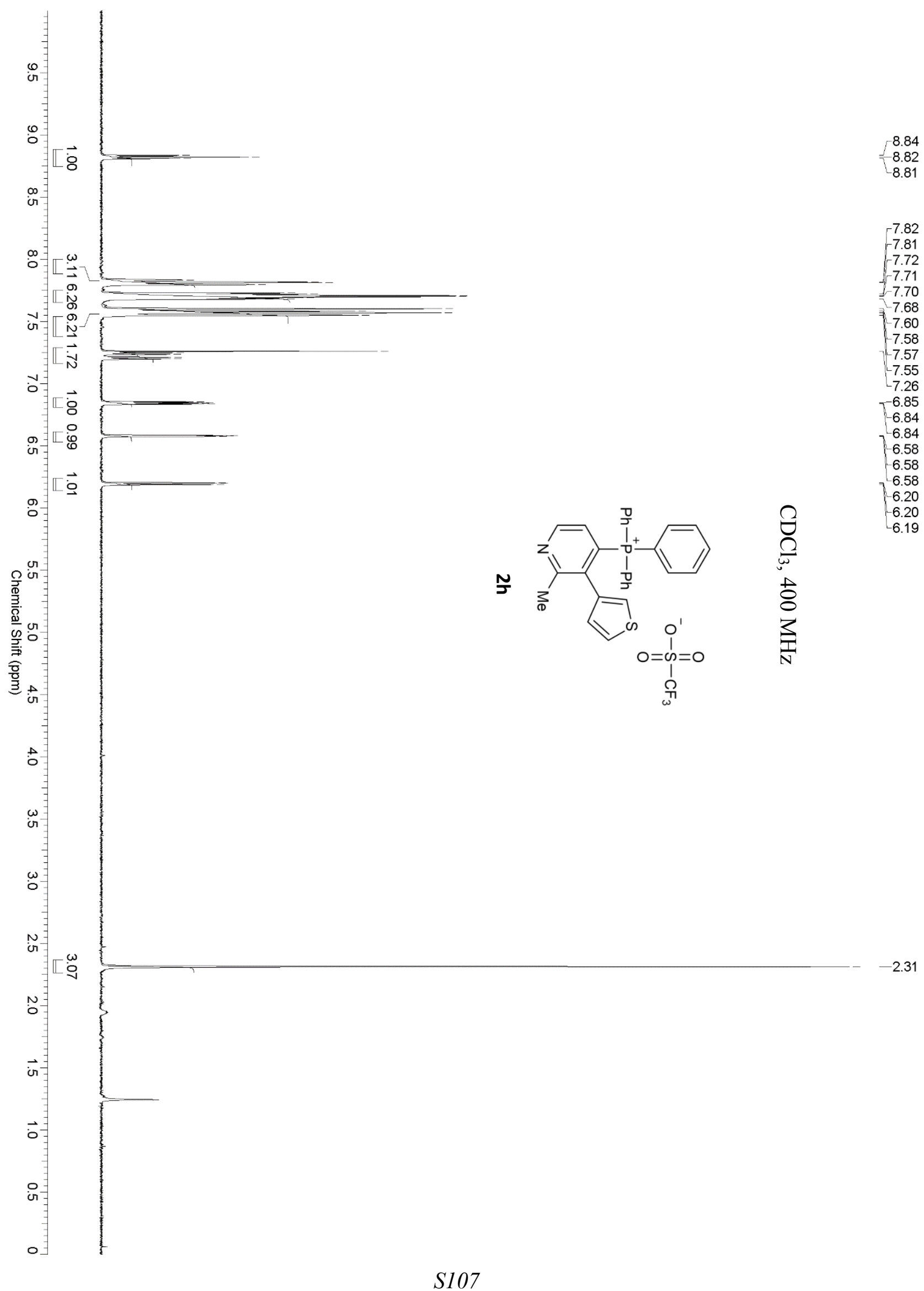




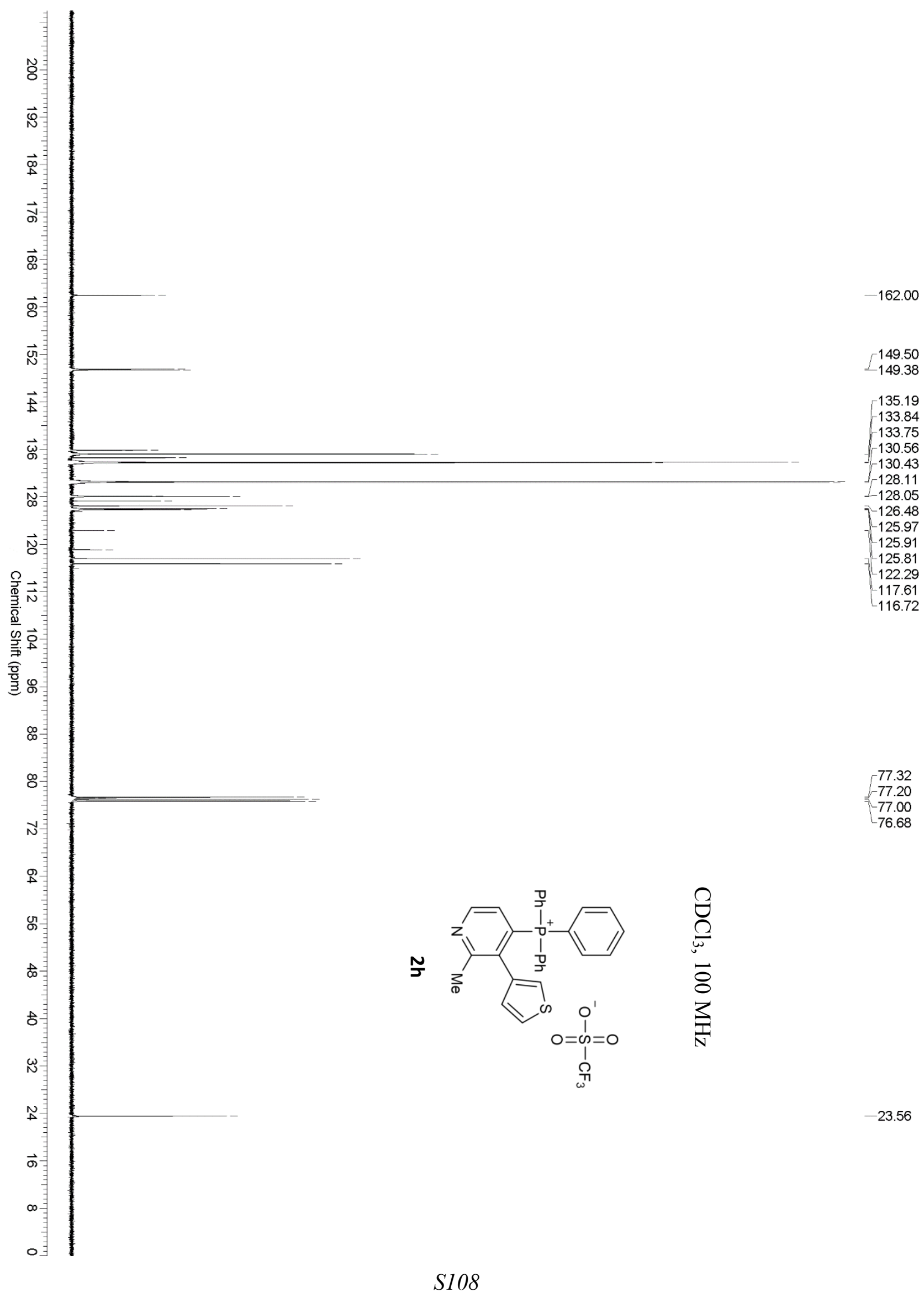




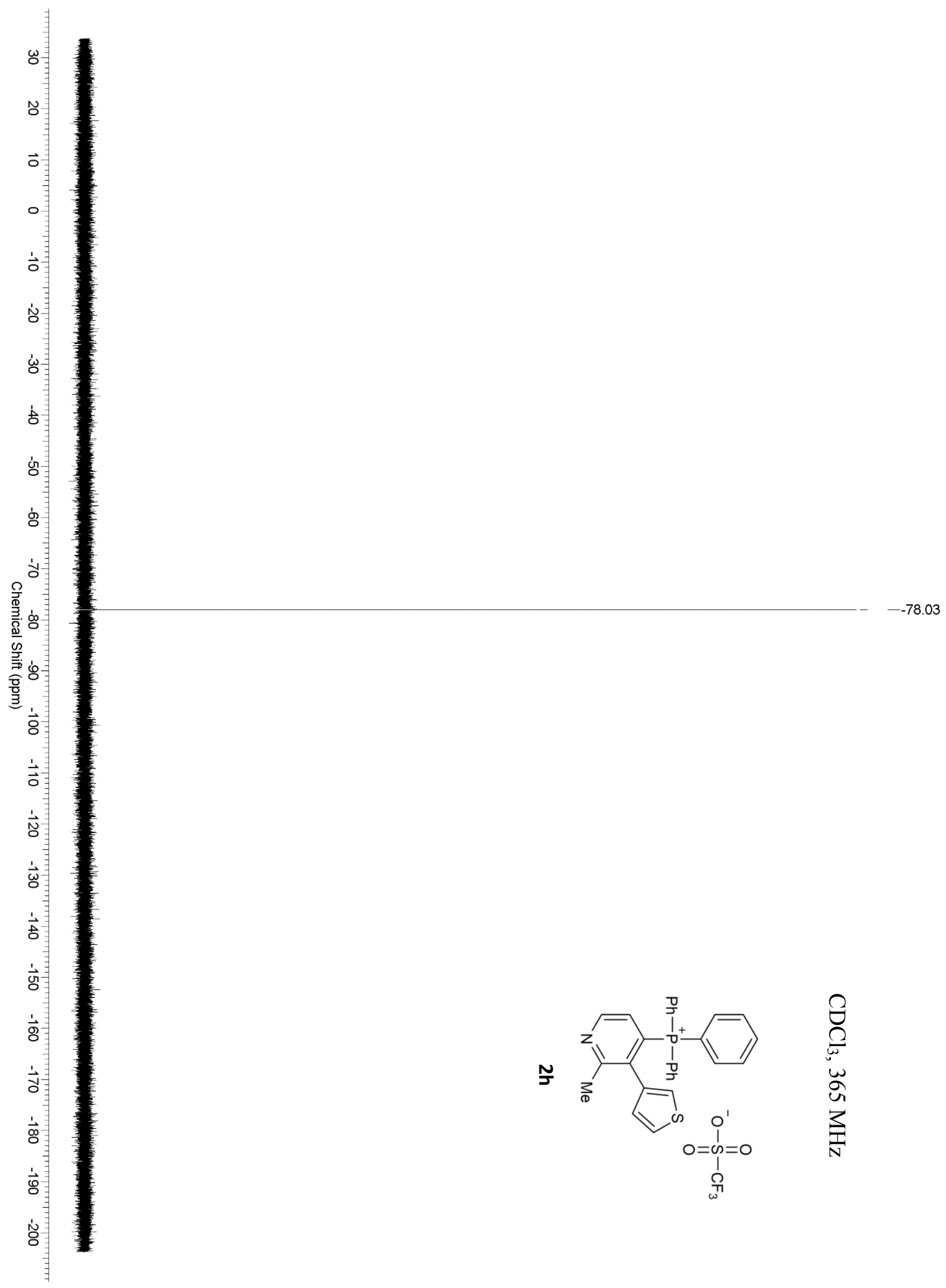




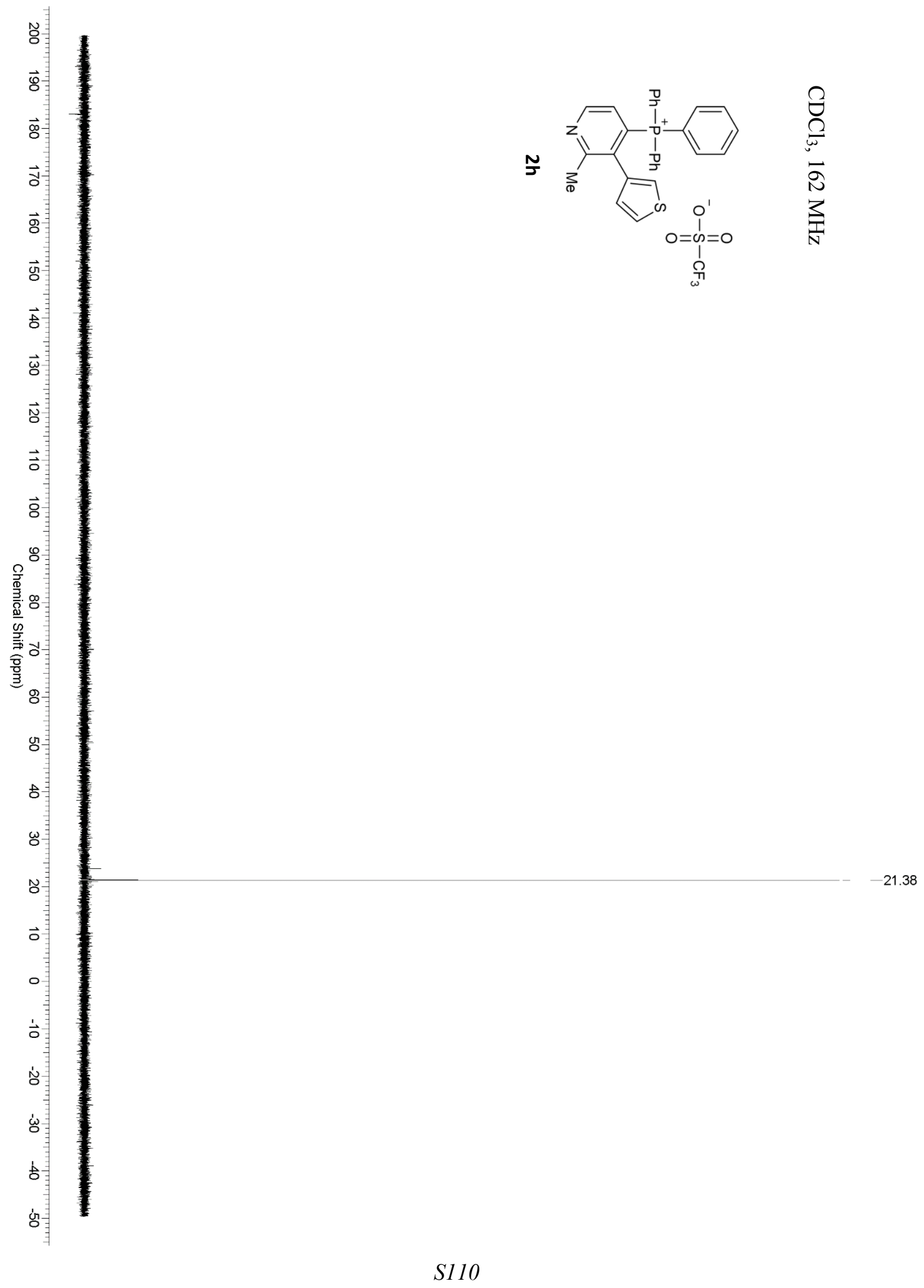




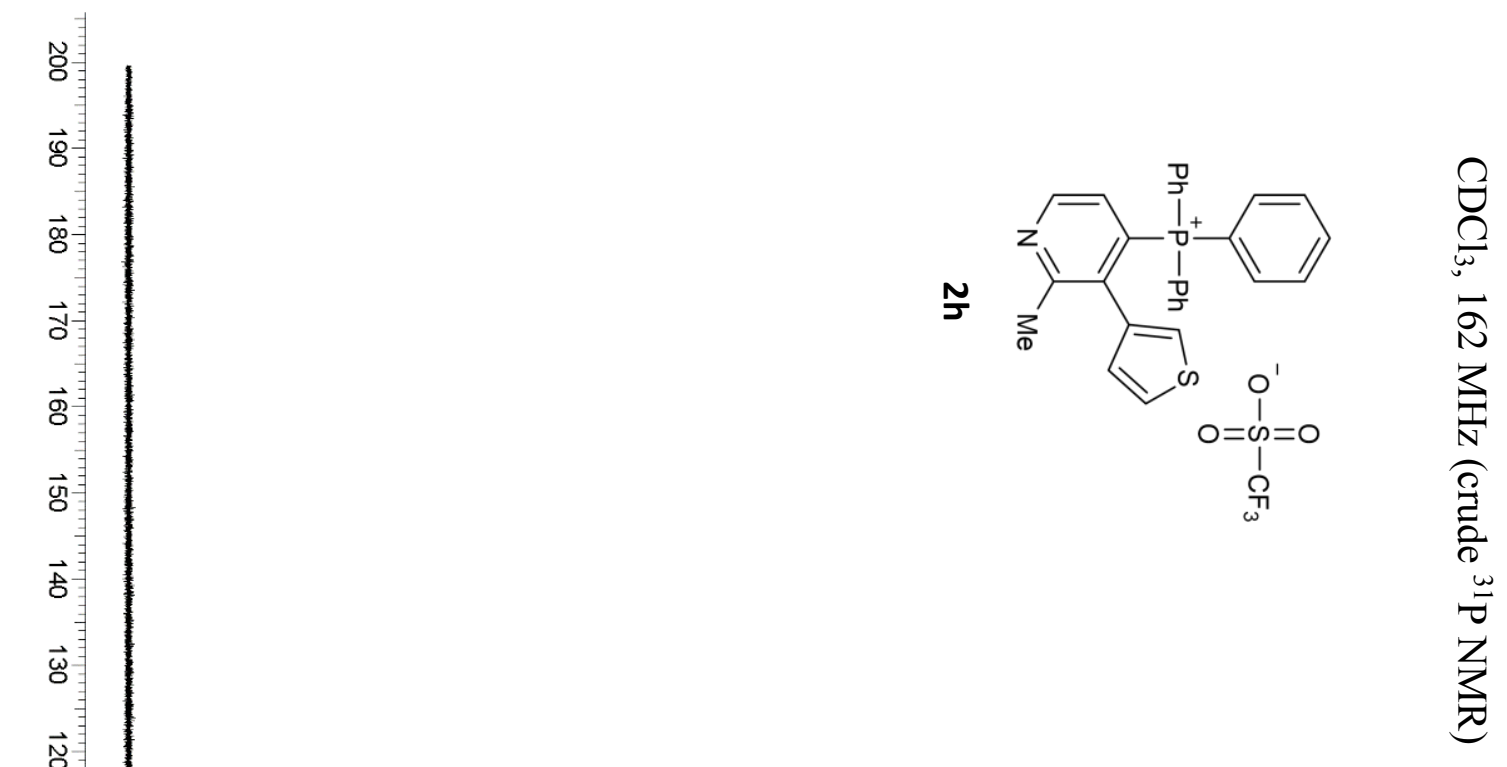

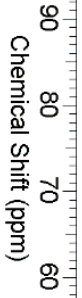

$\vec{o}$

$\overrightarrow{\mathrm{o}}$

응

古寻

wa

责围

$-{ }_{-1}^{-29.20}$

o

$\circ$

它暳

กิ่

客㓞

古寻

的丰 


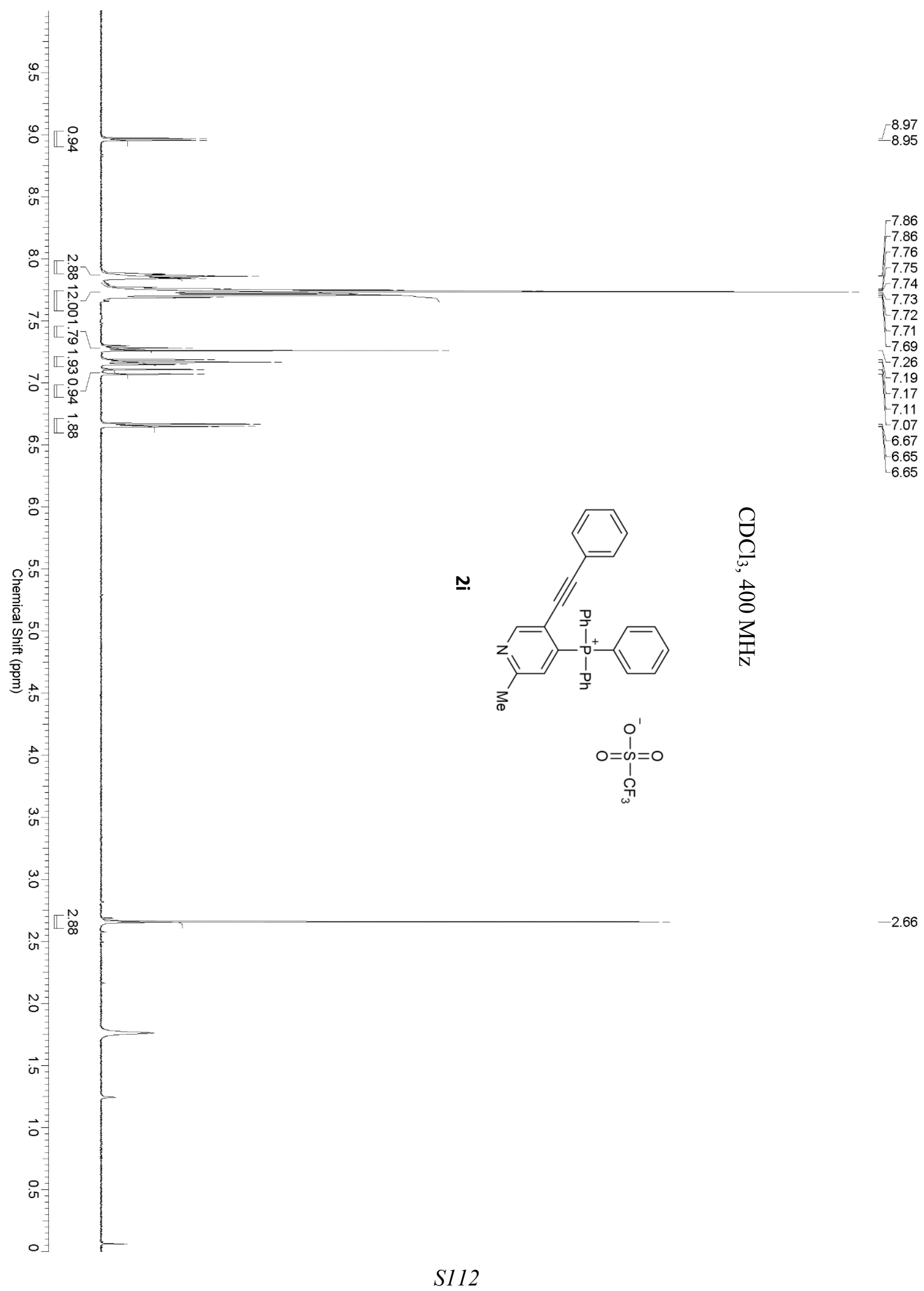




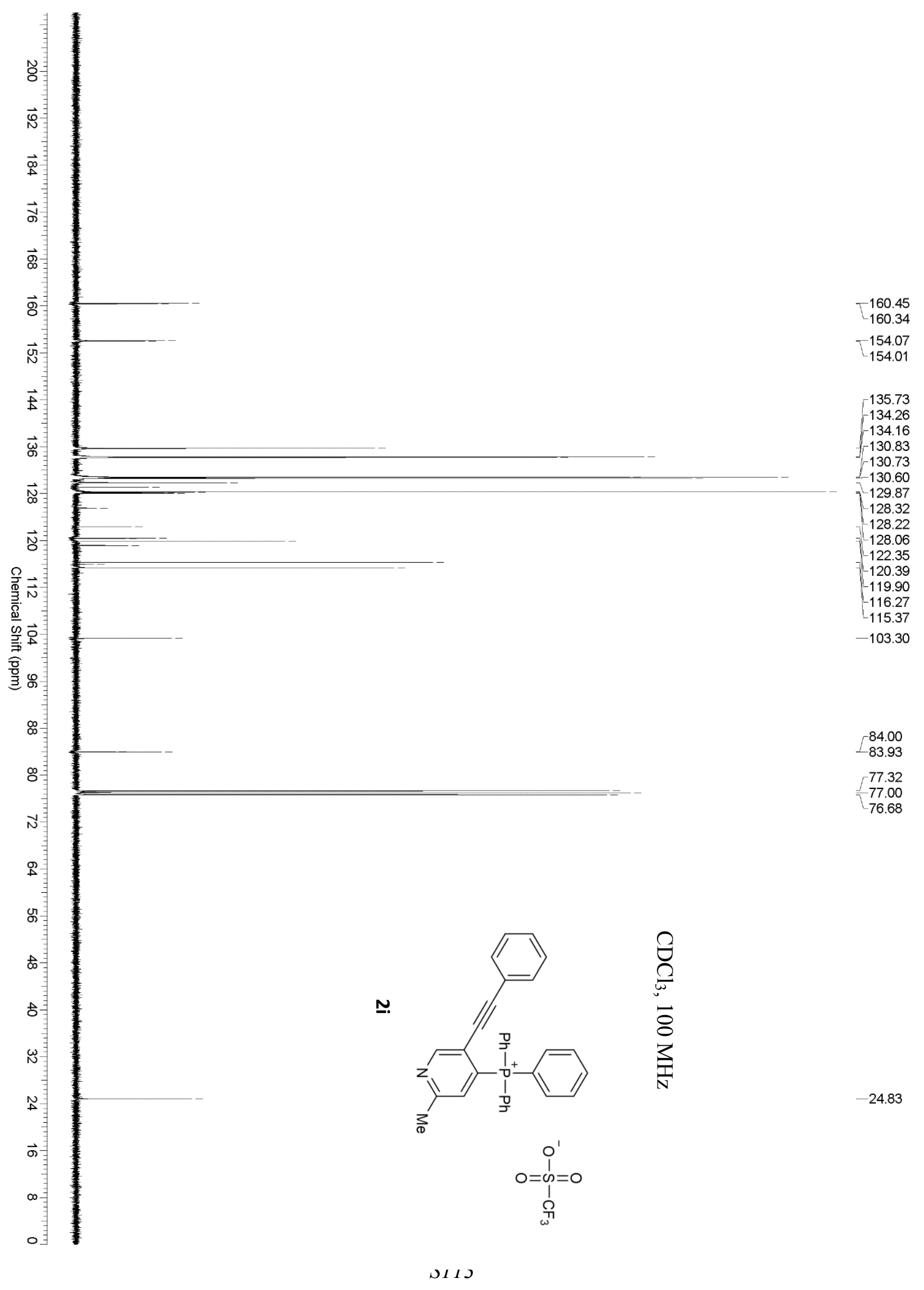




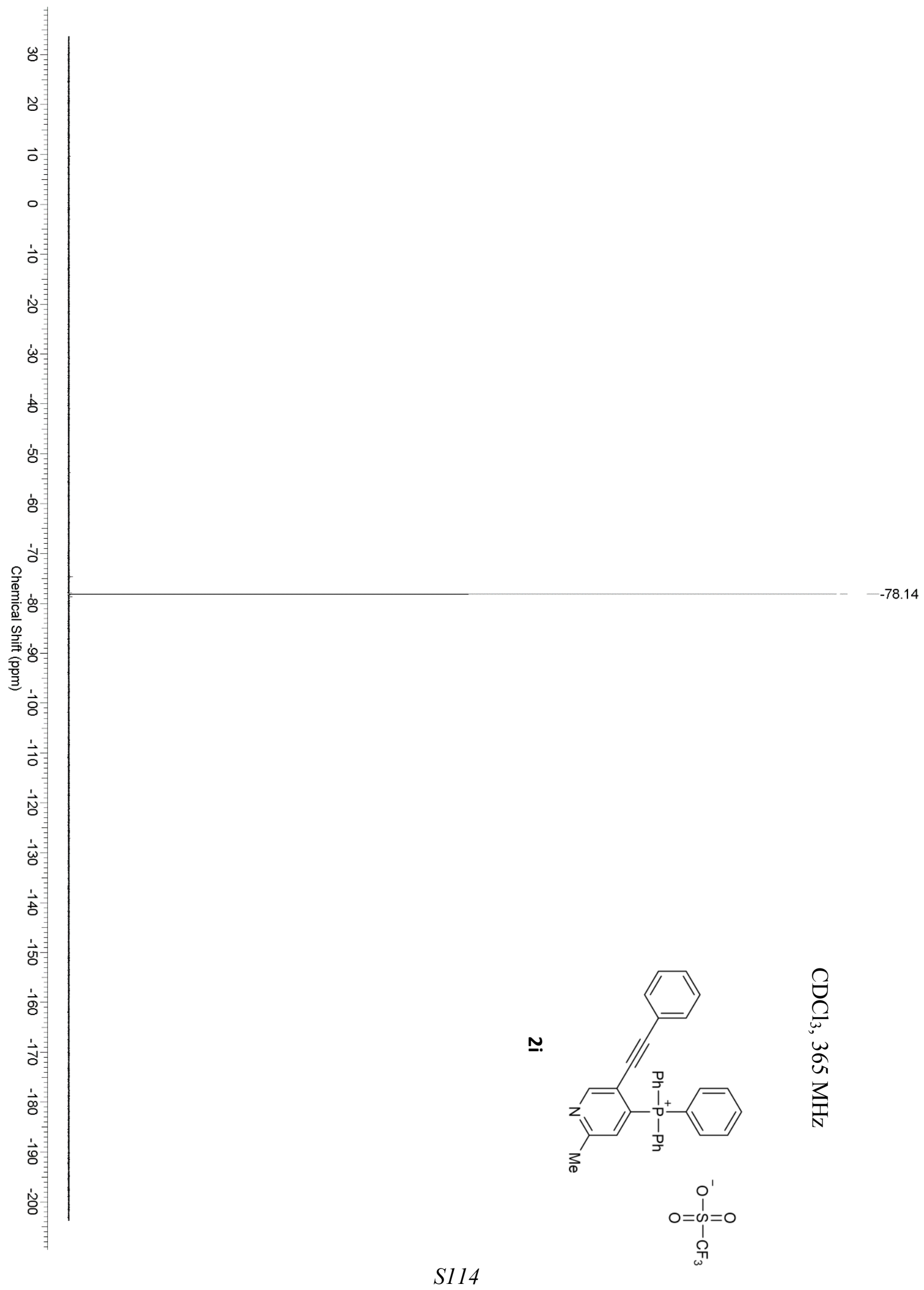




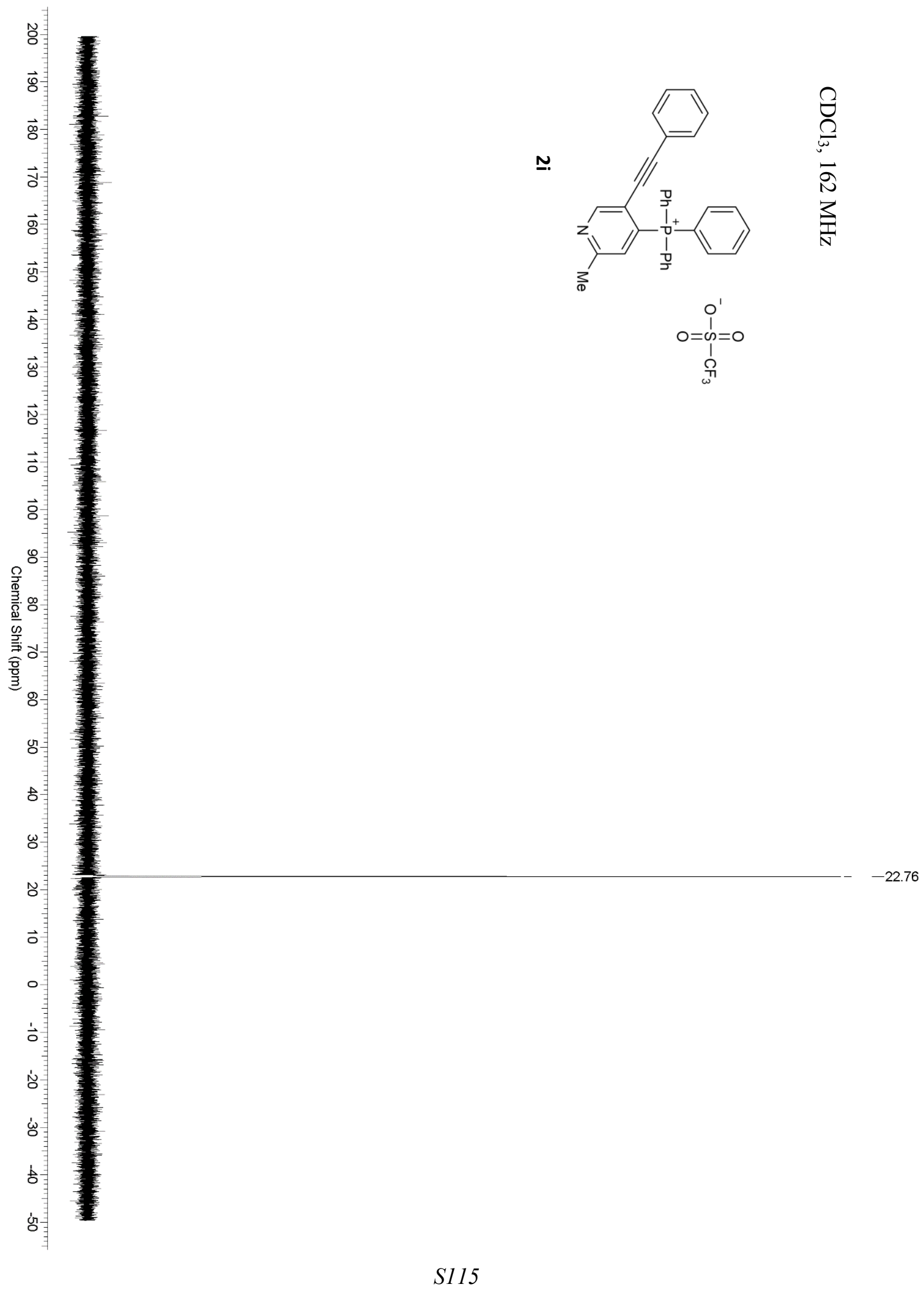




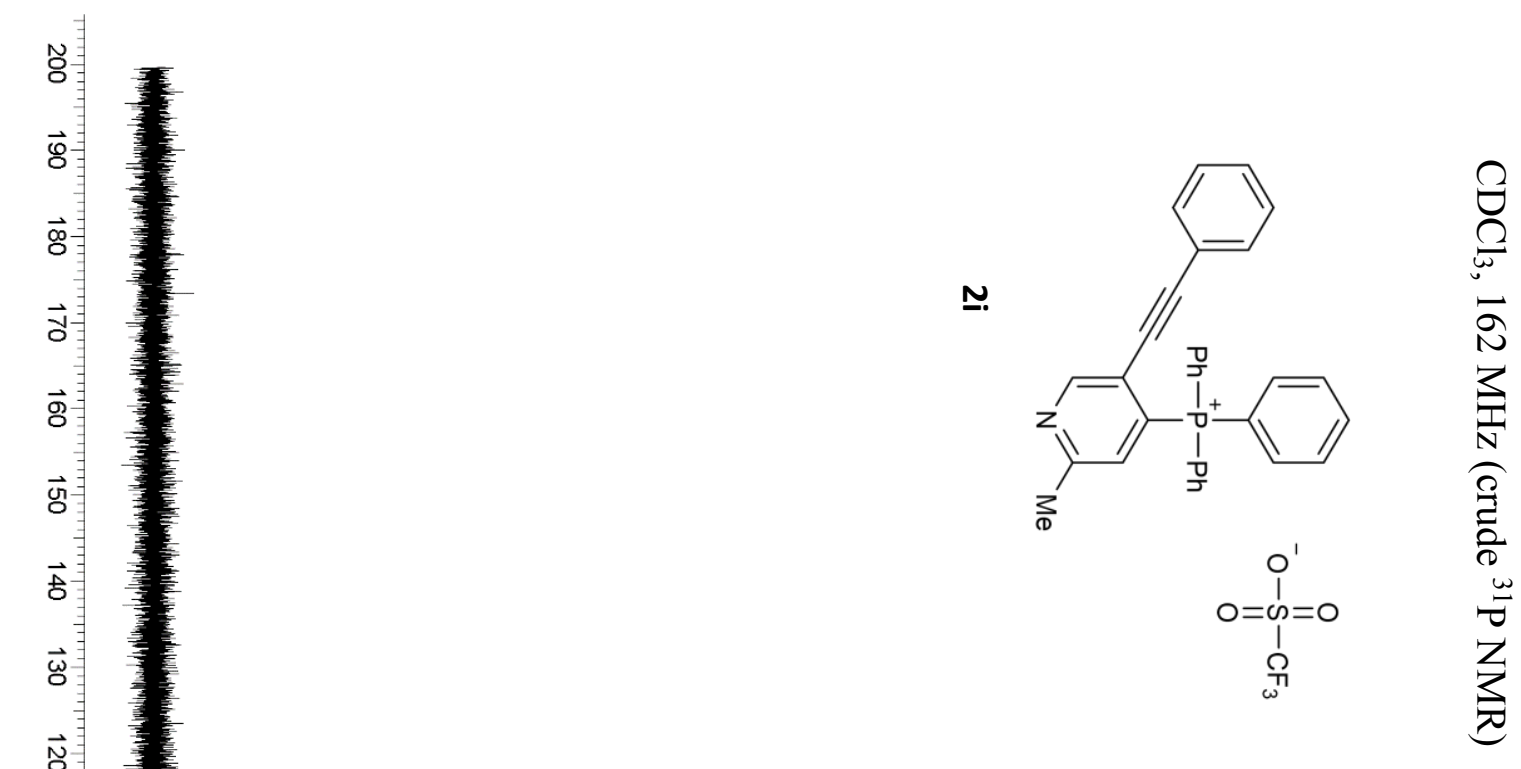

호

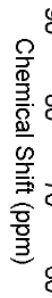

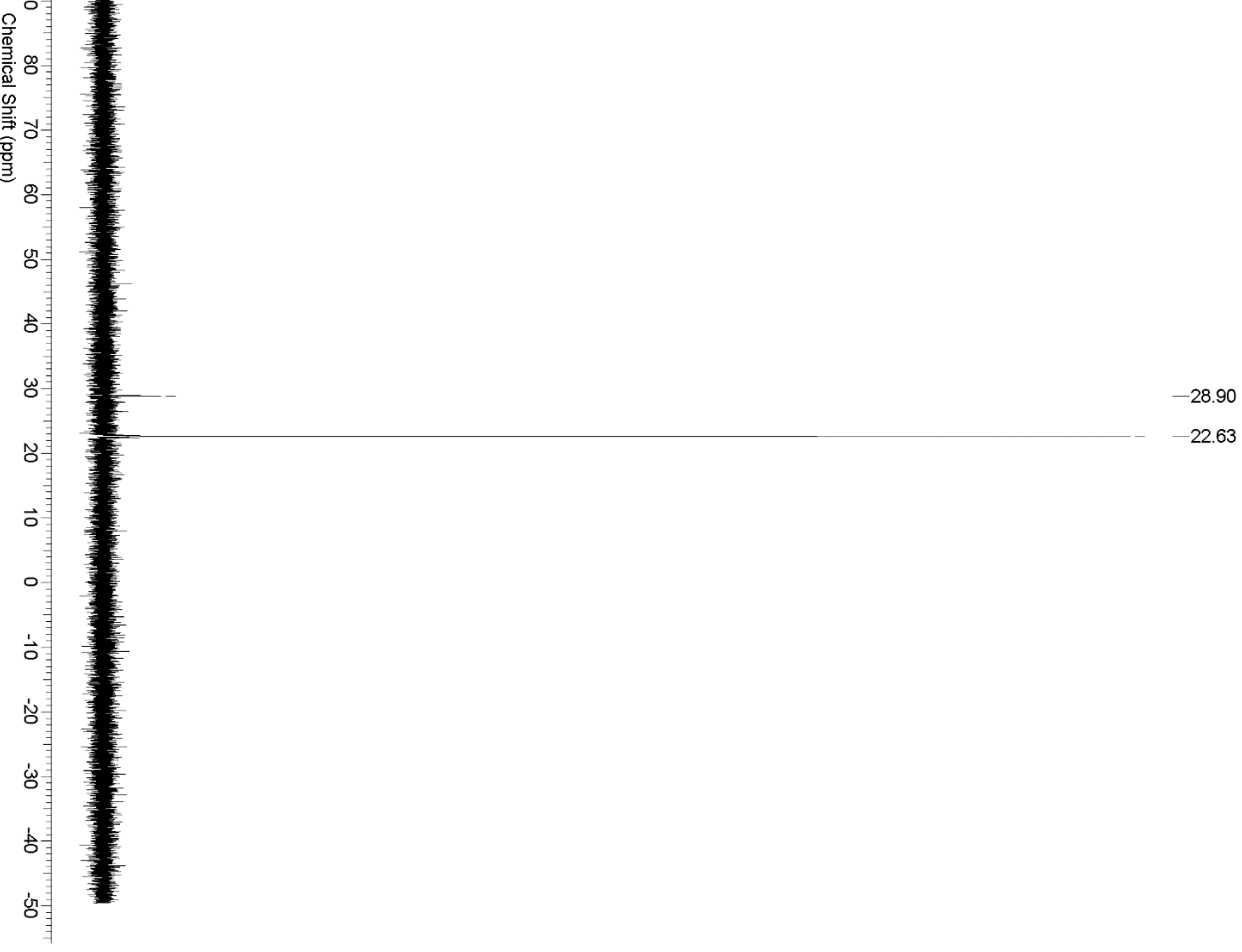




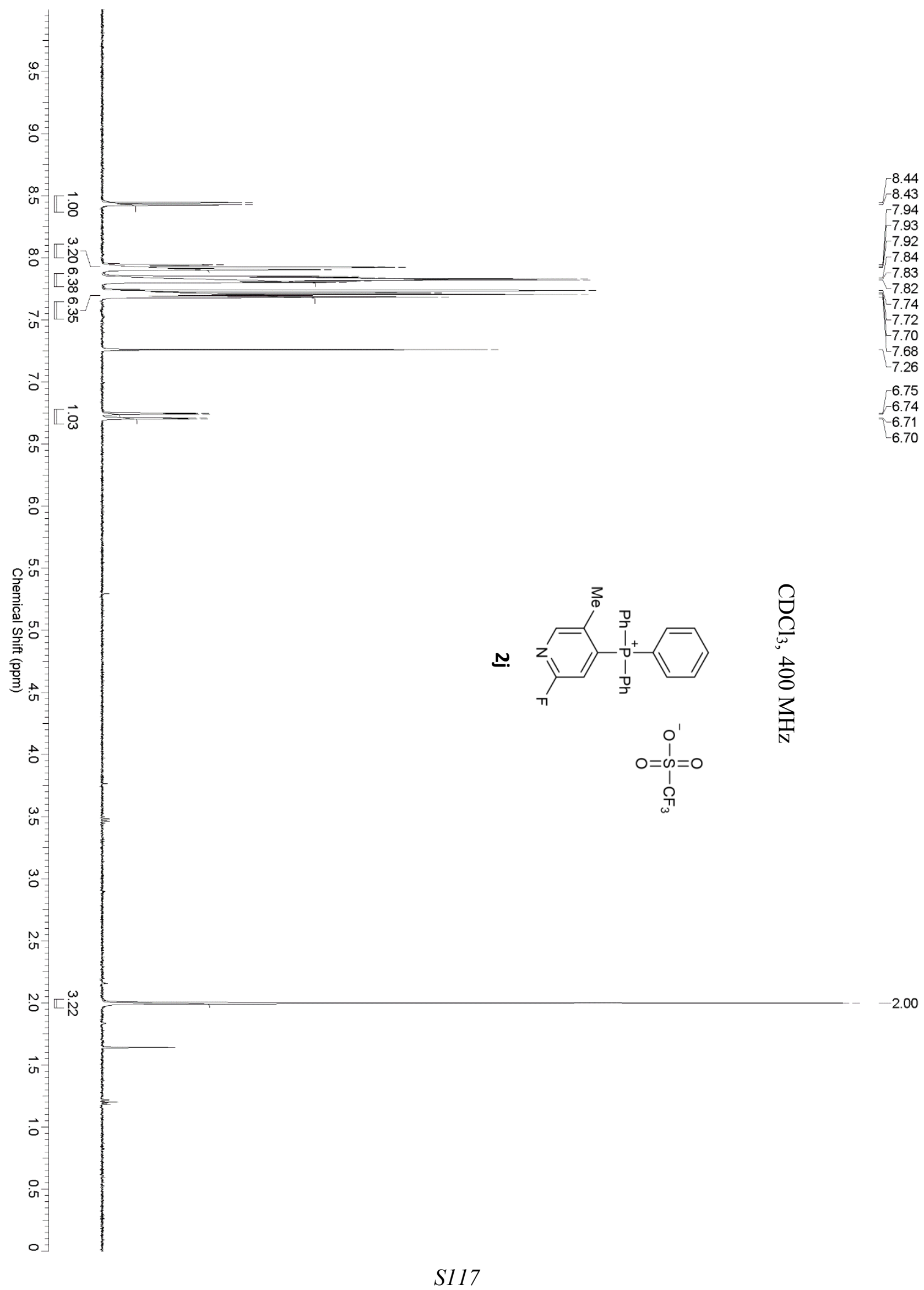




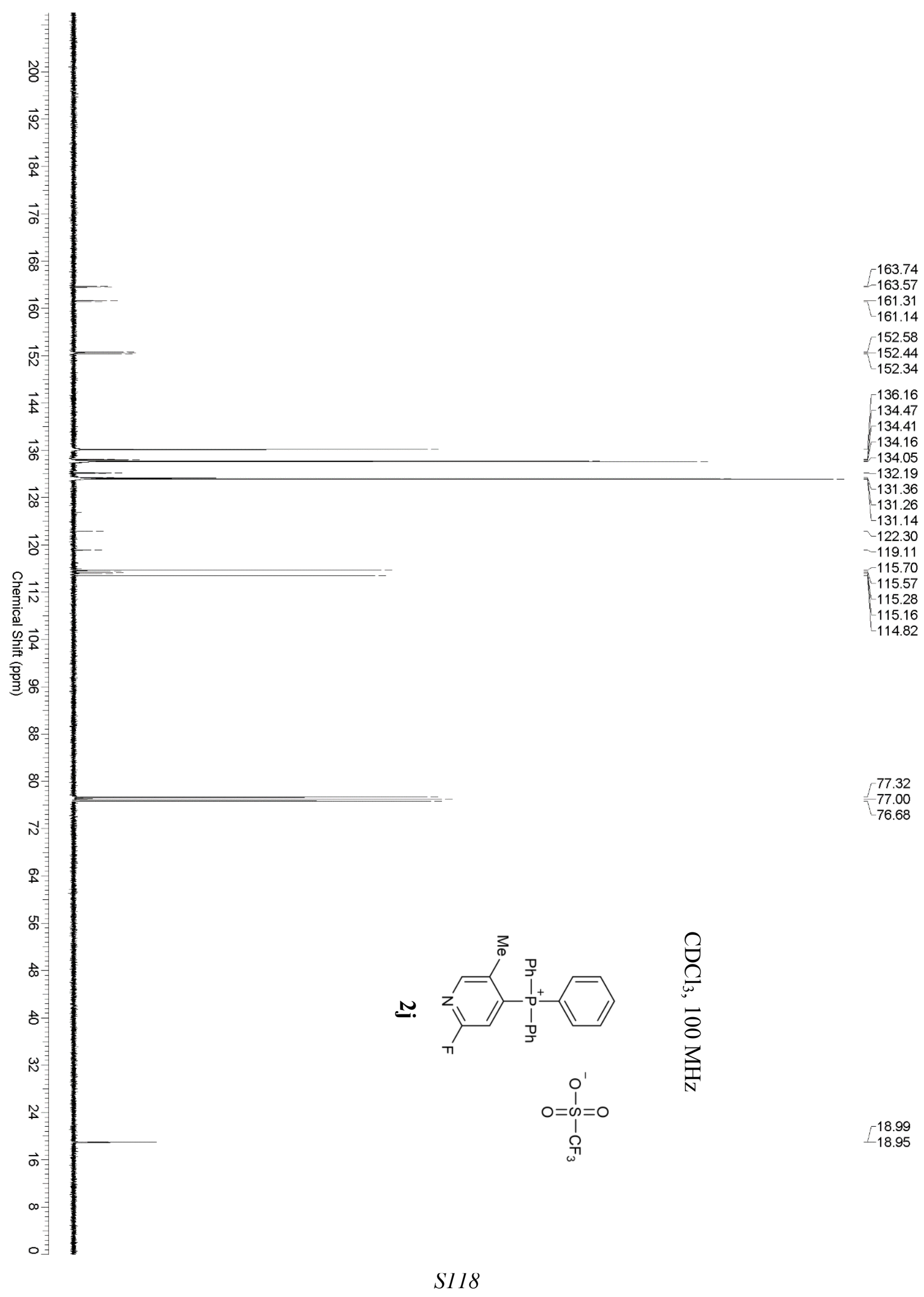




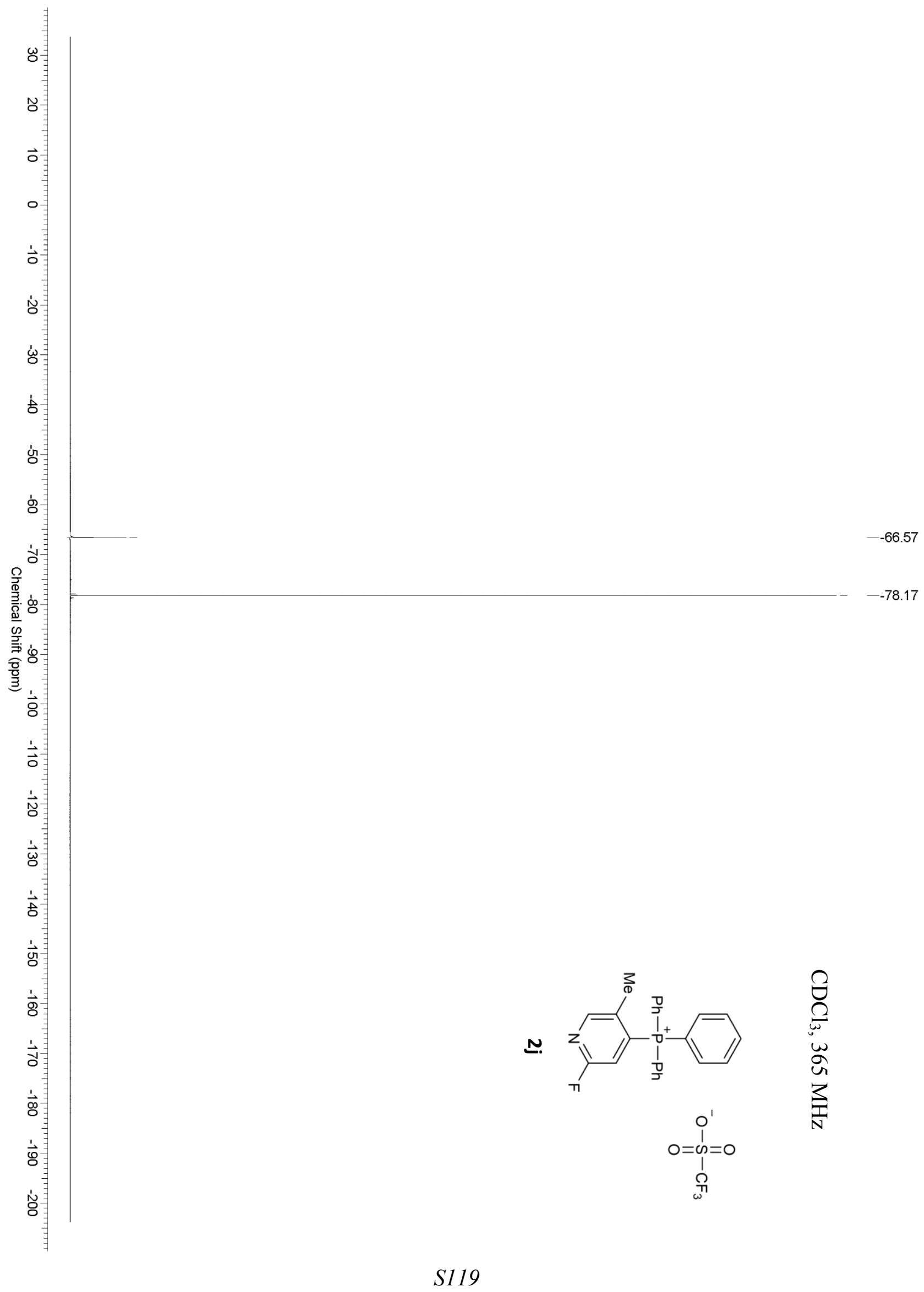




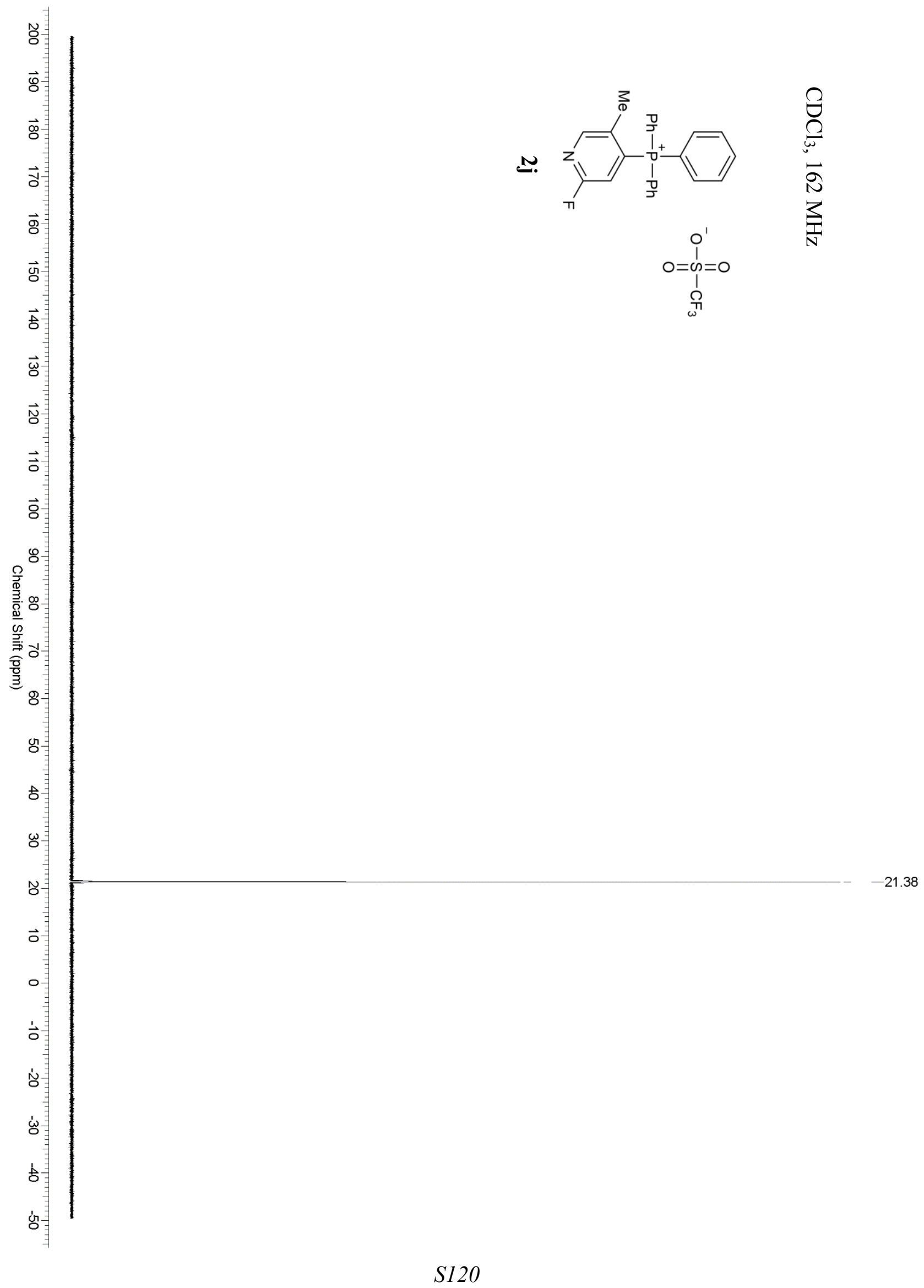




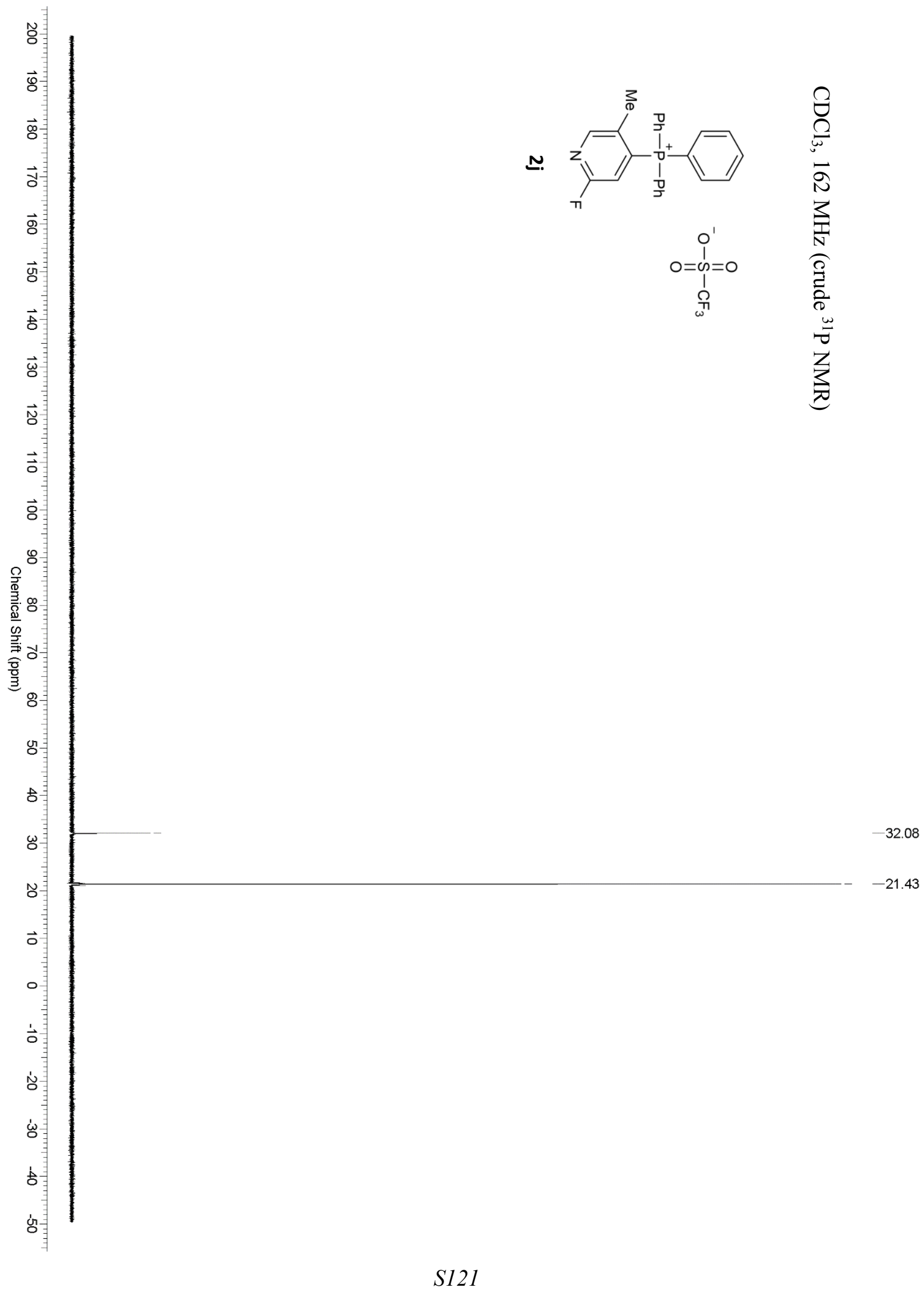




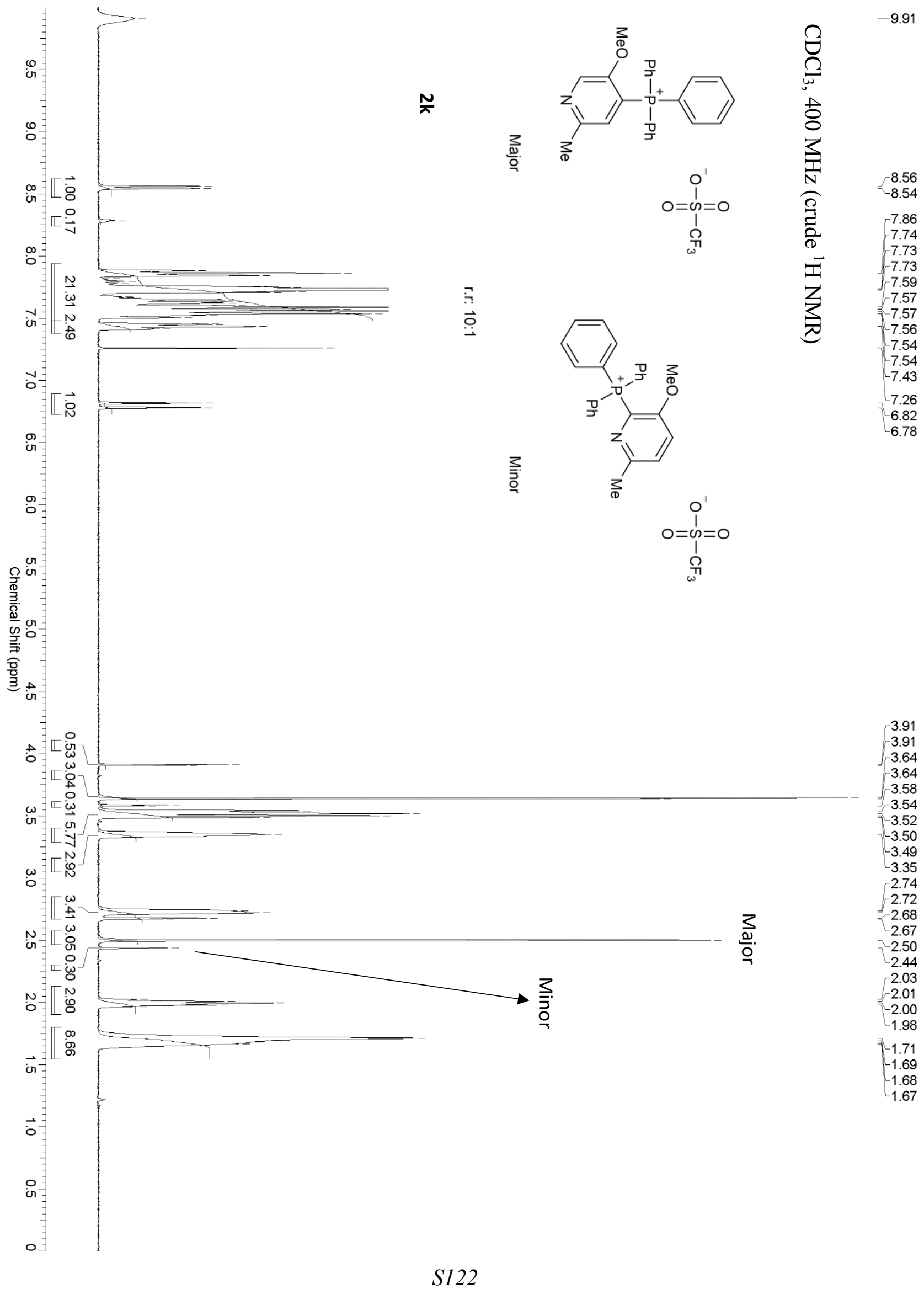




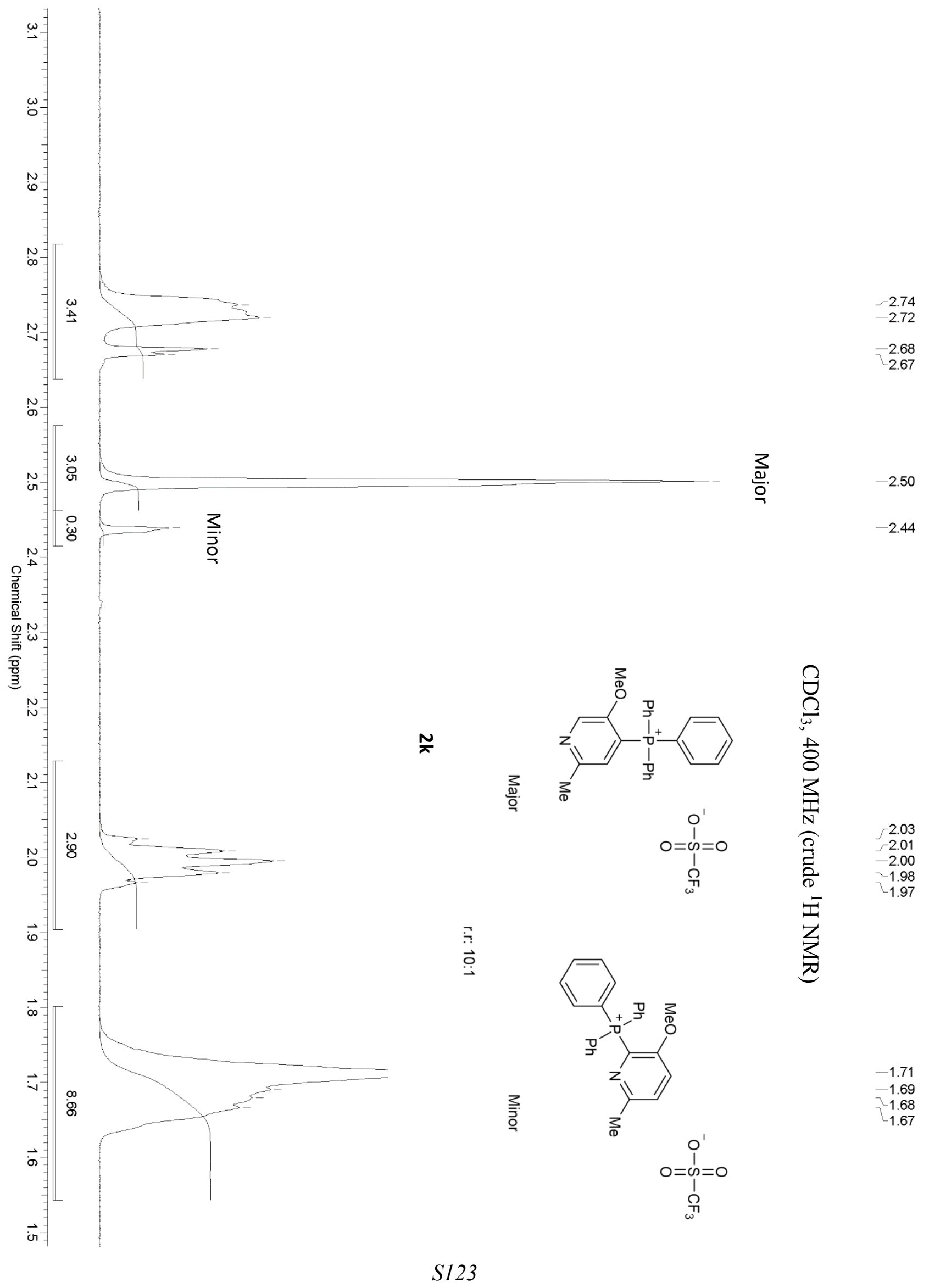




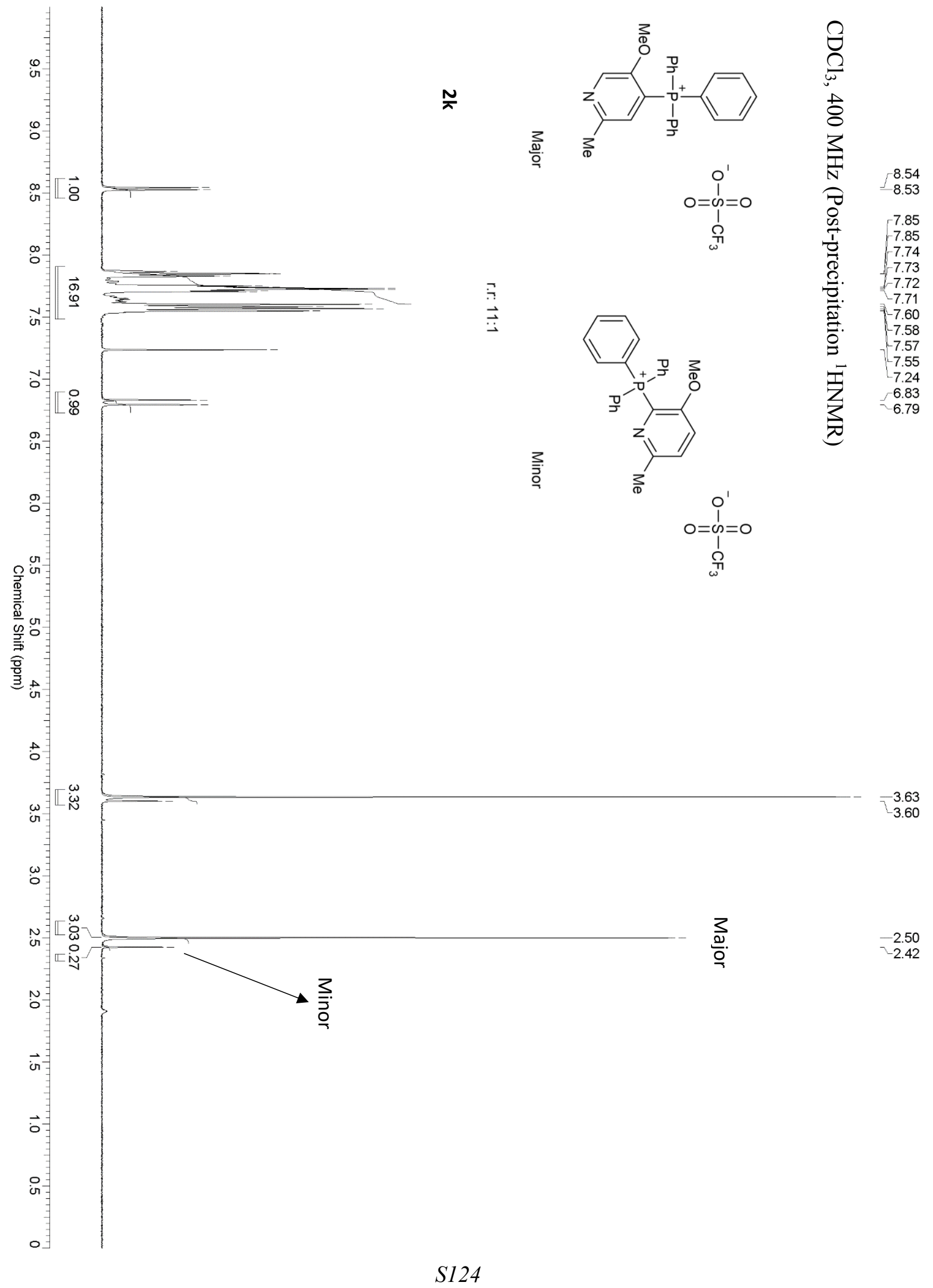




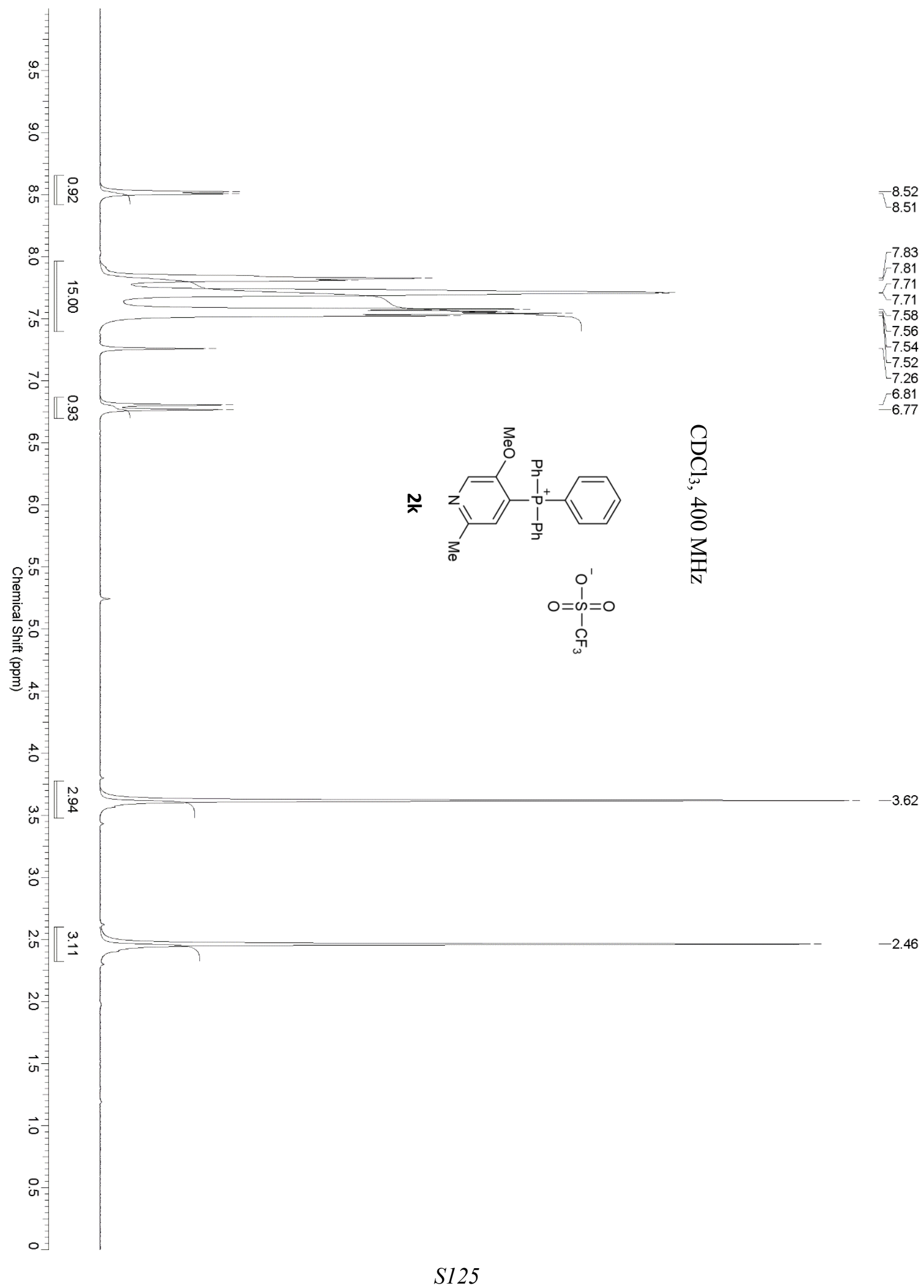




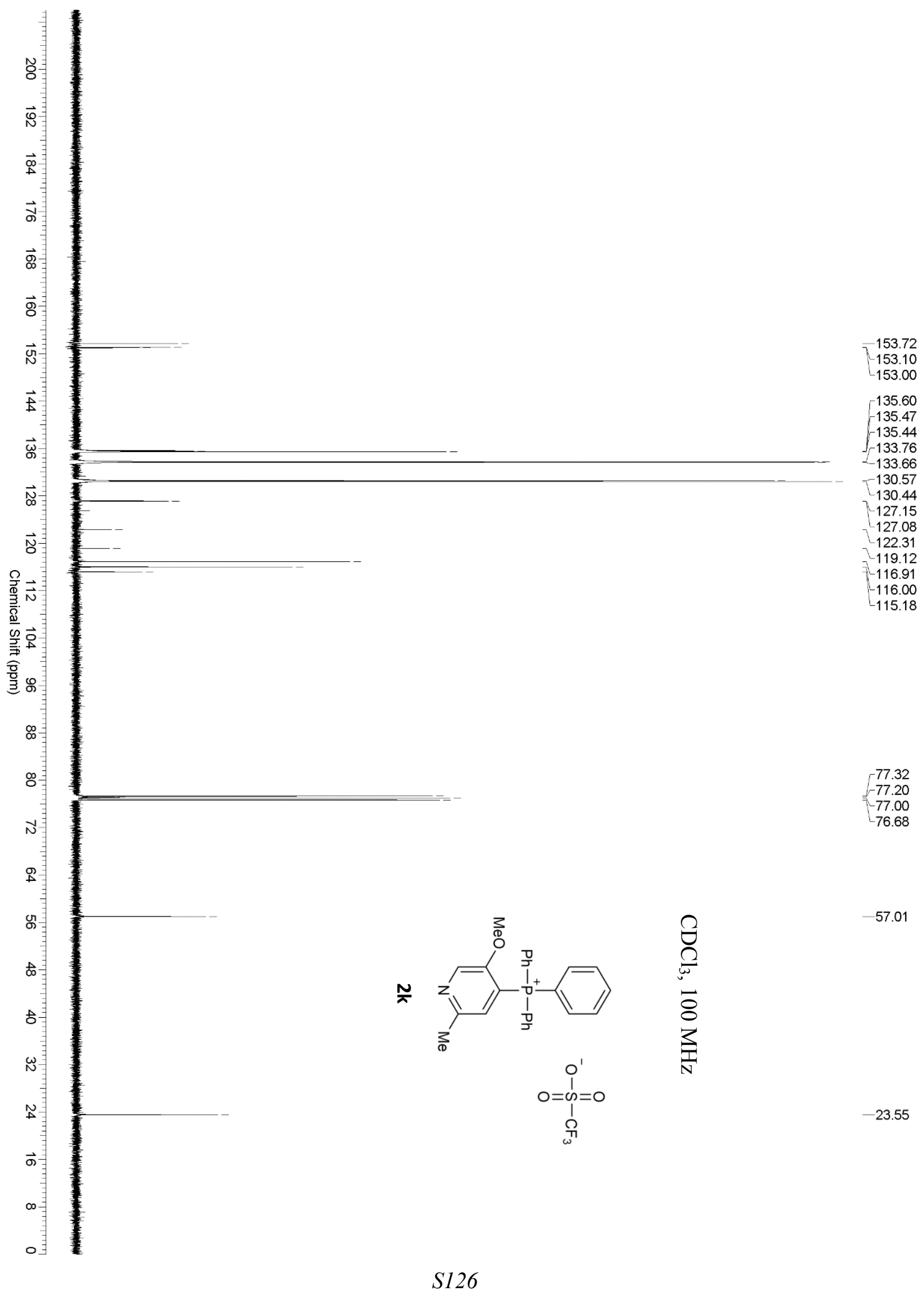




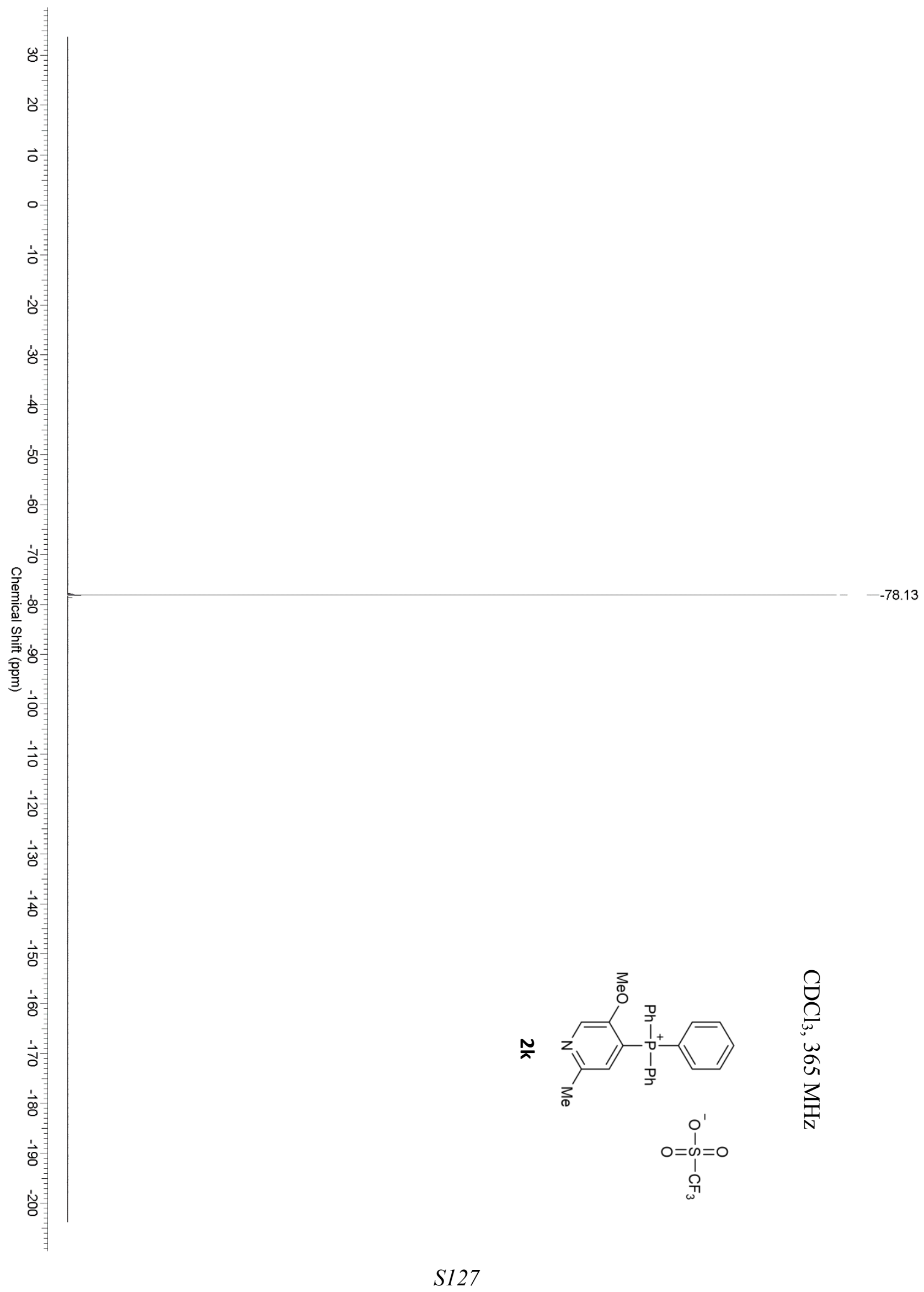




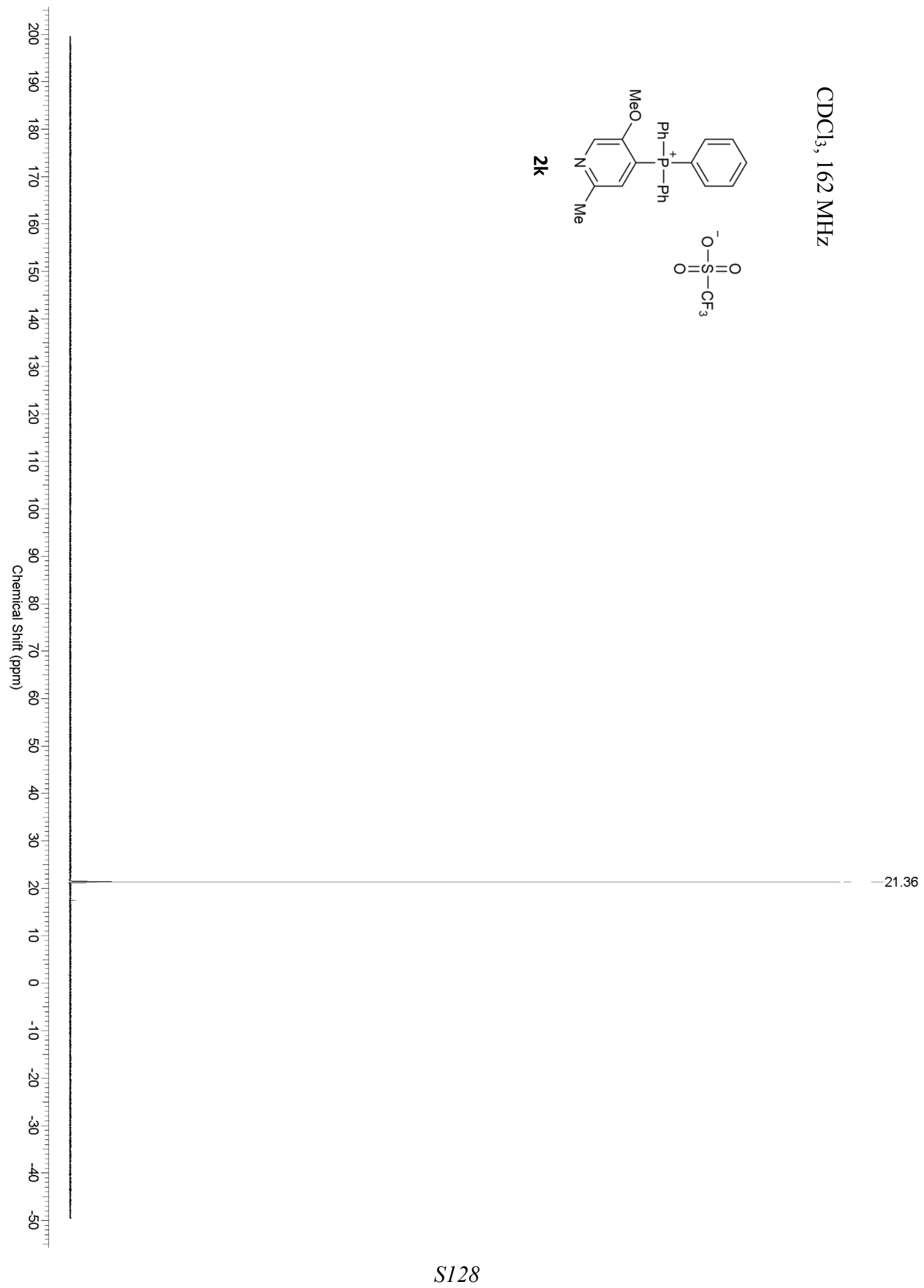




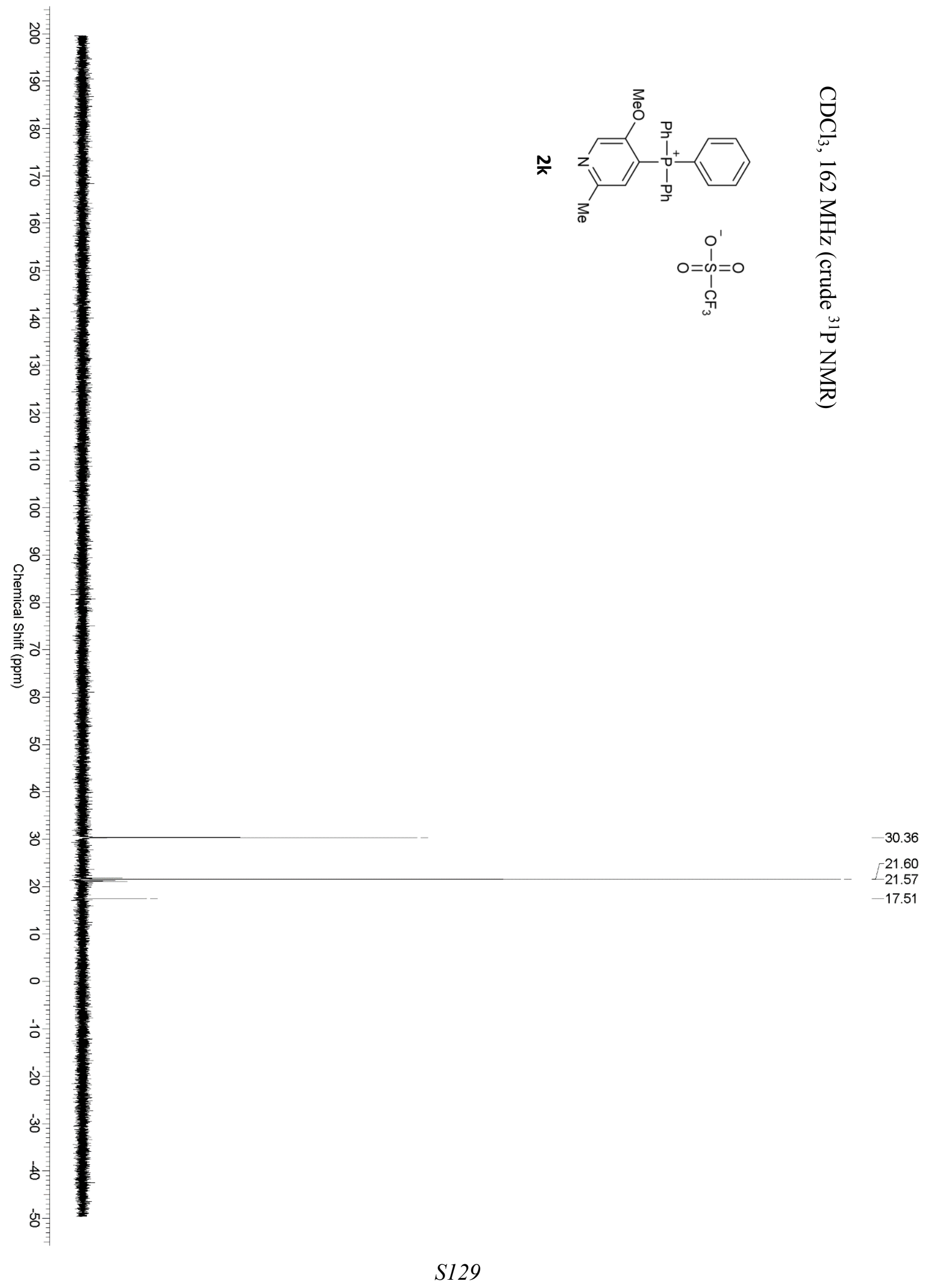




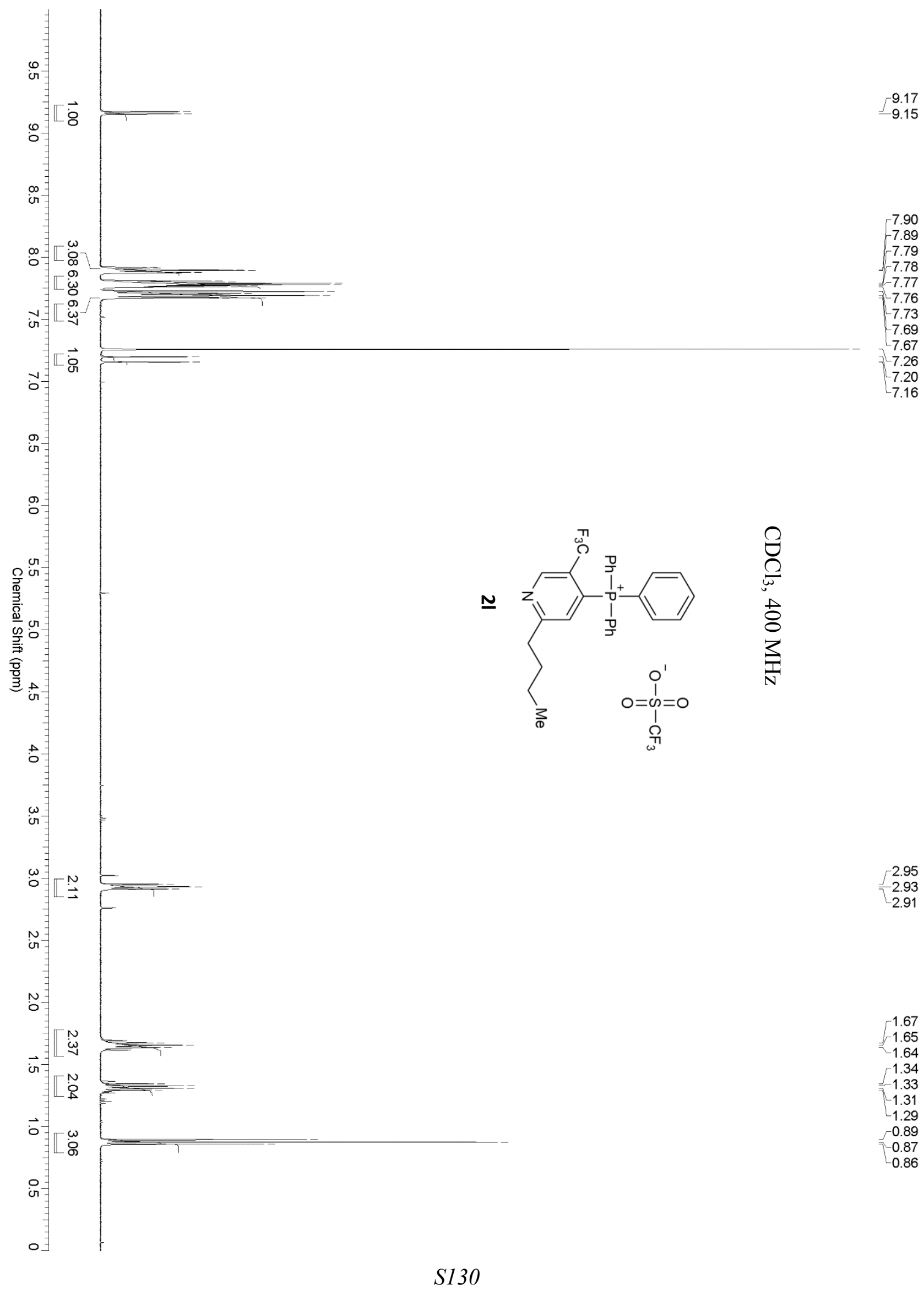




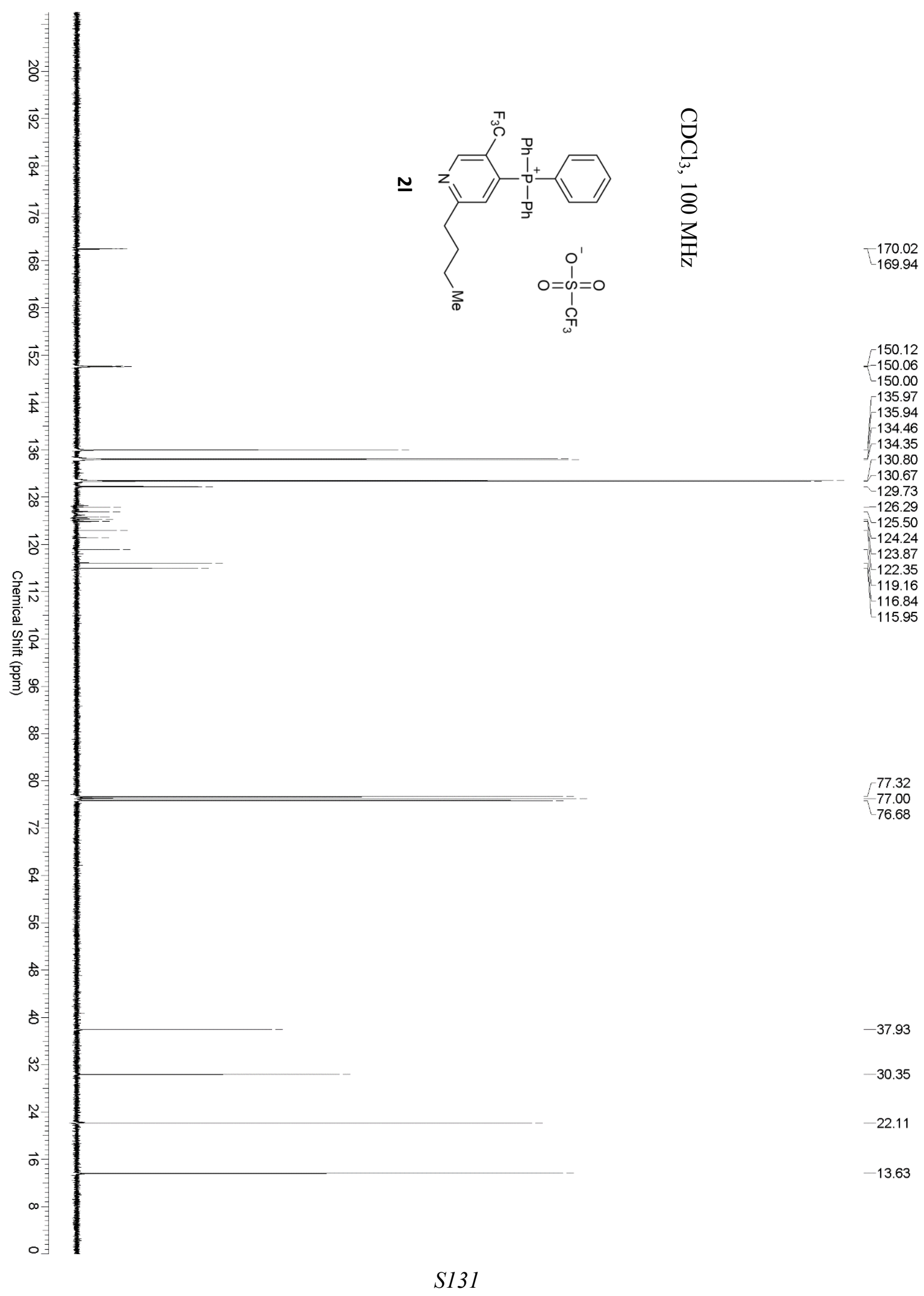




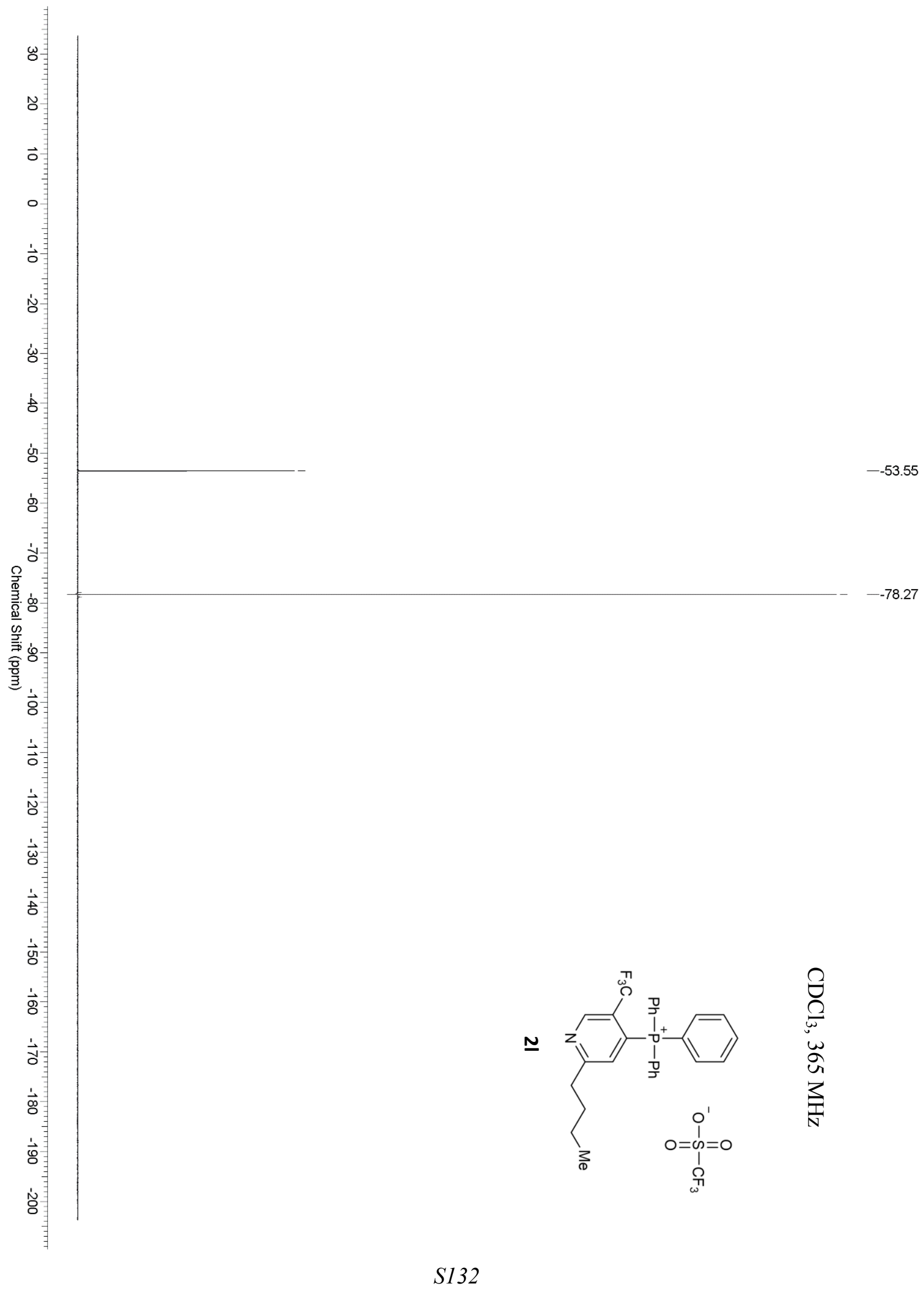




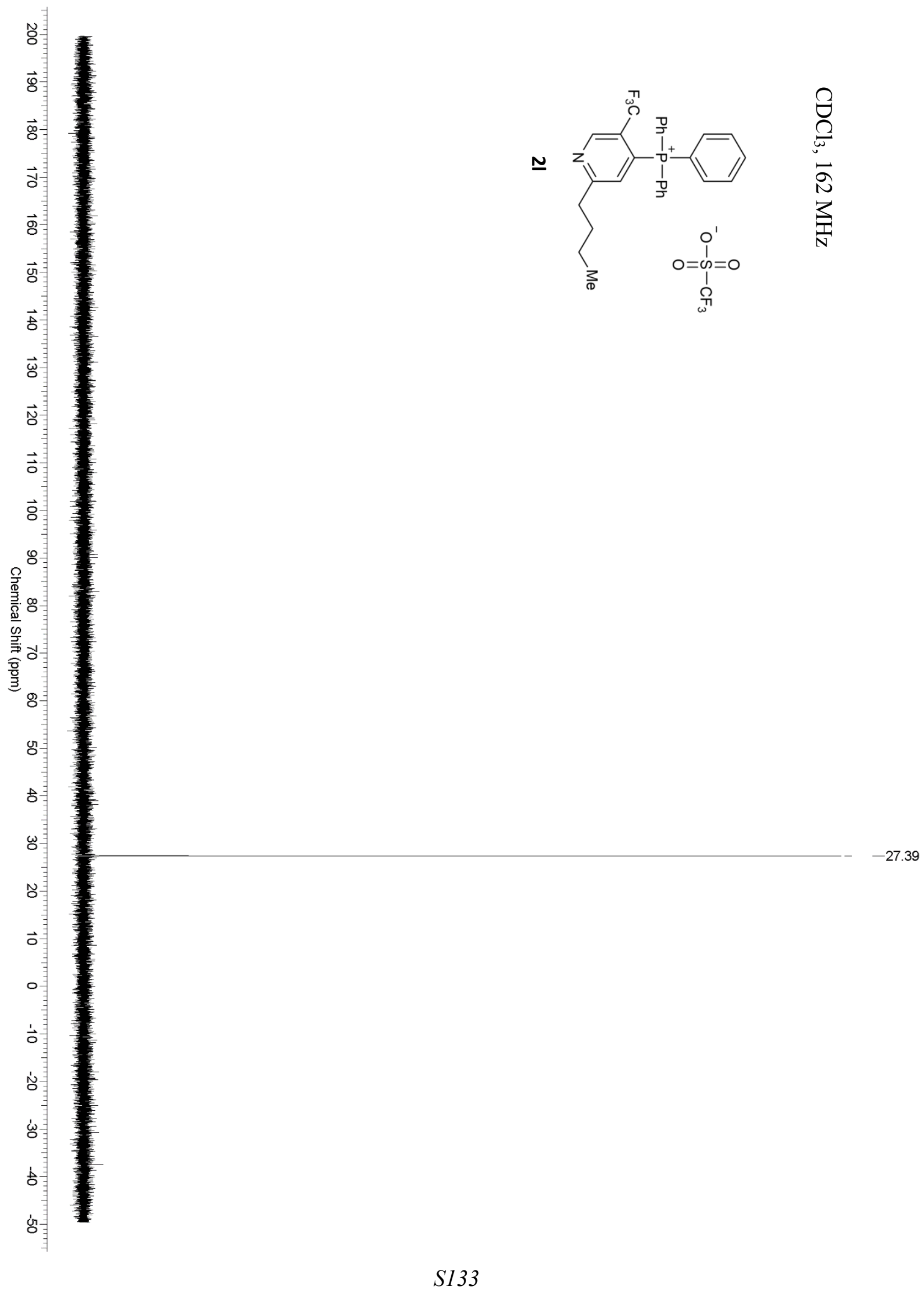




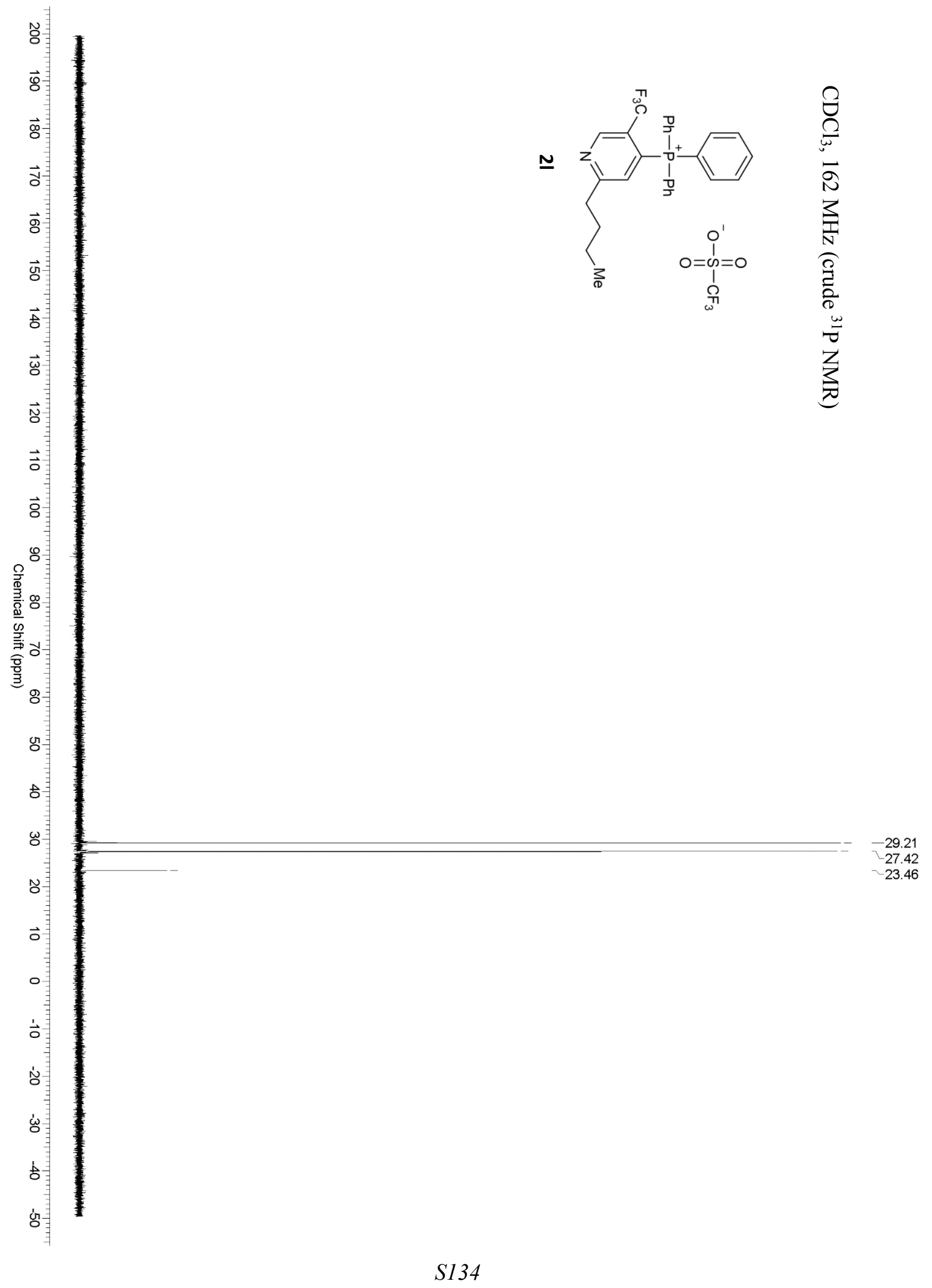




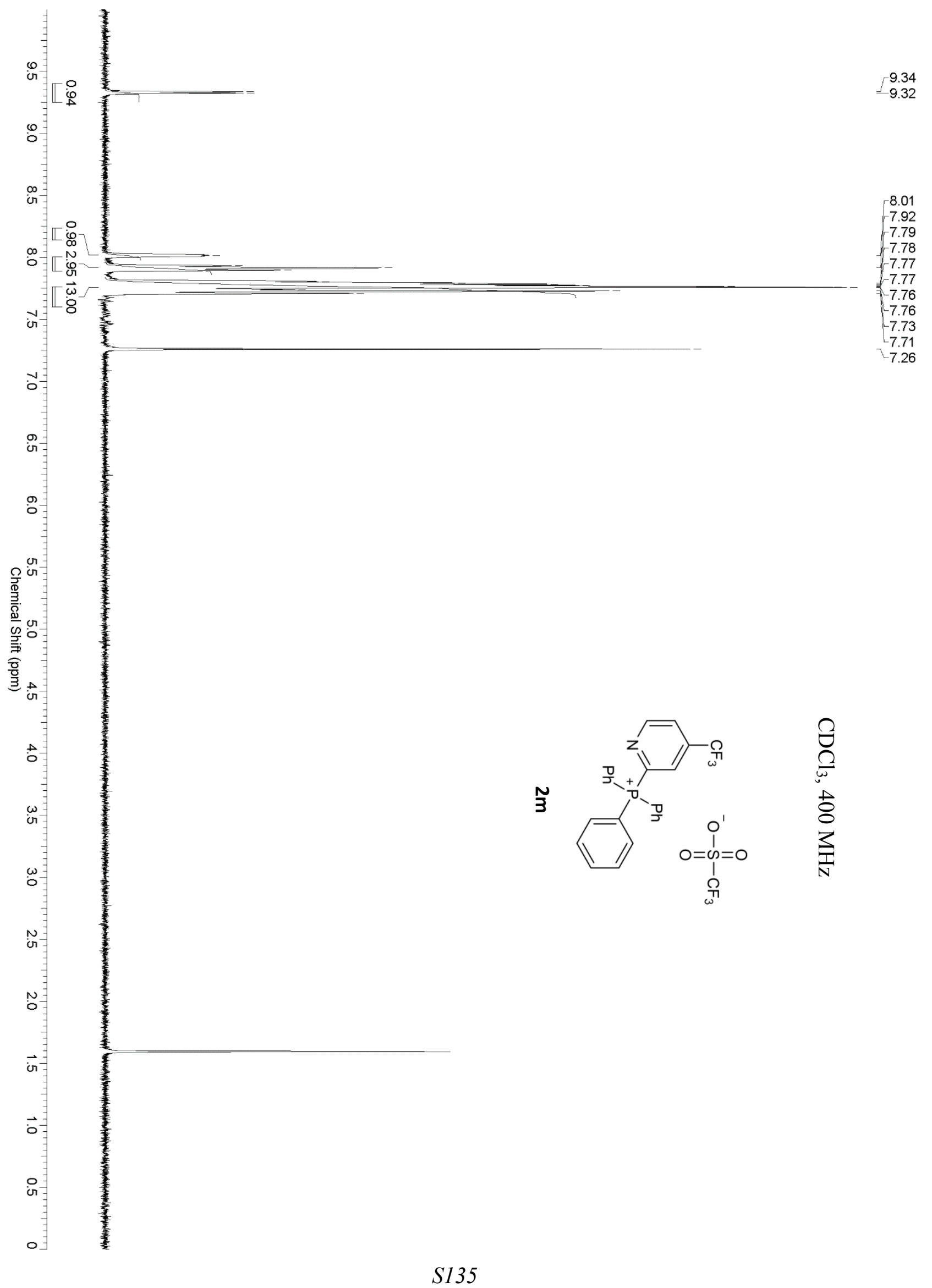




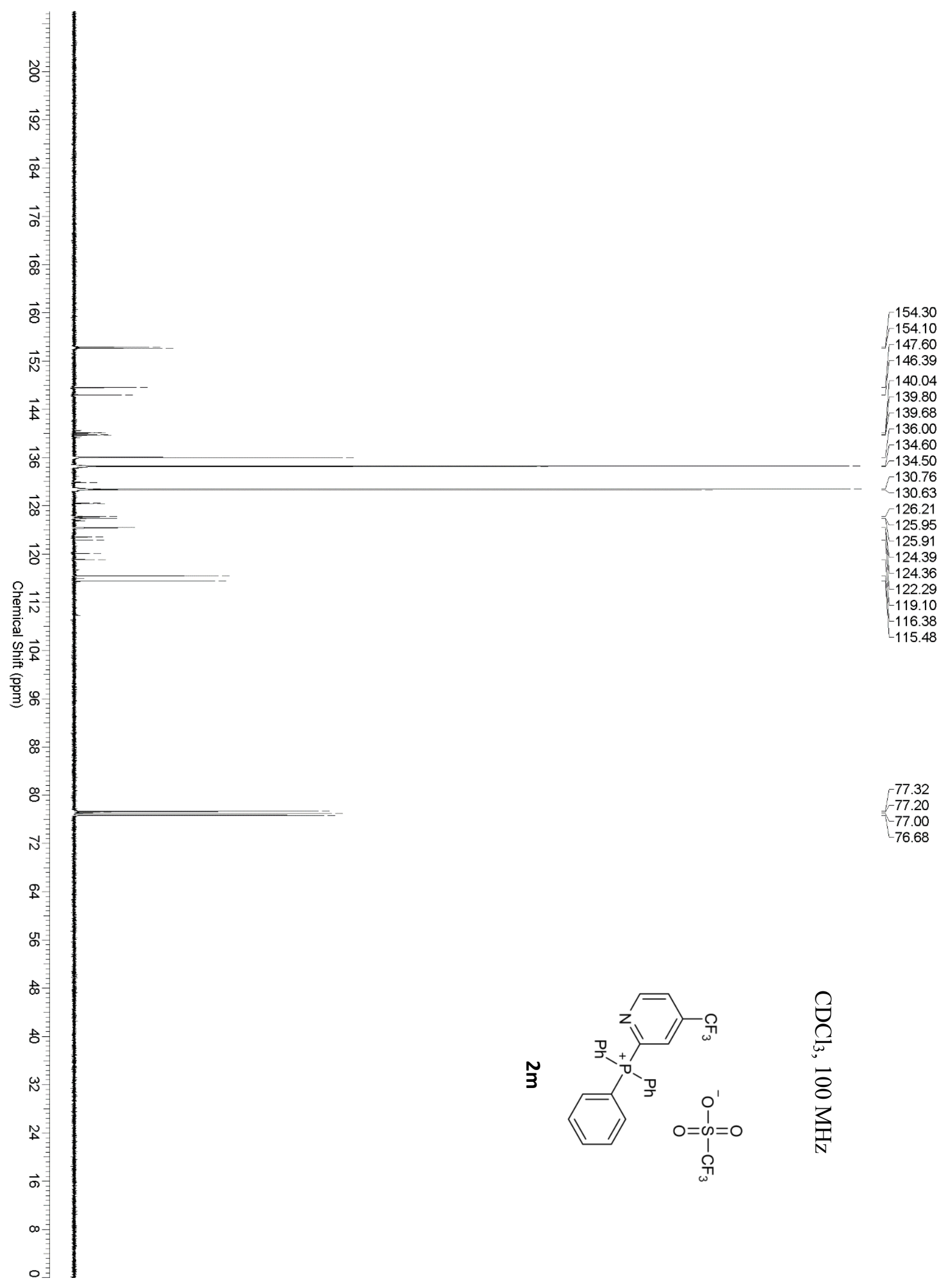




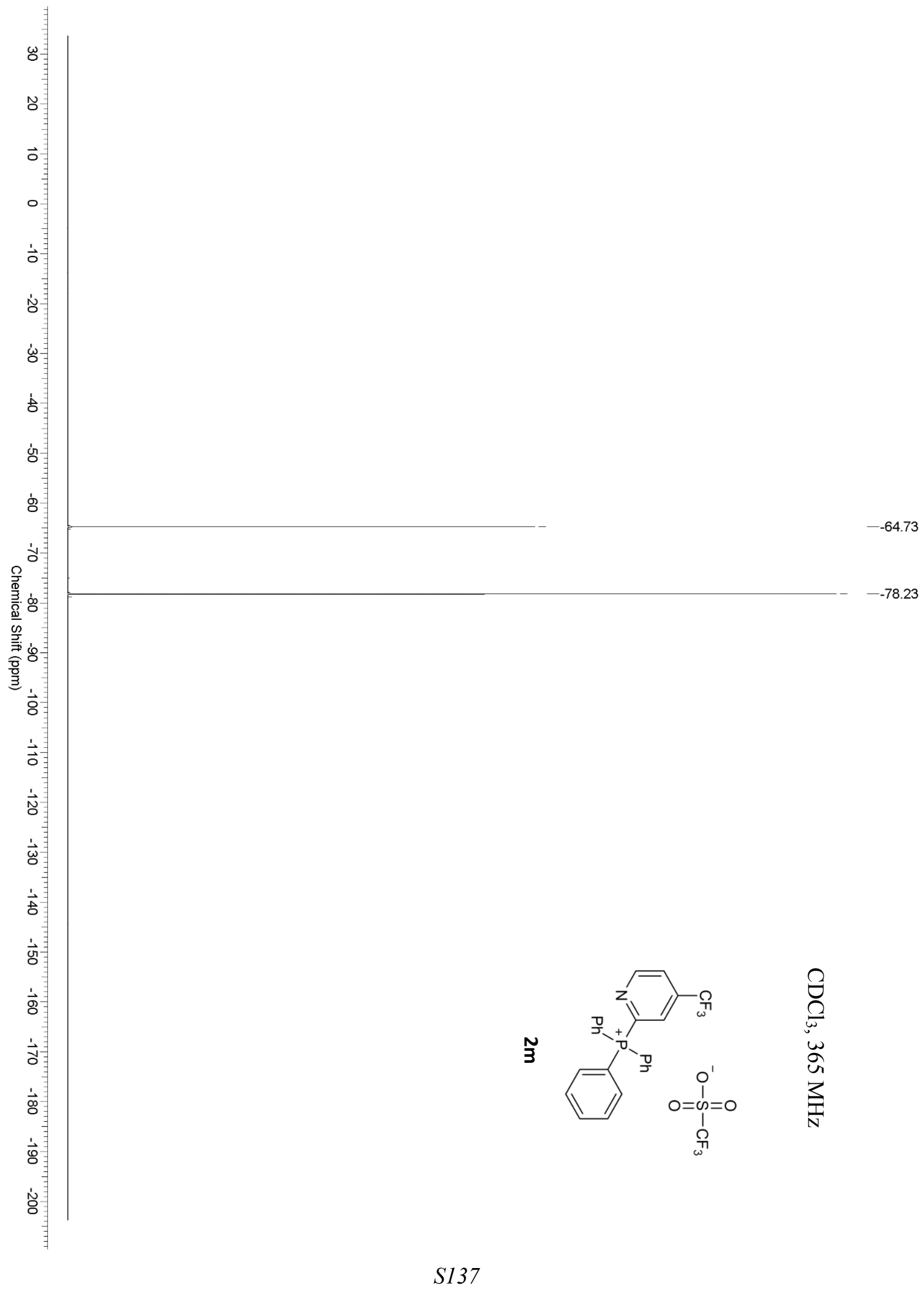




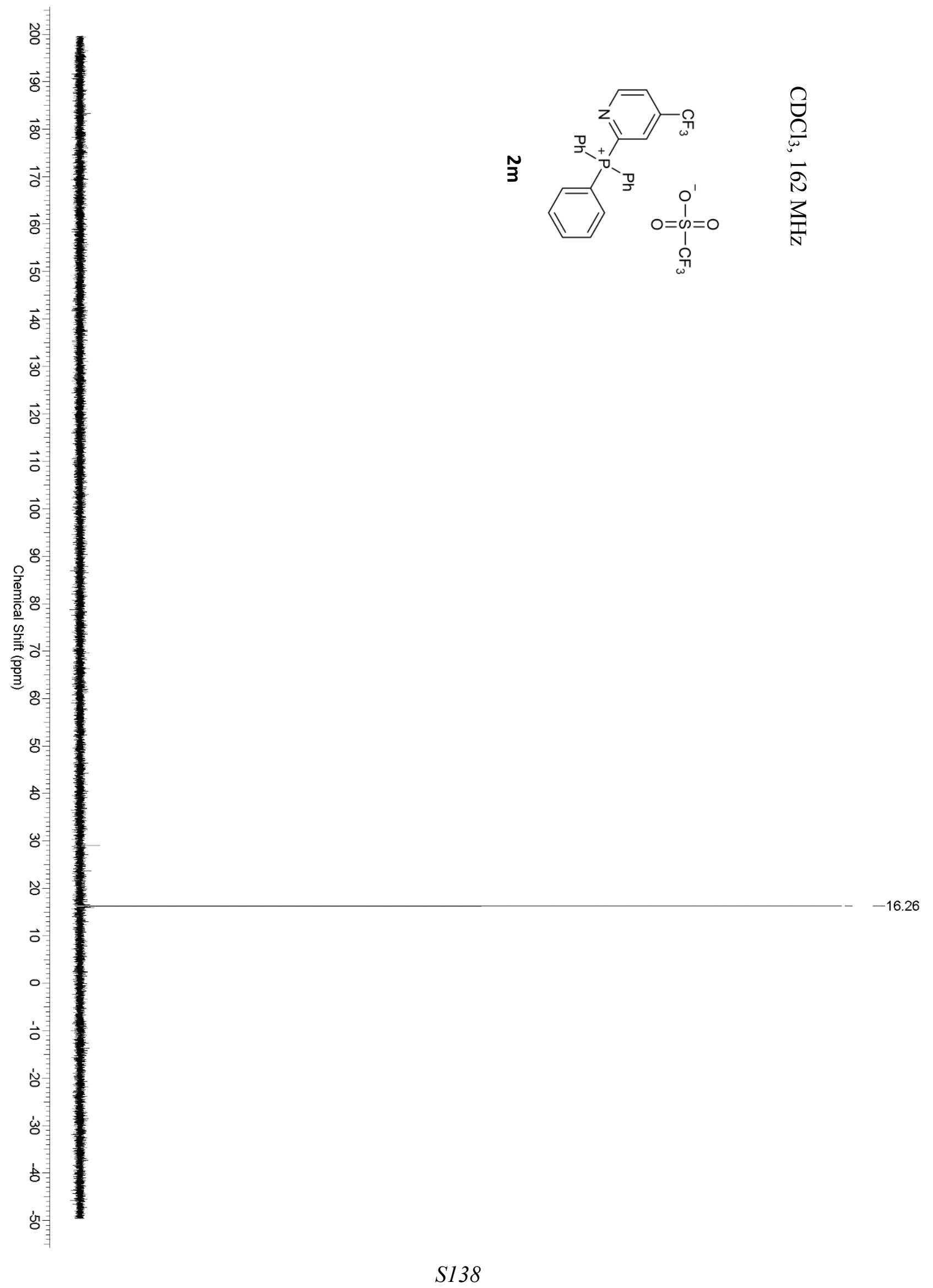




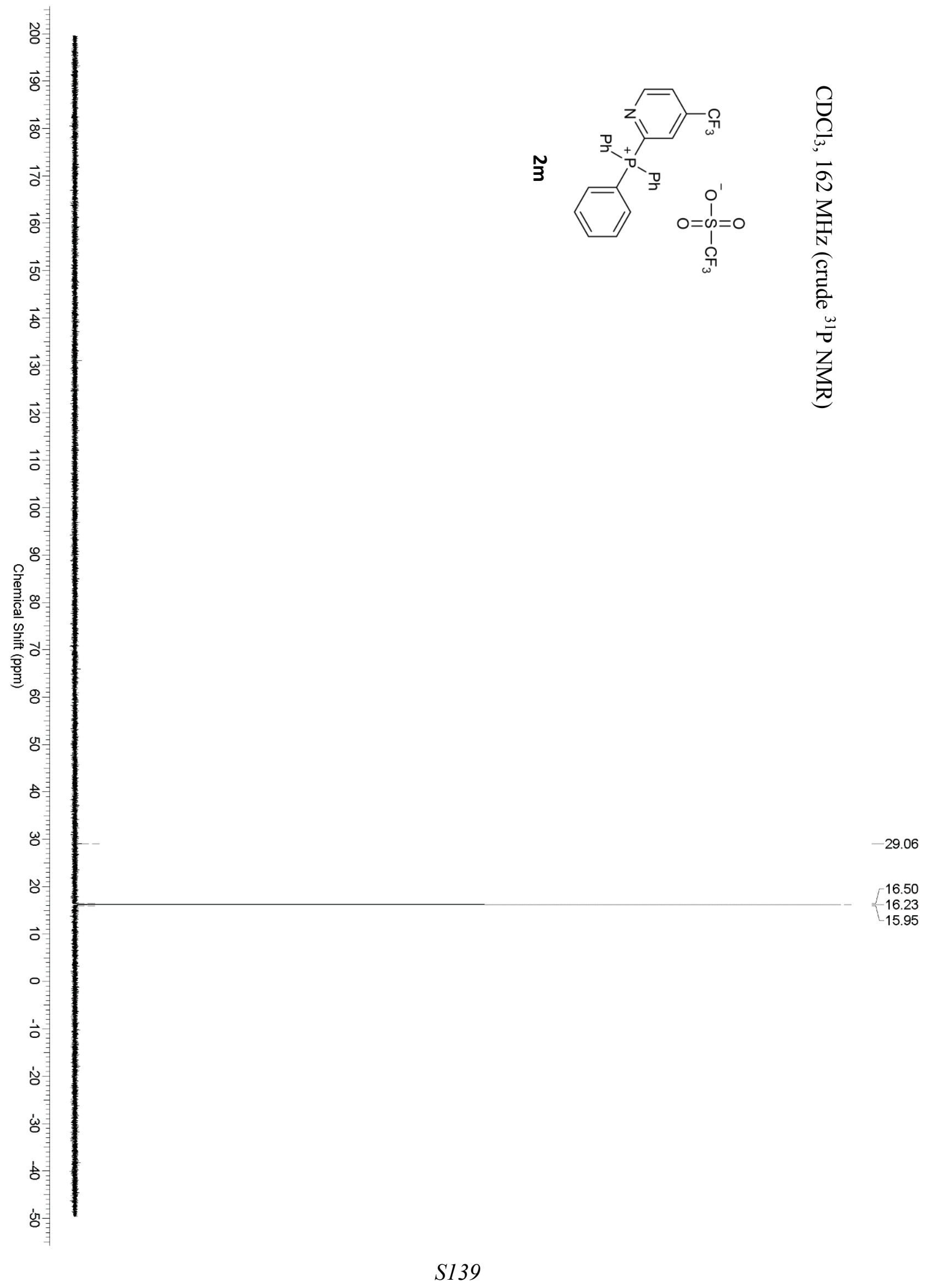




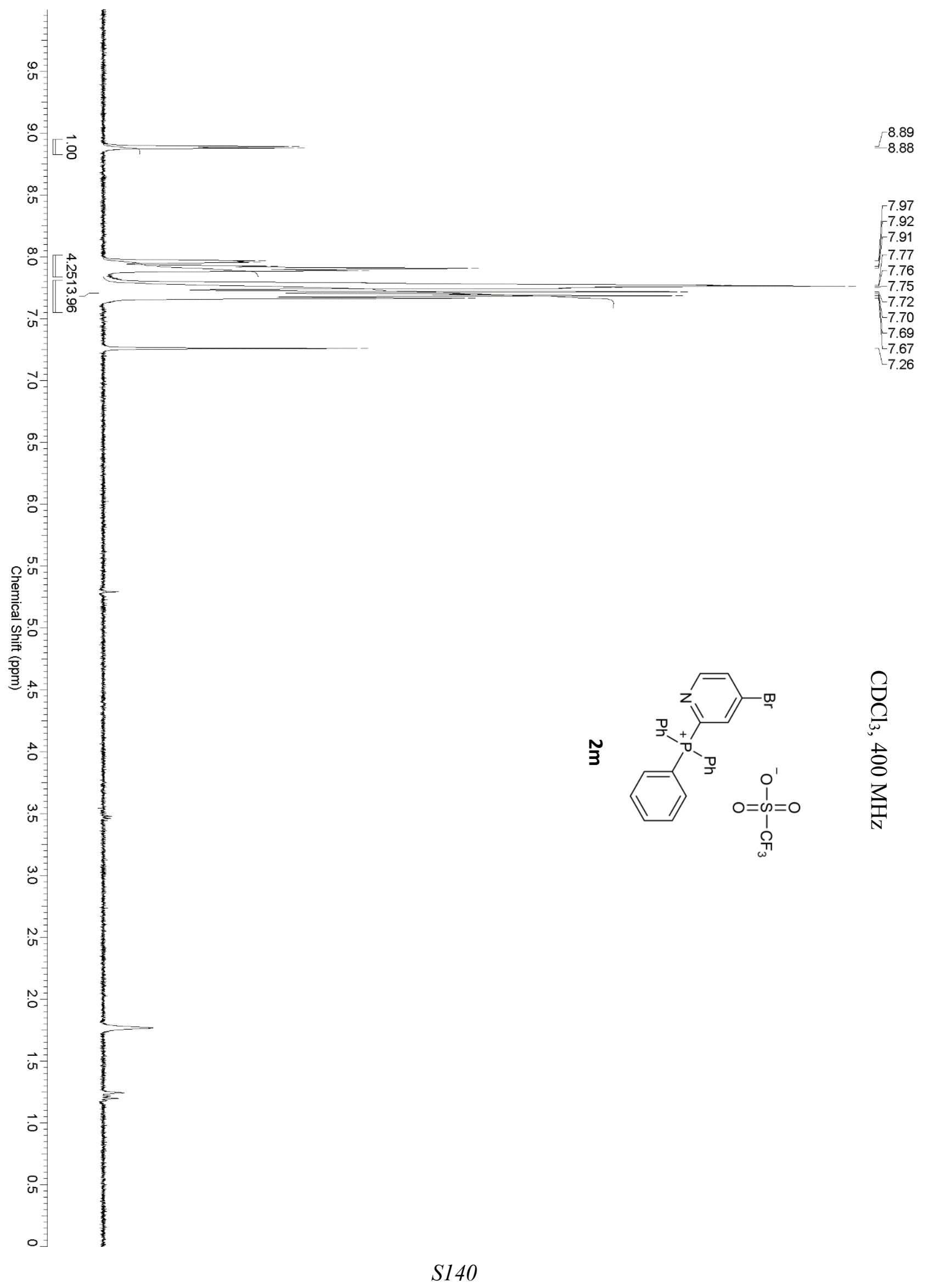




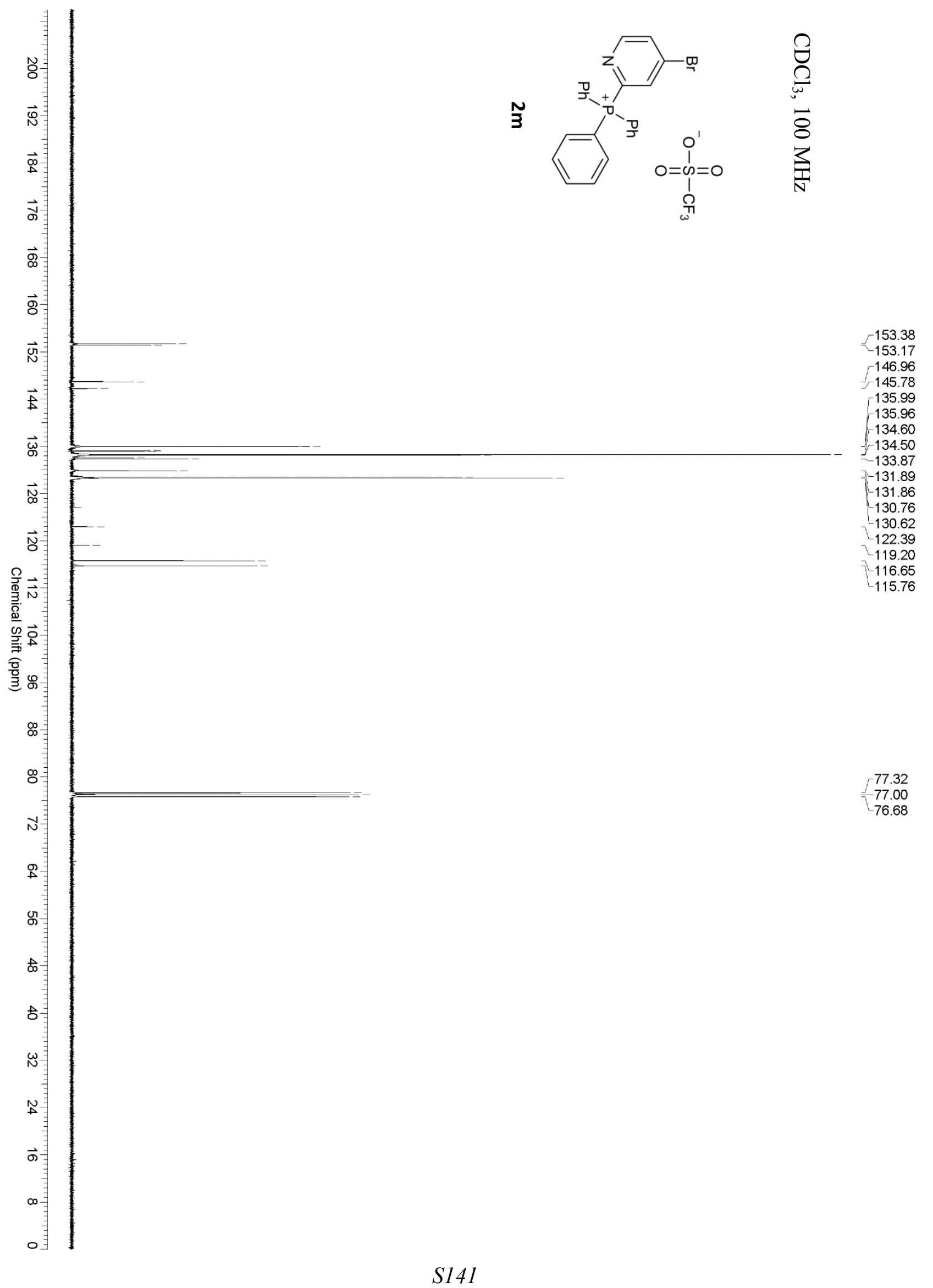




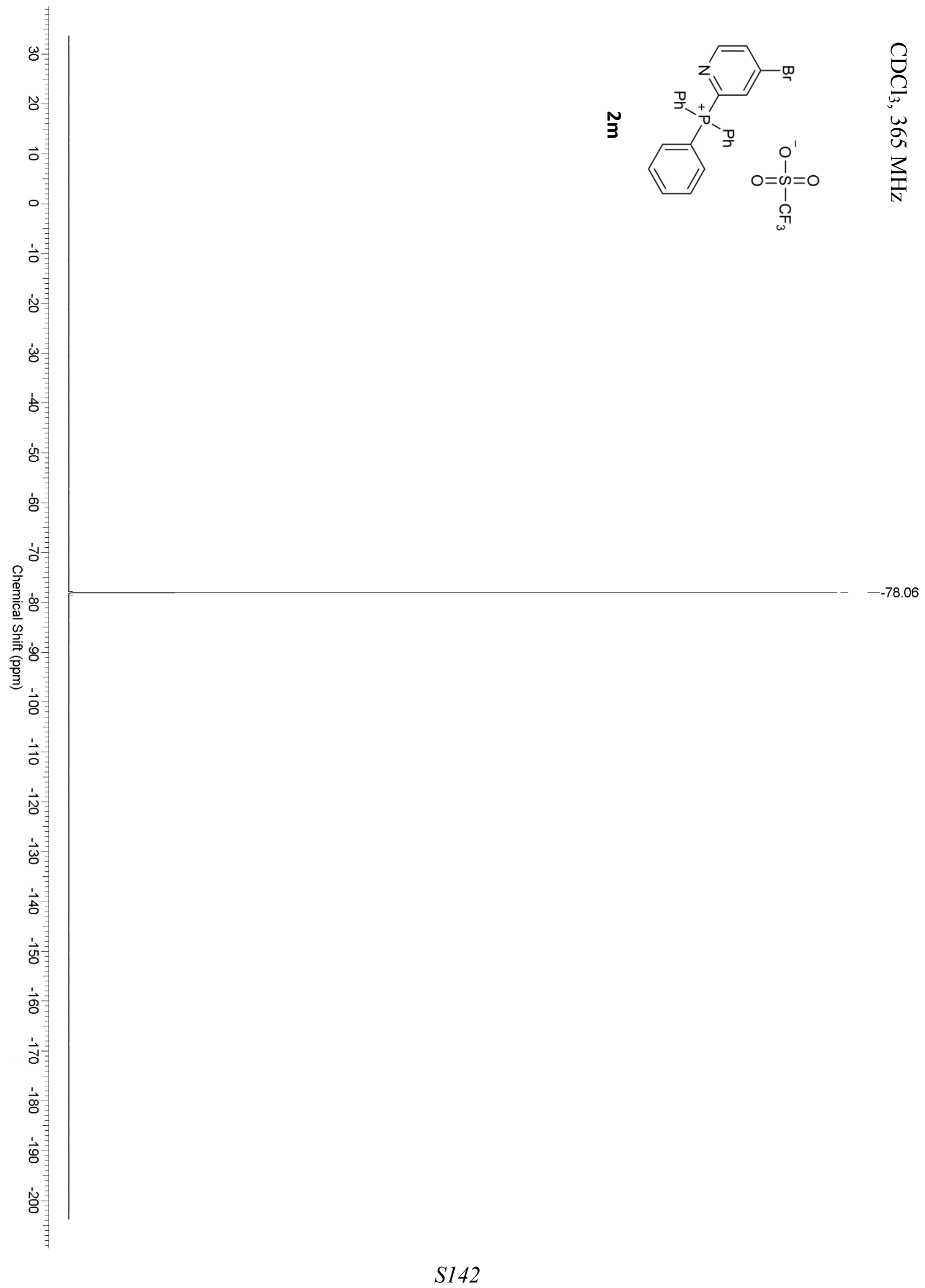



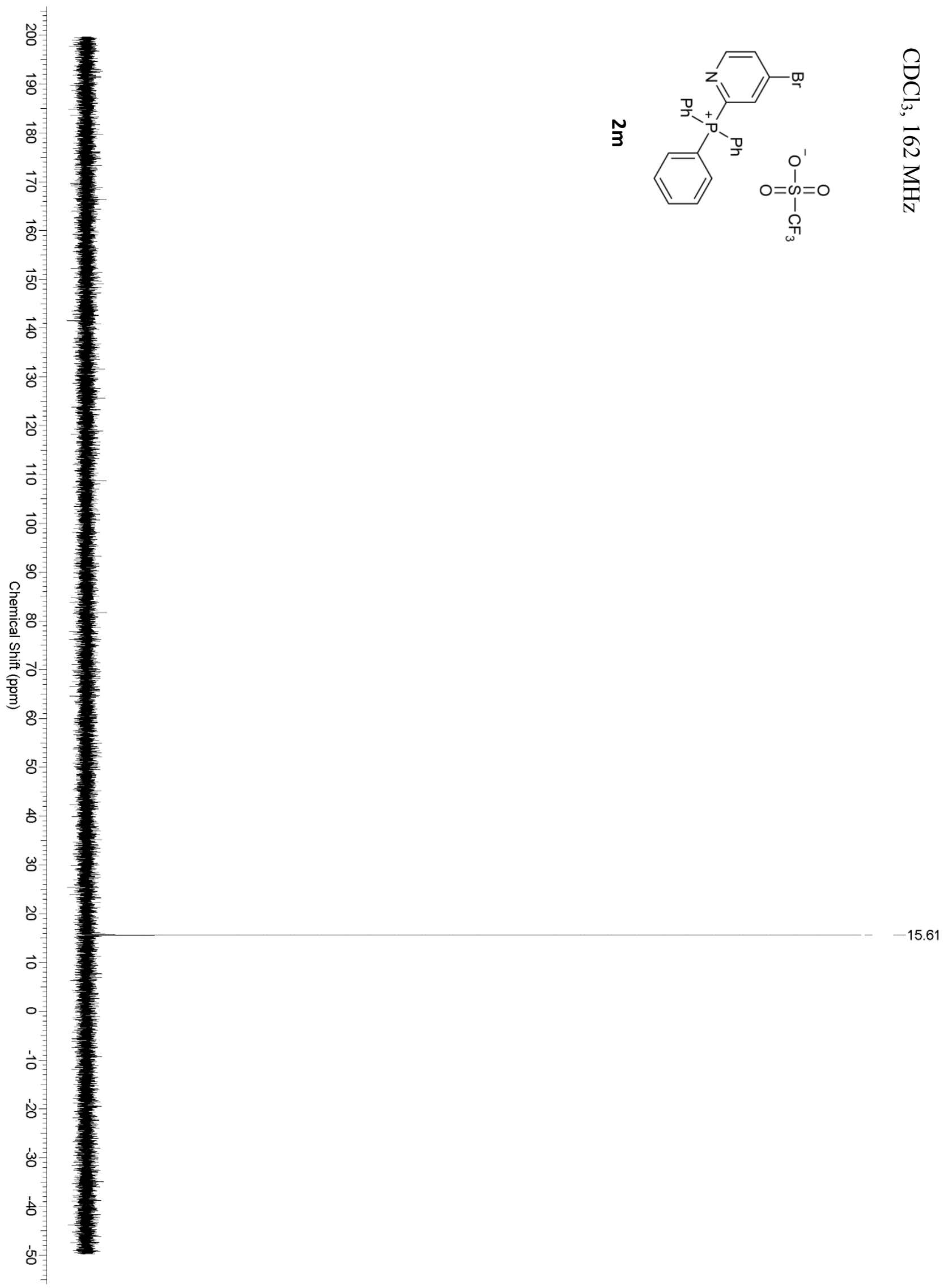


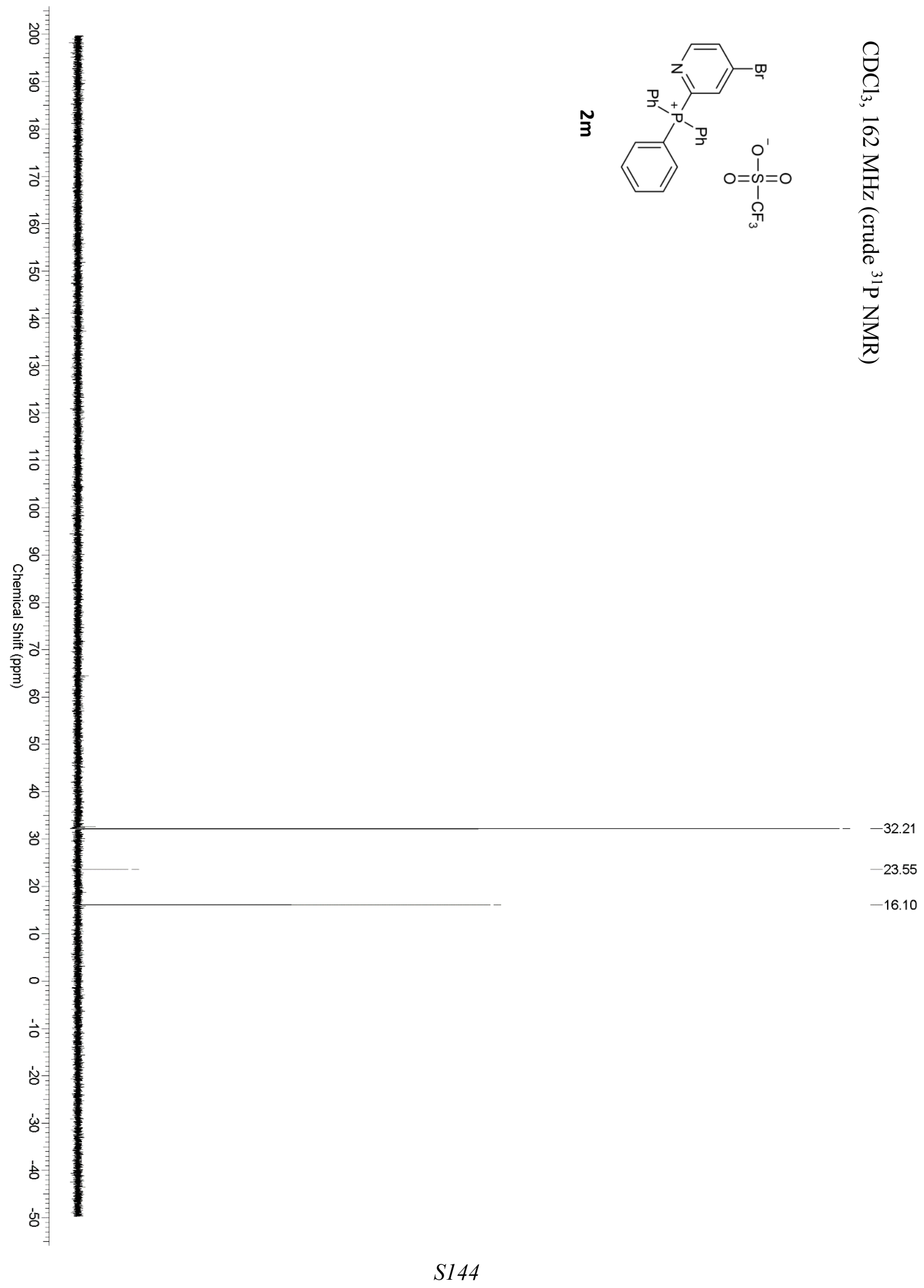




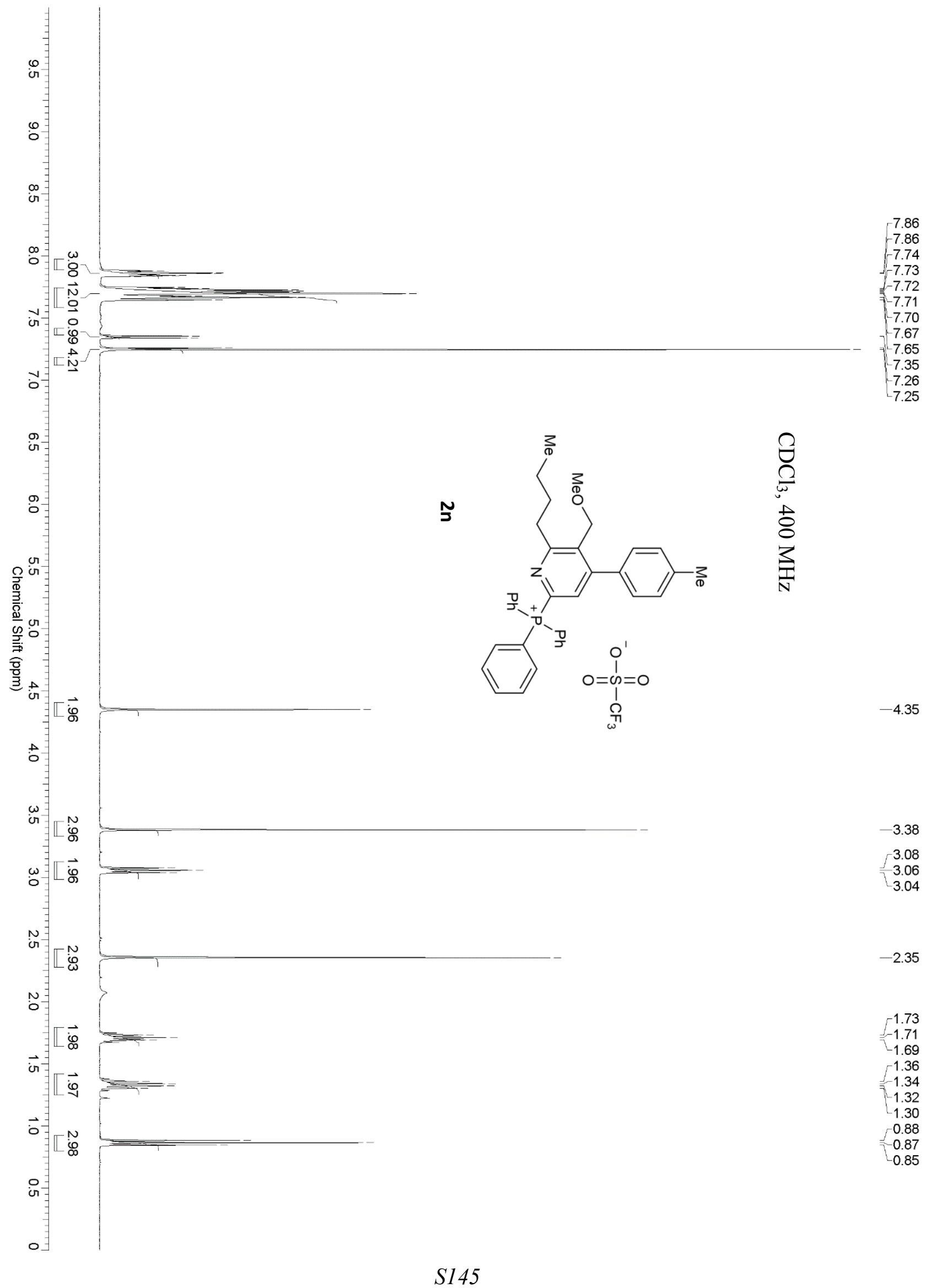




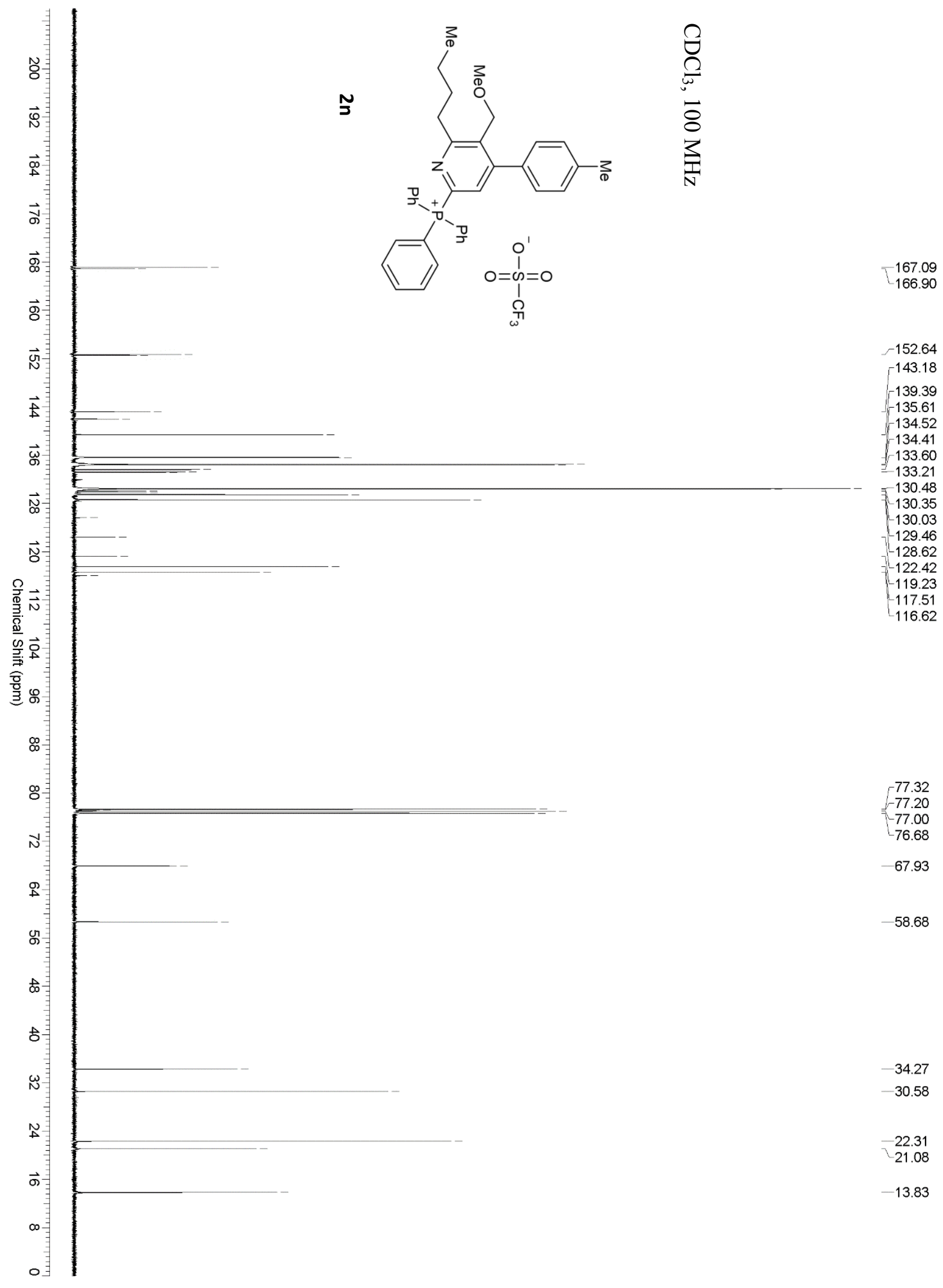




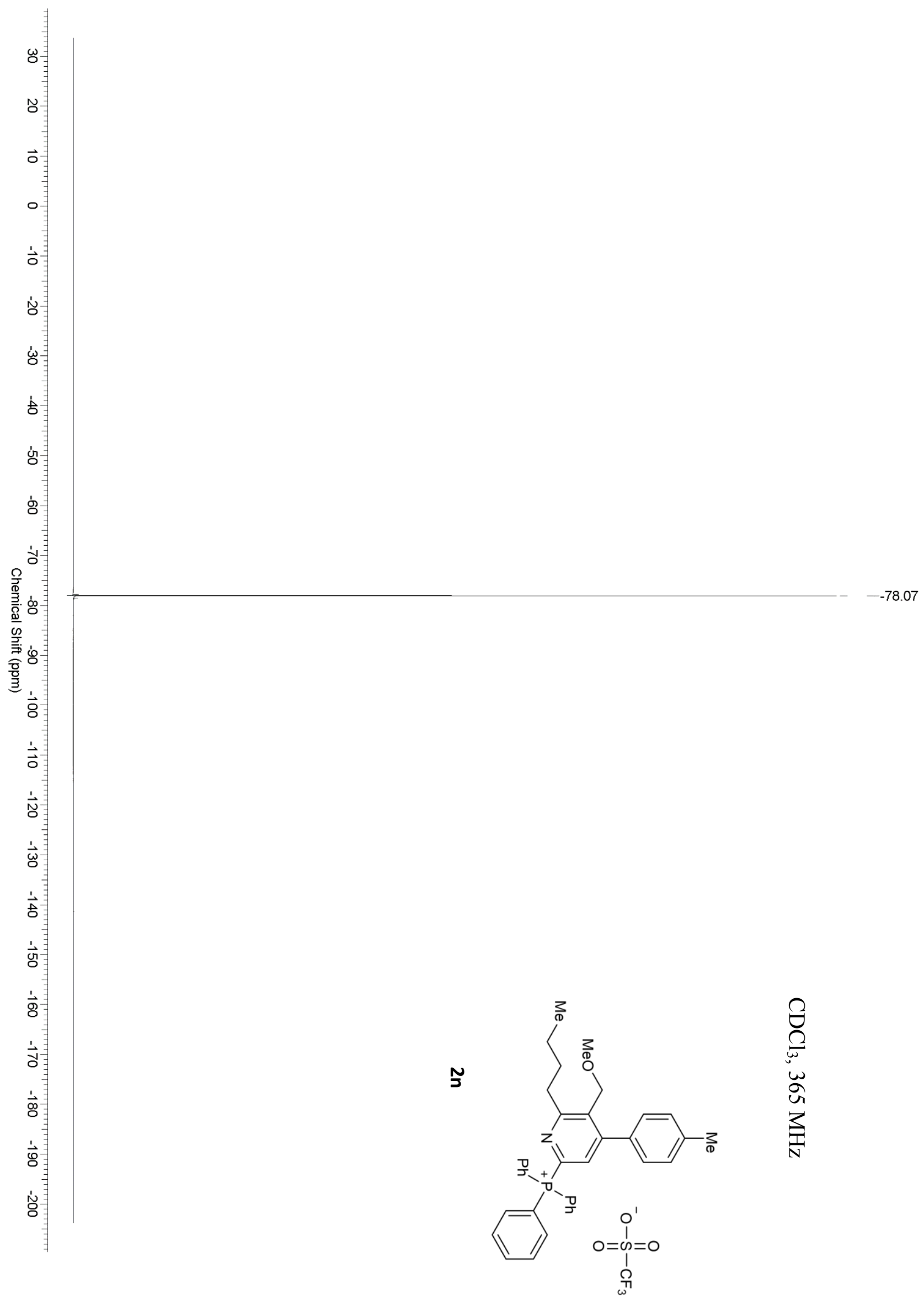




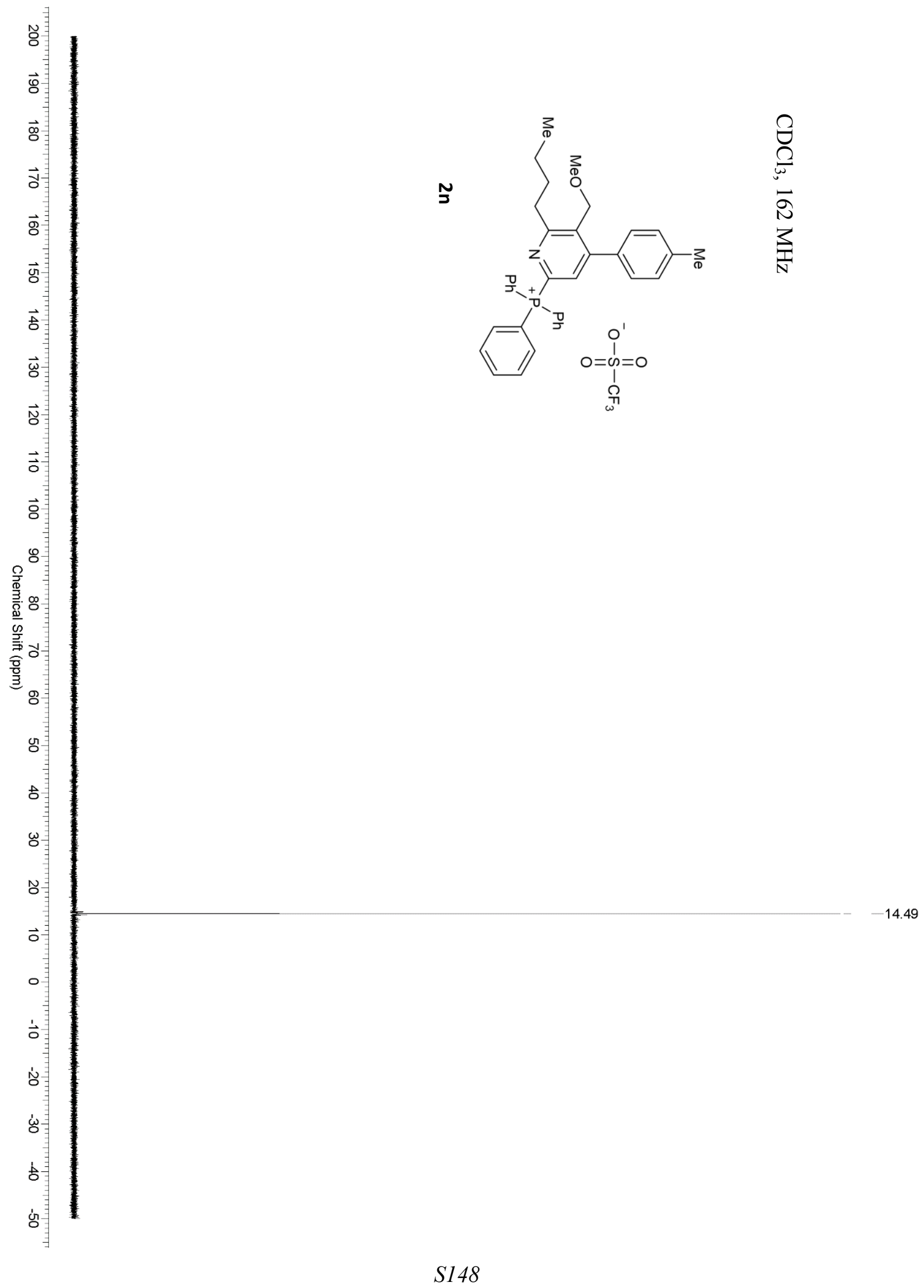




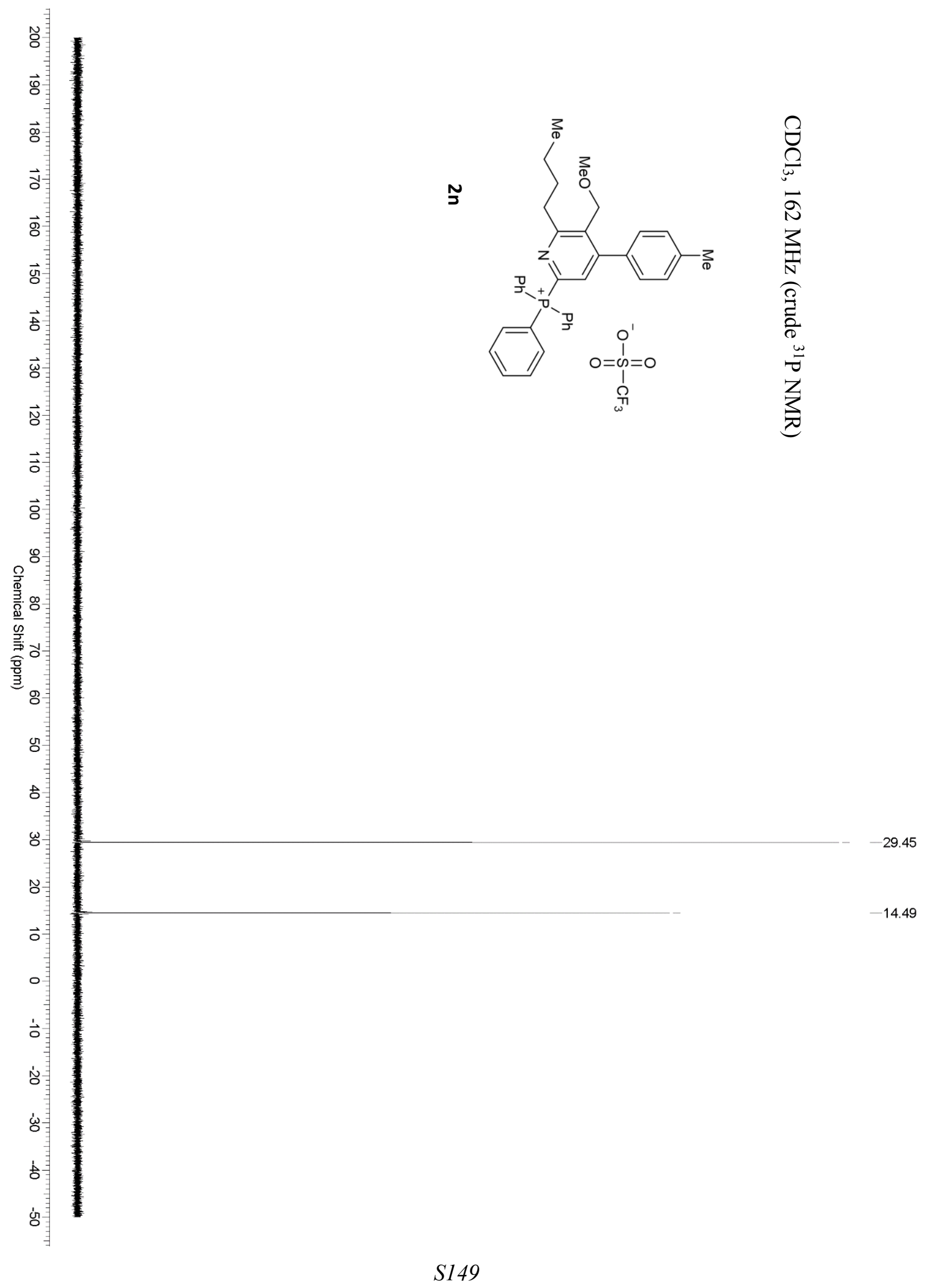



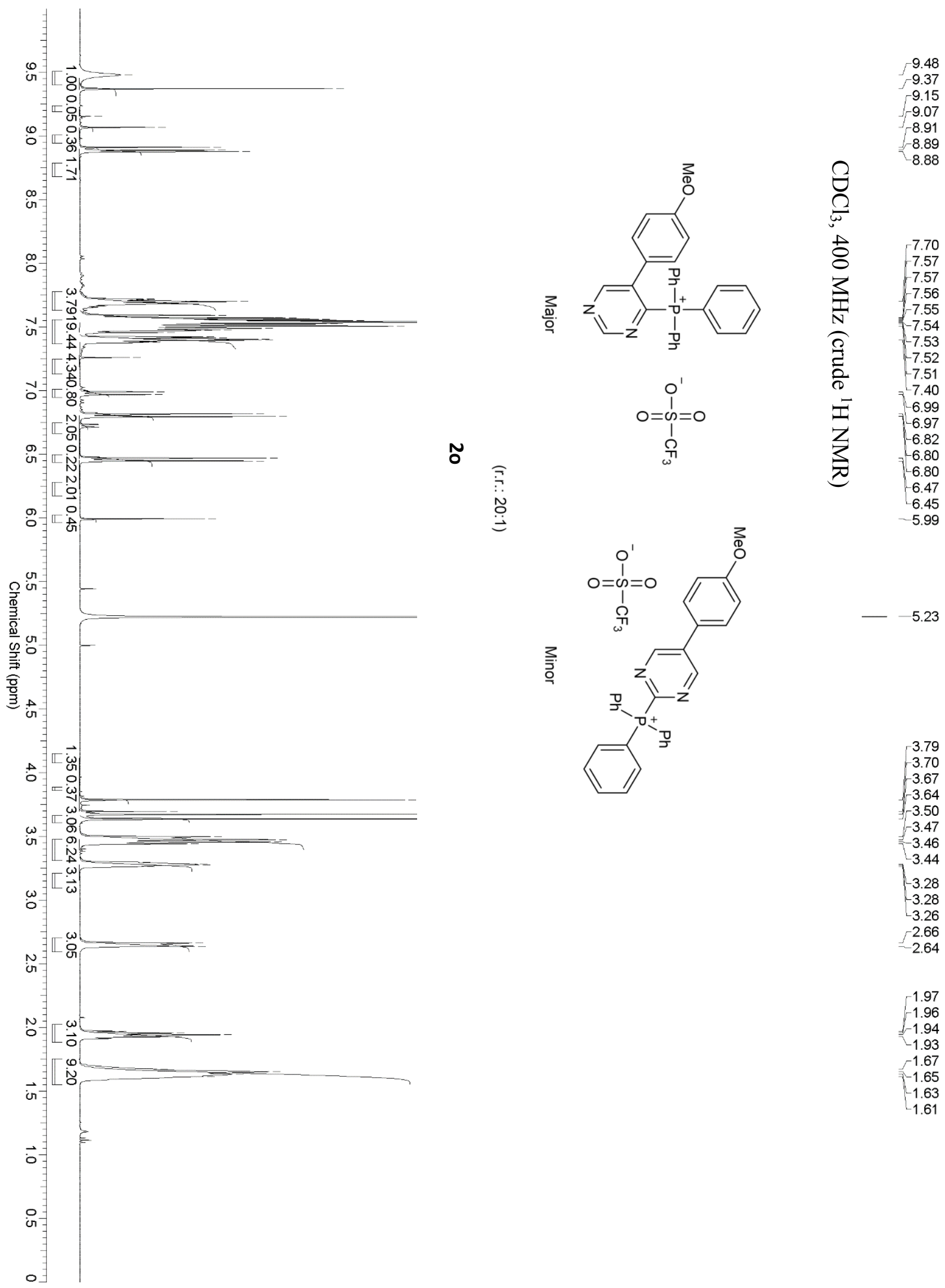

1.97
-1.96
-1.94
-1.93
-1.67
-1.65
-1.63
1.61 


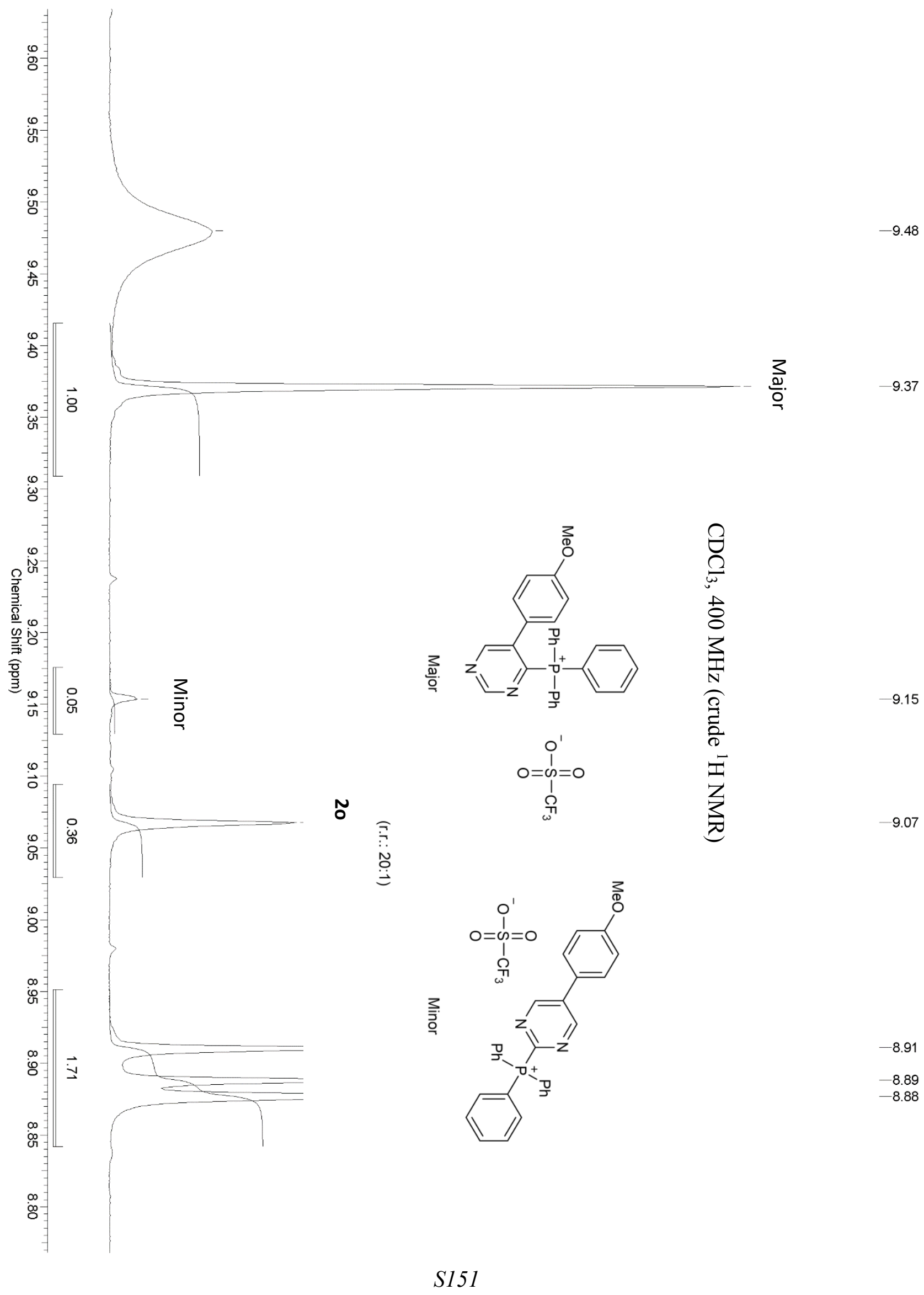




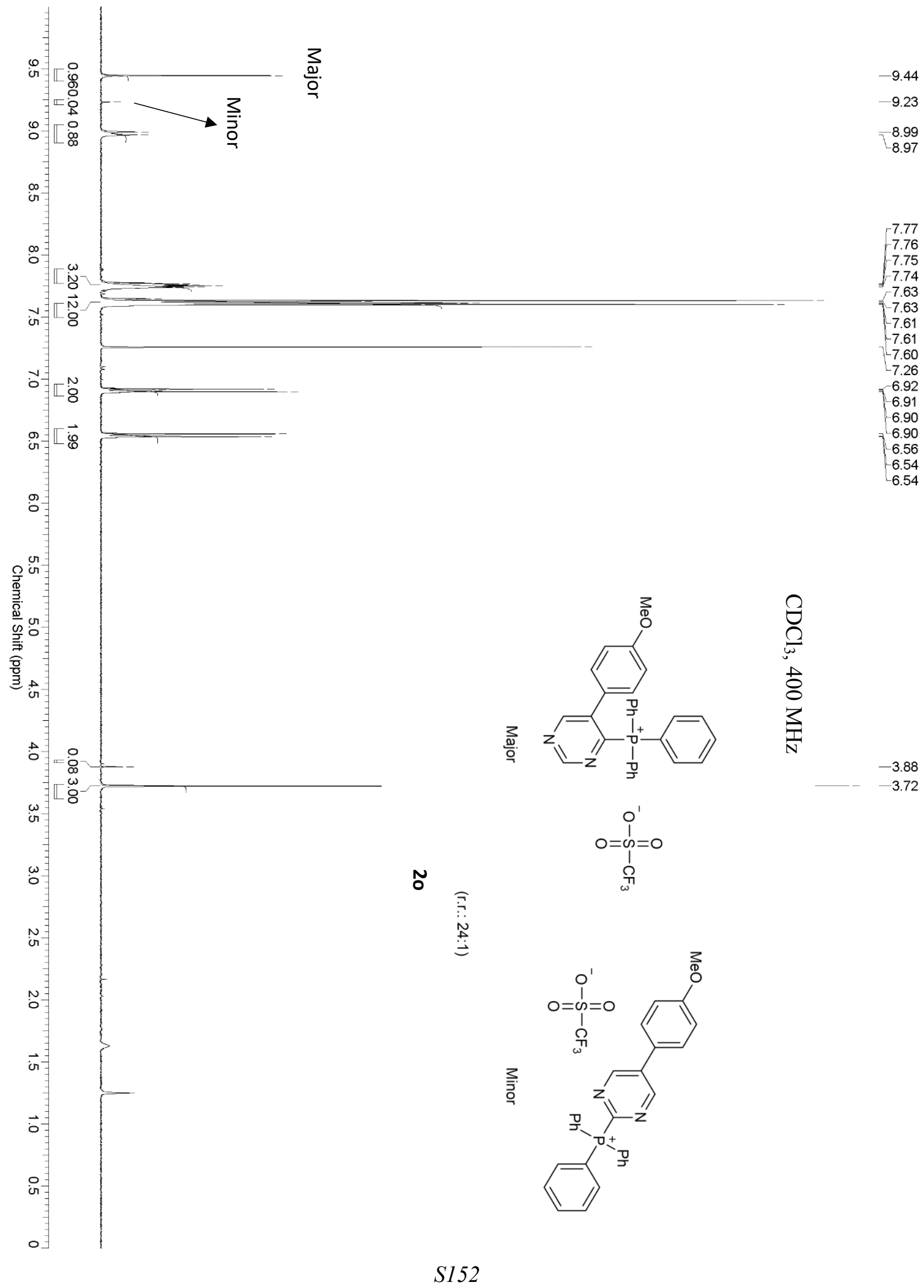




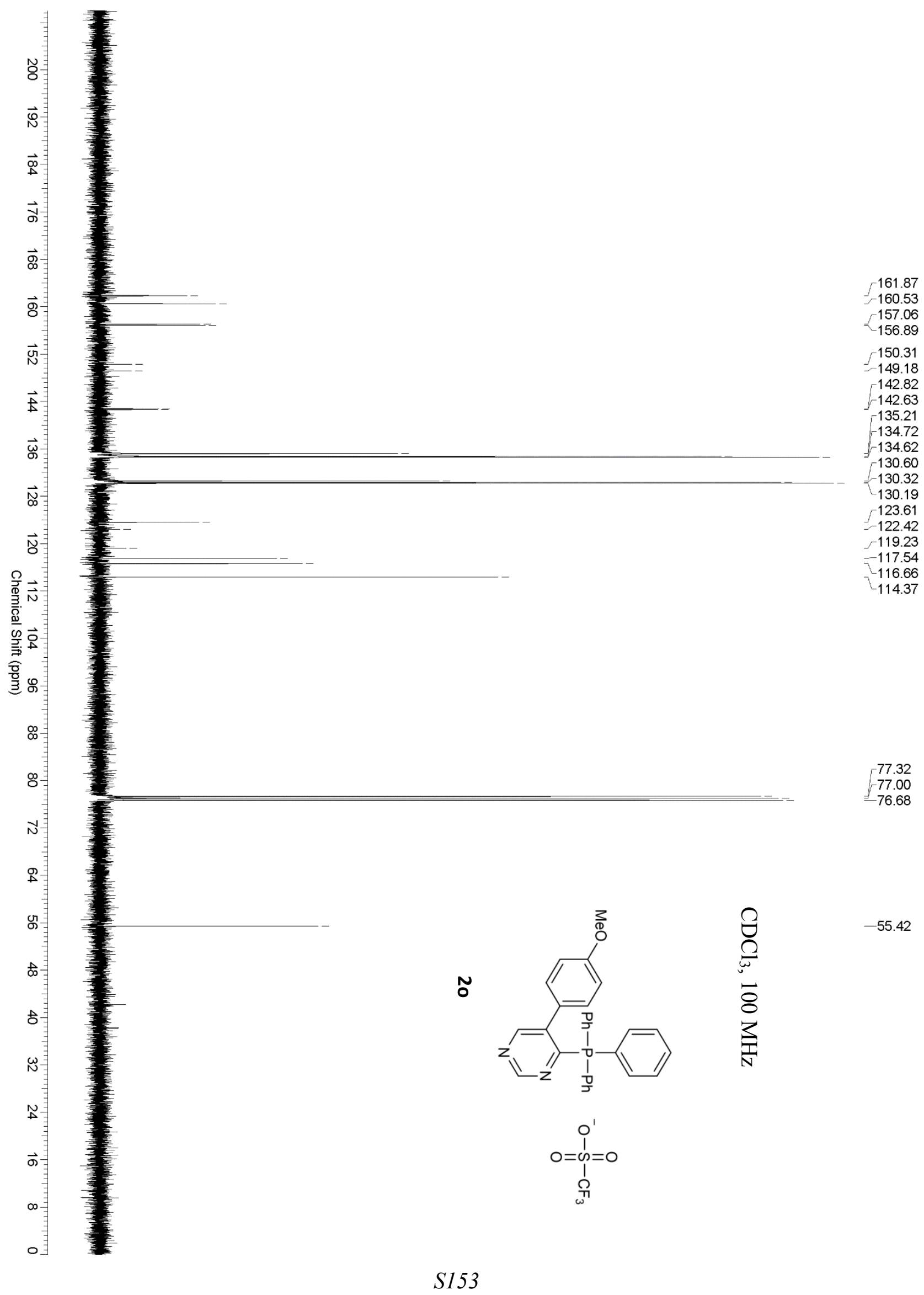




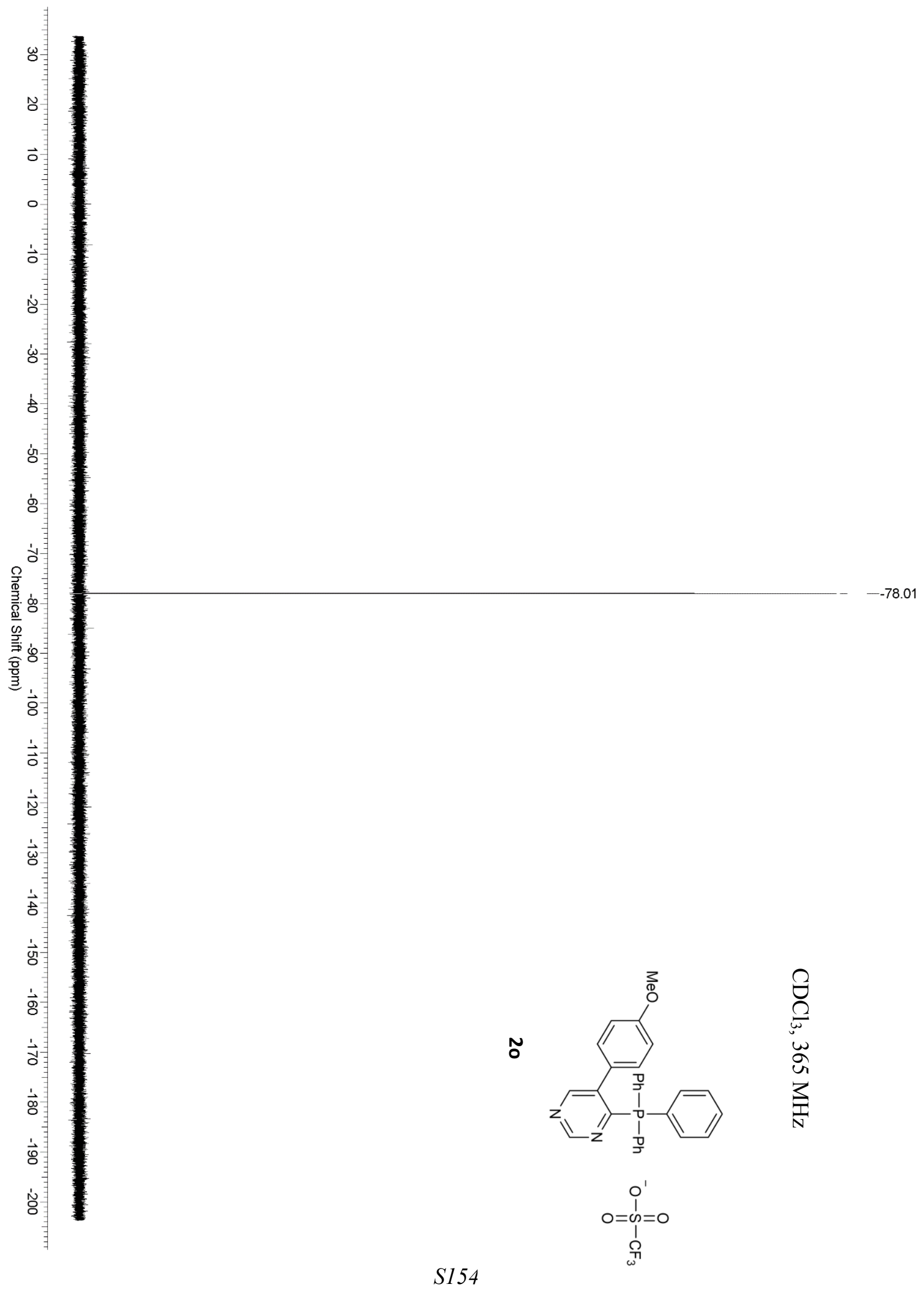




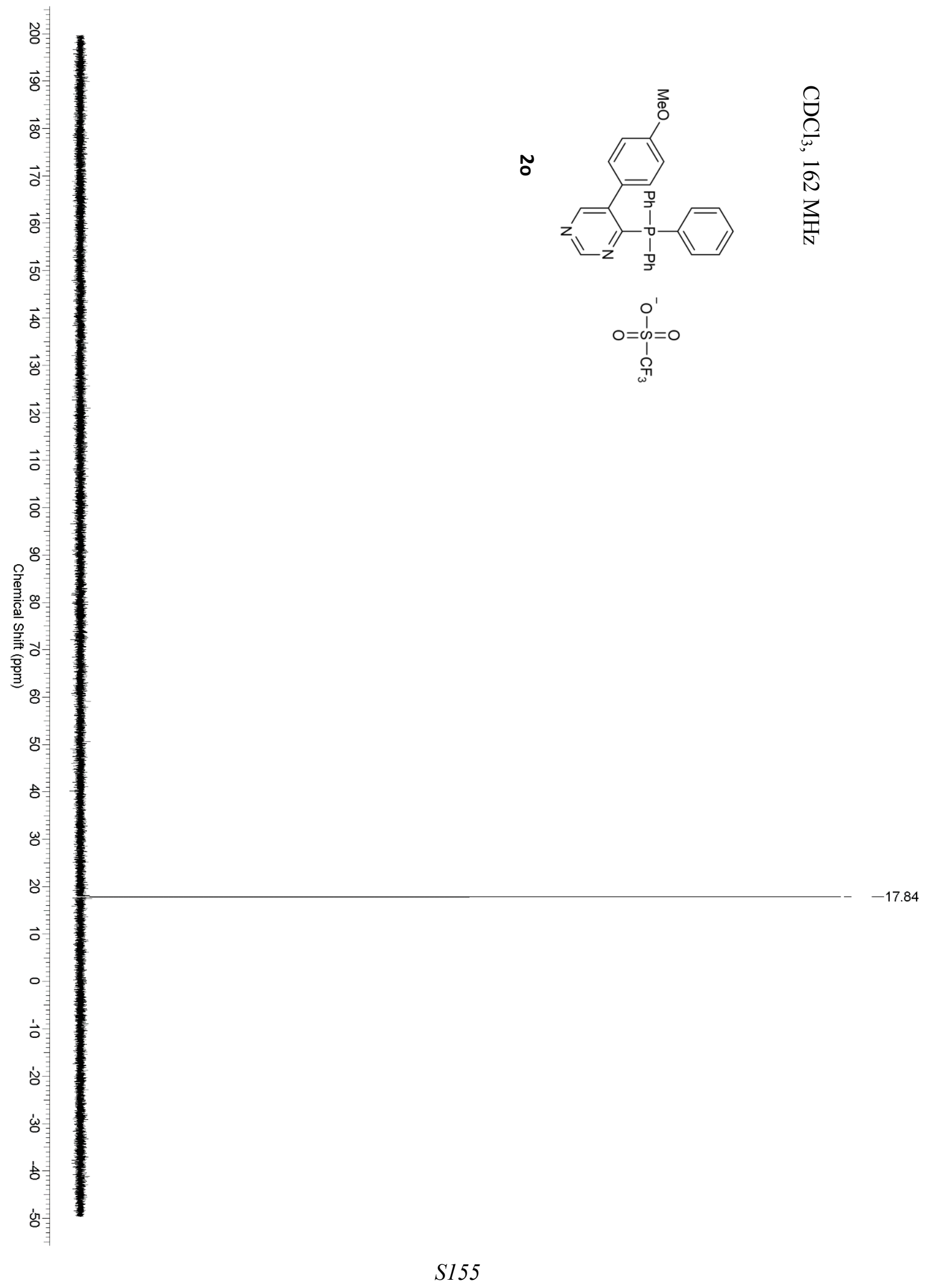




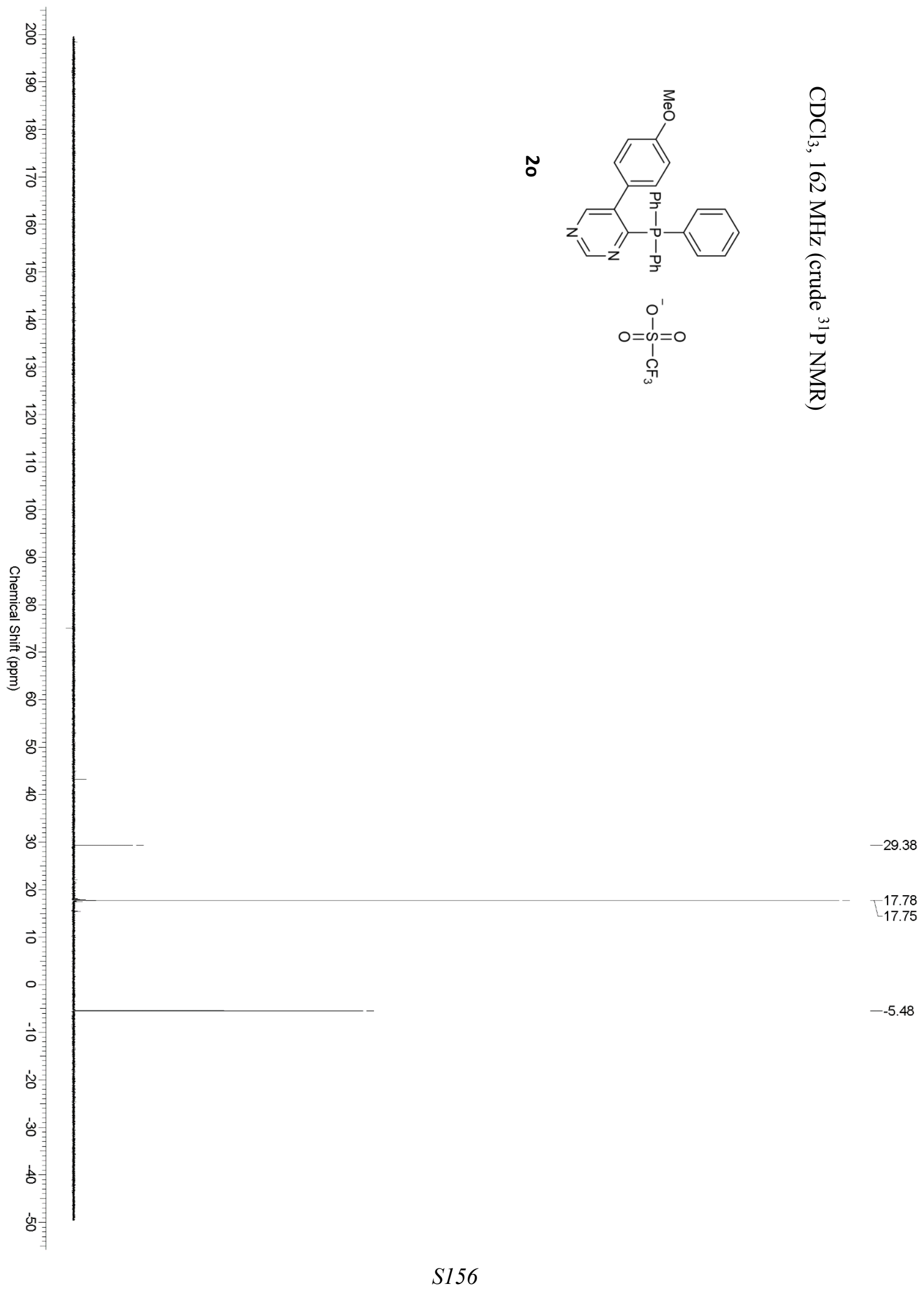




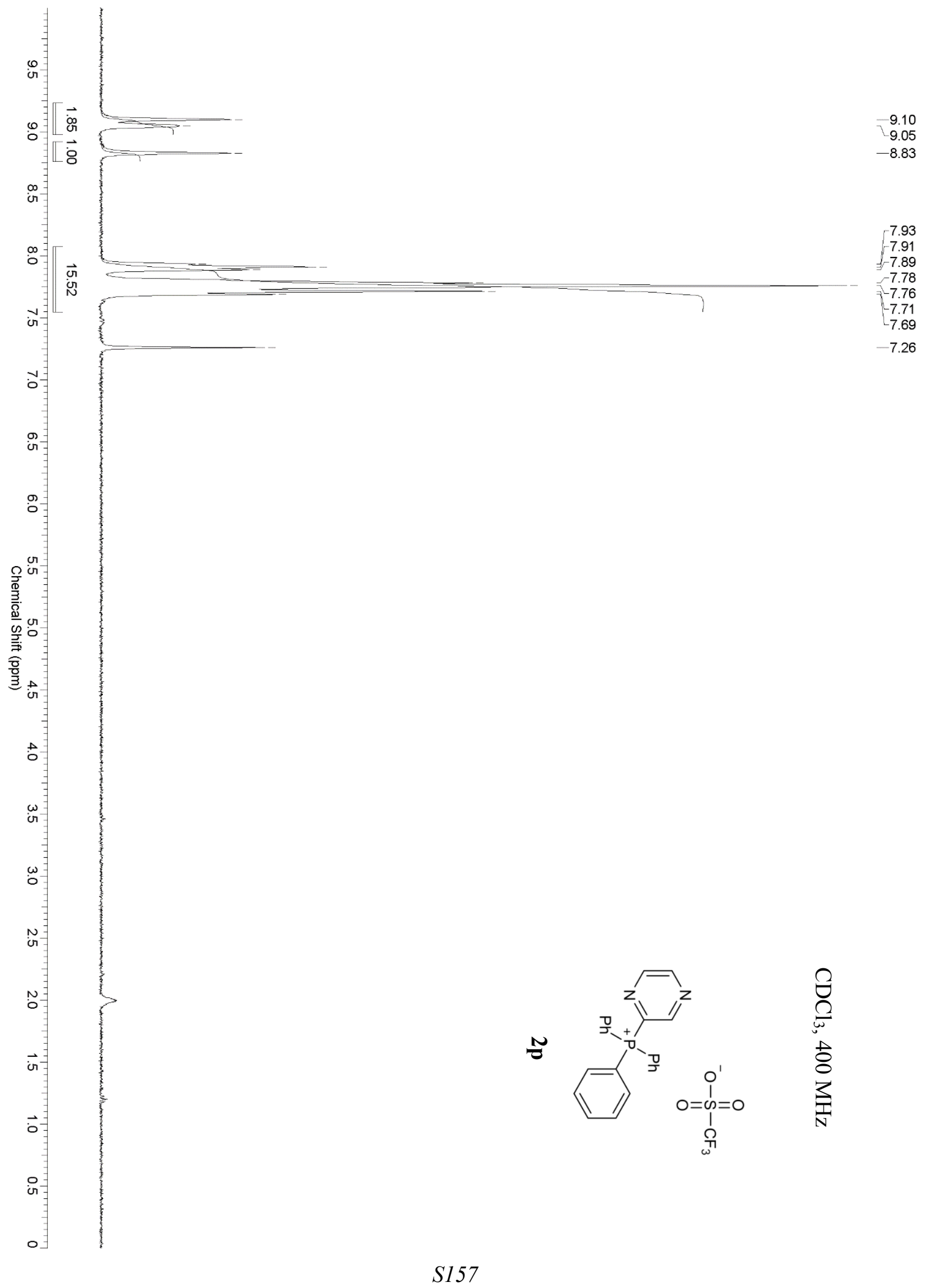




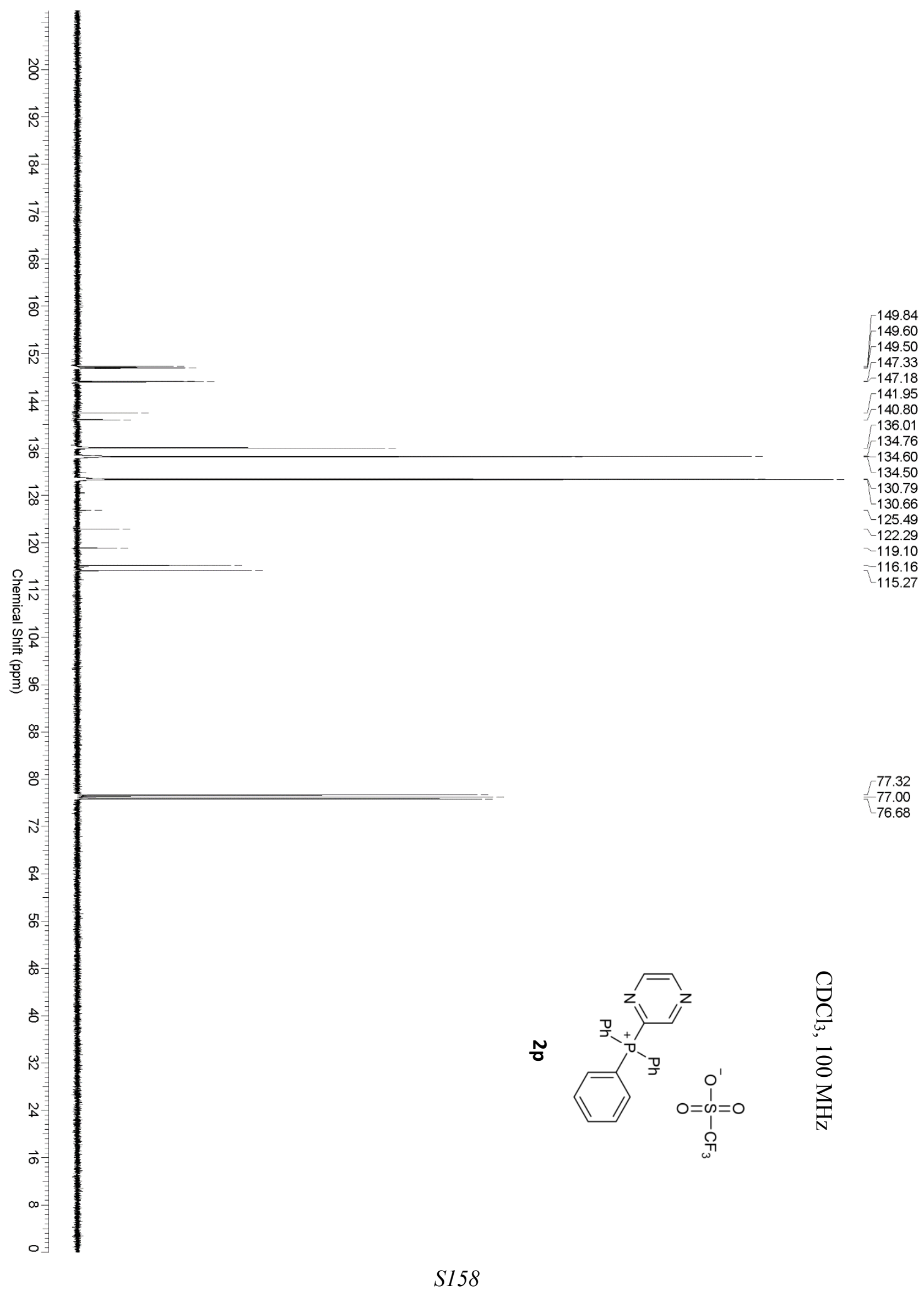




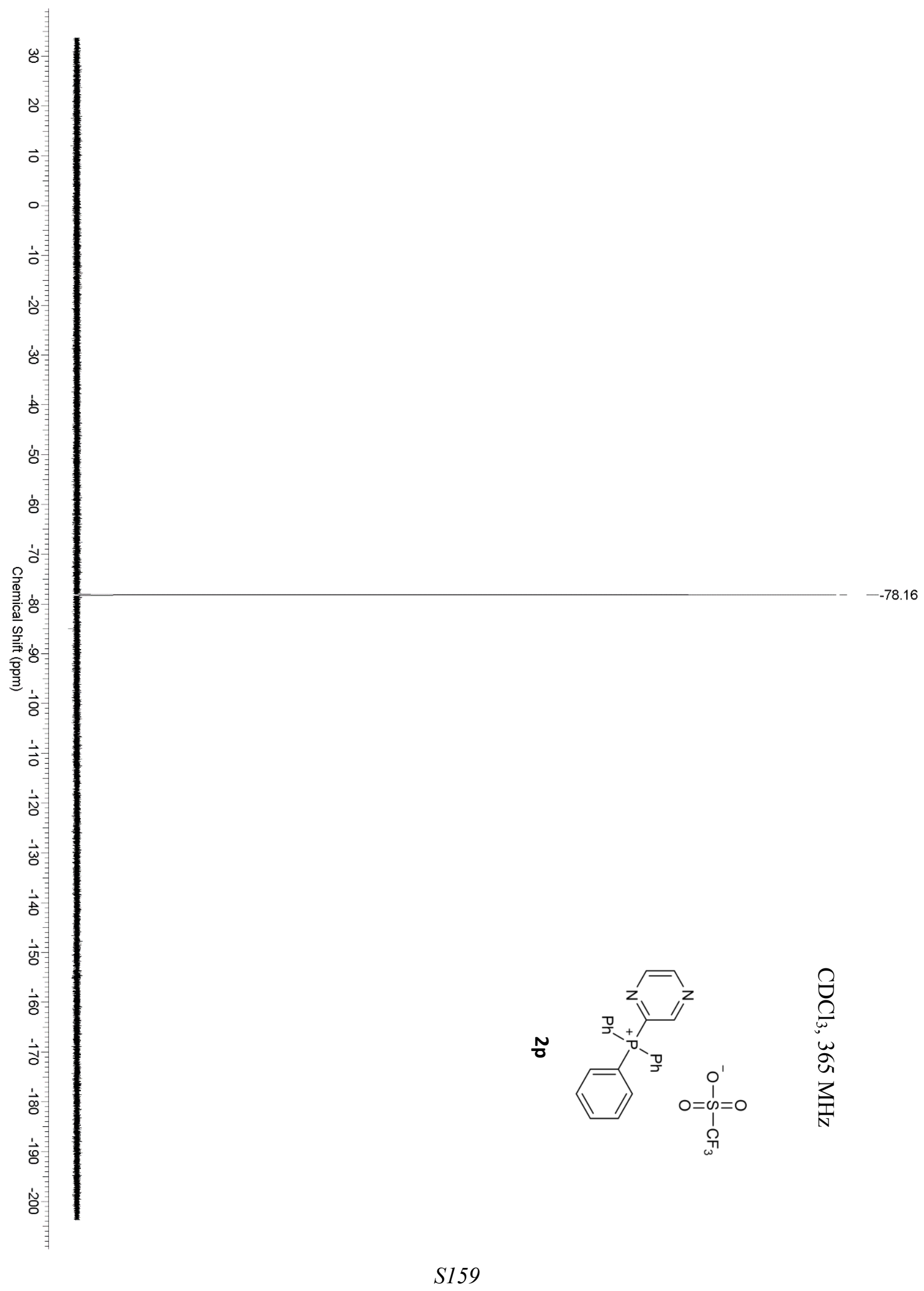




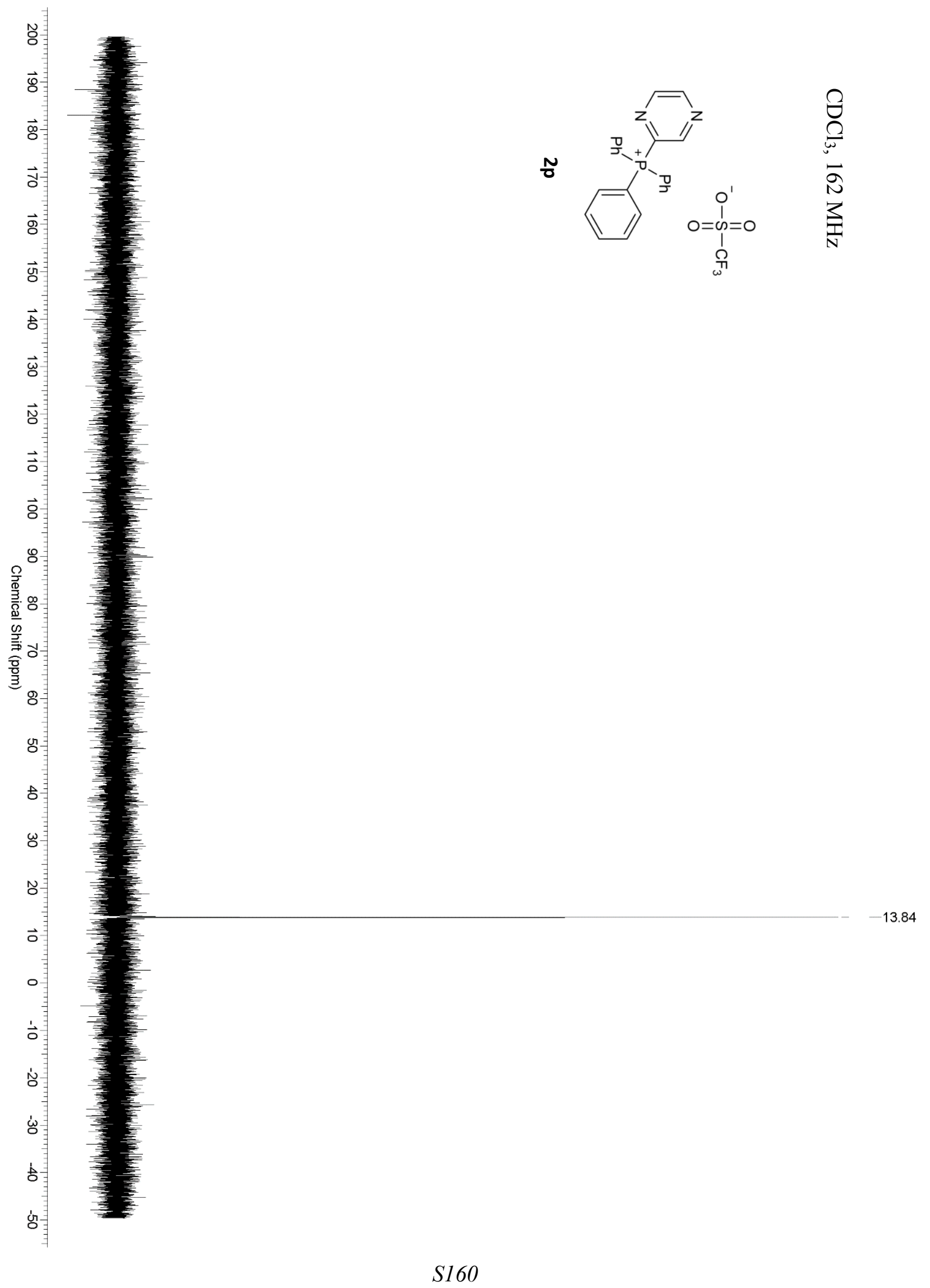




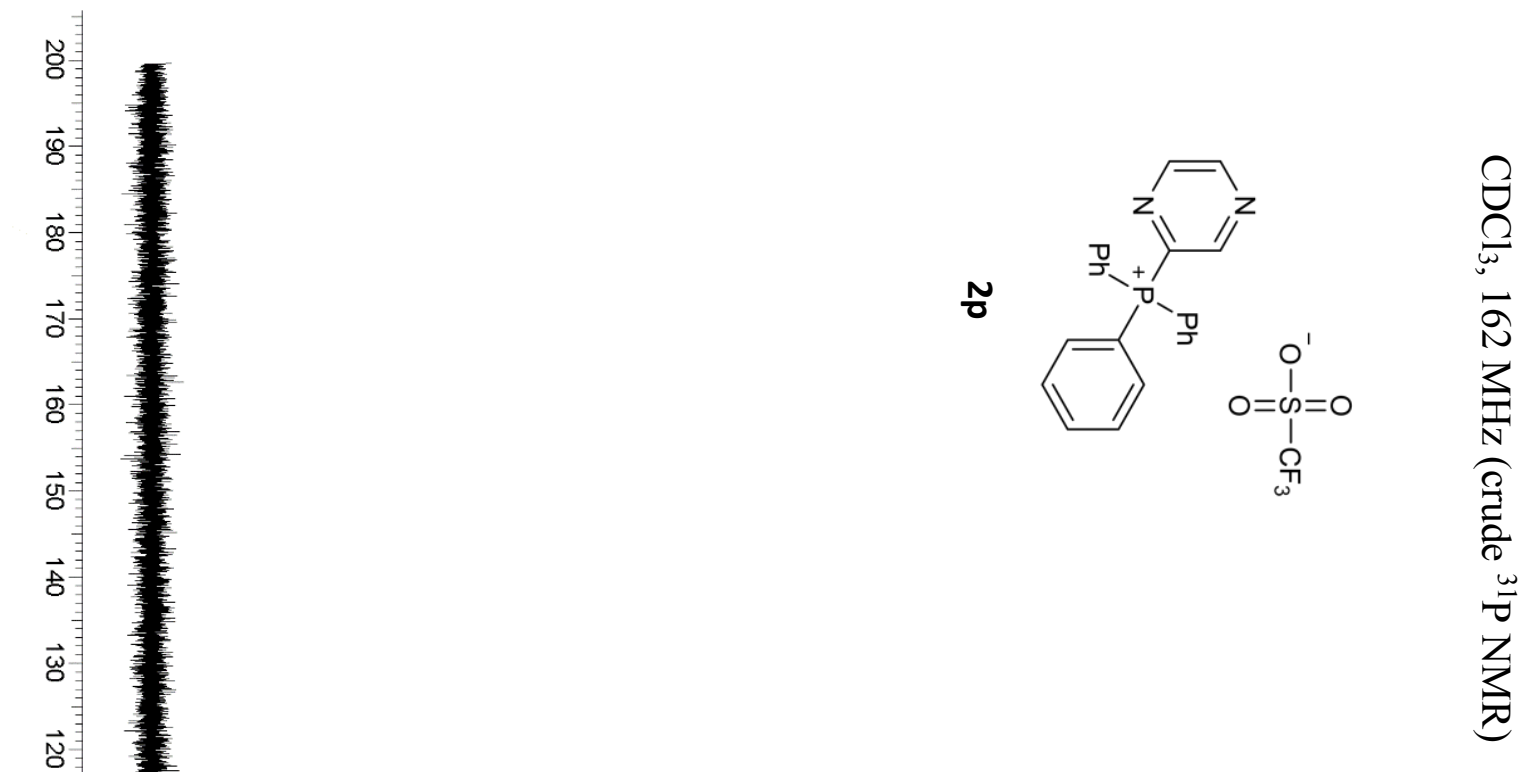




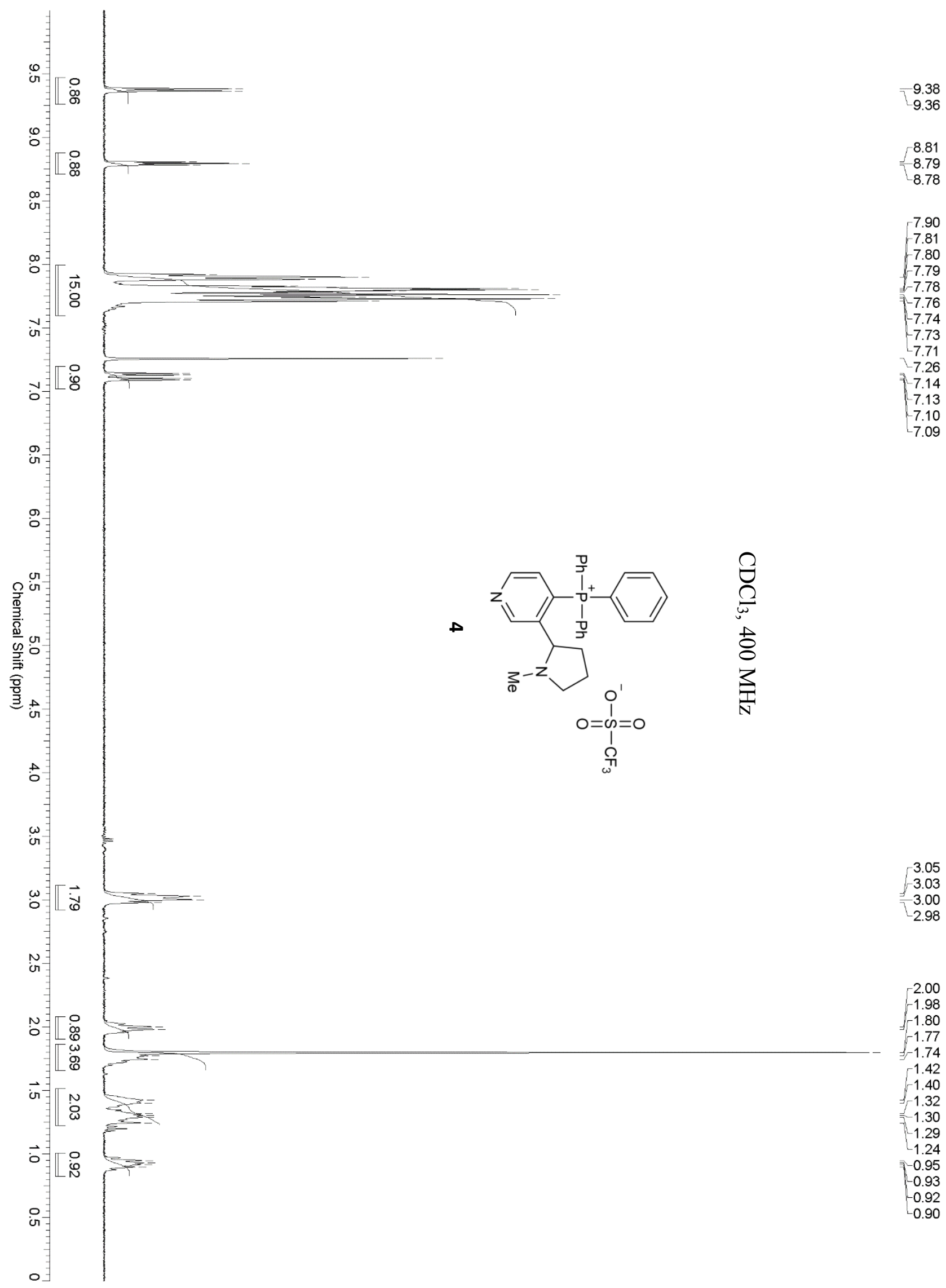




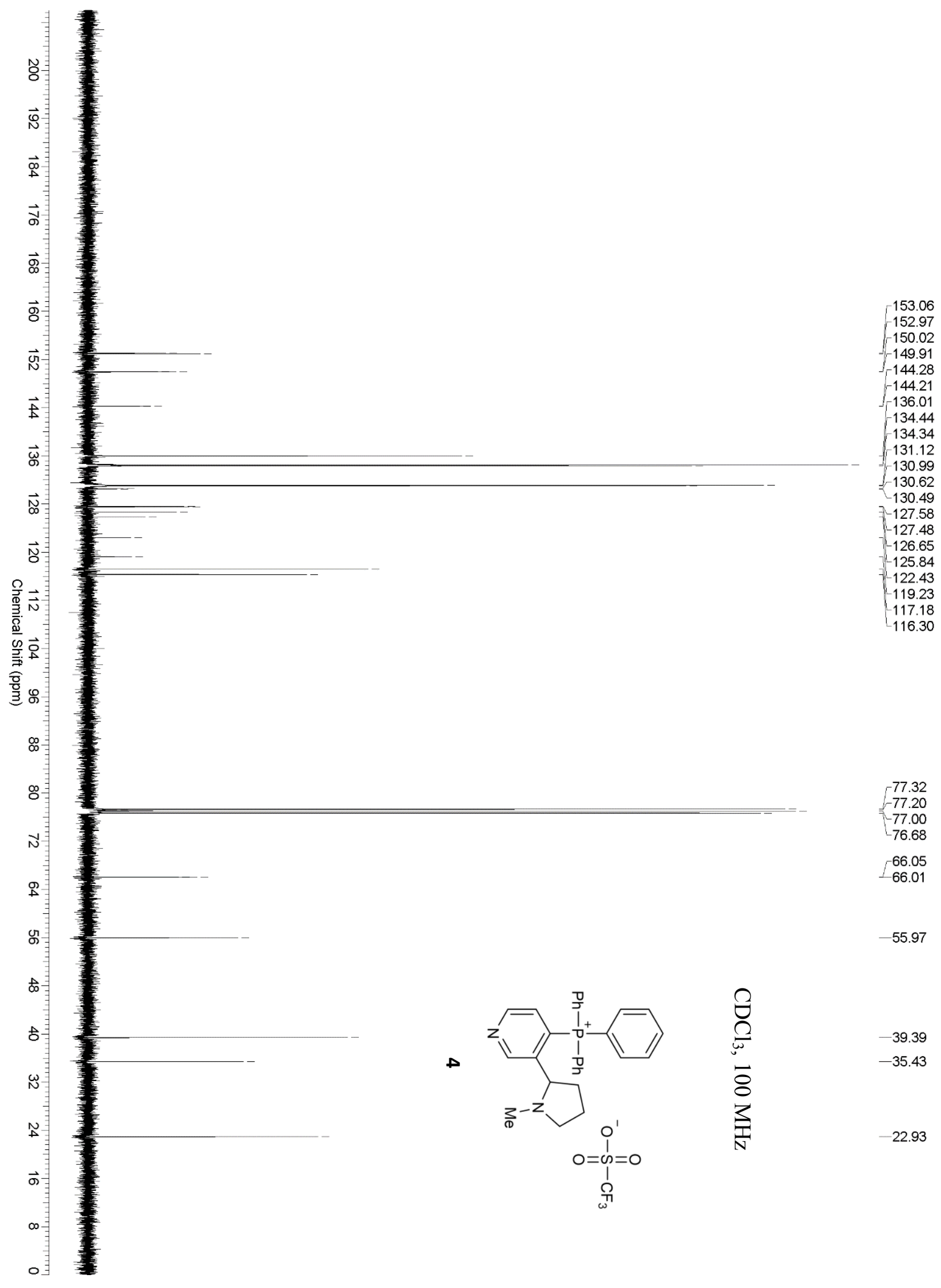




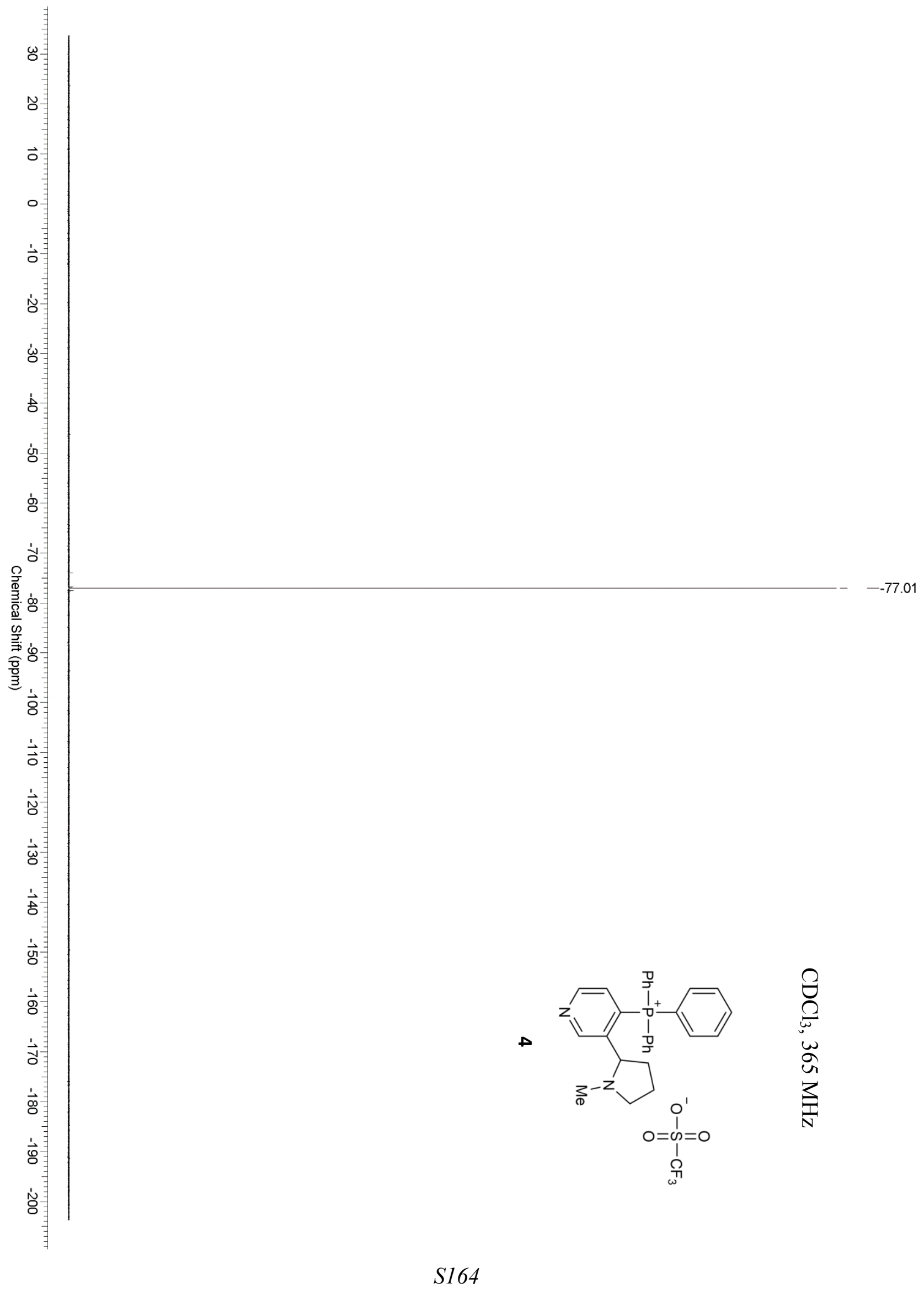




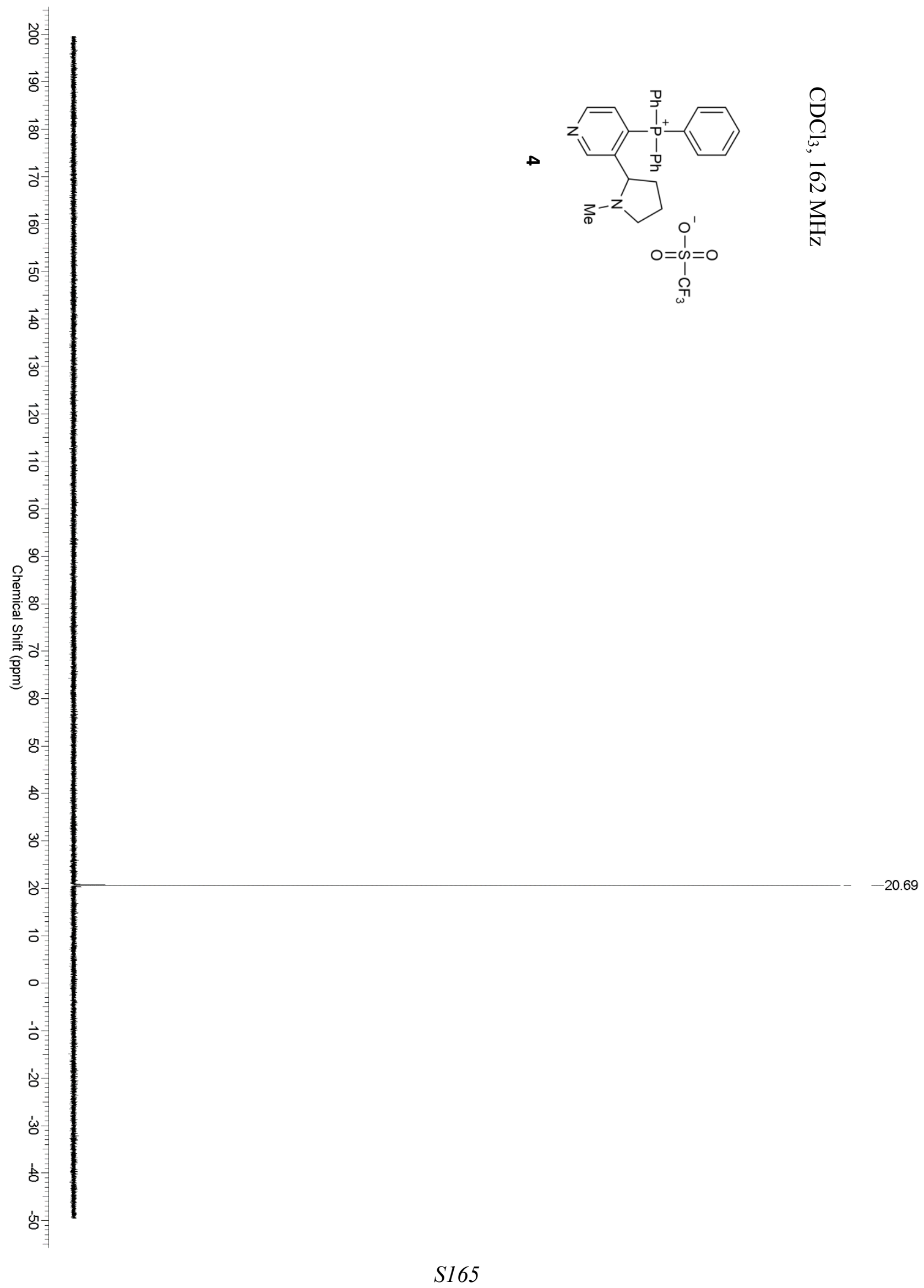




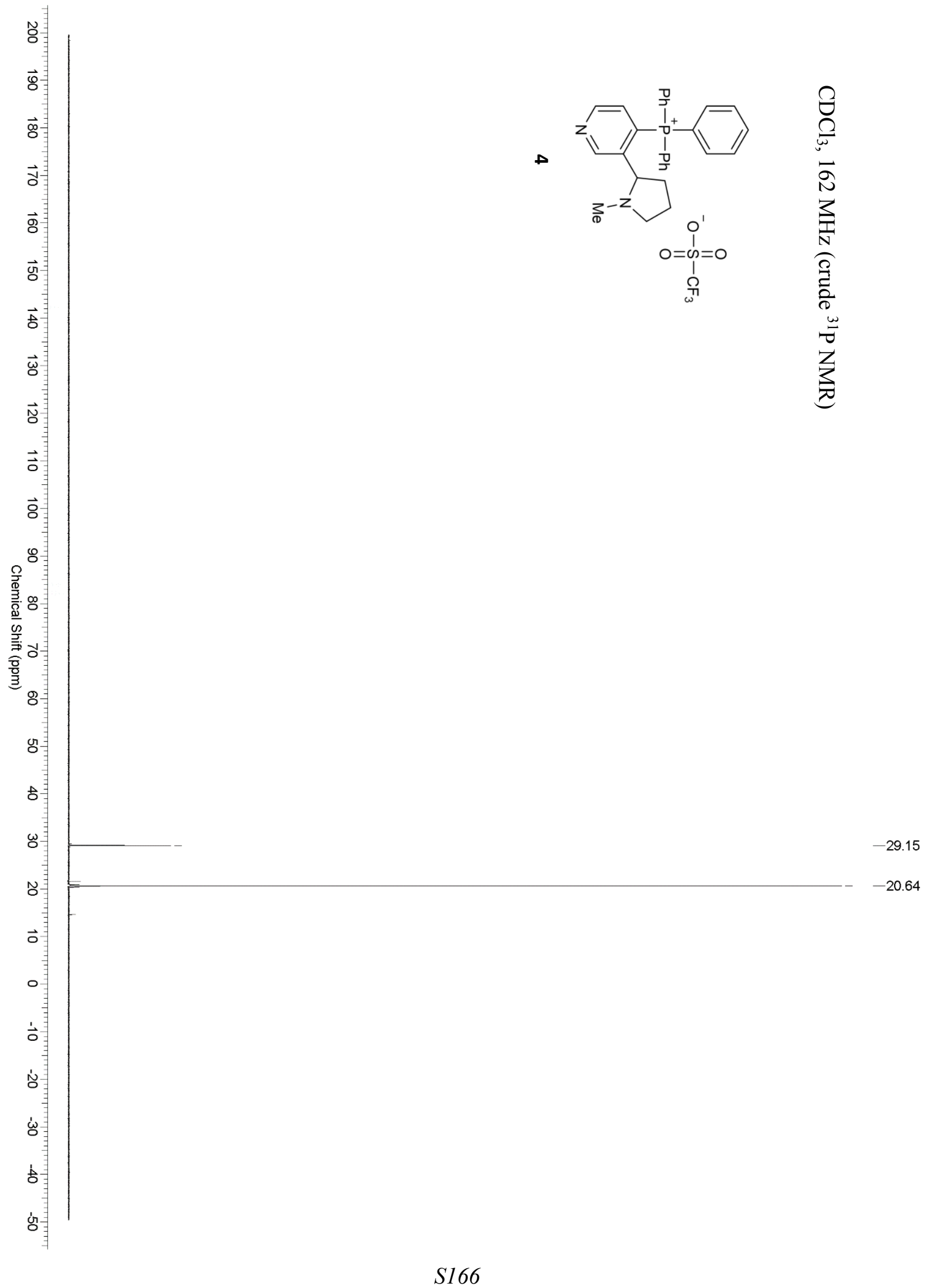




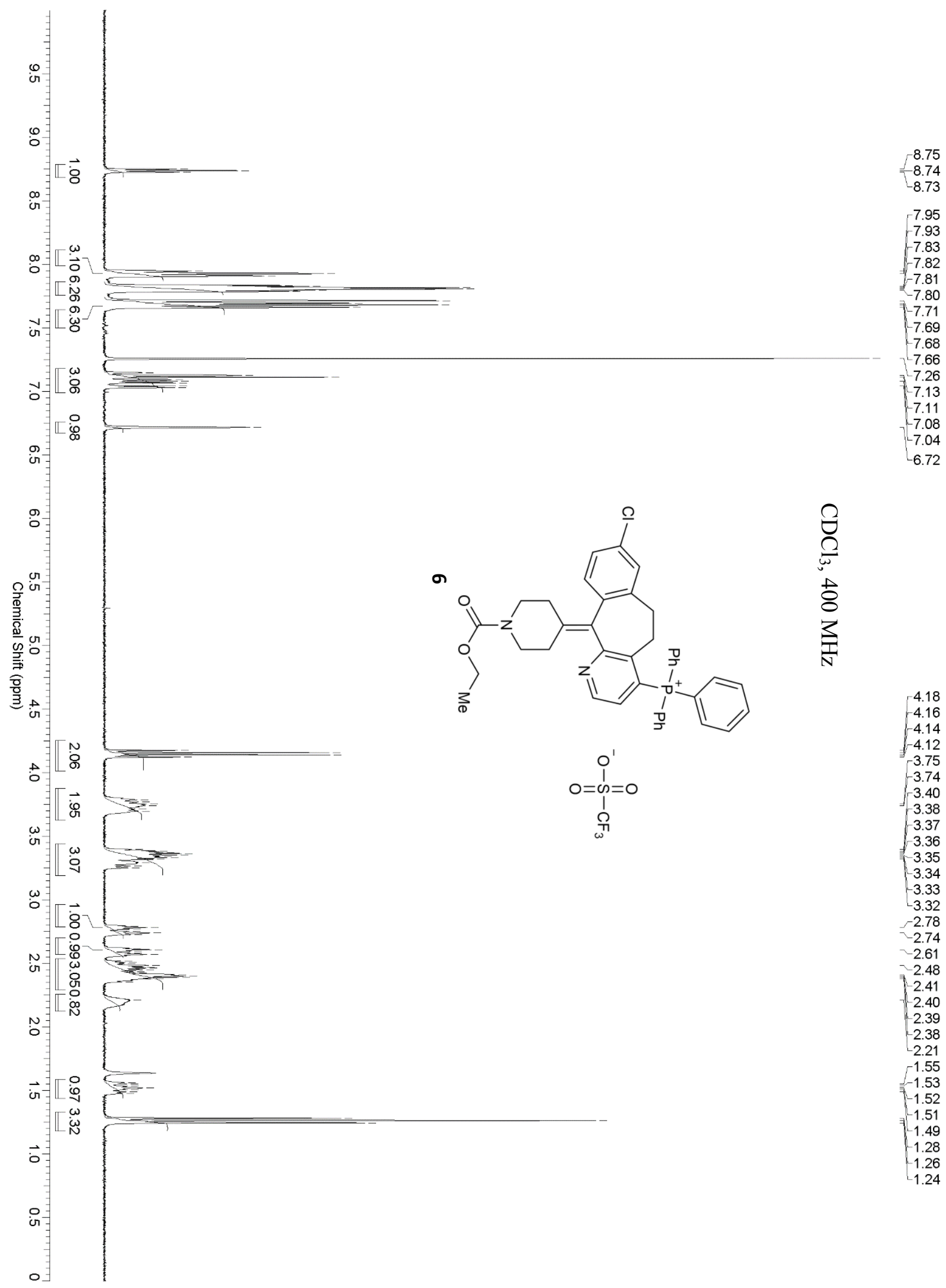




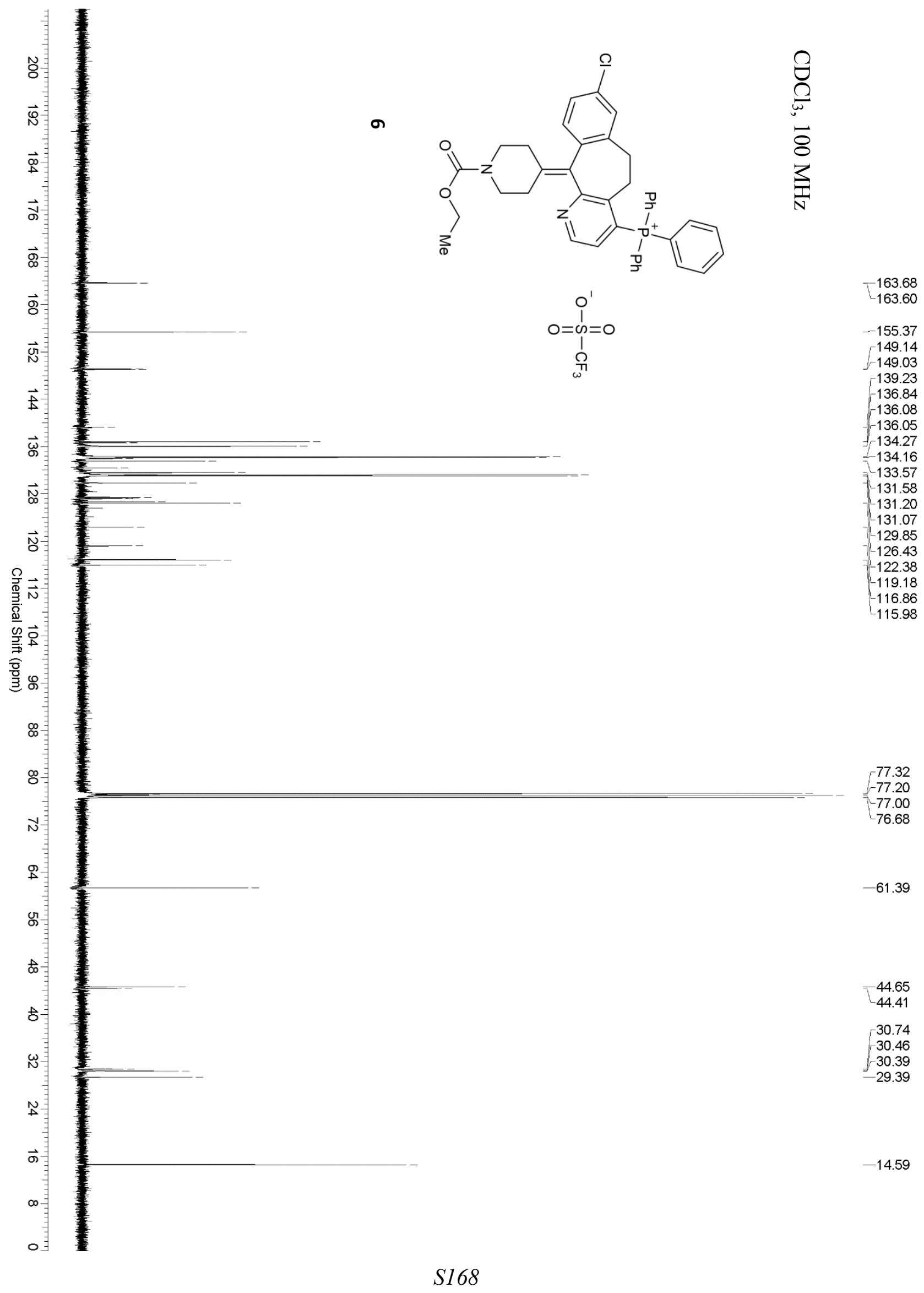




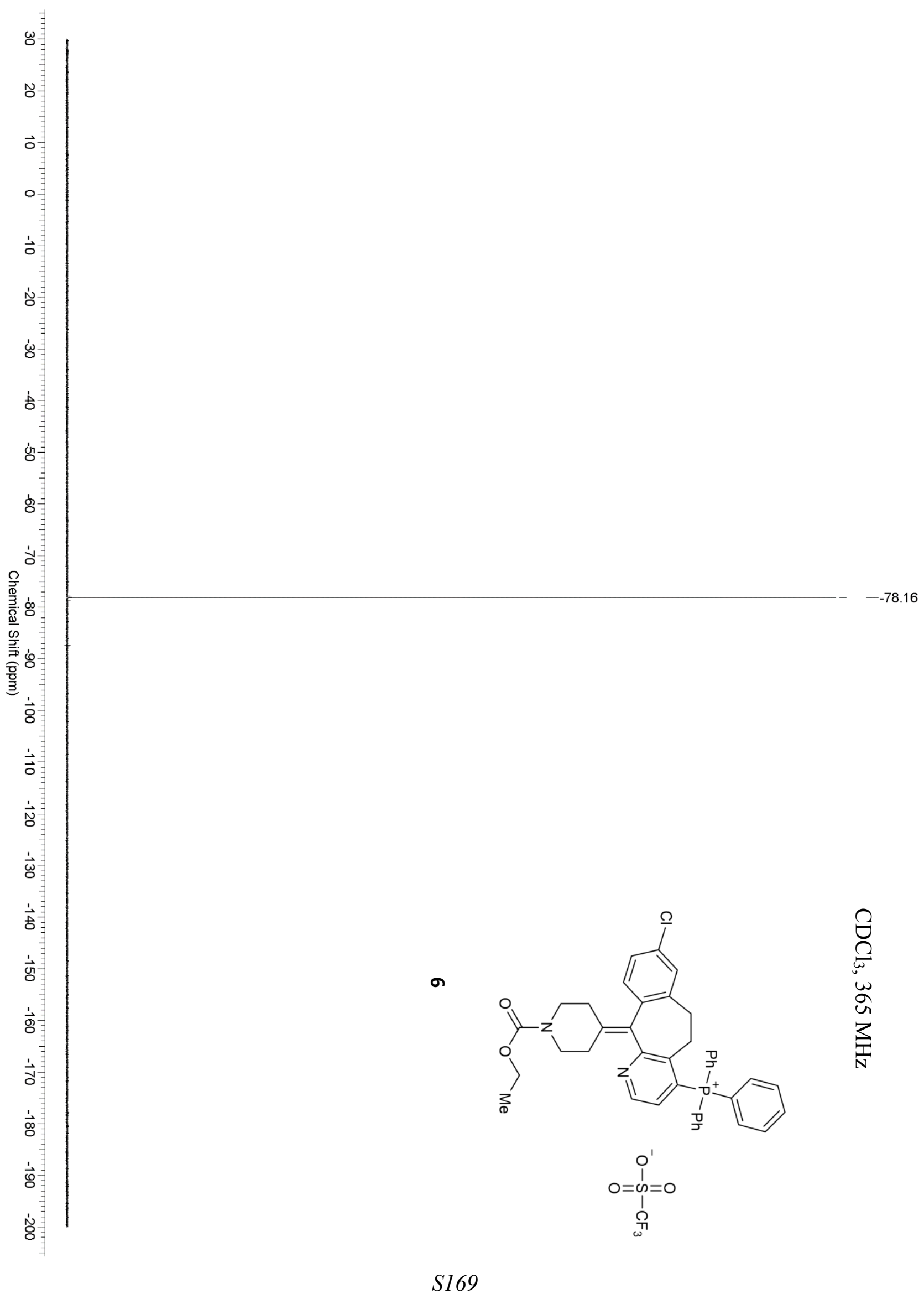




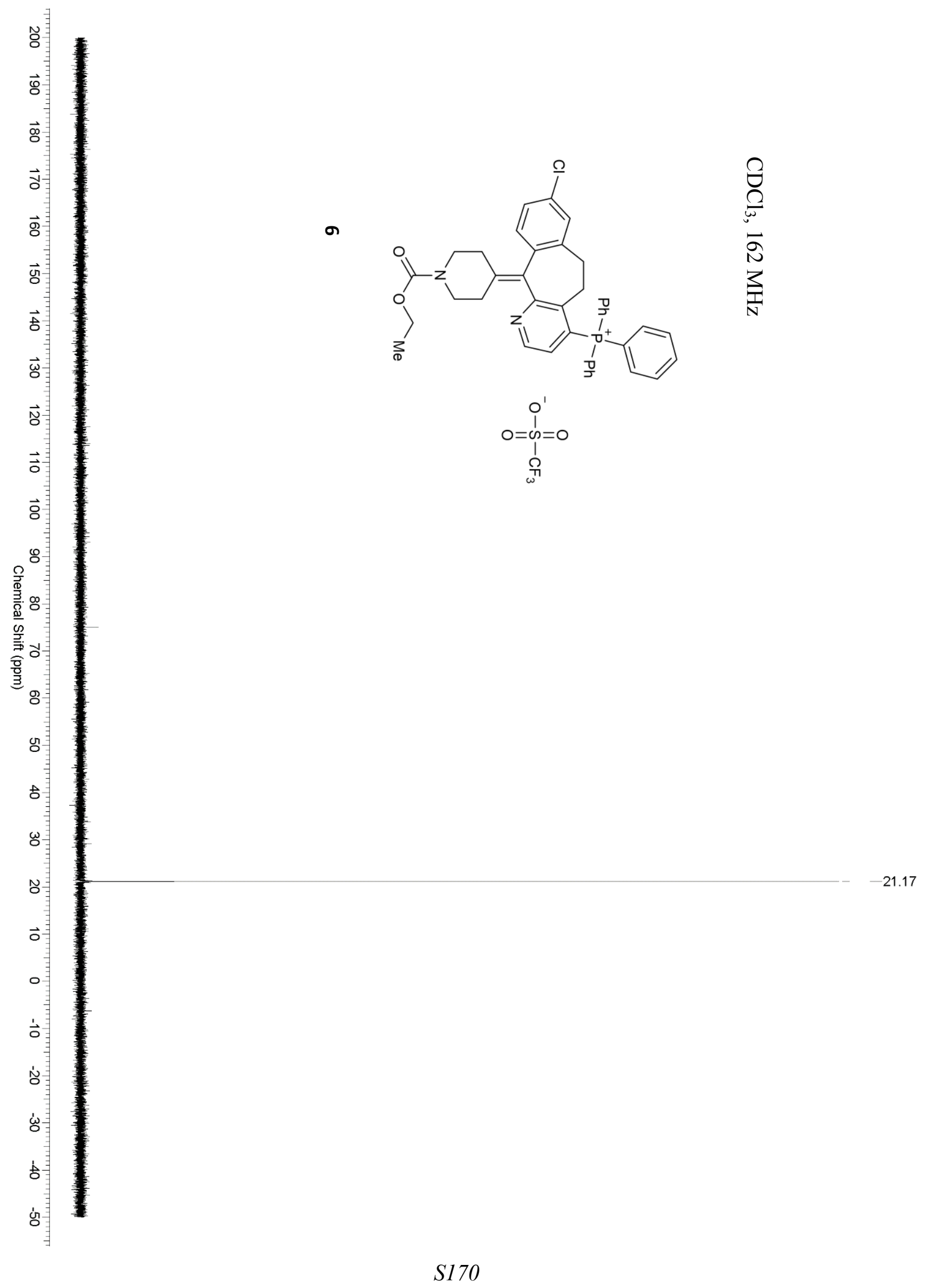




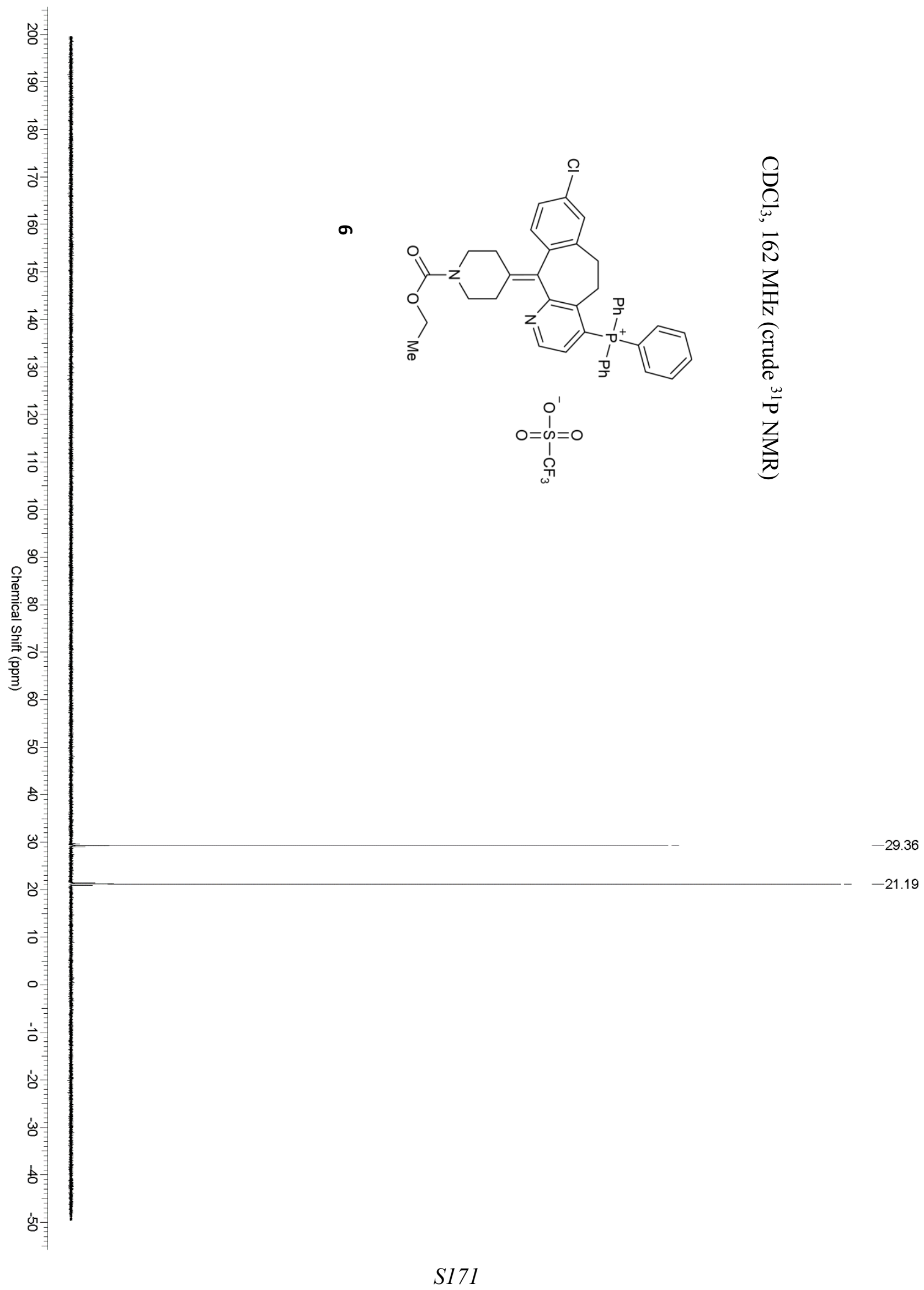




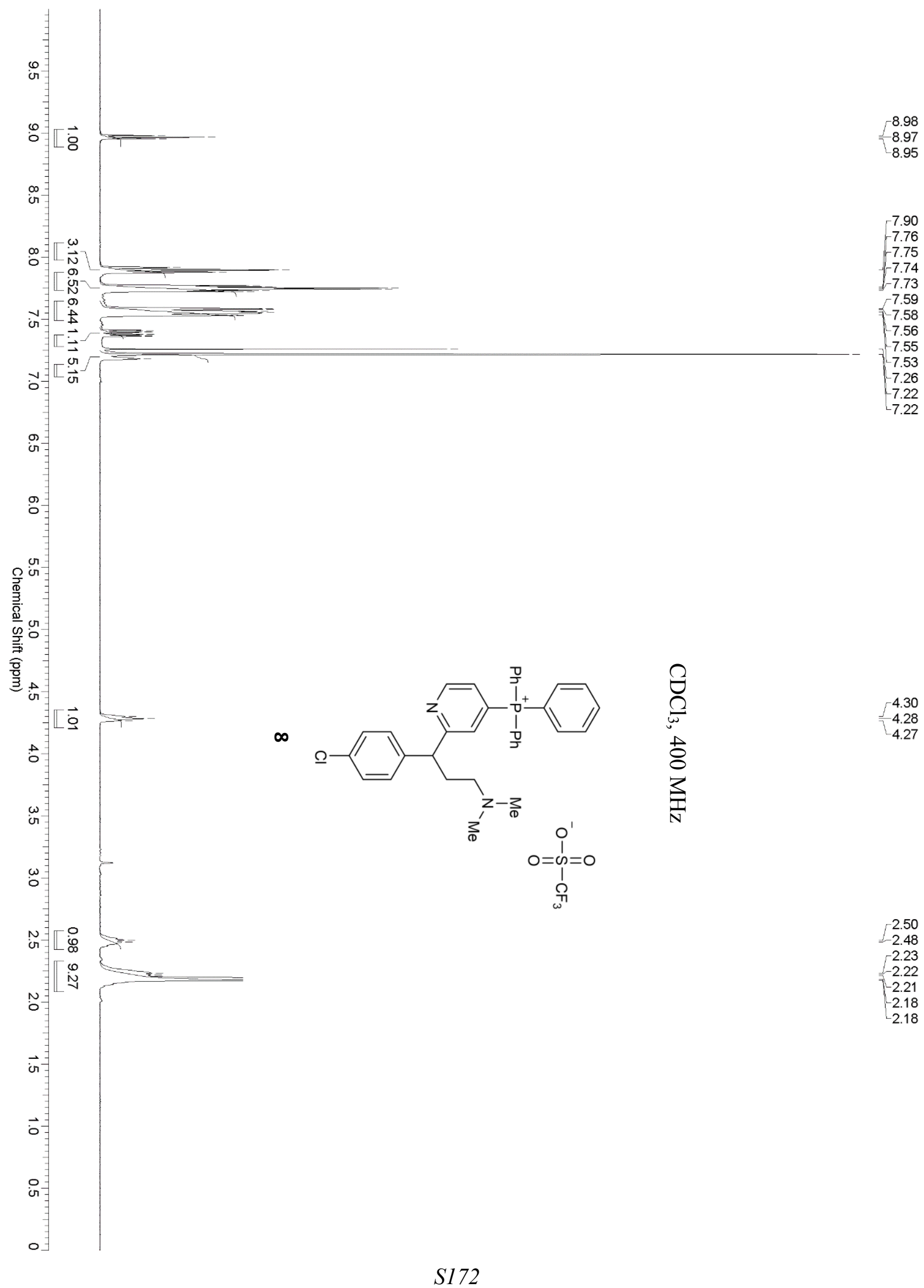




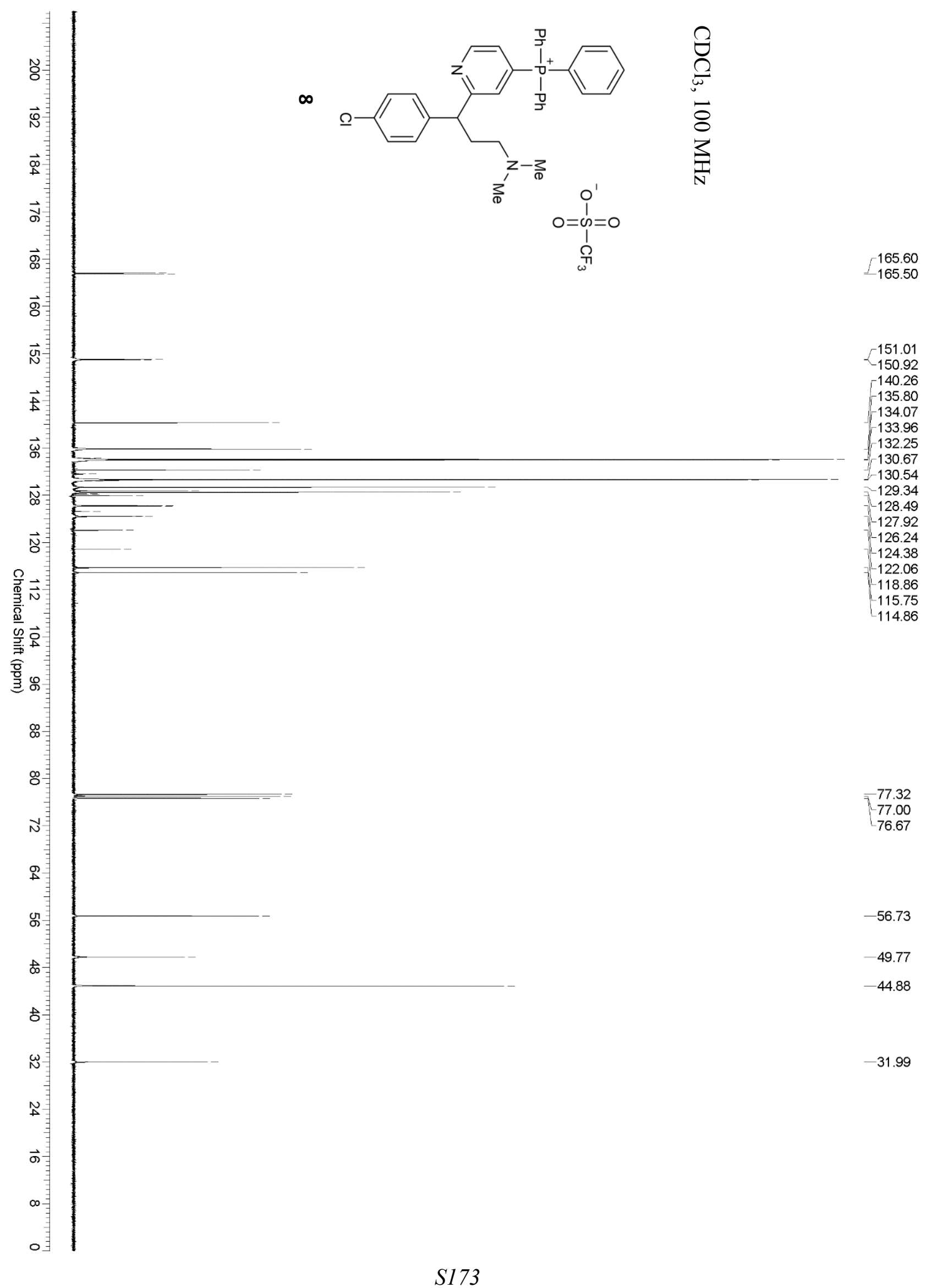




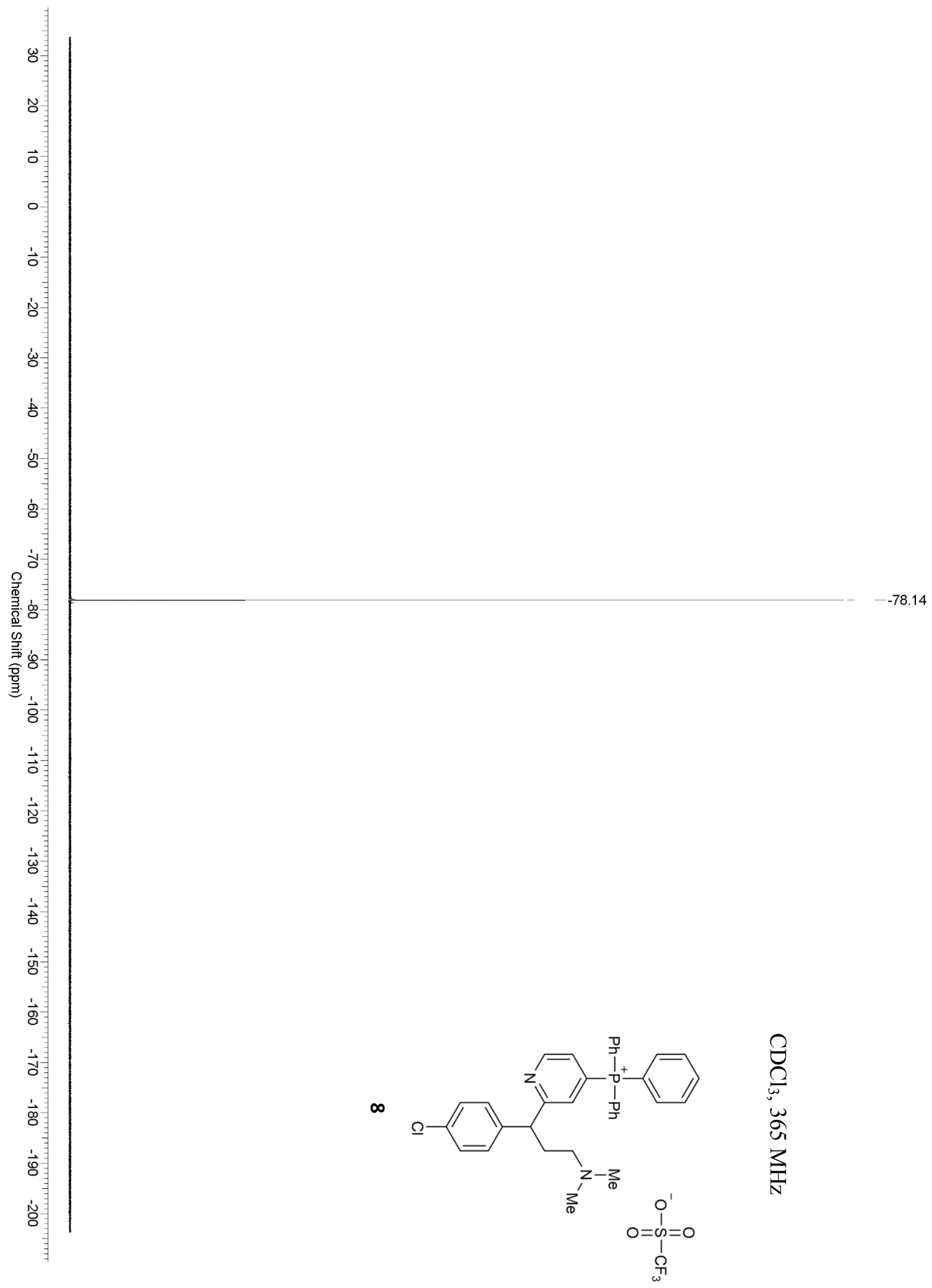




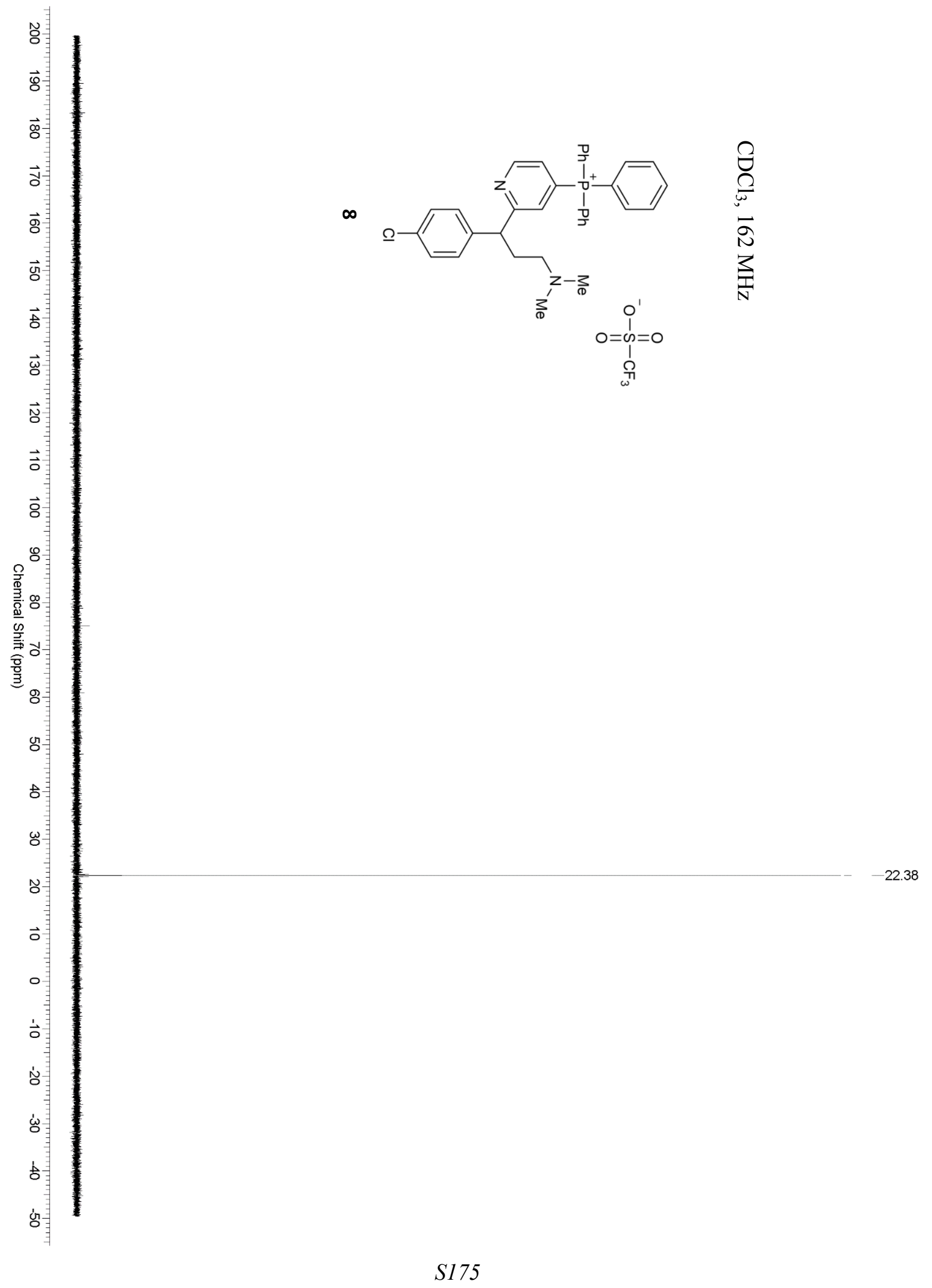




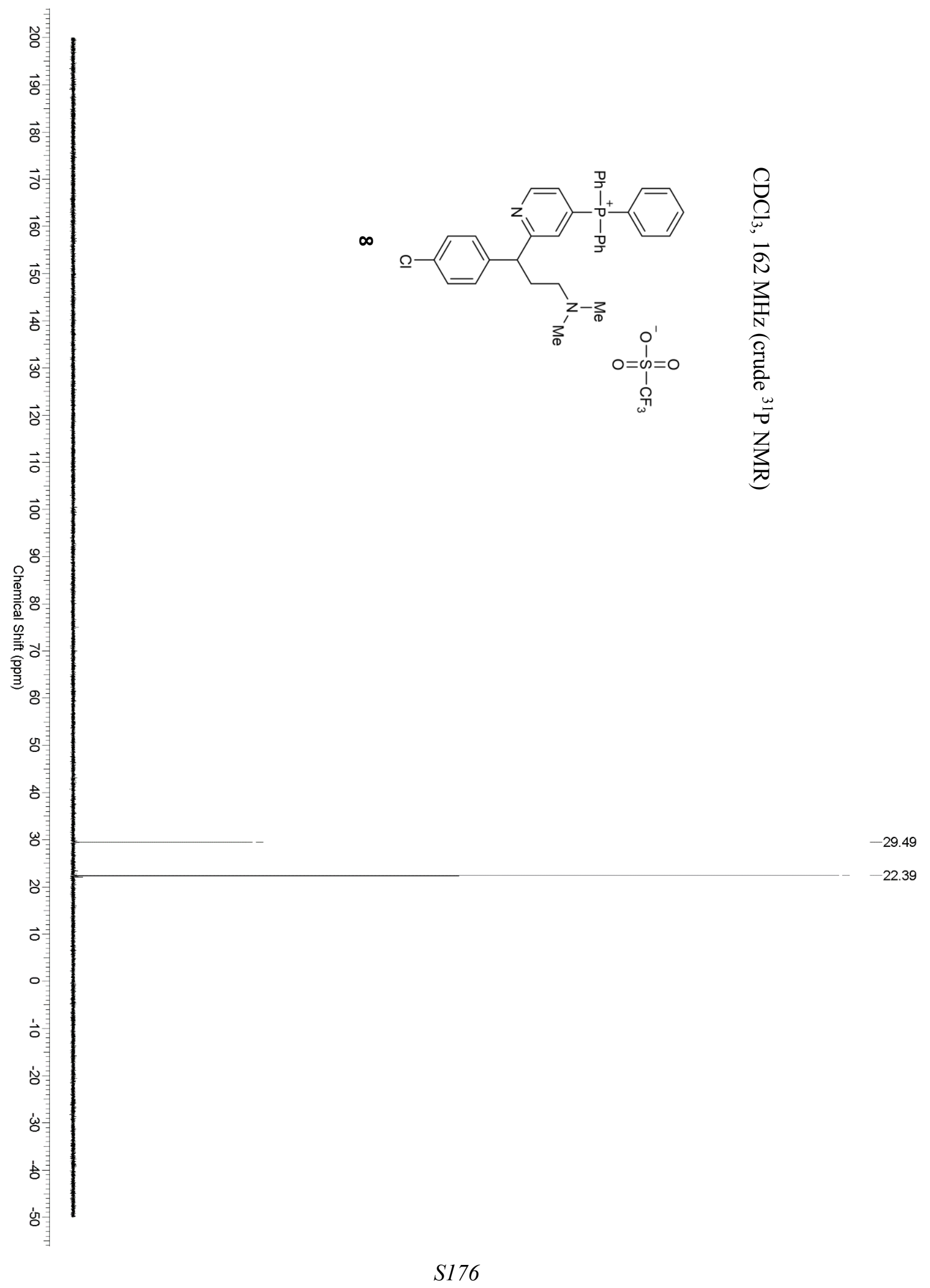




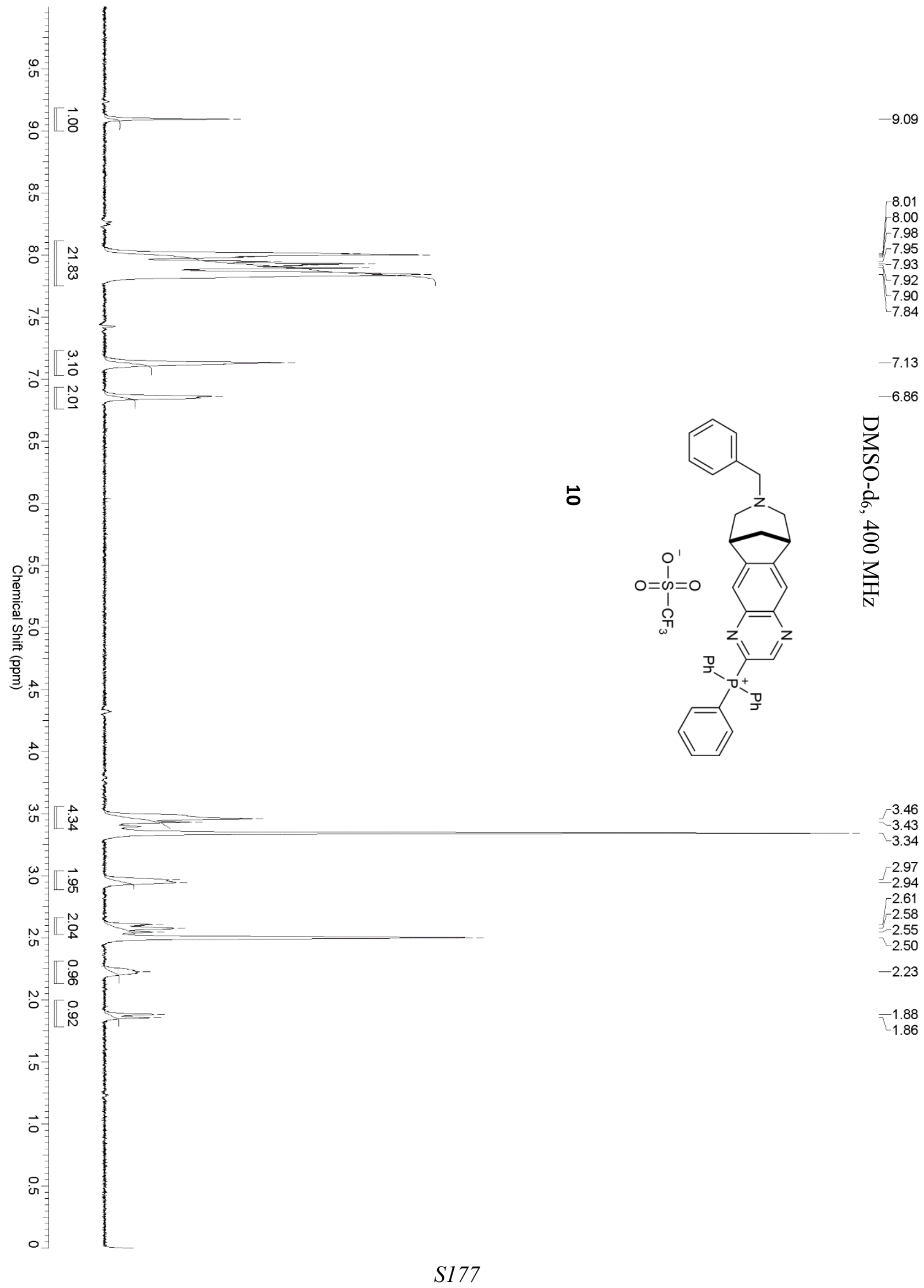




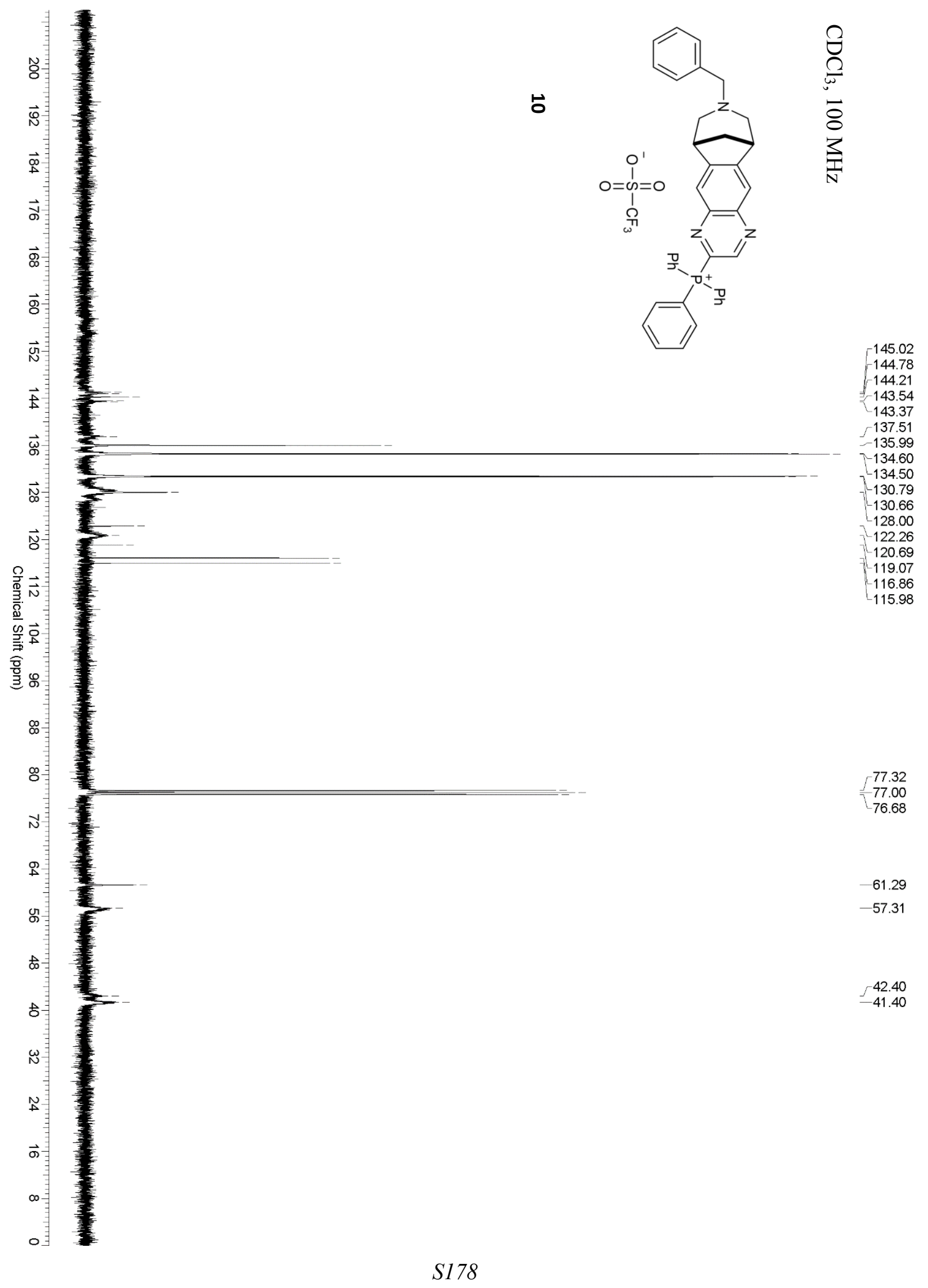




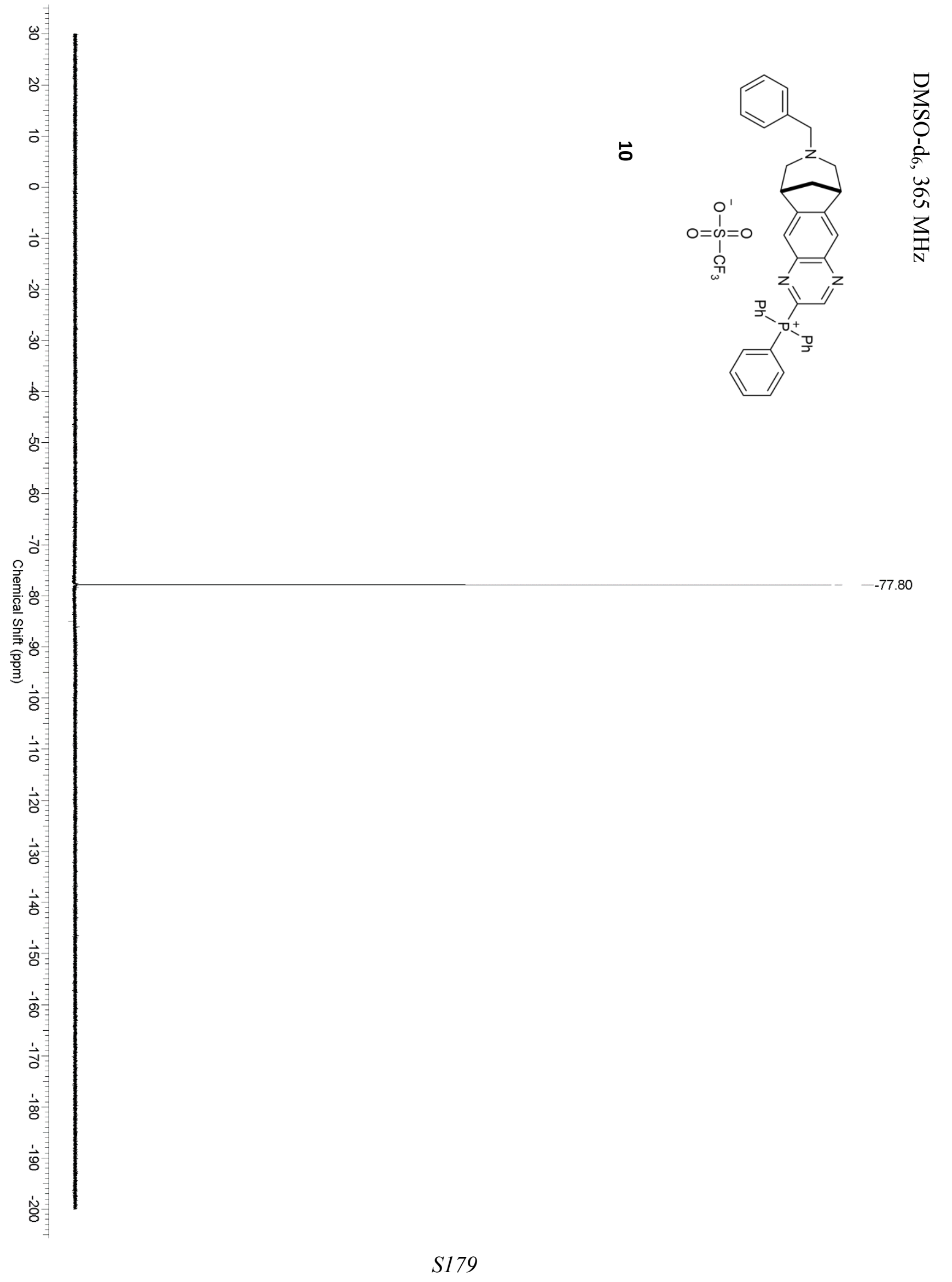




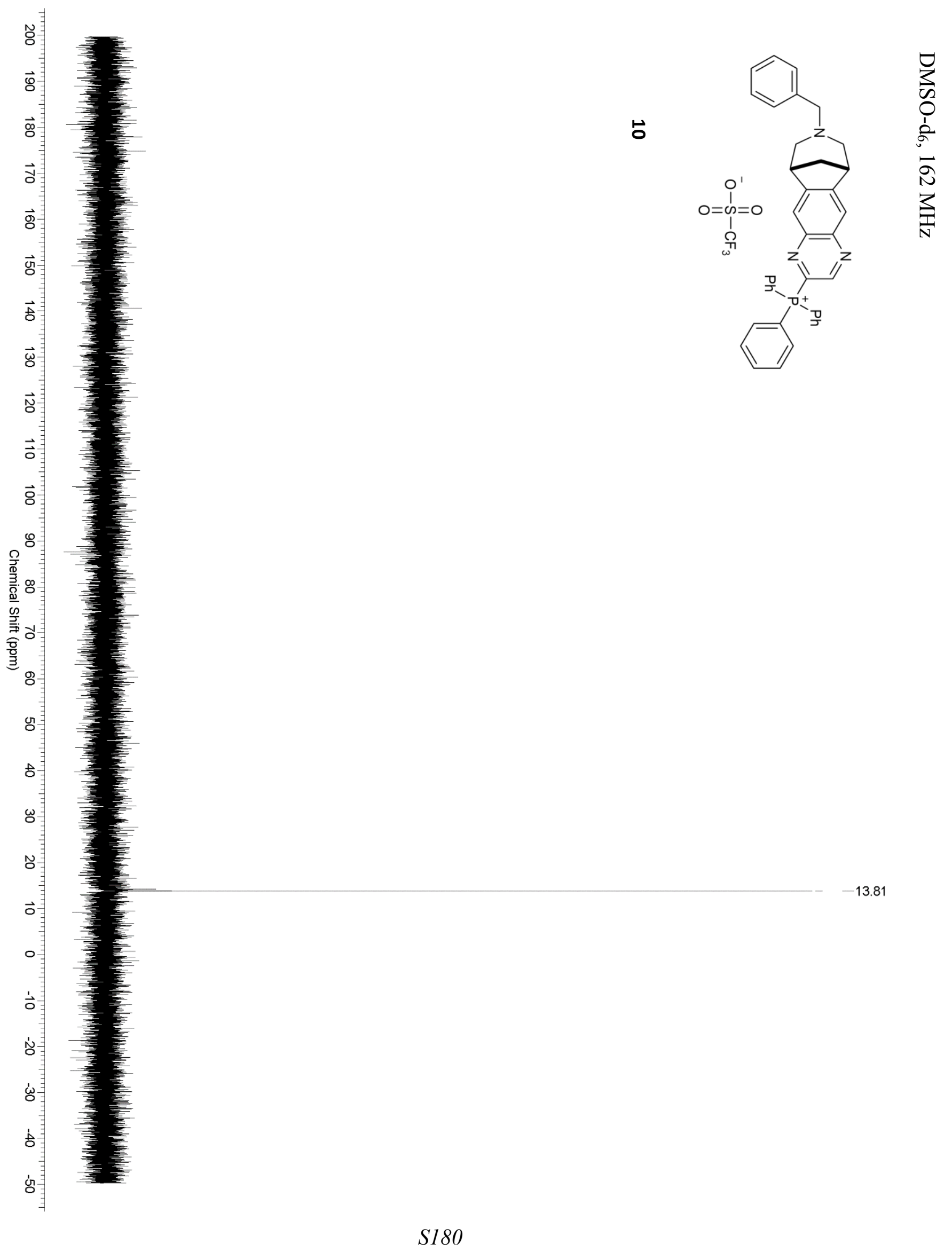




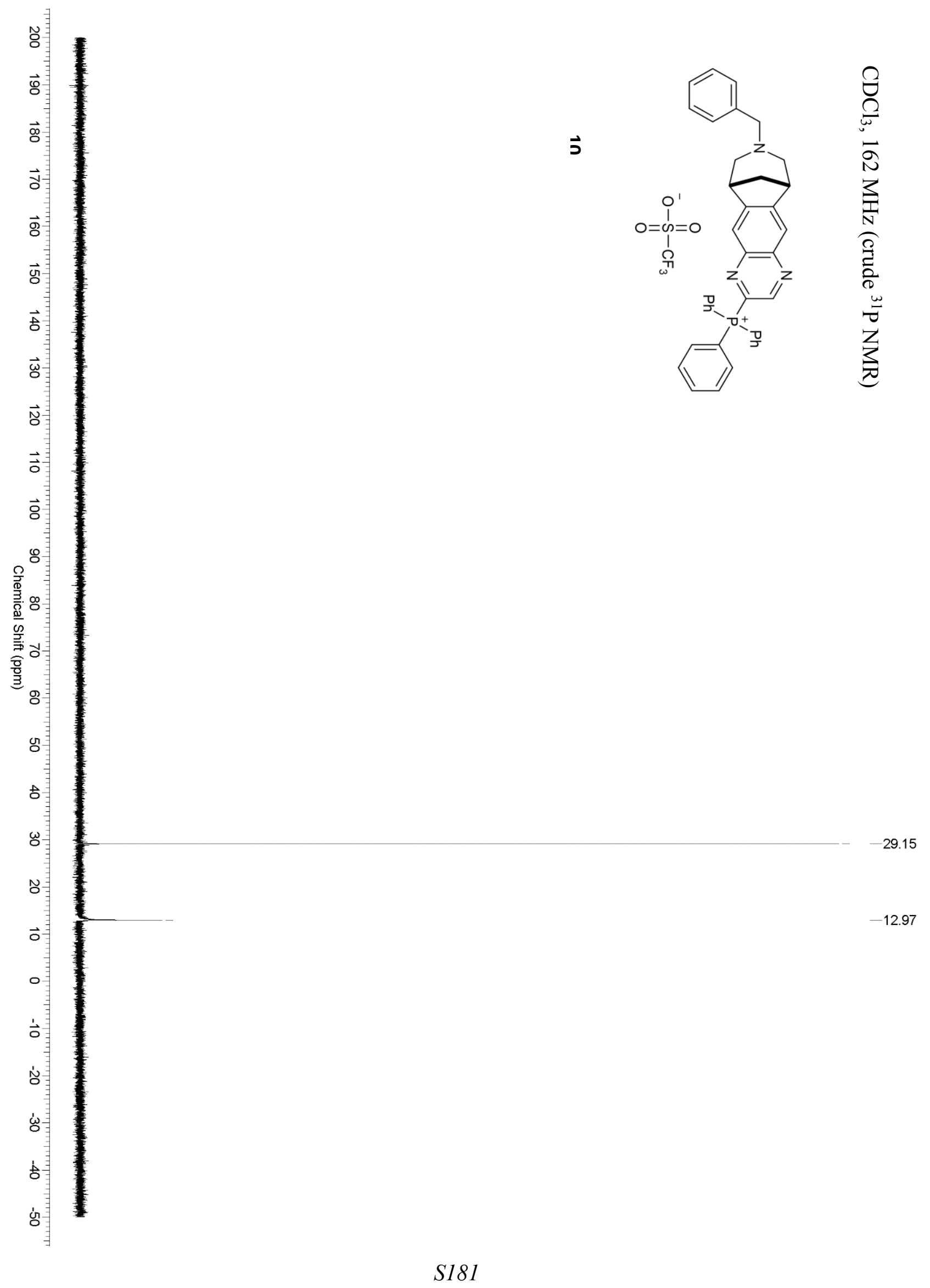




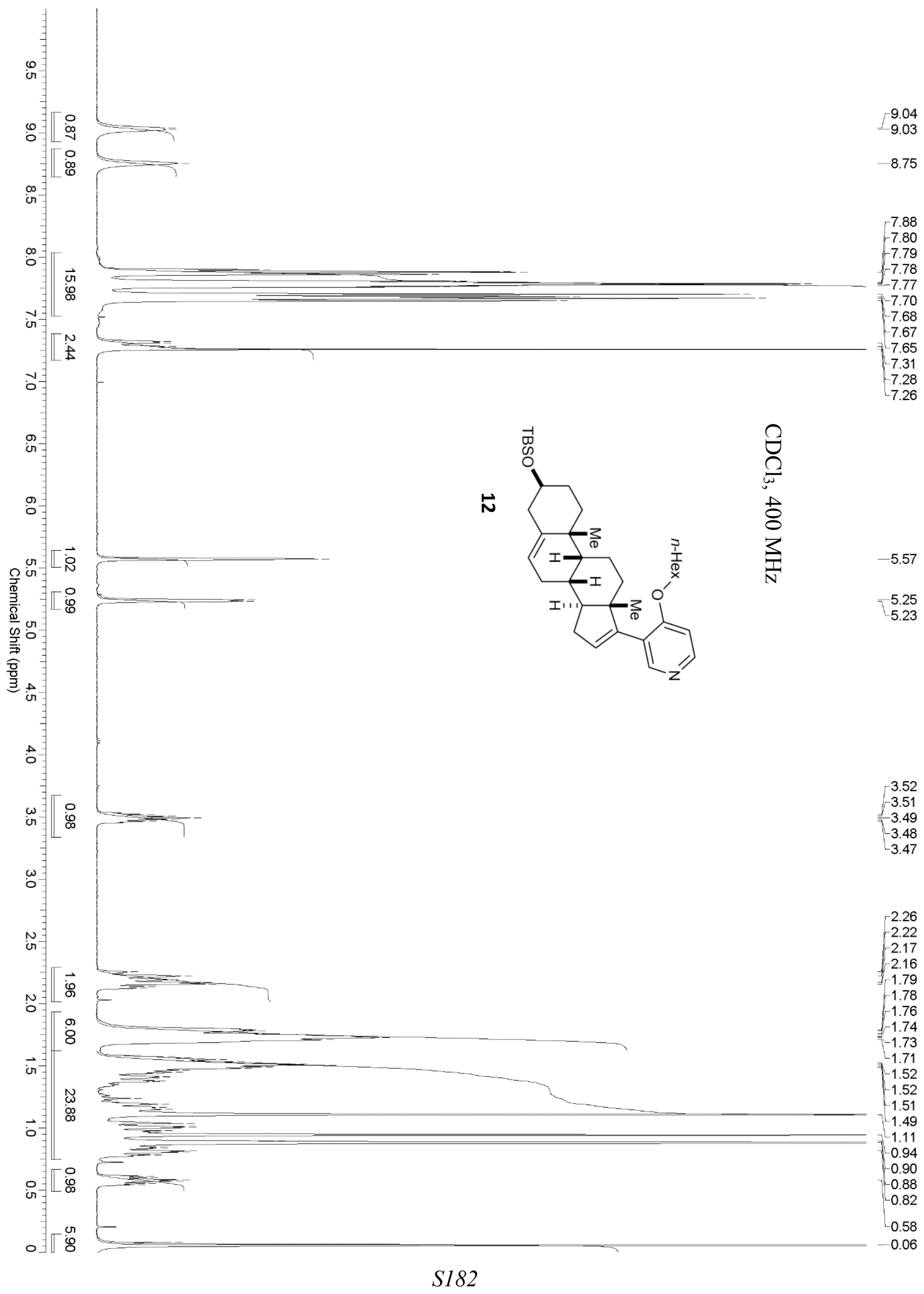




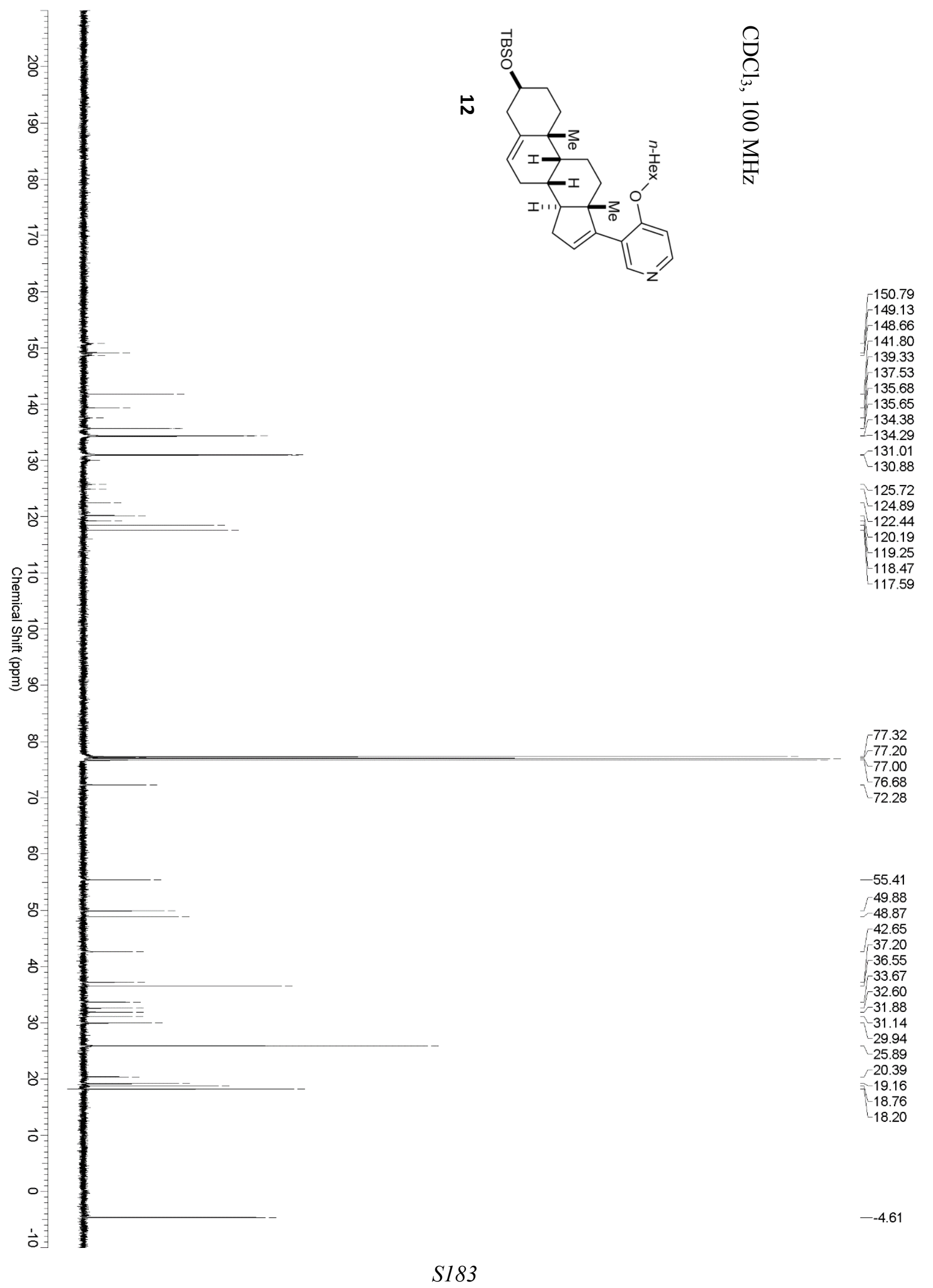




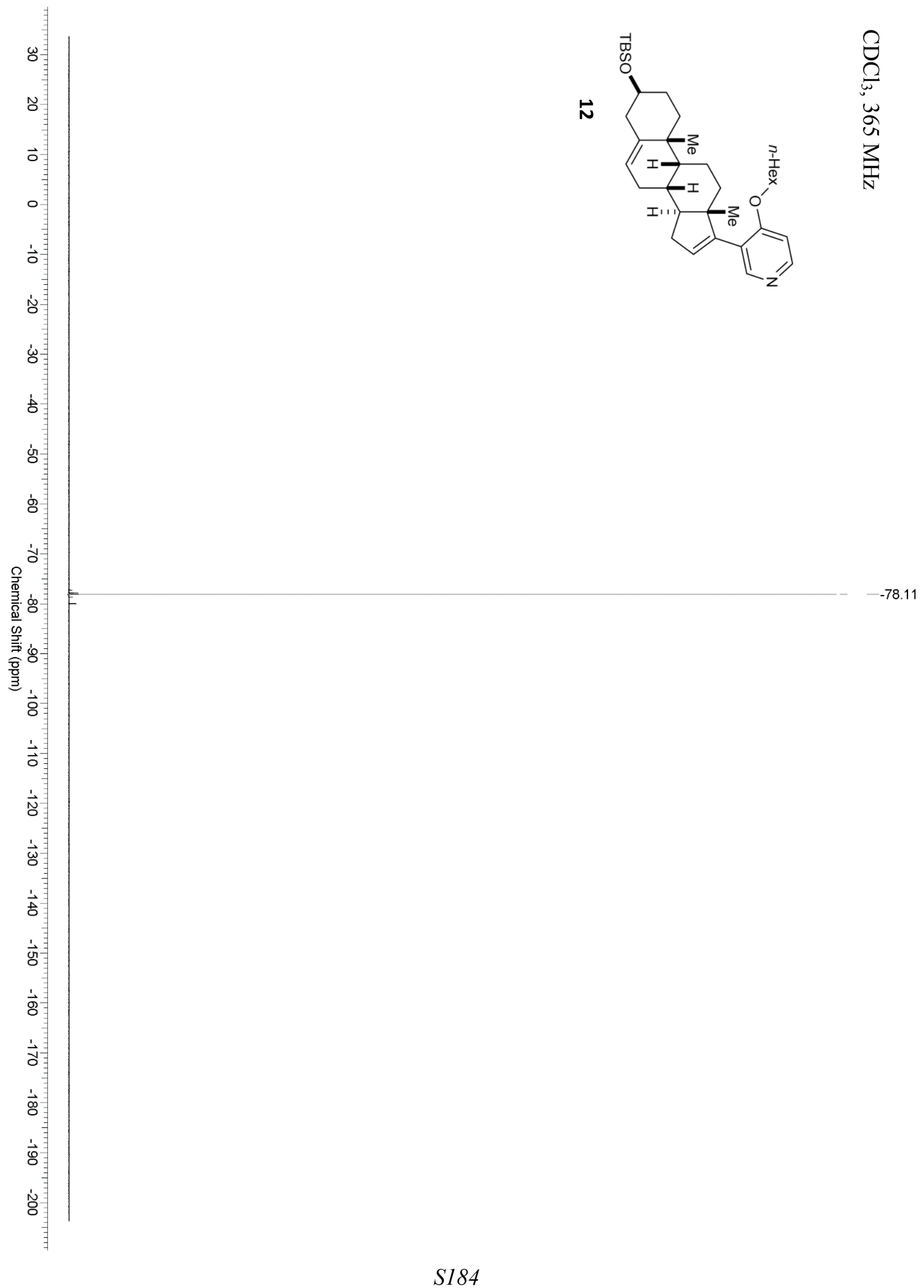




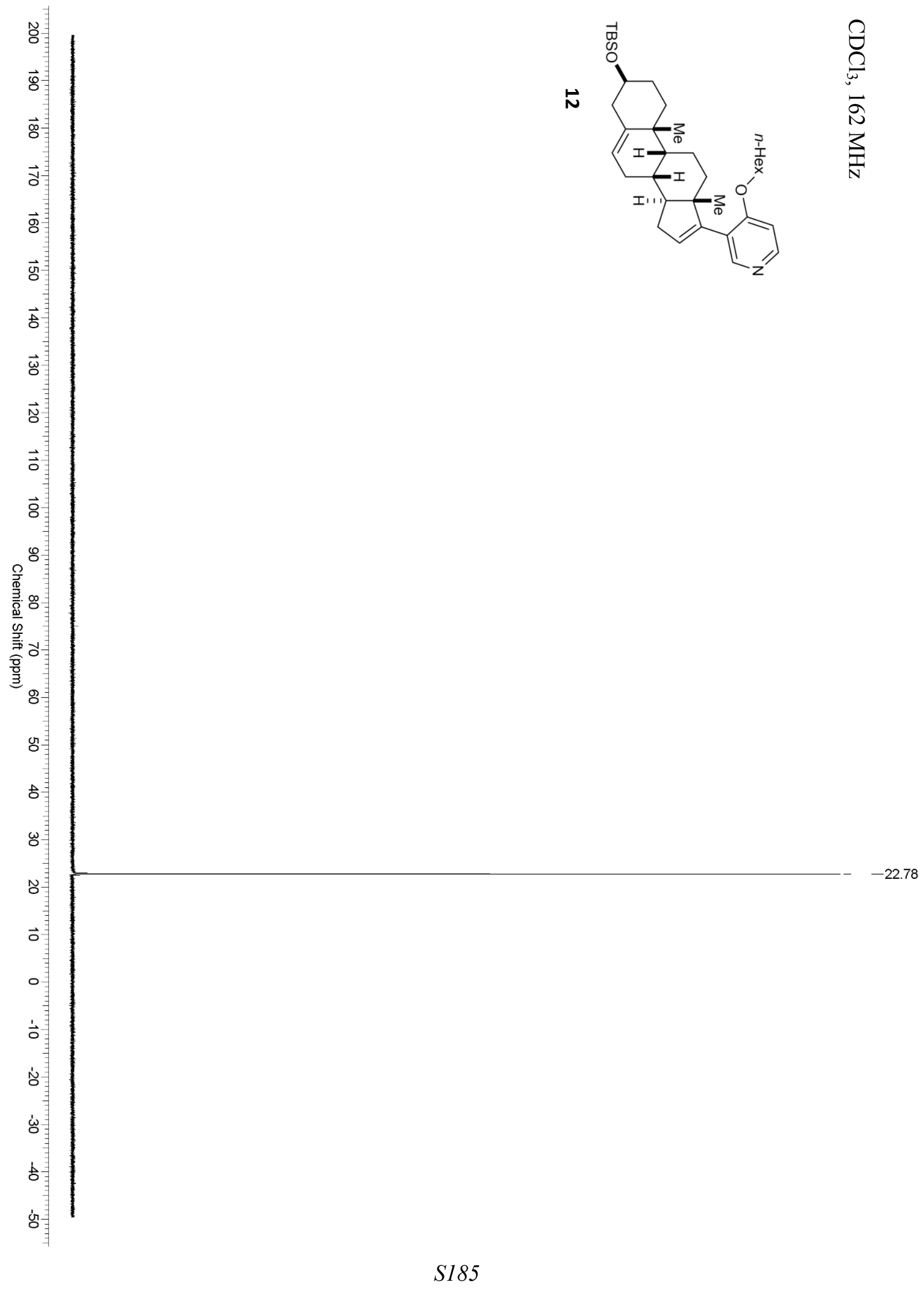




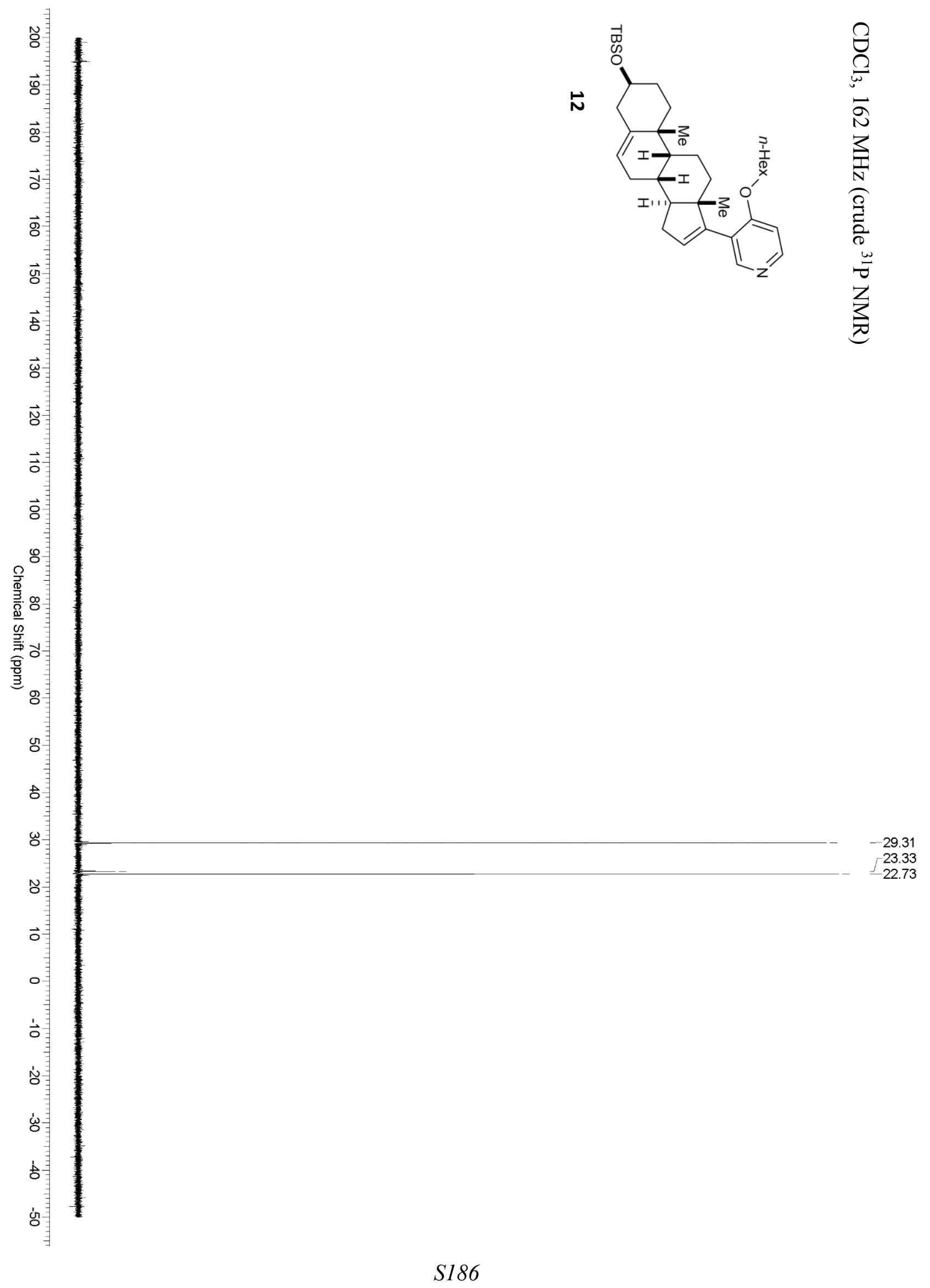




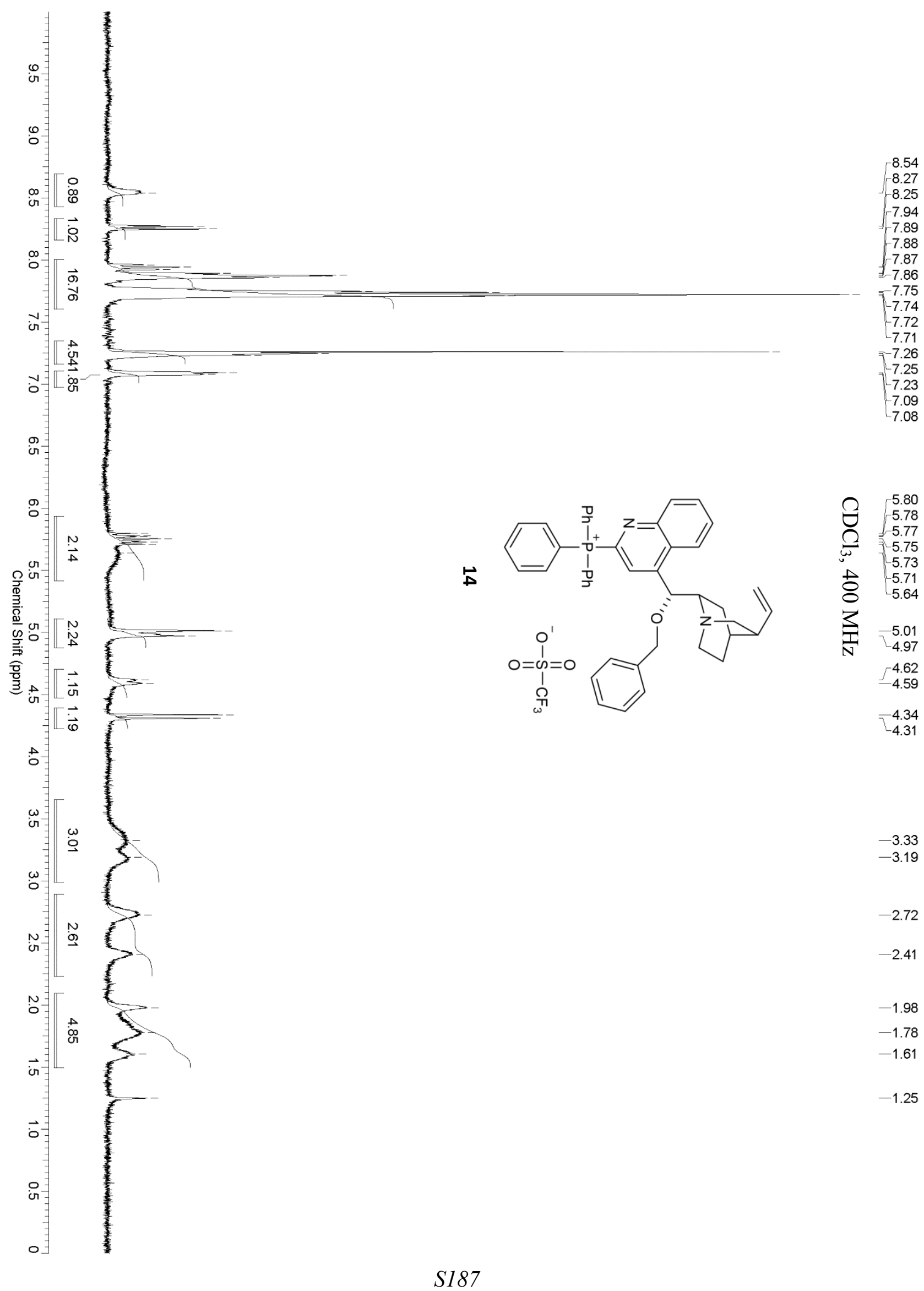




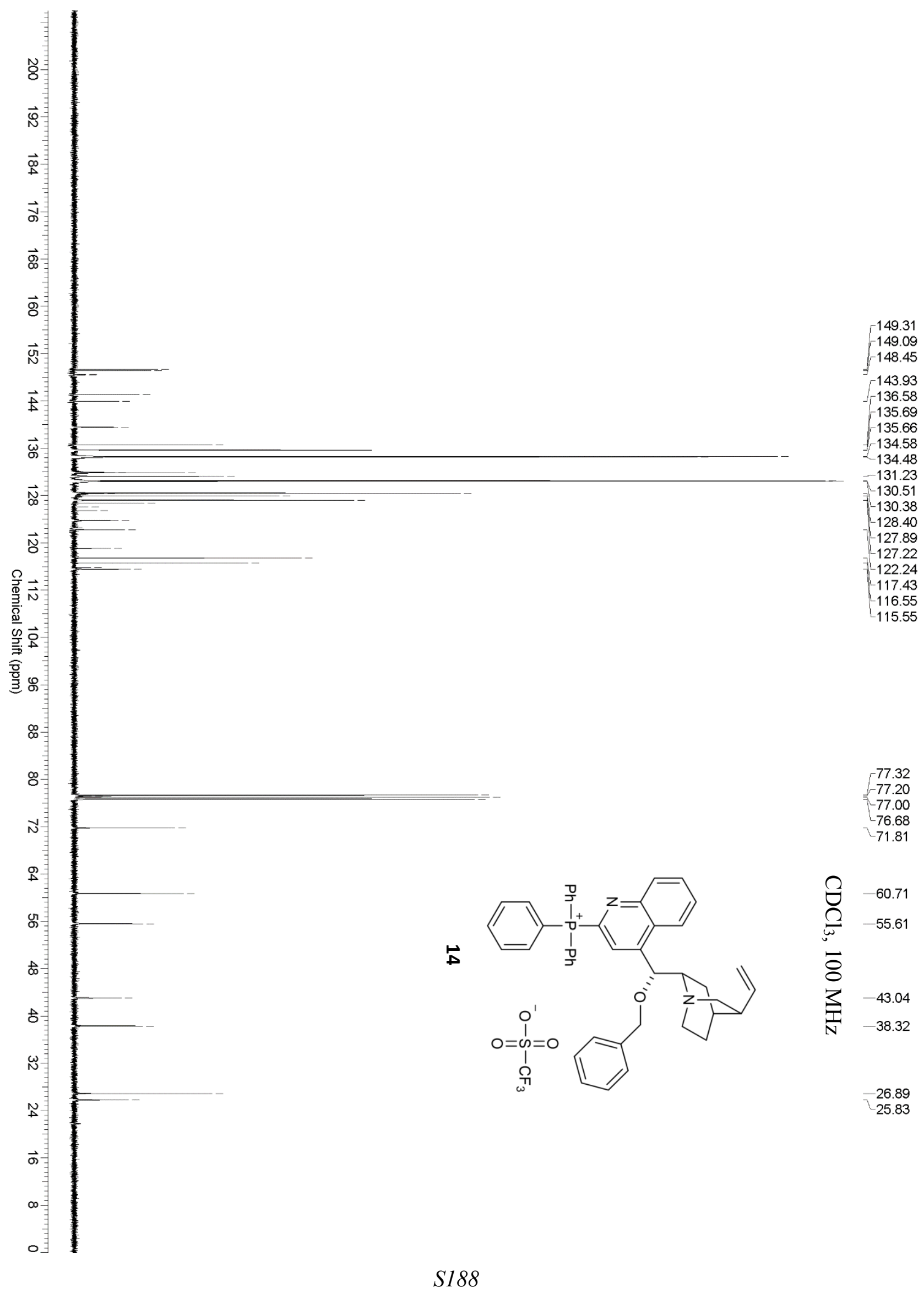




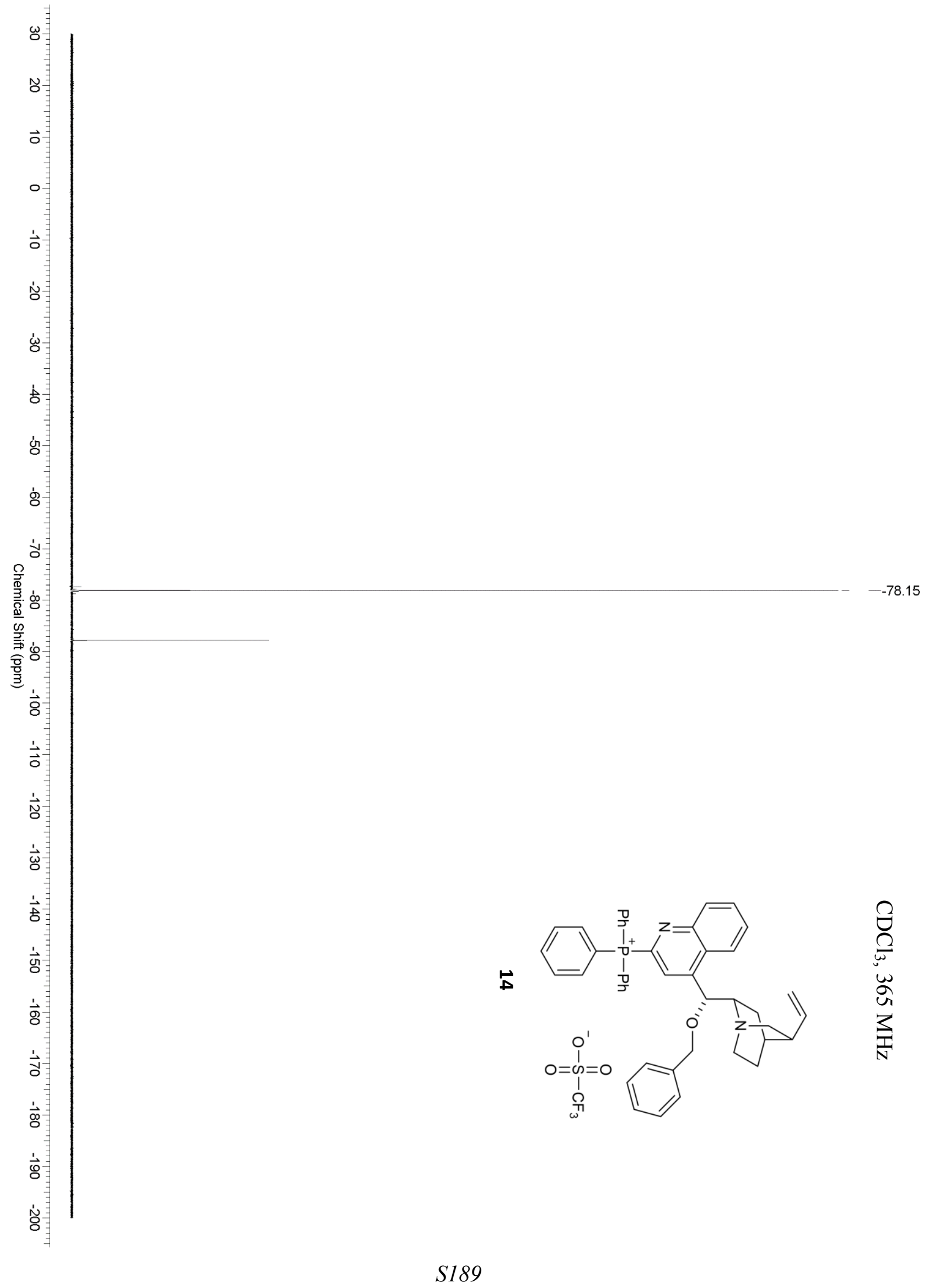




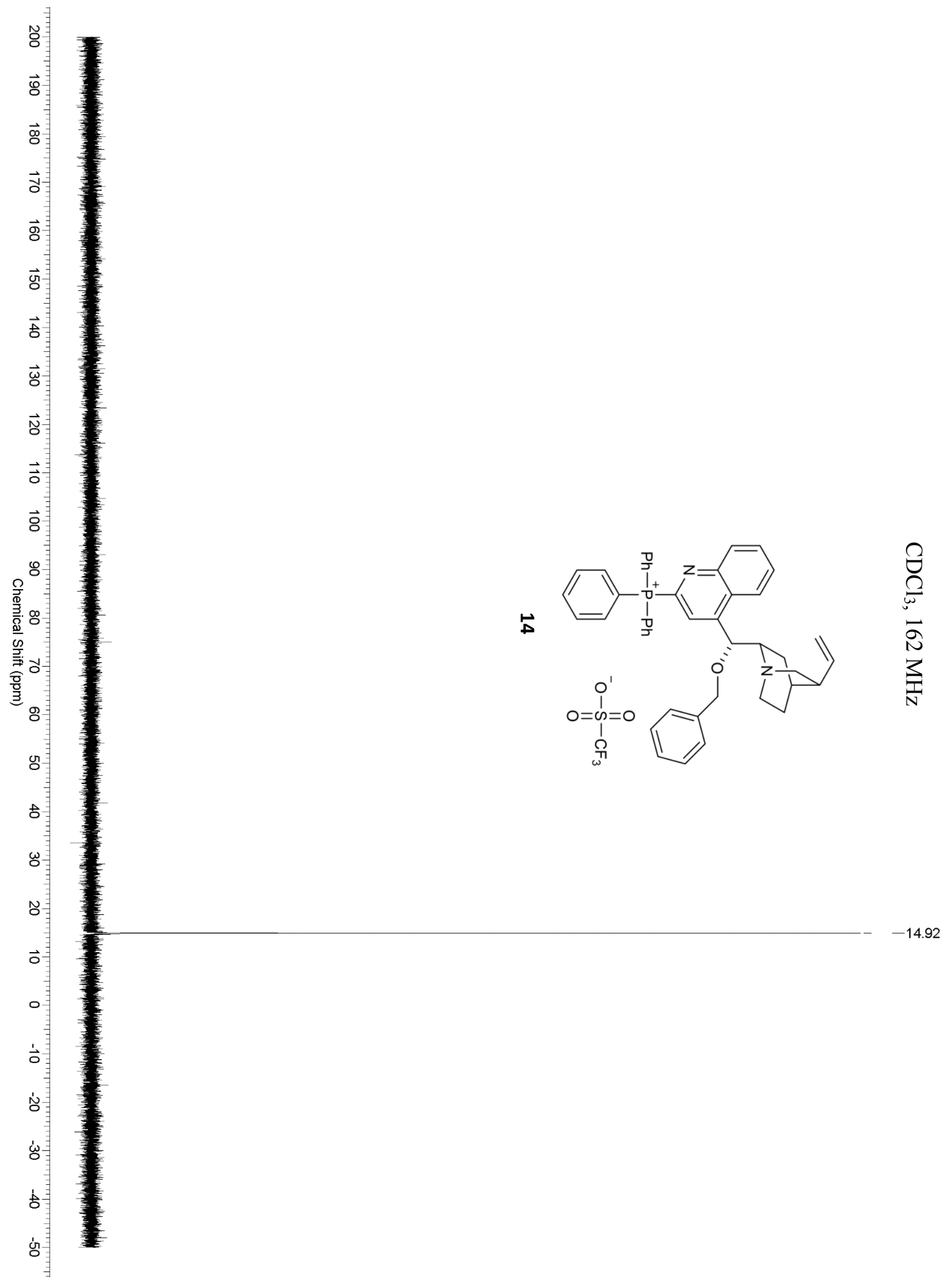




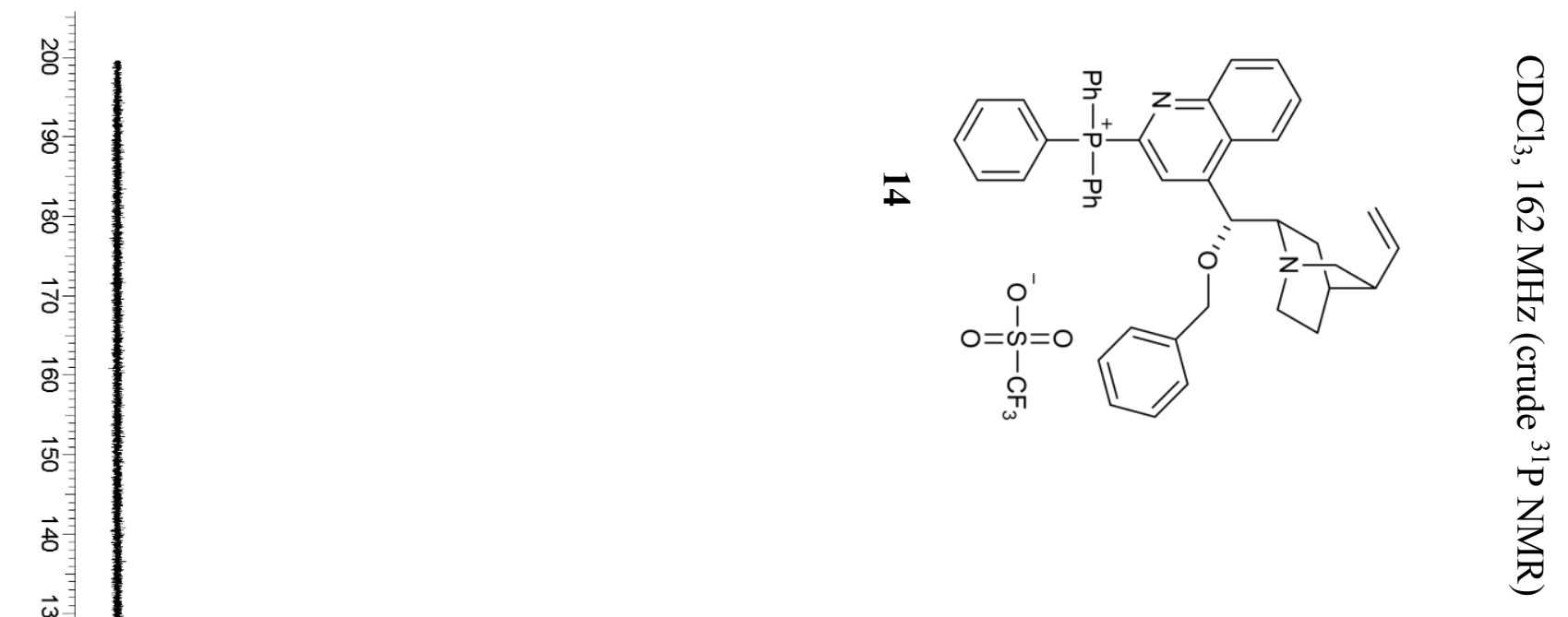

$\vec{N}$
$\vec{\partial}$
$\vec{\Delta}$

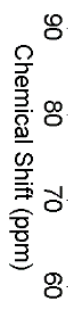

on

吕

$\omega$

w

읔

o

奉

宣青

定奉

ั่

山े

古

的春 


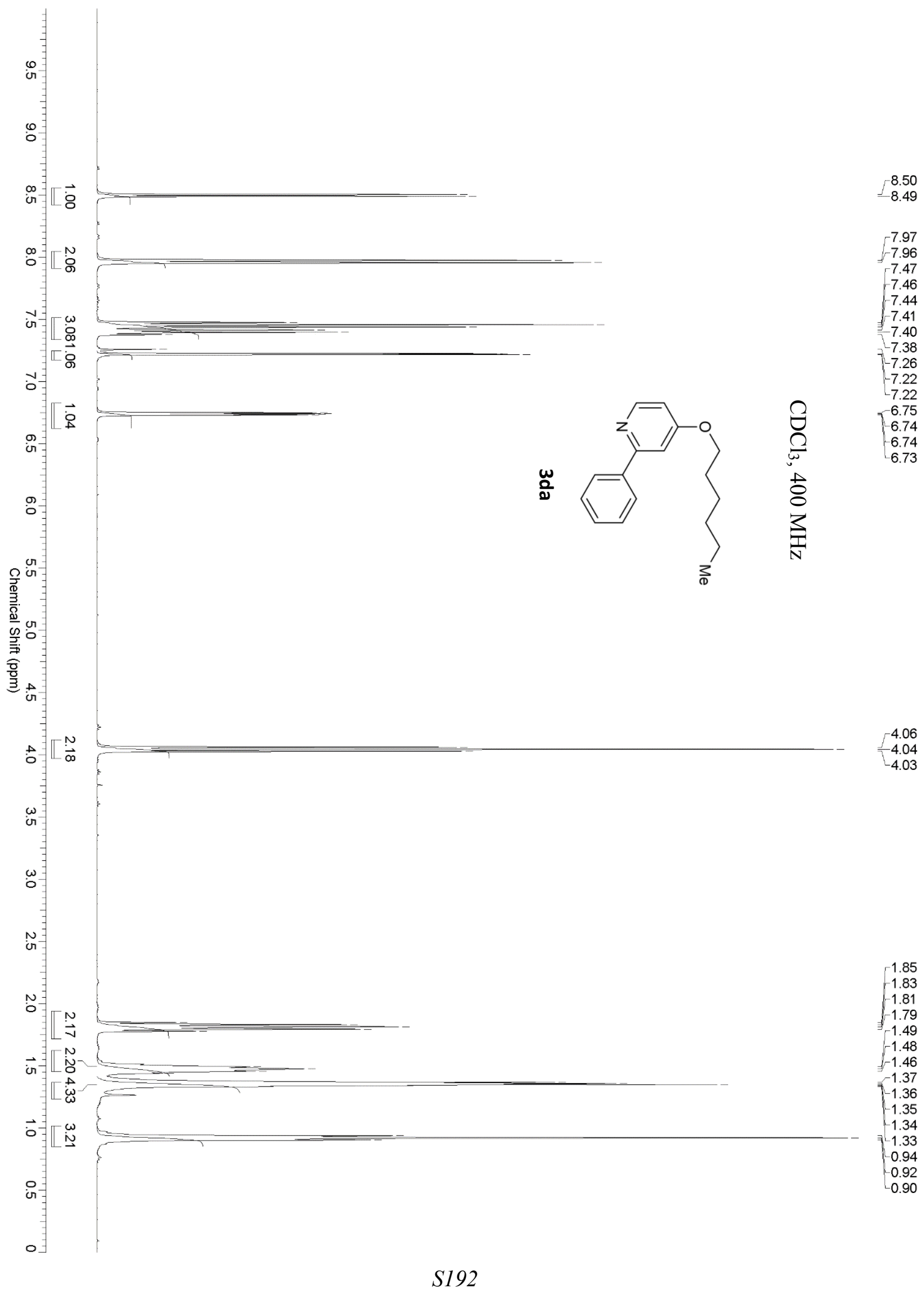




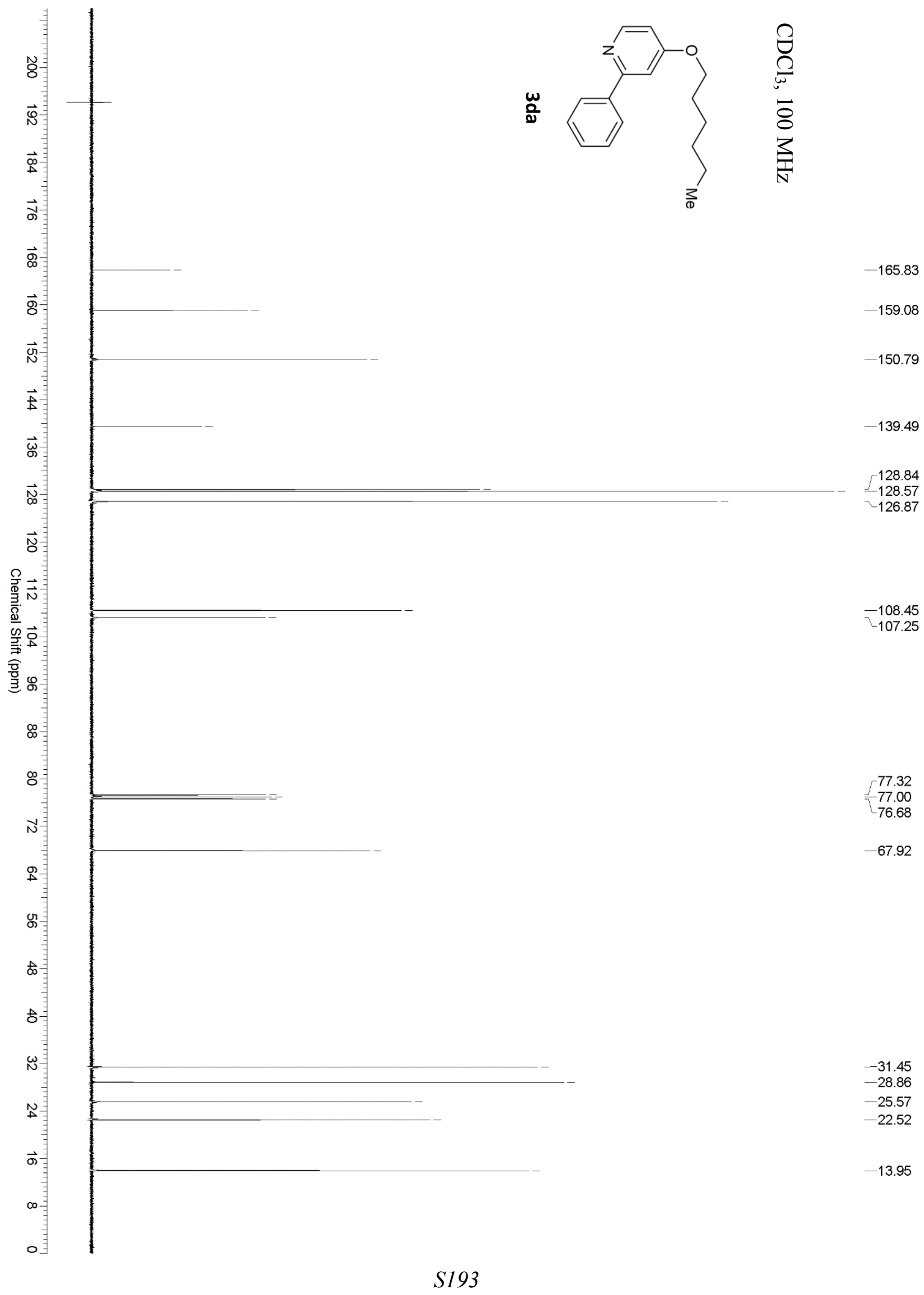




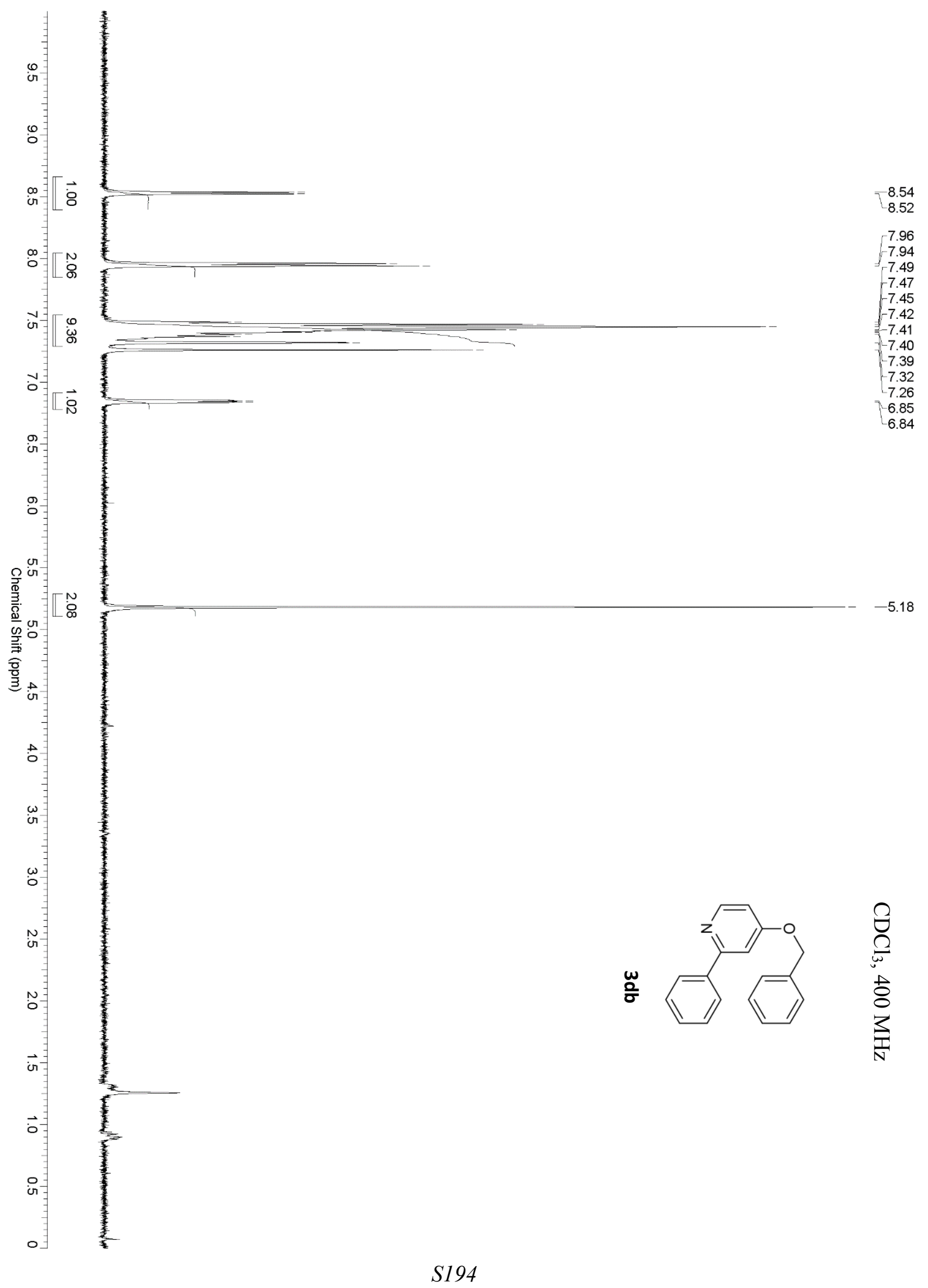




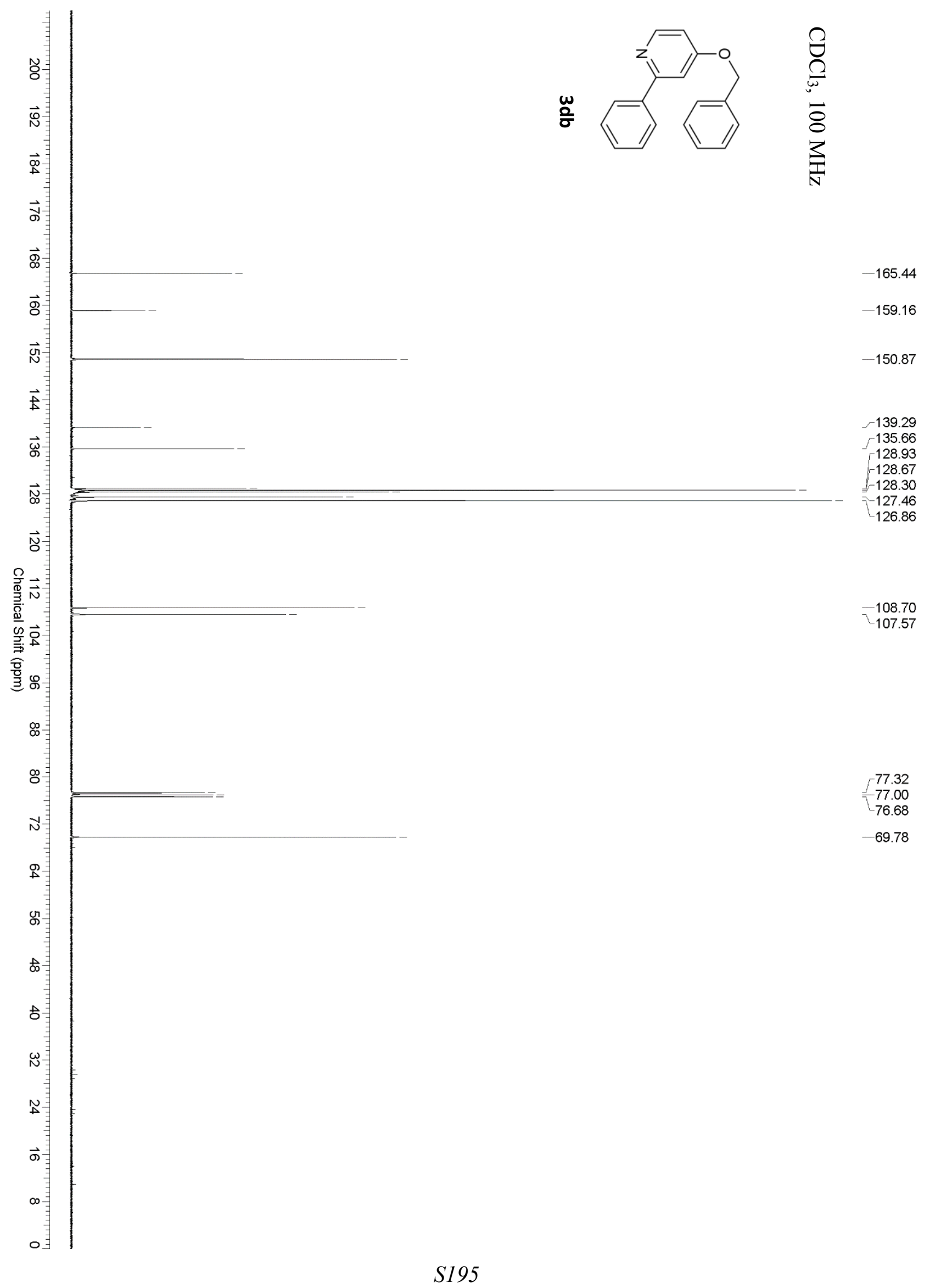




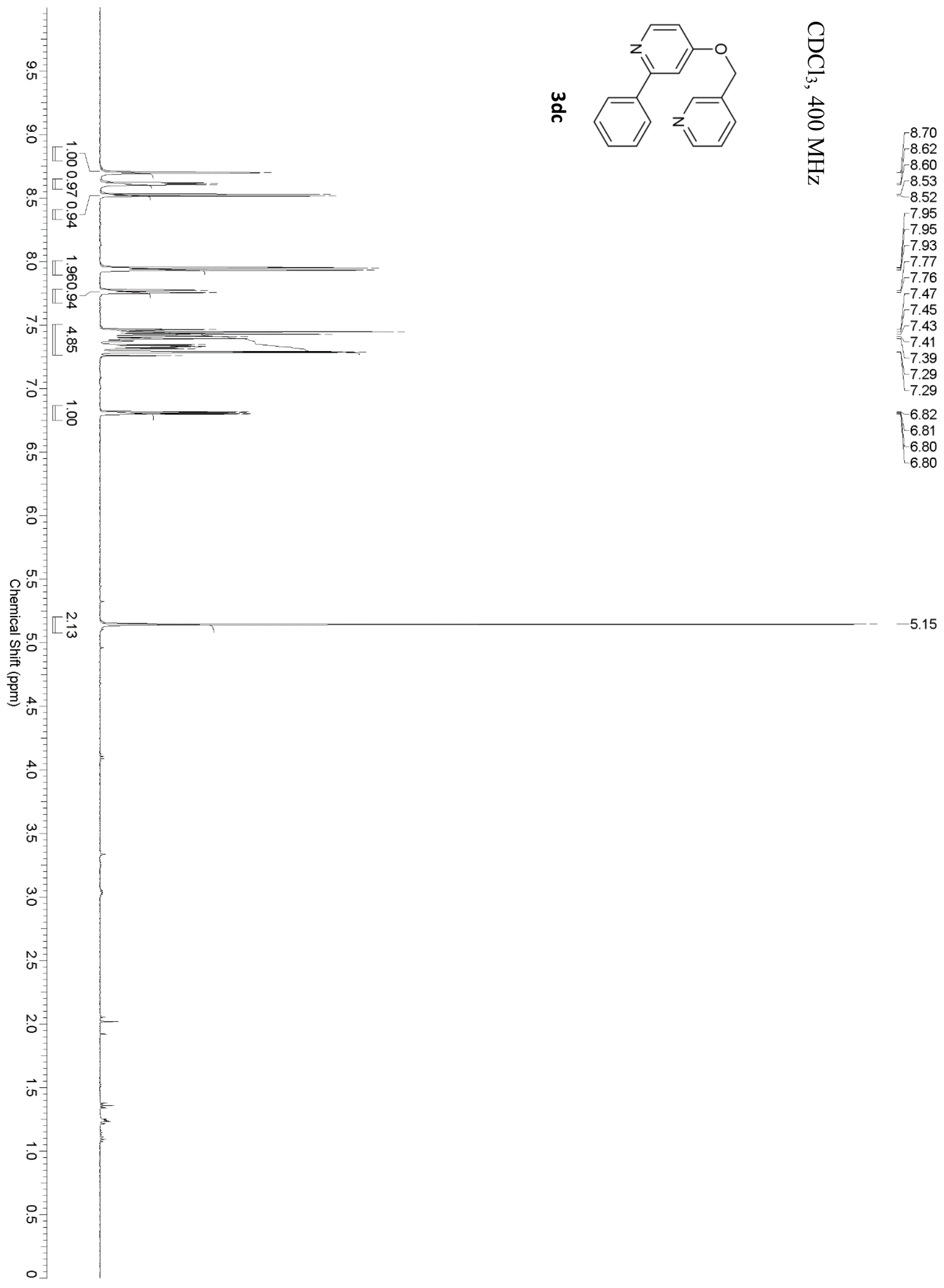




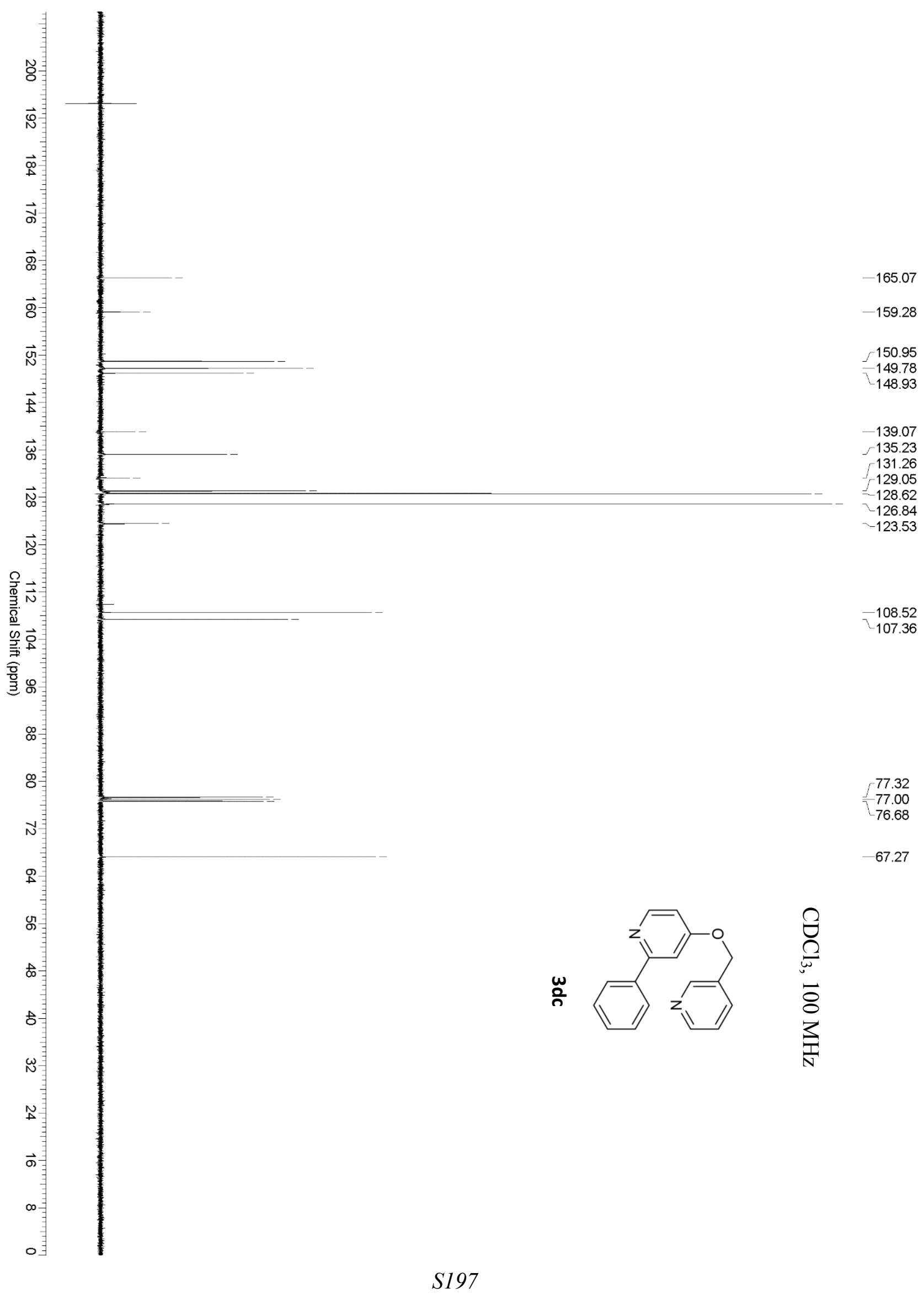




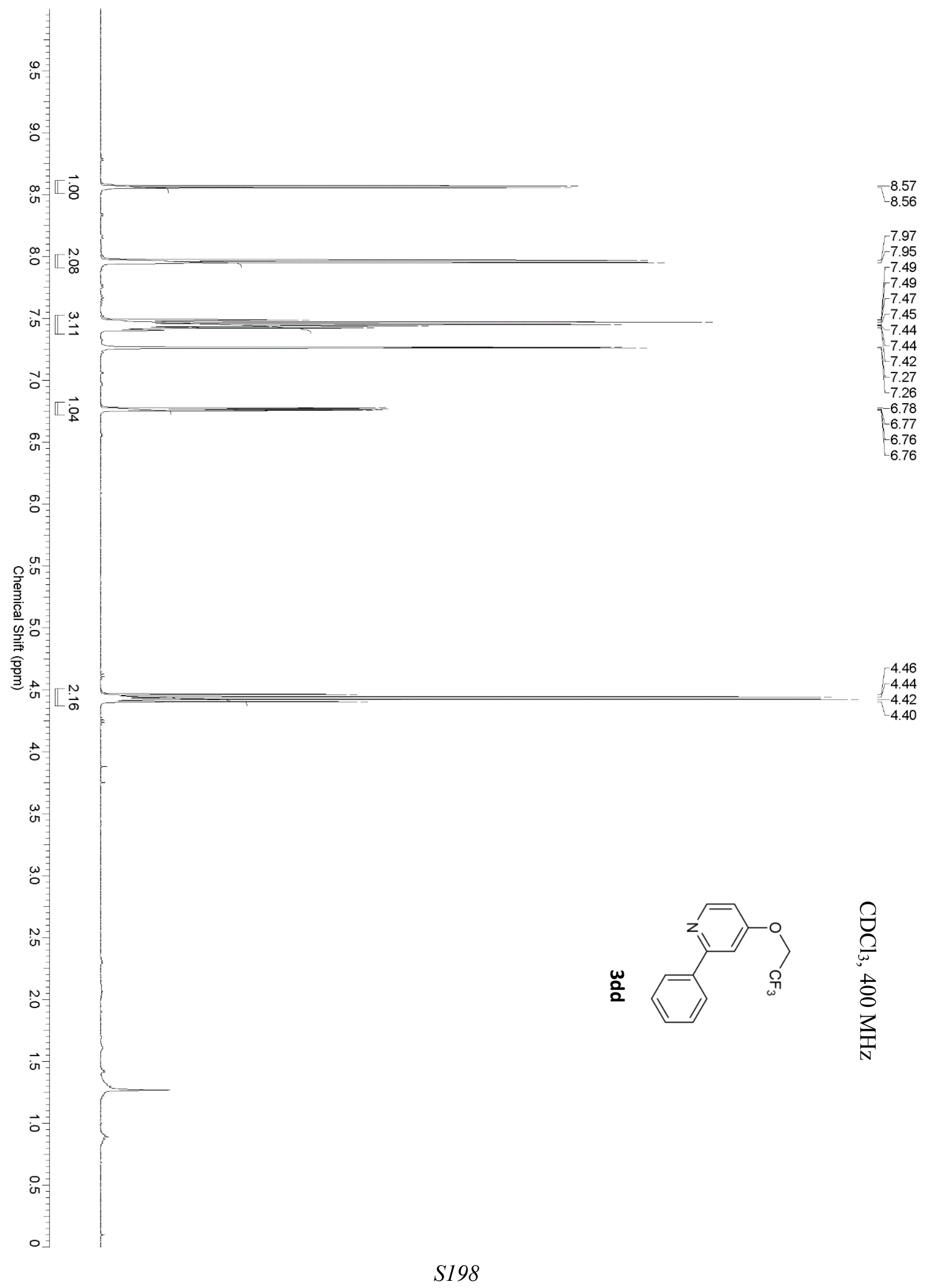




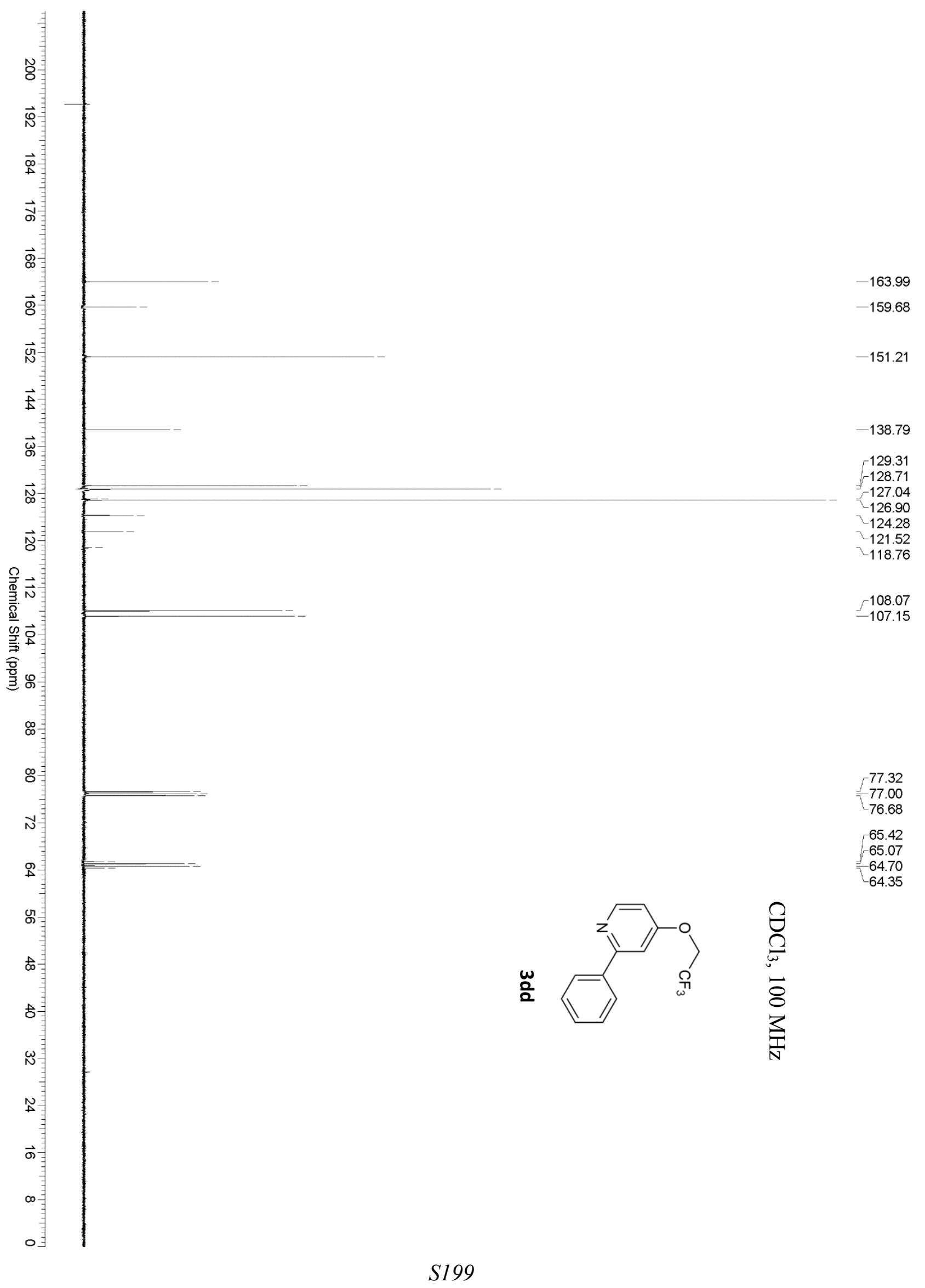




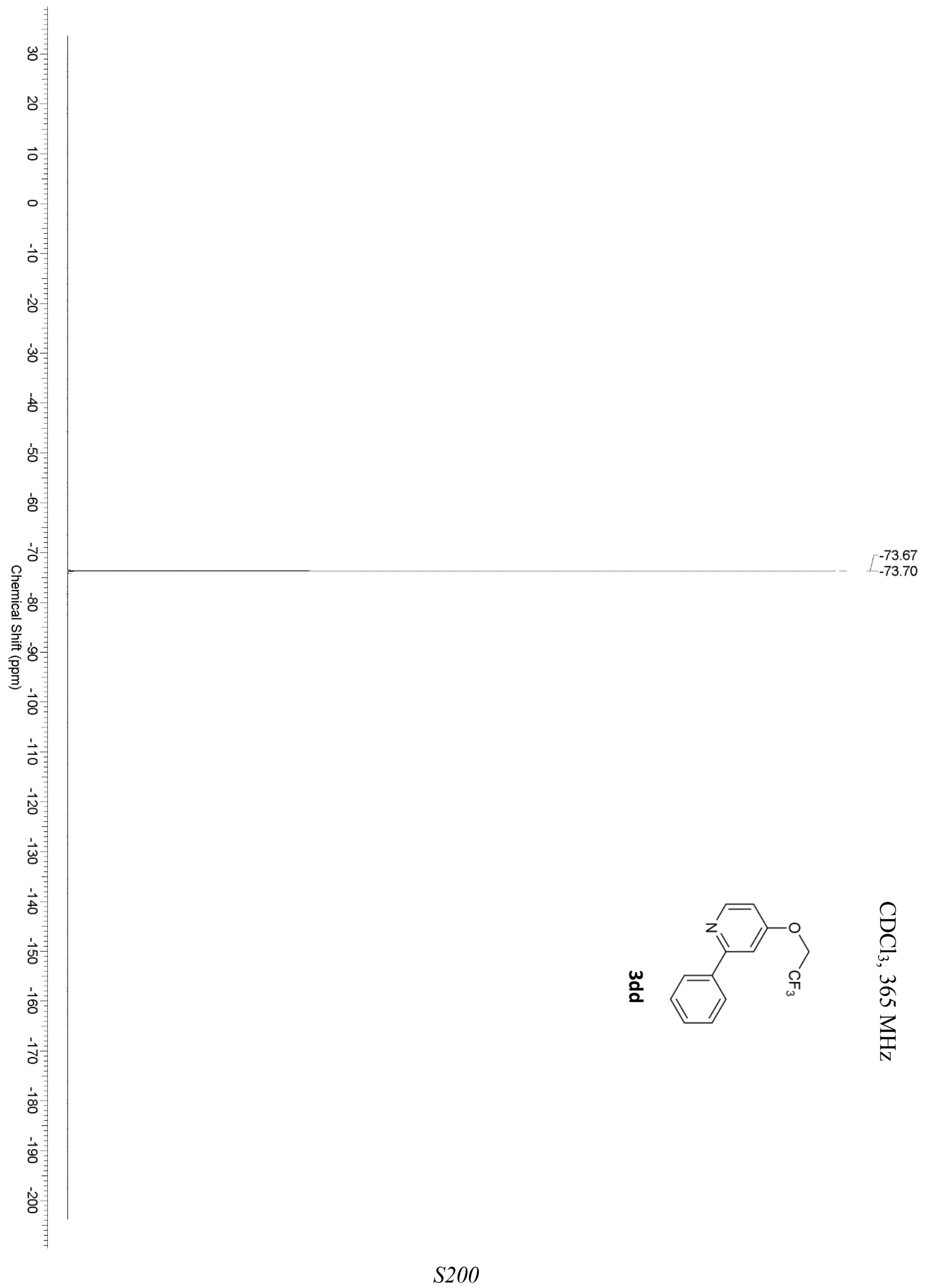




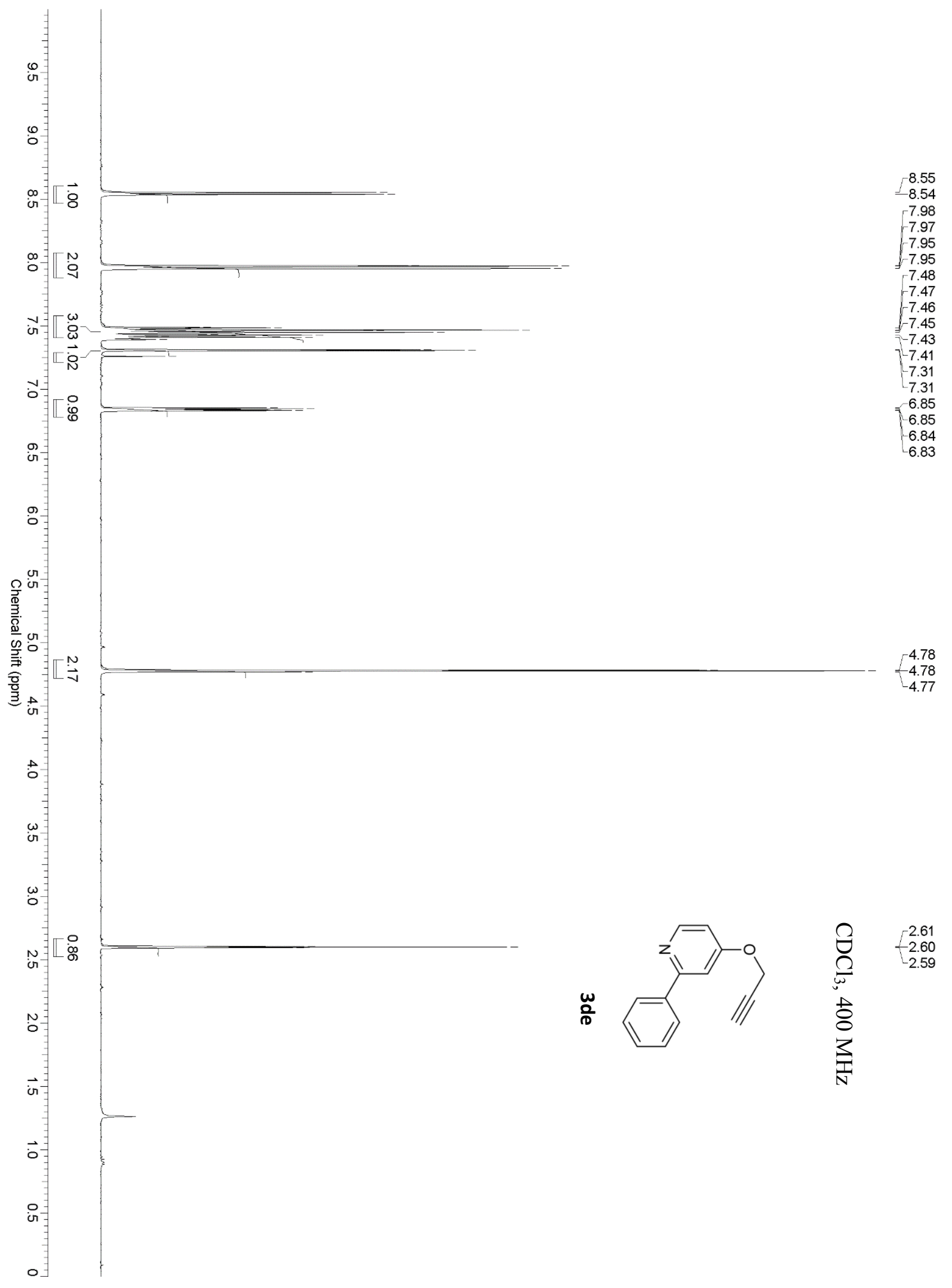




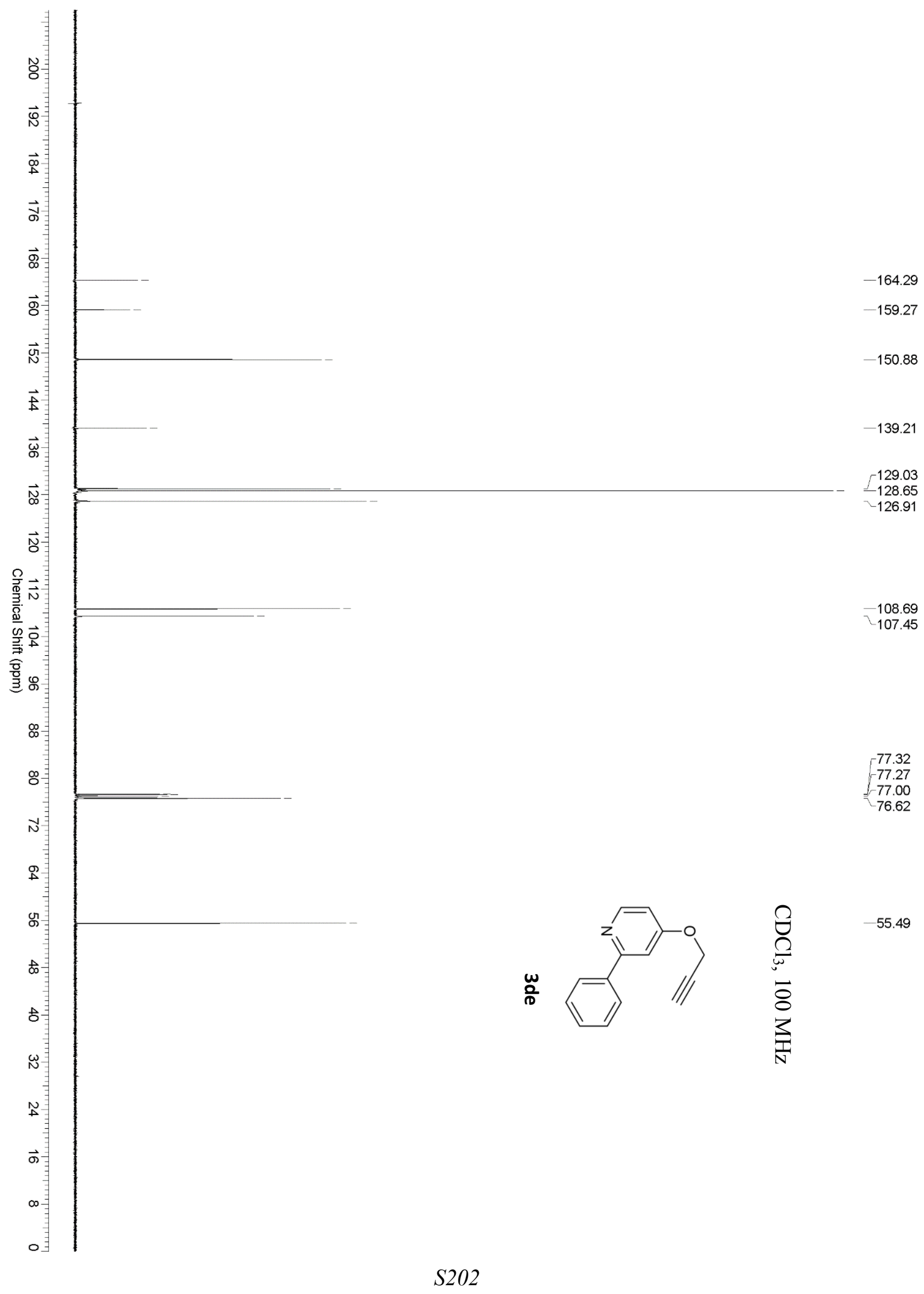




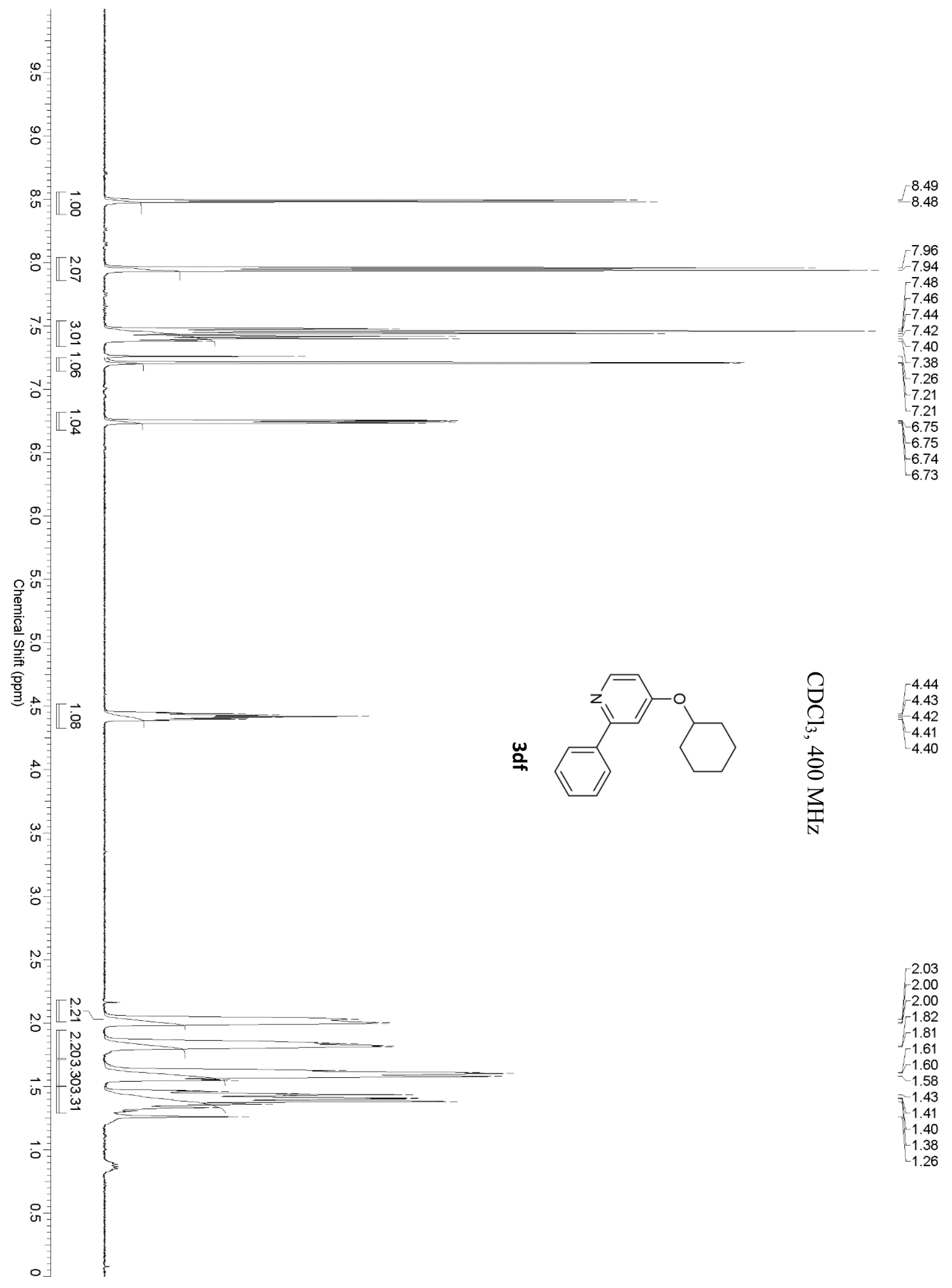




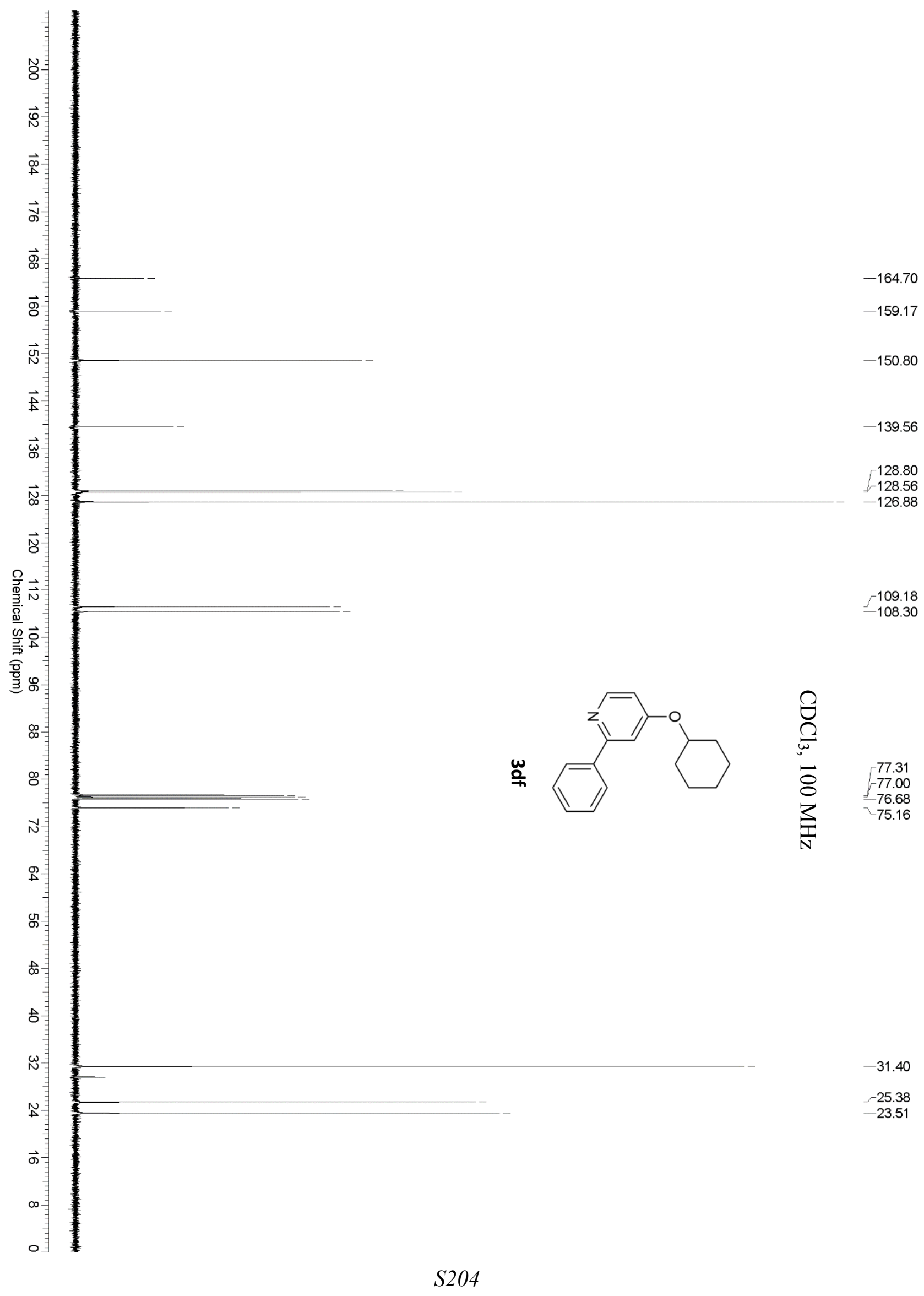




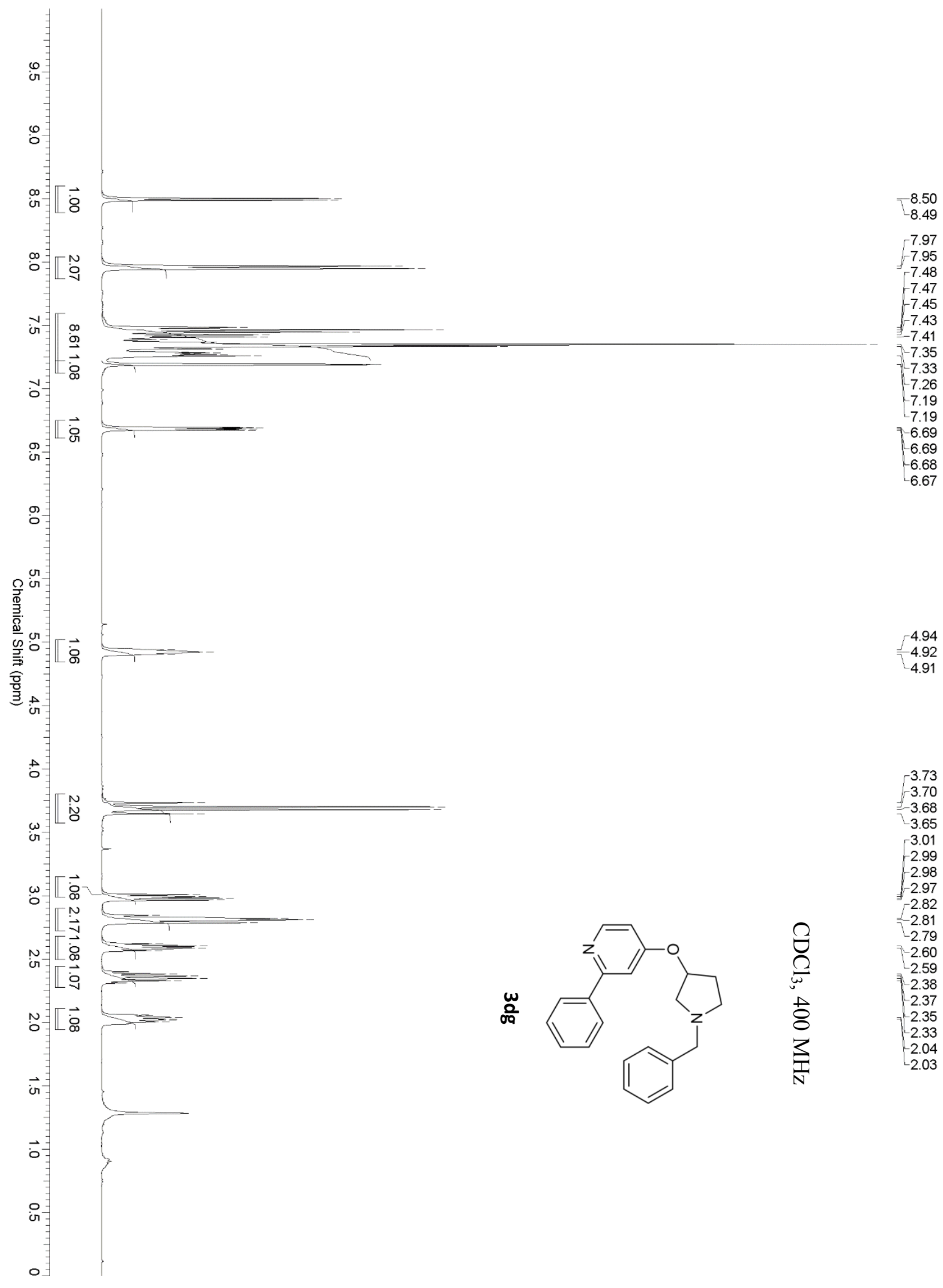




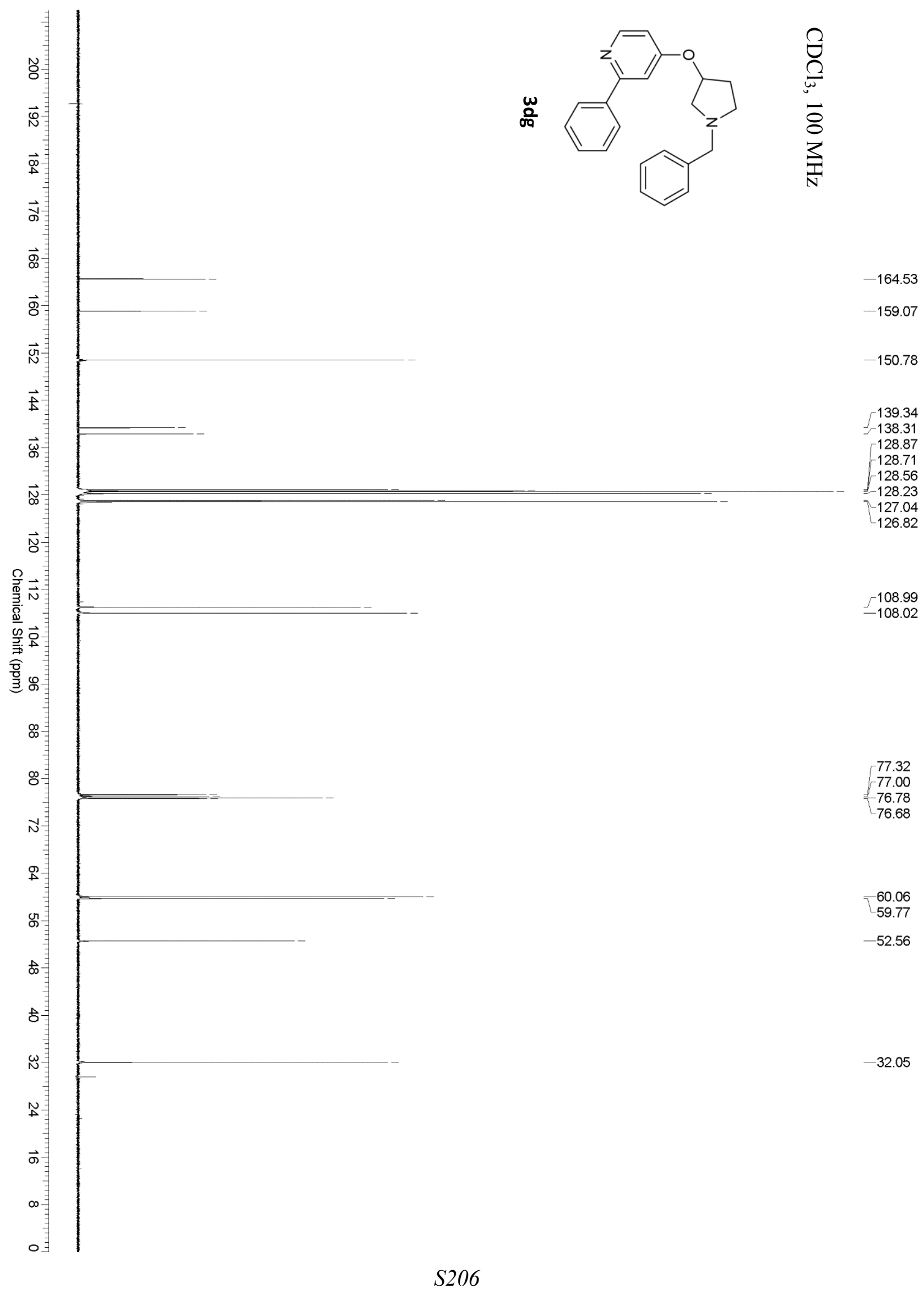




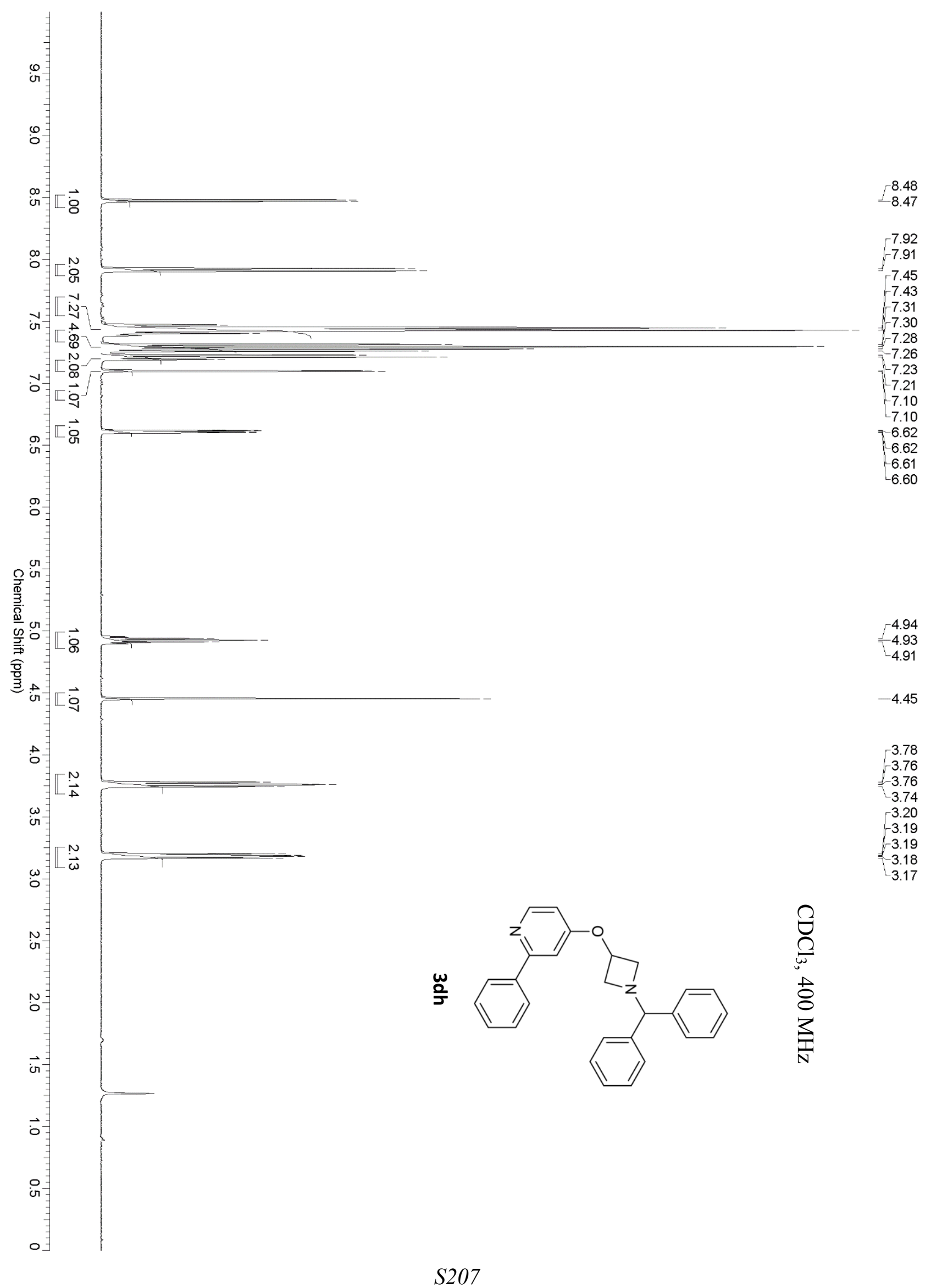




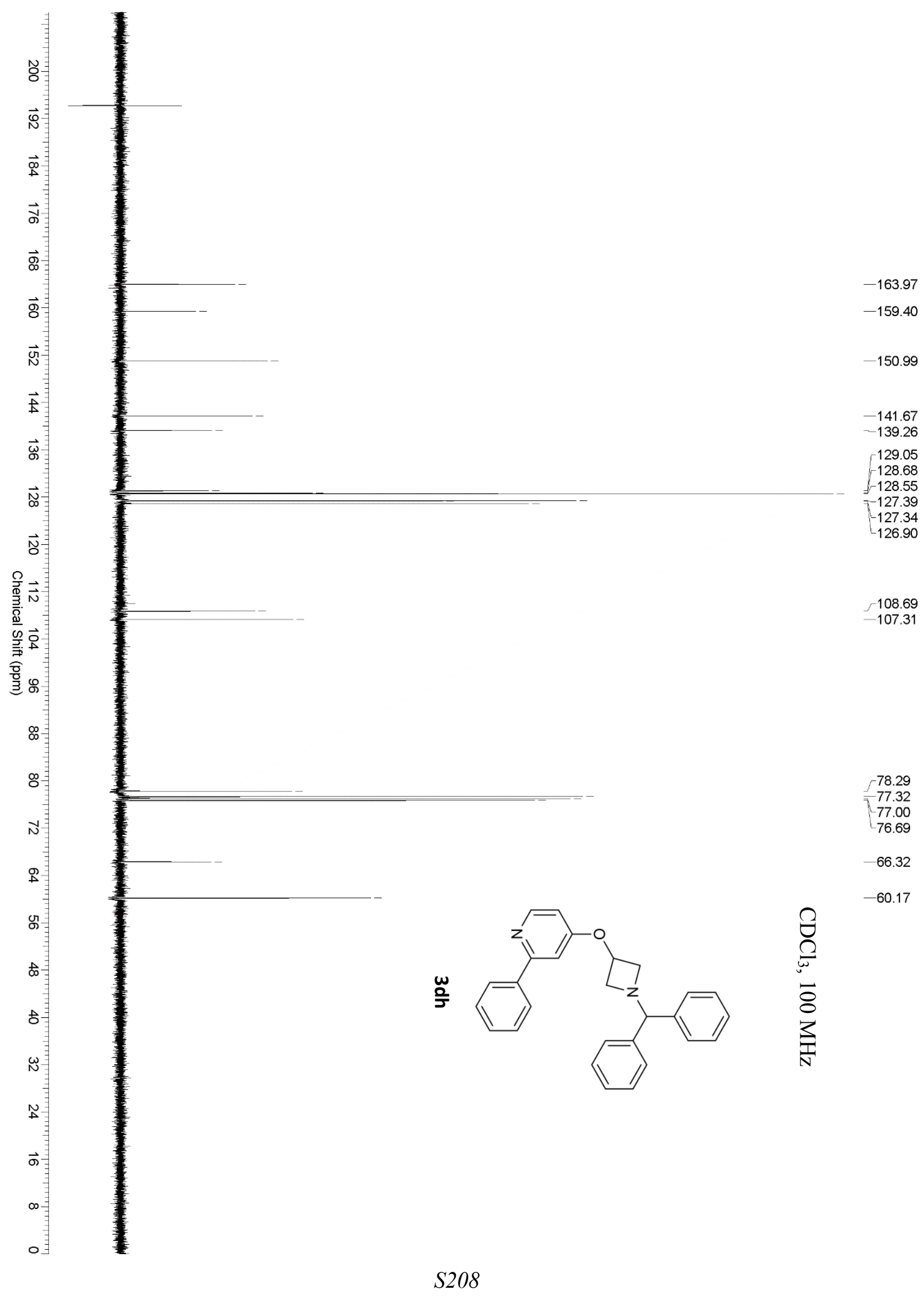




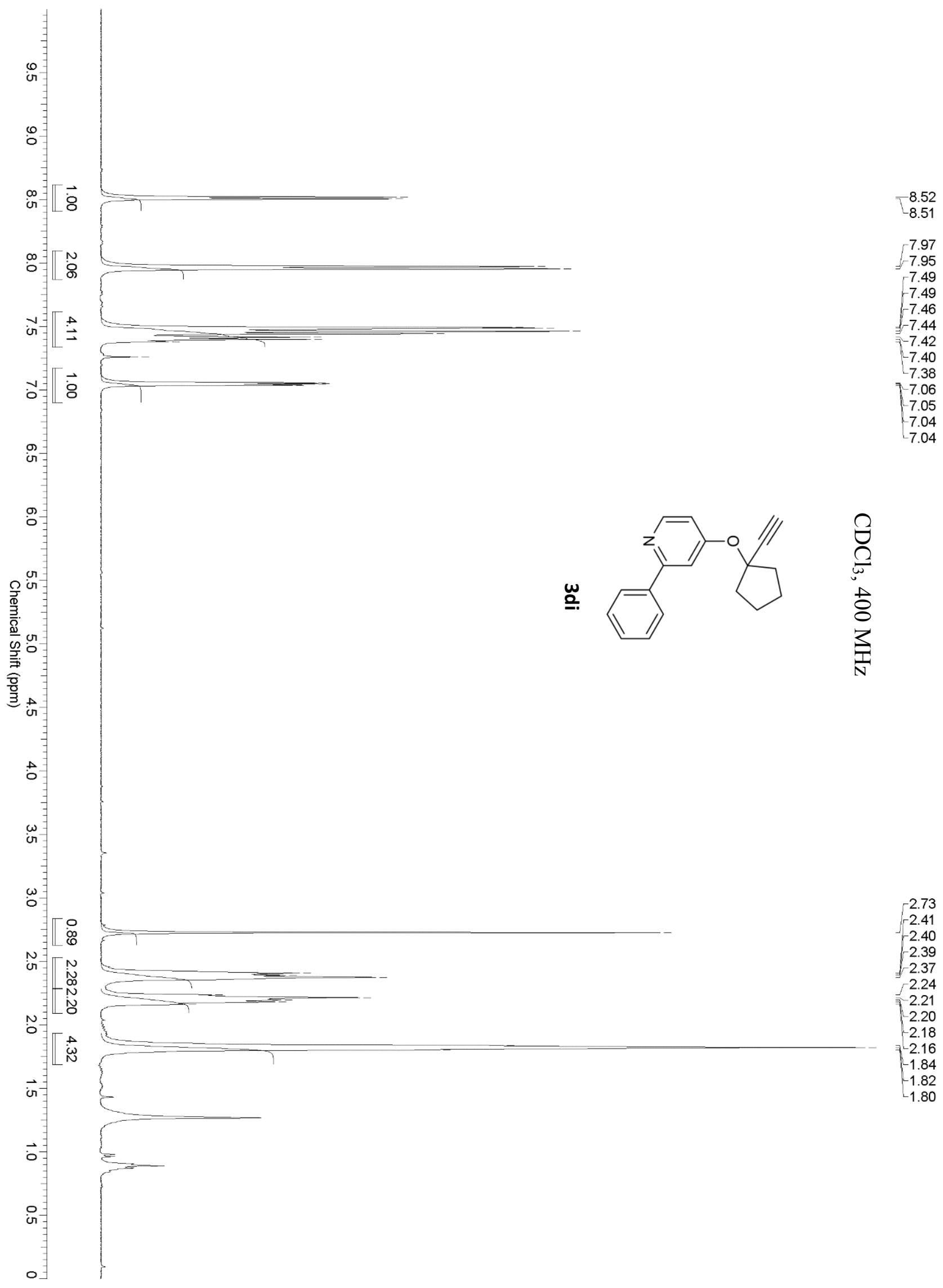




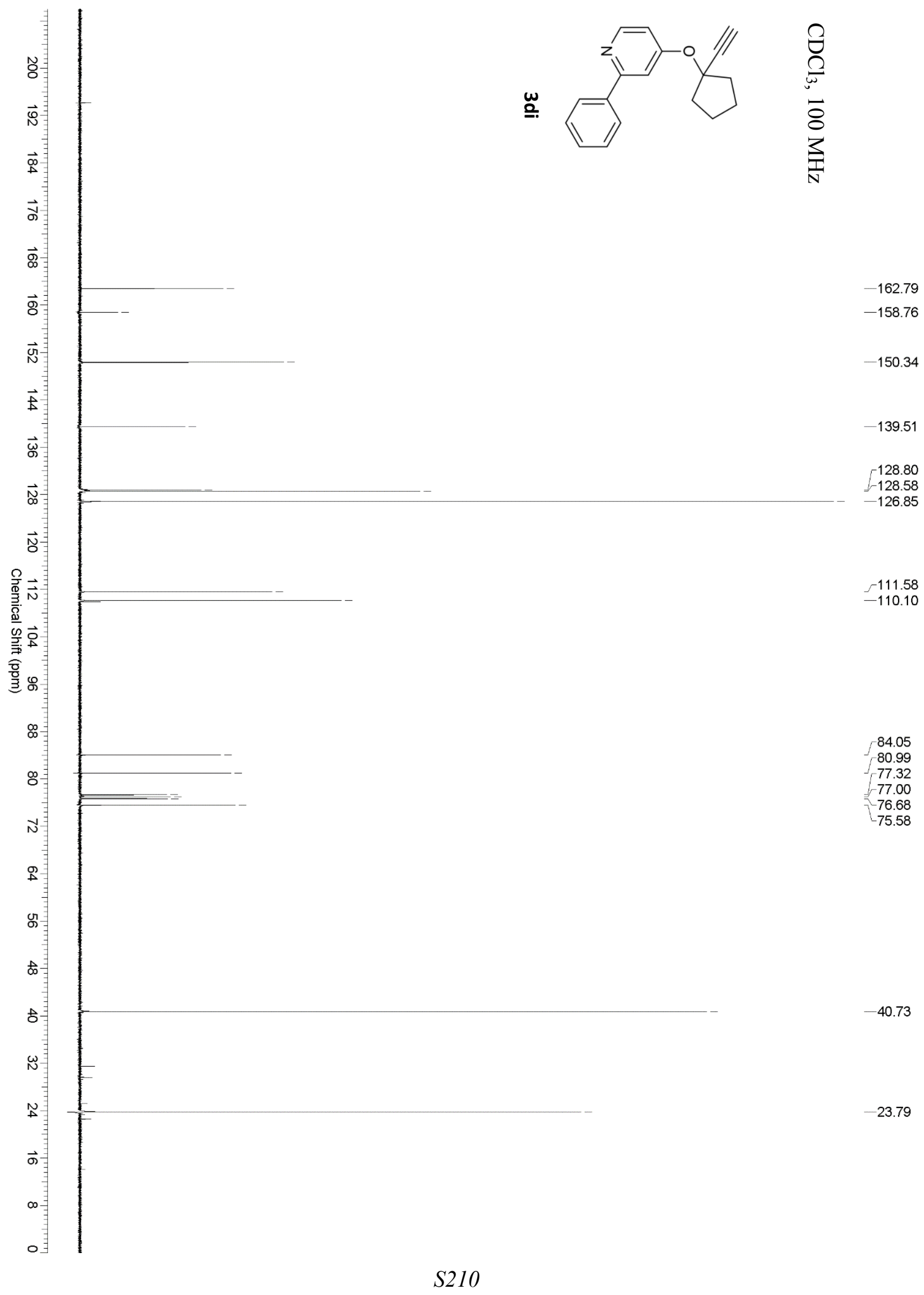




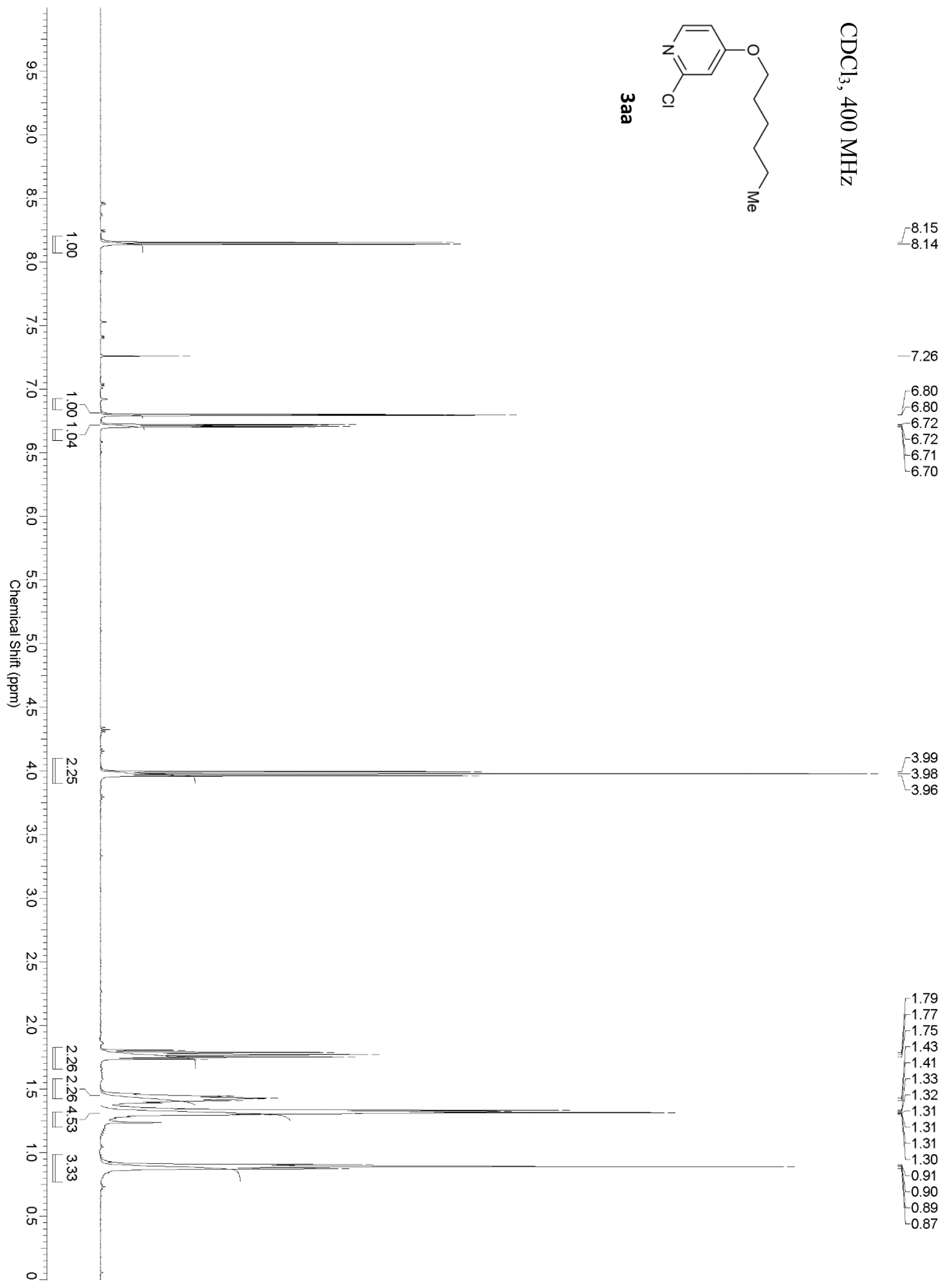




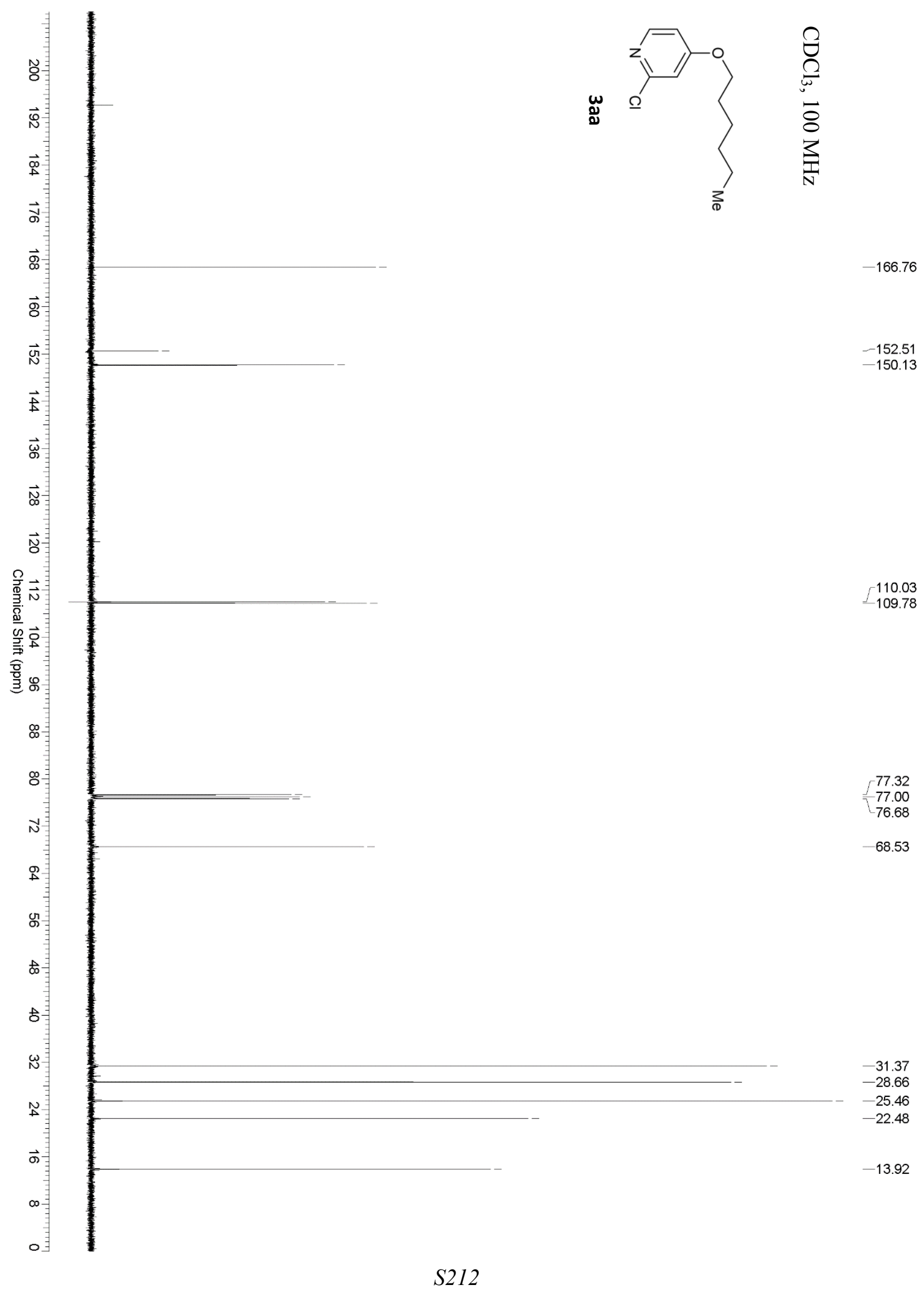




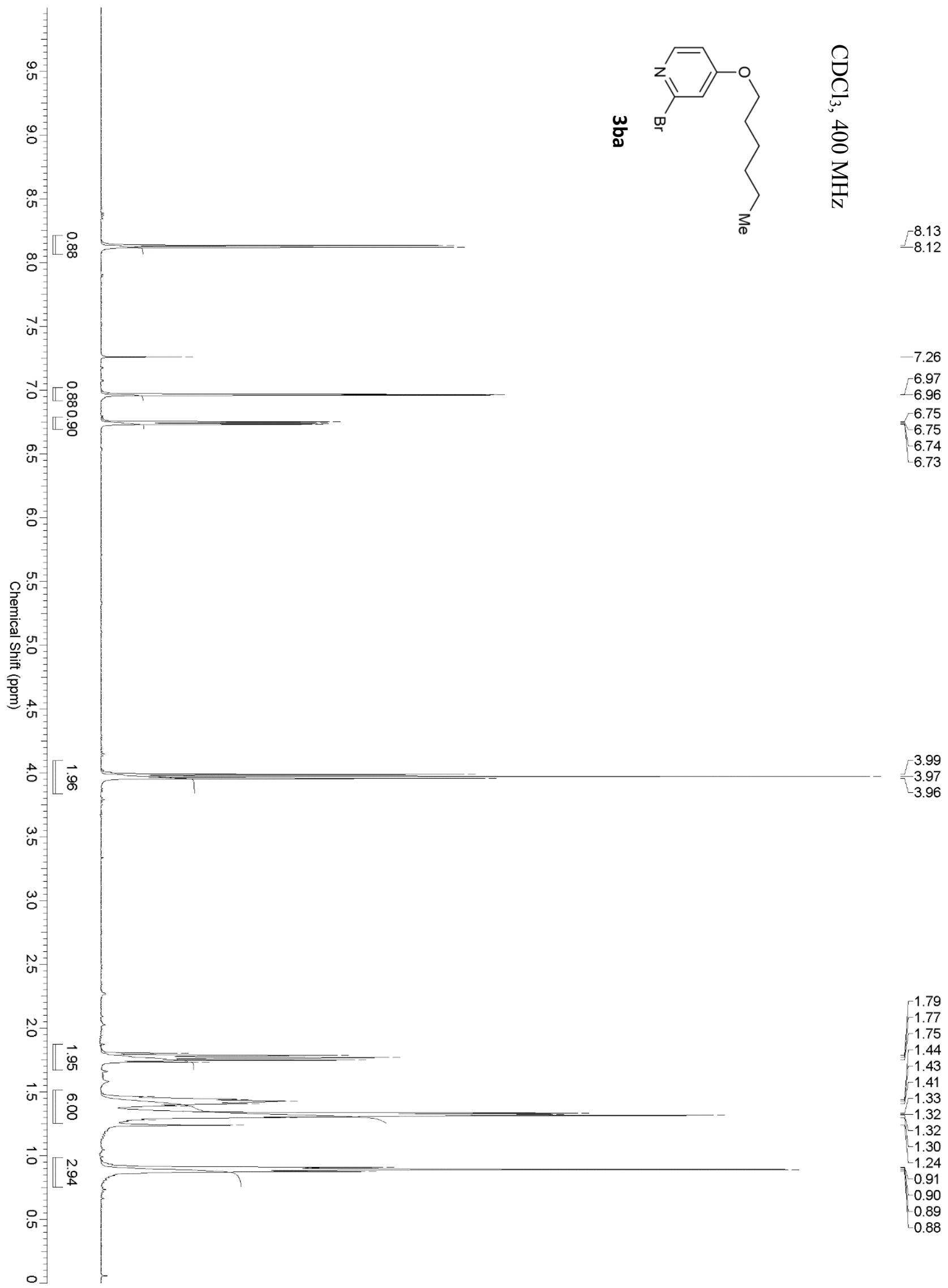




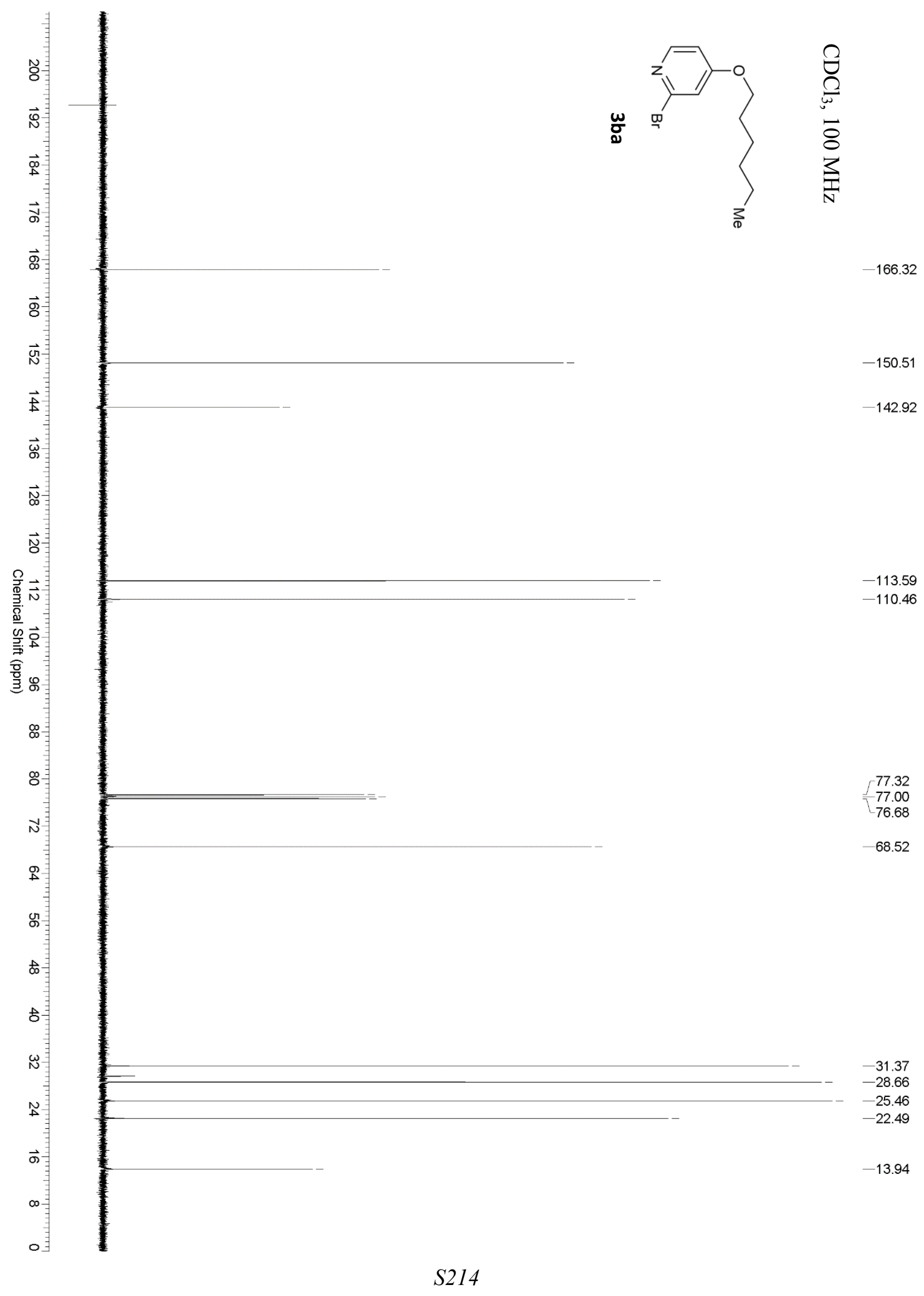




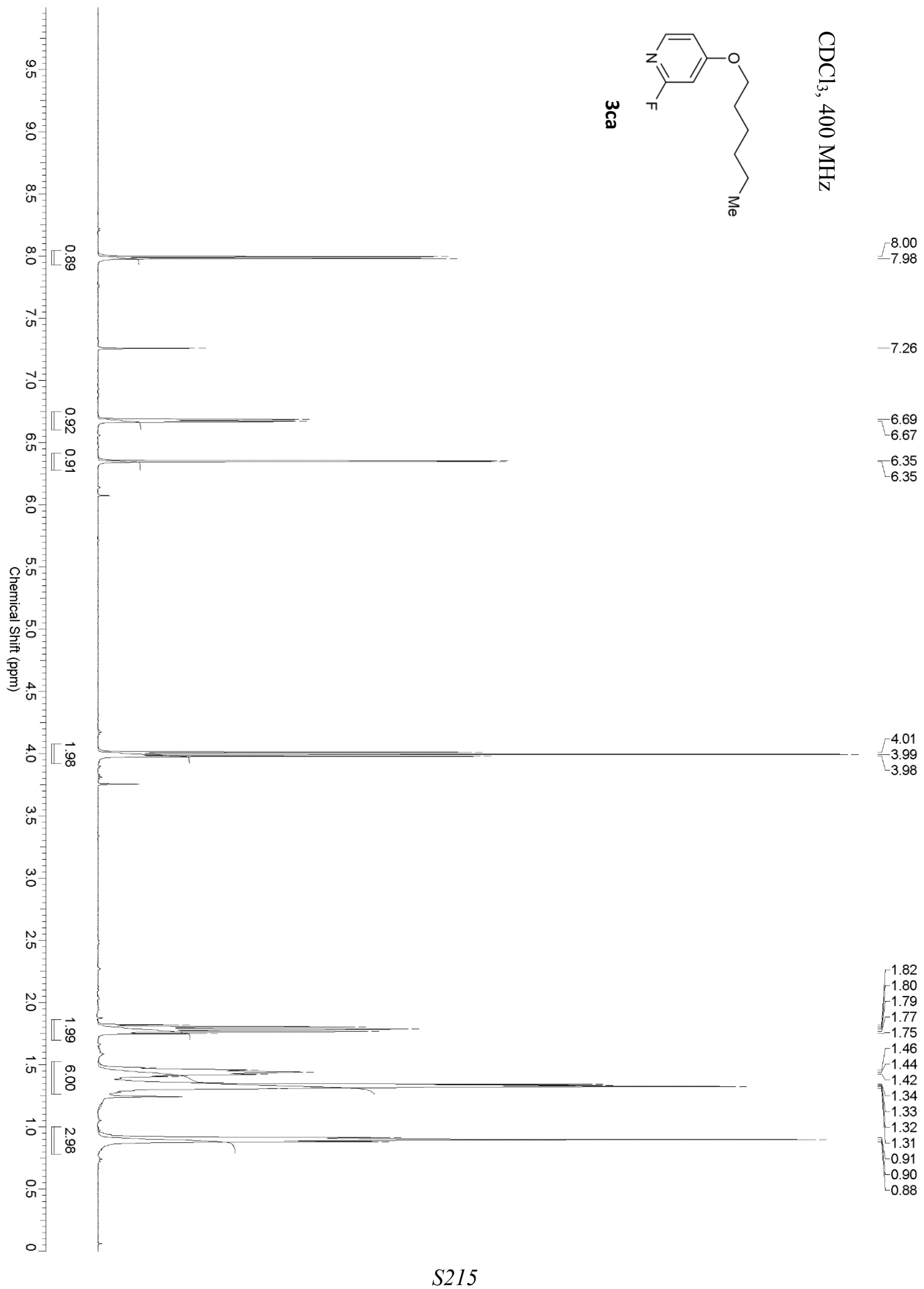




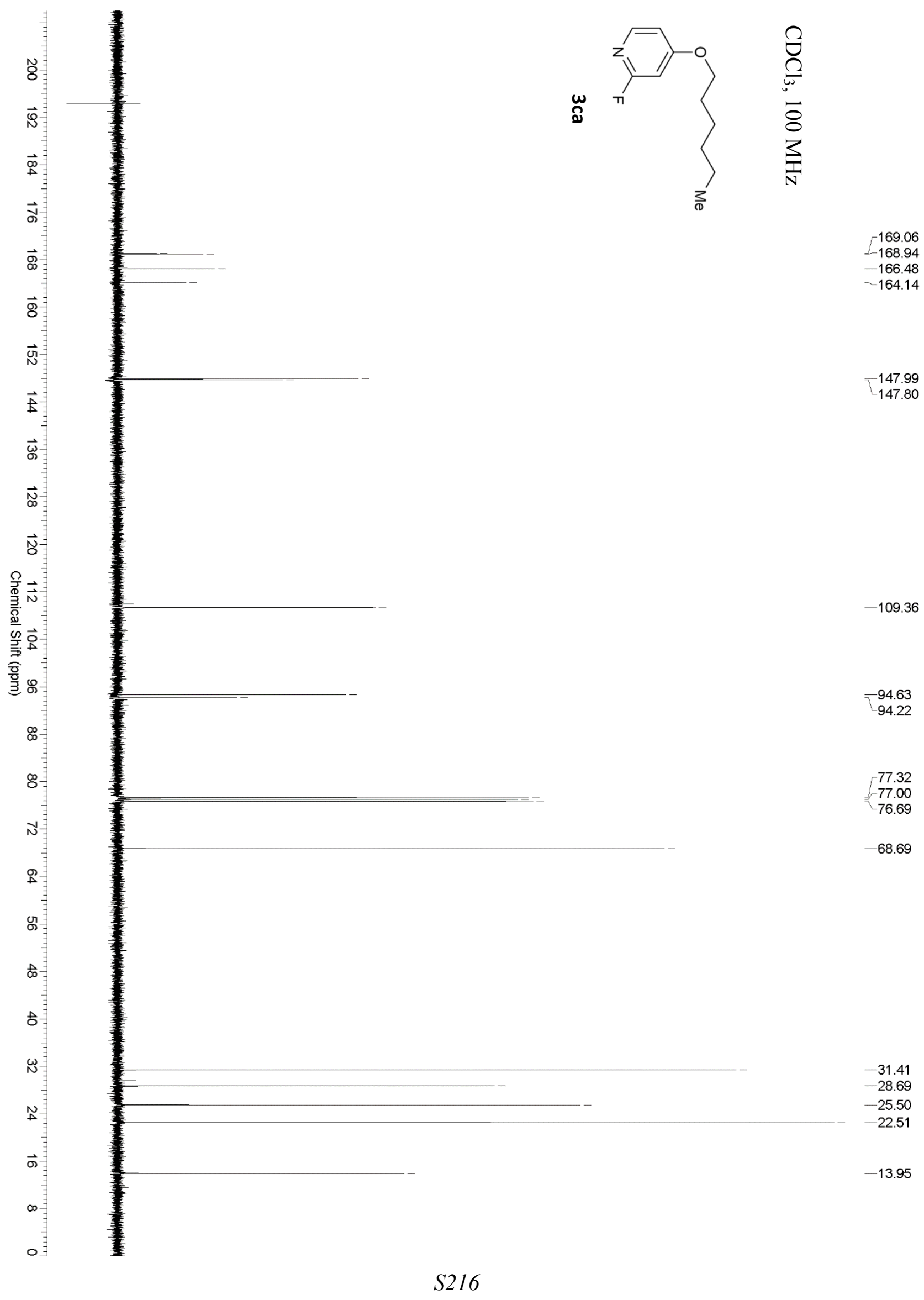




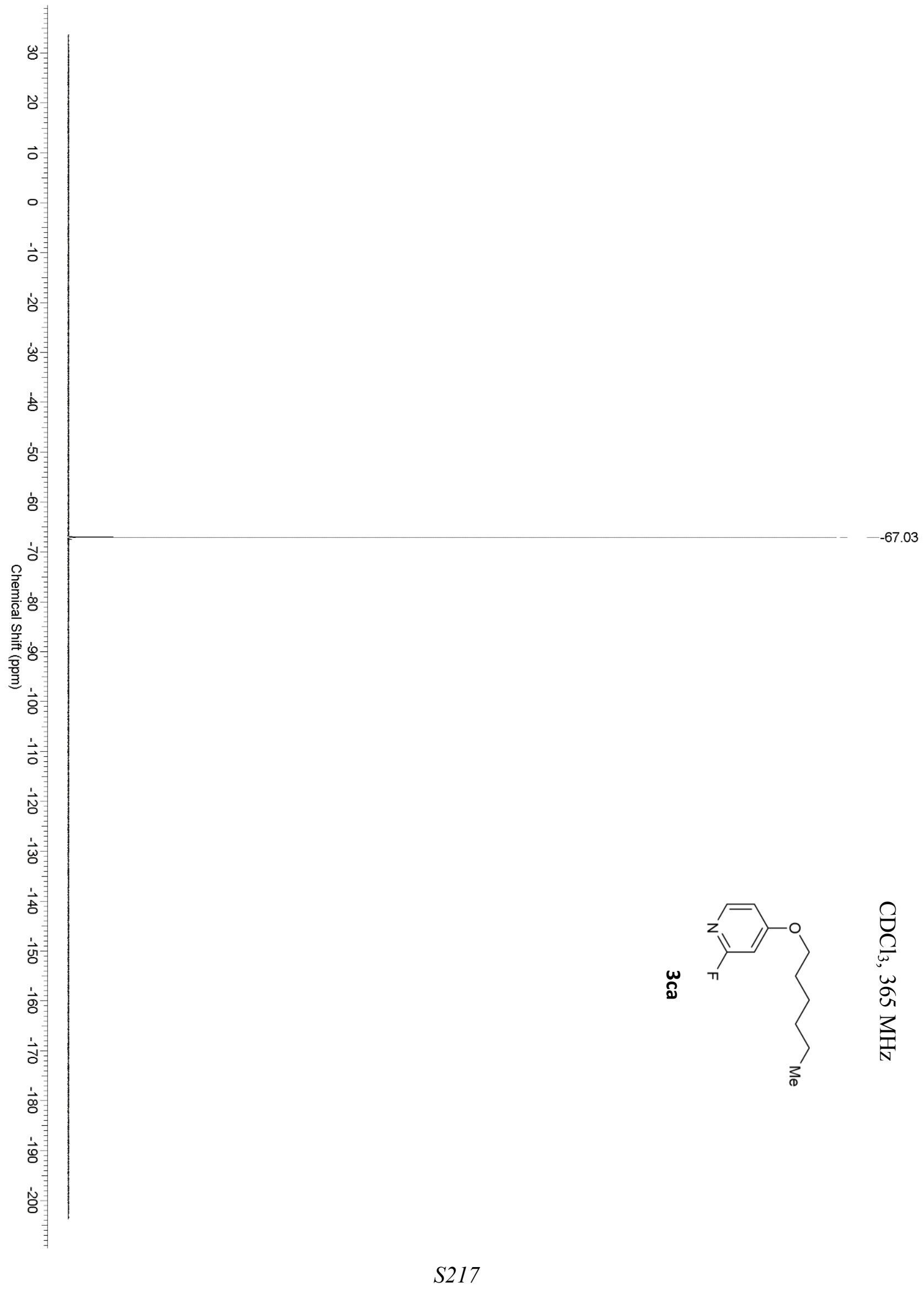




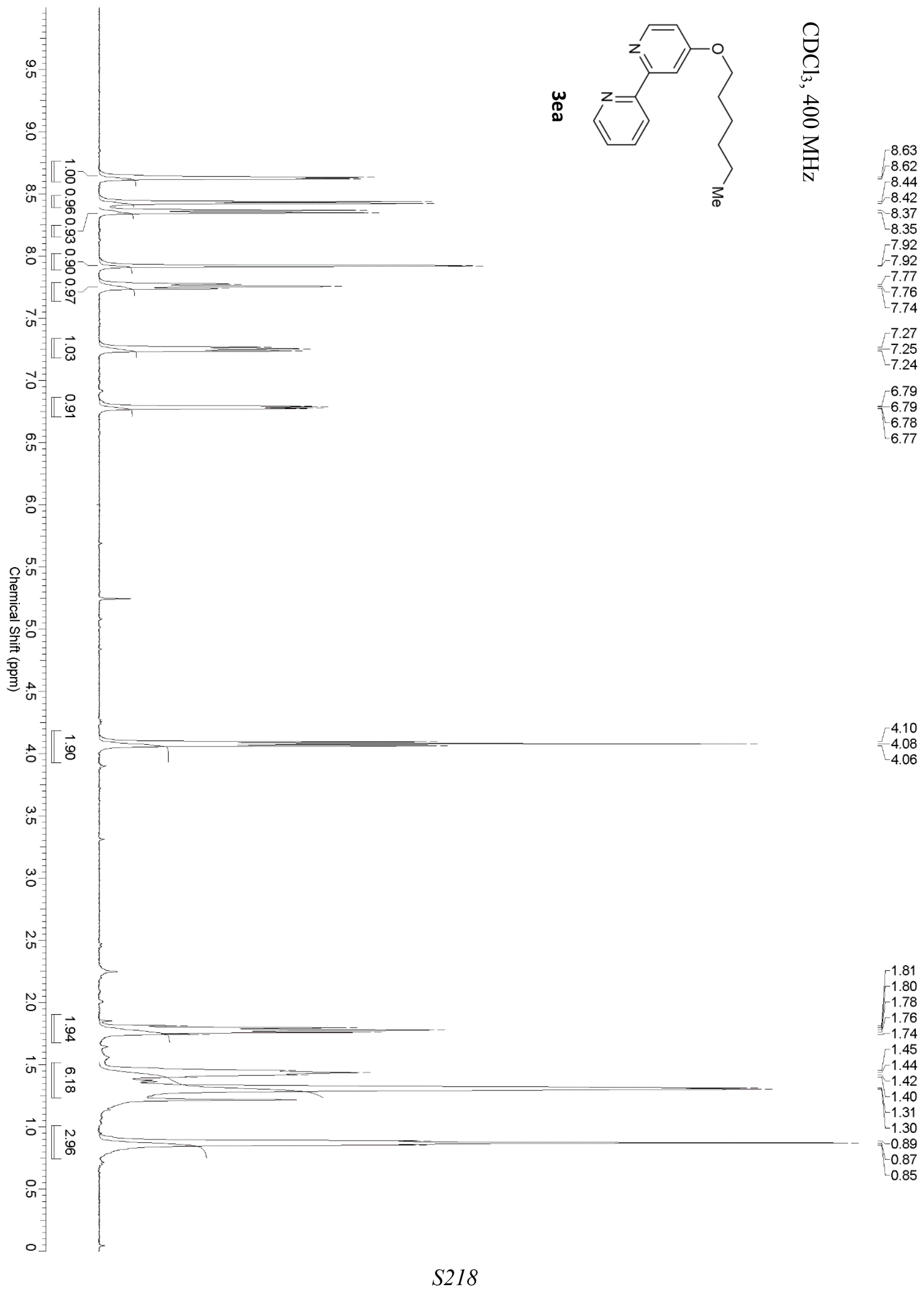




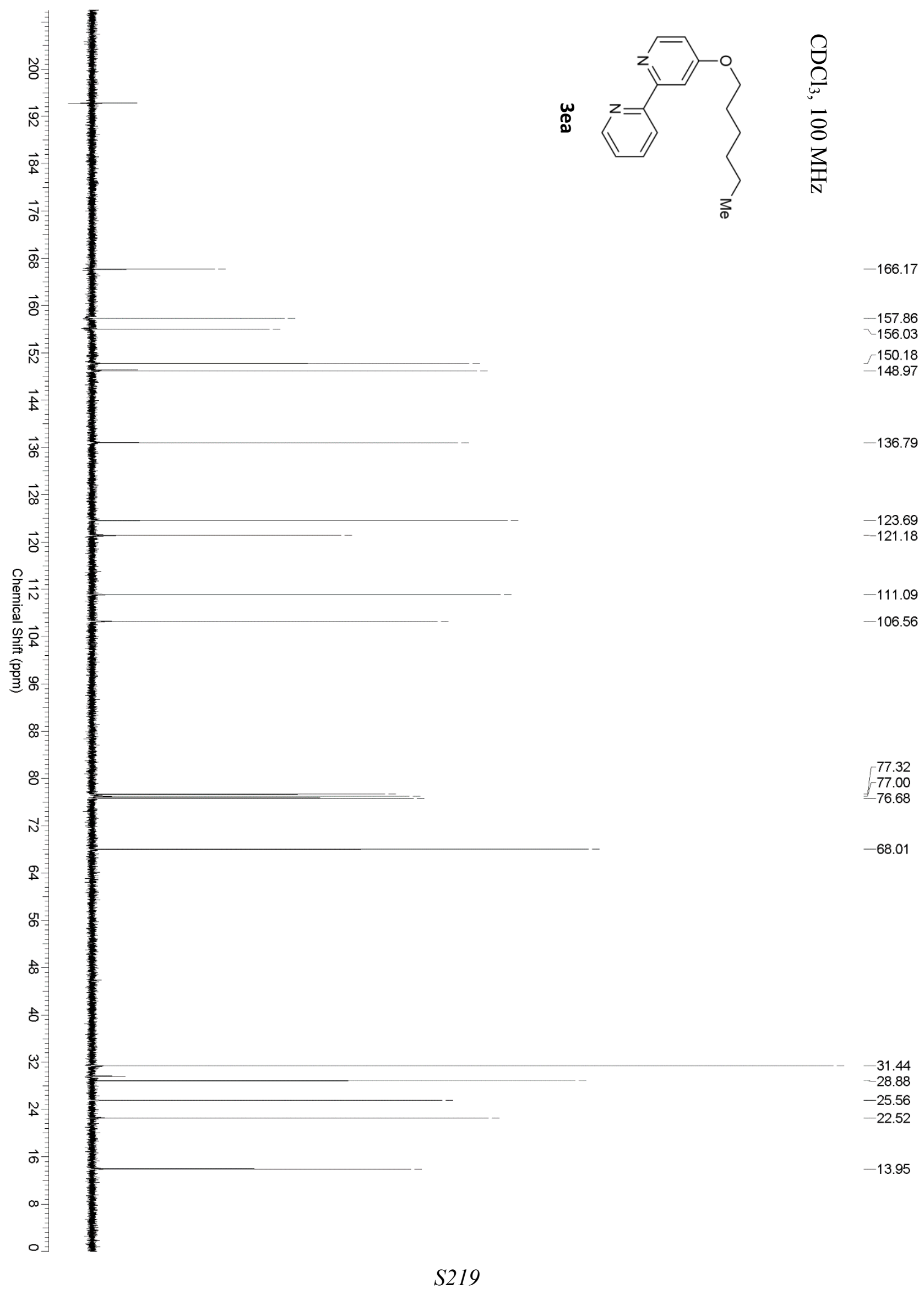




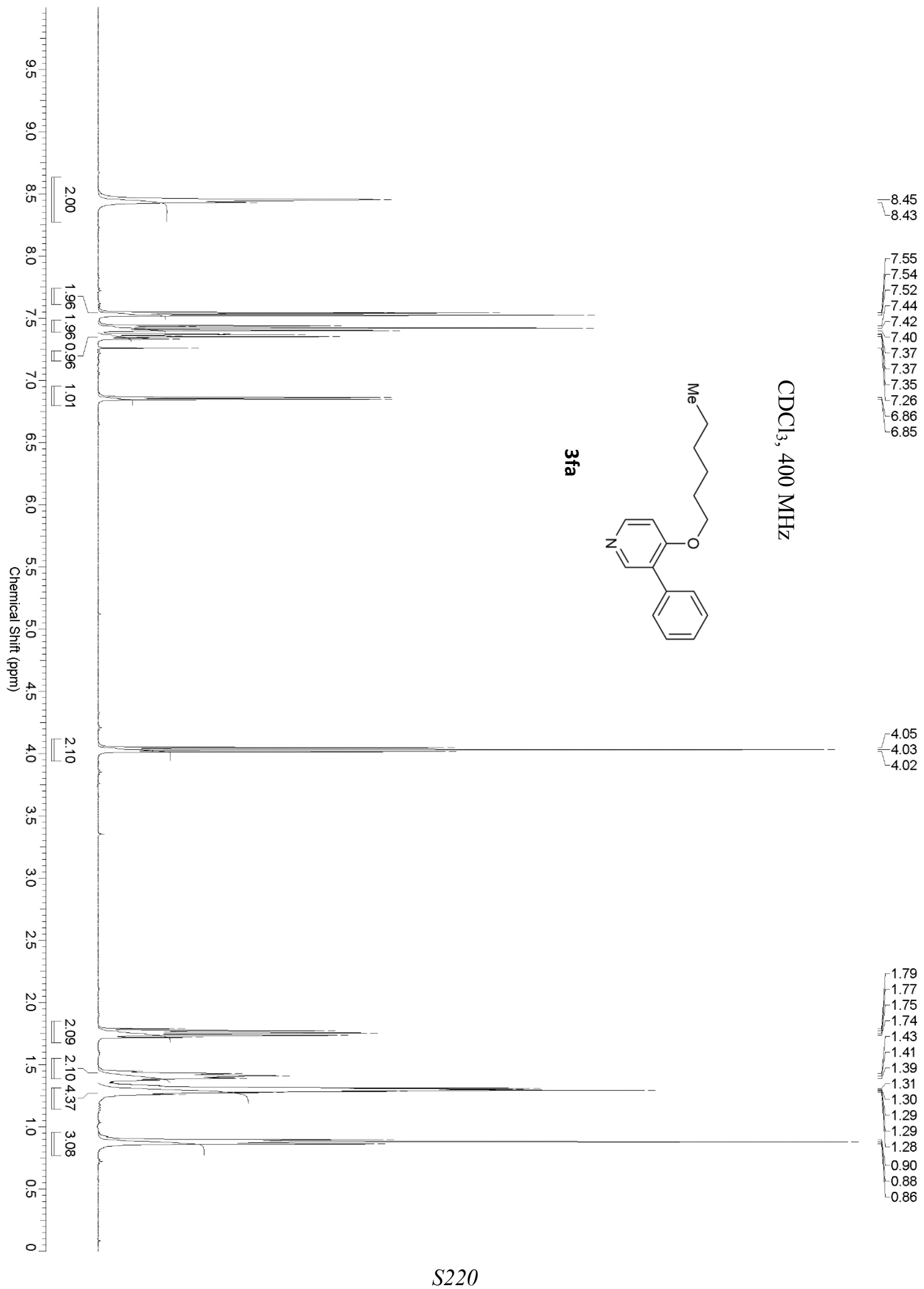




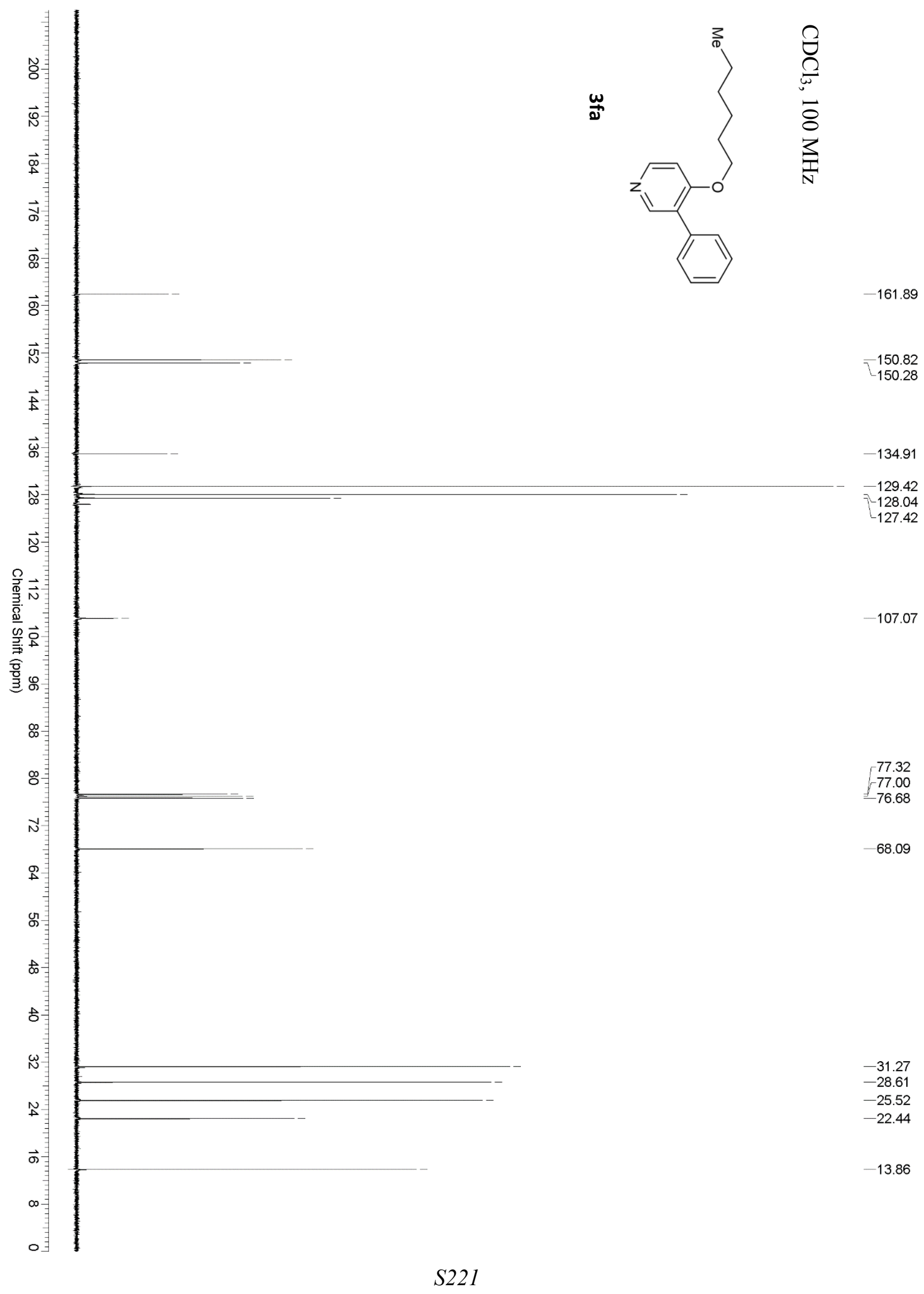




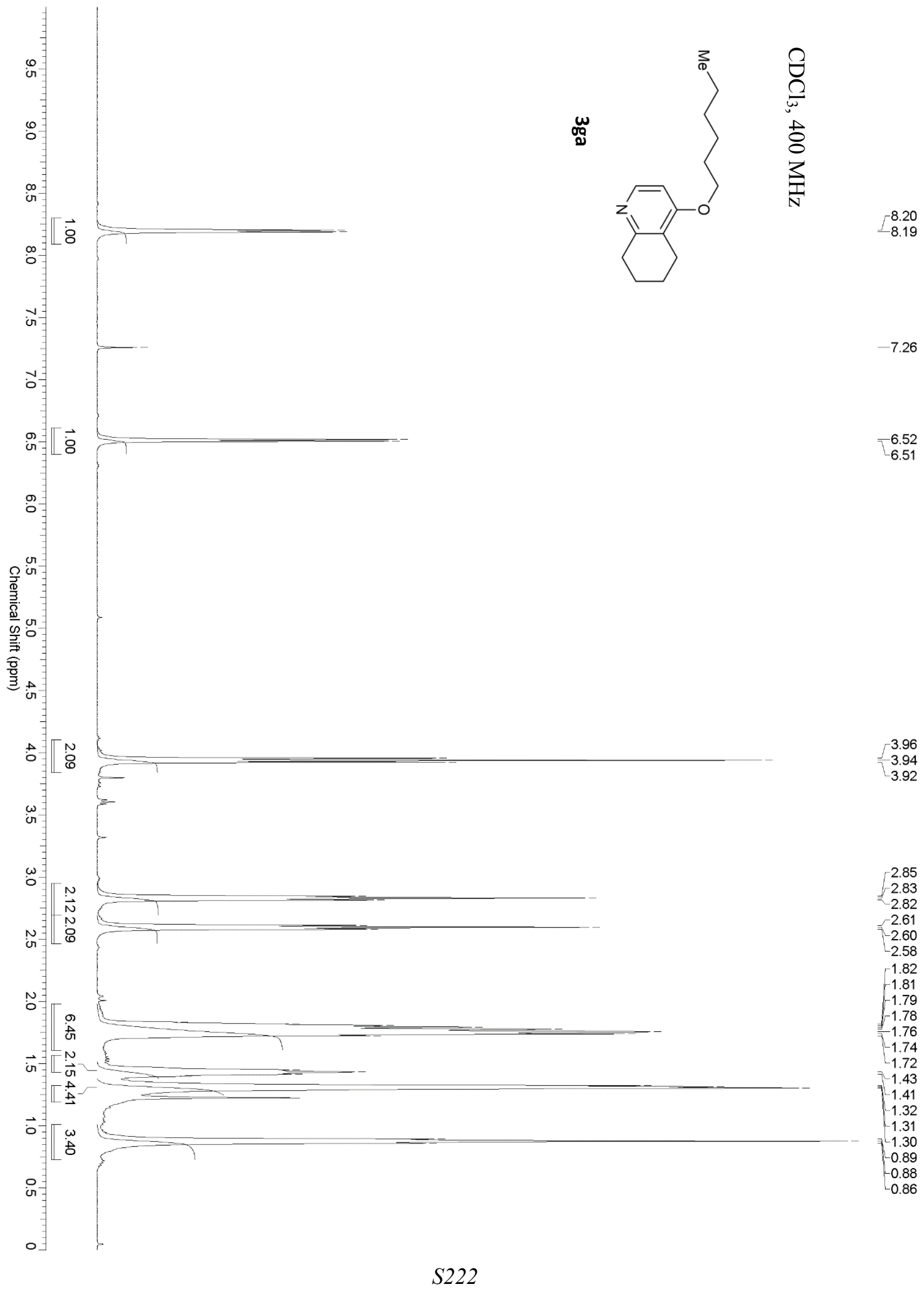




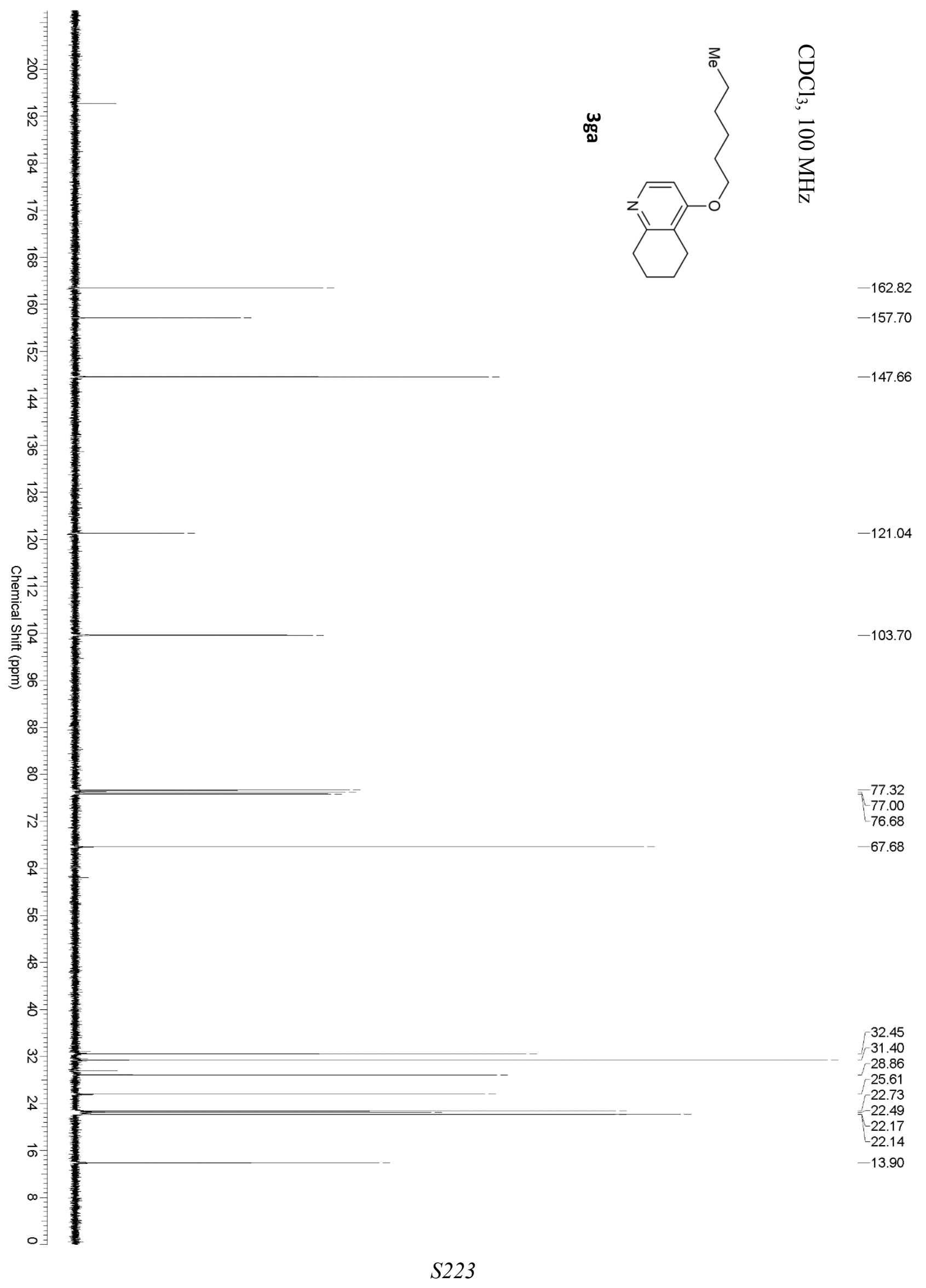




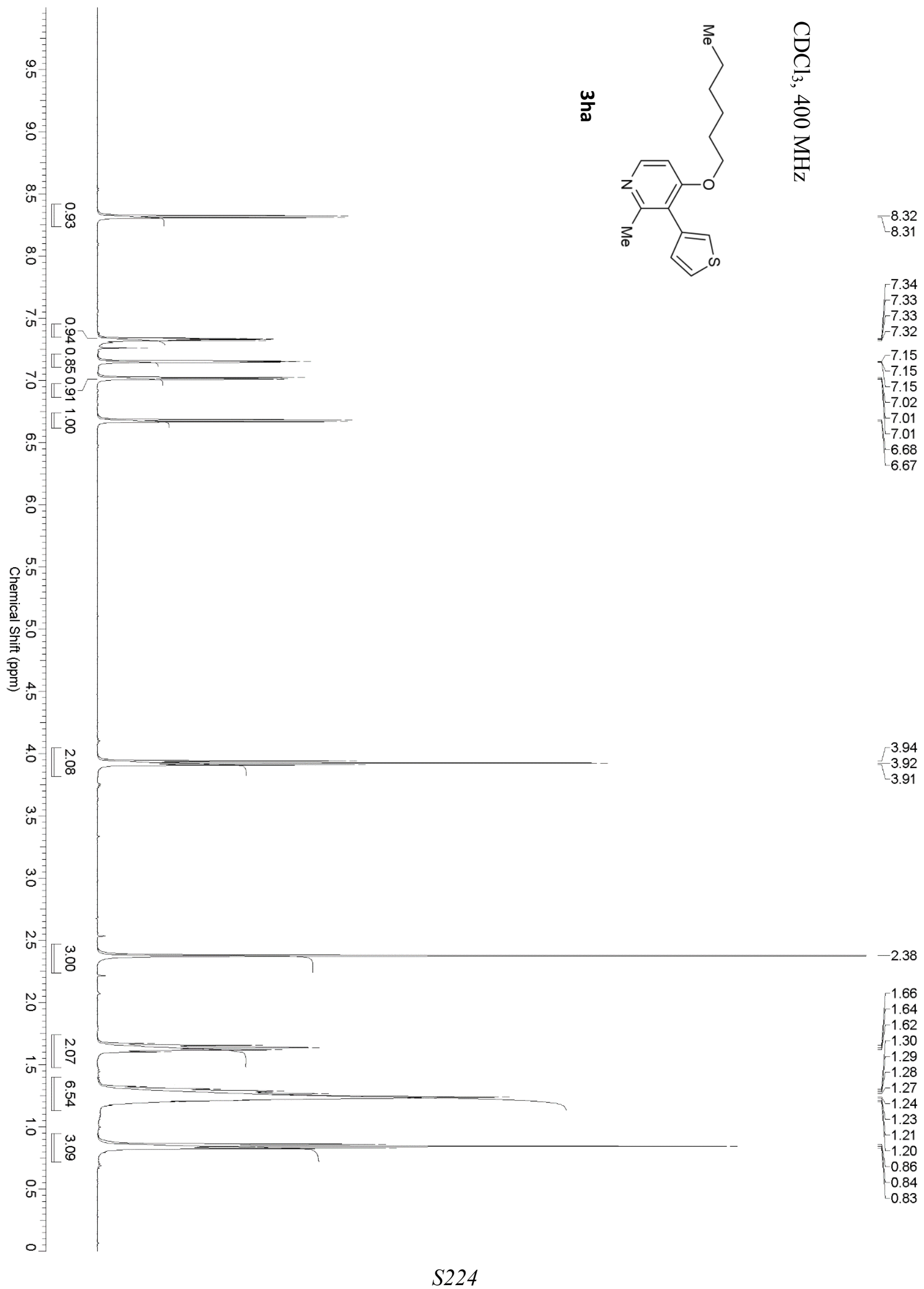




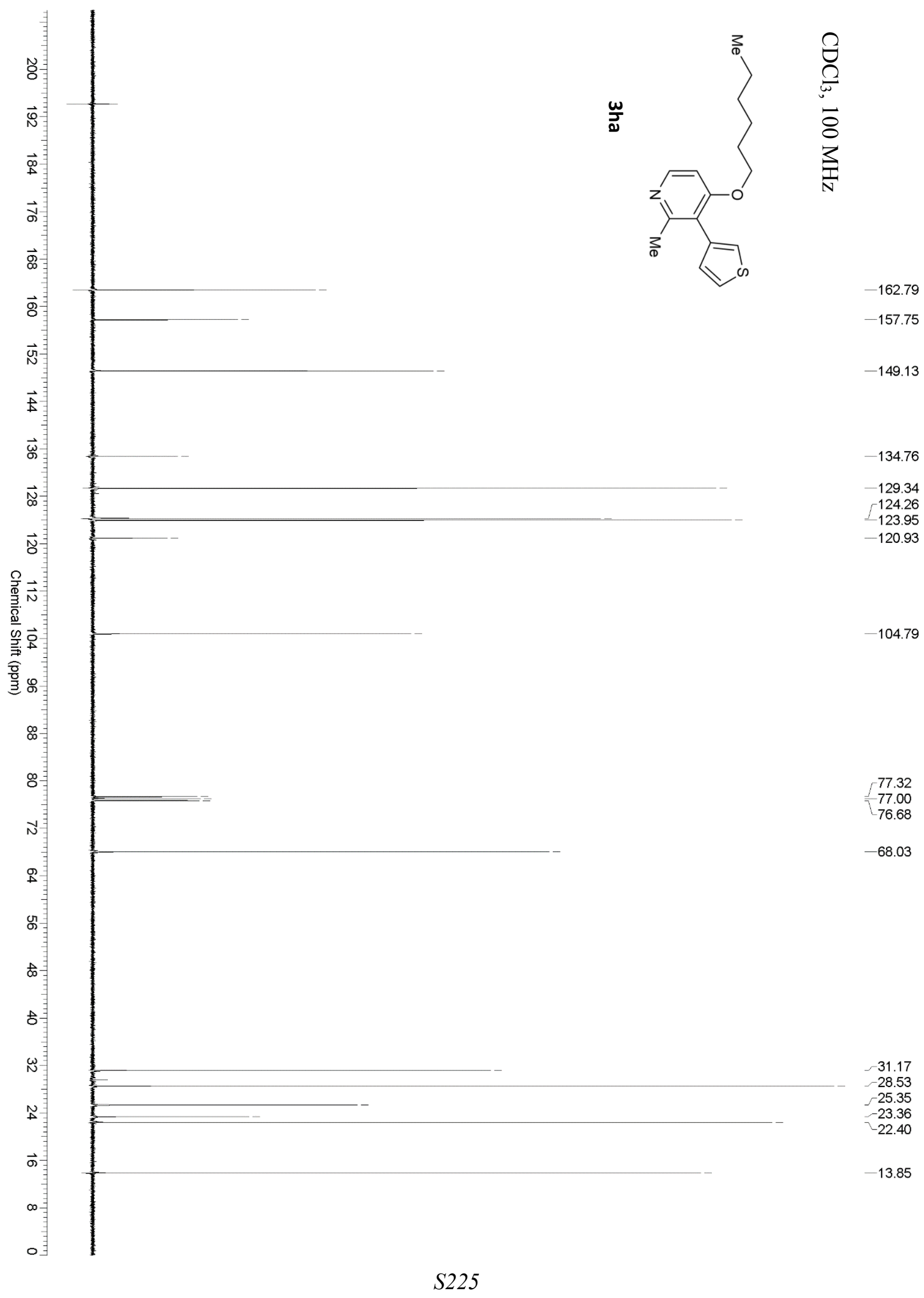




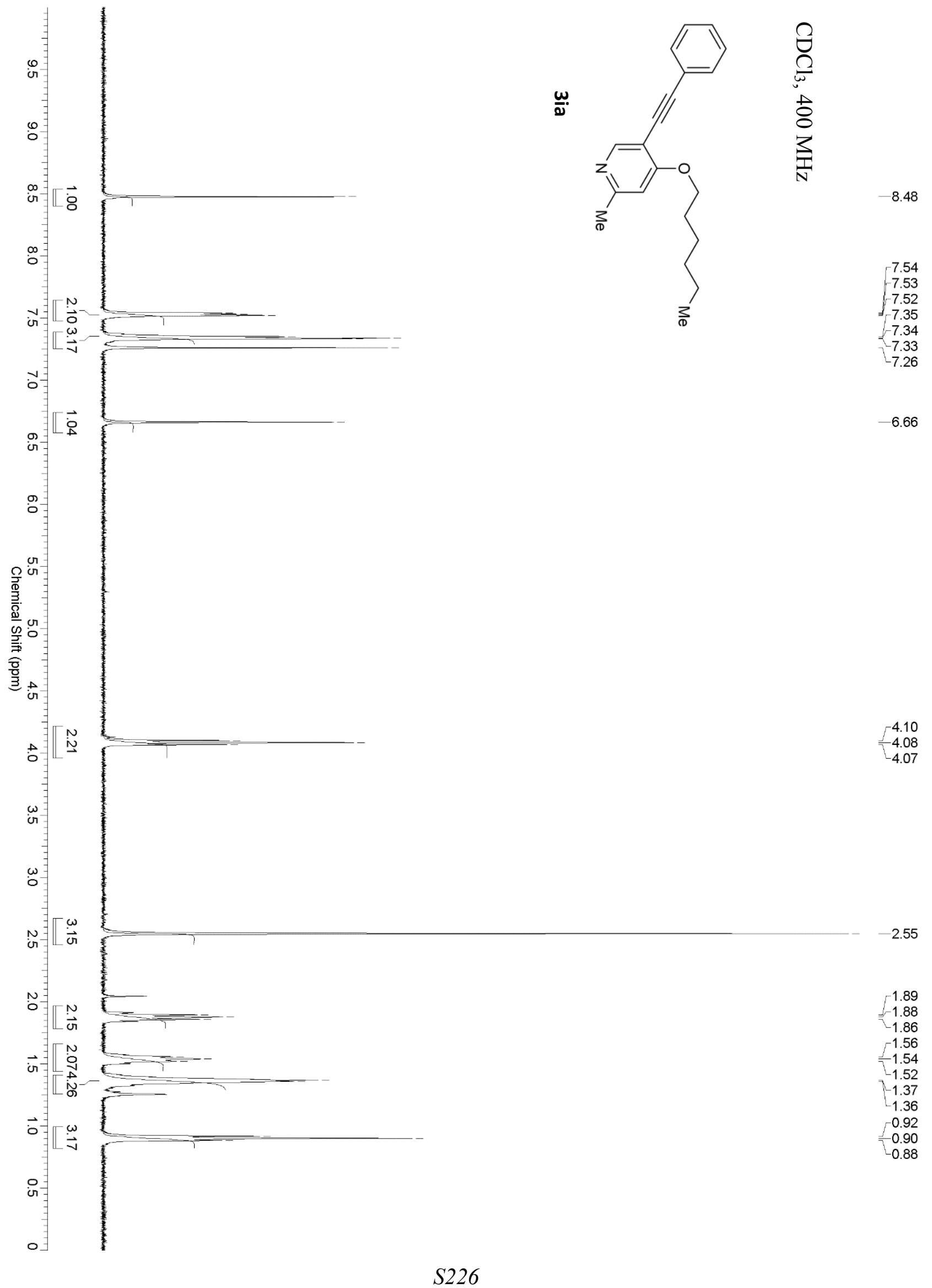




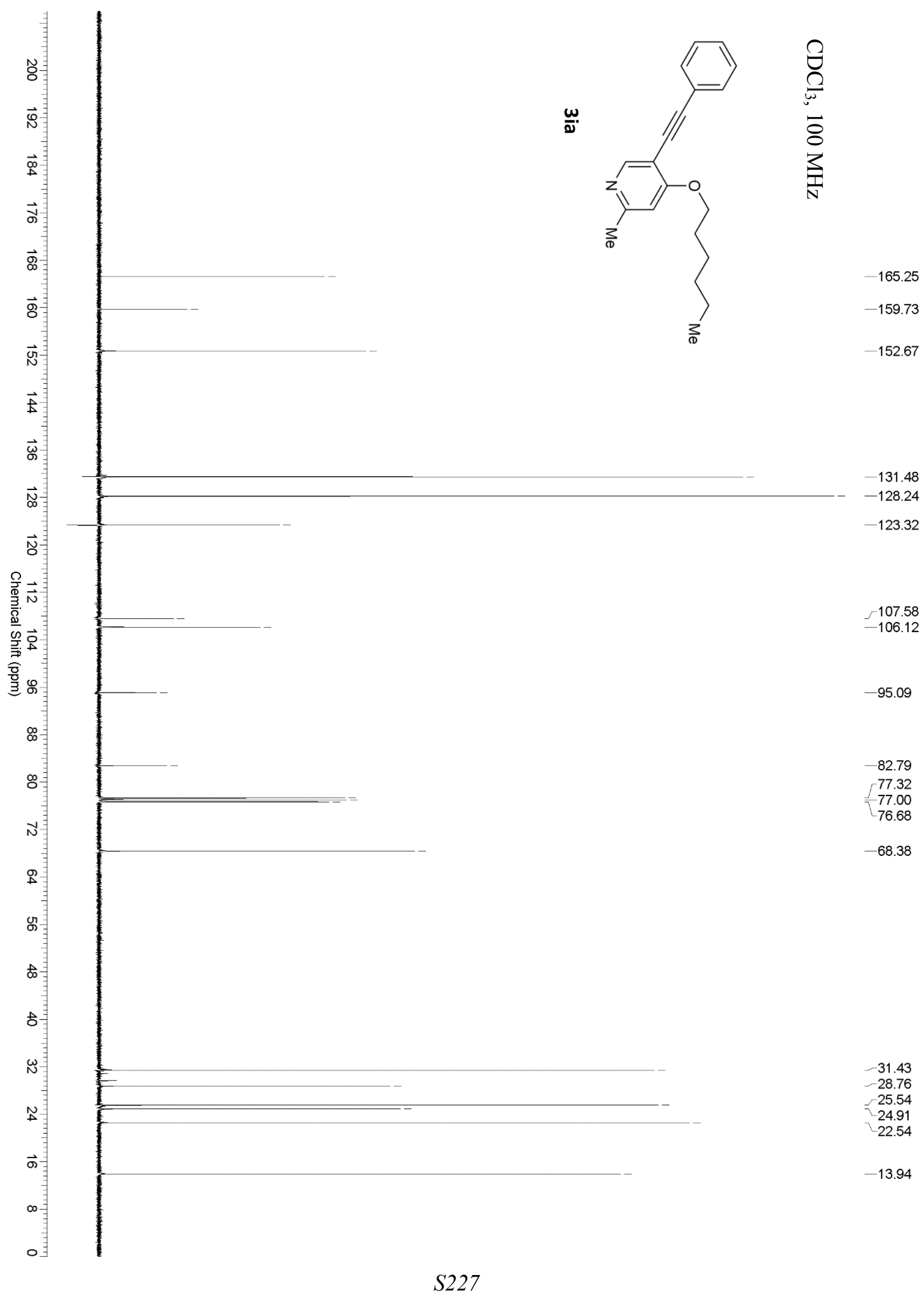




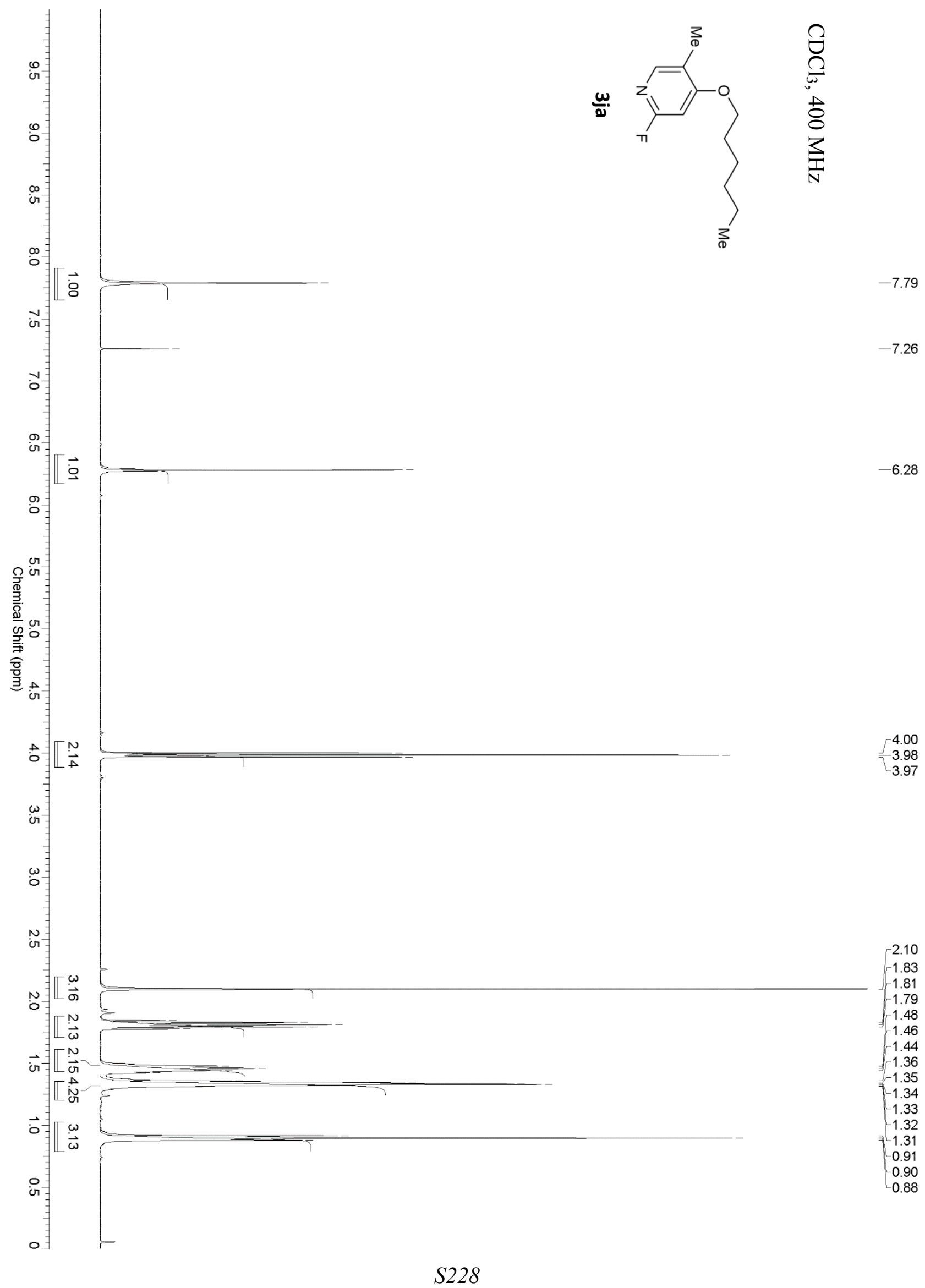




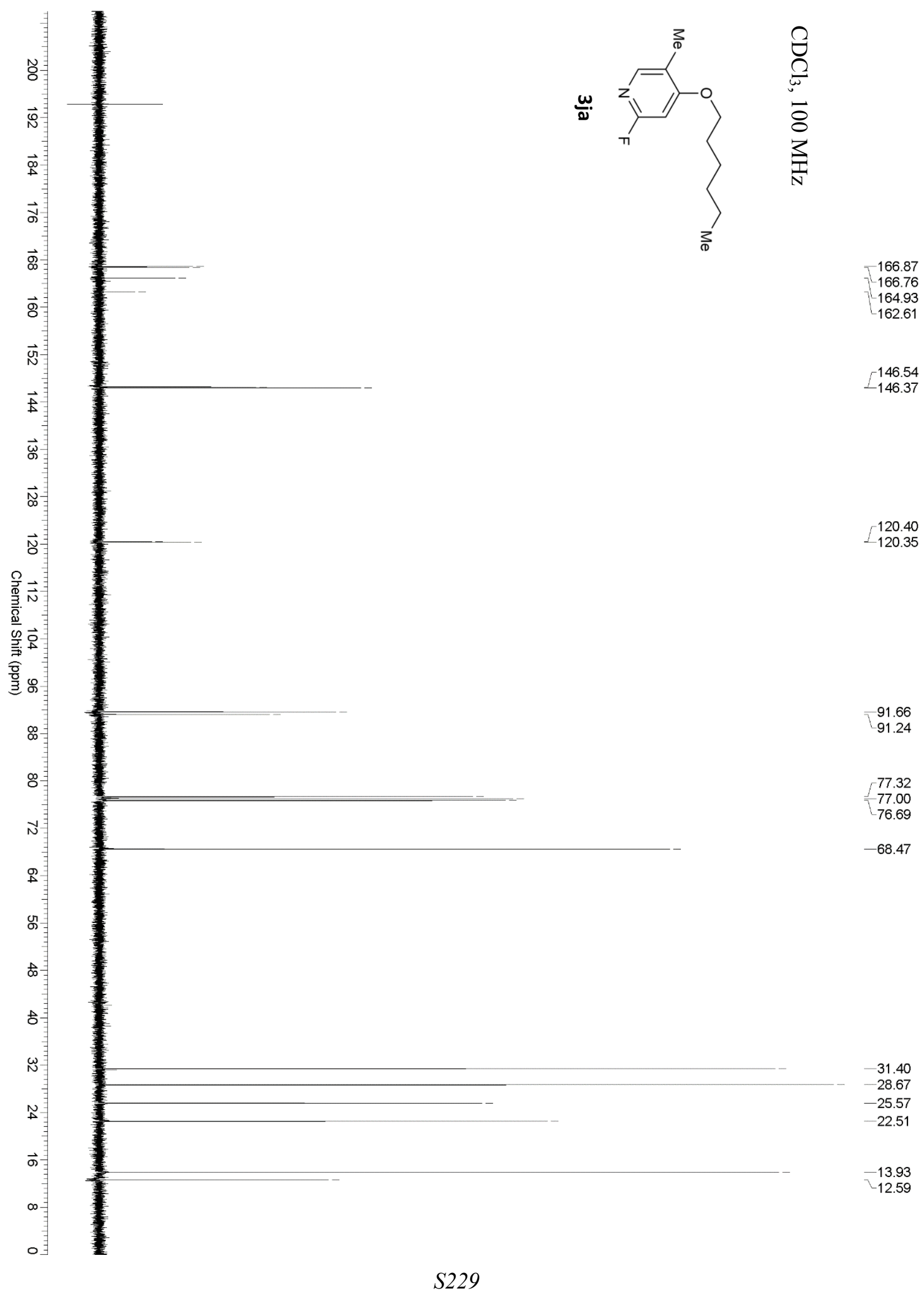




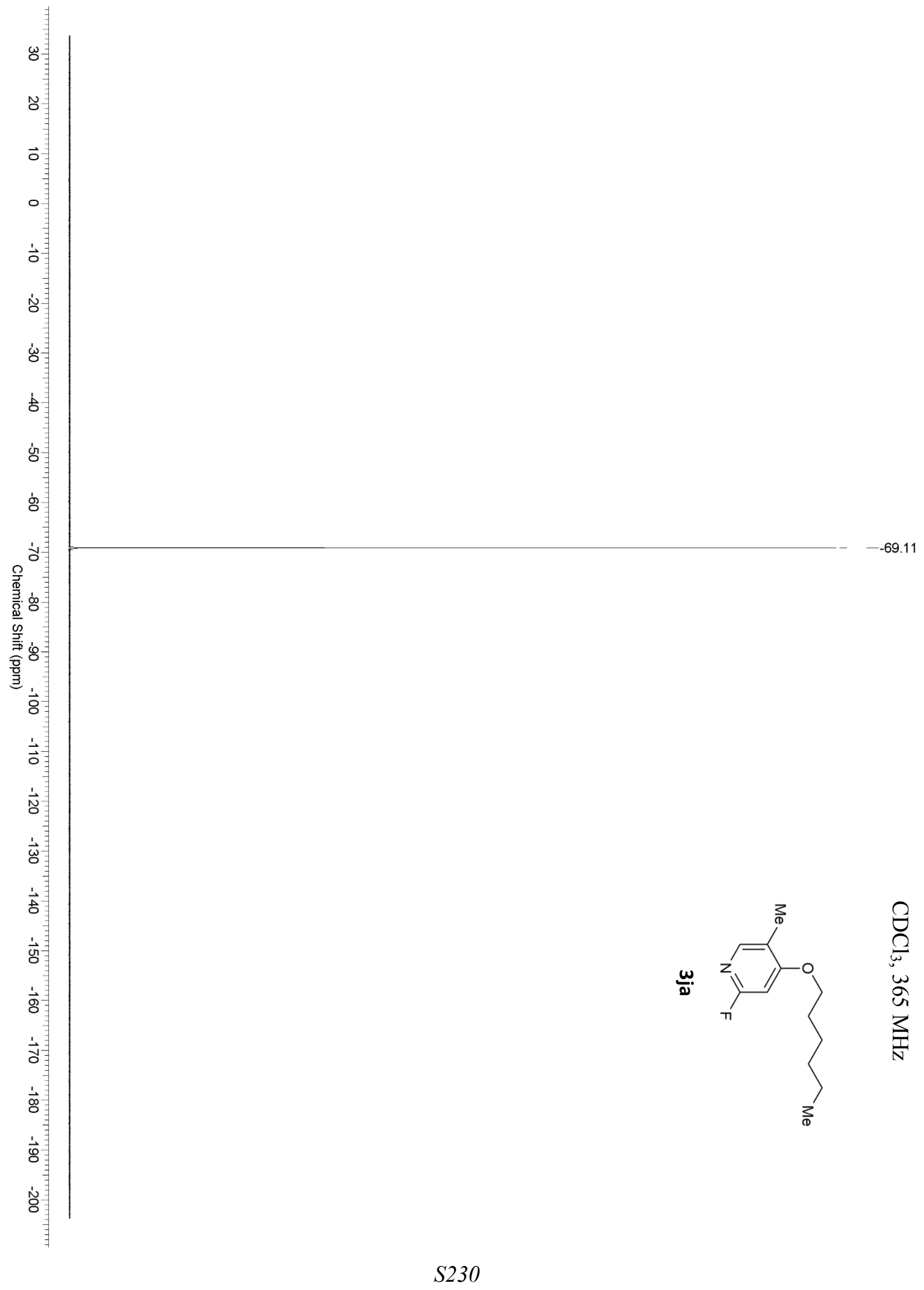




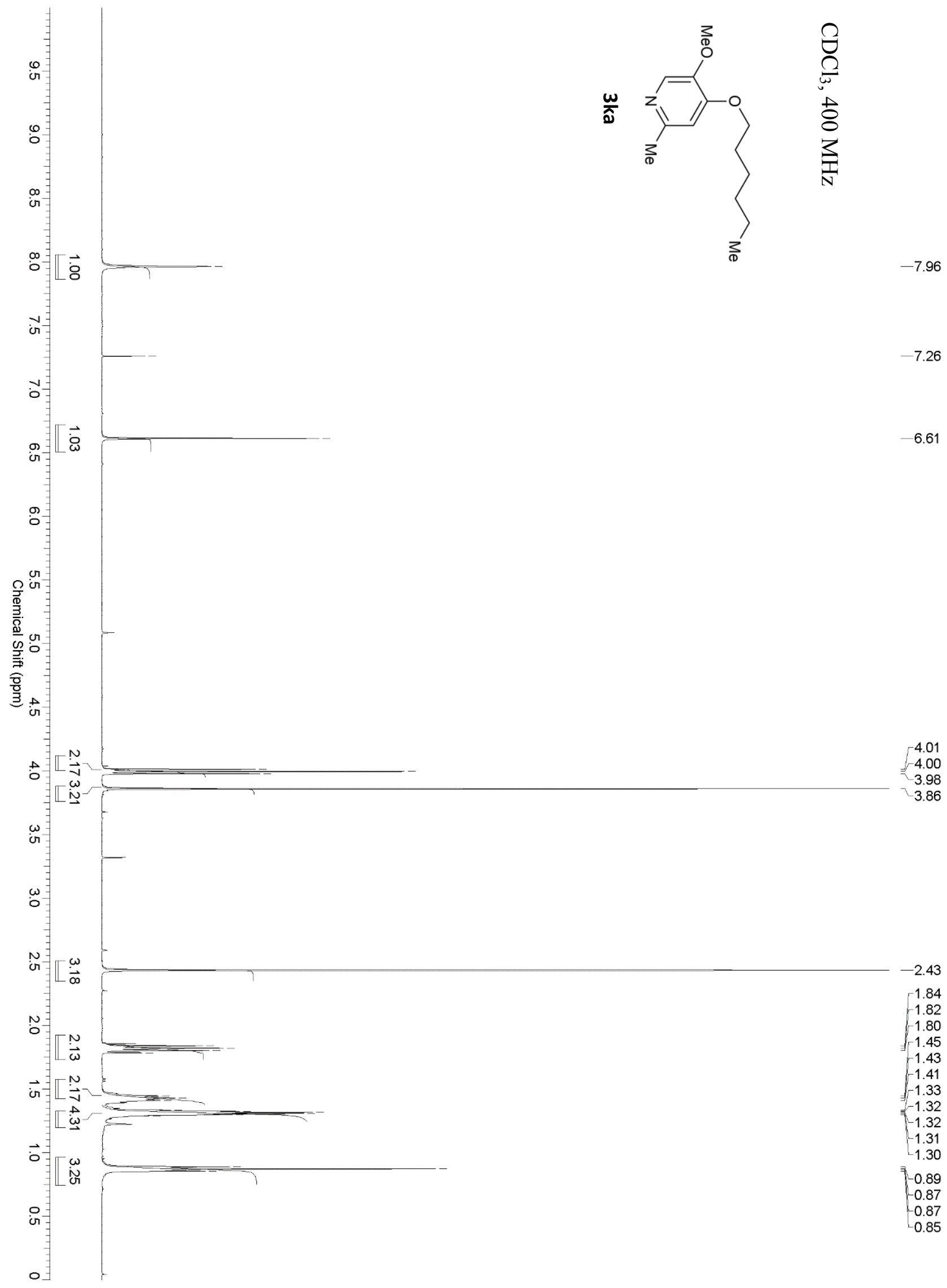




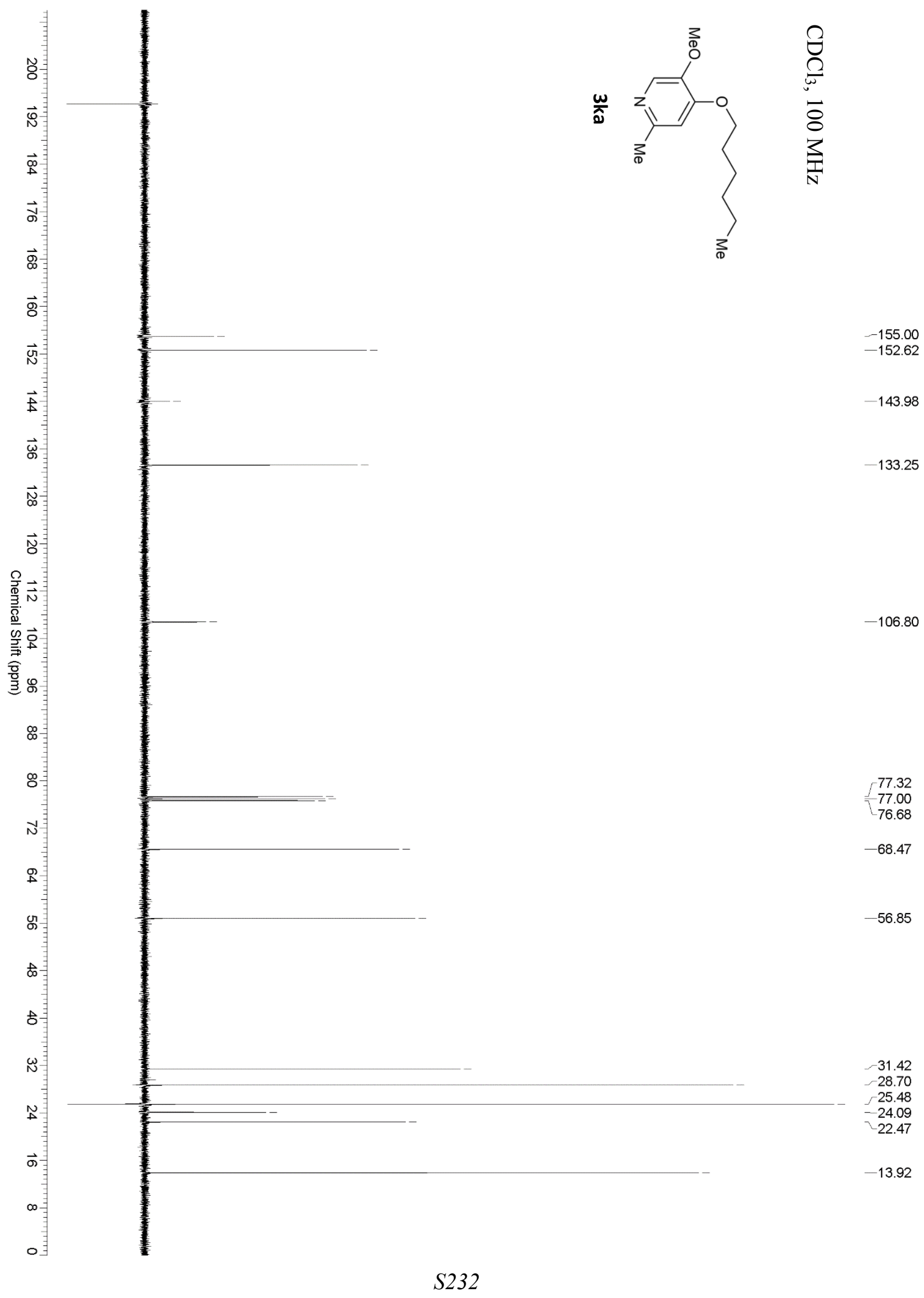




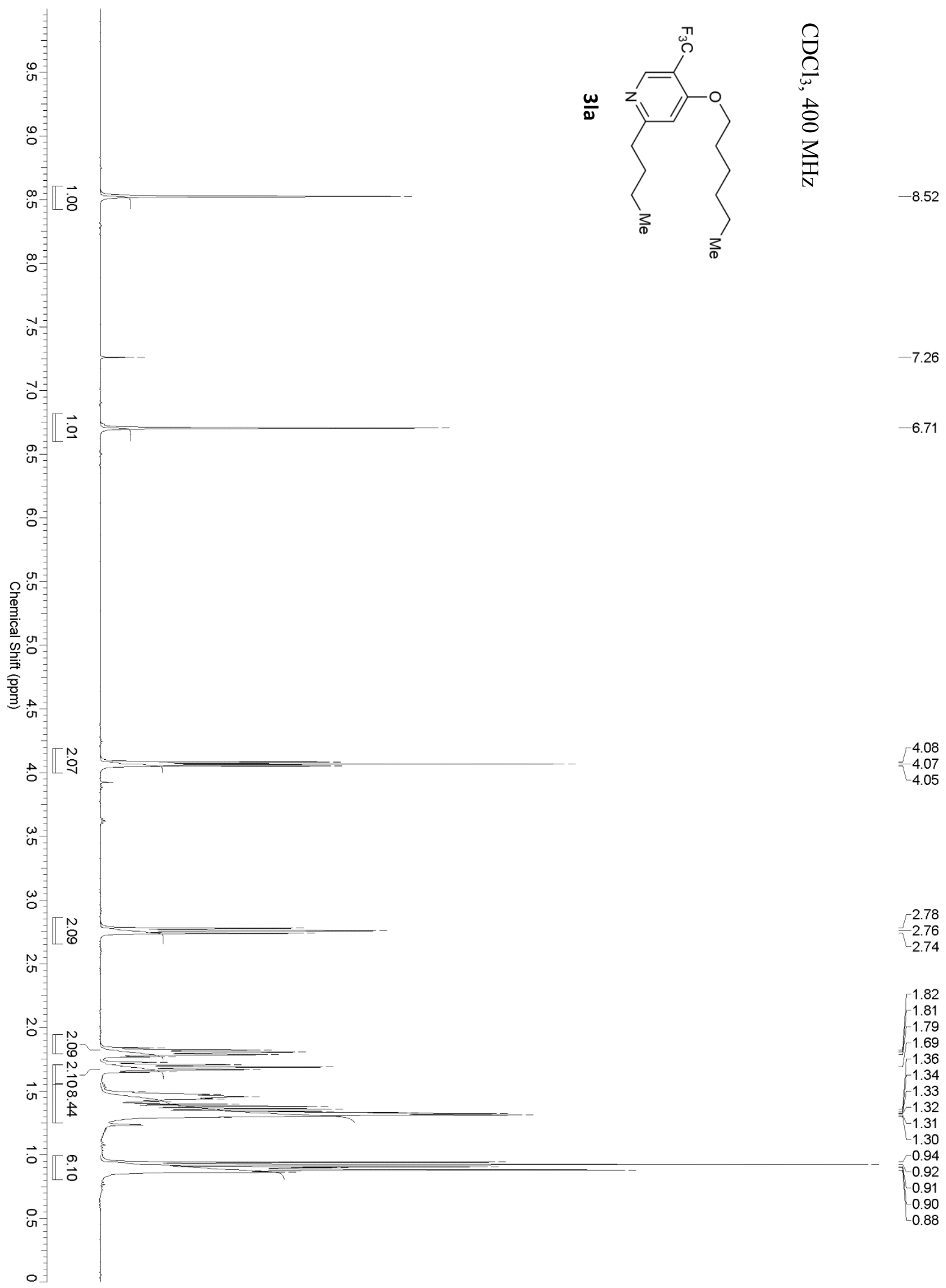




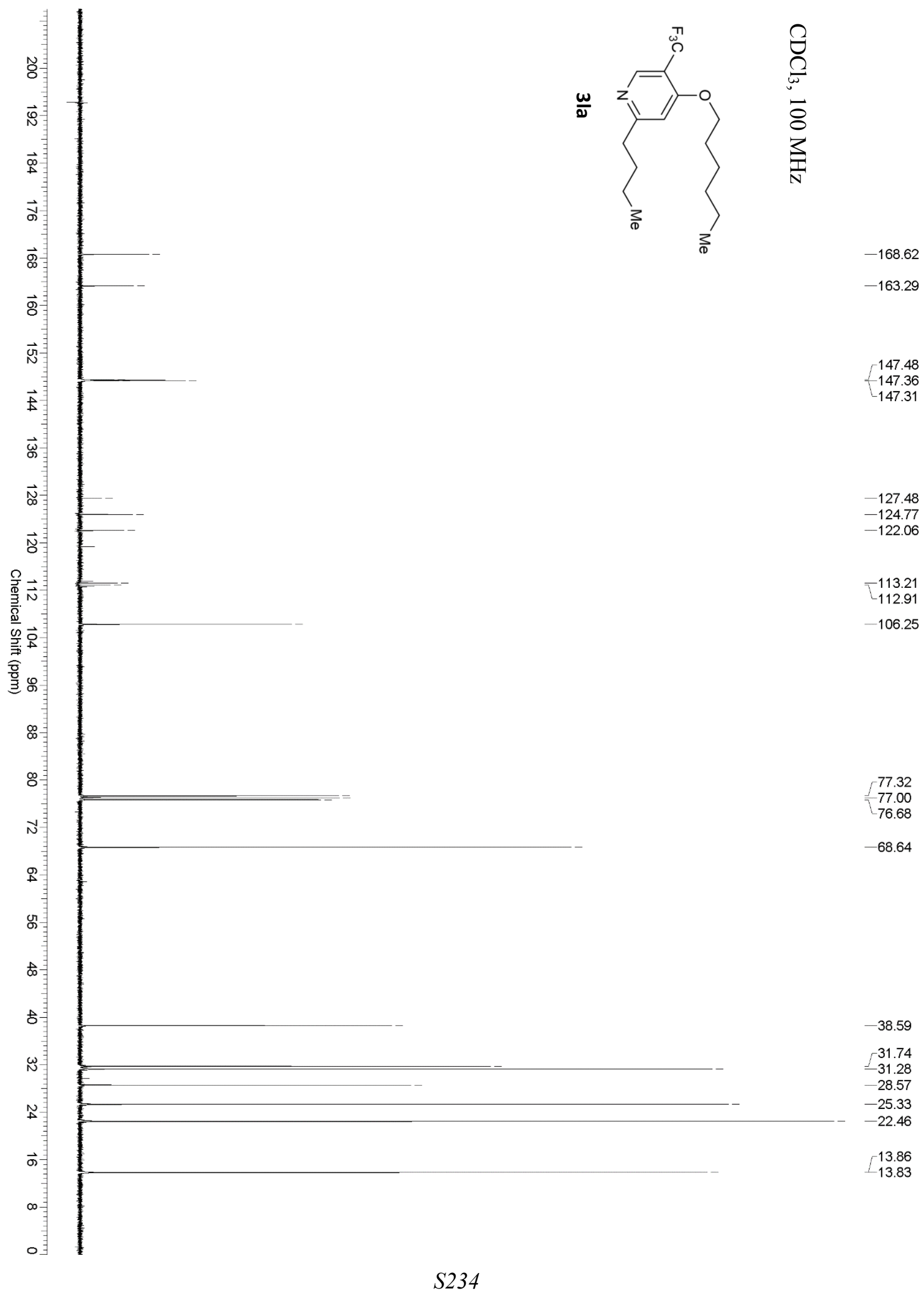




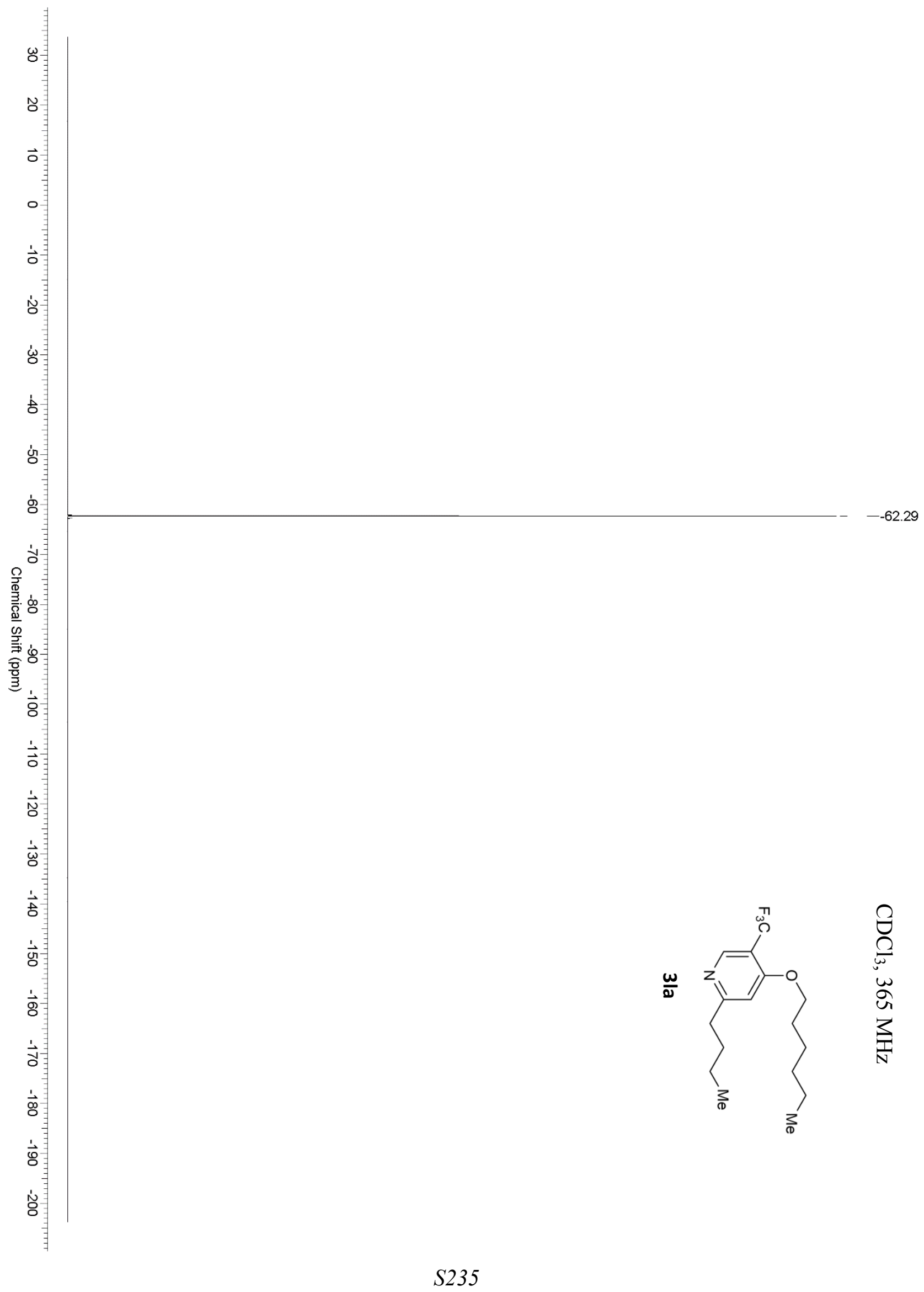




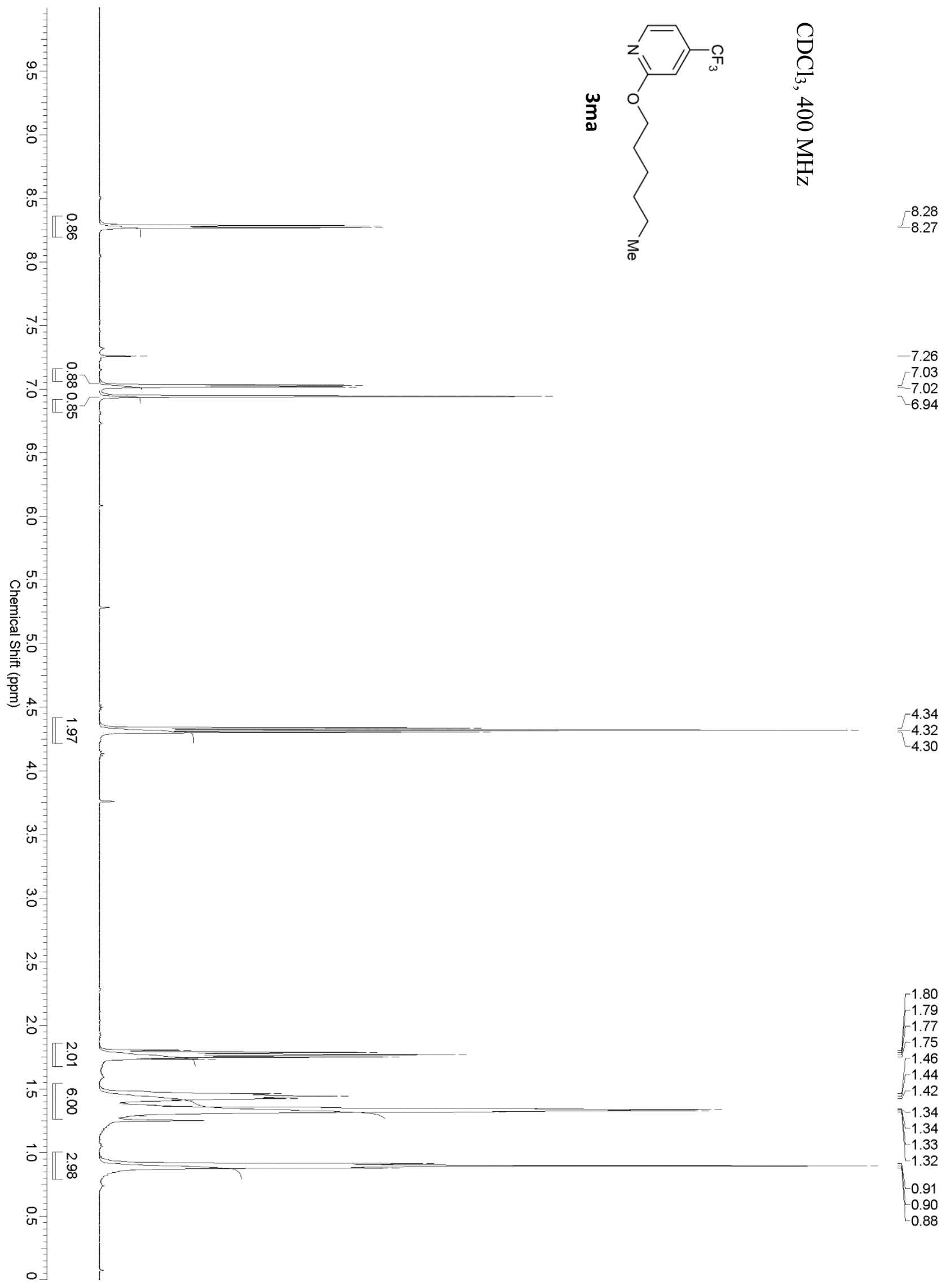




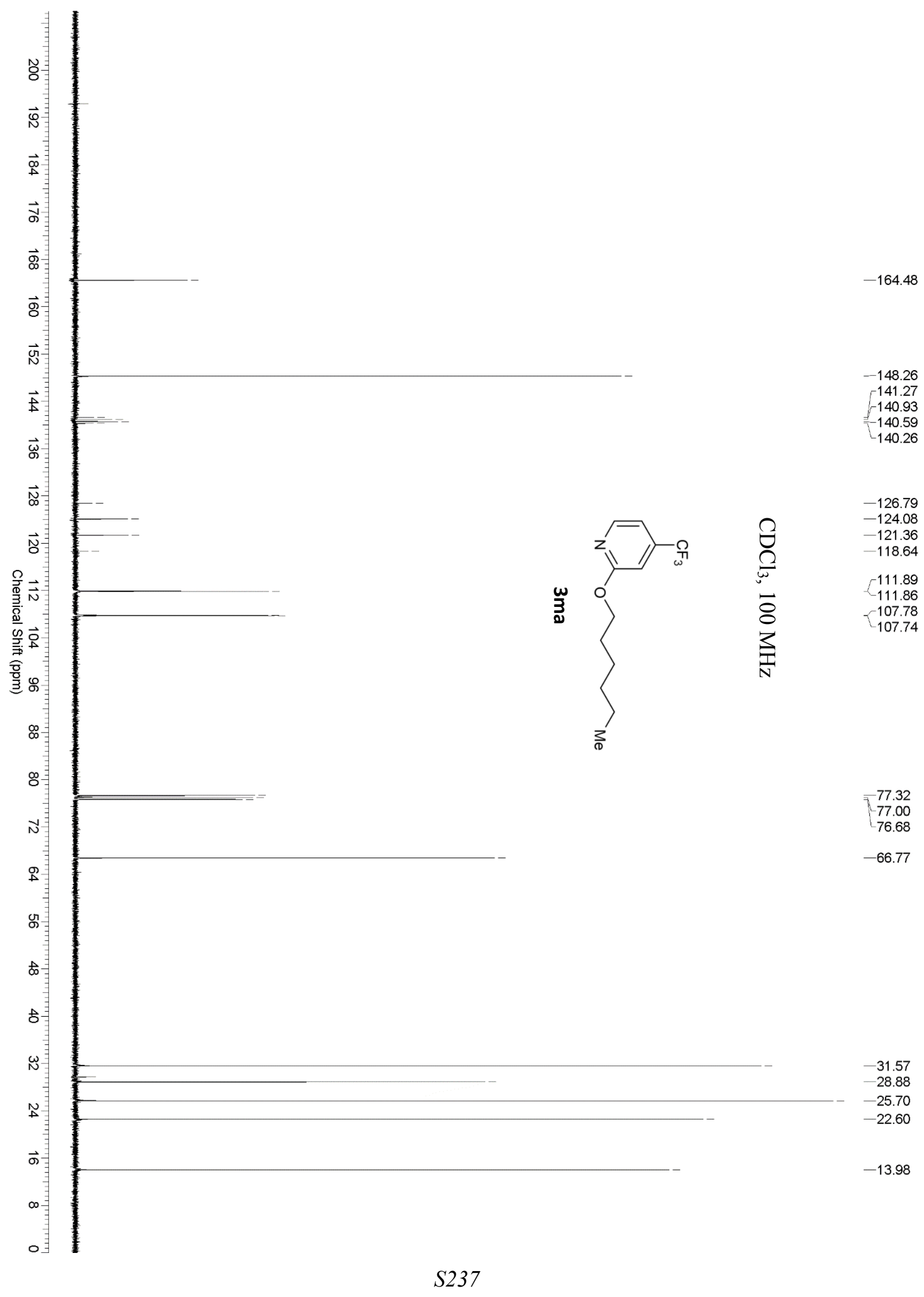




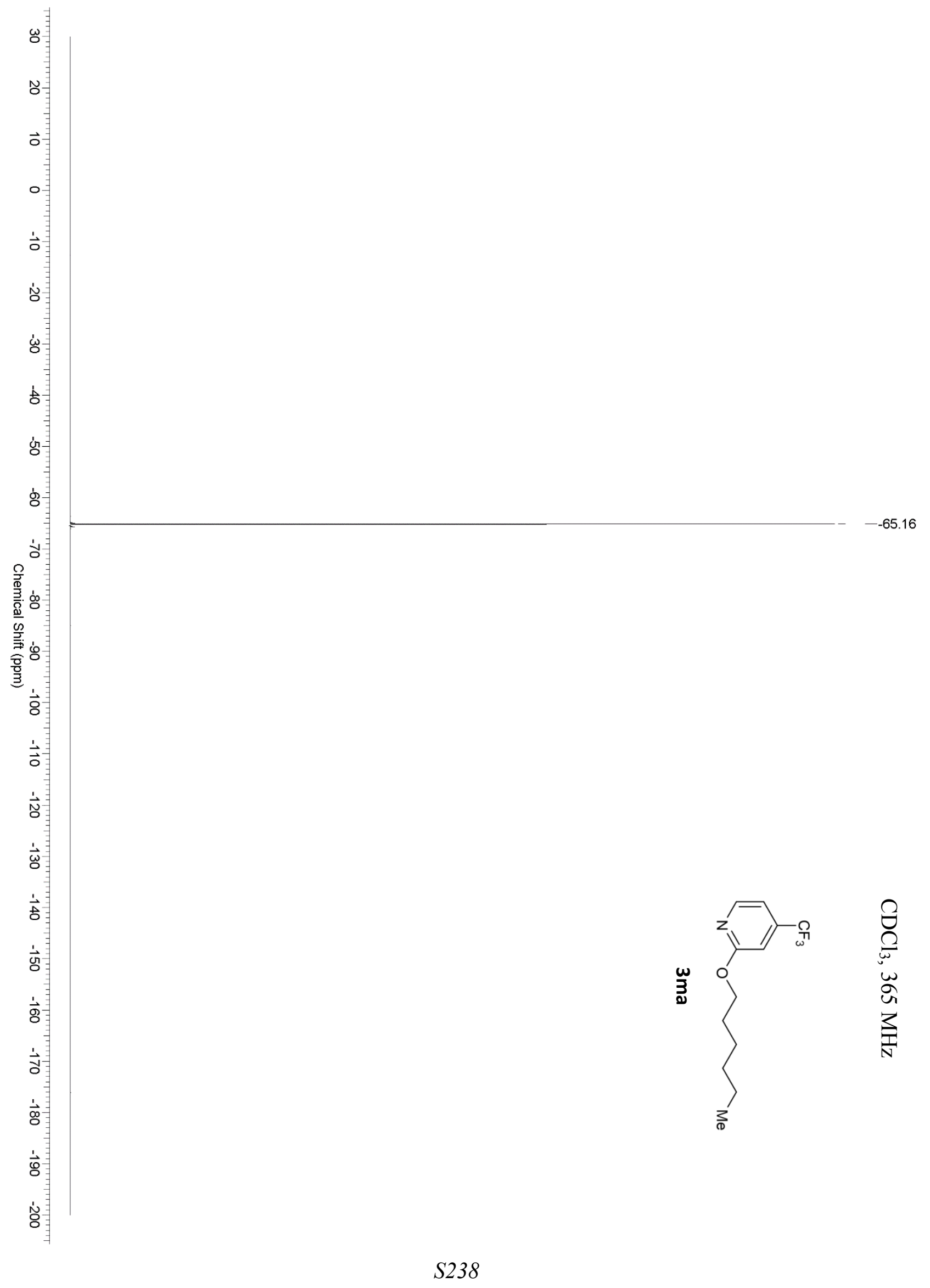




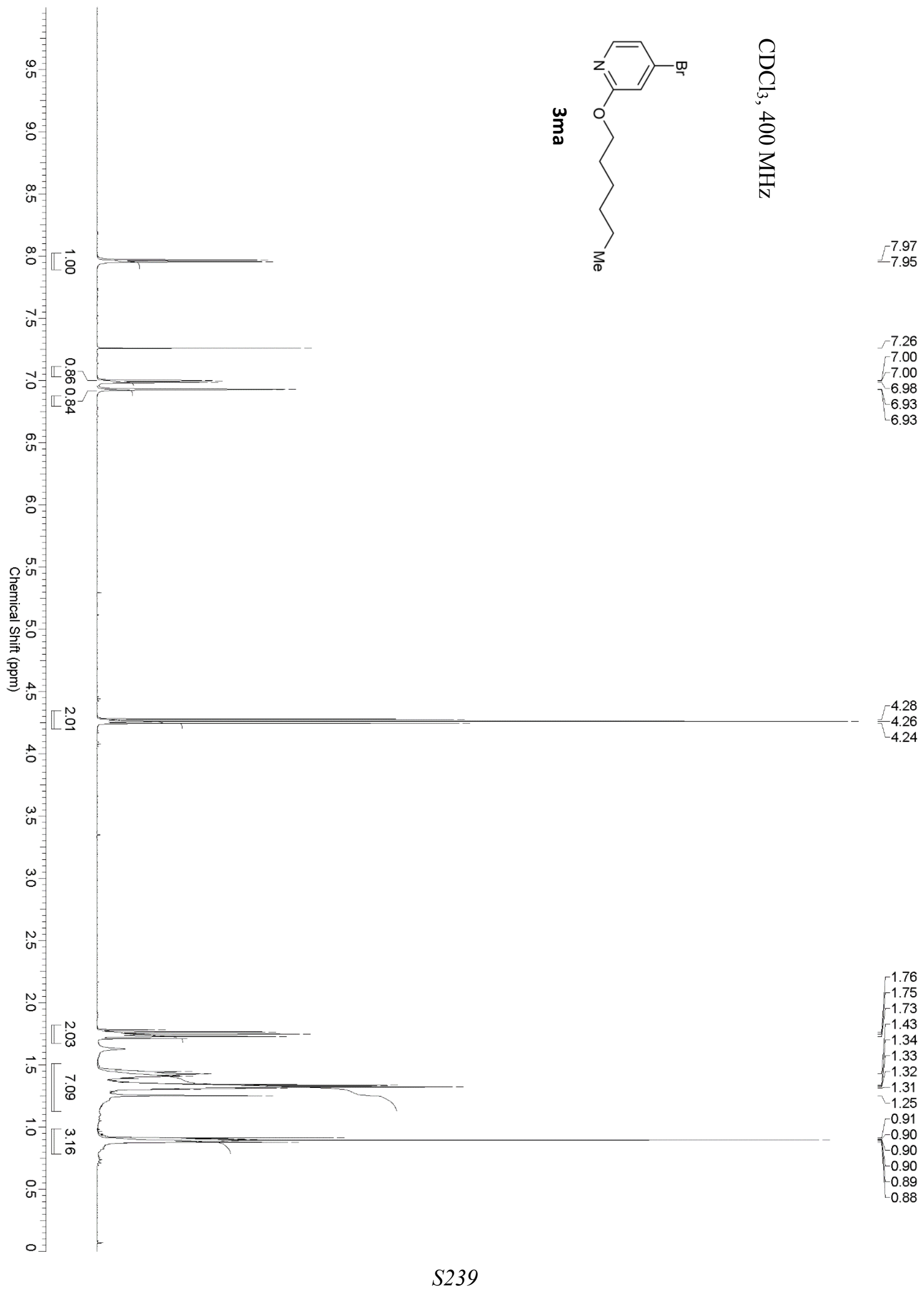




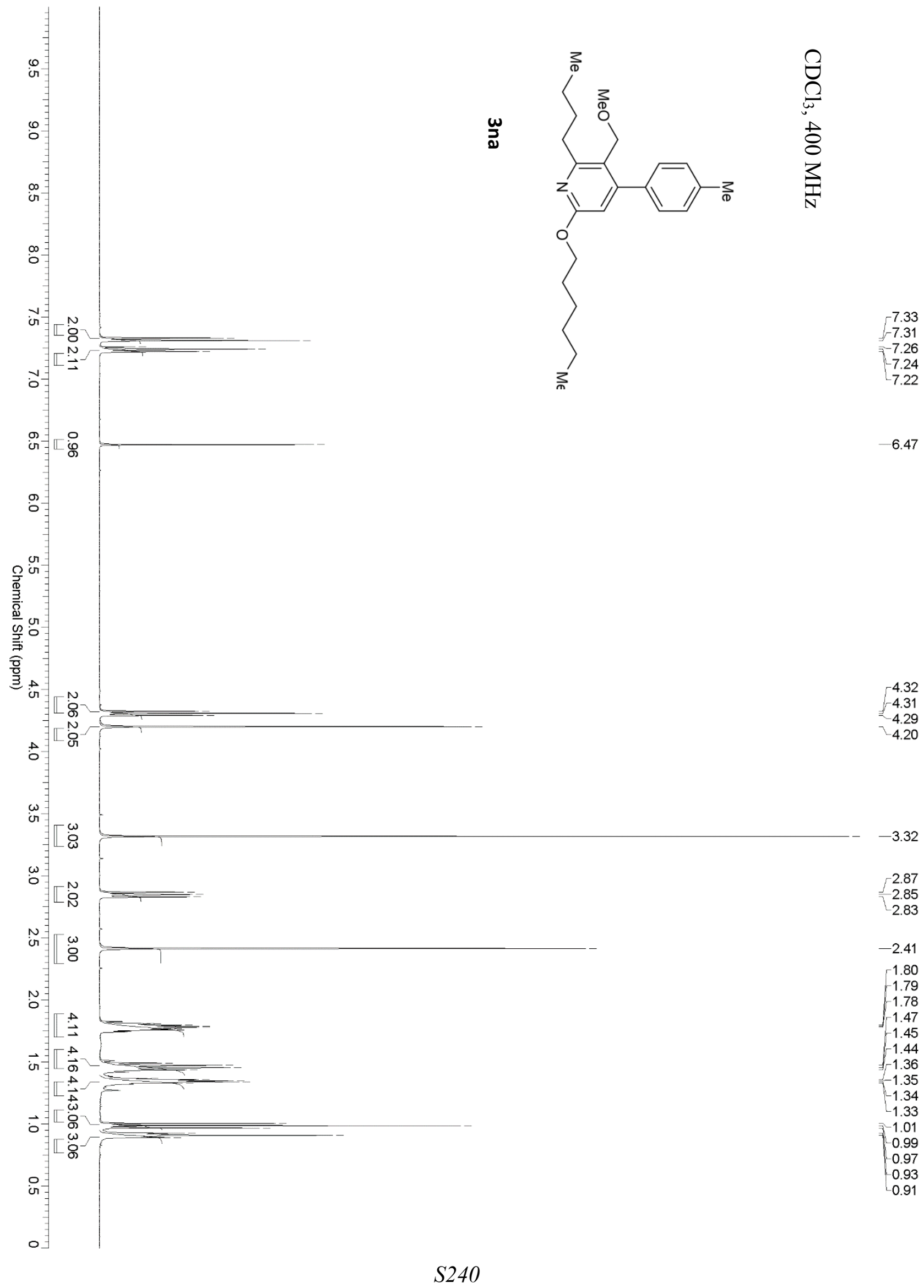




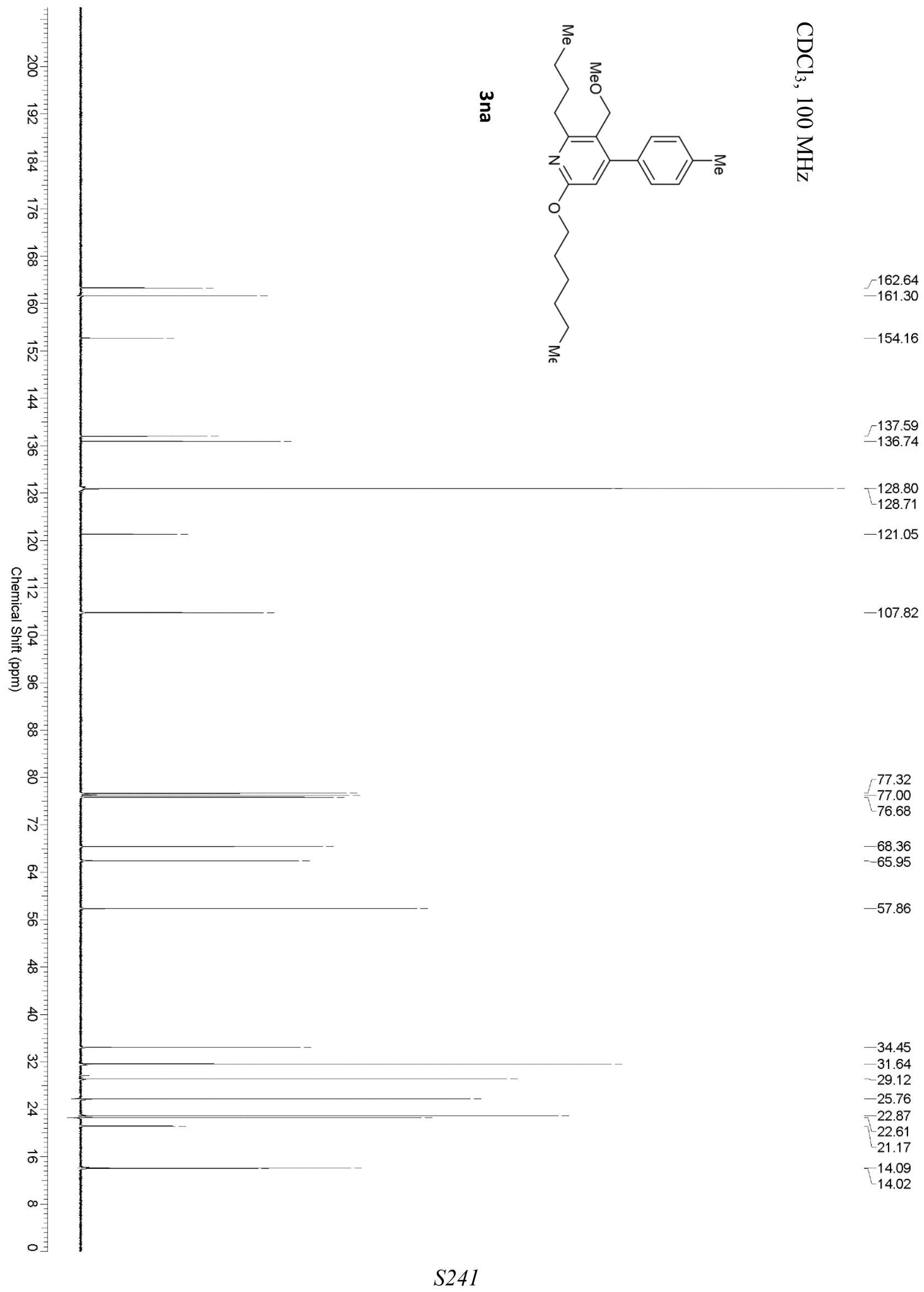



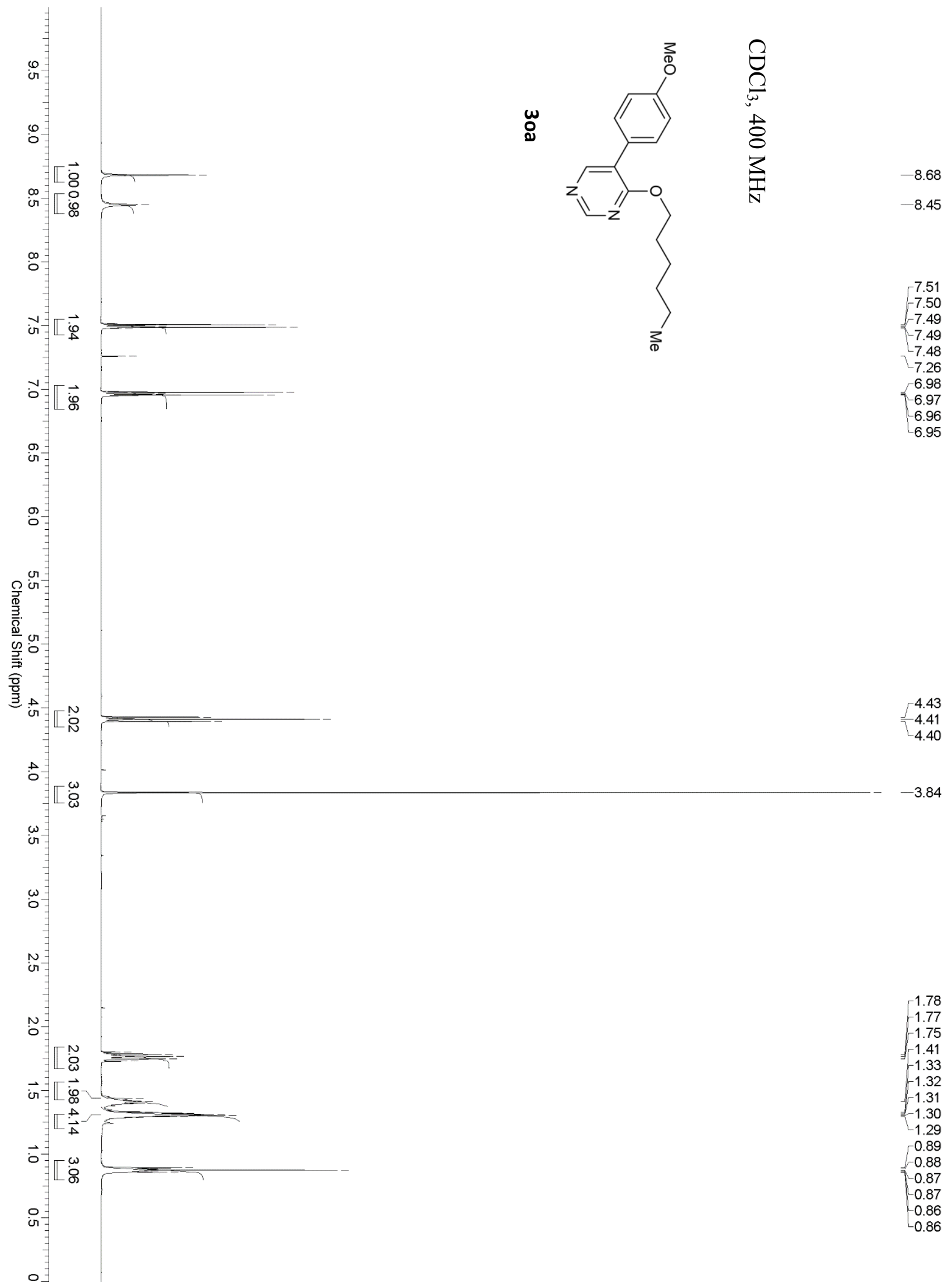

$-3.84$

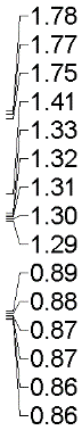




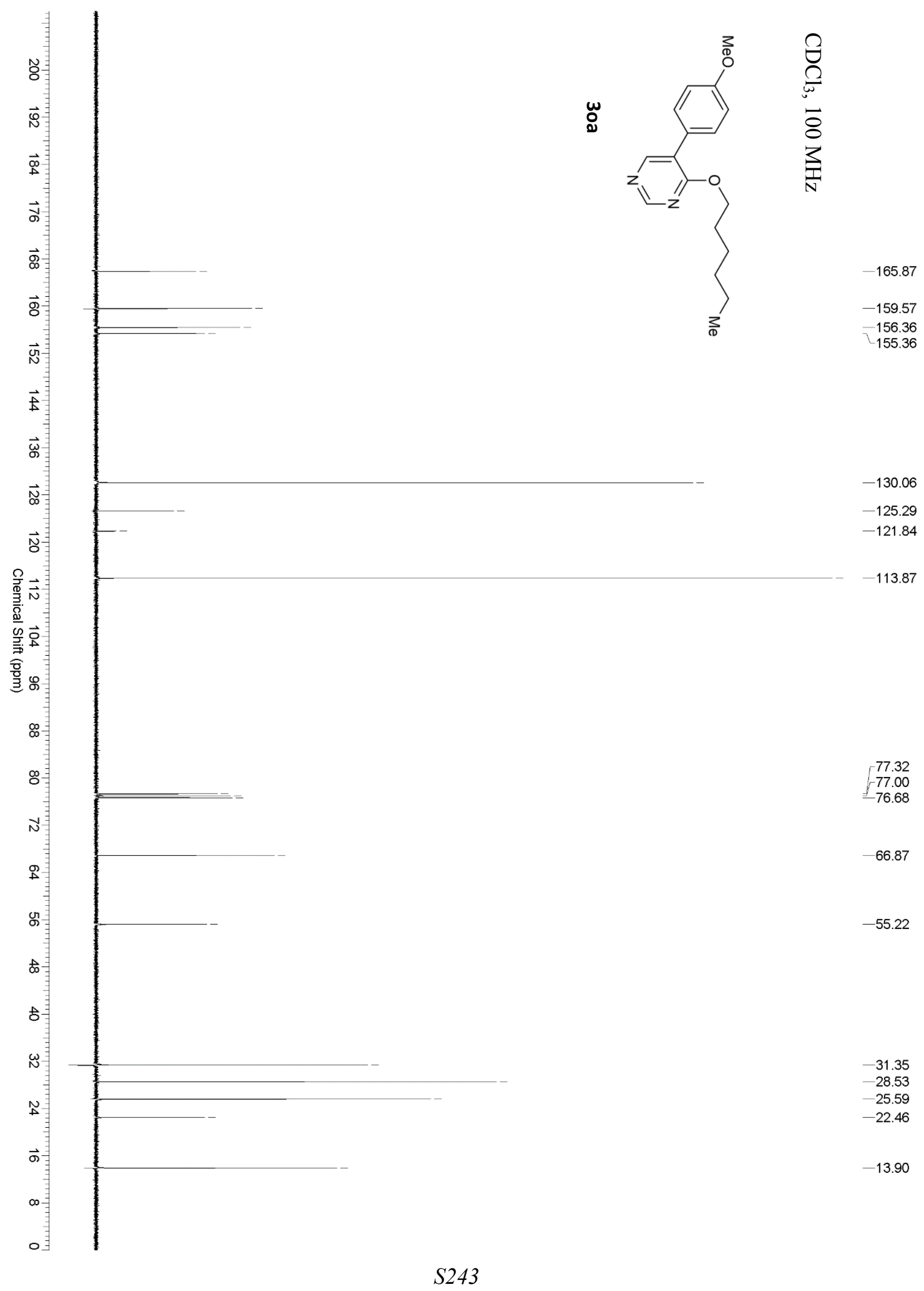




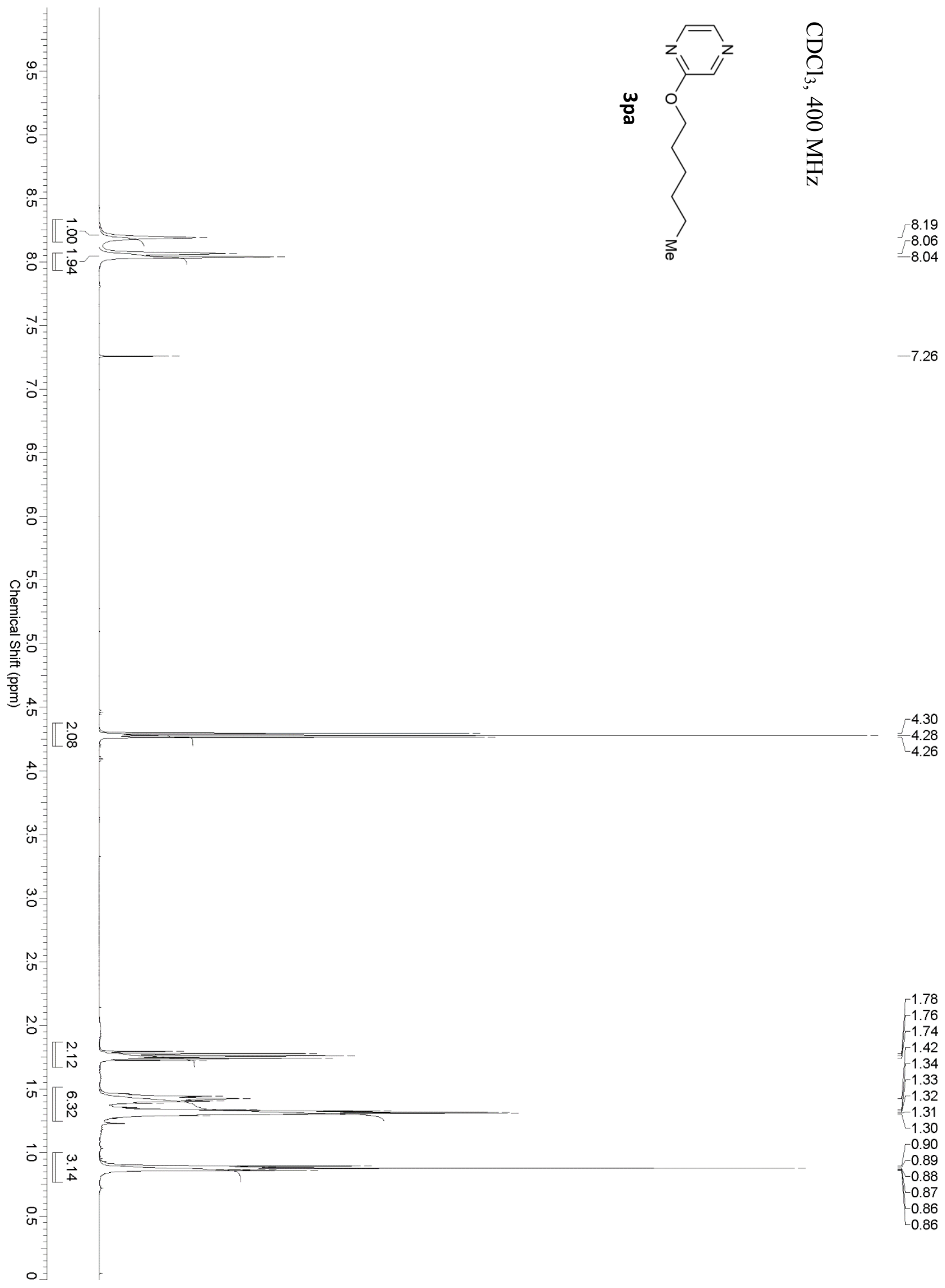




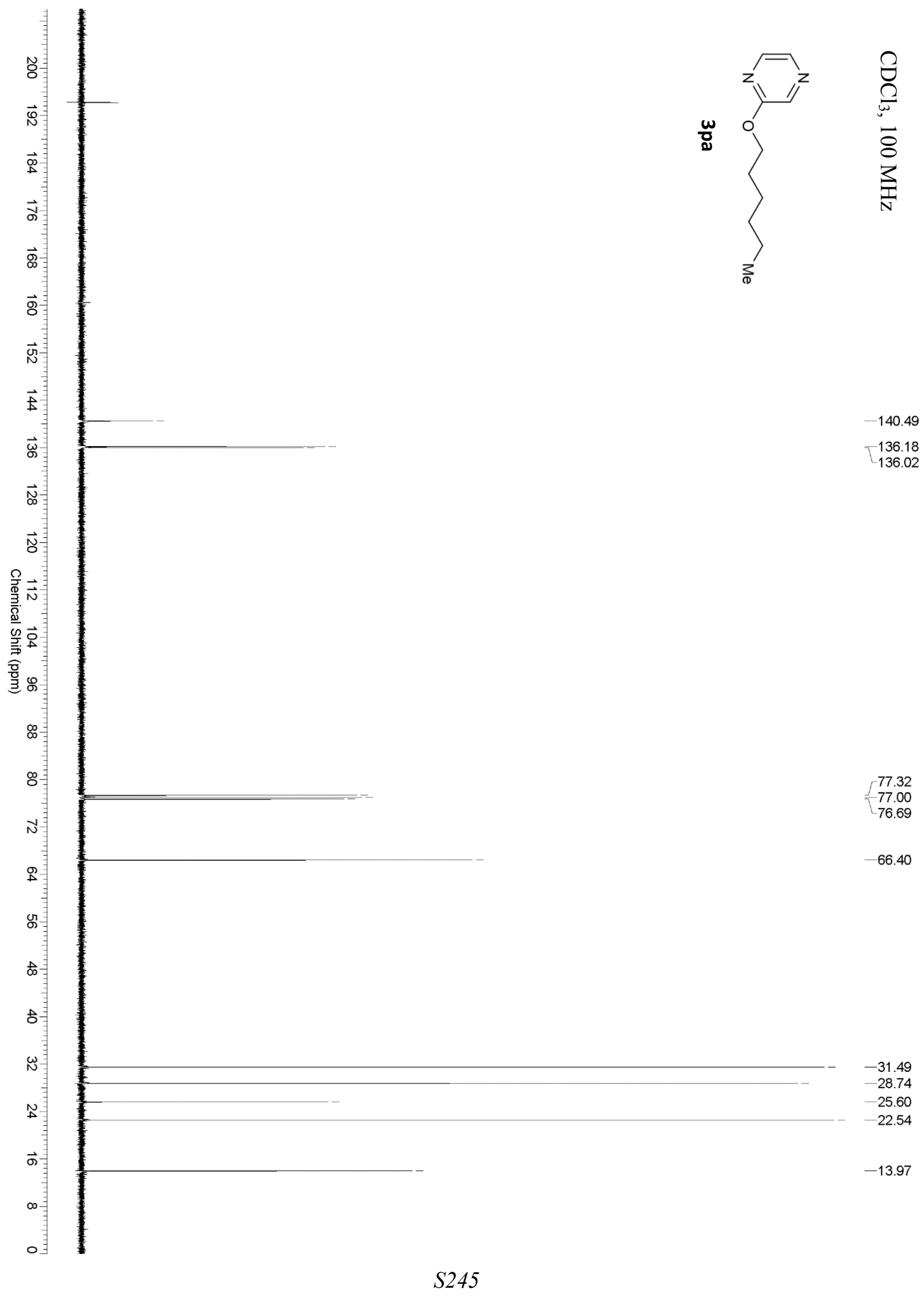




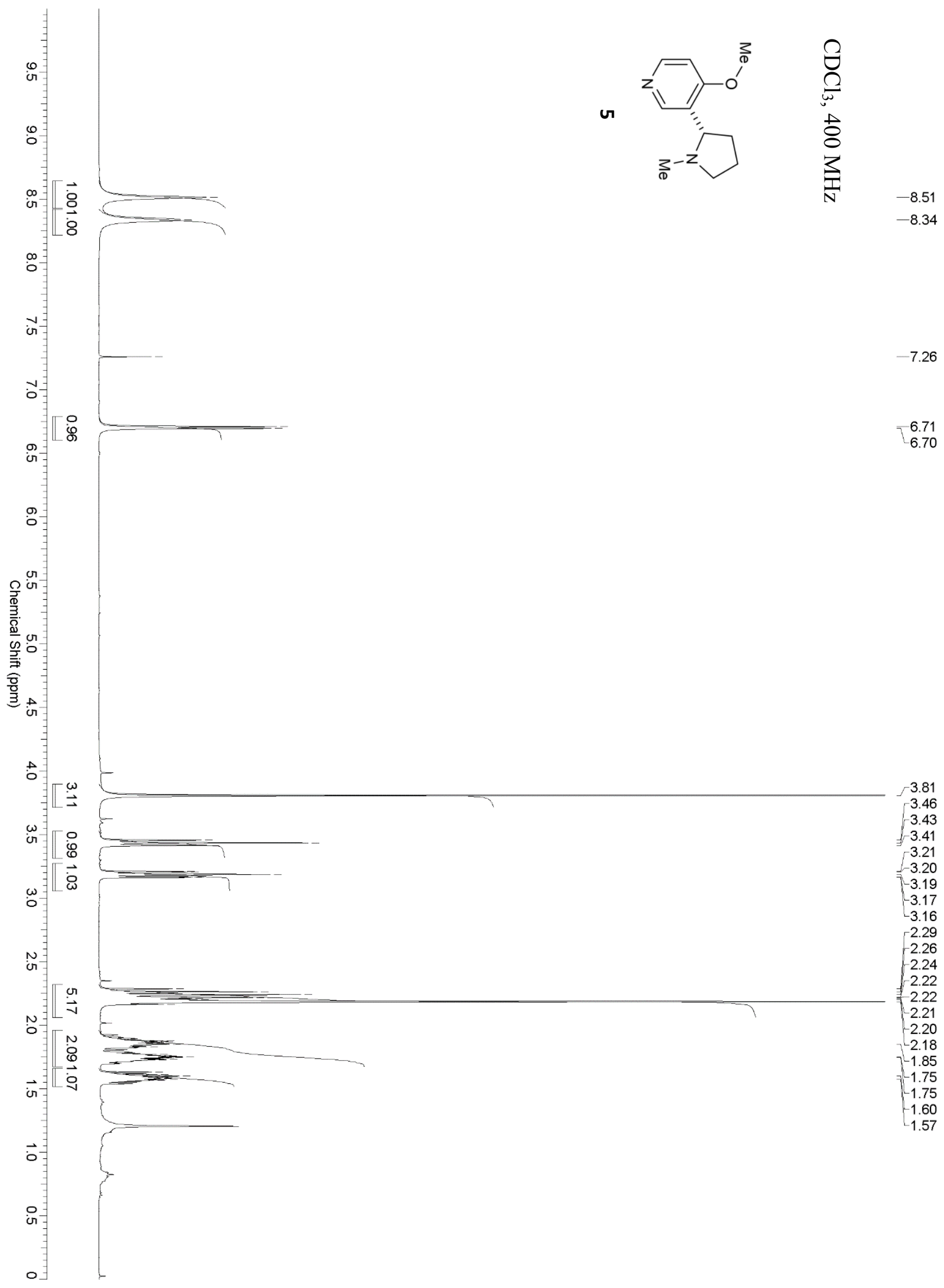




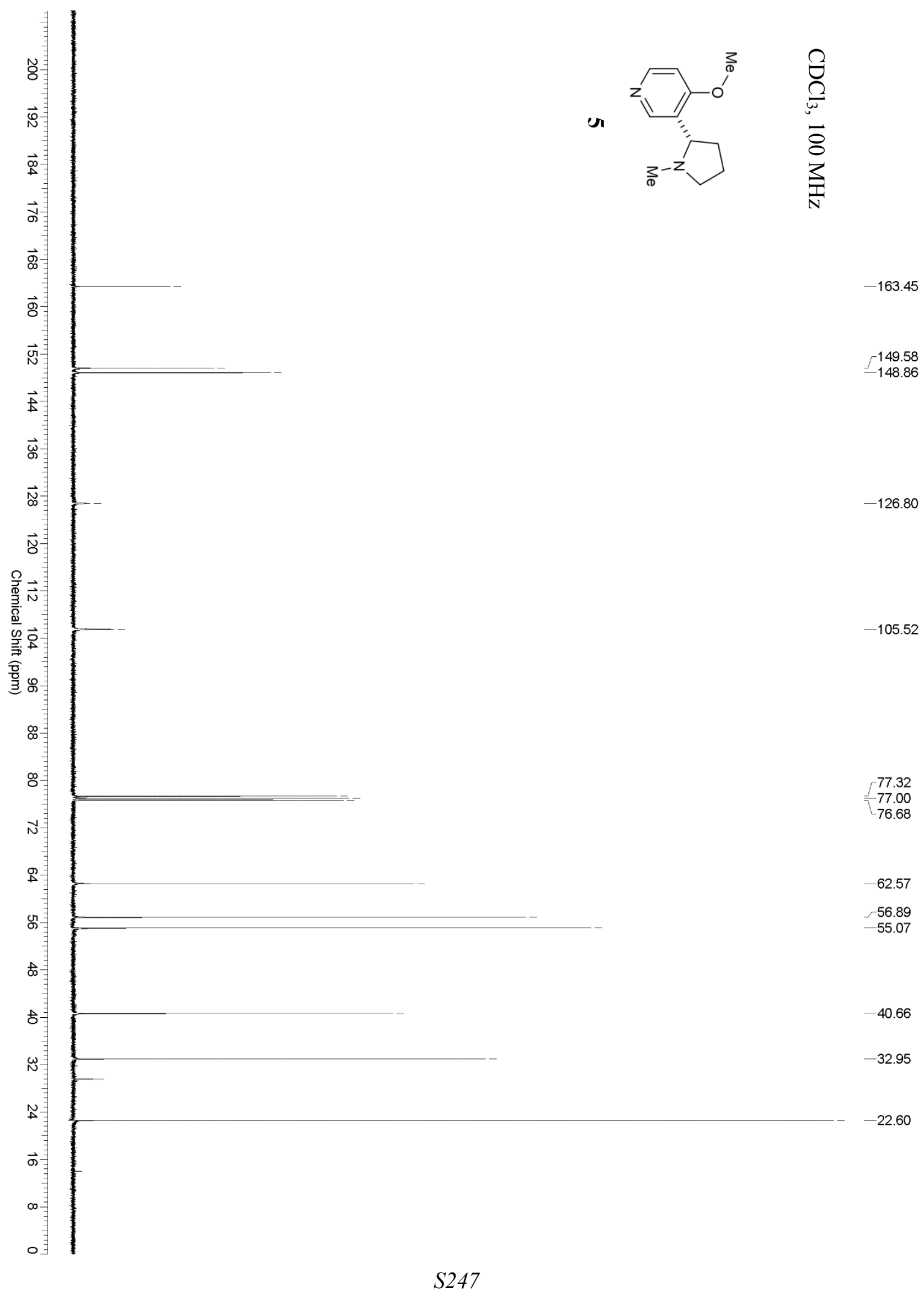




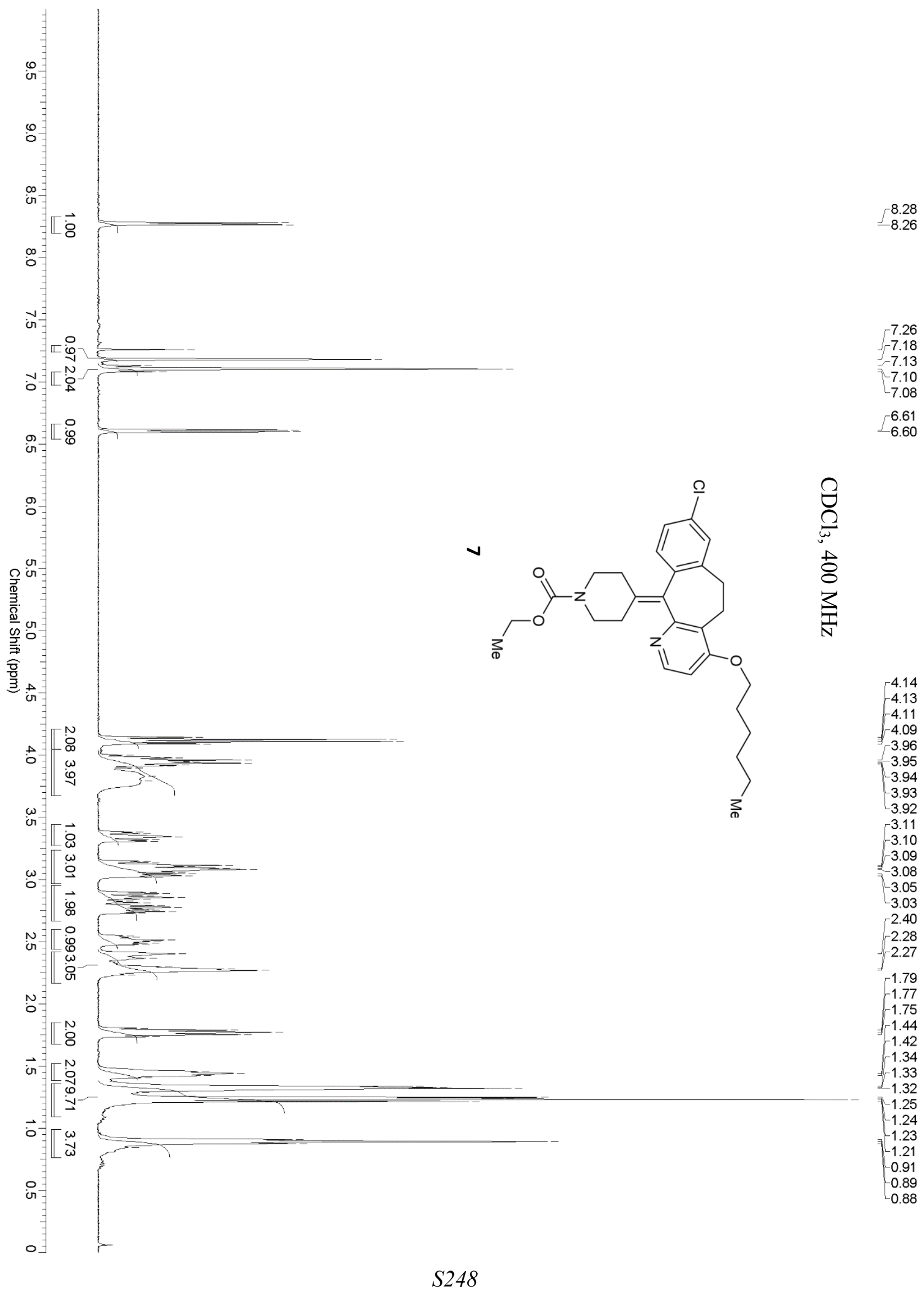




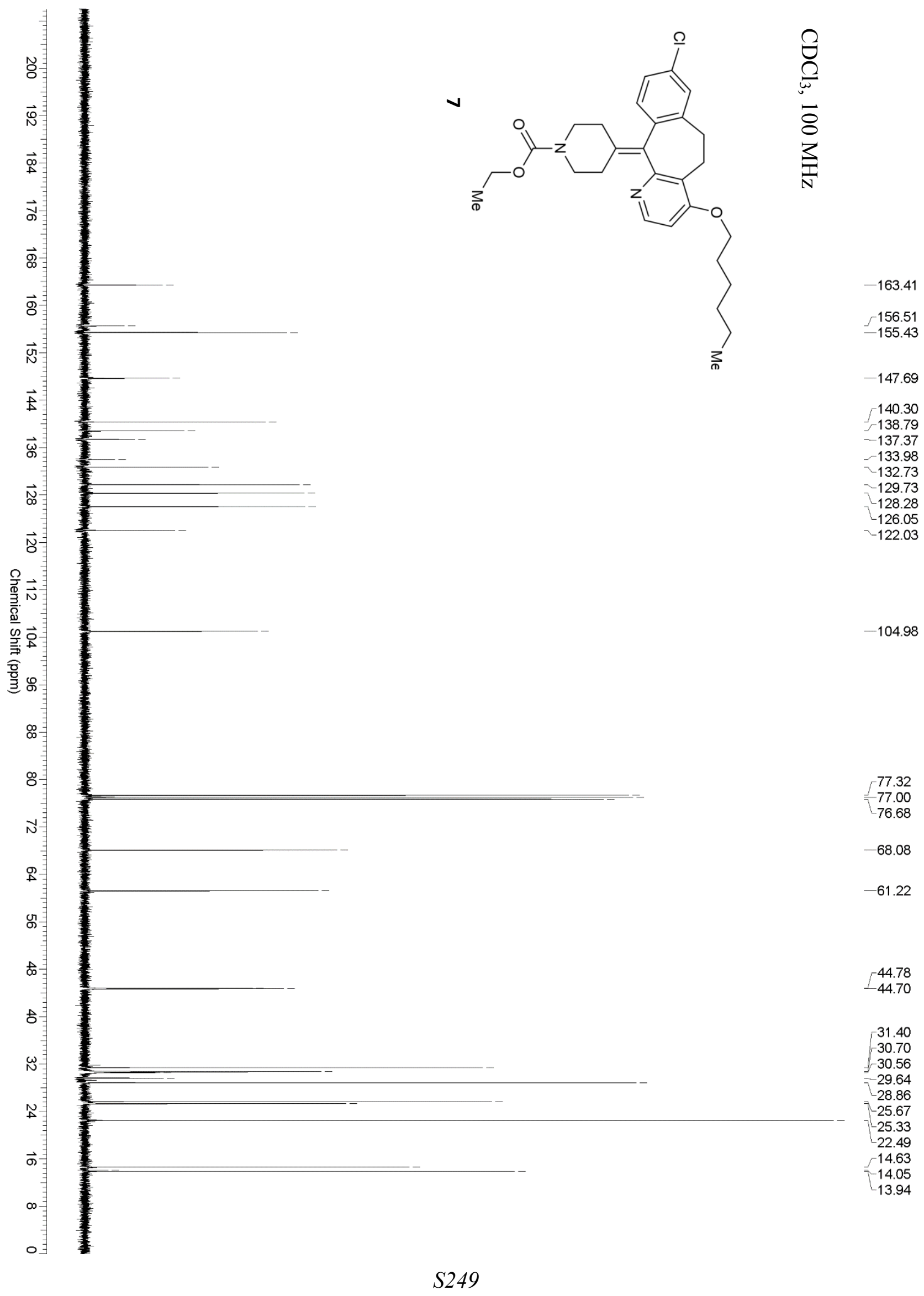




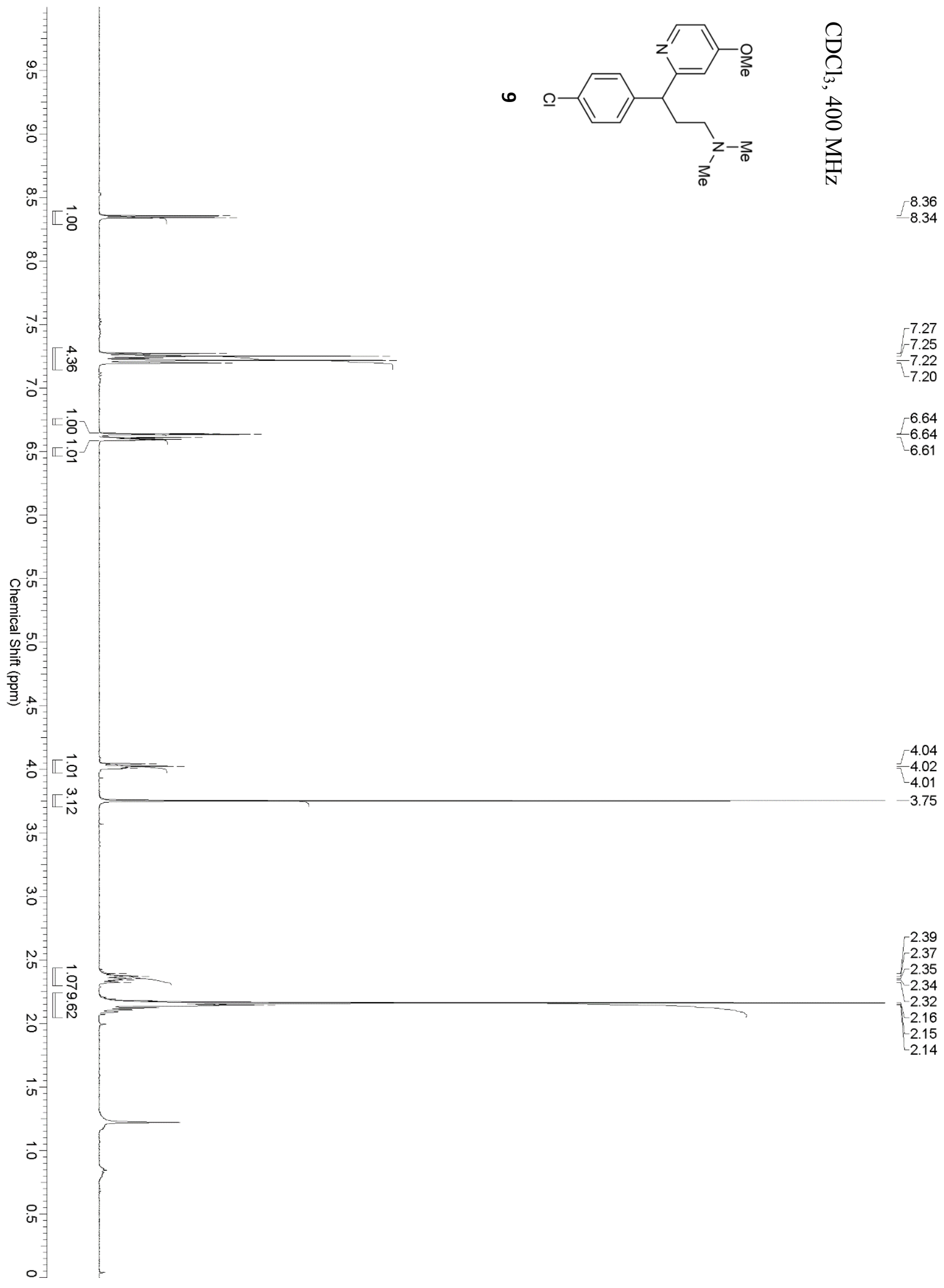




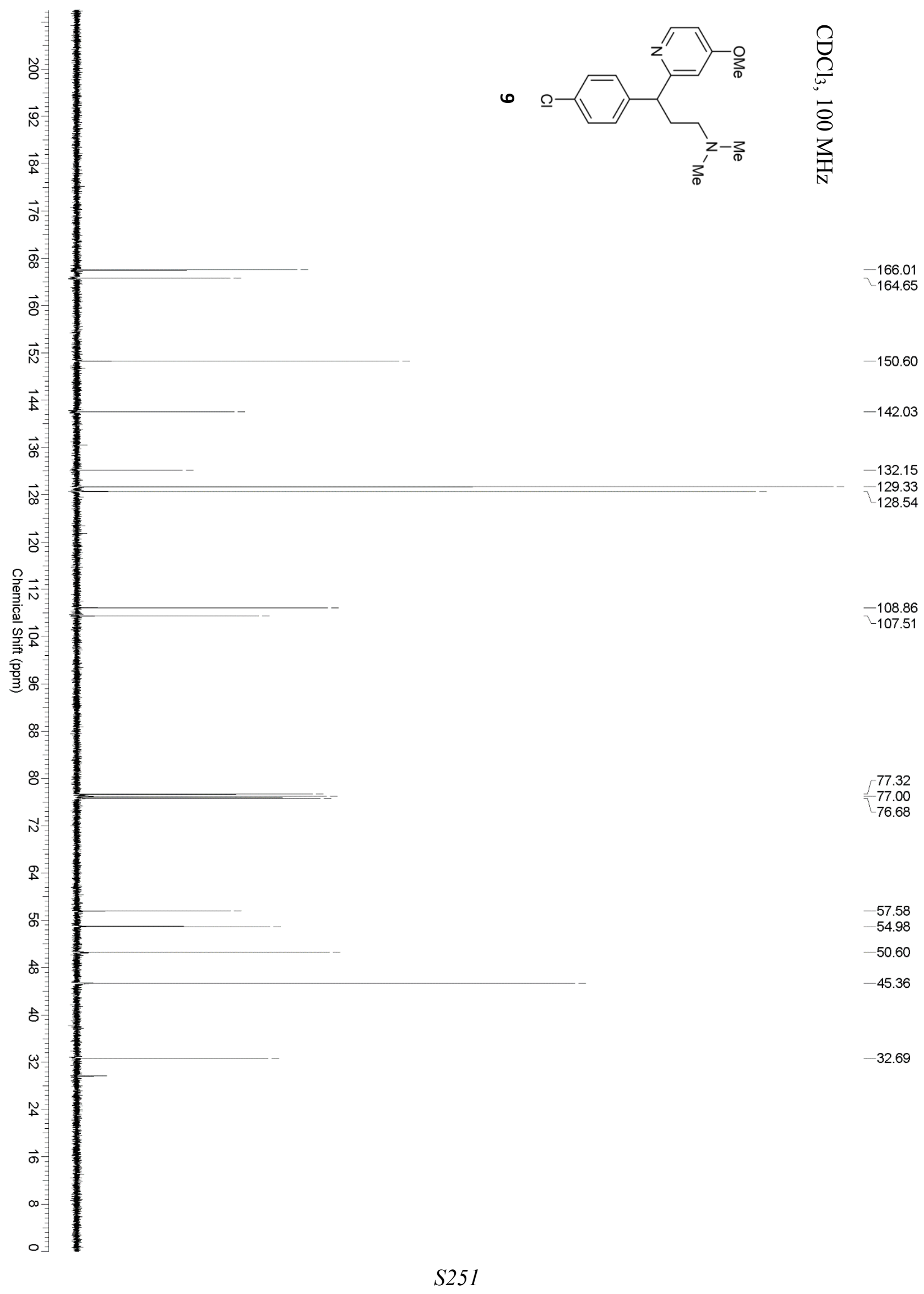




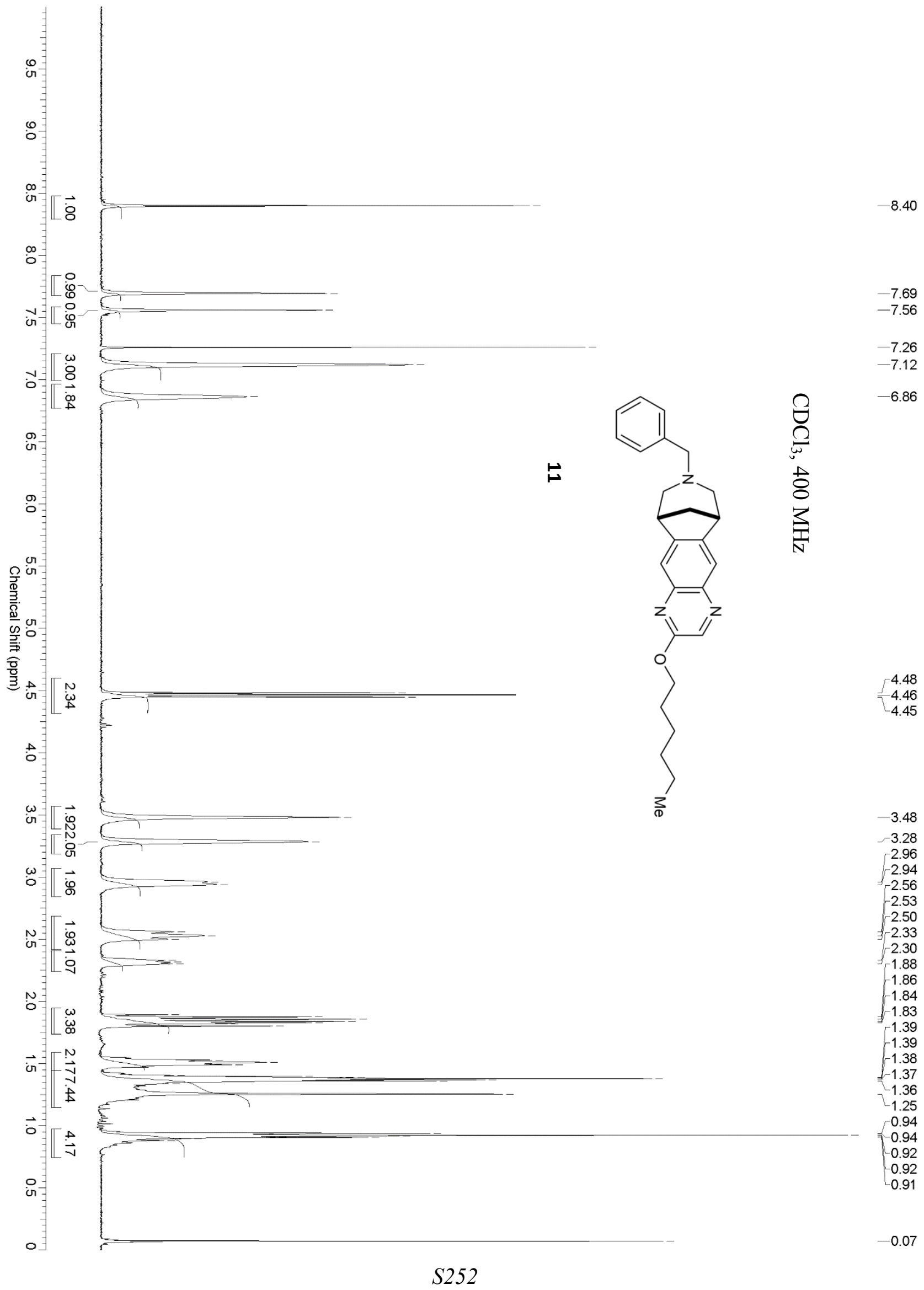




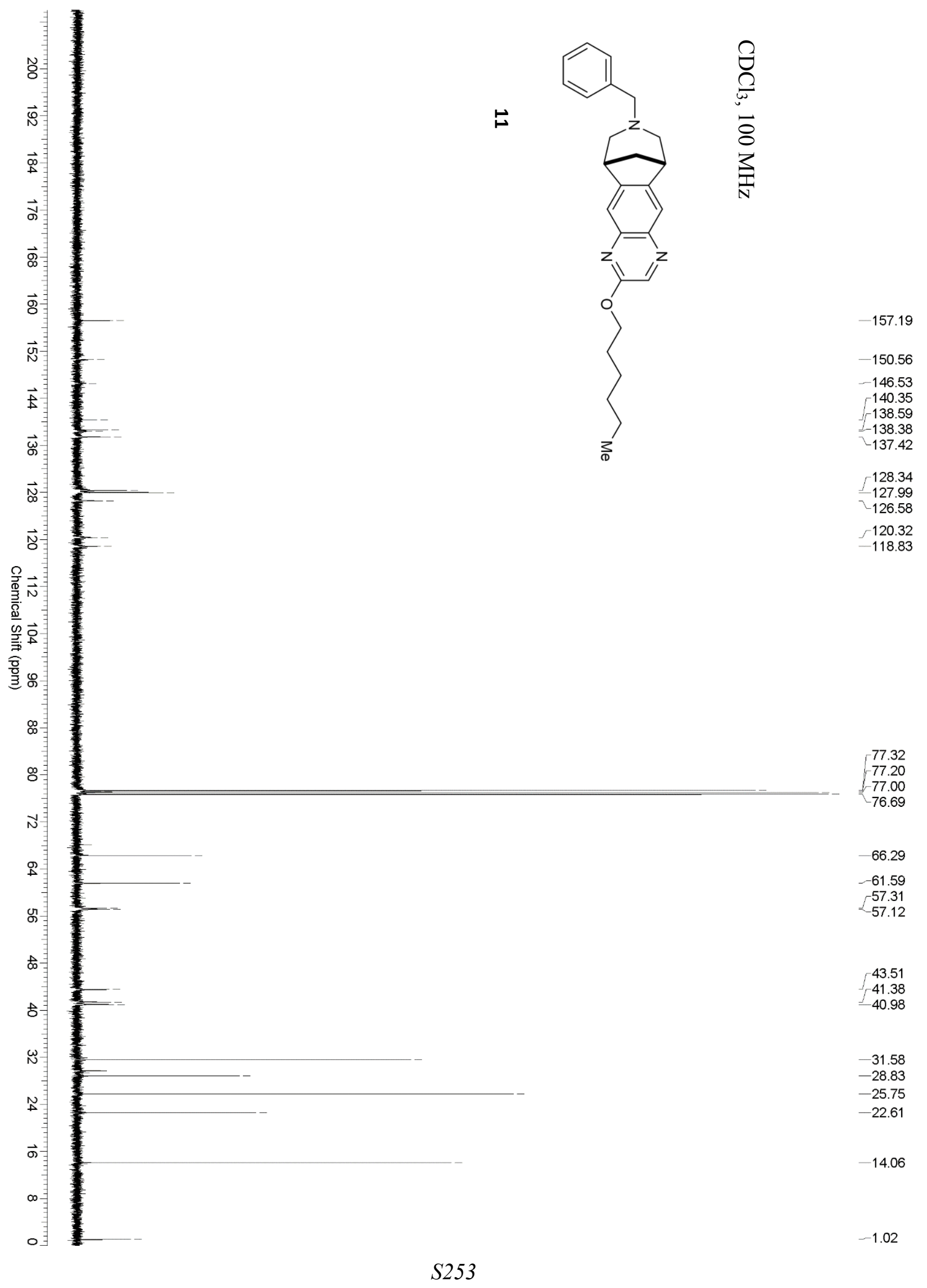




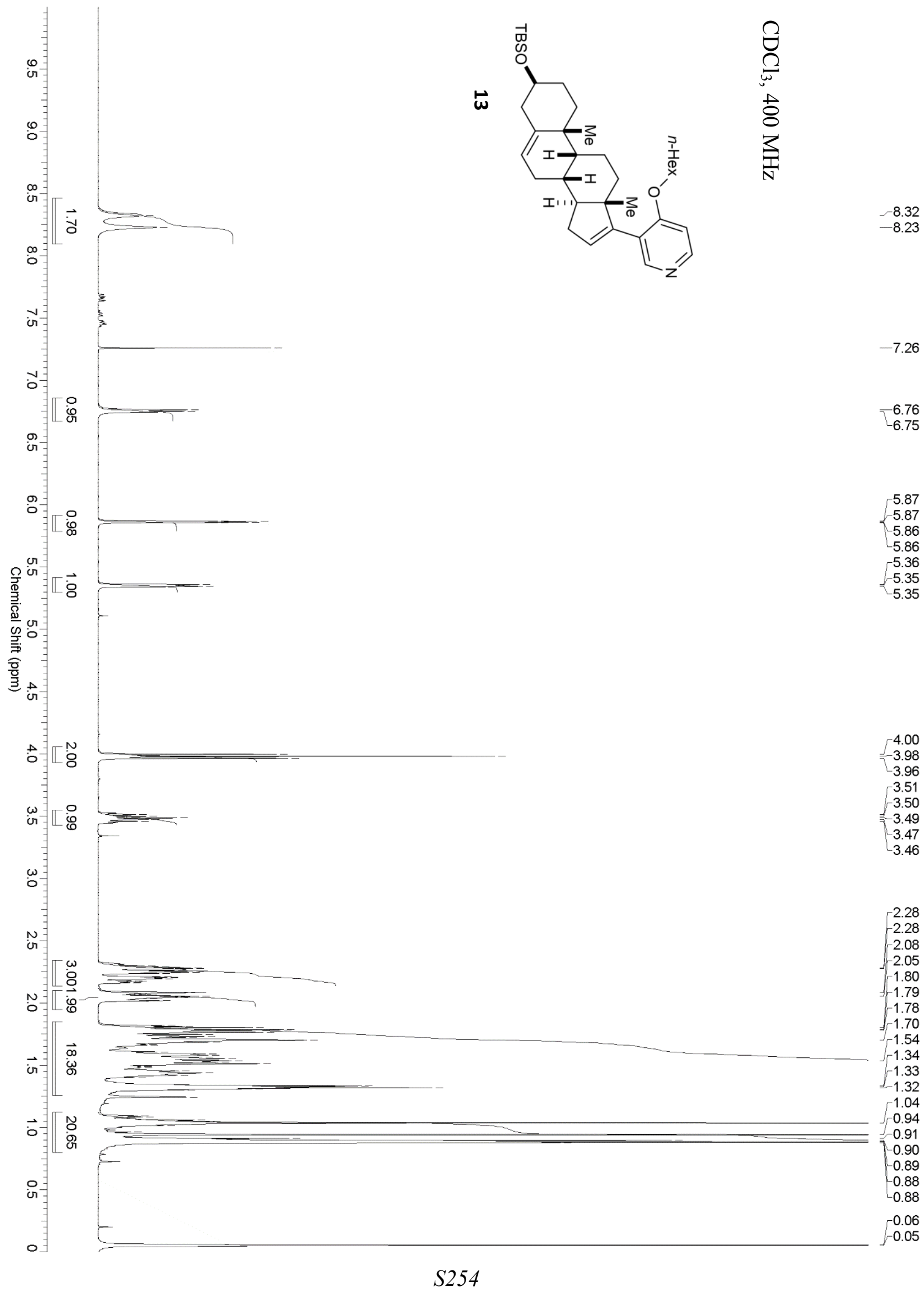




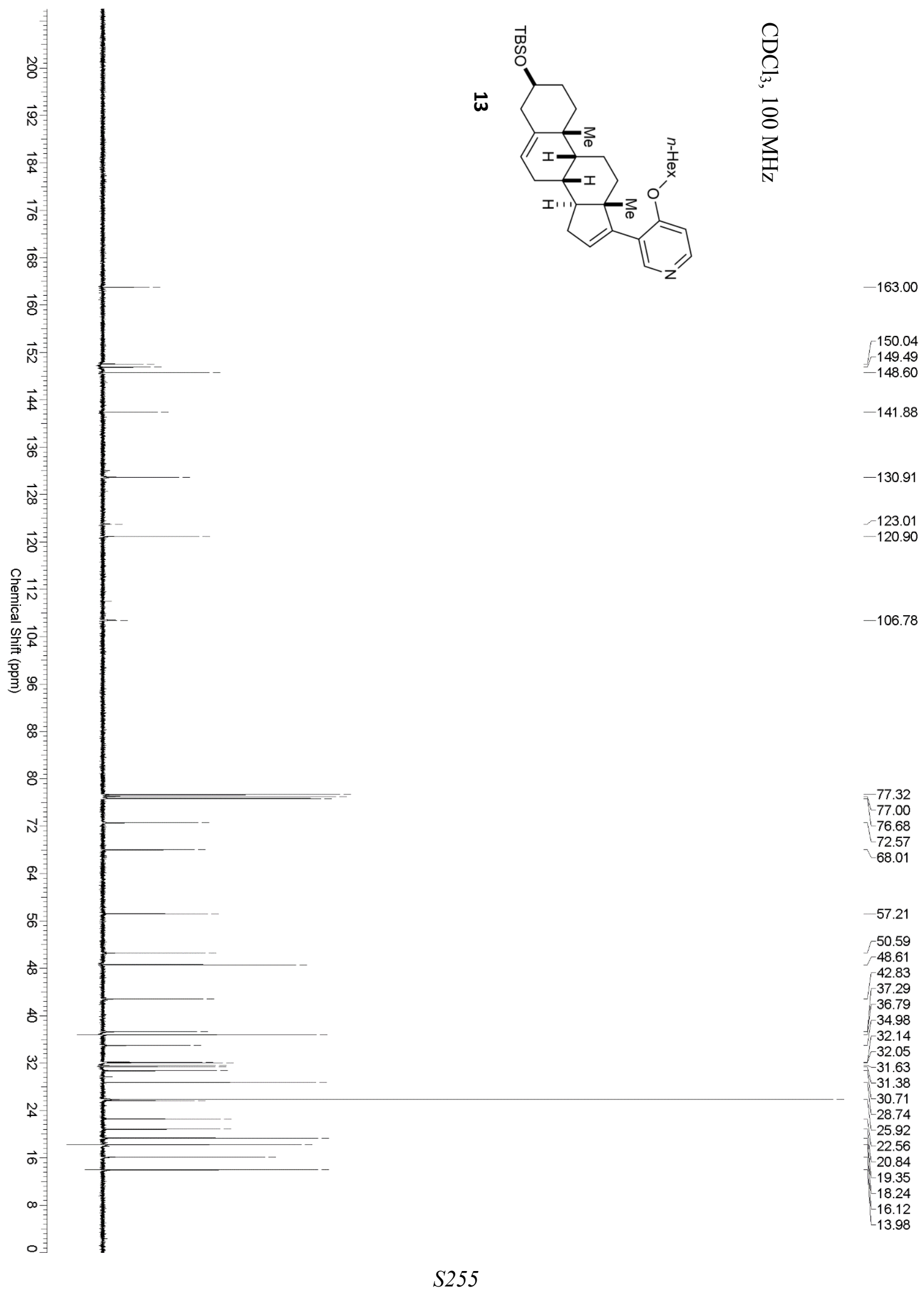




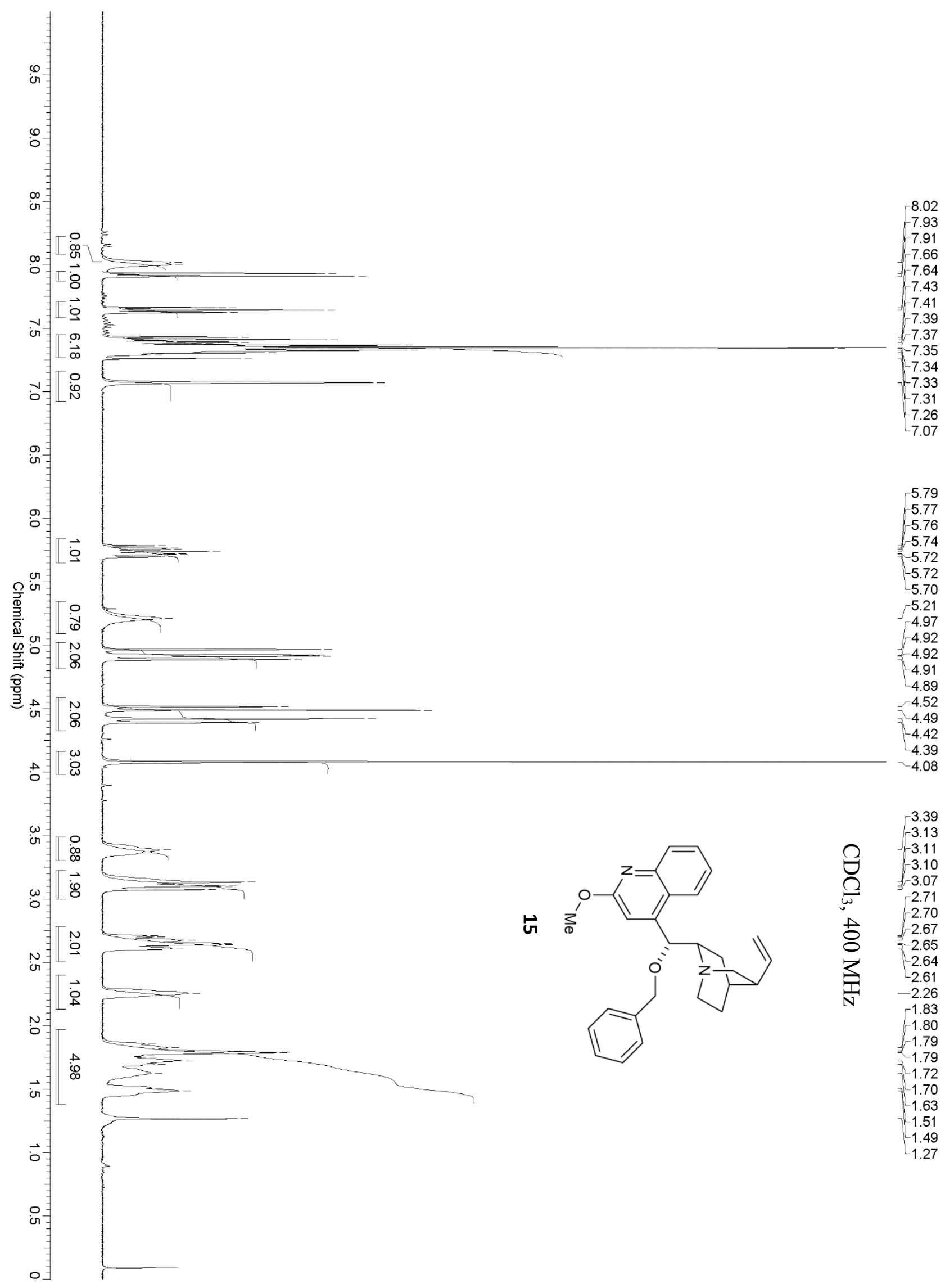




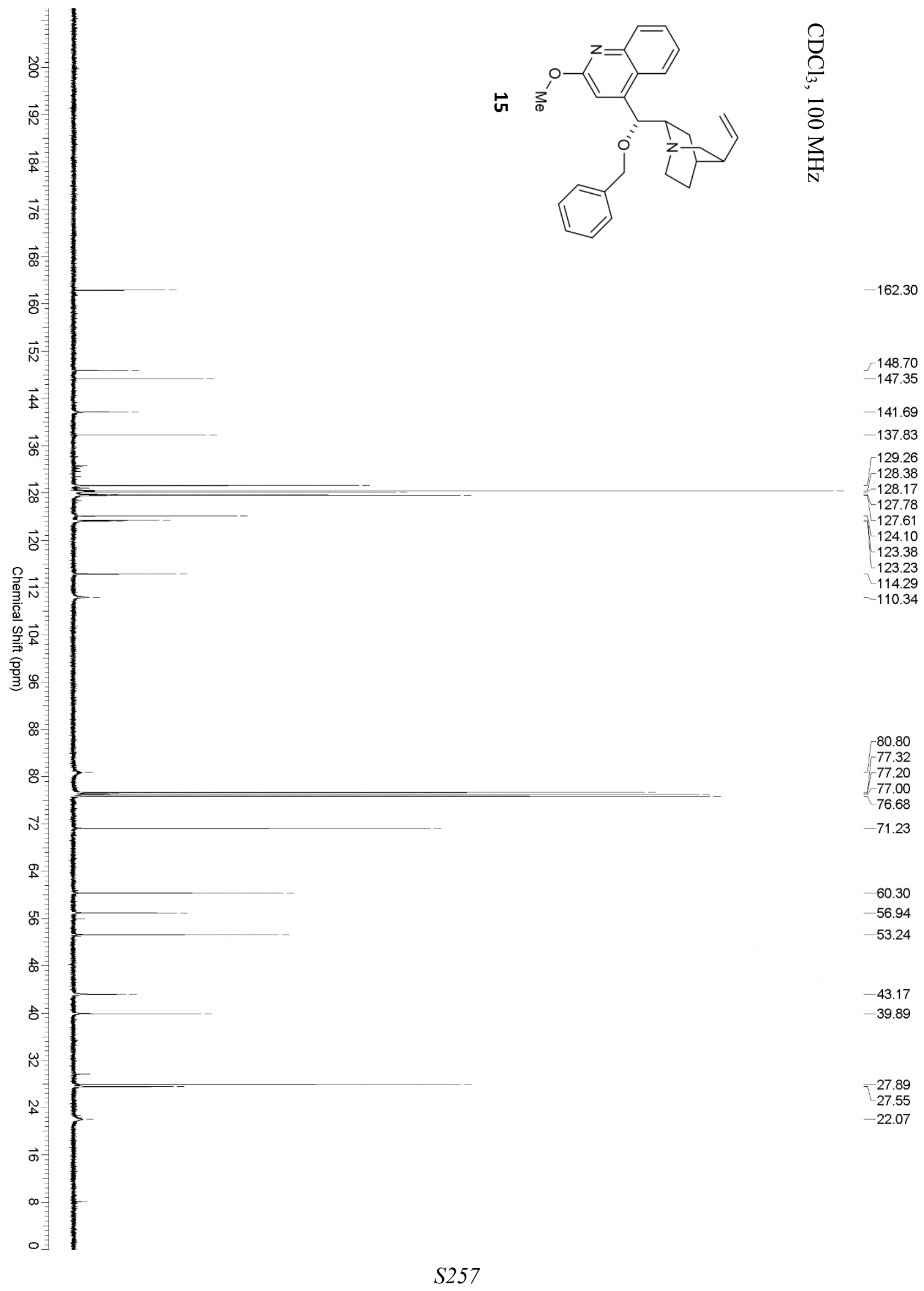




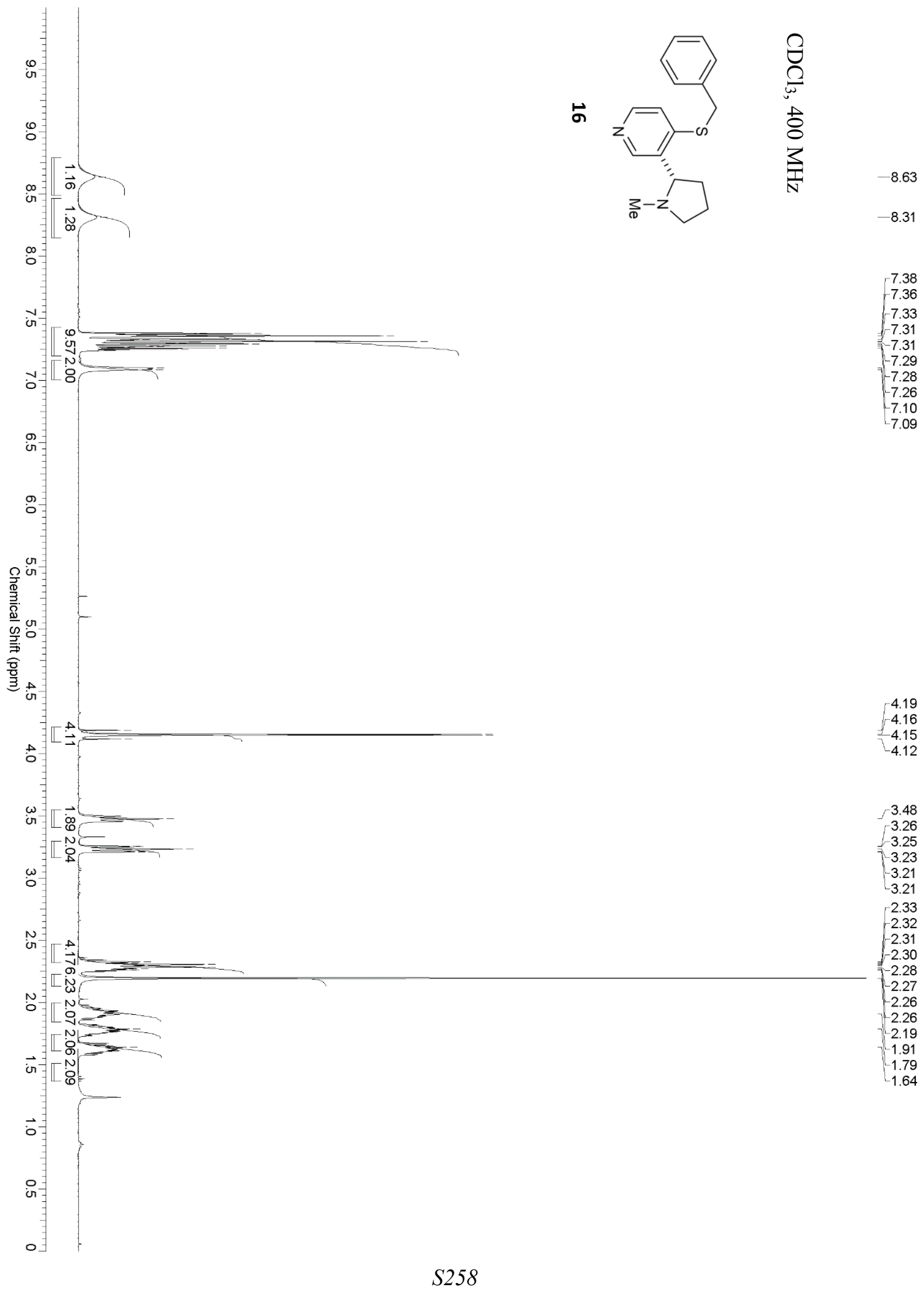




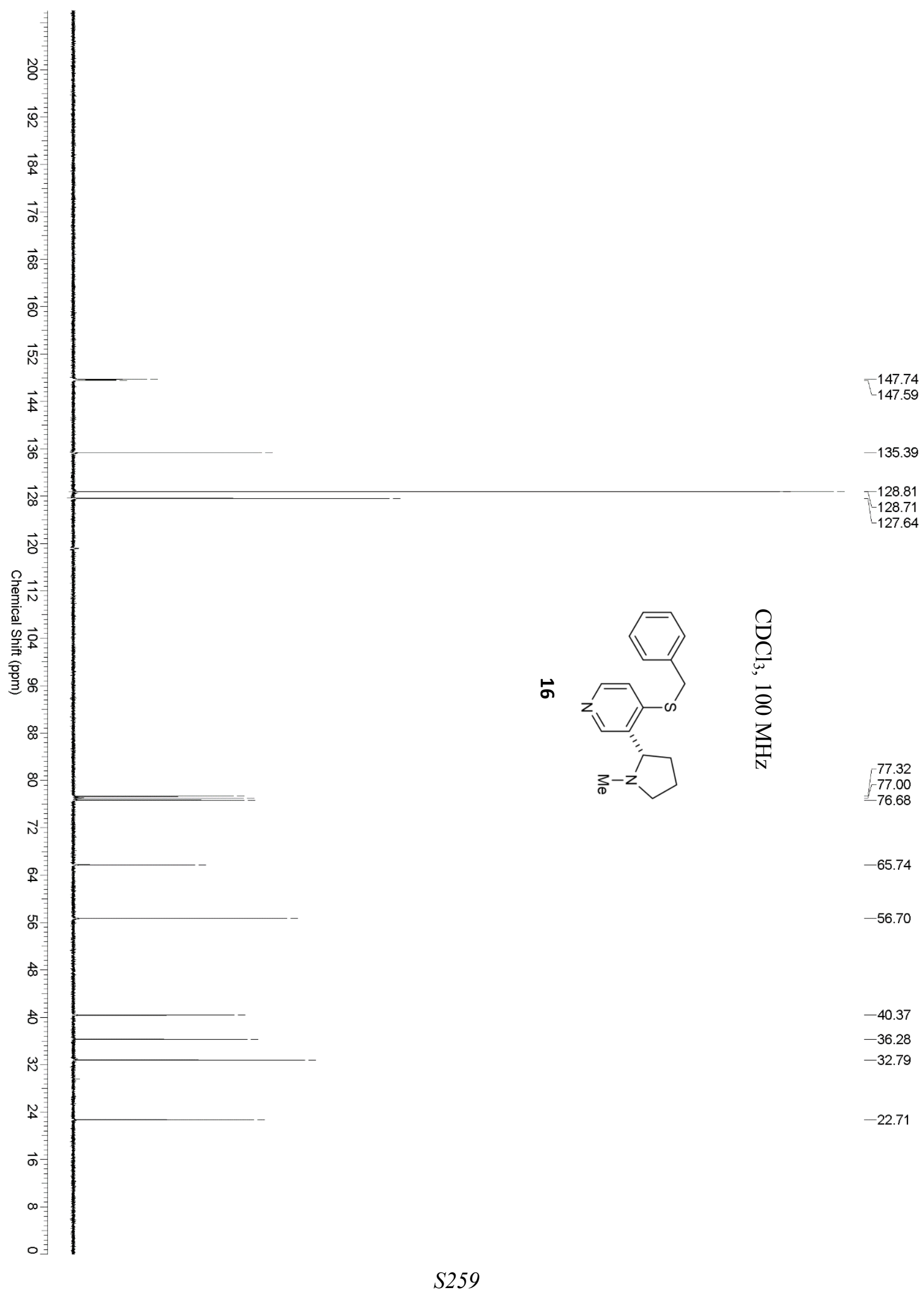




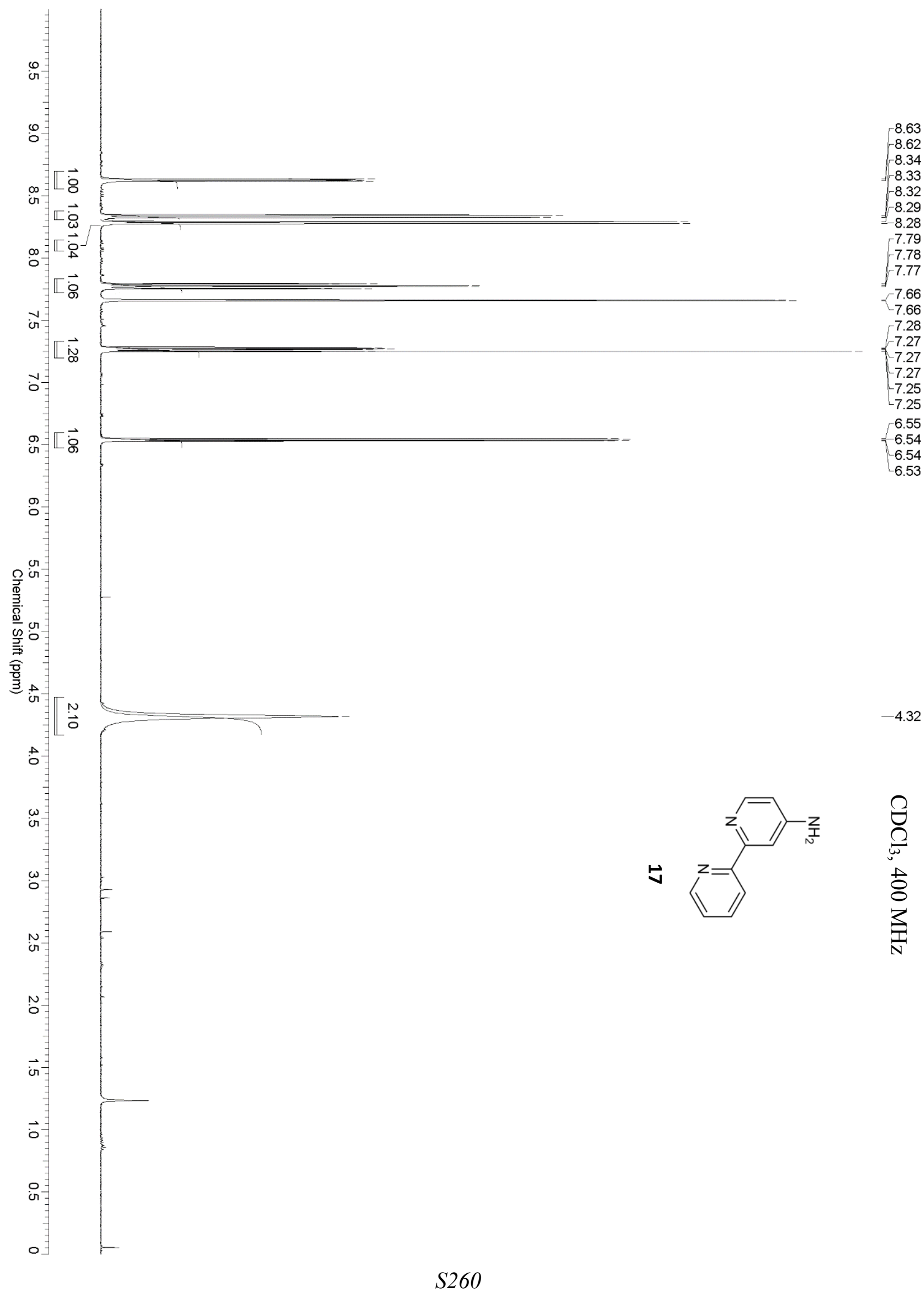




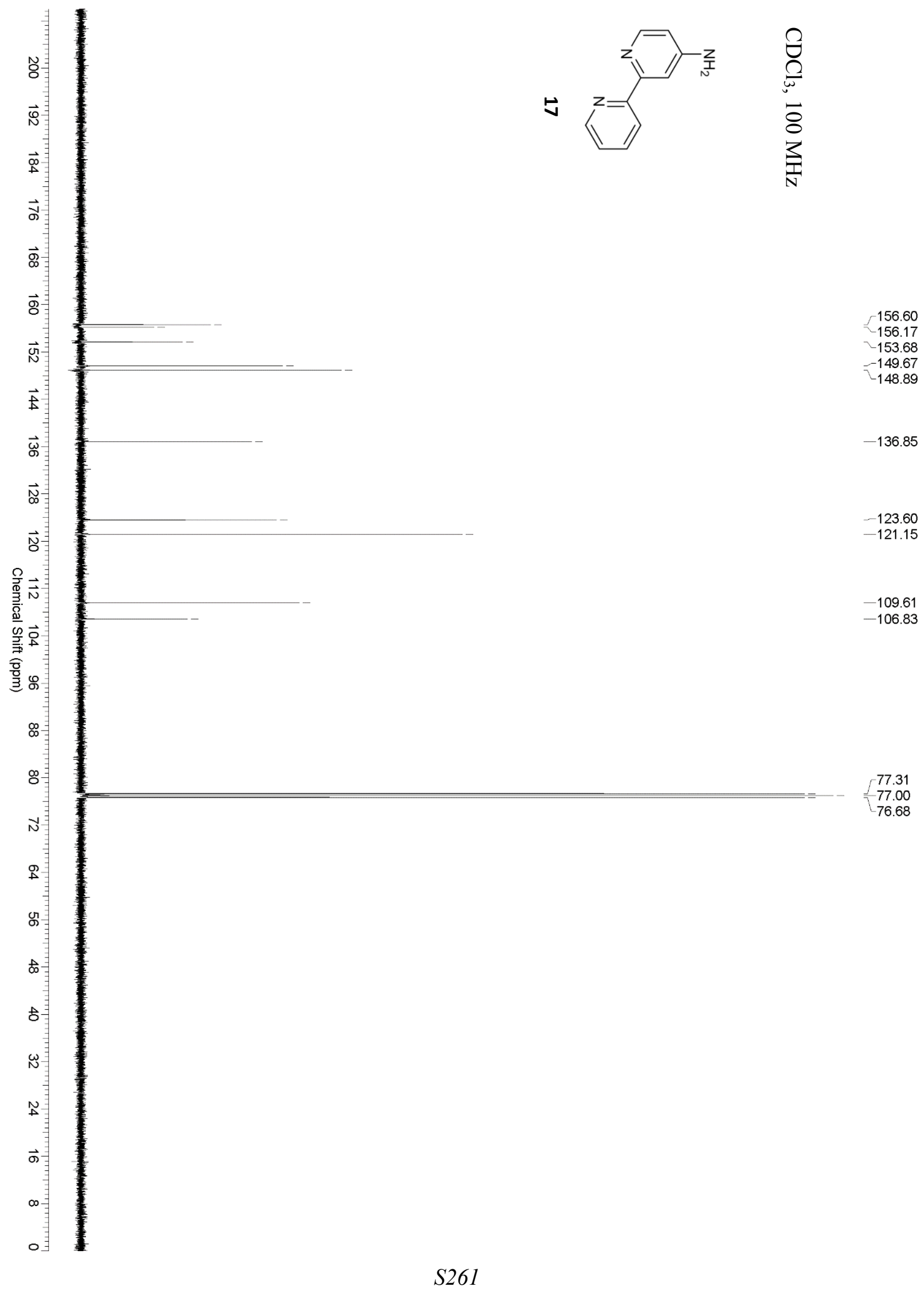




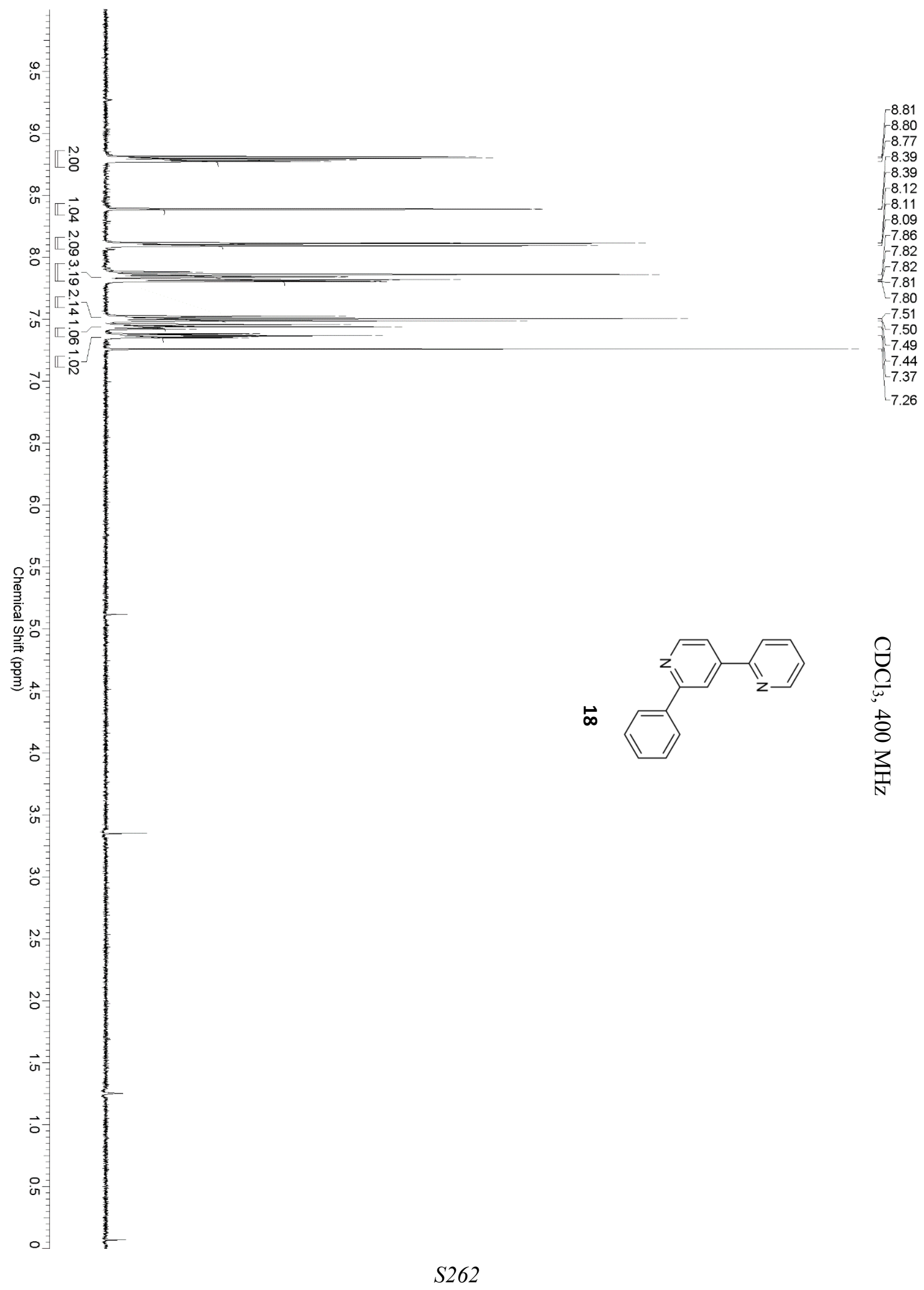




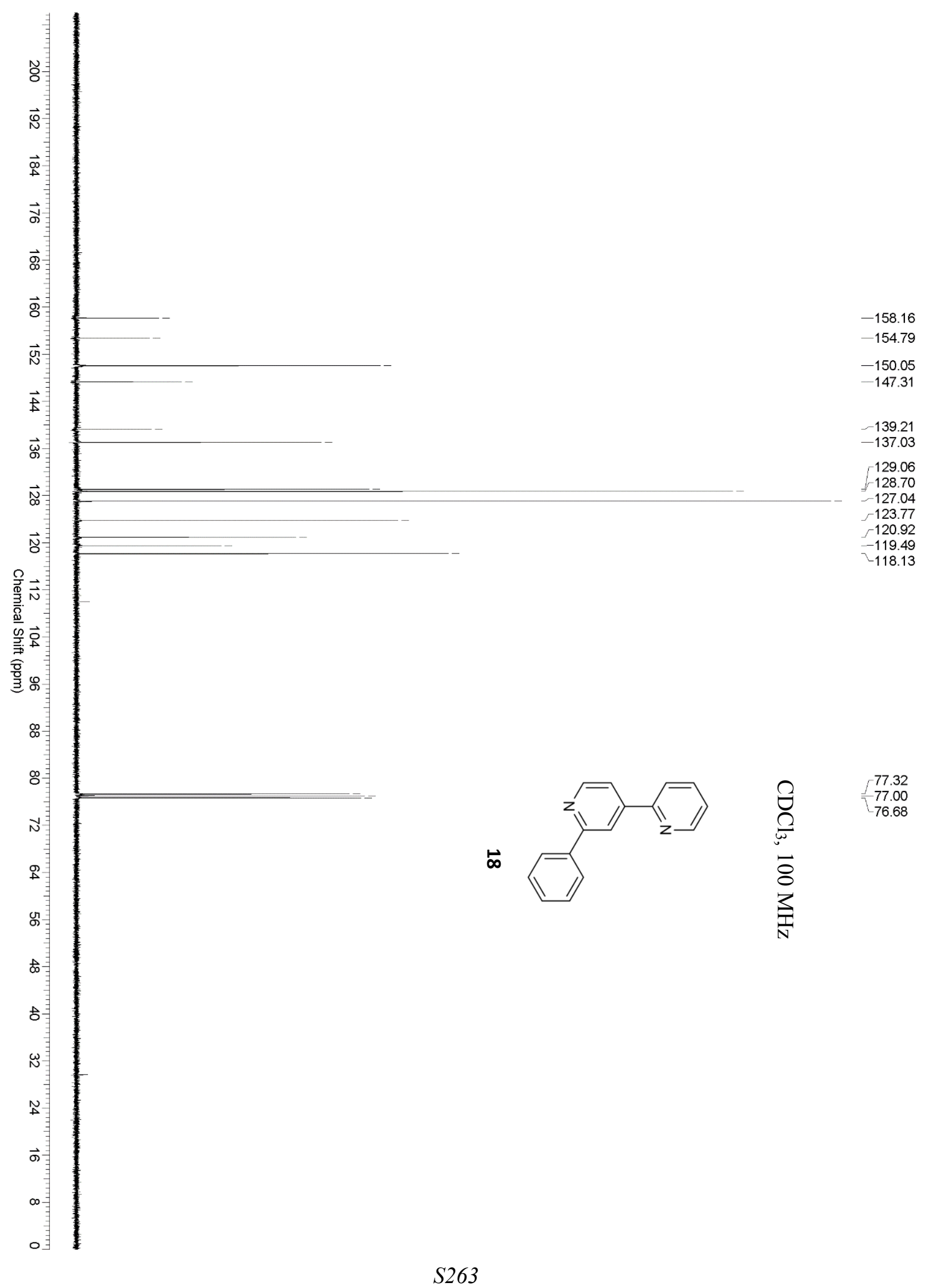




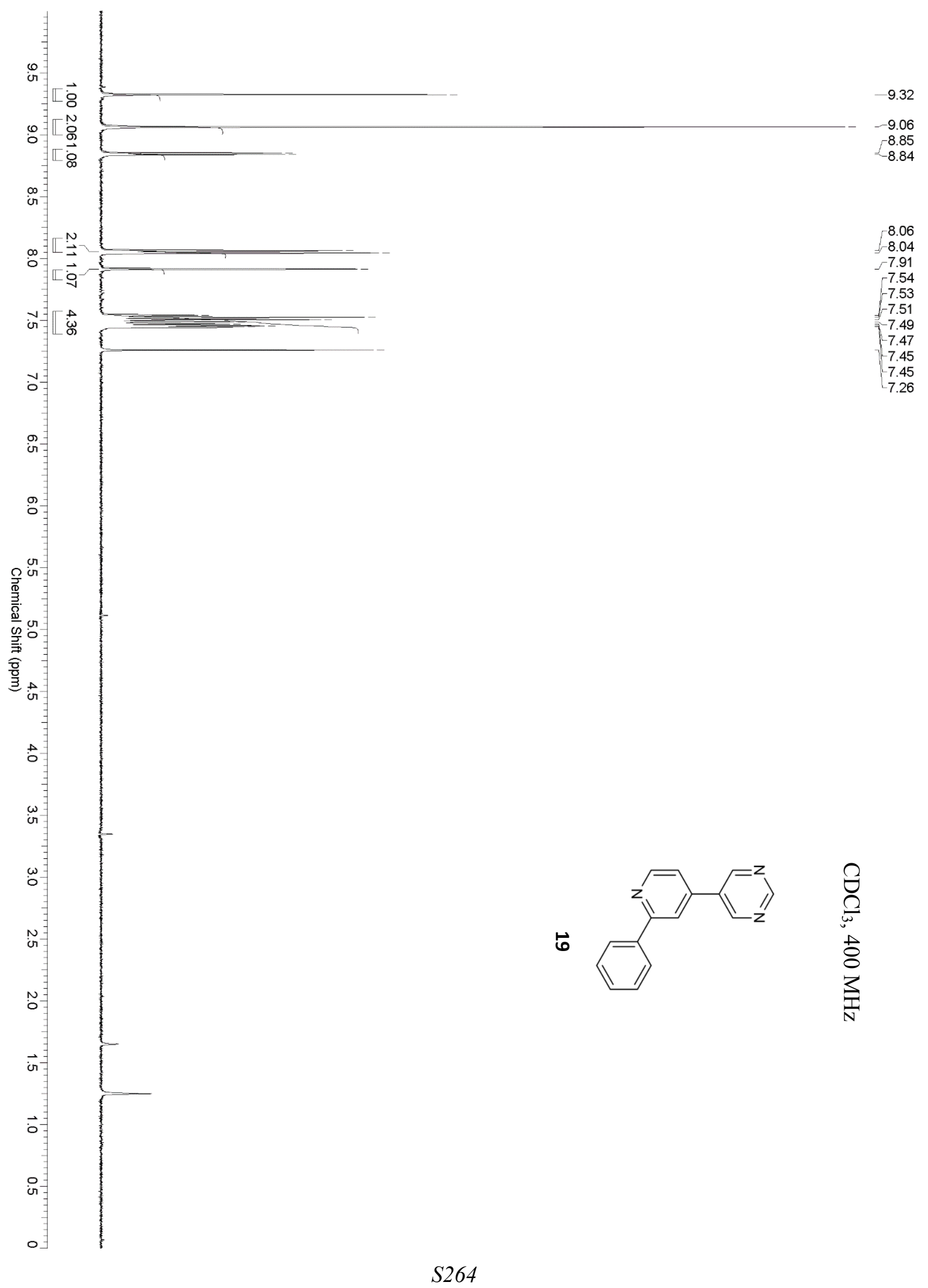




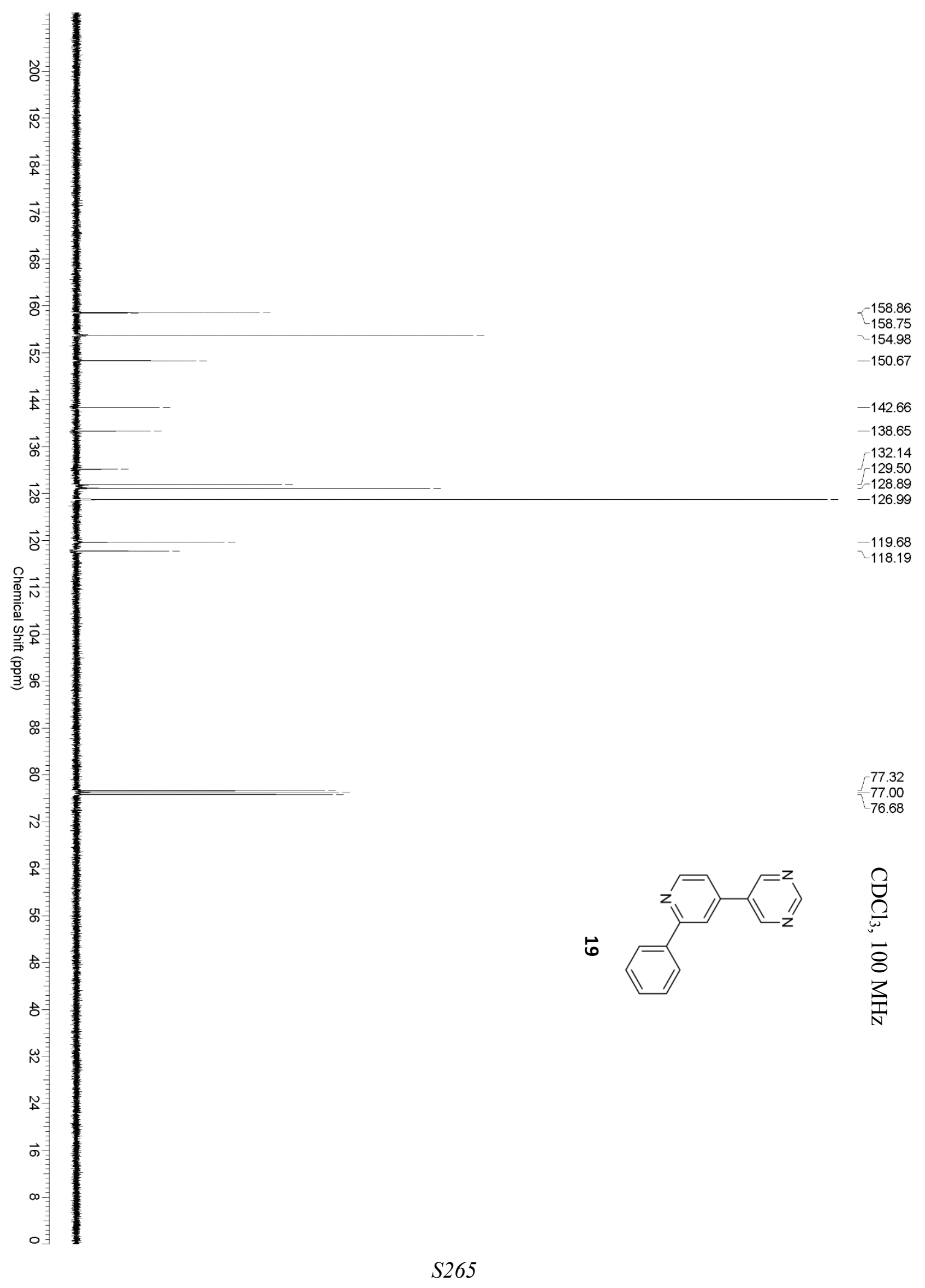




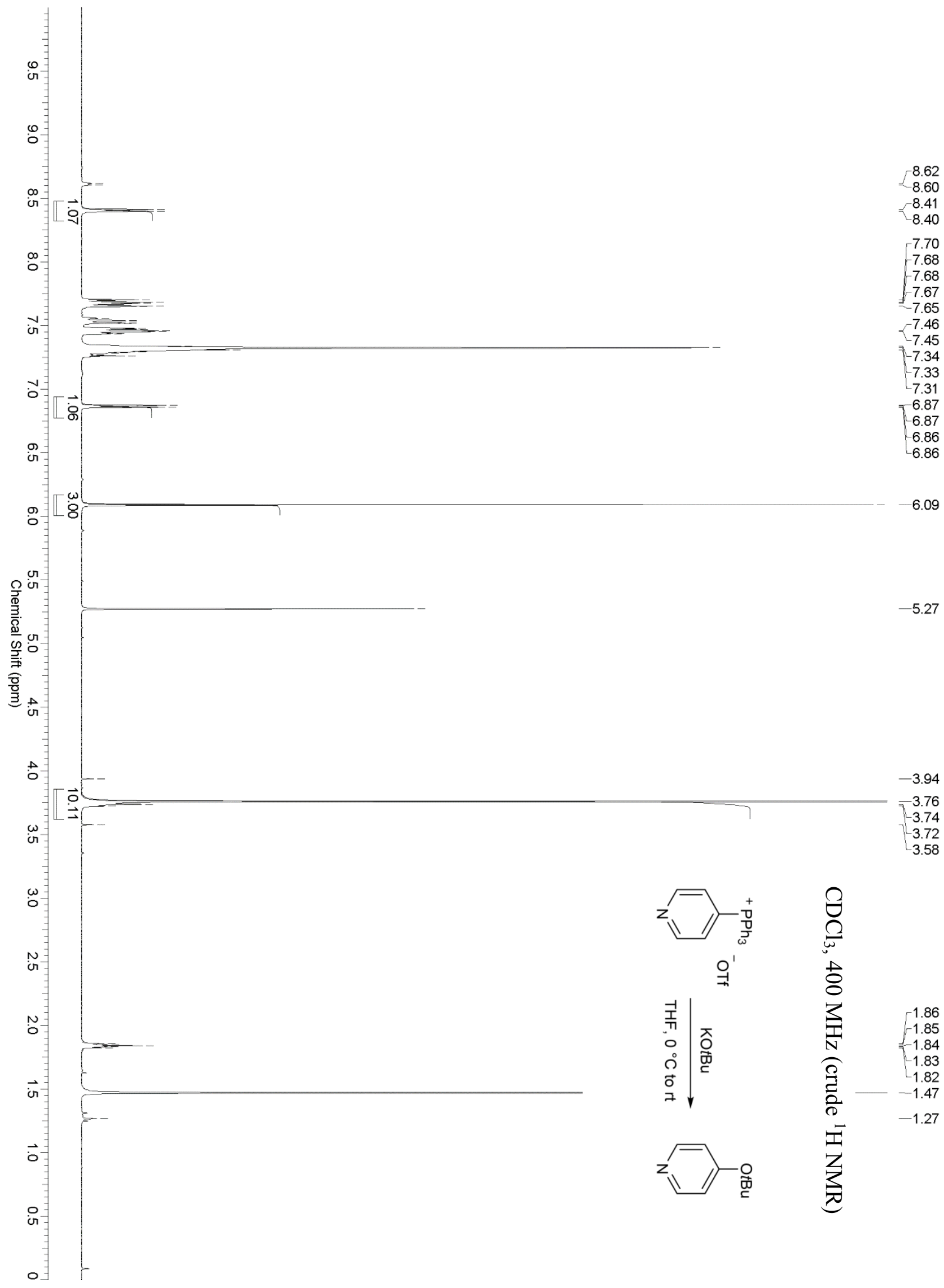




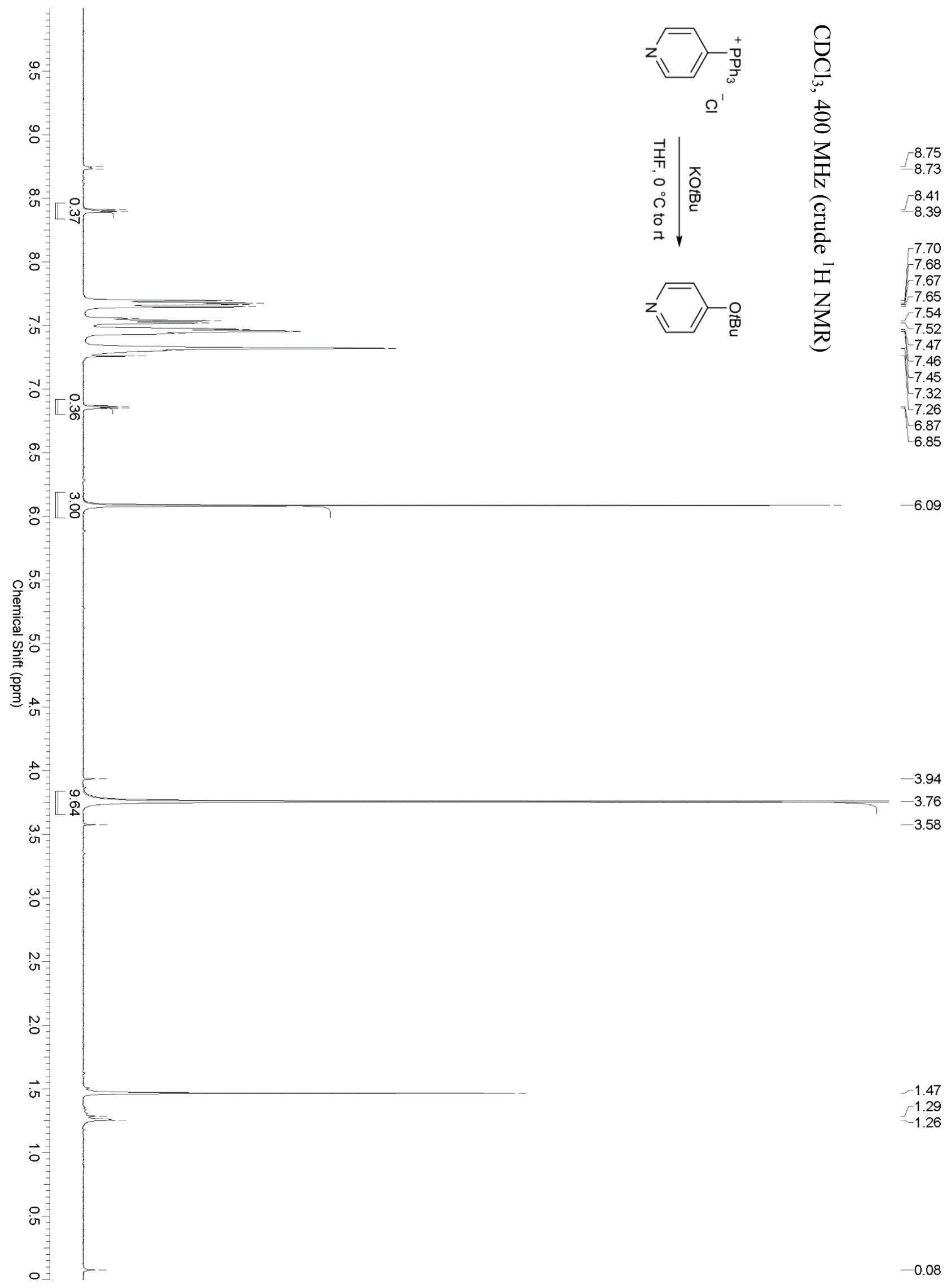




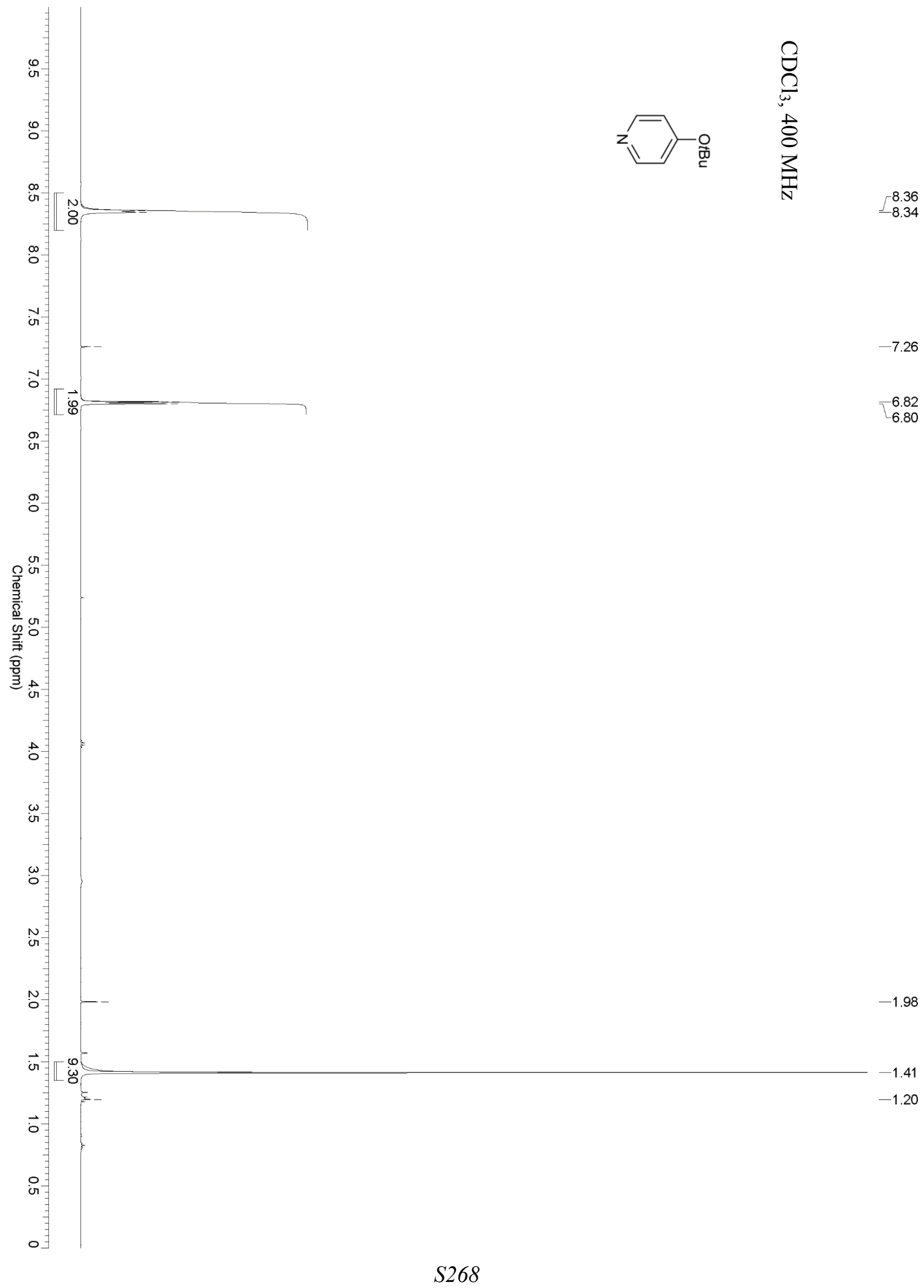




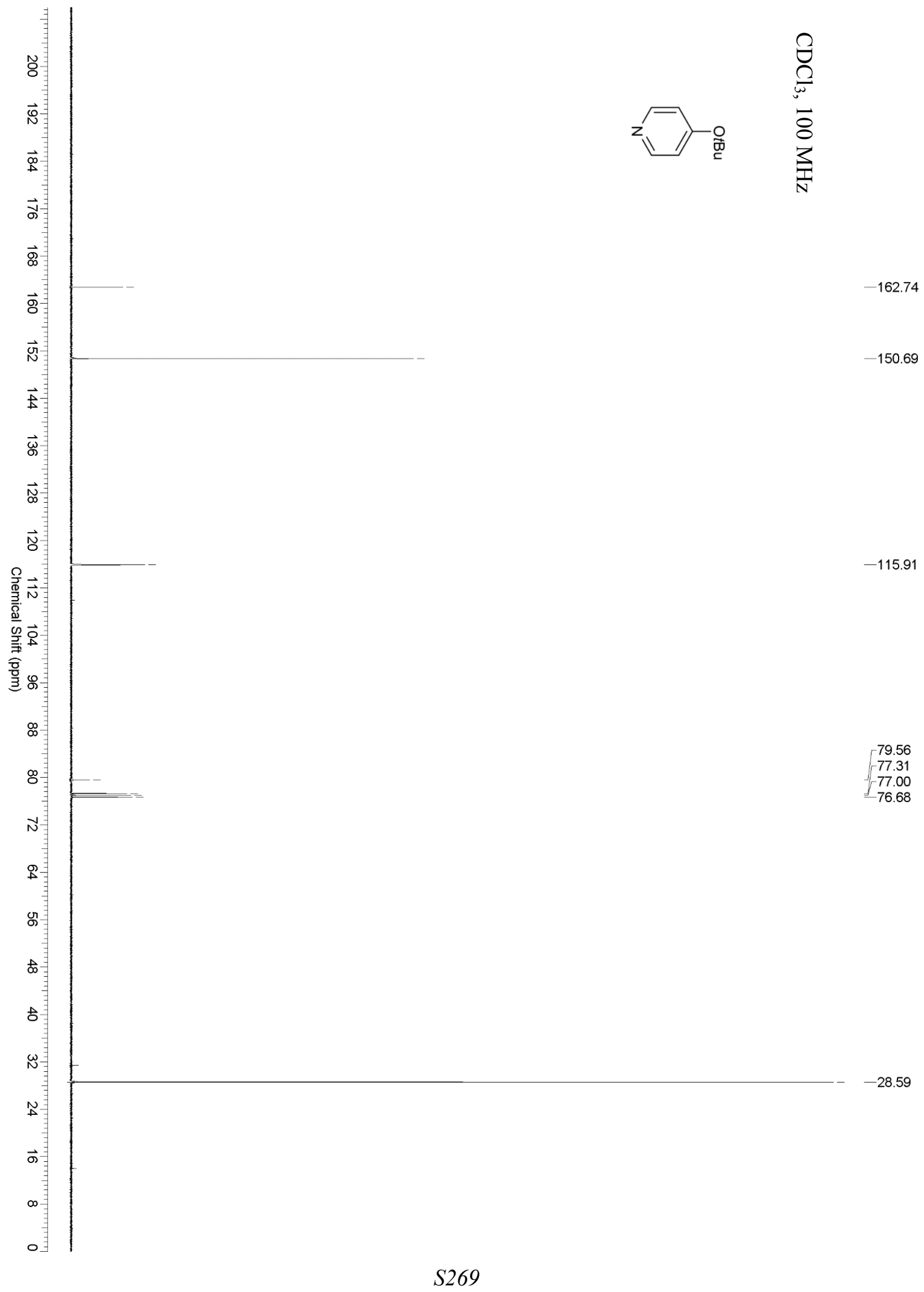

